Portland State University

PDXScholar

\title{
Molecular Coevolution of Pacific Northwest Hantaviruses and Their Host, The Deer Mouse, Peromyscus Maniculatus
}

Philip Darren Jones

Portland State University

Follow this and additional works at: https://pdxscholar.library.pdx.edu/open_access_etds

Part of the Biology Commons, Molecular Biology Commons, and the Virology Commons Let us know how access to this document benefits you.

\section{Recommended Citation}

Jones, Philip Darren, "Molecular Coevolution of Pacific Northwest Hantaviruses and Their Host, The Deer Mouse, Peromyscus Maniculatus" (2009). Dissertations and Theses. Paper 5369.

https://doi.org/10.15760/etd.7242

This Dissertation is brought to you for free and open access. It has been accepted for inclusion in Dissertations and Theses by an authorized administrator of PDXScholar. Please contact us if we can make this document more accessible: pdxscholar@pdx.edu. 


\title{
MOLECULAR COEVOLUTION OF PACIFIC NORTHWEST HANTAVIRUSES
}

\section{AND THEIR HOST, THE DEER MOUSE, PEROMYSCUS MANICULATUS}

by

\section{PHILIP DARREN JONES}

\author{
A dissertation submitted in partial fulfillment of the \\ requirements for the degree of
DOCTOR OF PHILOSOPHY
in
BIOLOGY

Portland State University

2009 


\section{DISSERTATION APPROVAL}

The abstract and dissertation of Philip Darren Jones for the Doctor of Philosophy in Biology were presented February 13, 2009, and accepted by the dissertation committee and the doctoral program.

COMMITTEE APPROVALS:

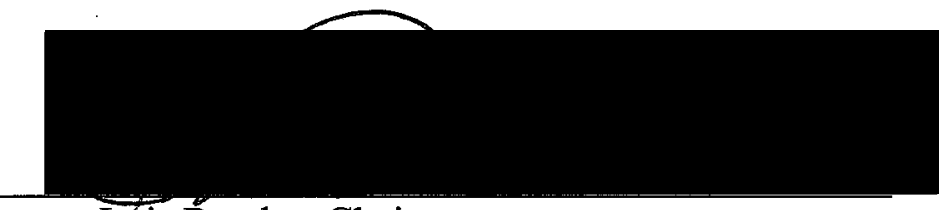

Luis Ruedas, Chair
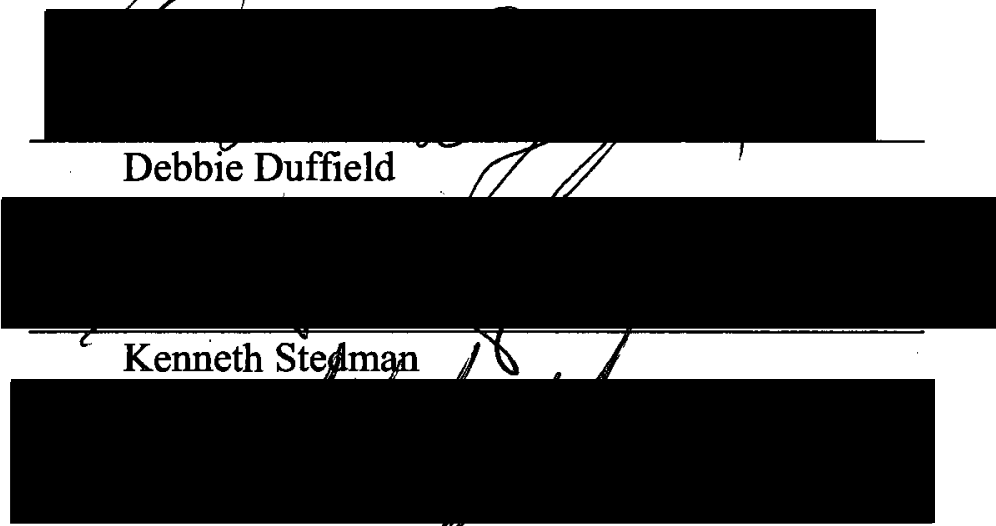

Dirk Iwata-Reuyl

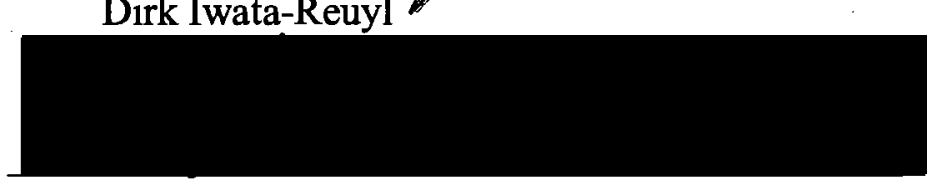

Niles Lehman

Representative of the Office of Graduate Studies

DOCTORAL PROGRAM APPROVAL:

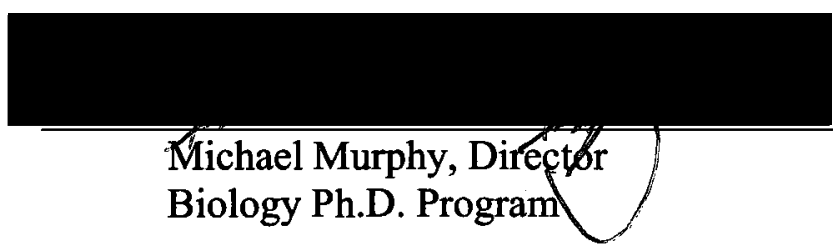




\begin{abstract}
An abstract of the dissertation of Philip Darren Jones for the Doctor of Philosophy in Biology presented February 13, 2009.
\end{abstract}

Title: Molecular Coevolution of Pacific Northwest Hantaviruses and their host, the Deer Mouse, Peromyscus maniculatus

Sin Nombre virus (SNV, family Bunyaviridae, genus Hantavirus), hosted by the deer mouse, Peromyscus maniculatus (family Cricetidae, Subfamily Neotominae), is the primary etiological agent of Hantavirus pulmonary syndrome (HPS) in the western United States. HPS, with known pathogenicity only to humans and for which there is no cure or prophylaxis, affects the epithelium of the lungs by making the capillaries leaky, thereby resulting in bilateral infiltrates, and eventually leading to respiratory failure and death by drowning in approximately $38 \%$ of hospitalized patients.

In the Americas, Peromyscus has been co-evolving with hantaviruses for approximately $12-20$ million years, since the first cricetids crossed Beringia, radiating and differentiating into the Neotominae and Sigmodontinae currently found in the New World. As it stands, the evolutionary relationships of deer mice remains unclear, consequently, so too is the associated viral phylogeny, with twelve named quasispecies in the genus Hantavirus presently characterized in North America, and twenty five quasispecies in the Western Hemisphere. Evidence of this opacity is seen in the new species that are regularly being discovered from among these host 
conspecifics suggesting that many uncharacterized hantaviruses remain to be described.

To improve our understanding of these co-evolutionary relationships, as well as the epidemiology of HPS, specimens of both host and virus were collected from throughout the Portland, Oregon region, as well as other locations across North America. Analyses of the ND3, ND4, ND4L, and arginine tRNA host mitochondrial genes yielded average genetic divergence estimates of 0.0413 among local (Portland, OR) deer mice relative to specimens from the type locality of the species, in Newfoundland. Phylogenetic analysis also revealed a deep division in the phylogeny of deer mice discriminating the distribution into two distinct clades on either side of the Mississippi River, and suggesting that western deer mice in fact are constituted by a formerly cryptic species distinct from those in Newfoundland and eastern North America. Sequencing and phylogenetic comparison of the Pacific Northwest hantaviral G1/G2 glycoprotein precursor encoding gene showed a large genetic distance $(\mathrm{p}=0.143)$ from Sin Nombre type specimens from New Mexico, suggesting that the Portland area virus minimally constitutes a new strain. 


\title{
Acknowledgments
}

Luis A. Ruedas, Ph.D. - Advisor

\author{
Advisory Committee: \\ Debbie Duffield, Ph.D. \\ Kenneth Stedman, Ph.D. \\ Dirk Iwata-Reuyl, Ph.D. \\ Niles Lehman, Ph.D.
}

Tissue Donations:

Al Lackey, Ph.D. - State University of New York, Oswego

Scott Burt, Ph.D. - Truman State University

Field:

Laboratory/Computational:

Laurie J. Dizney, Ph.D.

Zack Hyde

Lorelei E. Patrick, M.S.

Zachary T. Harlow

Barbara J. Shaw, Ph. D.

Larissa B. Garrett 


\section{Table of Contents}

Acknowledgements i

List of Tables

List of Figures a iv

Chapter 1: Introduction $\quad 1$

Chapter 2: Peromyscus in the Pacific Northwest 13

Chapter 3: Hantavirus in the Pacific Northwest 42

Chapter 4: Peromyscus and Hantavirus Co-evolution $\quad 65$

$\begin{array}{lll}\text { Epilogue: } \quad \text { Research Significance } & 70\end{array}$

$\begin{array}{ll}\text { Literature Cited } & 95\end{array}$

$\begin{array}{ll}\text { Appendix A: Peromyscus Distance Matrix } & 119\end{array}$

Appendix B: Hantavirus Distance Matrix $\quad 132$

Appendix C: Specimen Capture Data 140

Appendix D: Hantavirus Antibody Sero-positive Specimens 254

Appendix E: Rodent Specimen mtDNA Sequences 258

Appendix F: Hantavirus Specimen Sequences 319

Appendix G: Rodent Multiple Sequence Alignment 334

Appendix H: Hantavirus Multiple Sequence Alignment 408 


\section{List of Tables}

Table 1 Hantaviruses in the New World 93

Table 2 Hantavirus Antibody Sero-Positive Specimens

By Collection Site $\quad 94$

Table 3 Hantavirus Antibody Sero-Positive Specimens

By Season $\quad 94$ 


\section{List of Figures}

$\begin{array}{lll}\text { Figure } 1 \text { Worldwide Phylogeny of hantaviruses and Hosts } & 74\end{array}$

$\begin{array}{lll}\text { Figure } 2 \text { Hantavirus World Distribution Map } & 75\end{array}$

Figure $3 \quad$ Peromyscus maniculatus Range Map 76

Figure $4 \quad$ Pacific Northwest Peromyscus maniculatus

Subspecies Range Map $\quad 77$

$\begin{array}{lll}\text { Figure } 5 & \text { Specimen Collection Sites } & 78\end{array}$

$\begin{array}{lll}\text { Figure } 6 & \text { Small Mammal Trapping Web } & 79\end{array}$

$\begin{array}{lll}\text { Figure } 7 & \text { Peromyscus mtDNA Genome Map } & 80\end{array}$

$\begin{array}{lll}\text { Figure } 8 & \text { Peromyscus Pair-wise Distances } & 81\end{array}$

Figure 9 Peromyscus Pair-wise Distance Physical Map 82

$\begin{array}{lll}\text { Figure } 10 & \text { Peromyscus Distance Tree } & 83\end{array}$

Figure 11 Peromyscus Consensus Maximum Likelihood

Bootstrap Tree $\quad 84$

Figure 12 Peromyscus Distance Tree with Bootstrap Values 85

Figure 13 Peromyscus Bayesian Majority-Rule Consensus Tree 86

$\begin{array}{lll}\text { Figure } 14 & \text { Hantavirus Genome Map } & 87\end{array}$

Figure 15 Hantavirus Pair-wise Distances 88

Figure 16 Hantavirus Neighbor-Joining Distance Tree 89

Figure 17 Hantavirus Consensus M. L. Bootstrap Tree 90

Figure 18 Hantavirus Bayesian Majority-Rule Consensus Tree 91

Figure 19 Hantavirus / Host Coevolutionary Tree $\quad 92$ 


\section{Chapter 1: Introduction}

\section{Epizootiology-}

In the last few decades there has been what appears to be a significant increase in the emergence of pathogenic infectious diseases such as Sudden Acute Respiratory Syndrome (SARS; emerging in SE Asia in 2002), Ebola Virus (sub-Saharan Africa, 1976), Human Immunodeficiency Virus (HIV; sub-Saharan Africa, 1970's), Marburg Virus (Sub-Saharan Africa, 1970's), and tick-borne diseases such as Lyme disease (NE USA, 1990's)(Daszak et al., 2000). Among these recent discoveries are the hantaviruses, the causative agents of Hantavirus Pulmonary Syndrome (HPS; Western Hemisphere, 1993) and Hemorrhagic Fever with Renal Syndrome (HFRS; Eastern Hemisphere, 1950's). The appearance of these diseases are believed to be associated with a range of underlying causal factors including interactions with zoonotic pathogens, that would otherwise normally be contained within a host-parasite relationship, between wildlife and humans (Daszak et al., 2000). The term "emerging infectious disease" (EID) has been coined to describe the plethora of pathogens implicated in these recent discoveries, and is specifically used to define diseases that have recently increased in occurrence or geographic range, moved into new host populations, or only recently been discovered (Daszak et al., 2000).

In 1993 in the southwestern United States, an outbreak occurred of a previously unknown disease, now identified as Hantavirus Pulmonary Syndrome (HPS). The subsequent investigation led to the identification of the etiological agent-a novel hantavirus-Sin Nombre virus (SNV; Childs et al., 1994; Nichol et al., 1993). In the 
years since this discovery, the number of Hantavirus geno- and serotypes identified in the Americas has grown to at least 23 (Johnson et al., 1997; Morzunov et al., 1998; Pini et al., 2003; Rhodes et al., 2000; Sanchez et al., 2001; Schmaljohn et al., 1995; Yates et al., 2002), with this number on the increase as previously unknown hantaviruses are identified. In addition to Sin Nombre Virus some of the other most common hantaviruses known to cause HPS include; New York virus (NY), Bayou virus (BAY), Black Creek Canal virus (BCC), and Andes virus, with Sin Nombre virus being responsible for most of the 465 (Centers for Disease Control [CDC], 2007) known cases in the United States (Monroe et al., 1999; Peters et al., 1998; Schmaljohn et al., 1997). Recent surveys for Hantavirus antibodies have revealed Hantavirusinfected rodents throughout most of North America (Holmes et al., 2004; Monroe et al., 1999; Rhodes et al., 2000; Sanchez et al., 2001; Schmaljohn et al., 1995; Yates et al., 2002). Hantaviruses have been proposed to be transmitted through aggressive encounters - primarily between individuals of the same sex (usually males) —or by infectious aerosols generated from contaminated urine, feces, blood, or saliva (Schmaljohn et al., 1997; Olsson et al., 2002).

\section{Evolution-}

It has been hypothesized by many that for every type of Hantavirus there is a specific rodent host species whose members can potentially acquire a chronic, asymptomatic, lifelong infection from the virus (Kaufman et al., 1994; Morzunov et al., 1998; Netski et al., 1999). It has been shown through phylogenetic analyses that the topologies of the evolutionary relationships among hantaviruses were closely 
correlated with those of their known or suspected primary mammal reservoirs (Borucki et al., 2000; Monroe et al., 1999; Plyusnin and Morzunov 2000; Sironen et al., 2001; Yates et al., 2002).

In the Americas, the deer mouse has been co-evolving with hantaviruses for approximately $12-20$ million years, since the first cricetids crossed the Bering Land Bridge, radiating and differentiating into the Rodent subfamilies Neotominae and Sigmodontinae found in the New World today (Yates et al., 2002). While much is known about deer mouse phylogeny, it remains less than fully understood, and as a result of the documented host-parasite specificity among deer mice and their hantaviruses (Yates et al., 2002), the associated viral phylogeny may be unresolved as well. New species of Peromyscus are increasingly being revealed from among these former host conspecifics (Chirhart et al., 2001; Zheng et al., 2003), revealing the potential likelihood of many uncharacterized hantaviruses waiting to be described as well.

For many years it was believed that hantaviruses were maintained exclusively within Order Rodentia. Only very recently has that perception been challenged as genetically distinct hantaviruses have increasingly come to be found residing among members of the Order Soricomorpha (shrews) - family Soricidae (Xiao et al., 1994; Arai et al., 2007, 2008). In the former Soviet Union in the 1980's, antibodies to Hantavirus were found in members of families Soricidae and Talpidae (Gavrilovskaya et al., 1983; Tkachenko et al., 1983; Gligic et al., 1989). At that time molecular techniques were not yet available to delineate genetic identity of potentially hosted viruses to confirm whether these were truly distinct viral evolutionary lineages or 
merely cases of ecological spillover from a sympatric rodent host. As of yet, no human disease has been associated with shrew-borne hantaviruses (Arai et al. 2007).

Worldwide, most hantaviruses have been found in members of the rodent families Cricetidae (Subfamilies Neotominae and Arvicolinae) and Muridae (Subfamily Murinae), with genetically distinct viral lineages found associated with specific members of these taxa (Fig. 1). Hantaviruses are believed to have arisen at least 136 million years ago before the split of the Mammalian Orders Soricomorpha and Rodentia, evidenced by the presence of distinct hantaviral lineages found in both Orders (Carey et al., 1971; Plyusnin et al., 1996; Hughes and Friedman, 2000). Among the earliest described are those found in East Asia, including Hantaan virus from the Hantaan River valley, recognized during the Korean War, as well as Seoul virus, also found in Korea (Lee et al., 1978)(fig. 3). These East Asian viral species have been found to be reservoired within members of the family Muridae, Subfamily Murinae, as Apodemus agrarius (Hantaan), and Rattus norvegicus (Seoul; Lee et al., 1982). Another murine Hantavirus is the recently described Sangassou virus, shown to reside within the African wood mouse Hylomyscus simus, found in Sangassou, Guinea (Klempa et al., 2006). Reducing the clarity of this strict regional relationship, but upholding the host Subfamily paradigm, is the presence of Dobrava virus found in Northern Europe and hosted by the murine Apodemus flavicollis, a congener of the Hantaan virus host (Avsic-Zupanc et al., 1992; 2000). Phylogenetic analyses have supported placement of Dobrava virus in the murine-hosted viral clade (Avsic-Zupanc et al., 1992) (Fig. 1). European and North Asian serotypes such as Tula virus, Topografov virus, and Puumala virus, among others, have been found within 
arvicoline hosts such as Microtus and Clethrionomys species (Plyusnin et al., 1994; Vapalahti et al., 1999).

The more recently described members in Hantavirus are those found in the new world. In North and South America most of these infections are in species of the Subfamily Neotominae. Only a few Murid-hosted hantaviruses, consisting of Seoul virus in Rattus norvegicus, Subfamily Murinae, have been seen in the western hemisphere, primarily in urban environs (Monroe et al., 1999). Cricetid rodents found hosting hantaviruses are many (table 1). In North America most common among these are hosts in the genus Peromyscus (Monroe et al., 1999). The deer mouse, Peromyscus maniculatus, Subfamily Neotominae, has been identified as the reservoir for Sin Nombre virus (Childs et al., 1994). In-depth studies of Sin Nombre virus in North America have shown substantial genetic diversity and geographic structure of genetic variants within the viral quasispecies that may be the result of the genetic diversity in P. maniculatus (Monroe et al., 1999; Morzunov et al., 1996; Nichol et al., 1999; Plyusnin et al., 1996; Plyusnin et al., 2000; Salazar-Bravo et al., 2002). This rodent genetic diversity is paralleled by that of the virus (Dragoo et al., 2006). Peromyscus maniculatus as currently recognized have a vast range across the entire North American continent, excluding the southeastern U.S. (fig. 4). In addition, at least as many as 70 morphological subspecies are described in $P$. maniculatus (including sympatric subspecies), underscoring the variability in the species (Hall, 1981; Michaux et al., 2001).

As pointed out by Wilson and Ruff (1999), the basis of the Peromyscus taxonomic framework was built upon morphological analyses, long before the advent of 
molecular techniques. Recently, several former conspecifics, such as $P$. maniculatus keeni, $P$. maniculatus sitkensis; and congeners including $P$. oreas and $P$. sitkensis (now combined as P. keeni) have been removed from maniculatus and formally recognized as distinct taxa using chromosomal and molecular techniques (fig. 4) (Allard and Greenbaum, 1988; Avise et al., 1983; Calhoun and Greenbaum, 1991; Chirhart et al., 2005; Chirhart et al., 2001; Greenbaum and Baker, 1978; Gunn, 1988; Hogan et al., 1997; Hogan et al., 1993; Michaux et al., 2001; Smith et al., 2000; Zheng et al., 2003). Current literature has shown a wide range (2.5 - 16\%) of intrageneric genetic divergence, being the percent difference in pair-wise comparisons of sequences, within Peromyscus (Bradley and Baker, 2001). In point of fact, Bradley and Baker (2001) show Peromyscus boylii only $2.5 \%$ genetically divergent from $P$. simulus in a well accepted sister (non-conspecific) relationship. In addition, much of the current data in the literature strongly suggest that co-speciation between hantaviruses and their hosts characterize the long-term evolution of these genera (Morzunov et al., 1998; Yates et al., 2002; Dragoo et al., 2006).

While, as stated above, much analyses of $P$. maniculatus subspecies distributions have been morphologically based, analyses of hantaviruses have in contrast been undertaken using molecular techniques (Hjelle et al., 1995a; Hjelle et al., 1995b; Hjelle et al., 1994; Johnson et al., 1997; Li et al.,1995; Nichol et al., 1993; Nichol et al., 1999; Plyusnin and Morzunov, 2000; Dragoo et al., 2006). 


\section{Virology-}

Members of the genus Hantavirus of the family Bunyaviridae (5 genera, 250 species), are spherical, 100 nanometer diameter particles enclosed within a phospholipid bilayer envelope (Elliot et al., 1991; Nichol et al., 2005). Expressed within and extending above this envelope are two glycoproteins (G1 and G2) that facilitate cell entry via a clathrin coated pit (Elliot et al., 1991; Nichol et al., 2005). Within the envelope is a protein nucleocapsid that houses a segmented single-stranded negative-sense RNA genome $\sim 12,100$ nucleotides (nt) in length. These segments are small (S), medium (M), and large (L) (Plyusnin et al., 1996). The S segment, approximately 2,000nt in length, encodes the genes for the nucleocapsid proteins that encase the RNA genome in daughter viral particles. The medium, or M segment $(3,700 \mathrm{nt})$, encodes the envelope glycoprotein precursor that is then co-translationally cleaved to yield the G1 and G2 glycoproteins (Elliot, 1990; Vapalahti et al., 1992; Bohlman et al., 2002). The L segment (6,500nt) encodes the RNA-specific polymerase that is used to replicate viral progeny genomes (Elliott et al., 1991; Chizhikov et al., 1995).

\section{Purview of this study-}

The genomes, or subsets thereof, of many different hantaviruses have been sequenced to varying extents (Schmaljohn et al., 1990; Elliot et al., 1991; Monroe et al., 1999; Morzunov et al., 1996; Nichol et al., 1999; Plyusnin et al., 1996, 2000; Salazar-Bravo et al., 2002; Yates et al., 2002). The majority of studies have chosen to examine the M segment (at the very least) as it is responsible for the glycoproteins used for cell 
recognition, and thereby potentially the most variable since it is likely under the greatest amount of evolutionary pressure from the corresponding host. The L segment, coding for the RNA polymerase, is believed to be the most conserved of the Hantavirus genes (Schmaljohn, 1990; Elliott et al., 1991; Chizhikov et al., 1995). Many studies, especially those of hantaviruses with the greatest geographical proximity (and therefore a priori hypothesized to be the most genetically similar) to those found in the Pacific Northwest have used a fragment of the G1 and G2 encoding region of viral $\mathrm{M}$ segments, amplified by reverse transcriptase polymerase chain reaction (RT-PCR) (Avsic-Zupanc et al., 2000; Hjelle et al., 1995a; Hjelle et al., 1995b; Hjelle et al., 1994; Johnson et al., 1997; Li et al., 1995; Nichol et al., 1993; Nichol, 1999; Plyusnin and Morzunov, 2000). Accordingly, to ensure that the viral analyses contained herein might be comparable across even the most potentially closely related species, similar $\mathrm{M}$ segment amplicons were pursued in this research. The PCR products were then sequenced and compared for genetic divergence as a direct indicator of viral speciation and for phylogenetic comparisons.

Mitochondrial DNA (mtDNA) is often the genetic material of choice for small mammal phylogenetic analyses (Avise et al., 1983; Bradley et al., 2001). Again, in order to enable meaningful comparison of Pacific Northwest specimens of mice, genus Peromyscus, to the bulk of data previously published, mtDNA was chosen for the analyses presented herein (Avise et al., 1983; Allard et al., 1988; Hogan et al., 1993, 1997; Bradley et al., 2001; Chirhart et al., 2001; Michaux et al., 2001; Zheng et al., 2003). 
To date, characterized hantaviruses geographically most proximal to the Pacific Northwest have been found in Peromyscus; indeed, all viruses associated with rodents in the genus Peromyscus form a well-supported monophyletic clade (Botten et al., 2000; Monroe et al., 1999; Plyusnin and Morzunov, 2000; Weidmann et al., 2005; Yates et al., 2002). This viral monophyly likely reflects adaptation of the virus to the rodent host and not just speciation due to geographic isolation of the virus variants (Botten et al., 2000; Monroe et al., 1999; Plyusnin and Morzunov, 2000; Weidmann et al., 2005; Yates et al., 2002). For instance, P. maniculatus and P. leucopus are sympatric in many regions and share microhabitat, but virus mixing between species is not seen (Monroe et al., 1999). While extremely rare, cases of host switching (to hosts with no current hantavirus population), as well as spillover into neighboring species, do occur (Klingstrom et al., 2002; Nemirov et al., 2002; Vapalahti et al., 1999; Weidmann et al., 2005). Host switching is defined the result of formerly host-specific viruses having experienced genetic changes that allow the virus to be subsequently maintained in a different species population (Klingstrom et al., 2002; Weidmann et al., 2005). Spillover is the result of high population densities of the host species as well as another sympatric species leading, through increased encounters, to only a temporary presence (un-maintained) of the virus within that sympatric population, without any genetic change being seen in the virus (Nemirov et al., 2002; Vapalahti et al., 1999). A unique rodent host species would therefore be likely to have a novel Hantavirus as well. North American hantaviruses discovered among different rodent reservoir species have been found to range from one another in genetic percent divergence from as little as $5 \%$ to as much as $30 \%$ (Vincent et al., 2000). 
With so little known about Hantavirus distributions, it is important to understand the health risks faced by humans due to that lack of knowledge. Human mortality rates for HPS range from approximately $40 \%$ to $60 \%$ depending upon the strain of the virus encountered and the health and age of the patient (Peters, 1998; Schmaljohn and Hjelle, 1997). The current mortality rate among patients hospitalized and diagnosed with HPS in the U.S. is $38 \%$ but increases to $50 \%$ in Oregon. Three new cases occurred in Oregon during the span of this study with one of these three resulting in a fatality, underscoring the need for a clearer understanding of hantaviral species distributions.

No systematic studies of evolutionary relationships of deer mice have been undertaken in the Portland area or even Oregon in its entirety. Distribution maps show the Cascade Mountains forming a boundary between two subspecies of deer mice (fig. 4), with Peromyscus maniculatus rubidus on the West side, and Peromyscus maniculatus gambelii on the East (Michaux et al., 2001; Wilson and Ruff, 1999; Hall, 1981), but with the upper habitation zone limits of both subspecies surpassing the passes within the Cascade Mountains, it is therefore likely that they constitute an incomplete barrier to migration, and it is suspected that some overlapping of populations may have occurred (Hall, 1981; Verts and Carraway, 1998; Wilson and Ruff, 1999). No molecular phylogenetic studies of Portland, OR region Peromyscus have previously been undertaken although, as previously mentioned, current phylogenies are based solely upon morphology, leading to the specific and subspecific definitions described. 
The objective of this research was to elucidate, using molecular analyses, whether the Peromyscus species found in the Portland area had been misidentified as well as to provide an initial characterization of the evolutionary relationships of the as yet uncharacterized regional Hantavirus. Hypothesized as:

$\mathrm{H}_{0-1}: \quad$ Deer mice in the Portland, Oregon region are correctly classified as Peromyscus maniculatus rubidus.

Or, stated as a working hypothesis...

$\mathrm{H}_{1}$ : Based on molecular evidence, previously characterized Peromyscus maniculatus rubidus in the Portland, Oregon region, while a congener of $P$. maniculatus, is not conspecific.

$\mathrm{H}_{0-2}$ : The Hantavirus reservoired by deer mice in the Portland, Oregon region are in fact Sin Nombre virus.

Again, in working format...

$\mathrm{H}_{2}$ : The Hantavirus carried by the taxon currently recognized as Peromyscus maniculatus rubidus, constitutes a previously unrecognized species or strain of Hantavirus. 
Hall (1981) suggested that the partially sympatric subspecies $P$. $m$. rubidus and $P$. m. gambelii may in fact not be interbreeding, although there have been no studies to confirm or deny this hypothesis (Hall, 1981). If this truly is the case, then according to the biological species concept (Mayr, 1982) some of these populations may have differentiated into recognizable distinct species.

In light of the foregoing research described above showing the gaps in our understanding of the biogeography of Peromyscus, as well as their hantaviruses, and in conjunction with the additional resolution to be provided by molecular analyses over morphological analyses alone (Hoelzel and Green, 1992), the reasoning for this research is evident. This study has attempted to yield a sharper image of the speciation patterns in the Peromyscus maniculatus species group, as well as the hantaviruses putatively coevolving with them, with a focus on the region in and around Portland, Oregon, and the greater Pacific Northwest. 


\section{Chapter 2: Peromyscus in the Pacific Northwest}

\section{Abstract-}

The neotomine rodent Peromyscus maniculatus (deer mouse) is distributed and found throughout most of North America (fig. 3). With more than 70 subspecies described on a morphological basis (Hall, 1981; Osgood, 1909), evolutionary relationships among Peromyscus maniculatus subspecies, as well as those among the entire genus Peromyscus, remain complex and unclear. The research contained herein attempted to clarify some of these relationships, specifically the association of deer mice from the Pacific Northwest with their closest relatives, as well as their status within the larger generic taxonomic framework. To this end, phylogenetic analyses were performed on the mitochondrial DNA sequences of the ND3, ND4, ND4L, and Arginine tRNA from 84 individuals of Peromyscus representing the three putatively distinct subspecies from the Pacific Northwest. Specimens from beyond the Pacific Northwest region included subspecies from throughout the entire distribution of deer mice, including topotypical Peromyscus maniculatus maniculatus from Newfoundland. These sequences were compared to other congeners, including: Peromyscus keeni, P. sejugis, and P. leucopus, using Maximum Likelihood analysis with bootstrap support, genetic distance, and Bayesian analyses.

These molecular approaches to the problematic phylogenetic relationships among deer mice subspecies agreed in finding a taxonomic division in the molecular phylogeny of deer mice, separating $P$. maniculatus into two distinct clades. This division is equal in percent divergence to the divergence of $P$. maniculatus from $P$. keeni and $P$. sejugis. The division in the distribution of $P$. maniculatus occurs along 
the Mississippi River and continues from its source in central Minnesota (after a 97 $\mathrm{km}$ break in the barrier) north along the Red River to Lake Winnipeg, then along the Nelson River to Hudson Bay essentially dividing $P$. maniculatus into an eastern and western clade along this barrier. The western clade may represent a separateformerly cryptic - species, and is proposed in this investigation to be recognized as Peromyscus sonoriensis.

\section{Introduction-}

Description-

Peromyscus maniculatus, Order Rodentia, Family Cricetidae, Subfamily Neotominae, commonly known as the deer mouse, is a small, ubiquitous rodent with a range extending from the northeastern coast of North America across the continent to the west coast, north through most of Canada, and south into northern Mexico, but excluding most of the southeastern U.S. (fig. 3).

Peromyscus maniculatus varies in color from grayish to reddish-brown to black dorsally with a white venter, legs, and feet. The variation primarily depends on the habitat in which the population is found: color will generally tend toward the predominant local substrate. The tail is distinctly bi-colored, with dark above and white below (Huestis, 1931). Ears are large and semi-erect, and eyes are black and beady.

Adult Peromyscus maniculatus range from 10-30 grams with newborns at roughly 1.5-2 grams, and juveniles in the intervening range. Typically, adult deer mice will measure 106-222 $\mathrm{mm}$ in total length with tails from 46 to $123 \mathrm{~mm}$, hind feet 14-25 
$\mathrm{mm}$, and ears $12-21 \mathrm{~mm}$ (Hall, 1981). These wide ranges are seen as a result of the vast geographic range of the species (Hall, 1981). Local subspecies and populations exist within much smaller size ranges (Hall, 1981). A great deal of the variation seen throughout the deer mouse's range may also be seen on a smaller geographic scale in Oregon as a result of the highly disparate climates and habitats found on opposing sides of the Cascade mountains (Verts and Carraway, 1998; Woodley, 2007).

\section{Habitat and Distribution-}

Due to the vast range described above, $P$. maniculatus may be found in habitats as varied as agricultural fields, grassland, brushland, sub-alpine zones, northern boreal forest, desert, southern montane woodland, and dry subtropical climes. The species is most commonly found in prairies, bushy areas, and woodlands (King, 1968). In addition to these natural habitats, $P$. maniculatus is often seen in and around domestic dwellings.

The species has been divided into two subgroups: the grassland or prairie form in the Midwest and west, and the woodland form in the east and northeast (LTER, 1998). The woodland form of the east has a longer tail, ears, and hind foot than the more western prairie form. These differences have spawned controversy over whether or not they constitute distinct species.

Within the species' range as many as 70 subspecies of deer mice have been described (Hall, 1981; Michaux et al., 2001) with some of these being sympatric and not considered to be interbreeding (Hall, 1981). To be considered a single species, some level of gene flow must occur among individuals throughout the range (Mayr, 
1982). However, being a small organism with some relatively locally concentrated populations, it is clear that an individual $P$. maniculatus is incapable of migrating across the whole range. There also exist physical barriers within the range, such as the Rocky Mountains, Cascade Range, and Mississippi River, all of which serve to impede gene flow, thus isolating subspecies into allopatric populations. If these subspecies have not already diverged into separate species, they are therefore likely on that path.

Scope-

In consideration of the incomplete clarity of the phylogenetic relationships among members of the genus Peromyscus, this work was intended to use molecular data to test the monophyly of Peromyscus maniculatus, in particular, the subspecies from the Pacific Northwest currently assigned to $P$. maniculatus. While natural selection works upon phenotypes and not genotypes, the contents of genomes dictate those phenotypes. It is likely that molecular data will provide a more representative picture of evolutionary history than morphology because a portion phenotypic variation seen in/between species is the result of differential gene expression, not divergent genetic content.

\section{Research Design and Methods-}

Field Protocols-

Deer mice, Peromyscus, were collected from five sites in and around the Portland, Oregon metropolitan area from October 2002 through September 2004. Collection 
sites were chosen based on their relative size to contain the trapping web. To ensure that samples were taken from locations where humans would be most likely to encounter infected mice, public parks were selected for specimen collection (fig. 5) (Kuenzi et al., 2001). Sites chosen were: Forest Park, Multnomah Co., Tryon Creek State Park, Multnomah Co., Powell Butte Nature Park, Multnomah Co., Oxbow Regional Park, Multnomah Co., and Tualatin River National Wildlife Refuge (TRNWR), Washington Co.

The Forest Park site (5046892.5 N, 484267.53 E, UTM Zone 10, WGS-84), at a relatively flat 184 meters elevation near the top of the West Hills, on the northwest side of downtown Portland, consisted of a fairly dense second-growth mix of Douglasfir (Pseudotsuga menziesii), western hemlock (Tsuga heterophylla), western red cedar (Thuja plicata), Oregon big-leaf maple (Acer macrophyllum), and red alder (Alnus rubra). Sword fern (Polystichum munitum), vine maple (Acer cirinatum), Oregon grape (Berberis aquifolium), and English ivy (Hedera helix) dominated the understory. Several trails crossed the study site, and were used daily by hikers, bikers, and dogs. While currently in a relatively natural state, it is known that this site has been logged in the past and replanted, in an attempt to restore a natural habitat. Other than the few hiking trails running through the area, it appears to have been left to develop along a natural course for many decades. The Tryon Creek State Park site (5031184.0 N, 474109.6 E, UTM Zone 10, WGS-84), at $105 \mathrm{~m}$, on the south side of Portland contained essentially the same species composition as Forest Park, but with much greater proportion of ivy, a very undulating terrain, and two small creeks. The Tryon Creek research site was in an area of the park that did not have established trails 
to or within it. Powell Butte Nature Park, is an extinct volcanic cinder cone on the east side of Portland, and the most anthropogenically altered of the sites with a relatively open canopy made up of big leaf maples and alders, with stinging nettles (Urtica dioica) and Himalayan blackberry (Rubus discolor) in the understory. This site, on the south shoulder of the summit at 135 meters (5036804.5 N, $461386.22 \mathrm{E}$, UTM Zone 10, WGS-84), had been logged thoroughly in the past and was flanked on the north by a large, maintained grass clearing. The Tualatin River National Wildlife Refuge (TRNWR), on the banks of the Tualatin River, was dominated by English Hawthorn (Crataegus laevigata) and Himalayan blackberry; it was mostly level, with dense vegetation, and wetlands encroaching into the site $(34 \mathrm{~m})(5026923.0 \mathrm{~N}$, 486732.9 E, UTM Zone 10, WGS-84) during the rainy winter months. A newly established National Wildlife Refuge, the TRNWR had not yet opened to the public and had been temporarily left undisturbed to allow some reestablishment of the natural wildlife and ecology. Oxbow Regional Park on the Sandy River (site at 37 meters) (5037392.5 N, 445064.88 E, UTM Zone 10, WGS-84) is the only one of our sites containing a remnant patch of old growth forest. Douglas fir, western hemlock, and western red cedar almost completely shade the area. The ground is completely covered by sword fern, mosses, and large amounts of woody debris.

In addition to these five regular local collection centers, specimens were obtained from elsewhere in the range of Peromyscus maniculatus in order to provide comparative data (fig. 5). Peromyscus maniculatus tissues collected from the species type locality in northwestern Newfoundland were kindly donated by A. Lackey, State University of New York at Oswego. These tissues were included in the study to allow 
direct comparison of local $P$. maniculatus genotypes to those representative of the voucher P.m. maniculatus. Peromyscus specimens also were collected from habitats adjacent to both the east and west of the Mississippi River in northeastern Missouri (west site) and western Illinois (east site); these specimens were collected and tissues generously donated by S. Burt, Truman State University, Kirksville, Missouri. It was hypothesized that these allopatric Missouri and Illinois populations, kept separate by the barrier of the Mississippi River, would provide a distinct and visible break in the genetic continuum (gradient) believed to reflect the Peromyscus maniculatus subspecies distribution across the continent (LTER, 1998; Dragoo et al., 2006). This genetic continuum or gradient across the full range of $P$. maniculatus is seen as relative genetic similarity among subspecific populations, with that similarity inversely proportional to the geographic distance between subspecies.

Three types of live-capture traps were used to capture specimens: Sherman traps (H. B. Sherman Traps, Tallahassee, Florida), pitfall, and custom built mesh traps (O'Farrell et al., 1994). The Sherman traps were treadle-type collapsible aluminum boxes, models LFA and LFATDG, $8 \mathrm{~cm} \times 8 \mathrm{~cm} \times 23 \mathrm{~cm}$. The mesh live traps were designed to be approximately the same size as the Sherman live-traps with square wire cloth creating the box. The closure mechanism consisted of a treadle trigger, and gravity drop door, both of galvanized metal (O’Farrell et al., 1994). Pitfall traps consisted of a five-gallon bucket buried to the rim with a lid for a rain cover suspended above, leaving a $7-8 \mathrm{~cm}$ gap for specimen access. A trapping web design was selected over the more traditional trapping grid because absolute densities could be estimated from the capture data and because the estimation procedures required few 
assumptions about capture probabilities and animal movement patterns (Parmenter $e t$ al., 1998; Parmenter et al., 2003; Wilson and Anderson, 1985).

The trapping webs used for this study consisted of 12 spokes radiating at $30-$ degrees from one-another from a center point (fig. 6). Each spoke measures 100 meters from the center to end and contains 12 trapping locations along its length. The first four traps are at 5-meter intervals, while the subsequent traps are 10 meters apart. Each trap station had a mesh and Sherman live-trap; trap stations 7 and 12 on each line also had a medium $(48 \mathrm{~cm} \times 15 \mathrm{~cm} \times 15 \mathrm{~cm})$ or large $(81 \mathrm{~cm} \times 25 \mathrm{~cm} \times 30 \mathrm{~cm})$ Tomahawk live-trap. In addition, trap stations 4,7 , and 12 , of each line also had a pit fall trap as previously described. Lastly, 4 Sherman traps were located at the centerpoint of the web. This design results in 145 trap stations consisting of 144 mesh livetraps, 148 Sherman live-traps, 24 Tomahawk live-traps, and 36 pit fall traps (Parmenter et al., 1998; Parmenter et al., 2003) for a total of 353 traps. Mesh and Sherman traps were baited with a mixture of oats and peanut butter, while Tomahawk traps were baited with canned cat food. Pit falls remained unbaited. Poly-fiber nesting material was added to Sherman and mesh traps when warranted by weather. Traps were set out for four consecutive nights at each site with collection occurring at dawn the following day (Ritchie and Sullivan, 1989). After collection on the last morning, all Sherman and mesh traps were removed. To avoid extraneous captures, pitfalls were closed with a lid while not in use. Each park was trapped ten times over the course of this study, approximately every eight weeks. Total trap-nights were 65,200.

Captured animals were transferred from traps into clear, sealable plastic bags, labeled with the trap number, and transported to the center of the web for processing. 
Captures from Tomahawk traps were left in the trap and also transported to the center of the web center. Due to the possibility of contracting HPS, standard precautionary methods were implemented, including the use of full-face powered air purifying respirators (PAPR) with highly efficient particulate air (HEPA) filters, and latex gloves under leather gloves (Mills et al., 1995).

Each animal was identified to species and evaluated for gender, reproductive status, mass, relative age, and any other notable characteristics such as ectoparasites, wounds, or defects such as atypical coloration (appx. C). Heparinized glass micro-capillary tubes were used to draw blood from the retroorbital sinus and stored in cryovials, which were then placed in liquid nitrogen until testing (Kaufman et al., 1994). After blood samples were taken, deer mice were euthanized in a chloroform chamber (Mills et al., 1995).

This research was conducted under the auspices of federal and state permits, and complied with the American Society of Mammalogists Guidelines (Gannon et al., 2007) for the care and use of animals. Specimens were identified using museum tags including all relevant data mentioned above, sealed in plastic, then returned to the laboratory. Once in the laboratory, specimens were placed in a locked $-70^{\circ} \mathrm{C}$ ultracold freezer for storage and further analyses.

\section{Molecular Protocols-}

Field collected blood samples were tested for the presence of antibodies crossreactive to Sin Nombre virus (SNV) using Enzyme Linked Immunosorbent Assay (ELISA), by L. J. Dizney, Ph.D., of the Oregon State Health Laboratory, or Strip 
Immunoblot Assay, by B. Hjelle, Ph.D., of the University of New Mexico, (SIA; Kaufman et al., 1994; Mills et al., 1995; Morzunov et al., 1995; Rowe et al., 1995).

Kidney samples were extracted from all Peromyscus specimens that were seropositive for SNV antibodies as well as a random sample of those found negative (tables 2, 3; appx. D). Ultimately, including donated tissues from allopatric conspecifics $(n=9)$ and congeners $(n=4)$, tissues were collected for examination from 86 Peromyscus specimens.

Approximately 30-50 mg of tissue from each individual were used in DNA extraction. Whole cellular DNA was extracted and isolated from these tissue samples using the Qiagen DNeasy Tissue Kit (Valencia, CA) and protocols contained therein. To ensure comparison with sequence analyses in the literature, mitochondrial genes of the nicotinimide adenine dinucleotide (NADH) dehydrogenase subunit group were chosen as the targets for analysis. Specifically these consist of the ND3, ND4, ND4L, subunit mitochondrial genes and the Arginine tRNA mitochondrial gene that is located between the ND4L and the ND3 genes in the circular mitochondrial genome (Fig. 7). Amplification of gene targets was performed in two parallel but separate reactions for each DNA sample to obtain this entire gene set. Polymerase Chain Reactions (PCR) were set up utilizing Amersham Biosciènces (UK) "puReTaq ready-To-Go PCR Beads" (each bead contains $\sim 200 \mu \mathrm{M}$ final concentration dATP, dCTP, dGTP, and dTTP, $10 \mathrm{mM}$ Tris- $\mathrm{HCl}, 50 \mathrm{mM} \mathrm{KCl}, 1.5 \mathrm{mM} \mathrm{MgCl}_{2}$ ), $0.4 \mu 1$ (each) of $10.0 \mu \mathrm{M}$ primers, and $1.0 \mu \mathrm{l}$ of template DNA in a $25.0 \mu \mathrm{l}$ final reaction volume. The first of the two fragments was amplified using the specific primers 
5' - CGAACTAGTACAGCTGACTTCC - 3' (PI') for the (+) strand and 5' CCGTAGGAGTTTGATAGTTTTGCT - 3' (MANR) for the (-) strand (Arevalo et al., 1994). This PCR produced a mitochondrial DNA fragment of $734 \mathrm{bp}$. The second PCR reaction was set up with primers 5' AGCAAAACTATCAAACTCCTACGG - 3' (MANF) (+) and 5' TGGAGCTTCTACGTGGGCTTT - 3' (NAP2) (-), generating a 729 bp mitochondrial DNA fragment (Arevalo et al., 1994). Reaction mixtures were placed in an Eppendorf - Master Cycler 96 well Thermocycler for PCR amplification. Thermocycler protocol (STANDARD) was delineated as:

Denature: $\quad 95^{\circ} \mathrm{C} \quad 5 \mathrm{~min}$ 35 cycles: $\begin{cases}95^{\circ} \mathrm{C} & 45 \mathrm{sec} \\ 50^{\circ} \mathrm{C} & 30 \mathrm{sec} \\ 72^{\circ} \mathrm{C} & 90 \mathrm{sec}\end{cases}$

Extension: $\quad 72^{\circ} \mathrm{C} \quad 10 \mathrm{~min}$ Hold: $\quad 4^{\circ} \mathrm{C}$

To verify successful PCR, the products from the reactions were evaluated using electrophoresis. $5 \mu \mathrm{l}$ of amplified DNA product were combined with $12 \mu \mathrm{l}$ water and mixed. These $17 \mu \mathrm{l}$ solutions were then placed in each of the wells of an "E-Gel" (Invitrogen) 2\% agarose gel. Lane one of the 12-lane gel was left empty to receive 10 $\mu 1$ of $80 \mathrm{bp}-1 \mathrm{Kbp}$ DNA ladder (Fermentas (Glen Bernie, MD) MassRuler ${ }^{\mathrm{TM}}$ DNA Ladder, Low Range) as well as $7 \mu l$ water. The gel was run for 15 minutes. After the run was complete the E-Gel was removed and placed on an ultraviolet viewer to 
observe the fluorescently tagged DNA bands. In the E-Gel, fluorescent DNA stain is enclosed in the cartridge with the gel, allowing its interaction and binding with the DNA. Bands were seen, as expected, at the range of approximately $700 \mathrm{bp}-750 \mathrm{bp}$ as compared to the ladder.

After confirmation of PCR products the remaining reaction solutions were then subjected to PCR cleanup to remove excess polymerases, primers, dNTP's, and buffers using the QIAGEN (Valencia, CA) PCR Cleanup Kit and protocols contained therein.

The amplified products from each of these reactions were then utilized as template for sequencing using the modified Sanger chain terminator method (Sanger et al., $1975,1977)$. The sequencing reactions were set up with the $0.6 \mathrm{ml}$ tubes recessed in an ice-bath to limit reactivity of constituents until placed in the thermocycler. Each reaction vessel was prepared with $2.0 \mu \mathrm{l}$ Big Dye Terminator solution (Applied Biosystems), $2.1 \mu \mathrm{l}$ of $2.5 \mu \mathrm{M}$ appropriate primer, $1.0 \mu \mathrm{l} 5 \mathrm{x}$ reaction buffer, $4.8 \mu \mathrm{l}$ water, and $2.1 \mu \mathrm{l}$ purified PCR product for a final reaction volume of $12 \mu 1$. These sequencing reaction vessels were then placed in the Eppendorf thermocycler and exposed to the sequencing thermo protocol (SEQ) as:

25 Cycles $\begin{cases}96^{\circ} \mathrm{C} & 5 \mathrm{~min} \\ 96^{\circ} \mathrm{C} & 30 \mathrm{sec} \\ 50^{\circ} \mathrm{C} & 15 \mathrm{sec} \\ 60^{\circ} \mathrm{C} & 4 \mathrm{sec}\end{cases}$

Hold $\quad 4^{\circ} \mathrm{C}$ 
Upon completion of sequencing cycles, reactant solutions were plated in a 96 well micro titer plate that was then sealed and frozen at $-70^{\circ} \mathrm{C}$. The derived sequences were then shipped over night on dry ice to the Center for Genome Research and Bioinformatics (CGRB) Core Laboratories at Oregon State University, Corvallis, OR. There, sequences were analyzed on an Applied Biosystems (ABI) 3730 Capillary Sequence Machine.

\section{Sequence File Preparation-}

After the ABI 3730 generated the representative chromatograms they were downloaded via the internet from the CGRB Core Laboratories web site. Next, the sequence file chromatograms were uploaded into the software program SeqEd (Applied Biosystems v. 1.0.3). Within this program, sequences from corresponding primer pairs are viewed and edited for quality. First, the negative strand sequence, amplified from the reverse (ManR and Nap2) primers, is "reverse complimented" where the entire sequence is turned around to orient the last nucleotide first and the first nucleotide last. Then the bases of this reversed sequence are converted to their complementary nucleotide. Next, unidentifiable bases, artifacts of the sequencing process that occur at both the beginning and the end of the sequence, were removed. Sequences were aligned with SeqEd. These labeled mismatched bases were then examined based upon their corresponding peak in the sequence chromatogram. In most cases, one of the two opposing sequence chromatogram peaks was considerably cleaner, or stronger allowing the base to be "called" as the one indicative of the higher quality peak. In this way sequences were edited and aligned to produce a consensus 
sequence for the target gene group from each individual Peromyscus specimen. This task was undertaken twice for each Peromyscus specimen because the target sequence (mitochondrial NAD gene group) was originally amplified and sequenced in two separate fragment subsets.

The two consensus sequence fragments from each specimen were concatenated in Text-Edit (Apple-Inc.). The concatenated sequences were imported into MacClade (Maddison and Maddison, 2003) for multiple sequence alignment where sequences from all Peromyscus were aligned with one another as well as with other out-group species obtained from NCBI GenBank. The multiple sequence alignment was adjusted as to codon reading frame to minimize the occurrence of stop codons and thereby represent the natural reading frame. The NADH dehydrogenase genes coded within the sequence contained the Arginine transfer RNA gene (between ND3 and ND4L) as well which is not translated into protein. Therefore, to separate the noncoding tRNA from the rest of the sequence, that is read in a triplet codon-reading frame, single spaces were inserted flanking the tRNA gene. In doing so, the tRNA was considered separately during phylogenetic analysis.

\section{Phylogenetic Analysis-}

The multiple sequence alignment file was saved in MacClade in Nexus format to allow use in the software programs PAUP (Phylogenetic Analysis Using Parsimony v. 4.0b10) (Swofford, 2002), MrBayes (v3.1.2) (Huelsenbeck and Ronquist, 2001), and GARLI (Genetic Algorithm for Rapid Likelihood Inference v 0.951) (Zwickl, 2006). The first step of phylogenetic analysis entailed selection of an evolutionary model to 
enforce parameters of nucleotide substitution. To this end, the program MODELTEST v. 3.7 (Posada and Crandall, 1998) was utilized. Contained within the MODELTEST software package was the file modelblockPAUPb10. This file was inserted into the nexus sequence file and executed within PAUP. The purpose of this was to guide PAUP in analyzing the sequence data to choose from among 56 evolutionary models by using three different model selection frameworks: hierarchical likelihood ratio tests (hLRTs), Akaike information criterion (AIC), and Bayesian information criterion (BIC) (Posada and Crandall, 1998). The program also implements the assessment of model uncertainty and tools for model averaging and calculation of parameter importance, using the AIC or the BIC. Upon completion PAUP created an output file named model.scores that contained the results of the 56 analyses. The model.scores file was then read by MODELTEST that selected the model of nucleotide substitution that was the best fit. These analyses chose GTR $+\mathrm{I}+\Gamma$ (General Time Reversible + proportion Invariant + Gamma, 10 parameter) as the best fitting model. The GTR $+\mathrm{I}+\Gamma$ model is the most complex of the 56 model choices contained within the MODELTEST program where a proportion of sites are invariant (I) and a gamma-distributed substitution rate variation is implemented across sites $(\Gamma)$. This more complex model allows more free parameters for the analyses. A command block for the purpose of directing PAUP in application of the evolutionary model, known as a model block, was rendered by MODELTEST and contained within the output file. Other PAUP block commands were added to the Nexus file as well to command PAUP to conduct further analyses. 
These other commands included instructions for the type of analysis to be executed, in this case, Maximum Likelihood, which is a method for the inference of phylogeny. It evaluates a given hypothesis about the evolutionary history of the organisms involved (Peromyscus) in terms of the probability that the proposed model $(\mathrm{GTR}+\mathrm{I}+\Gamma)$ and the hypothesized history would give rise to the observed data set. The supposition is that a phylogenetic history with a higher probability of reaching the observed state (condition of genetic divergence represented by the nucleotide data) is preferred to a history with a lower probability, or simply put, the higher probability phylogenetic history is more likely. The Maximum Likelihood method searches for the tree with the highest probability of occurrence.

In addition to Maximum Likelihood, Distance analysis (in PAUP) was used to evaluate the relative amount of genetic divergence among the Peromyscus included in this study. Distance provides a simple comparative matrix of the nucleotide differences among organisms stated as percent divergence.

Bayesian analyses, by way of MrBayes Markov chain Monte Carlo (MCMC) (Hulsenbeck and Ronquist, 2001) were also performed. Bayesian analysis, as implemented by MrBayes, uses the likelihood function as well but instead of testing the probability of the data given the hypothesis, Bayesian evaluates the probability of the hypothesis given the data. In Maximum Likelihood, free parameters are optimized, maximizing the likelihood, but in a Bayesian MCMC approach, free parameters are probability distributions, which are then sampled (Hulsenbeck and Ronquist, 2001).

Bayesian analysis expresses its result as the posterior probability (PP) of the tree 
topologies and model parameters. The posterior probability is proportional to the likelihood, and, also proportional to the prior probability (Hulsenbeck and Ronquist, 2001). The best estimate of the phylogeny can be selected as the tree with the highest posterior probability (i.e., the MAximum Posterior probability [MAP] tree) (Rannala and Yang, 1996).

In a similar method to Bayesian posterior probabilities, Maximum Likelihood uses Bootstrapping (BS) (Felsenstein, 1985) to evaluate the probability that tree nodes are correct. These bootstrap values are reported as a percentage, being the percent of replicates that generated the same node. In the analyses conducted and described herein 1000 bootstrap replicates were generated using PAUP and 1000 BS replicates were performed using the program GARLI.

Studies have shown some incongruity between Bootstrap and Posterior Probability (Leache and Reeder, 2002; Whittingham et al., 2002). Although, when BS is plotted as a function of PP several examinations have revealed a correlation between BS and PP (Douady et al., 2003). Rannala and Yang (1996) found that within this correlation, posterior probabilities are consistently higher than corresponding bootstrap percentages. Wilcox et al. (2002) found that both PP and BS are overly conservative estimates. Therefore, considering the higher PP results of Rannala and Yang (1996) and the fact that both are overly conservative (Wilcox et al., 2002), it stands to reason that the Bayesian Posterior Probabilities may be slightly more representative of the true condition than Likelihood Bootstrap. That not withstanding, in a few circumstances, PP was found to have overestimated the hypothesis probability, and as such, over-reliance of Bayesian PP alone may be misleading (Douady et al., 2003). 
As a result of these findings both Bayesian Posterior Probabilities and Maximum Likelihood Bootstrapping have been included in these analyses to ensure the greatest possible representation of the Peromyscus phylogeny.

\section{Results-}

For comparison to five Rodent out-groups, consisting of Microtus pennsylvanicus, Oryzomys palustrus, Sigmodon hispidus, Rattus norvegicus, and Reithrodontomys megalotis, Peromyscus maniculatus mitochondrial ND3, ND4, ND4L, and arginine tRNA genes were sequenced (fig. 8) (appxs. E, G). Specimens included in these analyses were from the Portland, OR region $(n=46)$, the greater Pacific Northwest ( $\mathrm{n}=18$ ), East-central California ( $\mathrm{n}=1$, Peromyscus maniculatus rufinus), the Midwest ( $\mathrm{n}=9$, Peromyscus maniculatus bairdii, and P. leucopus), and Newfoundland $(\mathrm{n}=4$, Peromyscus maniculatus maniculatus), for a total of 78 sequence specimens generated by this study. Sequences for Peromyscus keeni, Peromyscus leucopus (one of six), and Peromyscus sejugis $(\mathrm{n}=2)$ were obtained from GenBank as well as the out-group sequences $(n=5)$, These specific out-group species were selected due to their condition as known reservoirs of Hantavirus species.

\section{Distance Analysis-}

Distance analyses ( $p$ distances) (figs. 8, 9; appx. A) for 1,450 nucleotides of the mitochondrial ND3, ND4, and ND4L genes, as well as the Arginine tRNA sequence, revealed an average pair-wise nucleotide genetic distance (defined as percent divergence) of $p=0.0413$ (4.13\%) between the Newfoundland voucher Peromyscus 
maniculatus maniculatus and the local Peromyscus maniculatus rubidus found west of the Cascade Mountains in Oregon (figs. 3, and 4). A relative pair-wise genetic distance of 0.0416 (figs. 8,9) was found between voucher P. m. maniculatus and the greater Pacific Northwest (PNW) Peromyscus maniculatus subspecies clade (figs. 1013) on the whole. Intra-PNW clade average genetic distance was found to be 0.0063 revealing no discernable structure signal (appx. A). In these analyses the PNW clade includes $P$. m. rubidus; $P$. m. austerus, from Washington, north of the Columbia River and west of the Cascade Mountains; P. m. gambeli, found east of the Cascade Mountains in Oregon and Northern California; and P. m. austerus, located in the San Juan Islands (fig. 4). At the same time, topo-typical P. m. maniculatus was shown to possess a genetic divergence of 0.0600 from the Pacific northwestern $P$. keeni (at least partially sympatric with the PNW $P$. $m$. clade), 0.0542 from $P$. maniculatus rufinus, the reservoir species of Sin Nombre Virus from the four corners region of New Mexico, and 0.0468 pair-wise genetic distance from $P . m$. bairdii from west of the Mississippi River in northeastern Missouri (figs. 8 and 9). This P. m. bairdiil P. $m$. maniculatus pair-wise genetic distance (0.0468), taken with consideration that the genetic distance between the Pacific Northwest Clade and P. m. bairdii from the west side of the Mississippi River is only 0.0171 , raises the possibility that a genetic break exists at or near the Mississippi River, dividing the $P$. maniculatus species into a western clade and an eastern Clade. Further to this point, when the $P$. maniculatus baridii subspecies from west of the Mississippi River are included with the Pacific Northwest specimens and compared as a group to the Newfoundland voucher specimens a pair-wise genetic distance of 0.0457 is resolved, strengthening the 
possibility of two separate major Peromyscus (maniculatus) clades (figs. 8 and 9). Interestingly, the calculated genetic distance between two widely accepted species, $P$. keeni, from Washington and British Columbia (Zheng et al., 2003), and Peromyscus sejugis, from San Diego and Santa Cruz Islands in the Gulf of California (Burt, 1932), is 0.0429 , less than the other $P$. maniculatus subspecies in the western clade from the supposed conspecific $P$. m. maniculatus (figs. 8 and 9). $P$. sejugis itself has a similar genetic distance (0.0511), and is also recognized as a separate congener of $P$. maniculatus (fig. 8). That a previously described isolated insular species is only marginally more divergent $(0.0511 / 0.0457)$ from the topo-type than are those from the rest of the western half of the continent as a whole raises the potential that speciation may have indeed occurred between the Newfoundland Peromyscus maniculatus maniculatus and the western $P$. maniculatus subspecies.

The Mississippi River only divides the majority of the United States, and not Canada, as it flows from West of Lake Superior in Central Minnesota to the Gulf of Mexico, therefore, it is an incomplete barrier for division of the entire North American Peromyscus maniculatus species distribution. Canadian populations may be similarly separated by a water barrier extending from Hudson Bay in the north to Lake Winnipeg (Lake Agassiz) via the Nelson River, and from there to the Lake of the Woods at the Canadian-Minnesota border, through the Boundary waters to the Mississippi River. The only opening in this barrier exists between the Red River, arising in Northern Minnesota, flowing to the southern tip of Lake Winnipeg, and the headwaters of the Mississippi River, a not so inconsequential 97 kilometers (60 mi.). Much more extensive sampling is required from these regions to further elucidate the 
possibility of the occurrence of a genetic break within the Peromyscus maniculatus species along this mostly complete barrier.

\section{Maximum Likelihood Analysis-}

Taxa including Peromyscus genus and related outgroups $(\mathrm{n}=88)$ from within the rodent families Cricetidae and Muridae were examined for their phylogenetic relationship using Maximum Likelihood in PAUP (Swofford, 2002). Likelihood analyses rendered a likelihood tree with a negative $\log$-likelihood $(-\operatorname{lnL})=8702.95284$ for the resolved tree topology. The likelihood tree was calculated using the tree bisection-reconnection (TBR) swapping algorithm. Nucleotide frequencies were $\mathrm{A}=0.35450, \mathrm{~T}=0.29800, \mathrm{C}=0.27910, \mathrm{G}=0.06840$. Among-site rate variation includes an assumed proportion of invariable sites of 0.2851 . A gamma distribution of rates across variable sites was implemented with a shape parameter of 0.7693 and four rate categories. These parameters are determined by the GTR $+\mathrm{I}+\Gamma$ model of evolution as chosen by Modeltest (Posada and Crandall, 1998).

Node support for the likelihood tree was generated using 1000 bootstrap replicates. The consensus bootstrap tree yielded strong support for resolved nodes (Fig. 11). Fig. 11 shows the Pacific Northwest Peromyscus Clade (PNW) nested within a greater clade containing all those representative Peromyscus taxa from west of the Mississippi River (western clade). The consensus tree demonstrates the western Peromyscus clustering in a very well supported, yet distinct, clade, sister to the Newfoundland topotype specimens. The western clade is also separate at the same level from the other nearest (geographically and genetically) related congeners including $P$. sejugis 
from San Diego and Santa Cruz Islands (Gulf of California) as well as $P$. keeni from the Pacific Northwest north of the Columbia River.

Consistent with widely accepted positions (Greenbaum et al., 1978; Avise et al., 1983) is the location in these analyses of Peromyscus leucopus, as a more distantly related member of the Peromyscus genus. As expected, all Peromyscus members represented in these analyses are seen as a separate monophyletic clade from the nearest regarded member of the Neotominae Subfamily that is included in these data, Reithrodontomys megalotis.

The genetic distance tree (fig. 10) resolved using the Neighbor Joining (NJ) method produced a tree with the same topology as the Bootstrapped Maximum Likelihood consensus tree (fig. 11). Branch lengths are shown above each corresponding branch with the change scale, representing the number of nucleotide changes from the previous node to the subsequent, located at the bottom-left of the figure. The Neighbor Joining analysis calculated a minimum evolution score of 3.18101 (analogous to sum of branch lengths) for these data.

A manually combined tree (fig. 12) includes branch lengths, likelihood scores, and bootstrap support at nodes present in both representative trees to allow direct comparison of these data.

Bayesian Analysis-

Bayesian analyses of these data were performed for 500,000 generations using two runs of four separate Markov chain Monte Carlo Metropolis coupling computations. Samples were taken from the tree-scape every 100 generations yielding 5,001 sample 
trees for each run, or 10,002 total sample trees. The final average standard deviation of split frequencies was 0.0077 after completion of the 500,000 generations. The Metropolis-coupled Markov Chain Monte Carlo (MCMCMC) analysis led to burn-in after 100,000 generations, therefore, the first 1,000 sample trees were excluded from the majority-rule consensus tree search, resulting in a consensus tree derived from a total of 8,002 sample trees or 800,000 generations.

The Bayesian majority-rule consensus tree (Fig. 13) resolved the exact same topology as all other phylogenetic analyses contained herein (Likelihood, and Neighbor-Joining Distance), showing all subspecies of Peromyscus from west of the Mississippi River (that were included in this study) as one whole clade - sister at the same level to the topo-type $P$. m. maniculatus from Newfoundland as well as $P$. keeni and $P$. sejugis.

\section{Discussion-}

Peromyscus maniculatus is a diverse species said to span most of the North American continent, excluding the southeastern United States and extreme northern Canada (Hall, 1981). Included within this distribution are as many as 70 morphological subspecies (Hall, 1981; Michaux et al., 2001). The vast majority of these sub specific relationships have not yet been evaluated by molecular analyses.

Being terrestrial and diminutive in size, deer mice possess limited vicariant abilities. With a home range of only $200-400 \mathrm{~m}^{2}$ and highly susceptible to ecological barriers, they would therefore have great difficulty maintaining gene flow over this entire distribution (Hall, 1981; Verts and Carraway, 1998; Wilson and Ruff, 1999; 
Michaux et al., 2001). Without this exchange of genetic material, it is inevitable that allopatric populations would begin to genetically drift apart, or diverge (Mayr, 1982; Kimura, 1983). Winding through the middle of the North American continent is the Mississippi River that, along with the Red River in north central Minnesota, that flows to Lake Winnipeg (Lake Agassiz) in Canada and is ultimately connected to Hudson Bay via the Nelson River, come within 97 kilometers (60 miles) of completely dividing the continent in two. This very nearly complete ecological barrier may provide the very blockade to genetic exchange of flanking allopatric sub specific populations that could drive such a genetic drift. The literature focusing on Peromyscus maniculatus phylogenetic relationships are found with references to two distinct morphotypes, each consisting of many subspecies from within the species as a whole, the putative forest-form, found east of the Mississippi River and these other bodies of water, and the grassland-form, seen from west of the Mississippi River to the Pacific Ocean (LTER, 1998, Dragoo et al., 2006).

In support of these previous findings, this study has resolved a similar division in the deer mouse distribution, in the form of a genetic break occurring at the Mississippi River. It should be noted however, that the discussion of a genetic break at this very specific location is based upon only four sequences from one site. It is apparent that the genetic break exists, only the exact location of it is in question. Further investigation into the populations distributed along this barrier would be needed to strengthen or refute these arguments.

Genetic distance analyses of the mitochondrial coding genes ND3, ND4, ND4L and the non-coding arginine tRNA, show the deer mouse subspecies nearest the 
western banks of the Mississippi River, $P . m$. bairdii, virtually as genetically divergent (p distance $=0.0468$, figs. 8,9 ) from the topotype $P$. m. maniculatus as are the western most deer mouse subspecies included in these analyses (\{P. m. rubidus, $P . m$. gambelii, $P$. $m$. austerus, $P$. m. rufinus $\}$ at $\mathrm{p}=0.0457$, figs. 8,9$)$. Conversely, the genetic distance between $P . m$. bairdii and these western subspecies is at most 0.0171 , confirming their closer alliance with those western subspecies (figs. 8, 9).

Interestingly, the haplotype distributions of the congener $P$. leucopus, with populations found on both sides of the Mississippi River, does not show this same division at the river (Morzunov et al., 1998). One possible explanation for this lack of genetic division could be the result of more recent range expansion by $P$. leucopus than the expansion that led to the division in $P$. maniculatus. Simply, not enough time has passed since that expansion to make the division significant enough to be detected. In fact, Morzunov et al. (1998) show haplotypes of $P$. leucopus with "hydrid" haplotypes in over lapping regions of haplotype distributions. To that point, Morzunov et al. (1998) show that hantaviruses found in P. leucopus, with their more rapid genetic divergence than their hosts, do in fact show this division, with separate viral lineages found on opposite sides of the Mississippi River, and do not overlap. The faster genetic divergence of the viruses carried by $P$. leucopus may be revealing, through their own lineal division, a forthcoming division in the distribution of $P$. leucopus.

Distance analysis, with its susceptibility to underestimation of evolution due to the possibility of multiple changes at a given single nucleotide locus, would alone not be enough to confirm a division in P. maniculatus, or more importantly, clarify that $P$. $m$. bairdii is indeed clustering with those subspecies further west and not with the 
Newfoundland topotype. Although, if underestimation is occurring, then correcting for that underestimation would only increase the resolved divergence, strengthening the argument for two separate east and west clades. Further phylogenetic analyses contained herein using methods less susceptible to underestimation of divergence reveal additional confirmation of the relationship espoused in these genetic distance analyses.

While considered an inferior phylogenetic method to the others used in these analyses - due to the simplicity of using least total branch length - Mihaescu et al. (2007) have shown that Neighbor Joining trees based upon genetic distance have proven to be reliable, and therefore worth inclusion in analyses. Genetic distance analysis may also suffer from one of the criticisms of Parsimony in that it may not be reflective of multiple changes within one nucleotide locus. This may result in an underestimate of the amount of change that may have historically occurred (Swofford, 1998). Conversely, with implementation of a more complex model (than used in Parsimony) such as GTR $+\mathrm{I}+\Gamma$ (used in these analyses), which allows a proportion of sites to be invariable (I), applies gamma-distributed variable rates $(\Gamma)$, and among-site rate variation is taken into account, thereby alleviating at least most, if not all of the underestimation (Foster, 2003).

As expected, the distance Neighbor-Joining tree (Fig. 10), derived from the genetic distance matrix, separates the western subspecies of $P$. maniculatus, including $P$. $m$. bairdii, from the topotypical P. maniculatus at the species level, the same as the division between these and other previously accepted western congeners including $P$. 
keeni, and $P$. sejugis. This association is repeated in other analyses as well. Fig. 11 shows the Bootstrapped Maximum Likelihood tree with the exact same topology as the NJ distance tree, showing all the subspecies from west of the Mississippi River contained within a single clade separate from, and at the same level as, the topotypical deer mouse. In point of fact, the node separating the western clade, the topotype, and P. keeni and P. sejugis in Fig. 11 was elucidated in $91 \%$ of 1000 bootstrap replicates.

There have been some purported advantages and disadvantages (Felsenstein, 1981; Cunningham et al., 1998; Lewis, (CH 5 Soltis book 1998); Leache and Reeder, 2002) of Maximum Likelihood methods. Specifically, Maximum Likelihood methods have recently been preferred over other common methods such as Maximum Parsimony. Likelihood analyses often have lower variance than Parsimony, meaning, it is frequently the estimation method least affected by sampling error (Felsenstein, 1981). Analyses using Likelihood tend to be robust to many violations of the assumptions in the evolutionary model, and even with very short sequences they tend to outperform alternative methods such as Parsimony (Foster, 2003). Also, Likelihood considers branch length. Conversely, Parsimony assumes that you would have the same expectation that a character would change along both long and short branches of the phylogenetic tree. Likelihood and distance methods, using models, instead consider that change is more probable along long branches than along short branches and provide accurate branch lengths even when a particular site has undergone more than one mutation (Foster, 2003).

There have also been a few disadvantages proposed for Maximum Likelihood as well. While not detrimental to the quality of results, but possibly restrictive on the 
number, depth, or types of analyses performed is the problem that Maximum Likelihood is very computationally intensive and thus extremely slow. Compared to Bayesian analysis, Maximum Likelihood has been shown to use $80 \%$ more computing time (Leache and Reeder, 2002). In these analyses, this has been alleviated to some extent by use of the research computer cluster at Portland State University. Another critique of Maximum Likelihood is that the result is dependent on the model of evolution used and therefore very susceptible to incorrect evolutionary model selection and application (Merl et al., 2005).

One million generations of Bayesian analysis also resolved a phylogenetic tree (Fig. 13) that possessed the same topology as the Likelihood and Distance trees. The Majority-Rule consensus tree derived from the 800,000 sample trees, once again, partitions all western subspecies within a single clade, separate from Newfoundland deer mice at the same (species) level as they are split from $P$. keeni and $P$. sejugis. The Majority-Rule consensus tree contained a Bayesian posterior probability for this bipartition of 100 , indicating the strongest possible probability of this node being representative of the natural state.

The results seen here displaying a clear division between the eastern and western clades are congruent with the conclusions reached by a concurrent study (Dragoo et al., 2006). Dragoo et al. (2006) suggest in their analyses that further data were needed from Oregon to further the understanding of these relationships. These analyses have provided those data. Like Dragoo et al. (2006), the data contained herein have shown that $P$. keeni of the Pacific Northwest allies very closely with the insular P. sejugis of San Diego and Santa Cruz Islands in the Gulf of California. Conversely, while 
Dragoo et al. (2006) suggest that coastal Pacific Northwest forms would ally more closely with $P$. sejugis and $P$. keeni than with $P$. maniculatus, these analyses, containing specimens from the coastal, insular, and non-coastal Pacific Northwest have resolved them as essentially equally distinct from $P$. maniculatus and the $P$. keeni/sejugis clade.

Therefore, it is concluded that deer mice in the western half of North America, possibly bound on the east by the Mississippi River and the northern bodies of water, and seen as the separate western clade resolved in Figs. 10-13, represent a formerly cryptic but distinct species from Peromyscus maniculatus in the east within the Peromyscus genus. As suggested elsewhere (Dragoo et al., 2006), this western clade may be representative of the previously morphologically described taxon Peromyscus sonoriensis (Le Conte, 1853). 


\section{Chapter 3: Hantavirus in the Pacific Northwest}

\section{Abstract-}

An outbreak of an illness in 1993 that came to be known as Hantavirus Pulmonary Syndrome (HPS) was later found to be caused by a novel Hantavirus, since named Sin Nombre virus. In the years since, as many as 34 previously unidentified hantaviruses have been discovered and characterized, with the vast majority of these occurring in the Western hemisphere. Until this study, no hantaviruses had been identified in Oregon in spite of the occurrence of five recognized cases of HPS in the state. To fill this gap, Peromyscus maniculatus (deer mice) were collected at 5 sites in parks and green-spaces in and around the Portland, OR region. Ultimately, 86 of 2084 (4.1\%) deer mice sampled were found to be sero-positive for antibodies to Hantavirus (tables 1, 2; appx. D).

Phylogenetic analyses were performed on 23 Hantavirus sequences obtained from sero-positive deer mice in the Portland, Oregon region, with additional sequences $(n=21)$ from other hantaviruses from GenBank included. Comparison of 617 nucleotides of the $\mathrm{G} 1 / \mathrm{G} 2$ glycoprotein precursor gene from the medium (M) segment genomic RNA, to other hantaviruses from around the world, found the Oregon viruses to be $11.2 \%$ divergent from the most closely related described Hantavirus, Convict Creek virus, from east-central California, $14.3 \%$ divergent from Sin Nombre virus, 21.5\% from New York virus, $23.6 \%$ from Blue River virus, and $41.4 \%$ from Hantaan virus. Maximum Likelihood and Bayesian analyses found the Oregon viruses to be monophyletic with the Convict Creek and Sin Nombre viruses. 
These data strongly suggest that the Hantavirus found in Oregon minimally constitutes a new strain, and may in fact be a new species. It is proposed here that this new virus be referred to as the Kalapuya virus.

\section{Introduction-}

Epizootiology-

In the last few decades there has been what appears to be a significant increase in the emergence of pathogenic infectious diseases such as Sudden Acute Respiratory Syndrome (SARS) (identified SE Asia, 2002), Ebola Virus (sub-Saharan Africa, 1976), Human Immunodeficiency Virus (HIV) (sub-Saharan, 1970's), Marburg Virus (Sub-Saharan Africa, 1970's), and tick-borne diseases such as Lyme disease (NE USA, 1990's). Among these recent discoveries are the hantaviruses, the causative agents of Hantavirus Pulmonary Syndrome (HPS) (Western Hemisphere, 1993) and Hemorrhagic Fever with Renal Syndrome (HFRS) (Eastern Hemisphere, 1950's). The appearance of these diseases is believed to be associated with a range of underlying causal factors including interactions with zoonotic pathogens, that would otherwise normally be contained within a host-parasite relationship, between wildlife and humans (Daszak et al., 2000). The term "emerging infectious disease" (EID) has been coined to describe any of the plethora of pathogens implicated in these recent discoveries, and is specifically used to define diseases that have recently increased in occurrence or geographic range, moved into new host populations, or only recently been discovered (Daszak et al., 2000). 
In 1993 in the southwestern United States, an outbreak occurred of a previously unknown disease, now called Hantavirus Pulmonary Syndrome (HPS). The subsequent investigation led to the identification of the causative agent-a novel Hantavirus-Sin Nombre virus (SNV) (Childs et al., 1994; Nichol et al., 1993). In the years since this discovery, the number of Hantavirus geno- and serotypes identified in the Western Hemisphere has grown to at least 23 (Johnson et al., 1997; Morzunov et al., 1998; Pini et al., 2003; Rhodes et al., 2000; Sanchez et al., 2001; Schmaljohn et al., 1995; Yates et al., 2002), with this number on the increase as previously unknown hantaviruses are identified. In addition to Sin Nombre Virus some of the other most common hantaviruses known to cause HPS include; New York virus (NY), Bayou virus (BAY), Black Creek Canal virus (BCC), and Andes virus, with Sin Nombre virus being responsible for most of the 465 (Centers for Disease Control [CDC] 2007) known cases in the United States (Monroe et al., 1999; Peters et al., 1998; Schmaljohn et al., 1997). Recent surveys for Hantavirus antibodies have revealed Hantavirus-infected rodents throughout most of North America (Holmes et al., 2004; Monroe et al., 1999; Rhodes et al., 2000; Sanchez et al., 2001; Schmaljohn et al., 1995; Yates et al., 2002). Hantaviruses are often transmitted among rodents through aggressive encounters-primarily between individuals of the same sex, usually males, and by infectious aerosols generated from contaminated urine, feces, blood, and saliva (Schmaljohn et al., 1997; Olsson et al., 2002). 
Description-

Members of the genus Hantavirus of the family Bunyaviridae (5 genera, 250 species), are spherical, 100 nanometer diameter particles enclosed within a phospholipid bilayer envelope (Elliot et al., 1991; Nichol et al., 2005). Present within and extending above this envelope are two glycoproteins (G1 and G2) that mediate false recognition of the viral particle as benign by the host target cell. The glycoproteins then facilitate cell entry via a clathrin coated pit (Elliot et al., 1991; Nichol et al., 2005). Within this envelope is a protein nucleocapsid that houses a segmented single-stranded negative-sense RNA genome $\sim 12,100$ nucleotides (nt) in length (fig. 14). These segments are small (S), medium (M), and large (L) (Plyusnin et al., 1996). The S segment, approximately 2,000nt in length, encodes the genes for the nucleocapsid proteins that encase the RNA genome in daughter viral particles. The medium, or M segment (3700nt), encodes the envelope glycoprotein precursor that is then co-translationally cleaved to yield the G1 and G2 glycoproteins (Elliot, 1990; Vapalahti et al., 1992; Bohlman et al., 2002). The L segment (6,500nt) encodes the RNA-specific polymerase that is used to replicate viral progeny genomes (fig. 14) (Elliott et al., 1991; Chizhikov et al., 1995).

\section{Natural History-}

It has been hypothesized by many that for every type of Hantavirus there is a specific rodent host species whose members can potentially acquire a chronic, asymptomatic, lifelong viral infection (Kaufman et al., 1994; Morzunov et al., 1998; 
Netski et al., 1999). It has been shown through phylogenetic analyses that the topologies of the evolutionary relationships among hantaviruses were closely correlated with those of their known or suspected primary mammal reservoirs (Borucki et al., 2000; Monroe et al., 1999; Plyusnin and Morzunov 2000; Sironen et al., 2001; Yates et al., 2002).

In the Americas, Peromyscus maniculatus has been co-evolving with hantaviruses for approximately 12-20 million years, since the first cricetids crossed the Bering Land Bridge, radiating and differentiating into the Rodent subfamilies Neotominae and Sigmodontinae found in the New World today (Yates et al., 2002). While much is known about deer mouse phylogeny, it remains less than fully understood, and as a result of the documented host-parasite specificity among deer mice and their hantaviruses (Yates et al., 2002), the associated viral phylogeny may be unresolved as well. New species of Peromyscus are increasingly being revealed from among these former host conspecifics (Chirhart et al., 2001; Zheng et al., 2003), revealing the potential likelihood of many uncharacterized hantaviruses waiting to be described as well.

For many years it was believed that hantaviruses were maintained exclusively within Order Rodentia. Only very recently has that perception been challenged as genetically distinct hantaviruses have increasingly come to be found residing among members of the Order Soricomorpha (shrews) - family Soricidae (Xiao et al., 1994; Arai et al., 2007, 2008). In the 1980's, in the former Soviet Union, antibodies to Hantavirus were found in members of families Soricidae and Talpidae (Gavrilovskaya et al., 1983; Tkachenko et al., 1983; Gligic et al., 1989). B y that time molecular 
techniques were not yet available to delineate genetic identity of potentially hosted viruses to confirm whether these were truly distinct viral evolutionary lineages or merely cases of ecological spillover from a sympatric rodent host. As of yet, no human disease has been associated with shrew-borne hantaviruses (Arai et al. 2007).

Worldwide, most hantaviruses have been found in members of the rodent families Cricetidae (Subfamilies Neotominae and Arvicolinae) and Muridae (Subfamily Murinae), with genetically distinct viral lineages found associated with specific members of these taxa (Monroe et al., 1999) (fig. 1). Hantaviruses are believed to have arisen at least 136 million years ago before the split of the Mammalian Orders Soricomorpha and Rodentia, evidenced by the presence of distinct hantaviral lineages found in both Orders (Carey et al., 1971; Plyusnin et al., 1996; Hughes and Friedman, 2000). Among the earliest described are those found in East Asia, including Hantaan virus from the Hantaan River valley, recognized during the Korean War, as well as Seoul virus, also found in Korea (Lee et al., 1978)(fig. 3). These East Asian viral species have been found to be reservoired within members of the family Muridae, Subfamily Murinae, as Apodemus agrarius (Hantaan), and Rattus norvegicus (Seoul; Lee et al., 1982). Another murine Hantavirus is the recently described Sangassou virus, shown to reside within the African wood mouse Hylomyscus simus, found in Sangassou, Guinea (Klempa et al., 2006). Reducing the clarity of this strict regional relationship, but upholding the host Subfamily paradigm, is the presence of Dobrava virus found in Northern Europe and hosted by the murine Apodemus flavicollis, a congener of the Hantaan virus host (Avsic-Zupanc et al., 1992; 2000). Phylogenetic analyses have supported placement of Dobrava virus in the murine-hosted viral clade 
(Avsic-Zupanc et al., 1992) (Figure 1). European and North Asian serotypes such as Tula virus, Topografov virus, and Puumala virus, among others, have been found within arvicoline hosts such as Microtus and Clethrionomys species (Plyusnin et al., 1994; Vapalahti et al., 1999).

The more recently described members in Hantavirus are those found in the new world. In North and South America most of these infections are in species of the Subfamily Neotominae. Only a few Murid-hosted hantaviruses, consisting of Seoul virus in Rattus norvegicus, Subfamily Murinae, have been seen in the western hemisphere, primarily in urban environs (Monroe et al., 1999). Cricetid rodents found hosting hantaviruses are many (table 1). In North America most common among these are hosts in the genus Peromyscus (Monroe et al., 1999). The deer mouse, Peromyscus maniculatus, Subfamily Neotominae, has been identified as the reservoir for Sin Nombre virus (Childs et al., 1994). In depth studies of Sin Nombre virus in North America have shown substantial genetic diversity and geographic structure of genetic variants within the viral quasispecies that may be the result of the genetic diversity in P. maniculatus (Monroe et al., 1999; Morzunov et al., 1996; Nichol et al., 1999; Plyusnin et al., 1996; Plyusnin et al., 2000; Salazar-Bravo et al., 2002). The rodent genetic diversity is paralleled by that of the virus (Dragoo et al., 2006).

\section{Scope-}

As described above, due to the recognized coevolution between Hantaviruses and their hosts, Hantaviruses are suspected to reside in nearly every (if not every) ecoregion where their potential host species are found. Therefore, the phylogenetic 
relationships among members of Hantavirus are incomplete, as many Hantaviruses are believed to remain undiscovered and thus undescribed. In light of this, the purpose of this work was to obtain in the field, Hantavirus potentially existing in the Portland, Oregon region, and to use molecular data to characterize and test the phylogenetic position of the Hantavirus strain found in the local deer mice.

\section{Research Design and Methods-}

Field Protocols-

(refer to page 16, Chap. 2)

\section{Molecular Protocols-}

Field collected blood samples were tested for the presence of antibodies crossreactive to Sin Nombre virus (SNV) using Enzyme Linked Immunosorbent Assay (ELISA), performed by L. J. Dizney of the Oregon State Health Laboratory, or Strip Immunoblot Assay (SIA), by B. Hjelle of the University of New Mexico (Kaufman et al., 1994; Mills et al., 1995; Morzunov et al., 1995; Rowe et al., 1995; Vincent et al., 2000).

Lung tissue was extracted from all 86 Peromyscus specimens found to be seropositive for SNV antibodies (tables 2, 3; appx. D).

Approximately 30-50 milligrams of tissue from each individual were used for RNA extraction. Frozen tissues were physically disrupted in liquid nitrogen $\left(\mathrm{IN}_{2}\right)$ by grinding with mortar and pestle. Ground tissues were then homogenized using the 
"QiaShredder" kit (QIAGEN). RNA was extracted and purified from these tissue samples using the QIAGEN “RNeasy Tissue Kit”. Also Hantaviral RNA was recovered from the blood serum of a human HPS patient from the Portland region. The viral RNA was obtained using the QIAGEN "QIAAmp Viral RNA Mini Kit" and protocols contained therein.

Two fragments of the hantaviral M segment (617 nucleotides combined), one each of the G1 (361nt) and G2 (256nt) glycoprotein precursor gene region, were chosen as the targets for analysis (Fig. 14). Viral RNA sequences were amplified in a one step process through primer specific reverse transcription (RT) followed by polymerase chain reaction (PCR) using the QIAGEN OneStep RT-PCR kit. Each reverse transcription and first round PCR reaction was set up in a single $50 \mu 1$ reaction volume containing $16.0 \mu \mathrm{l}$ RNase free water, $10.0 \mu \mathrm{l}$ 5x QIAGEN OneStep RT-PCR buffer, $2.0 \mu 1 \mathrm{dNTP}$ mix, $10.0 \mu 1$ 5x Q-Solution, $2.0 \mu \mathrm{l}$ QIAGEN OneStep RT-PCR enzyme mix, $1.0 \mu \mathrm{l}$ RNase inhibitor, $2.0 \mu \mathrm{l}$ each of first round primers at $10.0 \mu \mathrm{M}$ concentration, and $5.0 \mu 1$ of RNA template. Two of each of these reactions were prepared for each specimen, one for the G1 region and one for the G2 region. First round RT-PCR primers are G1 5'-ACAATGGGITCIATGGTITGTGTA-3' (SM1687C) and 5'-TTIAATITIICATCCAATCCA-3' (SM2255R) and G2 5'TGTGAITATCAAGGIAAIAC-3' (SM2779C) and 5'-ACIGTIGCICCATAIACAT3' (SM3128R) (Johnson et al., 1997). The RT-PCR reactions were set up on ice to prevent or slow any enzymatic catalysis from occurring before samples are placed in the thermocycler. 
The RT-PCR was performed by placing the samples in an Eppendorf-Mastercycler 96 well and running the RT-PCR program, defined as follows:

$30 \min @ 50^{\circ} \mathrm{C} \quad \mathrm{RT}$ reaction

$15 \min @ 95^{\circ} \mathrm{C} \quad \mathrm{RT}$ inactivated and DNA polymerase activated

1 min@ $94^{\circ} \mathrm{C} \quad$ Template denaturation

$1 \mathrm{~min} @ 62^{\circ} \mathrm{C} \quad$ Primer annealing $\} 25$ cycles

1 min@ $72^{\circ} \mathrm{C} \quad$ Extension

10 min@ $92^{\circ} \mathrm{C} \quad$ Final extension

After RT-PCR was complete, reaction products were cleaned using the PCR Purification kit from QIAGEN. To verify successful PCR, the amplified products were evaluated using gel electrophoresis (agarose). $5 \mu$ of amplified DNA product were combined with $12 \mu 1$ water and mixed. These $17 \mu 1$ solutions were then placed in each of the wells of an "E-Gel" (Invitrogen) 2\% agarose gel. Lane one of the 12lane gel was left empty to accommodate $10 \mu \mathrm{l}$ of $80 \mathrm{bp}-1 \mathrm{Kbp}$ DNA ladder (Fermentas MassRuler ${ }^{\mathrm{TM}}$ DNA Ladder, Low Range) as well as $7 \mu 1$ water. The gel was then placed in the Invitrogen E-Base and set to run for 30 minutes. After the run was complete the E-Gel was removed and placed on an ultraviolet viewer to illuminate the fluorescently labeled DNA bands.

When bands were found to be of the correct size (596 nucleotides (nt) for G1 and 350nt for G2), remaining products were then amplified further using hemi-nested primers inside those from RT-PCR. Second round nested primers for G1 (361nt) were 5'-GAITGIGAIACAGCAAAAGA-3' (SM1723C) and 5'-TCIGCACTIGCIGCCCA- 
3' (ASM2016R). Hemi-nested primers for the G2 coding region (256nt) were SM2779C (as above) and 5'-CCCCAIGCICCITCAAT-3' (SM3020R) (Johnson et al., 1997).

Second round hemi-nested PCR was set up utilizing Amersham Biosciences "puReTaq ready-To-Go PCR Beads" (each bead contains $\sim 200 \mu \mathrm{M}$ final concentration dATP, dCTP, dGTP, and dTTP, $10 \mathrm{mM}$ Tris- $\mathrm{HCl}, 50 \mathrm{mM} \mathrm{KCl}, 1.5 \mathrm{mM} \mathrm{MgCl}_{2}, 0.4 \mu \mathrm{l}$ (each) of $10.0 \mu \mathrm{M}$ primers, and $1.0 \mu 1$ of template DNA in a $25.0 \mu$ final reaction volume.

Thermocycler protocol (PCR2) was delineated as:

$5 \min @ 95^{\circ} \mathrm{C} \quad$ Initial Denaturation

$45 \sec @ 95^{\circ} \mathrm{C}$

$\left.\begin{array}{l}\begin{array}{l}\text { Template Denaturation } \\ \text { Primer Annealing } \\ \text { Extension }\end{array}\end{array}\right\} 35$ cycles

$10 \min @ 72^{\circ} \mathrm{C}$

Final Extension

Hold @ $4^{\circ} \mathrm{C}$

Again, to verify successful PCR, the products from the hemi-nested reactions were evaluated using electrophoresis as delineated above. Bands were resolved, as expected, at the range of approximately $361 \mathrm{nt}$ and $256 \mathrm{nt}$ as designated by the nucleotide ladder.

After confirmation of PCR products the remaining reaction solutions were then subjected to PCR cleanup again to remove excess polymerases, primers, dNTP's, and buffers using the QIAGEN PCR Cleanup Kit and protocols contained therein. 
The amplified products from each of these reactions were then utilized as template for sequencing using the modified Sanger chain terminator method. Each primer requires its own sequencing reaction resulting in four sequencing reactions for each original hantaviral sample ( $\mathrm{G} 1$ set and $\mathrm{G} 2$ set). The sequencing reactions were set up with $0.6 \mathrm{ml}$ micro centrifuge tubes recessed in an ice-bath to limit reactivity of constituents until placed in the thermocycler. Each reaction vessel was prepared with 2.0 $\mu \mathrm{l}$ Big Dye Terminator solution (Applied Biosystems), $2.1 \mu 1$ of $2.5 \mu \mathrm{M}$ appropriate primer (SM1723C, ASM2016R, SM2779C, SM3020R), $1.0 \mu 1$ 5x reaction buffer, $4.8 \mu 1$ water, and $2.1 \mu 1$ purified PCR product for a final reaction volume of 12 $\mu 1$. These sequencing reaction vessels were then placed in the Eppendorf thermocycler and exposed to the sequencing thermo protocol (SEQ) as:

25 Cycles $\left\{\begin{array}{cc}96^{\circ} \mathrm{C} & 5 \mathrm{~min} \\ 96^{\circ} \mathrm{C} & 30 \mathrm{sec} \\ 50^{\circ} \mathrm{C} & 15 \mathrm{sec} \\ 60^{\circ} \mathrm{C} & 4 \mathrm{sec}\end{array}\right.$

Hold : $\quad 4^{\circ} \mathrm{C}$

Upon completion of sequencing cycles, reactant solutions were plated in a 96 well micro titer plate that was then sealed and frozen at $-70^{\circ} \mathrm{C}$. The sequence reaction products were then shipped over night on dry ice to the Center for Genome Research and Bioinformatics (CGRB) Core Laboratories at Oregon State University, Corvallis, OR. There, sequences were analyzed on an Applied Biosystems (ABI) 3730 Capillary Sequence Machine. 


\section{Sequence File Preparation-}

After the ABI 3730 generated the representative chromatograms they were downloaded via the internet from the CGRB Core Laboratories web site. Next, the sequence file chromatograms were uploaded into the software program SeqEd (Applied Biosystems v. 1.0.3). Within this program, sequences from corresponding primer pairs are viewed and edited for quality. First, the negative strand sequence is "reverse complimented" where the entire sequence is turned around to orient the last nucleotide first and the first nucleotide last. Then the bases of this reversed sequence are converted to their complementary nucleotide. Next, unidentifiable bases that occur at both the beginning and the end of the sequence were removed. After this is done "comparative alignment" was selected in the program menu that asks the software to analyze and align the two sequences based upon where they are identical. To identify bases mismatched between the two sequences, a shadow sequence was generated that indicates those mismatches with an asterisk. These labeled mismatched bases were then examined based upon their corresponding peak in the sequence chromatogram. In most cases, one of the two opposing sequence peaks was considerably cleaner, or stronger allowing the base to be "called" as the one indicative of the higher quality peak. In this way sequences were edited and aligned to produce a consensus sequence for the target gene group from each hantaviral genomic segment. This task was undertaken twice for each viral sample representing the $\mathrm{G} 1$ and $\mathrm{G} 2$ coding regions, which were amplified and sequenced separately. 
The $\mathrm{G} 1$ and $\mathrm{G} 2$ consensus sequence fragments from each specimen were concatenated in a text-editing program. The concatenated sequences were imported into MacClade (Maddison and Maddison, 2003) for multiple sequence alignment where sequences from all Hantavirus samples were aligned with one another as well as with other out-group species obtained from NCBI GenBank. The multiple sequence alignment (showing coded amino acids) was adjusted as to codon reading frame to represent the natural transcriptional reading frame. To allow separate adjustment of the $\mathrm{G} 1$ and $\mathrm{G} 2$ reading frames single spaces were inserted between the fragments.

Phylogenetic Analysis-

(refer to pg. 21, Chap. 2)

\section{Results-}

Distance Analyses-

In these analyses Hantavirus sequences were obtained ( $\mathrm{n}=22$; appxs. $\mathrm{F}, \mathrm{H})$ from Peromyscus in the greater Portland, OR region. In addition, a twenty-third Hantavirus sequence was obtained from the blood of a current HPS patient. Based upon a 617 nucleotide fragment of the M segment RNA, glycoprotein precursor gene, Oregon hantaviruses were sequenced for comparison to other hantaviruses (sequences from GenBank, appx. F) from North America and the rest of the world. Genetic divergence (p distance) analysis was conducted using PAUP (Swofford, 1998). Figure 15 and appendix B contain pair-wise genetic distances for the Portland viruses relative to 
most other North American (Cricetidae-borne) hantaviruses and others from throughout the world (Muridae-borne). Both uncorrected and corrected genetic distance data are shown. Corrected genetic distances were calculated by application of the general time reversible model of evolution allowing a proportion of sites to be invariant with a gamma distribution of that variance across sites $(\mathrm{GTR}+\mathrm{I}+\Gamma)$. Most publications report uncorrected genetic distances, so these will be the focus here for more clarity of comparison.

The Portland area hantavirus referred to as Kalapuya virus (KV) here.(after the original inhabitants of the Willamette Valley - the Kalapuya tribe), possesses an uncorrected genetic distance of 0.112 (fig. 15) from its nearest known (geographically and genetically) congener, Convict Creek virus (CC) found in the eastern Sierra Nevada Mountains in east-central California (Schmaljohn et al., 1995). Intra-specific average nucleotide genetic distance for the Portland area hantaviruses was 0.017 , indicating the lack of diversity expected within a local population (Monroe et al., 1999). Included among these Kalapuya viruses is the sequence recovered from a Washington County, OR human patient serum. This sequence had a genetic distance of 0.014 from Kalapuya virus sequences found in mice from the patients home and 0.016 from the whole of KV. Viral sequences WC7 and WC13 were identical to one another and both most resembled the patient virus at $p=0.008$, less than half the distance of the patient virus from the other sequences recovered from the patients home, making it likely that one of the these two mice may have originally possessed the virus(es) that infected the patient (appx. B). 
The next nearest $(\mathrm{p}=0.143)$ described Hantavirus after $\mathrm{CC}$ is Sin Nombre virus (SNV) from the four corners region in the American Southwest (Childs et al., 1994). Peromyscus-borne hantaviruses in the American west are collectively known as the Sin Nombre-like group (Morzunov et al., 1997). Within this western North American region is El Moro Canyon virus (EMC) from southern California, but as seen in Figs. 16-18, El Moro Canyon virus is not within the Sin Nombre group and is in fact quite divergent with a $p=0.278$ from the Portland area virus and $p=0.269$ from Sin Nombre. Fig. 15 also reveals two other hantaviruses from west of the Mississippi River in Blue River virus (BR) hosted by Peromyscus leucopus (western haplotype), and Bayou virus (BV) reservoired by Oryzomys palustrus. Similar to El Moro Canyon virus, Bayou virus $(p=0.273)$ is quite divergent from Portland and also is not from within the Sin Nombre group (figs. 16-18). Blue River virus $(\mathrm{p}=0.236$ ) while also significantly divergent from Sin Nombre, is reservoired by a Peromyscine, and therefore is considered part of the Sin Nombre-like group (Morzunov et al., 1997). Of note, Blue River virus has also been described from Indiana (Morzunov et al., 1997) but BR sequences used in these analyses were derived from Oklahoman specimens.

Other more genetically distant hantaviruses seen in Fig. 15 are New York virus (NY) reservoired by the eastern haplotype of $P$. leucopus, and Black Creek Canal virus from south Florida found in Sigmodon hispidus. New York virus is shown to possess a genetic distance of 0.215 from Portland virus, similar to the other $P$. leucopus virus, Blue River at 0.236. The final Hantavirus seen in Fig. 15 is Prospect Hill virus found in the Northeastern United States and hosted by the arvicoline Microtus pennsylvanicus. Not known to cause human disease, Prospect Hill virus is 
the most genetically divergent Hantavirus in North America with a $\mathrm{p}=0.331$ from Kalapuya virus.

\section{Maximum Likelihood Analysis-}

The Hantavirus sequences examined with genetic distance methods were also subjected to Maximum Likelihood analysis. Using the GTR $+\mathrm{I}+\Gamma$ model of evolution as chosen by MODELTEST (Posada and Crandall, 1998), maximum likelihood analyses using PAUP (Swofford, 2002) resolved a tree with a score of $\operatorname{lnL}=8011.8281$ and an Akaike Information Criterion (AIC) of 16043.6562. The likelihood tree was calculated using the tree bisection-reconnection (TBR) swapping algorithm. Nucleotide frequencies were $A=0.33470, T=0.30010, C=0.16080$, $\mathrm{G}=0.20440$. Among-site rate variation includes an assumed proportion of invariable sites of 0.1364 . A gamma distribution of rates across variable sites was implemented with a shape parameter of 0.5469 and four rate categories. Node support for the likelihood tree was generated using 1000 bootstrap replicates.

Similar to the genetic distance tree in Fig. 16, the boot strapped Maximum Likelihood tree in Fig. 17 shows the Portland virus as sister to Convict Creek virus. This same clade is also nested in Sin Nombre virus. Each of these nodes is supported above 90 percent, with the separation of the Portland virus from Convict Creek in 990 of 1000 bootstrap replicates. Differing from the genetic distance tree, the likelihood tree places El Moro Canyon virus outside of the Sin Nombre-like Hantavirus clade, more in line with previous research (Hjelle et al., 1994; Morzunov et al., 1997; Vapalahti et al., 1999; Vincent et al., 2000). Supported at 97 percent the three 
arvicoline viruses, Prospect Hill, Tula virus, and Puumala virus, were separated in a single clade revealing their genetic similarity with one another but in contrast with their geographic disparity. The node containing these divisions was supported in 1000 of 1000 replicates. Bayou and Black Creek Canal viruses formed their own clade. Although only tepidly supported, all South American hantaviruses were contained within a single clade as well. Fig. 17 shows all of the New World Hantavirus clades nested within and monophyletic from Seoul virus. The cricetid (neotomine, sigmodontine, and arvicoline) hantaviruses in Fig. 17 are found nested within the Asian, European, and African murine hantaviruses (Hantaan, Dobrava, and Sangassou viruses).

\section{Bayesian Analysis-}

Summarized consensus tree data may be viewed in Fig. 18 with posterior probabilities shown for each bifurcation. The Majority-Rule Bayesian consensus tree yielded a topology very similar to the genetic distance tree (Fig. 16) and similar to the maximum likelihood (Fig. 17) phylogeny. In Fig. 18 the Portland virus is found sister to Convict Creek virus, which in turn is sister to Sin Nombre virus. These western United States hantaviruses are seen sister to the eastern U.S. hantaviruses, New York, and Blue River. These Sin Nombre-like hantaviruses form a single monophyletic clade sister to the clade found to contain the South American hantaviruses and the two southeastern U.S. hantaviruses, Bayou and Black Creek canal. In the same fashion as the likelihood tree in Fig. 17, the Bayesian tree in Fig. 18 also reveals the $R$. megalotis reservoired El Moro Canyon virus outside of each of these clades that are nested 
within EMC in the Bayesian tree but not the Neighbor-Joining distance tree (Fig. 16). In the Bayesian analysis the two major clades described above, along with El Moro Canyon virus, are nested in the three arvicoline hantaviruses, Prospect Hill, Puumala, and Tula. Again, as seen in the likelihood tree, the neotomine and arvicoline Hantavirus clades are nested in the murine hantaviruses from Europe, Asia, and Africa.

\section{Discussion-}

Phylogenetic analyses of Kalapuya virus (KV) were based upon 617 nucleotides of the medium (M) segment genomic RNA. This particular fragment of the genome was selected for these analyses because the envelope glycoproteins are used for host cell entry and quite possibly under the most selective pressure, making it a prime location for divergence in the genome. In addition, most literature on this subject use this region of the genome, making these data directly comparable to the rest of the literature. This fragment codes for two portions of the glycoprotein precursor gene, corresponding to segments of the G1 and G2 coding regions. Figures 17 and 18 have revealed the robustness of these analyses by showing the pair-wise genetic distances among commonly evaluated hantaviruses to be nearly identical to those resolved by Morzunov et al. (1998). For example, using M segment data, Morzunov et al. (1998) found Sin Nombre virus (SNV) to be at a genetic distance of 0.183 from another Sin Nombre group virus, New York virus, while these data herein show 0.189 , also similar is Sin Nombre to Blue River at 0.195 / 0.201, and Sin Nombre to E1 Moro Canyon at $0.288 / 0.269$. This congruity lends strength to the pair-wise genetic distances 
resolved by these analyses between the previously un-described Kalapuya virus and Sin Nombre virus $(\mathrm{p}=0.143)$, as well as the genetic distances between $\mathrm{KV}$ and Convict Creek virus $(\mathrm{CC})(\mathrm{p}=0.112)$ and $\mathrm{CC}$ and $\mathrm{SNV}(\mathrm{p}=0.130)$. Convict Creek virus has been characterized as at least a new strain of Hantavirus within the Sin Nombre group, if not a new species (Schmaljohn et al., 1995; Li et al., 1995). If Convict Creek virus has been classified as distinct from Sin Nombre, then, so too should Kalapuya virus, found at an even greater genetic distance from SNV than is CC.

Hjelle et al. (1995a), and Morzunov et al. (1998) have proposed that three lines of evidence be considered when evaluating hantaviruses for possible speciation. First is immunological by using plaque reduction neutralization assays (PRNA), where neutralizing species specific antibodies are bound to viral particles and infection susceptible cells are exposed to the resulting virus-antibody complex. Then speciation is defined by at least a four-fold, two-way difference between putative species, described as the known virus species having four times as much antibody binding (and therefore neutralization) as unknown comparative hantavirus species. The second uses ecological or physiological evidence as found in the viral host. For viral speciation to have occurred, it must be found in a distinct rodent host, known to reservoir no other Hantavirus. Morzunov et al. (1998) proposed the adoption of the definition of a different rodent host as "a reproductively and/or ecologically and/or geographically separated rodent species or subspecies, which carries a phylogenetically distinct Hantavirus and which is capable of being the sole carrier of this virus in a rodent community". Third, molecular evidence must reveal significant nucleotide differences from other described hantaviruses. Puthavathana et al. (1993) proposed, which has 
since been adopted elsewhere, that Hantavirus speciation is said to have occurred at more than a $25 \%$ nucleotide difference in the glycoprotein precursor gene open reading frame. A nucleotide difference of 15 to $25 \%$ was considered a "grey area" where phylogenetic analyses are then required for proper evaluation of speciation (Puthavathana et al., 1993).

In consideration of these criteria, the results of the analyses contained herein are discussed further. In terms of immunological evidence, even though Sin Nombre virus (SNV) has been adapted to cell culture, this examination is not currently possible as the Portland Hantavirus has yet to be adapted to cell culture, for which hantaviruses are notoriously difficult, thereby prohibiting the PRNA.

Criterion two, ecological or physiological evidence, is also difficult to evaluate. What is known, is the hosts of SNV and KV are geographically isolated from one another with the SNV host, $P$. m. rufinus, found in the four corners region, some 1,700 kilometers $(1,100$ miles) from western Oregon. In general terms, the vast difference in the disparate climes occupied by these conspecific hosts (temperate rain forest in western Oregon compared to scrub desert in the four corners region) leads to the logical conclusion that these two hosts, however defined, are also ecologically isolated from one another. Not known is the level of reproductive isolation that may exist between these two hosts, as those examinations have yet to be conducted. Not addressed by Hjelle et al. (1995a), but appropriately included in the discussion of different hosts, is the phylogenetic relationship between the two hosts based upon molecular evidence. As has been discussed previously, rodent phylogeny, and especially that among Peromyscus, is unresolved. Rodent phylogenies produced in 
these analyses, seen in Figs. 11 through 14 reveal the distinct possibility that Peromyscus maniculatus rubidus, from which the Kalapuya viruses were obtained, may in fact be a distinct species (along with other nearby allopatric subspecies) from the topo-typical Peromyscus maniculatus maniculatus. Making this assessment more difficult is the lack of a comparable sequence (mtDNA ND3, ND4, and ND4L) from the Convict Creek host to have allowed its inclusion in the Peromyscus phylogeny. The pair-wise genetic distance between the SNV reservoir, $P . m$. rufinus, and P. $m$. rubidus is seen at 0.025 (figs. 8,9 ). While this level of divergence is below the typical speciation threshold for rodents of roughly $4 \%$, Fig. 8 does show that $P$. m. rubidus is, as stated above, quite likely a separate species from $P$. m. maniculatus with a pair-wise genetic distance of $4.1 \%$. Moreover, the Bayesian tree seen in Fig. 13 shows the Pacific Northwest subspecies as a separate clade nested within P. m. rufinus.

The lack of complete data for more thorough analyses of the first two criteria has resulted in greater emphasis placed on the third criterion, molecular evidence. Molecular and phylogenetic evidence is the focus of these analyses and has revealed Kalapuya virus with a genetic distance from Sin Nombre virus of 0.143 , less than the definitive threshold for speciation as proposed by Puthavathana et al. (1993) of 25\%, is, however, near the lower limit of the "grey area" that was discussed by the same authors.

Maximum Likelihood and Bayesian phylogenetic analyses contained herein (Figs. 18-19) have strengthened the genetic distance analysis that found Kalapuya virus nested within and monophyletic to Convict Creek and Sin Nombre viruses. As all three are hosted by subspecies of $P$. maniculatus, and considering other North 
American hantaviruses, reservoired by the congener $P$. leucopus, are also recognized as part of the Sin Nombre group, this monophyly was expected.

In light of the pair-wise genetic distance as found at the lower limit of the grey area criterion and its relative phylogenetic location strongly within the Sin Nombre group, it may be argued by some that the Kalapuya virus has not yet speciated. That said, the level of genetic divergence from Sin Nombre virus does strongly argue that $\mathrm{KV}$ is on a separate evolutionary trajectory and are at the very least nearing speciation, if that level of distinction has not yet been achieved. The findings of the Peromyscus phylogenetic, geographic, and ecological relationships discussed above lend themselves to this argument. Further analysis of the complete Kalapuya virus genome, even greater elucidation of the host relationships through further molecular comparison as well as immunological analyses may in fact lead to such a specific classification in the near future. It is therefore concluded that Kalapuya virus - in honor of the first human inhabitants of the Portland, OR region, may in fact be a previously un-described species of Hantavirus, and minimally constitutes a new strain. 


\section{Chapter 4: Peromyscus and Hantavirus Coevolution}

\section{Abstract-}

It has been proposed that hantaviruses are found in a species-specific relationship with their hosts and have experienced a co-evolutionary history. Phylogenetic analyses performed here have been found in support of these studies and revealed the probability of the presence of a new deer mouse species, minimally in Oregon, if not including much of the western United States. As suggested by others, this western deer mouse in Oregon is referred to as Peromyscus sonoriensis rubidus. In light of the co-evolutionary relationship, as well as further phylogenetic analyses contained herein, the hantavirus reservoired by $P$. s. rubidus is in fact a new quasi-species of hantavirus, separate from Sin Nombre virus and Convict Creek virus, proposed as Kalapuya virus.

\section{Methods:}

Sets of hantavirus and host sequence specimens, as described in chapters 2 and 3 , were reduced to a subset of specimens consisting of only those from North America, including from elsewhere only Hantaan virus and its host, Rattus norvegicus for outgroup comparison. Bayesian analyses were performed on these two sets of data using the same Bayesian protocols as described in chapter 2 (pg. 36). The two resulting majority-rule trees were aligned for comparison after rotating the host tree to its mirror image. 


\section{Results:}

In addition to the previously discussed observations for individual phylogenies of hantaviruses and hosts a comparative Bayesian double phylogenetic tree of these hantaviruses and hosts is seen in Fig. 19. Included on the left of this figure are most of the hantaviruses from North America, excluding only those found in a reservoir already represented here by another Hantavirus, and the other significant mile-post Hantavirus, Hantaan virus from Korea, as the out-group. The right side of the figure shows the known reservoirs for these hantaviruses. With consistently strong posterior probabilities ( $\mathrm{PP}>96)$, the comparative phylogenies are also found to be near perfect mirror images of one another.

Only the location within the tree of El Moro Canyon virus relative to its known host, Reithrodontomys megalotis, is in dischord with parallel evolutionary histories. While $R$. megalotis is found more closely allied with other Peromyscus, El Moro Canyon virus is associated with hantaviruses (Bayou, Black Creek Canal) hosted by more distantly related Sigmodontines (O. palustrus, $S$. hispidus).

\section{Discussion-}

Peromyscus speciation-

Recently, Dragoo et al. (2006) recognized the hantavirus-host co-evolutionary framework in a more geographically comprehensive study in which the authors recognized six separate Peromyscus clades or lineages but, as noted by the authors, missing from their analyses are data from Oregon. Of those lineages found, one that was suggested existed in the Pacific Northwest coastal region. It was also proposed 
that an expanding Rocky Mountain clade is overlapping with this coastal clade, said to include $P$. keeni, $P$. sejugis, and P. maniculatus gambelii (Dragoo et al., 2006). Conflicting with this proposal is the fact that Peromyscus analysed in this study included P. m. gambelii from east of the Cascade Mountains in Oregon that were not seen to be significantly different from $P . m$. rubidus, on the west side of the Cascades. Also, these analyses found the Oregonian deer mice to be slightly more distant from the coastal $P$. keeni/sejugis clade $(0.0556)$ than the $P$. m. maniculatus $(0.0416)$ clade from central-northeastern North America, west of the St. Lawrence River, but very closely associated with the Rocky Mountain clade (0.0248). Contrary to Dragoo et al. (2006), Washington coastal $P$. m. austerus, which might have been assumed to reside with the coastal clade were not found associated with the coastal clade as hypothesized by those authors, but instead correlated very closely with the other conspecifics $P$. $m$. rubidus, and P. m. gambelii (Figs. 10-13) from Oregon, seen here linked to the Rocky Mountain clade (Figs. 8, 9). Therefore these data in conjunction with those found by Dragoo et al. (2006) suggest that there may in fact be several distinct deer mouse lineages in western North America, with those from Oregon, and possibly Washington as well, associating with the Rocky Mountain clade and not the Coastal clade. In addition, the analyses contained herein align with the findings of Hogan et al. (1997), and Dragoo et al. (2006) in suggesting that a deep genetic division occurs between the greater western North American and the eastern North American deer mouse clades, with this break (as proposed here) along the Mississippi River corridor. It has been proposed by Dragoo et al. (2006) that the Rocky Mountain clade may represent the previously described morpho-species Peromyscus sonoriensis (Le Conte, 1853). 


\section{Hantavirus speciation and Coevolution-}

Phylogenetic trees seen in Figs. 17-19 as well as the pair-wise genetic distances in Fig. 15 reveal Kalapuya virus (KV) closely associated with Convict Creek Virus (CC) $(\mathrm{p}=0.112)$ and Sin Nombre Virus (SNV) (0.143), all of which are reservoired by putative hosts ( $P . m$. sonoriensis and $P . m$. rufinus respectively) found to reside within the Rocky Mountain clade (proposed as P. sonoriensis).

The Hantavirus/host double phylogeny seen in Fig. 19 displays the suggested cophylogeny implied by the co-evolution seen between hantaviruses and hosts. The intrigue of this figure is borne-out by the virtually identical topologies of these two trees, only the split between El Moro Canyon virus (EMC) and the nested clade containing Bayou virus (BAY) and Black Creek Canal virus (BCC) differs from the reservoir tree topology for these taxa in that the reservoir tree places the hosts for BAY and BCC (Oryzomys palustrus and Sigmodon hispidus respectively) as a clade sister to, instead of nested within the EMC host (Reithrodontomys megalotis) as is seen for the virus tree. All other hantaviruses examined in Fig. 19, including Kalapuya virus, are found in a perfect mirror-image association with their putative reservoirs, representing mirrored, or co-evolution.

\section{Conclusions-}

Peromyscus maniculatus rufinus in the southwestern United States has for the past 14 years been considered to be the reservoir of Sin Nombre Hantavirus (Childs et al., 1994). Peromyscus maniculatus sonoriensis in eastern California has been recognized 
as the host for Convict Creek virus for the last 13 years (Schmaljohn et al., 1995). Armed with the knowledge that species of the genus Hantavirus are associated with specific host species, and that both virus and host evolve within this co-evolutionary framework (Monroe et al., 1999; Dragoo et al., 2006), and that the Portland (and likely all western) deer mouse range is composed of what may in actuality be a species distinct from P. maniculatus sensu stricto, it is reasonable to hypothesize that hantaviruses found in these reproductively isolated "P. maniculatus" populations also will have undergone a similar level of differentiation into a putatively novel viral species distinguishable from Sin Nombre virus at a molecular level. The data contained herein bear that out. It is therefore concluded that Kalapuya virus, found in Oregon deer mice, is a formerly undescribed species of Hantovirus, and the Oregon deer mouse, its reservoir, is in fact a separate species of the western deer mouse clade proposed as Peromyscus sonoriensis.

Also supported is the hypothesis that coevolution of North American hantaviruses with their hosts is seen in the presence of essentially identical phylogenetic topologies. 


\section{Epilogue: Research Significance}

In 1993 an outbreak of a previously unrecognized disease occurred in the four corners region of the southwestern United States. Subsequently, phylogenetic analyses of the etiological agent for this disease was identified as a formerly undescribed member of the viral genus Hantavirus (Family Bunyaviridae) (Childs et al., 1994). Sin Nombre virus was the moniker given to this new pathogen with the associated pathology described as Hantavirus pulmonary syndrome (HPS). With an initial mortality rate of more than 70\%, HPS is characterized by bilateral pulmonary infiltrates, resulting in pulmonary deficiency and sometimes, failure. While no direct treatment yet exists, mortality has recently been reduced to $38 \%$ through early recognition and subsequent fluid management.

Clouding the understanding of patterns of HPS occurrence is the fact that among the characterized hantaviruses $(>40)$ as many as 20 have not been known to cause disease. Even within the pathogenic group of hantaviruses there exist those with varying degrees of pathogenicity, where some may illicit only minor symptoms instead of the full illness.

The division between pathogenic versus non-pathogenic viral lineage is partially reflected in the reservoir phylogenies. Worldwide, hantaviruses have been recognized in specific mammalian hosts and in the New World, Plyusnin and Morzunov (2001) and others have found a great amount of association among hantaviruses and their hosts. In fact Yates et al. (2002) recognized that Sin Nombre virus, and therefore other Sin Nombre-group hantaviruses, were not introduced to the western hemisphere but instead, during the mid-Miocene, migrated and co-evolved in a very close 
relationship with their hosts as their reservoirs crossed the Bering land-bridge from Asia to North America, entered the New World, and then radiated and differentiated into the Neotominae and Sigmodontinae currently found there. In the process, those resident hantaviruses differentiated as well into the hantaviral lineages currently seen in the western hemisphere. For many years studies suggested these hosts resided only within Order Rodentia. Recently, however, new viral lineages have been described from Soricid reservoirs (Blarina spp. and Sorex spp.) (Arai et al., 2006, 2007) but to date, no Soricid-borne hantavirus has been associated with human disease. There exist, of course, Murid-borne hantaviruses as well that do not generate disease within humans, especially those found within the Arvicolinae such as one North American hantavirus, Prospect Hill virus, reservoired by the Arvicoline Microtus pennsylvanicus.

With the knowledge that hantaviruses are associated with specific mammalian hosts, and are not ubiquitously pathogenic, there arises, therefore, the need for a greater understanding of the distribution of hantavirus hosts (potential and recognized) as well as the putative virus strains themselves. The data contained herein contribute to that growing level of clarity of hantaviral-reservoir evolutionary, phylogenetic, and biogeographic associations. The theory espoused by Dragoo et al. (2006) that deer mouse phylogenies provide a framework, at least potentially, for the elucidation of the distribution of hantaviruses and their phylogenetic relationships to one another are supported by the findings reported here. In point of fact, Dragoo et al. (2006) state that missing from their data are specimens from Oregon of both deer mice and their potential hantaviruses. Again, at the conclusion of this study, those data are now 
provided. As acknowledged previously above, these analyses have shown that there exists an extreme likelihood that the deer mice from Oregon, likely in conjunction with other subspecies in the western United States, are in fact a new species separate from Peromyscus maniculatus sensu-stricto. In addition, the hantaviruses reservoired by the Oregon deer mice represent a new strain, if not a new species, as well. Taken in consideration with the variability of pathogenicity seen in the various Hantavirus strains and species, the new taxonomic designation for Oregon deer mice fills a gap in the current knowledge of those distributions in the Pacific Northwest. This becomes critical when the virulence of the Oregon Hantavirus is taken into account. As of this publication, nine cases of HPS have been documented in Oregon with four of those nine (0.44) resulting in death. While rare, any disease that is known to result in a $44 \%$ mortality rate warrants efforts to understand as much about its epizootiological, phylogenetic, and biogeographic characteristics as possible. Hantaviruses are referred to as emerging infectious diseases (EID), and as humans expand their environment further into natural habitat and more regularly encounter potential wildlife carriers of disease it is critical that the scientific community endeavor to facilitate the understanding of these disease parameters to gain a greater awareness of all the different quasispecies of hantavirus in nature today and the ranges in which they occur. This knowledge will allow a concentration of management efforts of the associated reservoir species, especially those that carry the most dangerous strains of hantavirus, to raise local community awareness and insofar as possible limit the amount of human exposure to the pathogens potentially carried by the mice. 
On a genetic scale, the viral sequences elucidated in these analyses were derived from the envelope glycoprotein-coding region of the viral tripartite genomic RNA. These co-translationally cleaved glycoproteins, G1 and G2, are expressed within and upon the phospholipid bilayer envelope that is procured from the infected cell, which surrounds and encloses the daughter virion. The envelope glycoproteins are believed to play a significant role in cellular recognition, binding of the virus particle to a clathrin coated pit, and subsequent cellular uptake of the virion.

Although attempts have been made, there currently exists no vaccine to inoculate against, or antiviral drug for the treatment of, HPS. Efforts to construct a vaccine to Hantavirus have involved utilization of G1 and G2 based upon previously mentioned fact that those glycoproteins play such a large role in the infection process. By focusing sequencing efforts on the envelope glycoprotein precursor coding gene, basal knowledge for the amino acid, and therefore the protein structure, may be extrapolated from those sequences for further application of the study of the interactions involved at the molecular level in the host cell mediated uptake of the virus particle at the clathrin coated pit.

Procurement of pathogens from their sources, in this case, hantaviruses from deer mice, followed by evaluation of their defining characteristics, epizootiology, and distributions are the first steps in working to avoid and/or offset their ultimate pathology. By identifying and characterizing the Hantavirus from Oregon, as well as its reservoir species, these analyses have contributed to the greater effort to reduce and understand Hantavirus Pulmonary Syndrome. 
Phylogeny of Hantaviruses: Based on Sequence of M Segment

(Subfamily, Hantavirus, Location, Host)

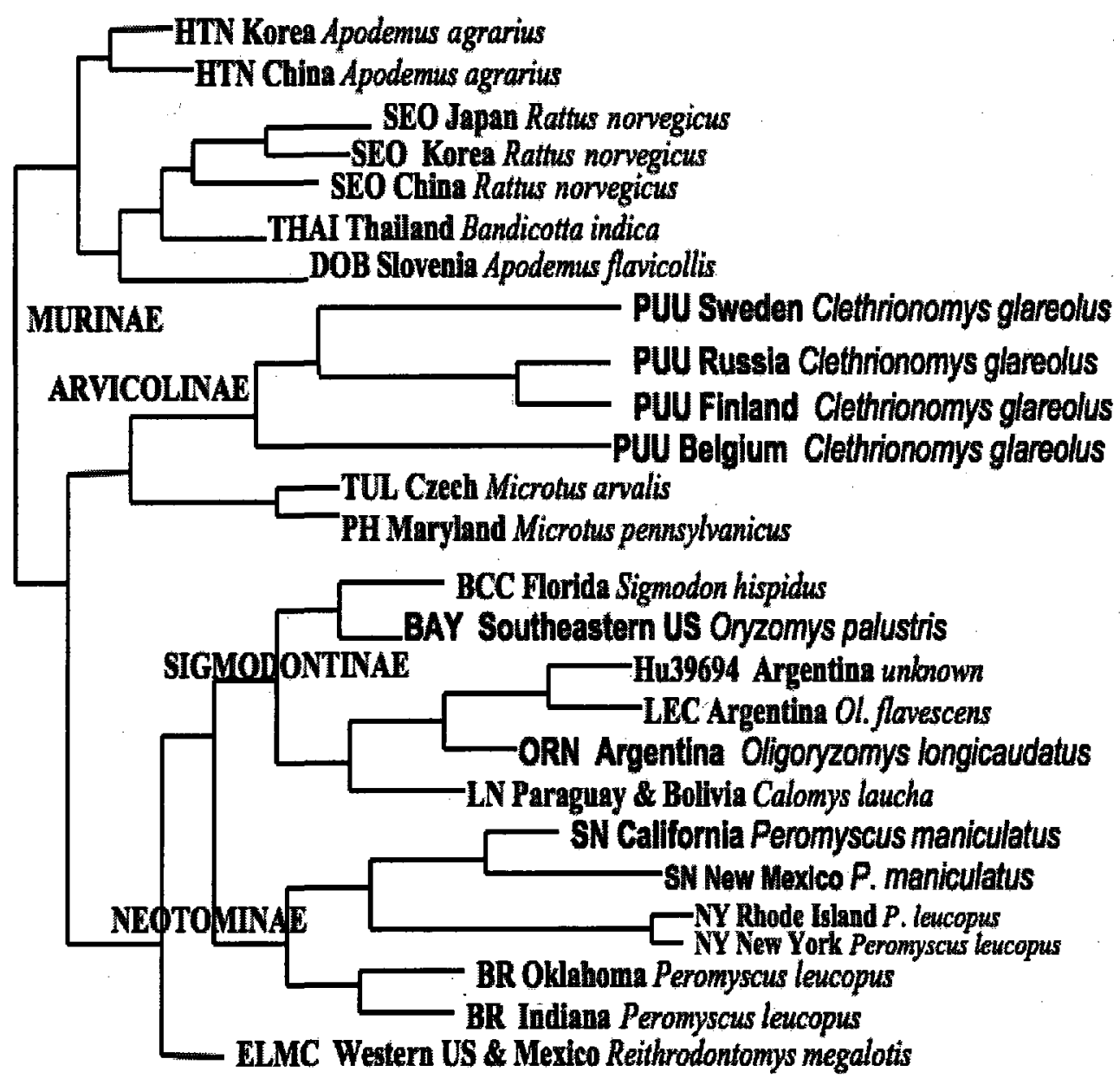

Figure 1: World Wide Hantavirus M Segment Phylogeny. SN=Sin Nombre, NY=New York, BR=Blue River, ELMC=El Moro Canyon, LN=Laguna Negra, $\mathrm{ORN}=$ Oran, $\mathrm{BAY}=$ Bayou, $\mathrm{BCC}=$ Black Creek Canal, $\mathrm{PH}=$ Prospect Hill, $\mathrm{TUL}=$ Tula, $\mathrm{PUU}=$ Puumala, DOB=Dobrava, $\mathrm{THAI}=$ Thai, $\mathrm{SEO}=$ Seoul, HTN=Hantaan.

Reproduced with permission from: Centers for Disease Control, National Center for Infectious Disease, Special Pathogens Branch, Division of Viral and Rickettsial Diseases - HPS Teaching Slideset. 


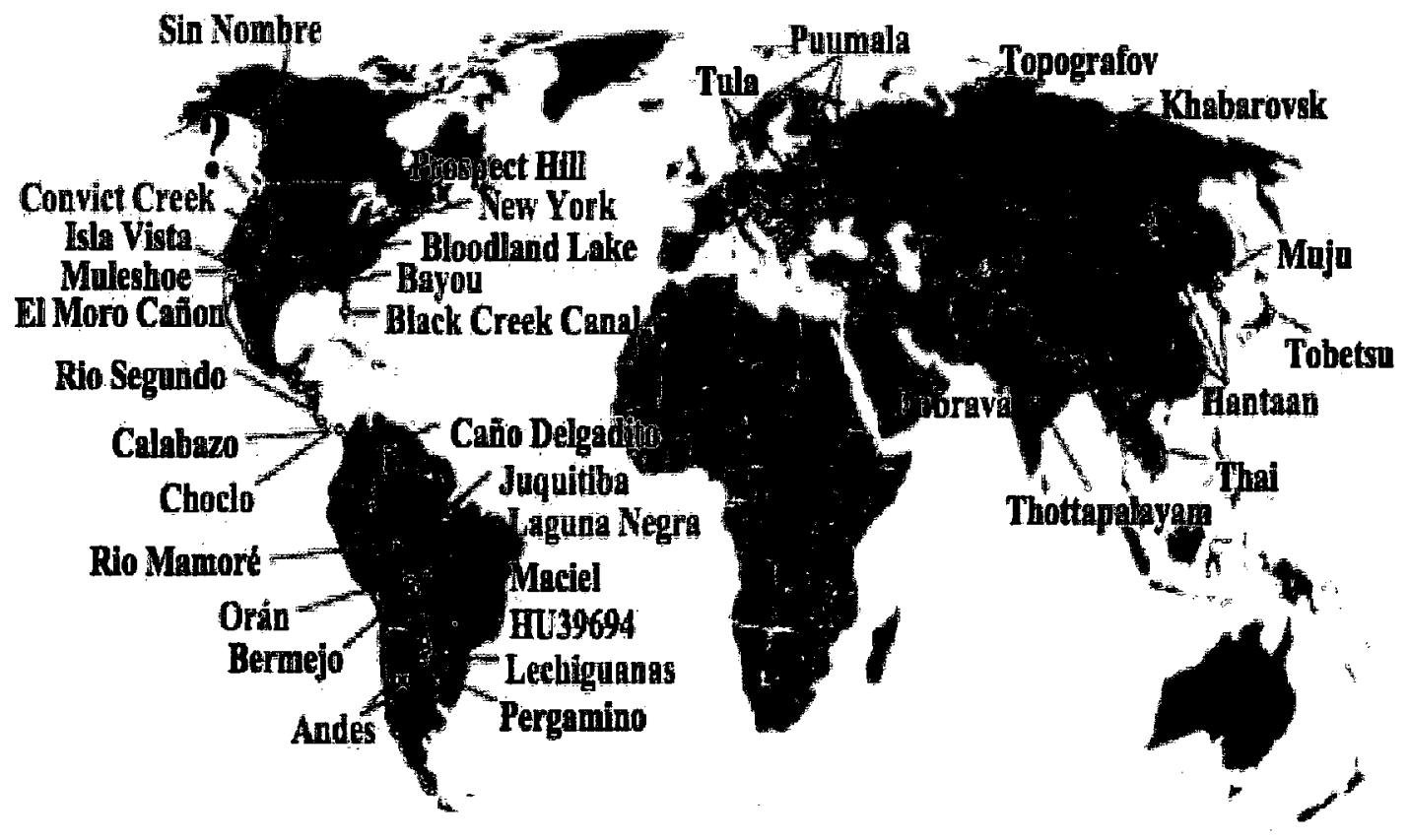

Figure 2: Hantavirus Species World Distribution. Known Hantavirus species and the regions where they are found. Viruses shown in black are not known to cause Hantavirus Pulmonary Syndrome (HPS) in humans. Viruses in magenta are known causative agents of HPS. Blue question mark represents the undescribed Hantavirus in the Portland, OR region. 


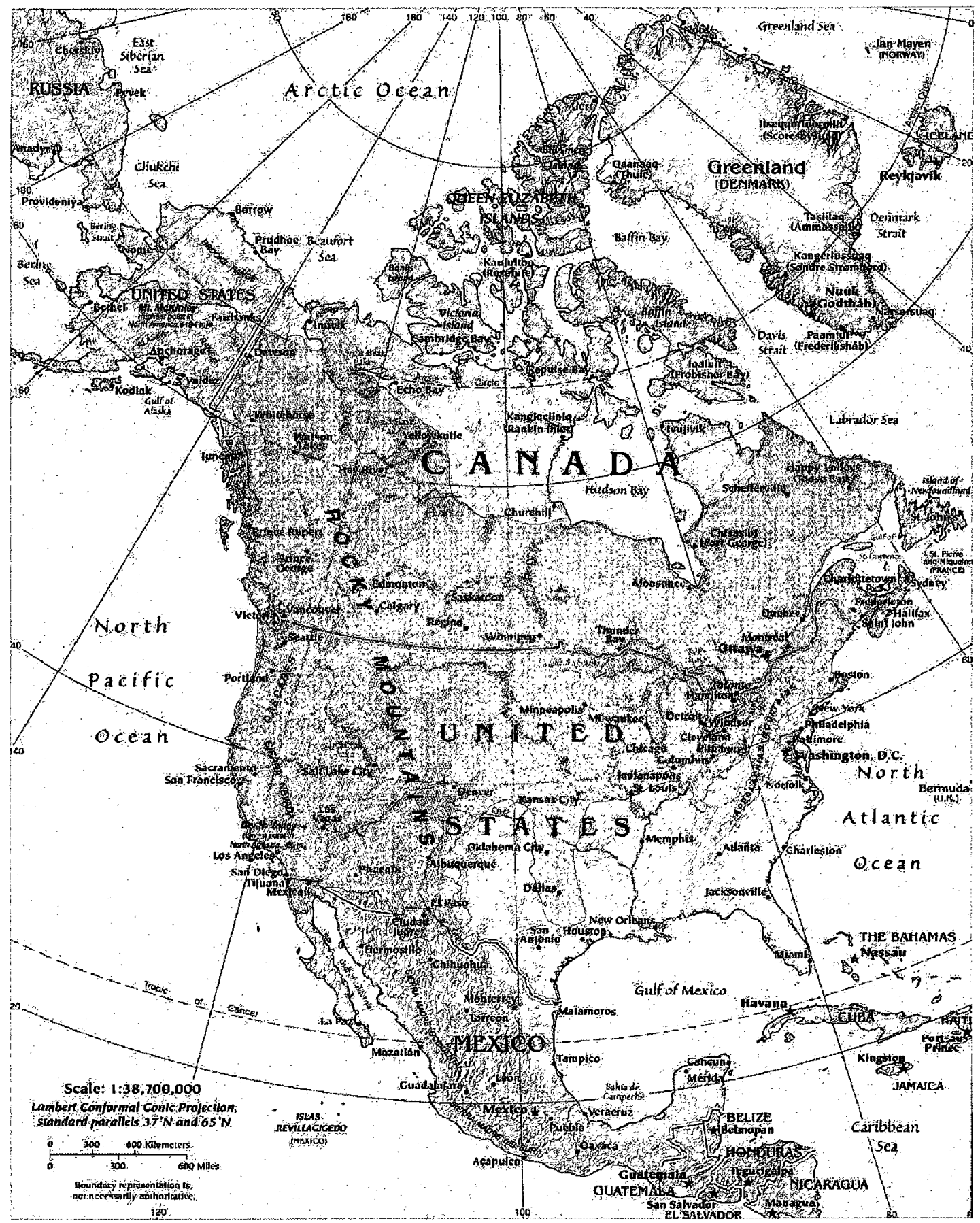

Figure 3: Peromyscus maniculatus Range Map. The purple overlay indicates the geographic distribution of the deer mouse, Peromyscus maniculatus, in North America. Re-drawn from Hall, 1981. 


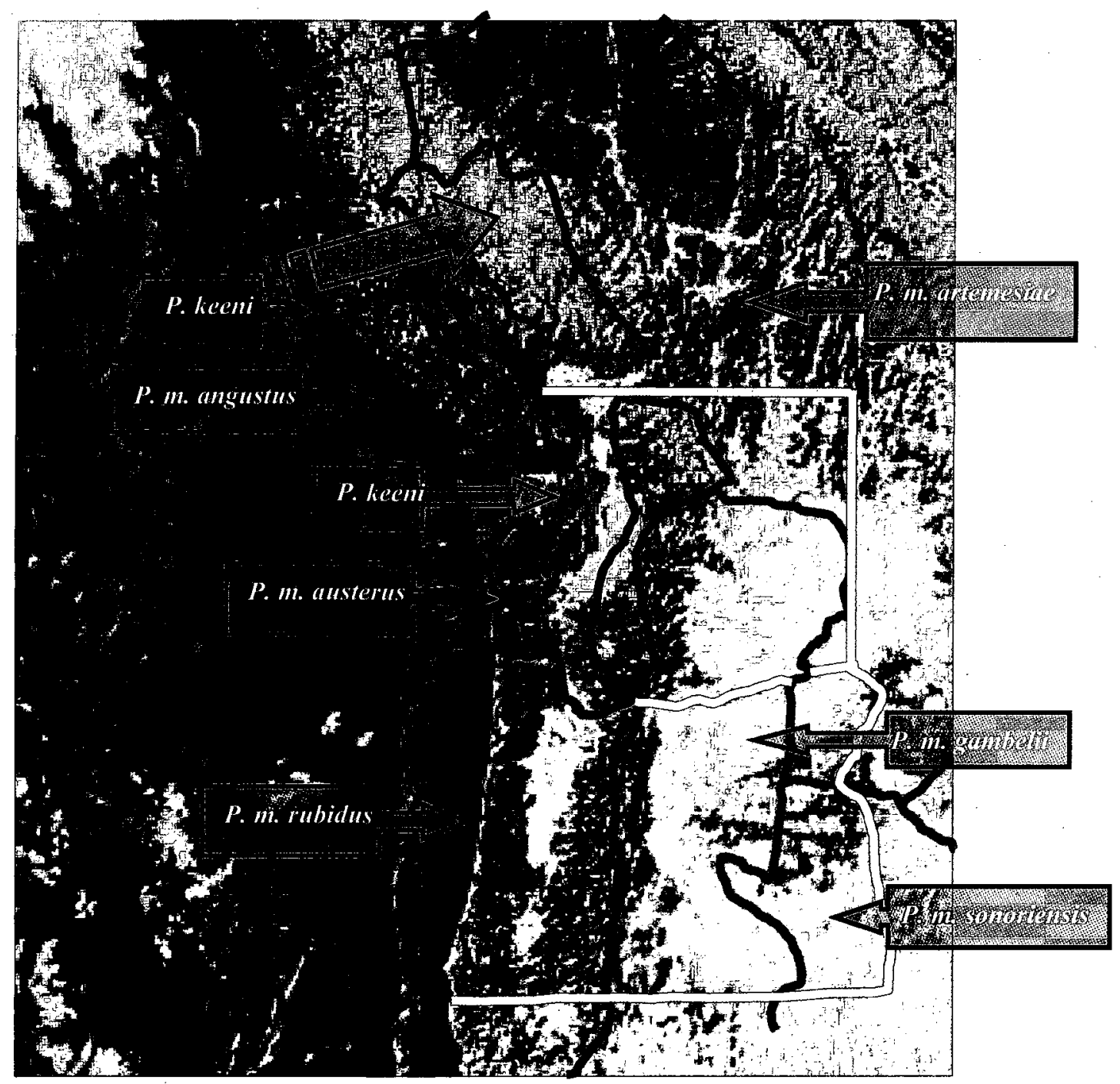

Figure 4: Pacific Northwest Subspecies Map. Distributions of subspecies of Peromyscus maniculatus in the Pacific Northwest of the U.S. and Canada, as well as the disjunct ranges of $P$. keeni. Range limits shown by blue lines. Yellow indicates Oregon and Washington state boundaries except where congruent with subspecies range limit. 

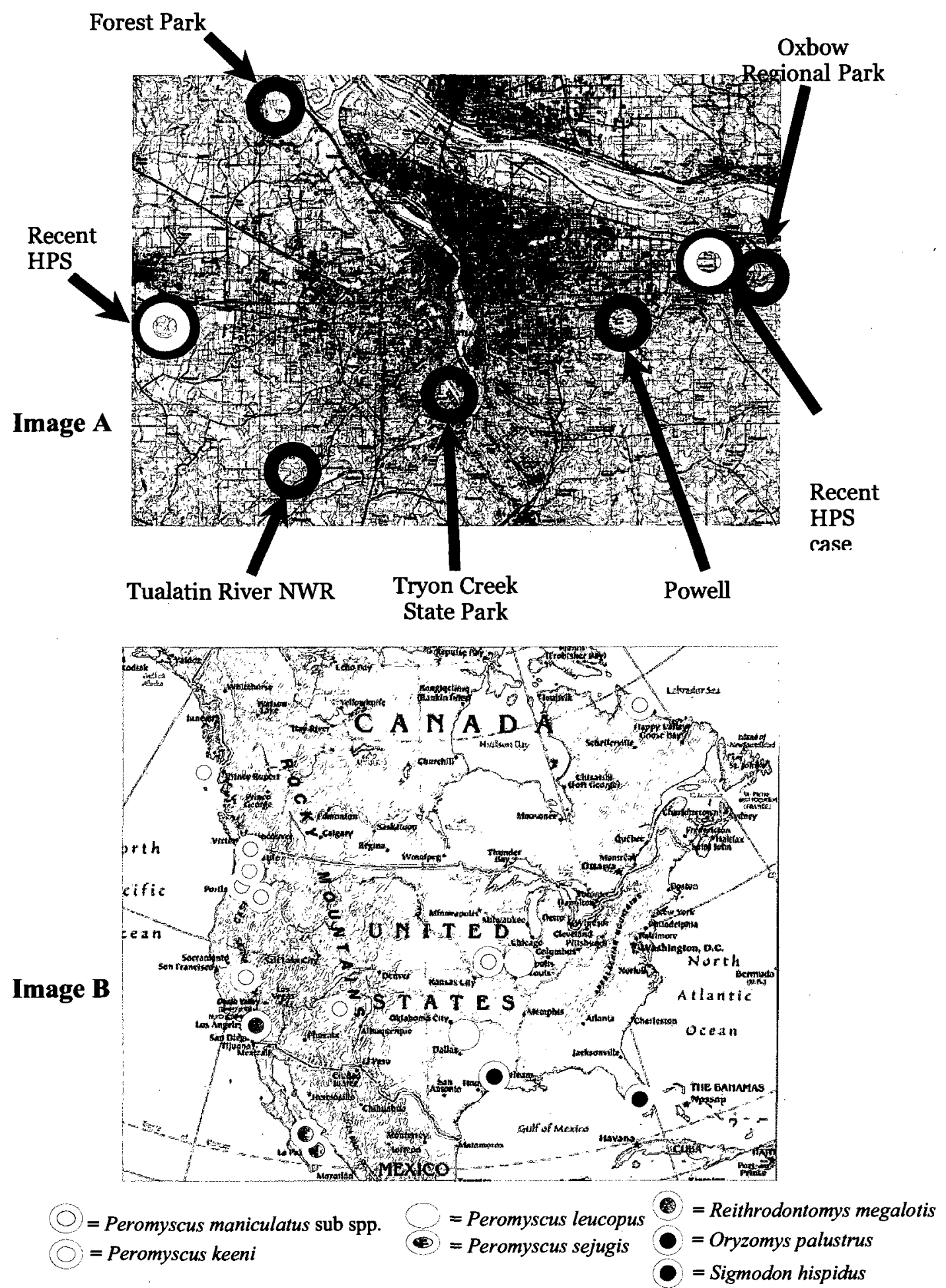

Figure 5: Specimen Collection Sites. Image A: map of Portland, OR, U.S.A. showing field collection sites of Peromyscus maniculatus in the metropolitan area. Concentric blue and light blue rings marks the locations of two recent HPS cases. Image B: locations of specimens from outside Portland, OR. Sequences not from Oregon, Missouri, Illinois, and Labrador were obtained from GenBank. 


\section{Centerpoint: 4 traps \\ o Mesh and Sherman traps \\ Trapping web \\ Mesh, Sherman, and pitfull traps \\ Mesh, Sherman; \\ Tomahawk, and piffall traps}

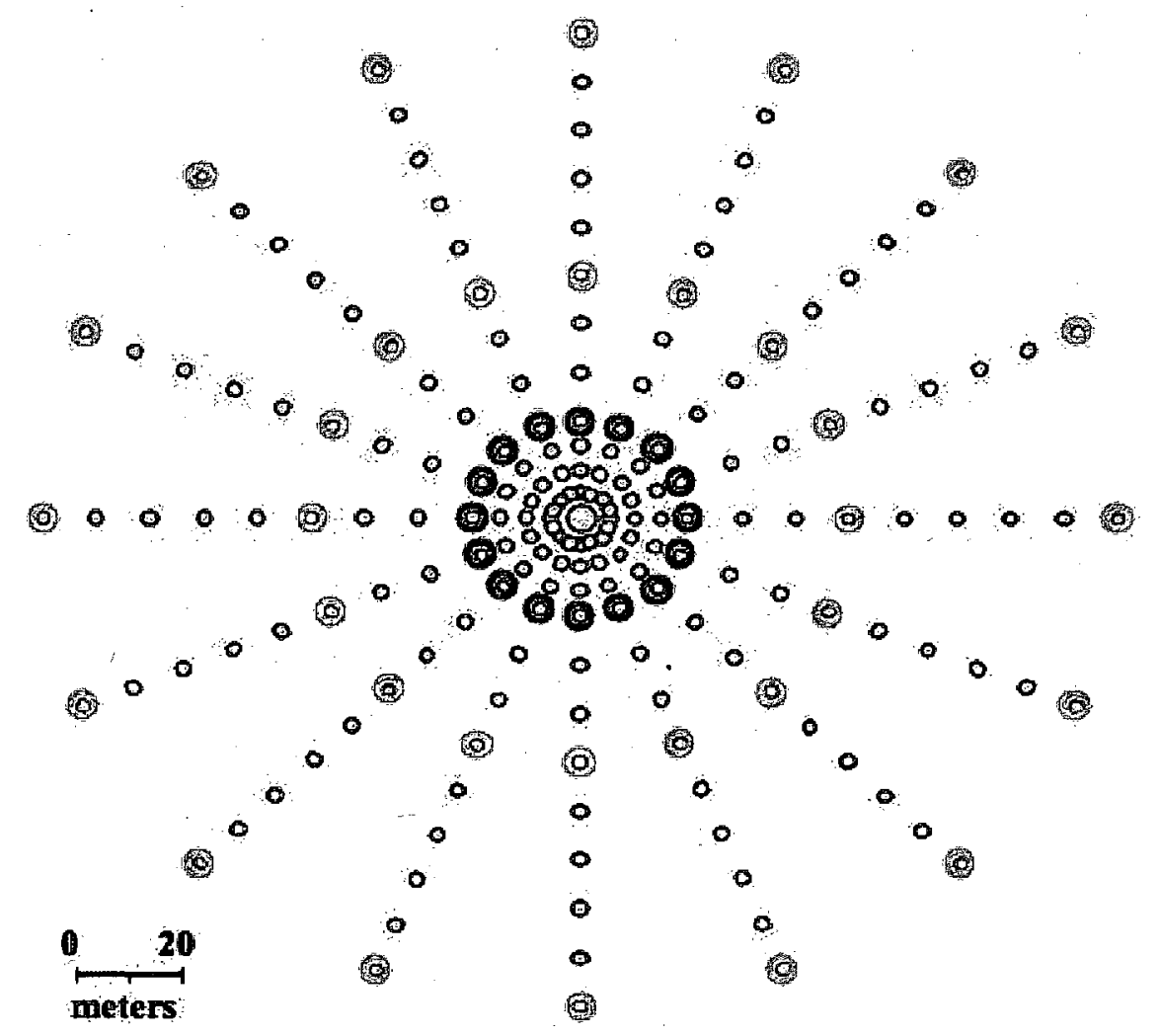

Figure 6: Small Mammal Trapping Web (used for this study). Consists of 12 trap lines radiating and numbered from center at $30^{\circ}$ apart, each containing 12 trap stations. Each line is $100 \mathrm{~m}$ in length from center point with trap stations 1-4 at fivemeter intervals on each line. Subsequent stations 5-12 are at $10 \mathrm{~m}$. Light blue dots are stations with Sherman and Mesh live traps. Those with dark blue rings include pitfall traps along with the Sherman and mesh live traps. Brown rings add a Tomahawk trap to the other three trap types. 

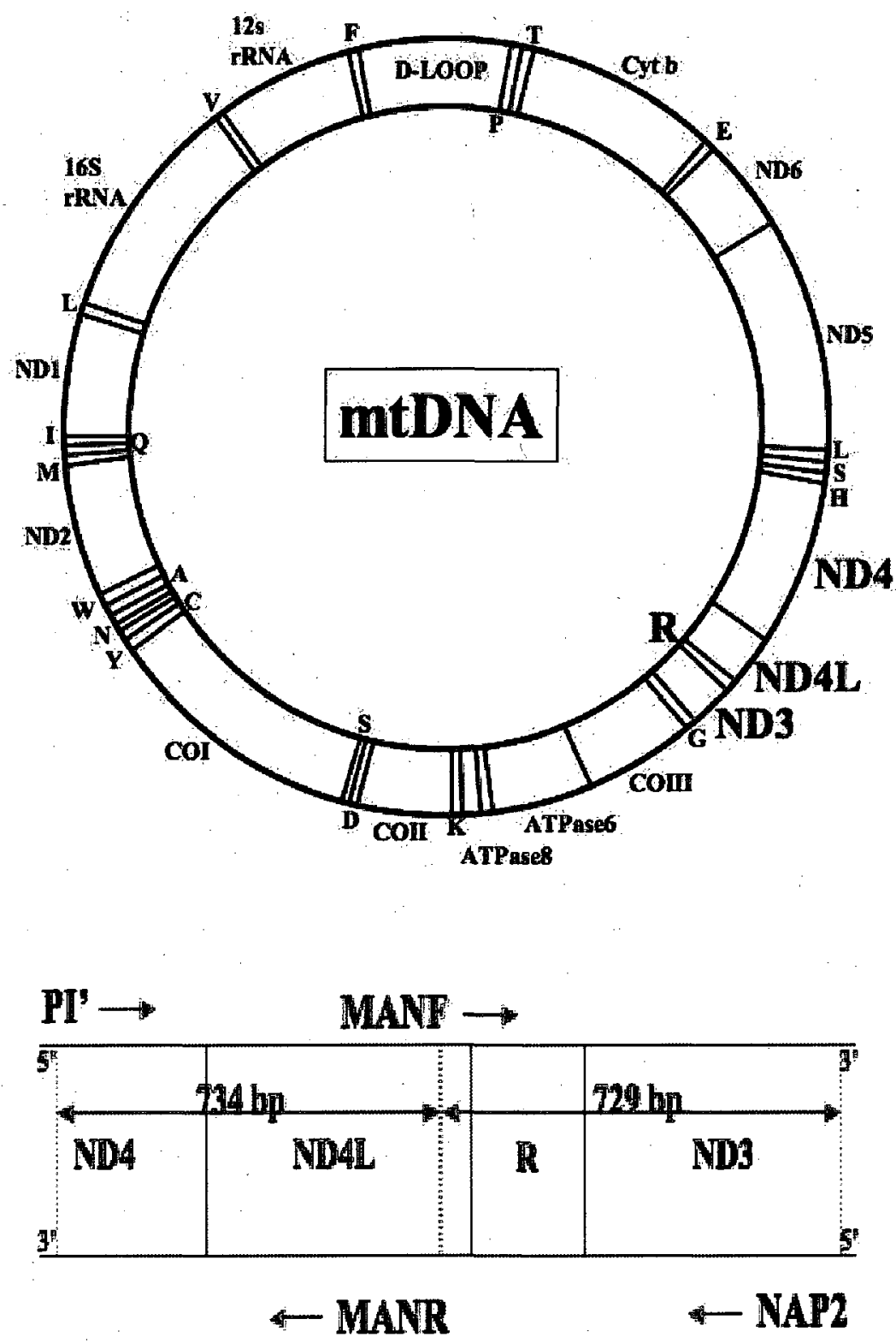

PI': 5' - CGAACTAGTACAGCTGACTTCC - 3'

MANR: 5' - CCGTAGGAGTTTGATAGTTTTGCT -3 , 7 Complimentary MANF: 5' - AGCAAAACTATCAAACTCCTACGG - 3' - (indicated by dotted NAP2: 5' - TGGAGCTTCTACGTGGGCTTT - 3' line)

Figure 7: Peromyscus Mitochondrial Genome Map. Upper: Circular mitochondrial genome at top shows locations of ND3, ND4, ND4L, and arginine tRNA (R) genes that were utilized in this study. Middle: Indicates locations of primers used for amplification and the relative size of amplicons (not to scale). Lower: Primer nucleotide sequences. 


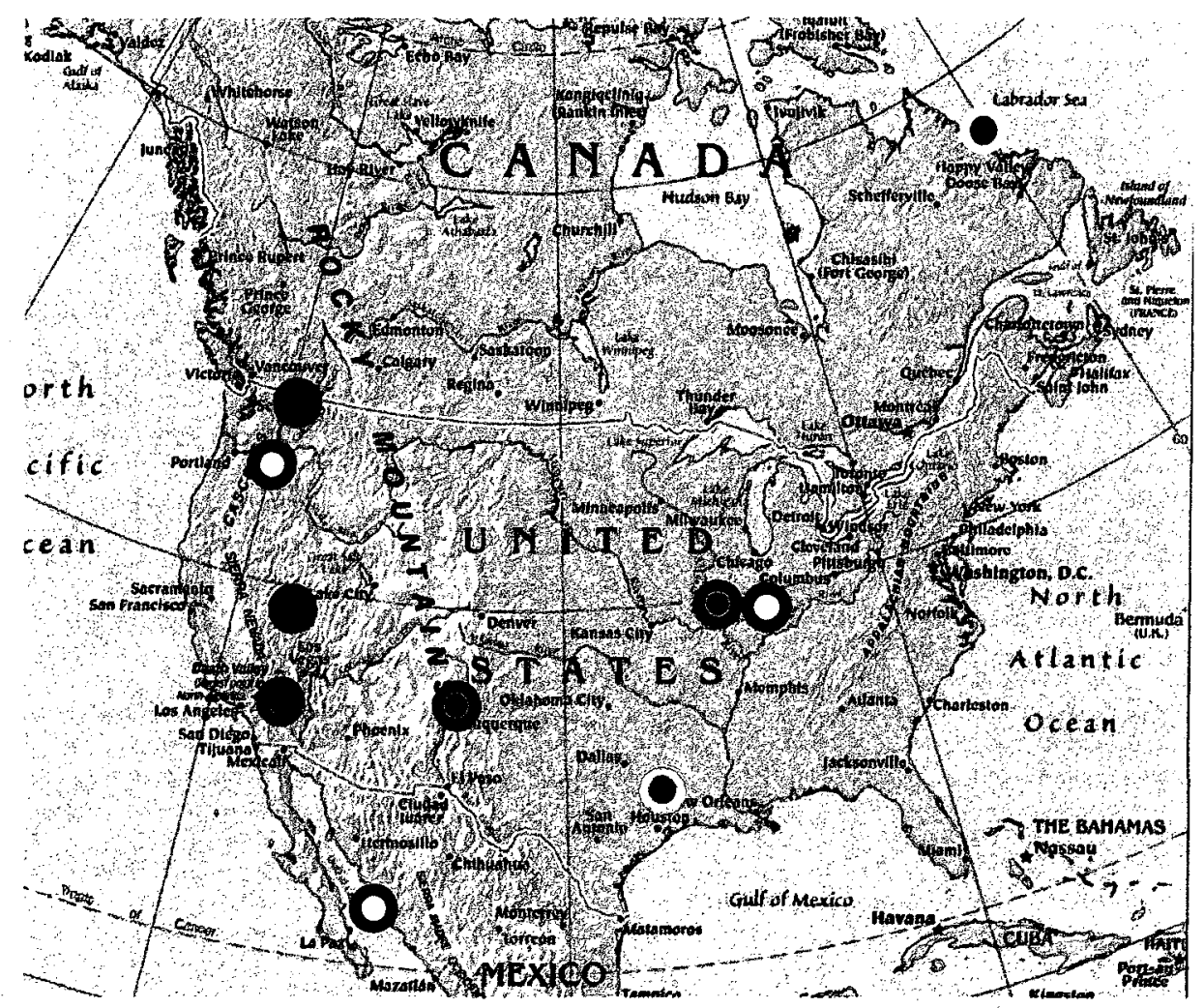

\begin{tabular}{|c|c|c|c|c|}
\hline & \multicolumn{2}{|c|}{ Pair-wise Taxa } & & Avg. Genetic Dist. \\
\hline & P. m. rubidus & P. m. maniculatus & & 0.0413 \\
\hline 0 & PNW clade & P.m.m. & e & 0.0416 \\
\hline & PNW + P.m.r. + P.m.bairdii & P.m.m. & & 0.0457 \\
\hline & P. keeni & $P$. sejugis & & 0.0429 \\
\hline & PNW clade & P.m.b. & & 0.0171 \\
\hline & PNW & P. keeni & & 0.0541 \\
\hline & $P$. sejugis & P.m.m. & & 0.0511 \\
\hline & P. m. rufinus & P.m.m. & & 0.0542 \\
\hline & P. m. bairdii & P.m.m. & & 0.0468 \\
\hline & P. keeni & P.m.m. & & 0.0600 \\
\hline & PNW & P. m. rufinus & & 0.0248 \\
\hline & P.m.m. & Peromyscus leucopus & 0 & 0.2062 \\
\hline & P. keeni & P. leucopus & & 0.2145 \\
\hline & PNW & R. megalotis & & 0.5613 \\
\hline & P.m.m. & R. megalotis & & 0.5906 \\
\hline & P. m. bairdii & R. megalotis & & 0.5783 \\
\hline
\end{tabular}

Figure 8: Peromyscus Pair-wise Distances. Pair-wise comparison of average percent genetic difference in mitochondrial ND3, ND4, and ND4L genes $(1,463 \mathrm{bp})$ between Peromyscus (including $R$. megalotis) species and subspecies $(\mathrm{n}=84)$. Non-Neotomine sequences not shown - see Appendix A for full pair-wise distance matrix. PNW clade=P. m. rubidus, P. m. gambelii, P. m. austerus. Color coded dots correspond to specimen collection locations on map. 


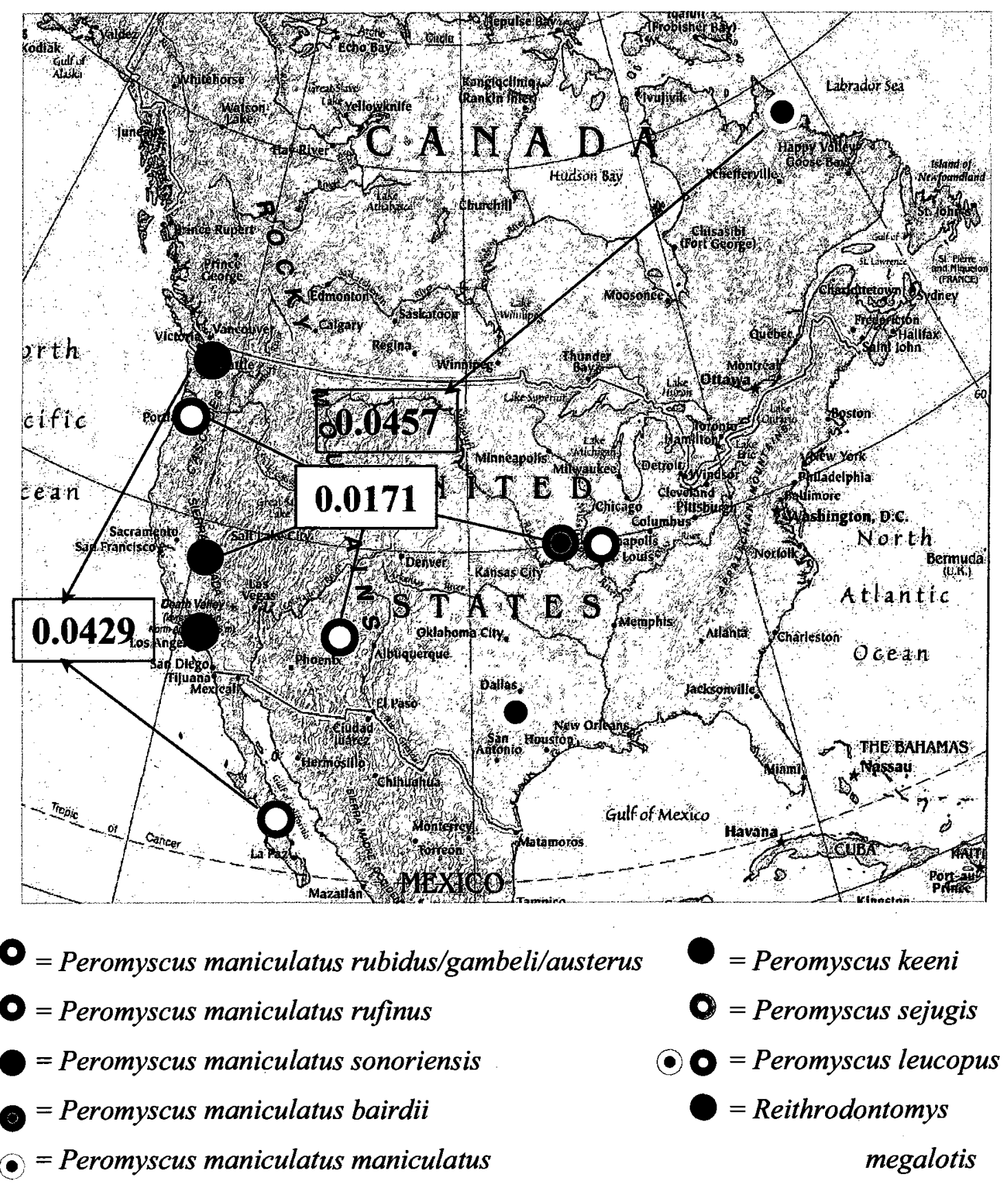

Figure 9: Peromyscus Pair-wise Nucleotide Distance Physical Map. Genetic distance of mitochondrial ND3, ND4, ND4L, and arginine tRNA genes of rodents relevant to the phylogenetic position of Peromyscus in the Portland, OR region. $P$. leucopus is represented by two separate haplotypes. Data in red indicates average intra-specific distance among western North American clade. 


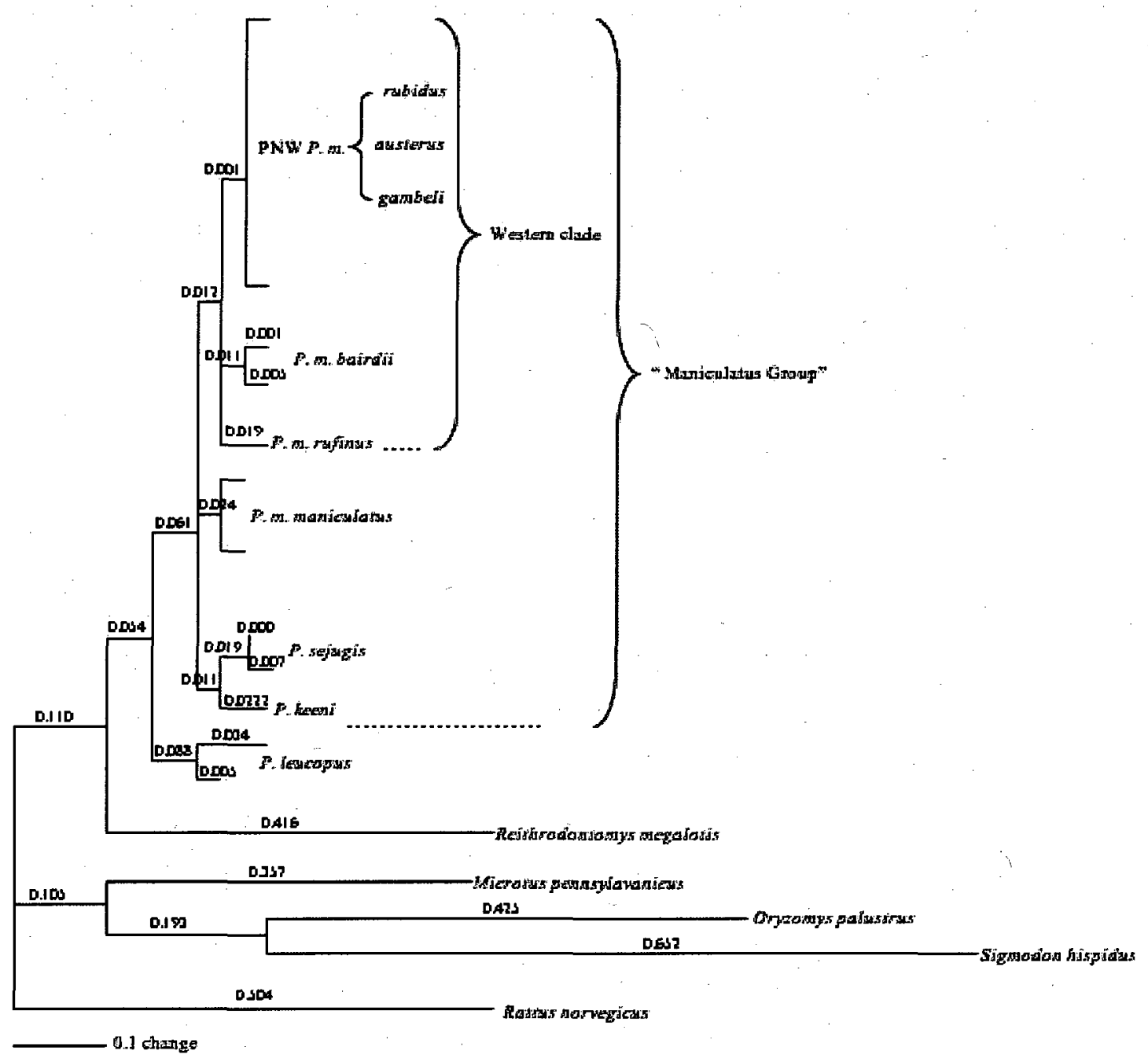

Figure 10: Peromyscus Distance Tree. Neighbor-Joining tree of Peromyscus and outgroups $(n=88)$ resolved from distance analysis of mitochondrial ND3, ND4, ND4L, and arginine tRNA genes $(1,463 \mathrm{bp})$. Numbers represent branch length as changes genetic distance from preceding node. PNW = Pacific Northwest. "Western clade" is west of the Mississippi River. "Maniculatus Group" includes PNW and Western clades. 


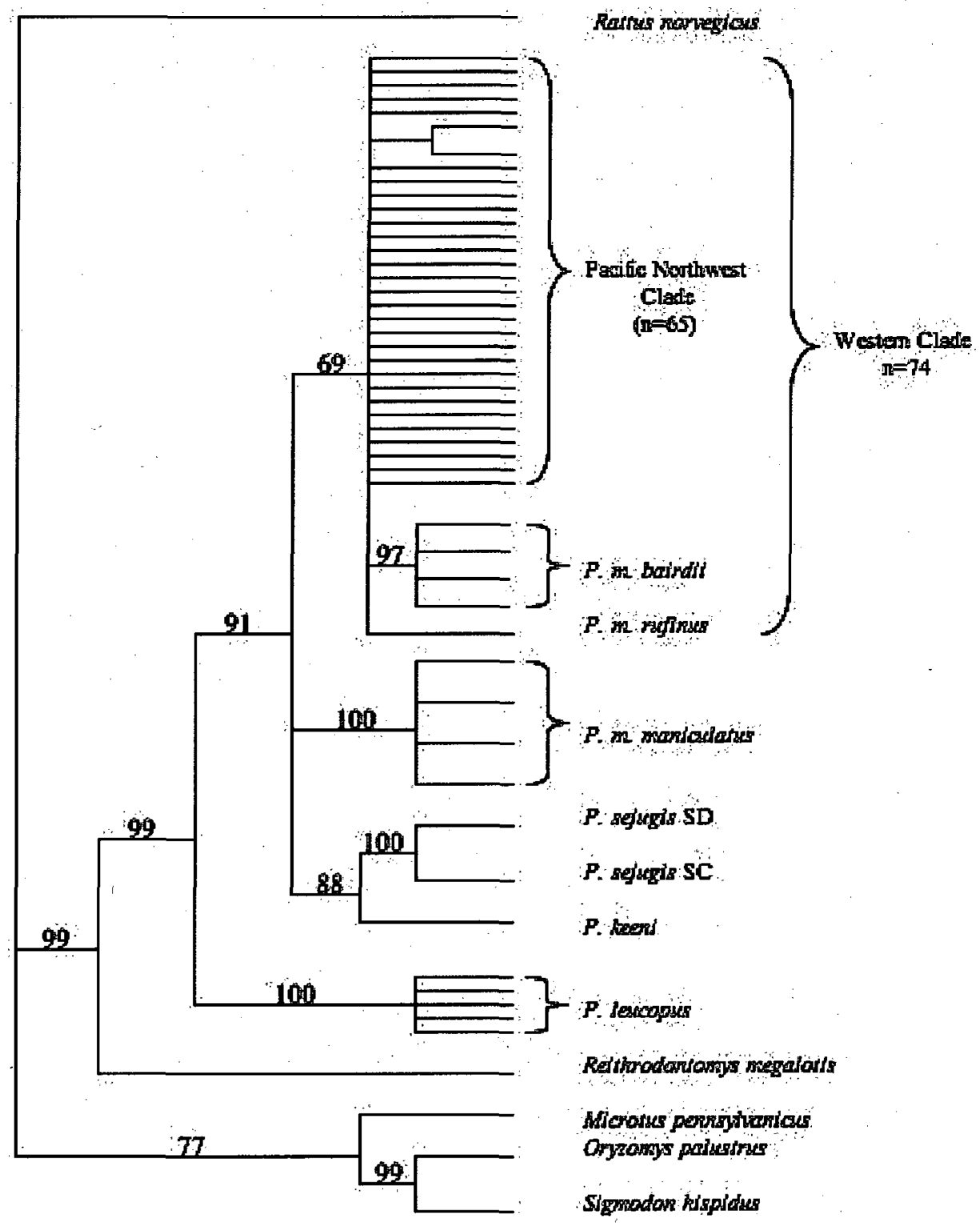

Figure 11: Peromyscus Maximum Likelihood Bootstrap Tree. ML tree of Peromyscus and outgroups $(\mathrm{n}=88)$ using $1,463 \mathrm{bp}$ of mitochondrial ND3, ND4, ND4L, and arginine tRNA genes. Numbers represent bootstrap support for nodes after 1000 replicates. $-\operatorname{lnL}=8702.95284$. Evolutionary model is $\mathrm{GTR}+\mathrm{I}+\Gamma$. $\mathrm{SD}=\mathrm{San}$ Diego Island. $\mathrm{SC}=\mathrm{Santa}$ Cruz Island.

\begin{tabular}{|cc|}
\hline $\begin{array}{c}\text { Among-site rate variation } \\
\text { Proportion of invariable sites }(\mathrm{I})= \\
\text { Variable sites }(\mathrm{G})\end{array}$ & $\mathbf{0 . 2 8 5 1}$ \\
Gamma distribution shape parameter $=$ & 0.7693 \\
\hline
\end{tabular}




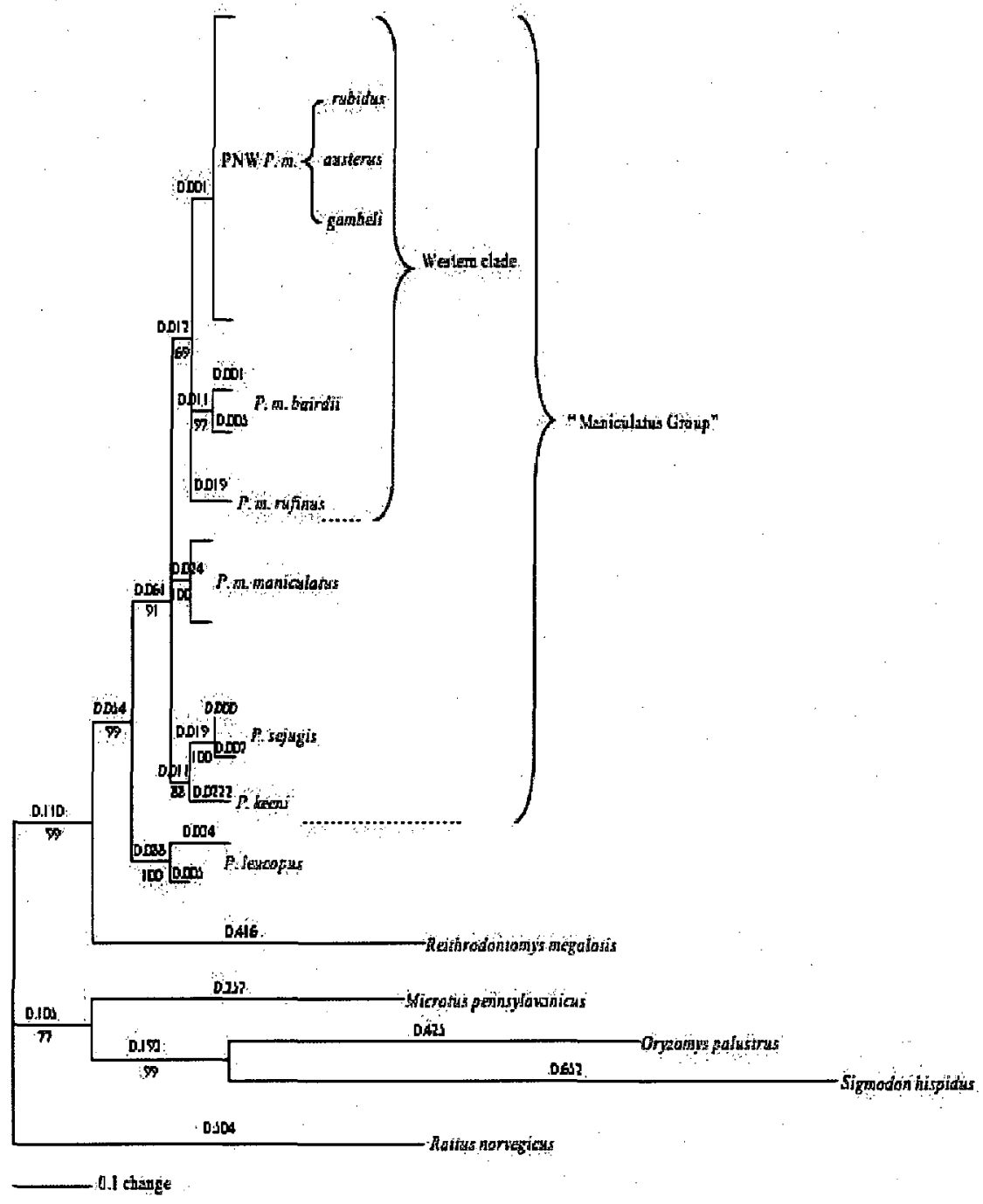

Figure 12: Peromyscus Bootstrap/Distance Tree. Combined tree of figures 9 and 10 for direct comparison based upon Peromyscus and out-group $(\mathrm{n}=88)$ mitochondrial ND3, ND4, ND4L, and arginine tRNA genes $(1,463 \mathrm{bp})$. Neighbor-Joining tree (fig. 10) with bootstrap values added from Maximum Likelihood tree (fig. 9) where nodes are identical. 


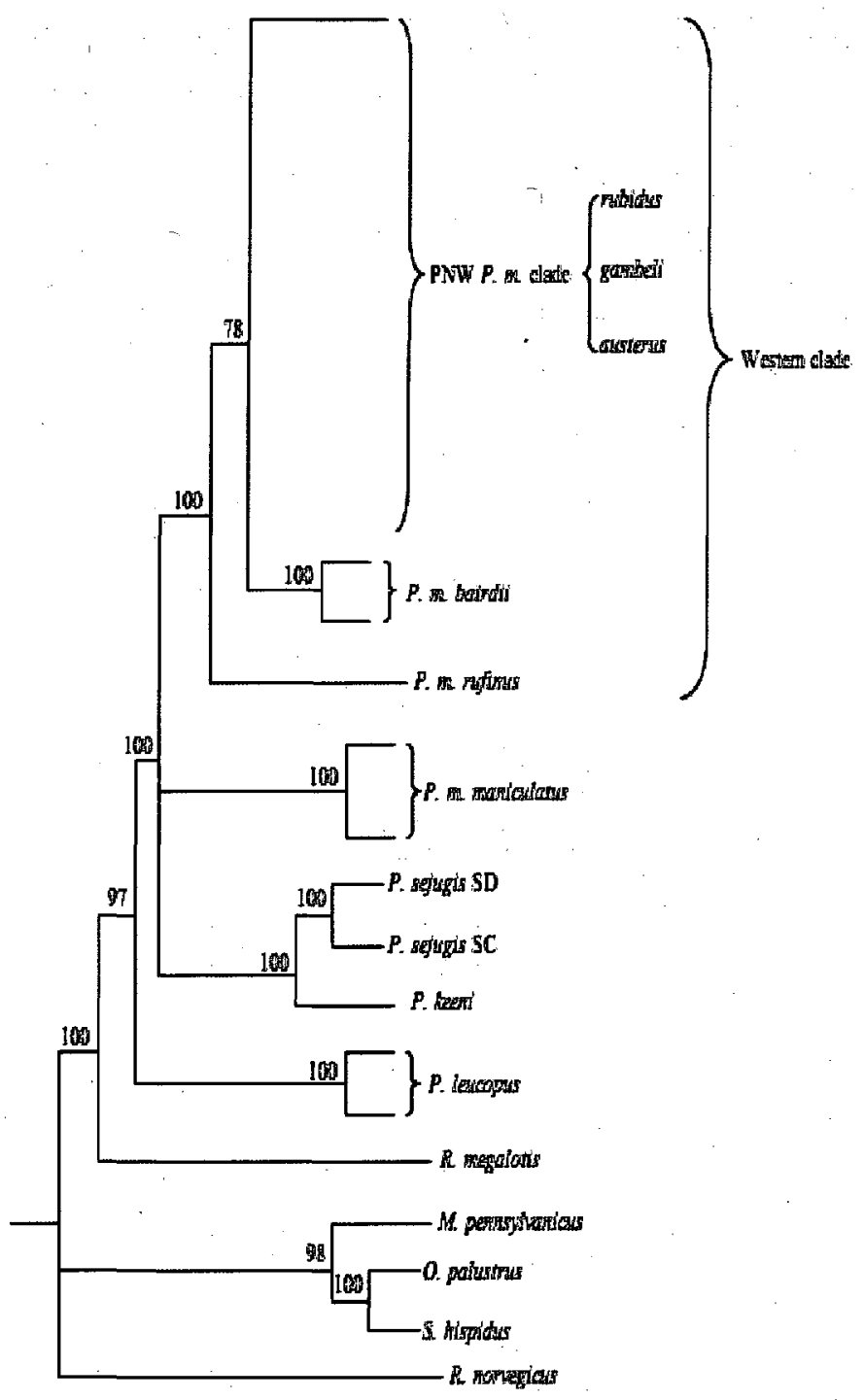

Figure 13: Peromyscus Bayesian Majority-Rule Consensus Tree. Based upon Peromyscus and outgroups $(\mathrm{n}=88)$ mitochondrial ND3, ND4, ND4L; and arginine tRNA genes (1,463bp). Numbers represent bipartition posterior probabilities from 500,000 generations in two runs of four separate Metropolis coupled Markov Chain Monte Carlo computations. Final average standard deviation of split frequencies is 0.0077 . Consensus tree derived from majority-rule search of 8,002 sample trees from 800,000 generations.

Component information (consensus fork) $=15$ (normalized $=0.652)$

Nelson-Platnick term information $=96$

Nelson-Platnick total information $=111$

Mickevich's consensus information $=0.347$

Colless weighted consensus fork (proportion max. information) $=0.371$

Schuh-Farris levels sum $=0$ (normalized $=0.000$ )

Rohlf's CI $(1)=0.615$

Rohlf's - $\ln \mathrm{CI}(2)=55.242(\mathrm{CI}(2)=1.02 \mathrm{e}-24)$ 

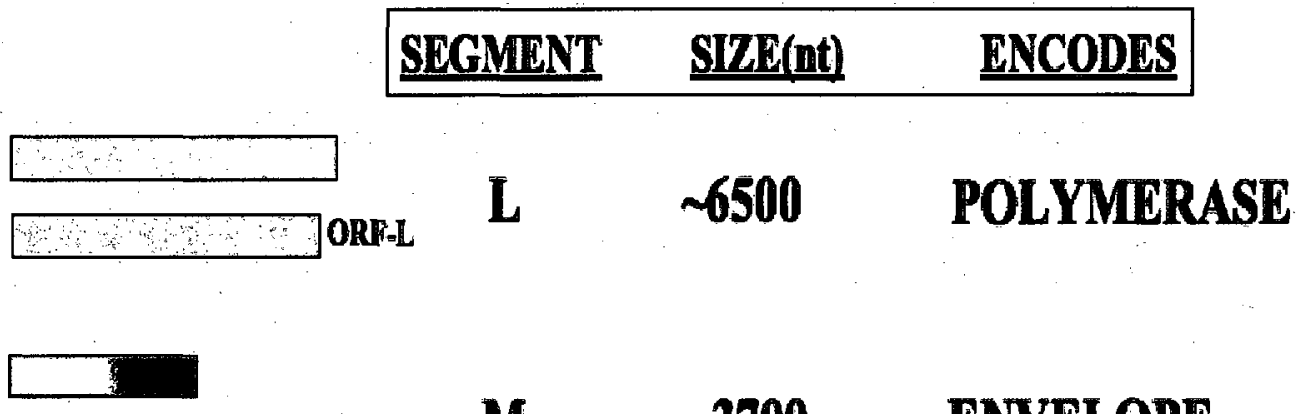

ORF-GI/G2

M $\quad \sim 3700 \quad$ ENVELOPE

ORF-N

S $\quad 2000 \quad$ NUCLEOCAPSID

ORF-N5x

?

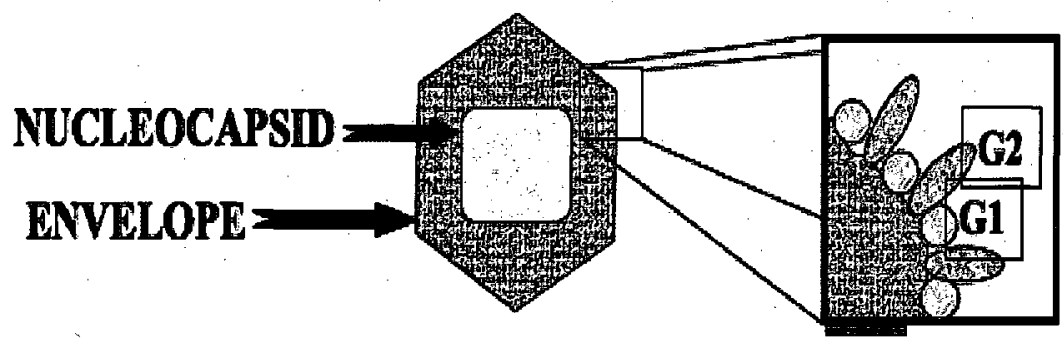

\section{HANTAVIRUS RNA SEGMENTS}

FIGURE 14: Hantavirus genome map. Family Bunyaviridae. Tripartite singlestranded negative sense RNA genome. $\sim 12,200$ nt total. Targeted G1/G2 glycoprotein precursor gene encoded in $\mathrm{M}$ segment. 


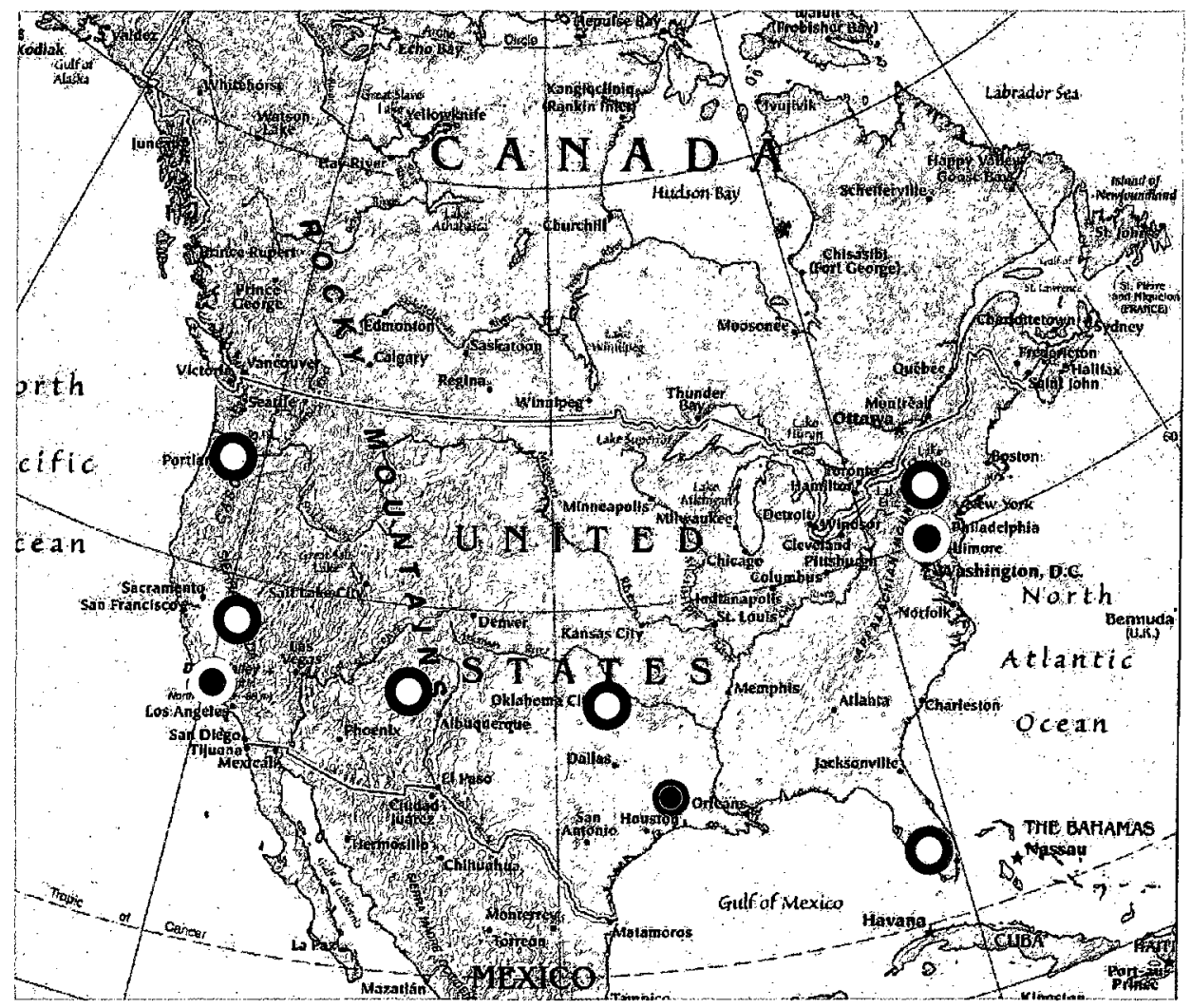

\begin{tabular}{|c|c|c|}
\hline $\begin{array}{l}\text { PAIR-WISE QUASI- } \\
\text { SPECIES } \\
\end{array}$ & $\begin{array}{c}\text { UNCORRECTED } \\
\text { DISTANCE }\end{array}$ & $\begin{array}{c}\text { CORRECTED } \\
\text { DISTANCE }(\mathrm{GTR}+\mathrm{I}+\Gamma)\end{array}$ \\
\hline $\mathbf{O K V}(\mathrm{OR}) / \mathrm{CC} \mathbf{O}$ & 0.112 & 0.158 \\
\hline KV/SNV $\mathbf{O}$ & 0.143 & 0.251 \\
\hline $\mathrm{KV} / \mathrm{NY}$ & 0.215 & 0.441 \\
\hline KV / BR & 0.236 & 0.578 \\
\hline $\mathrm{KV} / \mathrm{BAY}$ & 0.273 & 0.764 \\
\hline $\mathrm{KV} / \mathrm{BCC} \quad \mathbf{O}$ & 0.291 & 0.899 \\
\hline KV / EMC & 0.278 & 0.692 \\
\hline $\mathrm{KV} / \mathrm{PH}$ & 0.331 & 1.226 \\
\hline $\mathrm{SNV} / \mathrm{CC}$ & 0.130 & 0.228 \\
\hline $\mathrm{SNV} / \mathrm{NY}$ & 0.189 & 0.384 \\
\hline SNV / EMC & 0.269 & 0.662 \\
\hline
\end{tabular}

Figure 15: Hantavirus Pair-wise Distances: Pair-wise percent genetic distance among North American hantaviruses $(n=44)$ of $\mathrm{G} 1 / \mathrm{G} 2$ glycoprotein precursor gene $(617 \mathrm{nt})$. KV=Kalapuya virus; $C C=$ Convict $C$ reek virus; $S N V=S$ in Nombre virus; $N Y=$ New York virus; $B R=B l$ lue River virus; $\mathrm{BAY}=$ Bayou virus; $\mathrm{BCC}=\mathrm{Black}$ Creek Canal virus; $\mathrm{EMC}=\mathrm{E} 1$ Moro Canyon virus; $\mathrm{PH}=$ Prospect Hill virus. Corresponding colored dots represent specimen location. Rodent virus hosts: Yellow dot center $=P$. maniculatus, white $=P$. leucopus, aqua $=S$. hispidus, black $=O$. palustrus, red $=R$. megalotis . Corrected Distance derived with General Time Reversible model of evolution with a proportion of sites invariant and gamma distributed variance, as selected by MODELTEST (Posada and Crandall, 1998). 


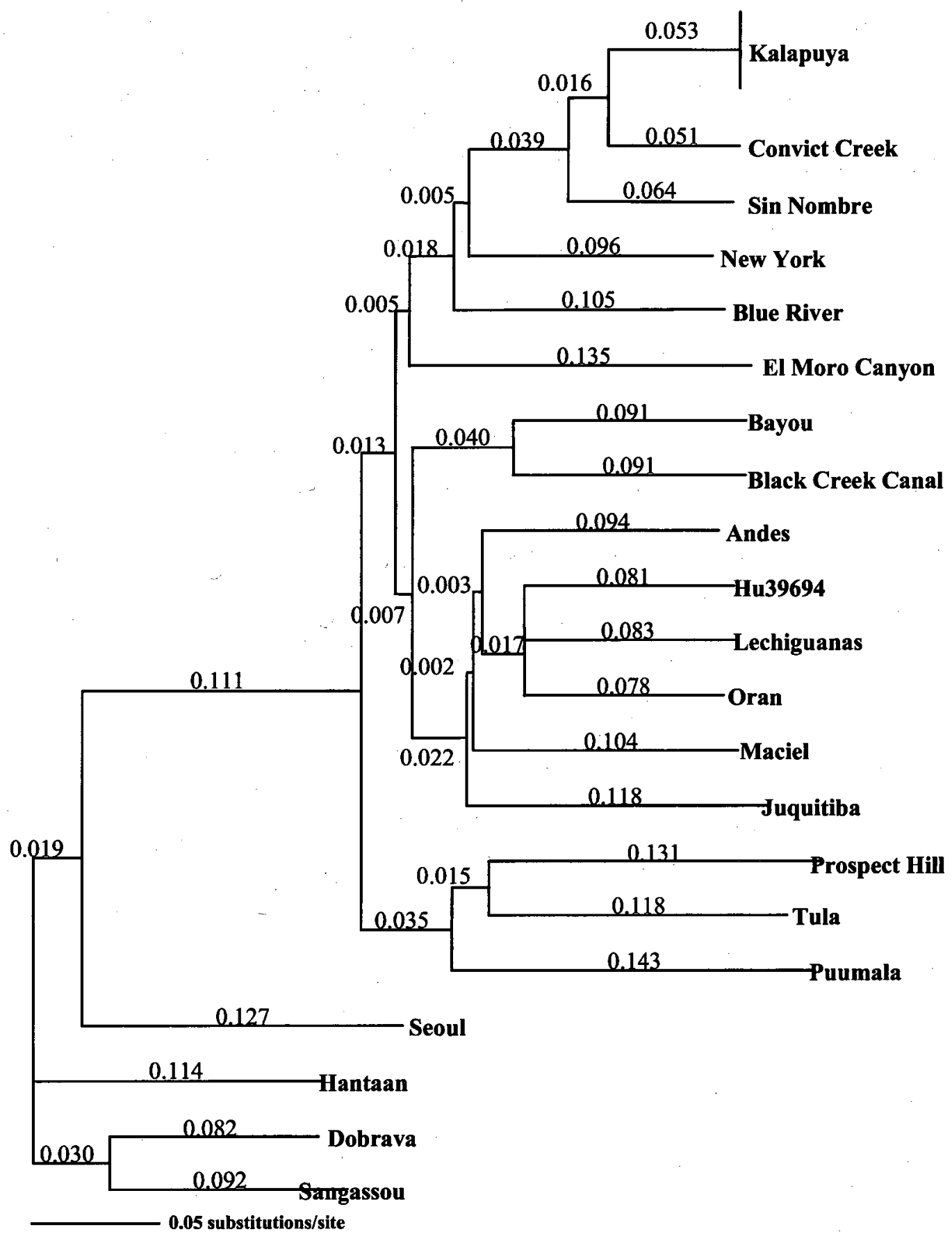

Figure 16: Hantavirus Neighbor-Joining Distance Tree. NJ tree of 617nt of Hantavirus $\mathrm{Gl} / \mathrm{G} 2$ glycoprotein precursor gene $(\mathrm{n}=44)$. Numbers represent branch length as changes from preceding node. 


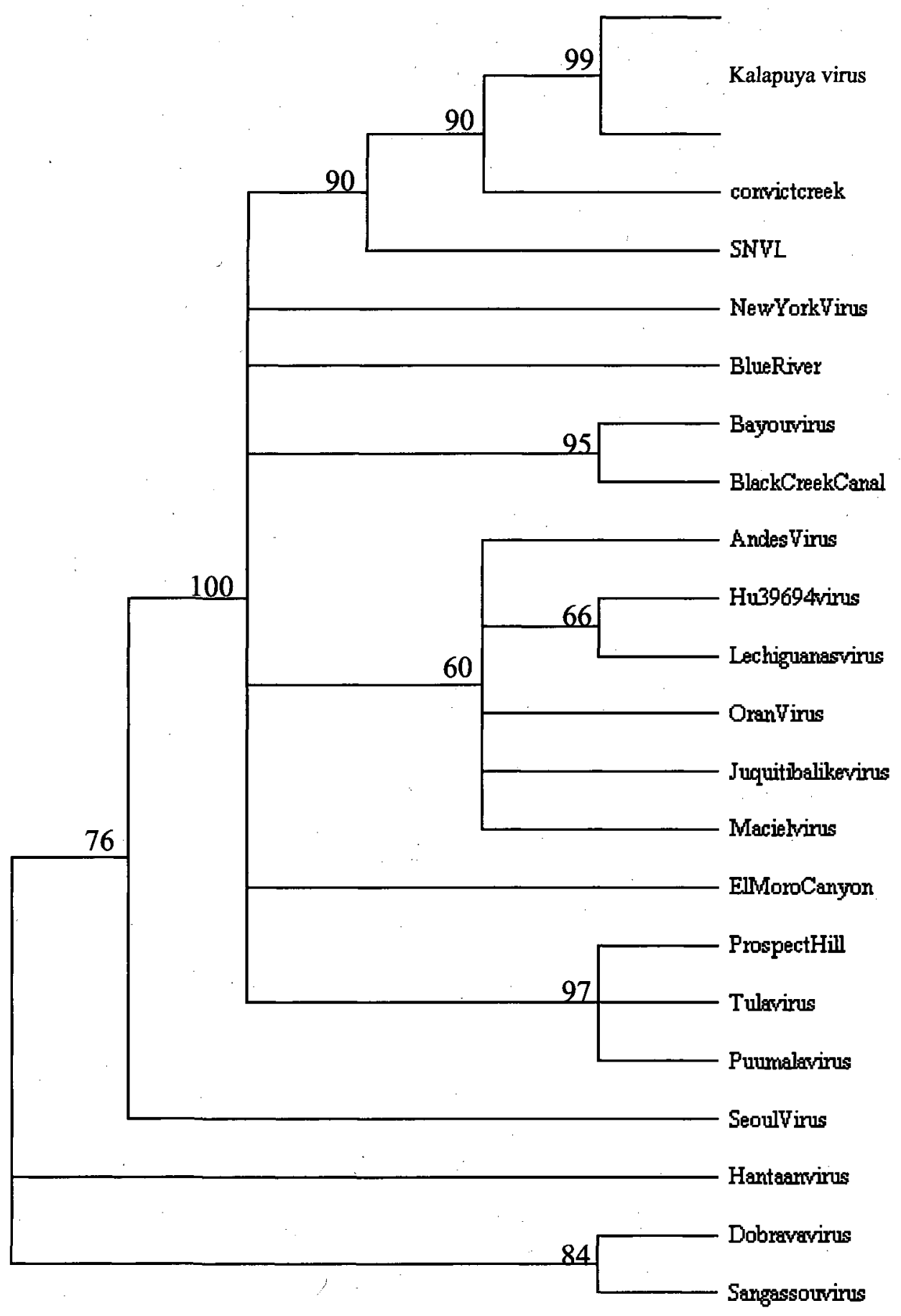

Figure 17: Hantavirus Maximum Likelihood Bootstrap Consensus Tree. Hantavirus ML tree based upon $617 \mathrm{nt}$ of M segment G1/G2 glycoprotein precursor gene $(\mathrm{n}=44)$. Numbers represent bootstrap support for nodes after 1000 replicates. Evolutionary model is GTR $+\mathrm{I}+\Gamma$. $-\operatorname{lnL}=8011.8281$. 


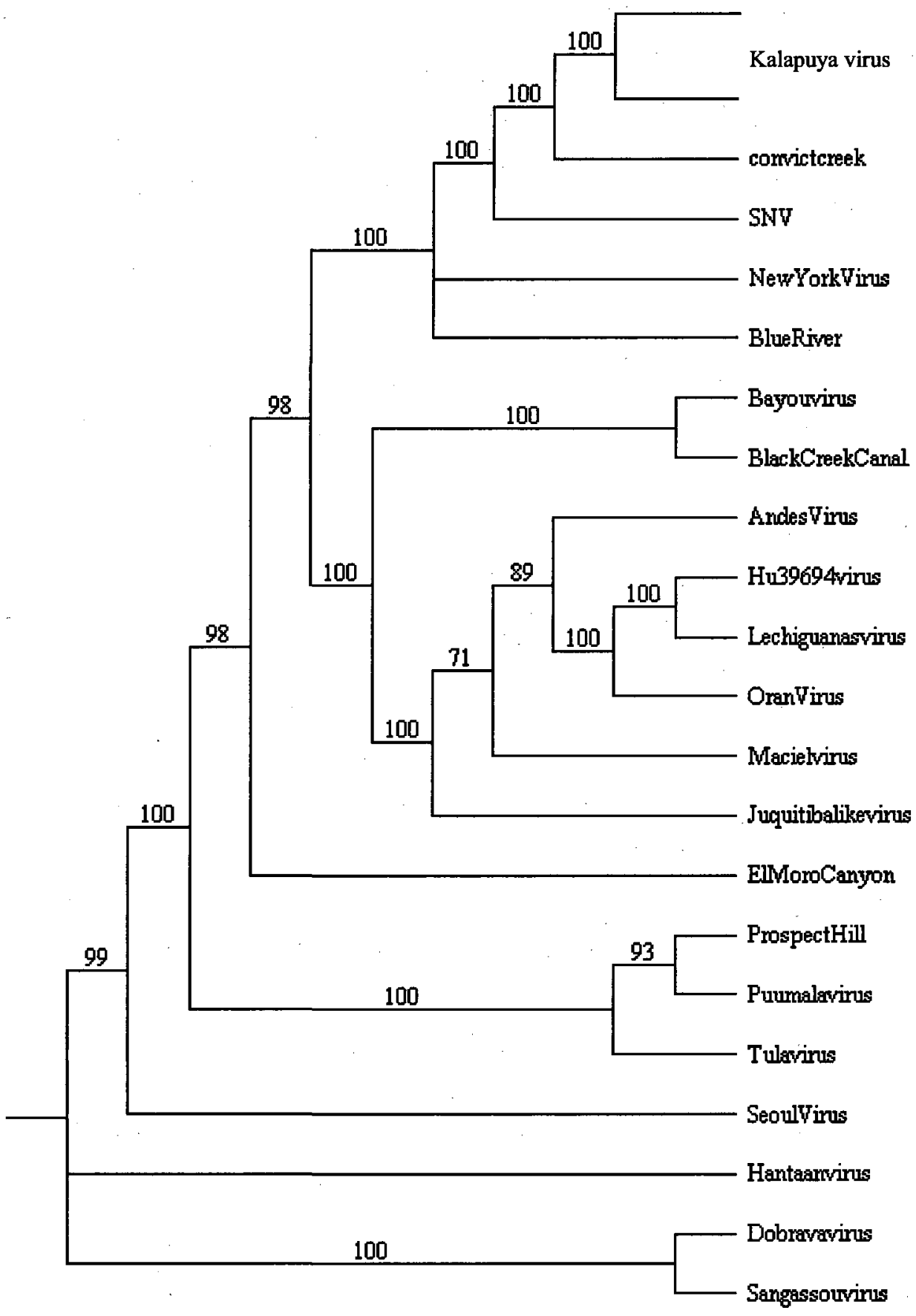

Figure 18: Hantavirus Bayesian Majority-Rule Consensus Tree: Tree based upon $617 \mathrm{nt}$ of $\mathrm{M}$ segment $\mathrm{Gl} / \mathrm{G} 2$ glycoprotein precursor gene $(\mathrm{n}=44)$. Numbers represent bipartition posterior probabilities from 500,000 generations in two runs of four separate Metropolis coupled Markov Chain Monte Carlo computations. Final average standard deviation of split frequencies is 0.0023 . Consensus tree derived from majority-rule search of 8,002 sample trees from 800,000 generations. 


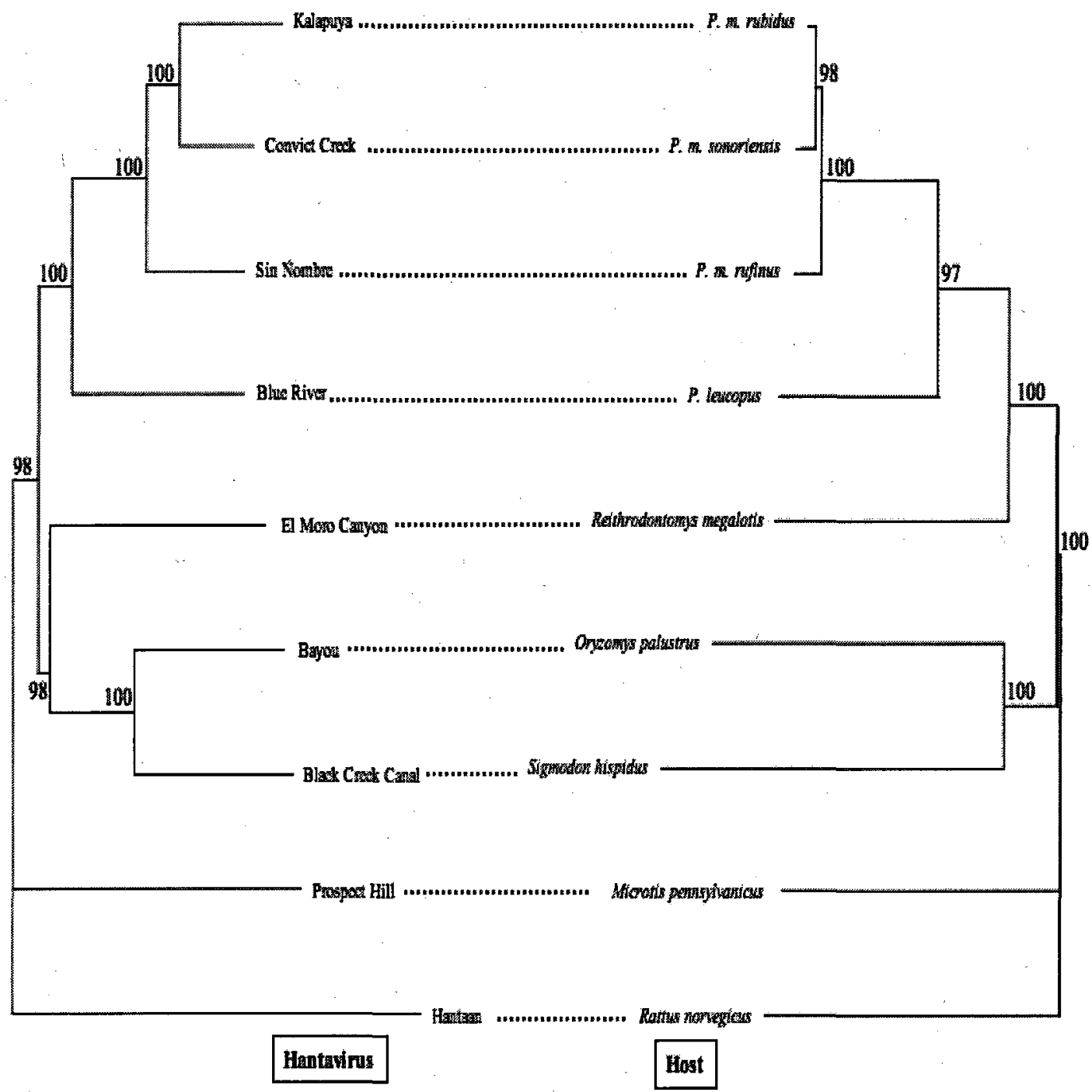

Figure 19: Hantavirus / Host Co-Evolutionary Tree. Comparative Bayesian trees of North American hantaviruses (and one Asian) and the rodent hosts for those hantaviruses. Hantavirus tree based upon $617 \mathrm{nt}$ of M segment G1/G2 glycoprotein precursor gene. Host tree based on 1,463bp of mitochondrial ND3, ND4, ND4L, and arginine tRNA genes. Numbers represent bifurcation posterior probabilities based upon 100,000 generations of Metropolis coupled Markov Chain Monte Carlo computations. 


\begin{tabular}{|c|c|c|c|}
\hline Virus & \begin{tabular}{|c|} 
Disea \\
se
\end{tabular} & Known or suspected host & Location \\
\hline \multicolumn{4}{|l|}{$\begin{array}{l}\text { Cricetidae } \\
\text { associated }\end{array}$} \\
\hline Sin Nombre & HPS & \begin{tabular}{||l} 
Peromyscus maniculatus \\
(grassland form)
\end{tabular} & $\begin{array}{l}\text { West \& Central U.S. and } \\
\text { Canada }\end{array}$ \\
\hline Monongahela & HPS & P. maniculatus (forest form) & Eastern U.S. and Canada \\
\hline New York & HPS & P. leucopus (eastern haplotype) & Eastern U.S. \\
\hline Blue River & & $\begin{array}{l}\text { P. leucopus (SW/NW } \\
\text { haplotypes) }\end{array}$ & Central U.S. \\
\hline Bayou & HPS & Oryzomys palustris & Southwestern U.S. \\
\hline $\begin{array}{l}\text { Black Creek } \\
\text { Canal } \\
\end{array}$ & HPS & $\begin{array}{l}\text { Sigmodon hispidus (eastern } \\
\text { form) }\end{array}$ & Florida \\
\hline Muleshoe & & S. hispidus (western form) & Southern U.S. \\
\hline Caño Delgadito & & S. alstoni & Venezuela \\
\hline Andes & HPS & Oligoryzomys longicaudatus & Argentina and Chile \\
\hline Oran & HPS & O. longicaudatus & Northwestern Argentina \\
\hline Lechiguanas & HPS & O. flavescens & Central Argentina \\
\hline Bermejo & & O. chacoensis & Northwestern Argentina \\
\hline Hu39694 & HPS & Unknown & Central Argentina \\
\hline Pergamino & & Akadon azarae & Central Argentina \\
\hline Maciel & & Bolomys obscurus & Central Argentina \\
\hline Laguna Negra & $\mathrm{HPS}$ & Calomys laucha & Paraguay and Bolivia \\
\hline Juquitiba & HPS & Unknown & Brazil \\
\hline Rio Mamore & & O. Microtus & Bolivia and Peru \\
\hline El Moro Canyon & & Reithrodontomys megalotis & Western U.S. and Mexico \\
\hline Rio Segundo & & R. mexicanus & Costa Rica \\
\hline
\end{tabular}

Table 1: Hantaviruses in the New World. Monroe et al., 1999. Western hemisphere Hantaviruses shown with known hosts, status of pathogenicity, and regions of host residence. 
Site

Forest Park

Tryon Creek State Park

Oxbow Regional Park

Tualatin River NWR

Powell Butte Park

Total
Infected Totals $(\mathrm{M} / \mathrm{F})$

$60(41 / / 19)$

$10(8 / / 2)$

$6(4 / / 2)$

$5(3 / / 2)$

$5(4 / / 1)$

Table 2: Infected Totals by Site. Hantavirus antibody sero-positive Peromyscus maniculatus rubidus from the Portland, OR region.

Season

Fall (Sep-Nov)

Winter (Dec-Feb)

Spring (Mar-May)

Summer (Jun-Aug)

Total

\section{Infected Totals $(\mathrm{M} / \mathrm{F})$}

$18(14 / / 4)$

$11(9 / / 2)$

$31(20 / / 11)$

$26(17 / / 9)$

$86(60 / / 26)$

Table 3: Infected Totals by Season. Hantavirus antibody sero-positive Peromyscus maniculatus rubidus from the Portland, OR region. 


\section{Literature Cited:}

Allard, M. W., and I. F. Greenbaum. 1988. Morphological variation and taxonomy of chromosomally differentiated Peromyscus from the Pacific Northwest. Canadian Journal of Zoology 66:2734-2739.

Arai, S., J. W. Song, L. Sumibcay, S. N. Bennett, V. R. Nerurkar, C. Parmenter, J. A. Cook, T. L. Yates, R. Yanagihara. 2007. Hantavirus in Northern Short-tailed Shrew, United States. Emerging Infectious Diseases 13(9):1420-1423.

Arai S., S. N. Bennett, L. Sumibcay, J. A. Cook, Jin-Won Song, A. Hope, C. Parmenter, V. R. Nerurkar, T. L. Yates, R. Yanagihara. 2008. Phylogenetically Distinct Hantaviruses In The Masked Shrew (Sorex Cinereus) And Dusky Shrew (Sorex Monticolus) In The United States. American Journal of Tropical Medicine \& Hygiene 44:126-137.

Avise, J. C., J. F. Shapira, S. W. Daniel, C. F. Aquadro, and R. A. Lansman. 1983. Mitochondrial DNA Differentiation during the Speciation Process in Peromyscus. Molecular Biology and Evolution 1(1):38-56.

Avsic-Zupanc, T., S. Y. Xiao, R. Stojanovic, A. Gligic, G. van der Groen, J. W. LeDuc. 1992. Characterization of Dobrava virus: a Hantavirus from Slovenia, Yugoslavia. Journal of Medical Virology 38 (2):132-137. 
Avsic-Zupanc, T., K. Nemirov, M. Petrovec, T. Trilar, M. Poljak, A. Vaheri, and A. Plyusnin. 2000. Genetic analysis of wild-type Dobrava Hantavirus in Slovenia: co-existence of two distinct genetic lineages within the same natural focus. Journal of General Virology 81,1747-1755.

Baker, R. H. 1983. Michigan Mammals. Wayne State University, Detroit, Michigan.

Beer, J. 1959. A collection of deer mice from Otter Rock, Oregon. The Murrelet 40:28-29.

Bohlman, M. C., S. P. Morzunov, J. Meissner, M. B. Taylor, K. Ishibashi, J. Rowe, S. Levis, D. Enria, S. C. St. Jeor. 2002. Analysis of Hantavirus Genetic Diversity in Argentina: S Segment-Derived Phylogeny. Journal of Virology, 76.8.3765-3773.

Borucki, M. K., J. D. Boone, J. E. Rowe, M. C. Bohlman, E. A. Kuhn, R. DeBaca, S. C. St. Jeor. 2000. Role of Maternal Antibody in Natural Infection of Peromyscus maniculatus with Sin Nombre Virus. Journal of Virology 74(5): 2426-2429. 
Botten, J., K. Mirowsky, D. Kusewitt, M. Bharadwaj, J. Yee, R. Ricci, R. M.

Feddersen, B. Hjelle. 2000. Experimental Infection model for Sin Nombre Hantavirus in the deer mouse (Peromyscus maniculatus). Proceedings of the National Academy of Sciences 97(19):10578-10583.

Bradley, R. D., R. J. Baker. 2001. A test of the genetic species concept: cytochromeb sequences and mammals. Journal of Mammalogy 82(4):960-973.

Buckley, T. R., and C. W. Cunningham. 2002. The effect of nucleotide substitution model assumptions on estimates of nonparametric bootstrap support. Molecular Biology and Evolution 19:394-405.

Burt, W. H. 1932. Descriptions of heretofore unknown mammals from islands in the Gulf of California, Mexico. Transactions of the San Diego Society of Natural History 7:161-182.

Calhoun, S. W., I. F. Greenbaum. 1991. Evolutionary implications of genic variation among insular populations of Peromyscus maniculatus and Peromyscus oreas. Journal of Mammalogy 72(3):248-262.

Carey D. E., R. Reuben, K. N. Panicker, R. E. Shope, R. M. Myers. 1971. Thottapalayam virus: A presumptive Arbovirus isolated from a shrew in India. 
Indian Journal of Medical Research 59:1758-1760.

Centers for Disease Control. Figure 1. HPS Teaching Slideset - Phylogeny of Hantaviruses: Based on Sequence of M Segment. http://www.cdc.gov/ncidod/diseases/hanta/hps/noframes/hpsslideset/hpsslides1 $-12 . \mathrm{htm}$

Childs, J. E., T. G. Ksiazek, C. F. Spiropoulou, J. W. Krebs, S. Morzunov, G. O. Maupin, K. L. Gage, P. E. Rollin, J. Sarisky, R. E. Enscore, J. K. Frey, C. J. Peters, S. T. Nichol. 1994. Serologic and genetic identification of Peromyscus maniculatus as the primary rodent reservoir for a new Hantavirus in the southwestern United States. Journal of Infectious Diseases 169:1271-1280.

Chirhart, S. E., R. Arianpour, R. L. Honeycutt, I. F. Greenbaum. 2001. Mitochondrial DNA sequence variation and the specific identification of deer mice (Peromyscus) from Triangle Island, British Columbia, Canada. Canadian Journal of Zoology 79:2257-2260.

Chirhart, S. E., R. L. Honeycutt, I. F. Greenbaum. 2005. Microsatellite variation and evolution in the Peromyscus maniculatus species group. Molecular Phylogenetics and Evolution 34:408-415.

Chizhikov, V. E., C. F. Spiropoulou, S. P. Morzunov, M. C. Monroe, C. J. Peters, S. 
T. Nichol. 1995. Complete Genetic Characterization and Analysis of Isolation of Sin Nombre Virus. Journal of Virology 69(12):8132-8136.

Cunningham, C. W., H. Zhu, D. M. Hillis. 1998. Best-fit Maximum Likelihood models for phylogenetic inference - test with known phylogenies. Evolution 52(4):978-987.

Daszak, P., A. A. Cunningham, A. D. Hyatt. 2000. Emerging Infectious Diseases of Wildlife- Threats to Biodiversity and Human Health. Science 287:443-449.

Daszak, P., A. A. Cunningham, A. D. Hyatt. 2001. Anthropogenic Environmental Change and the Emergence of Infectious Diseases in Wildlife. Acta Tropica 78:103-116.

Douady, C. J., F. Delsuc, Y. Boucher, W. F. Doolittle, E. J. P. Douzery. 2003. Comparison of Bayesian and Maximum Likelihood Bootstrap Measures of Phylogenetic Reliability. Molecular Biology and Evolution 20(2):248-254. 2003.

Douglass, R. J., T. Wilson, W. J. Semmens, S. N. Zanto, C. W. Bond, R. C. VanHorn, J. N. Mills. 2001. Longitudinal Studies of Sin Nombre Virus in Deer MouseDominated Ecosystems of Montana. American Journal of Tropical Medical Hygiene 65(1):33-41. 
Dragoo, J. W., A. Lackey, K. E. Moore, E. P. Lessa, J A. Cook, T. L. Yates. 2006. Phylogeography of the deer mouse (Peromyscus maniculatus) provides a predictive framework for research on hantaviruses. Journal of General Virology 87:1997-2003.

Efron, B., E. Halloran, and S. Holmes. 1996. Bootstrap confidence levels for phylogenetic trees. Proceedings of the National Academy of Science USA 93:13429-13434.

Elliot, R. M.. 1990. Review article: Molecular biology of the Bunyaviridae. Journal of General Virology (1990), 71, 501-522.

Elliott, R. M., C. S. Schmaljohn, and M. S. Collett. 1991. Bunyaviridae genome structure and gene expression. Current Topics in Microbiology and Immunology 169:91-141.

Falls, J. B. 1968. Activity. Pp. 543-570, in Biology of Peromyscus (Rodentia) (J.A. King, ed.) The American Society of Mammalogists, Special Publication, 2:1593.

Felsenstein, J. 1981. Evolutionary trees from DNA sequences: a maximum likelihood approach. Journal of Molecular Evolution 17:368-376. 
Felsenstein, J. 1985. Confidence limits on phylogenies: an approach using the bootstrap. Evolution 39:783-791.

Felsenstein, J., and H. Kishino. 1993. Is there something wrong with the bootstrap on phylogenies-a reply. Systematic Biology 42:193-200.

Foster, P.G. 2003. Likelihood in molecular phylogenetics. The Natural History Museum of London.

Gavrilovskaya I. N., N. S. Apekina, Y. A. Myasnikov, A. D. Bernshtein, E. V. Ryltseva, E. A. Gorbachkova. 1983. Features of circulation of hemorrhagic fever with renal syndrome (HFRS) virus among small mammals in the European U.S.S.R. Archaean Virology 75:313-6.

Gligic A., R. Stojanovic, M. Obradovic, D. Hlaca, N. Dimkovic, G. Diglisic. 1989. Hemorrhagic fever with renal syndrome in Yugoslavia: epidemiologic and epizootiologic features of a nationwide outbreak. European Journal of Epidemiology 8:816-25.

Goldman, N. and Z. Yang. (1994) A codon-based model of nucleotide substitution for protein-coding DNA sequences. Molecular Biology and Evolution 11(5):72536. 
Goldman, N., J. P. Anderson, and A. G. Rodrigo. 2000. Likelihood-based tests of topologies in phylogenetics. Systematic Biology 49:652-670.

Greenbaum, I. F., R. J. Baker. 1978. Determination of the Primitive Karyotype for Peromyscus. Journal of Mammalogy 59(4):820-834.

Gunn, S. J. 1988. Chromosomal variation and differentiation among insular populations of Peromyscus from the Pacific Northwest. Canadian Journal of Zoology 66:2726-2733.

Hall, E. R. 1981. The Mammals of North America Volume II. John Wiley and Sons Press, NY, NY.

Hill, R. W. 1983. Thermal physiology and energetics of Peromyscus; ontogeny, body temperature, metabolism, insulation, and microclimatology. Journal of Mammalogy 64:19-37.

Hillis, D. M., and J. J. Bull. 1993. An empirical-test of bootstrapping as a method for assessing confidence in phylogenetic analysis. Systematic Biology 42:182192. 
Hjelle, B., F. Chavez-Giles, N. Torrez-Martinez, T. Yates, J. Sarisky, J. Webb, M. Ascher. 1994. Genetic identification of a novel Hantavirus of the harvest mouse Reithrodontomys megalotis. Journal of Virology 68:6751-6754.

Hjelle, B., B. Anderson, N. Torrez-Martinez, W. Song, W. L. Gannon, T. L. Yates. 1995a. Prevalence and Geographic genetic variation of Hantaviruses of New World harvest mice (Reithrodontomys): Identification of a divergent genotype from a Costa Rican Reithrodontomys mexicanus. Virology 207:452-459.

Hjelle, B., J. Krolikowski, N. Torrez-Martinez, F. Chaves-Giles, C. Vanner, E. Laposata. 1995b. Phylogenetically distinct Hantavirus implicated in a case of Hantavirus Pulmonary Syndrome in the Northeastern United States. Journal of Medical Virology 46:21-27.

Hoelzel, A. R., A. Green 1992. Analysis of Population-Level Variation by Sequencing PCR-Amplified DNA. Ch. 6:159-187.

Hogan, K. M., M. C. Hedin, H. S. Koh, S. K. Davis, I. F. Greenbaum. 1993. Systematic and taxonomic implications of karyotypic, electrophoretic, and mitochondrial-DNA variation in Peromyscus from the Pacific Northwest. Journal of Mammalogy 74(4):819-831. 
Hogan, K. M., S. K. Davis, I. F. Greenbaum. 1997. Mitochondrial-DNA analysis of the systematic relationships within the Peromyscus maniculatus species group. Journal of Mammalogy 78(3):733-743.

Holmes, E. C. 2004. The phylogeography of human viruses. Molecular Ecology 13:745-756.

Huelsenbeck, J.P. and F. Ronquist. 2001. MRBAYES: Bayesian inference of phylogenetic trees. Bioinformatics 17(8):754-755.

Huelsenbeck, J. P., F. Ronquist, R. Nielsen, and J. P. Bollback. 2001. Bayesian inference of phylogeny and its impact on evolutionary biology. Science 294:2310-2314.

Huelsenbeck, J.P., B. Larget, R. E. Miller, and F. Ronquist. 2002. Potential applications and pitfalls of Bayesian inference of phylogeny. Systematic Biology 51(5):673-688.

Huestis, R. R. 1931. Seasonal pelage differences in Peromyscus. Journal of Mammalogy, 12:372-375. 
Hughes, A. L., and R. Friedman. 2000. Evolutionary Diversification of ProteinCoding Genes of hantaviruses. Molecular Biology and Evolution 17:15581568.

Johnson, A. M., M. D. Bowen, T. G. Ksiazek, R. J. Williams, R. T. Bryan, J. N. Mills, C. J. Peters, S. T. Nichol. 1997. Laguna Negra virus associated with HPS in Western Paraguay and Bolivia. Virology 238:115-127.

Kaufman, G. A., D. W. Kaufman, B. R. McMillan, D. E. Brillhart. 1994. Prevalence of Hantavirus Antibodies in Natural Populations of Deer Mice in North Central Kansas. Prairie Naturalist 26(3):209-216.

Kimura, M. 1983. The Neutral Theory of Molecular Evolution. Cambridge: Cambridge University Press.

King, J. A. (ed.). 1968. Biology of Peromyscus (Rodentia). The American Society of Mammalogists, Special Publication, 2:1-593.

Klempa, B., E. Fichet-Calvet, E. Lecompte, B. Auste, V. Aniskin, H. Meisel, C. Denys, L. Koivogui, J. Meulen, and D. H. Krüger. 2006. Hantavirus in African Wood Mouse, Guinea. Emerging Infectious Diseases 12(5):838-840. 
Klingstrom, J., P. Heymann, S. Escutenaire, K. B. Sjolander, F. DeJaegere, H. Henttonen, A. Lundkvist. 2002. Rodent host specificity of European hantaviruses: evidence of Puumala virus interspecific spillover. Journal of Medical Virology 68(4):581-588.

Ksiazek, T. G., C. J. Peters, P. E. Rollin, S. Zaki, S. T. Nichol, C. Spiropoulou, S. Morzunov, H. Feldmann, A. Sanchez, A. S. Khan, B. W. J. Mahy, K.

Wachsmuth, J. C. Butler. Identification of a new North American Hantavirus that causes acute pulmonary insufficiency. American Journal of Tropical Medical Hygiene 52:117-123.

Kuenzi, A. J., R. J. Douglass, D. White Jr., C. W. Bond, J. N. Mills. 2001. Antibody to Sin Nombre Virus in rodents associated with peridomestic habitats in west central Montana. Am. J. Trop. Med. Hyg., 64(3,4):137-146.

Larget, B., and D. L. Simon. 1999. Markov chain Monte Carlo algorithms for the Bayesian analysis of phylogenetic trees. Molecular Biology and Evolution 16:750-759.

Le Conte, J. 1853. Description of three new species of American Arvicolae, with remarks upon some other American rodents. Proceedings of the National Academy of Sciences, Philadelphia 6:404-415. 
Leache', A. D., and T. W. Reeder. 2002. Molecular systematics of the Eastern Fence Lizard (Sceloporus undulatus): a comparison of parsimony, likelihood, and Bayesian approaches. Systematic Biology 51:44-68.

Lee, H. W., P. W. Lee, and K. M. Johnson. 1978. Isolation of the etiologic agent of Korean hemorrhagic fever. Journal of Infectious Diseases 137:298 308.

Lee, H. W., L. J. Baek, and K. M. Johnson. 1982. Isolation of Hantaan virus, the etiologic agent of Korean hemorrhagic fever, from wild urban rats. Journal of Infectious Diseases 146(5):638-44.

Lewis, P. O. 1998. Maximum likelihood as an alternative to parsimony for inferring phylogeny using nucleotide sequence data. Pages $132-163$ in: Soltis, D. E., Soltis, P. S., and Doyle, J. J., Molecular Systematics of Plants II. Kluwer, Boston.

Li, D., A. L. Schmaljohn, K. Anderson, C. S. Schmaljohn. 1995. Complete nucleotide sequences of the $\mathrm{M}$ and $\mathrm{S}$ segments of two Hantavirus isolates from California: Evidence for reassortment in nature among viruses related to Hantavirus pulmonary syndrome. Virology 206:973-983. 
LTER. 1998. (Sevilleta Long-Term Ecological Research Project). 1998. University of New Mexico. http://sevilleta.unm.edu/data/species/mammal/profile/deermouse.html

Maser, C., B. R. Mate, J. F. Franklin, C. T. Dyrness. 1981. Natural history of Oregon Coast mammals. Gen. Tech. Rep. PNW-133. Portland, OR: U.S. Department of Agriculture, Forest Service, Pacific Northwest Forest and Range Experiment Station. 496 p. [10238]

Mayr, E. 1982. Speciation and macroevolution. Evolution 36:1119-11323.

Michaux, J., A. Reyes, F. Catzeflis. 2001. Evolutionary history of the most speciose mammals: Molecular phylogeny of Muroid rodents. Molecular Biology and Evolution 18:2017-2031.

Mihaescu, R., D., Levy, L. Pachter. 2007. Why Neighbor-Joining Works. Department of Mathematics and Computer Science, U.C. Berkeley arXiv:cs/0602041v3.

Mills, J. N., T. L. Yates, J. E. Childs, R. R. Parmenter, T. G. Ksiazek, P. E. Rollin, C. J. Peters. 1995. Guidelines For Working With Rodents Potentially Infected With Hantavirus. Journal of Mammalogy 76(3):716-722. 
Monroe, M. C., S. P. Morzunov, A. M. Johnson, M. D. Bowen, H. Artsob, T. Yates, C. J. Peters, P. E. Rollin, T. G. Ksiazek, S. T. Nichol. 1999. Genetic Diversity and Distribution of Peromyscus-Borne hantaviruses in North America. Emerging Infectious Diseases 5(1).

Morzunov, S. P., S. C. St. Jeor, T. L. Yates, J. E. Rowe, T. G. Ksiazek, C. J. Peters, S. T. Nichol. 1996. Co-evolution of North American Peromyscus-borne hantaviruses with specific rodent hosts. W 11-5, p. 23. In Abstracts of the Xth International Congress of Virology 1996. Virology Division, International Union of Microbiological Societies, Jerusalem Israel.

Morzunov, S. P., J. E. Rowe, T. G. Ksiazek, C. J. Peters, S. C. St. Jeor, S. T. Nichol. 1998. Genetic Analysis of the Diversity and Origin of Hantavirus in Peromyscus leucopus Mice in North America. Journal of Virology 72(1):5764.

Nei, M. and S. Kumar. 2000. Molecular Evolution and Phylogenetics. New York: Oxford University Press.

Nemirov, K., H. Henttonen, A. Vaheri, A. Plyusnin. 2002. Phylogenetic evidence for host switching in the evolution of hantaviruses carried by Apodemus mice. Virus Research 90:207-215. 
Netski, D., B. H. Thran, S. C. St. Jeor. 1999. Sin Nombre Virus Pathogenesis in Peromyscus maniculatus. Journal of Virology 73(1):585-591.

Nichol, S. T., C. F. Spiropoulou, S. Morzunov, P. E. Rollin, T. G. Ksiazek, H. Feldmann, A. Sanchez, J. Childs, S. Zaki, C. J. Peters. 1993. Genetic identification of a Hantavirus associated with an outbreak of acute respiratory illness. Science 262:914-917.

Nichol, S. T. 1999. Genetic analysis of hantaviruses and their host relationships. In "Factors in the control and emergence of rodent borne viral diseases" (J.-F. Saluzzo and B. Dodet, Eds.), pp. 99-109. Elsevier, Paris.

Nichol S. T., B. J. Beaty, R. M. Elliott, R. Goldbach, A. Plyusnin, C. S. Schmaljohn, R. B. Tesh. 2005. Bunyaviridae. In Virus taxonomy. VIIIth report of the International Committee on Taxonomy of Viruses Edited by: Fauquet C. M., M. A. Mayo, J. Maniloff, U. Desselberger, L. A. Ball. Amsterdam: Elsevier Academic Press, 695-716.

Olsson, G. E., N. White, C. Ahlm, F. Elgh, A. C. Verlemyr, P. Juto, and R. T. Palo. 2002. Demographic Factors Associated With Hantavirus Infection in Bank Voles (Clethrionomys glareolus). Emerging Infectious Diseases 8(9). 
Osborne, T.O., and W. A. Sheppe. 1971. Food habits of Peromyscus maniculatus on a California beach. Journal of Mammalogy 52:844-845.

Osgood, W. H. 1909. Revision of the mice of the American genus Peromyscus. North American Fauna 28:1-285.

Parmenter, C. A, T. L. Yates, R. R. Parmenter, J. N. Mills, J. E. Childs, M. L. Vampell, J. L. Dunnum, J. Milner. 1998. Small mammal survival and trapability in mark-recapture monitoring programs for Hantavirus. Journal of Wildlife Diseases 34(1):1-12.

Parmenter, R. R., T. L. Yates, D. R. Anderson, K. P. Burnham, J. L. Dunnum, A. B. Franklin, M. T. Friggens, B. C. Lubow, M. Miller, G. S. Olson, C. A. Parmenter, J. Pollard, E. Rexstad, T. M. Shenk, T. R. Stanley, G. C. White. 2003. Small Mammal Density Estimation: A Field Comparison of Grid vs. Web. Ecological Monographs, 73(1):1-26.

Peters, C. J. 1998. Hantavirus pulmonary syndrome in the Americas. In "Emerging infections 2" (WM Scheld, WJ Craig, and JM Hughes Eds.), pp. 17-64 American Society of Microbiology, Press Washington DC.

Pini, N., S. Levis, G. Calderón, J. Ramirez, D. Bravo, E. Lozano, C. Ripoll, S. St. Jeor, T. G. Ksiazek, R. M. Barquez, D. Delia Enria. 2003. Hantavirus Infection in 111 
Humans and Rodents; Northwestern Argentina. Emerging Infectious Diseases 9(9):1070-1076.

Plyusnin, A., O. Vapalahti, H. Lankinen, H. Lehväslaiho, N. Apekina, Y. Myasnikov, H. Kallio-Kokko, H. Henttonen, A. Lundkvist, M. Brummer-Korvenkontio. 1994. Tula virus: a newly detected Hantavirus carried by European common voles. Journal of Virology. 68(12):7833-9.

Plyusnin, A., O. Vapalahti, A. Vaheri. 1996. Hantaviruses: genome structure, expression and evolution. Journal of General Virology 77:2677-2687.

Plyusnin, A., S. P. Morzunov. 2000. Evolution and genetic diversity of hantaviruses and their rodent hosts. Curr. Top. Microbiol. Immunol. 256:45-47.

Posada D., and K. A. Crandall. 1998. Modeltest: testing the model of DNA substitution. Bioinformatics 14:817-818.

Puthavathana, P., M. Dobbs, L.-J. Baek, Y.-K. Chu, H. W. Lee, and C. Y. Kang. 1993. Comparison of nucleotide sequences among hantaviruses belonging to the same serotype: an analysis of amplified DNA by thermal cycle sequencing. Virus Research 30:161-169. 
Rannala B., Z. Yang. 1996. Probability distribution of molecular evolutionary trees: a new method for phylogenetic inference. Journal of Molecular Evolution 43:304-311.

Rhodes III, L. V., C. Huang, A. J. Sanchez, S. T. Nichol, S. R. Zaki, T. G. Ksiazek, J. G. Humphreys, J. J. Freeman, K. R. Knecht. 2000. Hantavirus Pulmonary Syndrome associated with Monongahela virus, Pennsylvania. Centers for Disease Control 6(6).

Rowe, J. E., S. C. St. Jeor, J. Riolo, E. W. Otteson, M. C. Monroe, W. W. Henderson, T. G. Ksiazek, P. E. Rollin, S. T. Nichol. 1995. Coexistence of several novel hantaviruses in rodents indigenous to North America. Virology 213:122-130.

Ruedas, L. A., J. Salazar-Bravo, D. S. Tinnin, L. Caceres, A. Garcia, L. J. Eskew, P. Kitsutani, M. A. Diaz, T. G. Ksiazek, S. T. Nichol, P. E. Rollin, F. Gracia, G. Suzan, B. Armiens, C. J. Peters, T. L. Yates, J. N. Mills. 2004. Community Ecology of Small Mammal Populations in Panama Following an Outbreak of Hantavirus Pulmonary Syndrome. Journal of Vector Ecology 29(1):177-91.

Salazar-Bravo, J., J. W. Dragoo, M. D. Bowen, C. J. Peters, T. G. Ksiazek, T. L. Yates. 2002. Natural nidality in Bolivian hemorrhagic fever and the systematics of the reservoir species. Infections, Genetics, and Evolution 1:191-199. 
Sanchez, A. J., K. D. Abbott, S. T. Nichol. 2001. Genetic identification and characterization of Limestone Canyon Virus, a Unique Peromyscus-borne Hantavirus. Virology 286:345-353.

Sanger, F., A. R. Coulson. 1975. A rapid method for determining sequences in DNA by primed synthesis with DNA polymerase. Journal of Molecular Biology, 94(3):441-448.

Sanger, F., S. Nicklen, A. R. Coulson. 1977. DNA sequencing with chainterminating inhibitors, Proc Natl Acad Sci U.S.A., 74(12): 5463-5467.

Schmaljohn, C. S. 1990. Nucleotide sequence of the L genome segment of Hantaan virus. Nucleic Acid Research 18:6728.

Schmaljohn, A., D. Li, D. L. Negley, D. S. Bressler, M. J. Turell, G. W. Korch, M. S. Ascher, C. S. Schmaljohn. 1995. Isolation and initial characterization of a newfound Hantavirus from California. Virology 206:963-972.

Schmaljohn, C., B. Hjelle. 1997. Hantaviruses: A Global Disease Problem. United States Army Medical Research Institute of Infectious Diseases.

Sironen, T., A. Vaheri, A. Plyusnin. 2001. Molecular Evolution of Puumala 
Hantavirus. Journal of Virology 75(23):11803-11810.

Smith, L. R., D. W. Hale, I. F. Greenbaum. 2000. Systematic implications of chromosomal data from two insular species of Peromyscus from the Gulf of California. The Journal of Heredity 91(2):162-164.

Soltis, Pamela S., and Douglas E. Soltis. 2003. Applying the Bootstrap in Phylogeny Reconstruction. Statistical Science, Vol. 18, No. 2, 256-267.

Swofford, D. L. 1998. Phylogenetic Analysis Using Parsimony (and other methods). Sinauer Associates, Sunderland MA.

Swofford, D. L. 2002. PAUP*. Phylogenetic analysis using parsimony (*and other methods). Version 4.0b10. Sinauer Associates, Sunderland, Mass.

Tkachenko, E. A., A. P. Ivanov, M. A. Donets, Y. A. Miasnikov, E. V. Ryltseva, L. K. Gaponova, V. N. Bashkirtsev, N. M. Okulova, S. G. Drozdov, R. A. Slonova. 1983. Potential Reservoir and Vectors of Haemorrhagic fever with renal syndrome (HFRS) in the U. S. S. R. Annals of the Belgian Society Tropical Medicine. 63(3):267-9. 
Vapalahti, O., A. Lundkvist, V. Federov, C. J. Conroy, S. Hirvonen, A. Plyusnina, K. Nemirov, K. Fredga, J. Cook, J. Niemimaa, A. Kaikusalo, H. Henttonen, A. Vaheri, A. Plyusnin. 1999. Isolation and characterization of a Hantavirus from Lemmicus sibiricus: evidence for host switch during Hantavirus evolution. Journal of Virology 73(7):5586-5592.

Verts, B.J., and L. N. Carraway. 1998. Land Mammals of Oregon. University of California Press. pp. 270-273.

Vincent, M. J., E. Quiroz, F. Gracia, A. J. Sanchez, T. G. Ksiazek, P. T. Kitsutani, L. A. Ruedas, D. S. Tinnin, L. Caceres, A. Garcia, P. E. Rollin, J. N. Mills, C. J. Peters, S. T. Nichol. 2000. Hantavirus Pulmonary Syndrome in Panama: Identification of Novel Hantaviruses and Their Likely Reservoirs. Virology 277:14-19.

Waddell, P. J., H. Kishino, and R. Ota. 2001. A phylogenetic foundation for comparative mammalian genomics. Genome Informatics Series 12:141-155.

Weidmann, M., P. Schmidt, M. Vackova, K. Krivanec, P. Munclinger, F. T. Hufert. 2005. Identification of genetic evidence for Dobrava Virus spillover in rodents by nested reverse transcription (RT)-PCR and TaqMan RT-PCR. Journal of Clinical Microbiology 43(2):808-812. 
Wilson, D. E., and S. Ruff. 1999. The Smithsonian Book of North American Mammals. Smithsonian Institution pgs.575-577.

Wilson, K. R., D. R. Anderson. 1985. Evaluation of A Density Estimator Based on a Trapping Web and Distance Sampling Theory. Ecology 66(4):1185-1194.

Woodley, S. A. 2007. Morphological Variation in the Deer Mouse, Peromyscus maniculatus in Oregon: Effects on Morphology from Environmental Factors. Unpublished Masters Thesis. Department of Biology, Portland State University, Portland, OR.

Xiao, S.-Y., J. W. LeDuc, Y. K. Chu, and C. S. Schmaljohn. 1994. Phylogenetic analyses of virus isolates in the genus Hantavirus, family Bunyaviridae. Virology 198:205-217.

Yates, T. L., J. N. Mills, C. A. Parmenter, T. G. Ksiazek, R. R. Parmenter, J. R. Vande Castle, C. H. Calisher, S. T. Nichol, K. D. Abbott, J. C. Young, M. L. Morrison, B. J. Beaty, J. L. Dunnum, R. J. Baker, J. Salazar-Bravo, C. J. Peters. 2002. The ecology and evolutionary history of an emergent disease: Hantavirus Pulmonary Syndrome. Bioscience 52(11):989-998.

Yang, Z., and B. Rannala. 1997. Bayesian phylogenetic inference using DNA sequences: a Markov Chain Monte Carlo method. Molecular Biology and 
Evolution 14:717-724.

Zheng, X., B. S. Arbogast, G. J. Kenagy. 2003. Historical demography and genetic structure of sister species: deermice (Peromyscus) in the Northern American temperate rain forest. Molecular Ecology 12:711-724. 
Appendix A: Peromyscus Distance Matrix. Pair-wise nucleotide percent difference (distance) of mitochondrial ND3, ND4, ND4L, and arginine tRNA genes (1,463bp) among species and subspecies of Peromyscus (Cricetidae) and other out-group Muridae $(\mathrm{n}=88)$.

\begin{tabular}{|c|c|c|c|c|c|c|c|c|c|}
\hline & WC7 & WC11 & WC12 & WC13 & ljd3256 & ljd3304 & ljd3306 & ljd3308 & ljd3317 \\
\hline WC7 & & & & & & & & & ! \\
\hline wC11 & 0.0014 & & & & & & & & \\
\hline WC12 & 0.0000 & 0.0014 & & & & & & & \\
\hline WC13 & 0.0000 & 0.0014 & 0.0000 & & & & & & \\
\hline ljd3256 & 0.0043 & 0.0028 & 0.0043 & 0.0043 & & & & & \\
\hline ljd3304 & 0.0043 & 0.0028 & 0.0043 & 0.0043 & 0.0057 & & & & \\
\hline ljd3306 & 0.0036 & 0.0021 & 0.0036 & 0.0036 & 0.0050 & 0.0007 & & & \\
\hline ljd3308 & 0.0029 & 0.0014 & 0.0029 & 0.0029 & 0.0043 & 0.0043 & 0.0036 & & \\
\hline ljd3317 & 0.0014 & 0.0028 & 0.0014 & 0.0014 & 0.0029 & 0.0028 & 0.0021 & 0.0014 & \\
\hline ljd3324 & 0.0029 & 0.0014 & 0.0028 & 0.0028 & 0.0028 & 0.0028 & 0.0036 & 0.0029 & 0.0014 \\
\hline ljd3327 & 0.0036 & 0.0036 & 0.0036 & 0.0036 & 0.0050 & 0.0050 & 0.0043 & 0.0007 & 0.0021 \\
\hline ljd3330 & 0.0058 & 0.0050 & 0.0058 & 0.0058 & 0.0073 & 0.0028 & 0.0021 & 0.0058 & 0.0043 \\
\hline ljd3333 & 0.0036 & 0.0021 & 0.0036 & 0.0036 & 0.0050 & 0.0007 & 0.0000 & 0.0036 & 0.0021 \\
\hline ljd3344 & 0.0036 & 0.0028 & 0.0036 & 0.0036 & 0.0050 & 0.0007 & 0.0000 & 0.0036 & 0.0021 \\
\hline ljd3348 & 0.0095 & 0.0080 & 0.0095 & 0.0095 & 0.0110 & 0.0110 & 0.0103 & 0.0081 & 0.0080 \\
\hline IJD620 & 0.0058 & 0.0064 & 0.0058 & 0.0058 & 0.0073 & 0.0072 & 0.0065 & 0.0043 & 0.0050 \\
\hline LDD774 & 0.0050 & 0.0057 & 0.0050 & 0.0050 & 0.0050 & 0.0065 & 0.0057 & 0.0036 & 0.0042 \\
\hline LJD886 & 0.0028 & 0.0035 & 0.0028 & 0.0028 & 0.0028 & 0.0028 & 0.0036 & 0.0029 & 0.0021 \\
\hline LJD2088 & 0.0080 & 0.0079 & 0.0080 & 0.0080 & 0.0079 & 0.0094 & 0.0087 & 0.0065 & 0.0065 \\
\hline LJD1960 & 0.0043 & 0.0086 & 0.0043 & 0.0043 & 0.0043 & 0.0058 & 0.0050 & 0.0029 & 0.0057 \\
\hline LJD1669 & 0.0058 & 0.0101 & 0.0058 & 0.0058 & 0.0057 & 0.0072 & 0.0065 & 0.0043 & 0.0072 \\
\hline LJD1524 & 0.0021 & 0.0021 & 0.0021 & 0.0021 & 0.0036 & 0.0021 & 0.0014 & 0.0021 & 0.0007 \\
\hline LJD1523 & 0.0000 & 0.0014 & 0.0000 & 0.0000 & 0.0043 & 0.0043 & 0.0036 & 0.0029 & 0.0014 \\
\hline LDD1122 & 0.0029 & 0.0014 & 0.0029 & 0.0028 & 0.0043 & 0.0028 & 0.0021 & 0.0029 & 0.0021 \\
\hline LDD2522 & 0.0043 & 0.0050 & 0.0043 & 0.0043 & 0.0028 & 0.0043 & 0.0050 & 0.0043 & 0.0028 \\
\hline LDD1637 & 0.0043 & 0.0043 & 0.0043 & 0.0043 & 0.0072 & 0.0072 & 0.0065 & 0.0043 & 0.0043 \\
\hline LD1638 & 0.0036 & 0.0036 & 0.0050 & 0.0050 & 0.0065 & 0.0050 & 0.0043 & 0.0036 & 0.0036 \\
\hline LD2161 & 0.0029 & 0.0014 & 0.0029 & 0.0029 & 0.0029 & 0.0043 & 0.0036 & 0.0029 & 0.0014 \\
\hline LJD2651 & 0.0058 & 0.0043 & 0.0058 & 0.0058 & 0.0072 & 0.0073 & 0.0065 & 0.0043 & 0.0043 \\
\hline LJ2737 & 0.0080 & 0.0087 & 0.0080 & 0.0080 & 0.0095 & 0.0095 & 0.0087 & 0.0065 & 0.0072 \\
\hline LJD2834 & 0.0036 & 0.0021 & 0.0036 & 0.0036 & 0.0050 & 0.0007 & 0.0000 & 0.0036 & 0.0021 \\
\hline LD2850 & 0.0043 & 0.0029 & 0.0043 & 0.0043 & 0.0058 & 0.0014 & 0.0007 & 0.0043 & 0.0029 \\
\hline LD2898 & 0.0080 & 0.0065 & 0.0080 & 0.0080 & 0.0095 & 0.0095 & 0.0087 & 0.0065 & 0.0065 \\
\hline LD2914 & 0.0014 & 0.0000 & 0.0014 & 0.0014 & 0.0029 & 0.0029 & 0.0021 & 0.0014 & 0.0000 \\
\hline LD2936 & 0.0028 & 0.0021 & 0.0035 & 0.0035 & 0.0028 & 0.0028 & 0.0036 & 0.0029 & 0.0021 \\
\hline LJD3000 & 0.0021 & 0.0007 & 0.0021 & 0.0021 & 0.0036 & 0.0036 & 0.0029 & 0.0014 & 0.0007 \\
\hline LD2126 & 0.0095 & 0.0095 & 0.0095 & 0.0095 & 0.0110 & 0.0110 & 0.0102 & 0.0080 & 0.0080 \\
\hline LJD2138 & 0.0050 & 0.0043 & 0.0057 & 0.0057 & 0.0072 & 0.0072 & 0.0057 & 0.0050 & 0.0043 \\
\hline LD2154 & 0.0036 & 0.0021 & 0.0036 & 0.0036 & 0.0050 & 0.0007 & 0.0000 & 0.0036 & 0.0021 \\
\hline LJD2158 & 0.0095 & 0.0087 & 0.0102 & 0.0102 & 0.0117 & 0.0117 & 0.0102 & 0.0080 & 0.0087 \\
\hline LJD2836 & 0.0095 & 0.0080 & 0.0095 & 0.0095 & 0.0110 & 0.0110 & 0.0103 & 0.0080 & 0.0080 \\
\hline
\end{tabular}




\begin{tabular}{|c|c|c|c|c|c|c|c|c|c|}
\hline LD2852 & 0.0087 & 0.0073 & 0.0088 & 0.0088 & 0.0103 & 0.0103 & 0.0095 & 0.0073 & 0.0073 \\
\hline LD2860 & 0.0029 & 0.0014 & 0.0029 & 0.0029 & 0.0028 & 0.0043 & 0.0036 & 0.0029 & 0.0014 \\
\hline LD2865 & 0.0050 & 0.0036 & 0.0050 & 0,0050 & 0.0050 & 0.0050 & 0.0058 & 0.0043 & 0.0036 \\
\hline LJD2890 & 0.0058 & 0.0043 & 0.0058 & 0.0058 & 0.0073 & 0.0073 & 0.0065 & 0.0043 & 0.0043 \\
\hline LJD2943 & 0.0029 & 0.0014 & 0.0029 & 0.0029 & 0.0029 & 0.0043 & 0.0036 & 0.0029 & 0.0014 \\
\hline Jefferson 3 & 0.0043 & 0.0049 & 0.0043 & 0.0043 & 0.0043 & 0.0043 & 0.0050 & 0.0028 & 0.0035 \\
\hline ADAMS6 & 0.0050 & 0.0057 & 0.0050 & 0.0050 & 0.0065 & 0.0065 & 0.0058 & 0.0036 & 0.0042 \\
\hline clatsop 1 & 0.0029 & 0.0029 & 0.0030 & 0.0029 & 0.0045 & 0.0045 & 0.0037 & 0.0015 & 0.0029 \\
\hline clatsop2 & 0.0029 & 0.0029 & 0.0029 & 0.0029 & 0.0044 & 0.0030 & 0.0022 & 0.0029 & 0.0029 \\
\hline clatsop3 & 0.0029 & 0.0029 & 0.0029 & 0.0029 & 0.0044 & 0.0030 & 0.0022 & 0.0029 & 0.0029 \\
\hline clatsop4 & 0.0029 & 0.0029 & 0.0029 & 0.0029 & 0.0044 & 0.0030 & 0.0022 & 0.0029 & 0.0029 \\
\hline LostForest1A & 0.0085 & 0.0084 & 0.0099 & 0.0099 & 0.0082 & 0.0084 & 0.0069 & 0.0069 & 0.0084 \\
\hline LostForest2 & 0.0109 & 0.0100 & 0.0116 & 0.0116 & 0.0124 & 0.0124 & 0.0117 & 0.0087 & 0.0130 \\
\hline LostForest3 & 0.0058 & 0.0107 & 0.0065 & 0.0064 & 0.0057 & 0.0072 & 0.0065 & 0.0043 & 0.0078 \\
\hline LostForest4 & 0.0102 & 0.0115 & 0.0109 & 0.0109 & 0.0117 & 0.0117 & 0.0110 & 0.0087 & 0.0100 \\
\hline sj1Pmausterus & 0.0080 & 0.0065 & 0.0080 & 0.0080 & 0.0095 & 0.0095 & 0.0088 & 0.0065 & 0.0065 \\
\hline sj2Pmausterus & 0.0080 & 0.0102 & 0.0080 & 0.0087 & 0.0102 & 0.0103 & 0.0095 & 0.0072 & 0.0079 \\
\hline sj2aPmausterus & 0.0051 & 0.0036 & 0.0051 & 0.0051 & 0.0066 & 0.0065 & 0.0058 & 0.0036 & 0.0036 \\
\hline sj3Pmausterus & 0.0065 & 0.0050 & 0.0065 & 0.0065 & 0.0080 & 0.0080 & 0.0073 & 0.0050 & 0.0050 \\
\hline sj5Pmausterus & 0.0058 & 0.0043 & 0.0058 & 0.0058 & 0.0073 & 0.0073 & 0.0065 & 0.0043 & 0.0043 \\
\hline sj6Pmausterus & 0.0058 & 0.0043 & 0.0058 & 0.0058 & 0.0073 & 0.0073 & 0.0065 & 0.0043 & 0.0043 \\
\hline sj7Pmausterus & 0.0058 & 0.0050 & 0.0058 & 0.0058 & 0.0073 & 0.0072 & 0.0065 & 0.0043 & 0.0043 \\
\hline OSM723Pmman & 0.0391 & 0.0410 & 0.0389 & 0.0387 & 0.0405 & 0.0424 & 0.0417 & 0.0391 & 0.0391 \\
\hline OSM726Pmman & 0.0383 & 0.0402 & 0.0380 & 0.0379 & 0.0397 & 0.0416 & 0.0408 & 0.0383 & 0.0383 \\
\hline OSM727Pmman & 0.0391 & 0.0410 & 0.0389 & 0.0387 & 0.0405 & 0.0424 & 0.0417 & 0.0391 & 0.0391 \\
\hline OSM737Pmman & 0.0383 & 0.0402 & 0.0380 & 0.0379 & 0.0397 & 0.0416 & 0.0408 & 0.0383 & 0.0383 \\
\hline U40255PsejugSD & 0.0498 & 0.0517 & 0.0497 & 0.0496 & 0.0496 & 0.0513 & 0.0505 & 0.0478 & 0.0500 \\
\hline U40253PsejugSC & 0.0595 & 0.0613 & 0.0593 & 0.0591 & 0.0591 & 0.0610 & 0.0602 & 0.0573 & 0.0594 \\
\hline JEB1309APtruei & 0.2475 & 0.2391 & 0.2439 & 0.2439 & 0.2437 & 0.2391 & 0.2410 & 0.2435 & 0.2391 \\
\hline AF374578PKEEN & 0.0542 & 0.0541 & 0.0540 & 0.0538 & 0.0559 & 0.0538 & 0.0529 & 0.0521 & 0.0522 \\
\hline U40252Pleuc & 0.2439 & 0.2378 & 0.2427 & 0.2418 & 0.2421 & 0.2421 & 0.2447 & 0.2392 & 0.2349 \\
\hline JAM160Pleuc & 0.1936 & 0.1923 & 0.1940 & 0.1933 & 0.1935 & 0.1935 & 0.1949 & 0.1894 & 0.1882 \\
\hline JAM161Pleuc & 0.2121 & 0.2095 & 0.2116 & 0.2108 & 0.2110 & 0.2110 & 0.2128 & 0.2034 & 0.2053 \\
\hline JAM162Pleuc & 0.2027 & 0.1978 & 0.2031 & 0.2024 & 0.2026 & 0.2026 & 0.2040 & 0.1982 & 0.1979 \\
\hline JAM163Pleuc & 0.2041 & 0.2027 & 0.2045 & 0.2038 & 0.2039 & 0.2039 & 0.2054 & 0.1952 & 0.1985 \\
\hline JAM164Pleuc & 0.2095 & 0.2080 & 0.2099 & 0.2092 & 0.2094 & 0.2094 & 0.2109 & 0.2003 & 0.2039 \\
\hline JAM168Pmbairdï & 0.0157 & 0.0140 & 0.0156 & 0.0156 & 0.0156 & 0.0171 & 0.0164 & 0.0141 & 0.0140 \\
\hline JAM167Pmbairdii & 0.0164 & 0.0147 & 0.0163 & 0.0163 & 0.0163 & 0.0179 & 0.0171 & 0.0148 & 0.0148 \\
\hline JAM 159Pmbairdii & 0.0157 & 0.0156 & 0.0157 & 0.0157 & 0.0157 & 0.0172 & 0.0165 & 0.0141 & 0.0141 \\
\hline JAM166Pmbairdii & 0.0196 & 0.0180 & 0.0196 & 0.0196 & 0.0196 & 0.0212 & 0.0204 & 0.0180 & 0.0180 \\
\hline M.pennsylvanicus & 0.6270 & 0.6193 & 0.6269 & 0.6267 & 0.6331 & 0.6247 & 0.6284 & 0.6334 & 0.6185 \\
\hline Oryzomyspalustris & 0.9310 & 0.9429 & 0.9310 & 0.9309 & 0.9690 & 0.9224 & 0.9283 & 0.9556 & 0.9407 \\
\hline Sigmodonhispidus & 1.0163 & 1.0279 & 1.0164 & 1.0163 & 1.0275 & 1.0370 & 1.0348 & 1.0494 & 1.0251 \\
\hline Rmegalotis & 0.5375 & 0.5552 & 0.5378 & 0.5381 & 0.5554 & 0.5736 & 0.5733 & 0.5550 & 0.5548 \\
\hline Rattusnorvegicus & 0.7430 & 0.7559 & 0.7469 & 0.7426 & 0.7854 & 0.7527 & 0.7559 & 0.7855 & 0.7507 \\
\hline Pmrufinus & 0.0239 & 0.0244 & 0.0238 & 0.0237 & 0.0237 & 0.0253 & 0.0246 & 0.0223 & 0.0228 \\
\hline
\end{tabular}


$\begin{array}{lllllllllll}\operatorname{ljd} 3324 & \operatorname{ljd} 3327 & \operatorname{ljd} 3330 & \mathrm{ljd} 3333 & \operatorname{ljd} 3344 & \mathrm{ljd} 3348 & \text { LJD620 } & \text { LJD774 } & \text { LJD886 } & \text { LJD2088 } & \text { LJD1960 }\end{array}$

WC7

WC11

wC12

WC13

ljd3256

ljd3304

ljd3306

ljd3308

ljd3317

ljd3324

ljd3327

ljd3330

ljd3333

ljd3344

ljd3348

LJD620

LD774

LJD886

LJD2088

LDD1960

LDD1669

LDD1524

LDD1523

LDD1 122

LD2522

LD1637

LDD1638

LJD2161

LJD2651

LJD2737

LJD2834

LJD2850

LJ2898

LDD2914

LD2936

LD3000

LD2126

LD2138

LD2154

LD2158

LD2836

LJD2852

LJD2860

LJD2865

LJD2890

0.0036

$0.0058 \quad 0.0065$

$\begin{array}{lll}0.0036 & 0.0043 & 0.0021\end{array}$

$\begin{array}{llll}0.0036 & 0.0043 & 0.0021 & 0.0000\end{array}$

$\begin{array}{lllll}0.0095 & 0.0088 & 0.0111 & 0.0103 & 0.0102\end{array}$

$\begin{array}{llllll}0.0058 & 0.0050 & 0.0087 & 0.0065 & 0.0065 & 0.0110\end{array}$

$\begin{array}{llllll}0.0036 & 0.0043 & 0.0065 & 0.0058 & 0.0057\end{array}$

0.0000

0.0080

0.0029

0.0043

0.0021

0.0028

0.0029

0.0014

0.0058

0.0036

0.0029

$$
0.0058
$$

0.0080

0.0036

0.0043

0.0080

0.0014

$$
0.0007
$$

0.0021

0.0095

0.0057

0.0036

0.0102

0.0095

0.0088

0.0088

0.0029

0.0021

0.0058
0.0043

0.0036

0.0072

0.0036

$\begin{array}{llll}0.0051 & 0.0072 & 0.0065 & 0.0065\end{array}$

$\begin{array}{llll}0.0028 & 0.0036 & 0.0014 & 0.0014\end{array}$

$\begin{array}{lllll}0.0036 & 0.0058 & 0.0036 & 0.0036\end{array}$

$\begin{array}{llll}0.0043 & 0.0050 & 0.0021 & 0.0028\end{array}$

0.0050

0.0050

0.0072

$0.0065 \quad 0.0065$

0.0065

$0.0036 \quad 0.0036$

0.0036

$0.0051 \quad 0.0073$

$0.0073 \quad 0.0095$

$0.0043 \quad 0.0021$

$\begin{array}{ll}0.0050 & 0.0029\end{array}$

$\begin{array}{ll}0.0087 & 0.0087\end{array}$

0.0095

$0.0021 \quad 0.0043$

$\begin{array}{ll}0.0036 & 0.0058 \\ & \end{array}$

0.0087

0.0110

0.0058

0.0080

$0.0043 \quad 0.0021$

0.0088

$0.0088 \quad 0.0111$

0.0081

0.0111

0.0036

0.0058

0.0050

0.0051

0.0080

0.0058
$0.0050 \quad 0.0050$

$0.0043 \quad 0.0057$

$0.0065 \quad 0.0065$

$0.0088 \quad 0.0087$

$0.0000 \quad 0.0000$

$0.0007 \quad 0.0007$

$0.0021 \quad 0.0021$

$0.0036 \quad 0.0036$

$0.0029 \quad 0.0029$

0.0103

0.0087

0.0095

0.0102

0.0096

0.0080

0.0088

0.0095

0.0095

0.0110

0.0095

0.0118

0.0096

0.0065

0.0119

0.0103

0.0111

0.0118

0.0080

0.0095

0.0088

0.0029

0.0110

0.0103

0.0036

0.0029

0.0096

0.0096

$0.0036 \quad 0.0036$

0.0057

0.0058

0.0118

0.0096

0.009
0.0050

$0.0057 \quad 0.0035$

$\begin{array}{lll}0.0094 & 0.0072 & 0.0079\end{array}$

0.0094

0.0080

$0.0080 \quad 0.0014$

$0.0072 \quad 0.0036$

$0.0080 \quad 0.0043$

$0.0087 \quad 0.0050$

0.0065

0.0072

0.0072

0.0079

0.0059

0.0073

0.0079

0.0065

0.0073

0.0080

0,0043

0.0057

0.0050

0.0110

0.0087

0.0065

0.0117

0.0110

0.0103

0.0058

0.0080

0.0073

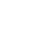




\begin{tabular}{|c|c|c|c|c|c|c|c|c|c|c|c|}
\hline LJD2943 & 0.0029 & 0.0036 & 0.0058 & 0.0036 & 0.0036 & 0.0096 & 0.0058 & 0.0050 & 0.0029 & 0.0080 & 0.0043 \\
\hline Jefferson 3 & 0.0014 & 0.0036 & 0.0072 & 0.0050 & 0.0050 & 0.0095 & 0.0042 & 0.0021 & 0.0014 & 0.0079 & 0.0064 \\
\hline ADAMS6 & 0.0050 & 0.0043 & 0.0065 & 0.0058 & 0.0057 & 0.0058 & 0.0050 & 0.0042 & 0.0050 & 0.0072 & 0.0086 \\
\hline clatsop1 & 0.0029 & 0.0022 & 0.0060 & 0.0037 & 0.0037 & 0.0069 & 0.0052 & 0.0044 & 0.0037 & 0.0067 & 0.0074 \\
\hline clatsop2 & 0.0029 & 0.0037 & 0.0045 & 0.0022 & 0.0022 & 0.0099 & 0.0067 & 0.0059 & 0.0037 & 0.0082 & 0.0089 \\
\hline clatsop3 & 0.0029 & 0.0037 & 0.0045 & 0.0022 & 0.0022 & 0,0099 & 0.0067 & 0.0059 & 0.0037 & 0.0082 & 0.0089 \\
\hline clatsop4 & 0.0029 & 0,0037 & 0.0045 & 0.0022 & 0.0022 & 0.0099 & 0.0067 & 0.0059 & 0.0037 & 0.0082 & 0.0089 \\
\hline LostForest1 A & 0.0099 & 0.0070 & 0.0085 & 0.0069 & 0.0068 & 0.0097 & 0.0097 & 0.0067 & 0.0084 & 0.0052 & 0.0085 \\
\hline LostForest2 & 0.0116 & 0.0110 & 0.0125 & 0.0110 & 0.0123 & 0.0116 & 0.0116 & 0.0108 & 0.0131 & 0.0146 & 0.0169 \\
\hline LostForest3 & 0.0050 & 0.0050 & 0.0072 & 0.0065 & 0.0064 & 0.0095 & 0.0093 & 0.0056 & 0.0078 & 0.0079 & 0.0043 \\
\hline LostForest4 & 0.0109 & 0.0095 & 0.0117 & 0.0110 & 0.0109 & 0.0109 & 0.0086 & 0.0078 & 0.0101 & 0.0124 & 0.0139 \\
\hline sj1Pmausterus & 0.0080 & 0.0073 & 0.0095 & 0.0088 & 0.0088 & 0.0043 & 0.0095 & 0.0072 & 0.0080 & 0.0103 & 0.0080 \\
\hline sj2Pmausterus & 0.0080 & 0.0080 & 0.0117 & 0.0087 & 0.0095 & 0.0088 & 0.0109 & 0.0102 & 0.0094 & 0.0110 & 0.0109 \\
\hline sj2aPmausterus & 0.0050 & 0.0043 & 0.0080 & 0.0058 & 0.0058 & 0.0073 & 0.0065 & 0.0058 & 0.0050 & 0.0088 & 0.0050 \\
\hline sj3Pmausterus & 0.0065 & 0.0058 & 0.0095 & 0.0073 & 0.0073 & 0.0088 & 0.0080 & 0.0073 & 0.0065 & 0.0103 & 0.0065 \\
\hline sj5Pmausterus & 0.0058 & 0.0051 & 0.0088 & 0.0065 & 0.0065 & 0.0081 & 0.0073 & 0.0065 & 0.0058 & 0.0095 & 0.0058 \\
\hline sj6Pmausterus & 0.0058 & 0.0050 & 0.0088 & 0.0065 & 0,0065 & 0.0081 & 0.0073 & 0.0065 & 0.0058 & 0.0095 & 0.0058 \\
\hline sj7Pmausterus & 0.0058 & 0.0050 & 0.0087 & 0.0065 & 0.0065 & 0.0081 & 0.0072 & 0.0065 & 0.0058 & 0.0095 & 0.0058 \\
\hline OSM723Pmman & 0.0389 & 0.0400 & 0.0428 & 0.0419 & 0.0414 & 0.0454 & 0.0419 & 0.0393 & 0.0384 & 0.0452 & 0.0412 \\
\hline OSM726Pmman & 0.0381 & 0.0392 & 0.0419 & 0.0410 & 0.0406 & 0.0446 & 0.0411 & 0.0384 & 0.0376 & 0.0443 & 0.0403 \\
\hline OSM727Pmman & 0.0389 & 0.0400 & 0.0428 & 0.0419 & 0,0414 & 0.0454 & 0.0419 & 0.0393 & 0.0384 & 0.0452 & 0.0412 \\
\hline OSM737Pmman & 0.0381 & 0.0392 & 0.0419 & 0.0410 & 0.0406 & 0.0446 & 0.0411 & 0.0384 & 0.0376 & 0.0443 & 0.0403 \\
\hline U40255PsejugSD & 0.0495 & 0.0486 & 0.0515 & 0.0507 & 0.0504 & 0.0543 & 0.0531 & 0.0500 & 0.0490 & 0.0542 & 0.0520 \\
\hline U40253PsejugSC & 0.0592 & 0.0582 & 0.0612 & 0.0605 & 0.0600 & 0.0642 & 0.0627 & 0.0595 & 0.0584 & 0.0640 & 0.0617 \\
\hline JEB1309APtruei & 0.2393 & 0.2435 & 0.2446 & 0.2427 & 0.2389 & 0.2316 & 0.2436 & 0.2408 & 0.2392 & 0.2363 & 0.2466 \\
\hline AF374578PKEEN & 0.0539 & 0.0530 & 0.0520 & 0.0531 & 0.0527 & 0.0550 & 0.0553 & 0.0524 & 0.0533 & 0.0528 & 0.0583 \\
\hline U40252Pleuc & 0.2387 & 0.2403 & 0.2435 & 0.2458 & 0.2432 & 0.2456 & 0.2454 & 0.2430 & 0.2348 & 0.2267 & 0.2463 \\
\hline JAM160Pleuc & 0.1905 & 0.1905 & 0.1932 & 0.1952 & 0.1946 & 0.1948 & 0.1903 & 0.1870 & 0.1891 & 0.1835 & 0.1864 \\
\hline JAM161Pleuc & 0.2079 & 0.2045 & 0.2116 & 0.2138 & 0.2122 & 0.2123 & 0.2075 & 0.2040 & 0.2063 & 0.2002 & 0.2039 \\
\hline JAM162Pleuc & 0.1995 & 0.1997 & 0.2026 & 0.2043 & 0.2040 & 0.2038 & 0.2000 & 0.1966 & 0.1988 & 0.1924 & 0.1945 \\
\hline JAM163Pleuc & 0.2009 & 0.1962 & 0.2036 & 0.2057 & 0.2051 & 0.2052 & 0.2008 & 0.1973 & 0.1995 & 0.1933 & 0.1952 \\
\hline JAM164Pleuc & 0.2063 & 0.2014 & 0.2091 & 0.2111 & 0.2105 & 0.2105 & 0.2062 & 0.2027 & 0.2049 & 0.1985 & 0.2005 \\
\hline JAM168Pmbairdii & 0.0156 & 0.0148 & 0.0173 & 0.0164 & 0.0164 & 0.0164 & 0.0172 & 0.0148 & 0.0155 & 0.0148 & 0.0141 \\
\hline JAM167Pmbairdii & 0.0163 & 0.0156 & 0.0180 & 0.0171 & 0.0171 & 0.0171 & 0.0179 & 0.0156 & 0.0163 & 0.0155 & 0.0148 \\
\hline JAM 159Pmbairdii & 0.0157 & 0.0149 & 0.0189 & 0.0165 & 0.0165 & 0.0196 & 0.0173 & 0.0164 & 0.0156 & 0.0164 & 0.0141 \\
\hline JAM166Pmbairdii & 0.0196 & 0.0188 & 0.0213 & 0.0204 & 0.0204 & 0.0188 & 0.0213 & 0.0188 & 0.0196 & 0.0188 & 0.0180 \\
\hline M.pennsylvanicus & 0.6101 & 0.6371 & 0.6183 & 0.6280 & 0.6282 & 0.6317 & 0.6110 & 0.6115 & 0.6099 & 0.6284 & 0.6102 \\
\hline Oryzomyspalustris & 0.9251 & 0.9618 & 0.9381 & 0.9276 & 0.9280 & 0.9341 & 0.8914 & 0.9159 & 0.9251 & 0.9415 & 0.9421 \\
\hline Sigmodonhispidus & 1.0402 & 1.0571 & 1.0263 & 1.0339 & 1.0345 & 0.9834 & 1.0347 . & 1.0225 & 1.0402 & 0.9954 & 1.0400 \\
\hline R.megalotis & 0.5625 & 0.5571 & 0.5670 & 0.5730 & 0.5734 & 0.5385 & 0.5619 & 0.5825 & 0.5631 & 0.5411 & 0.5769 \\
\hline Rattusnorvegicus & 0.7747 & 0.7857 & 0.7479 & 0.7555 & 0.7538 & 0.7734 & 0.7533 & 0.7521 & 0.7539 & 0.7465 & 0.7872 \\
\hline Pmrufinus & 0.0222 & 0.0231 & 0.0254 & 0.0247 & 0.0245 & 0.0262 & 0.0236 & 0.0197 & 0.0220 & 0.0245 & 0.0260 \\
\hline
\end{tabular}


$\begin{array}{lllllllllll}\text { LJD1669 } & \text { LJD1524 } & \text { LJD1523 } & \text { LJD1122 } & \text { LDD2522 } & \text { LJD1637 } & \text { LDD1638 } & \text { LD2161 } & \text { LD2651 } & \text { LJD2737 } & \text { LDD2834 }\end{array}$

WC7

wCll

WC12

WC13

ljd3256

ljd3304

ljd3306

ljd 3308

ljd3317

ljd3324

ljd3327

ljd 3330

ljd3333

ljd3344

ljd3348

LJD620

LD774

LJD886

LJD2088

LJD1960

LJD1669

LJD1524

LDD1523

LD1122

LJD2522

LJD1637

LDD1638

LJD2161

LD2651

LD2737

LD2834

LD2850

LJD2898

LD29 14

LJD2936

LD3000

LJD2126

LJD2138

LJD2154

LJD2158

LJD2836

LD2852

LD2860

LJD2865

LJD2890

LD2943

DD2161

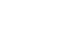




\begin{tabular}{|c|c|c|c|c|c|c|c|c|c|c|c|}
\hline Jefferson 3 & 0.0079 & 0.0036 & 0.0043 & 0.0050 & 0.0028 & 0.0057 & 0.0050 & 0.0043 & 0.0058 & 0.0065 & 0.0050 \\
\hline ADAMS6 & 0.0086 & 0.0043 & 0.0050 & 0.0057 & 0.0064 & 0.0050 & 0,0072 & 0.0051 & 0.0036 & 0.0072 & 0.0058 \\
\hline clatsopl & 0.0090 & 0.0022 & 0.0029 & 0.0037 & 0.0052 & 0.0045 & 0.0037 & 0.0022 & 0.0030 & 0.0075 & 0.0037 \\
\hline clatsop2 & 0.0105 & 0.0007 & 0.0029 & 0.0037 & .0 .0052 & 0.0060 & 0.0037 & 0.0022 & 0.0060 & 0.0090 & 0.0022 \\
\hline clatsop3 & 0.0105 & 0.0007 & 0.0029 & 0.0037 & 0.0052 & 0.0059 & 0.0037 & 0.0022 & 0.0060 & 0.0090 & 0.0022 \\
\hline clatsop4 & 0.0105 & 0.0007 & 0.0029 & 0.0037 & 0.0052 & 0.0060 & 0.0037 & 0.0022 & 0.0060 & 0.0090 & 0.0022 \\
\hline LostForest 1A & 0.0082 & 0.0069 & 0.0084 & 0.0083 & 0.0084 & 0.0069 & 0.0145 & 0.0101 & 0.0080 & 0.0081 & 0.0069 \\
\hline LostForest2 & 0,0169 & 0.0116 & 0.0109 & 0.0109 & 0.0146 & 0.0109 & 0.0139 & 0.0103 & 0.0110 & 0.0139 & 0.0117 \\
\hline LostForest3 & 0.0043 & 0.0050 & 0.0057 & 0.0064 & 0.0057 & 0.0058 & 0.0087 & 0.0058 & 0.0058 & 0.0101 & 0.0065 \\
\hline LostForest4 & 0.0138 & 0.0094 & 0.0101 & 0.0109 & 0.0116 & 0.0102 & 0.0132 & 0.0103 & 0.0102 & 0.0109 & 0.0110 \\
\hline sj1Pmausterus & 0.0065 & 0.0073 & 0.0080 & 0.0080 & 0.0095 & 0.0080 & 0.0088 & 0.0081 & .0 .0050 & 0.0103 & 0.0088 \\
\hline sj2Pmausterus & 0.0124 & 0.0080 & 0.0080 & 0.0095 & 0.0102 & 0.0095 & 0.0095 & 0.0081 & 0.0081 & 0.0132 & 0.0087 \\
\hline sj2aPmaustenus & 0.0065 & 0.0043 & 0.0051 & 0.0050 & 0.0065 & 0.0065 & 0.0058 & 0.0051 & 0.0051 & 0.0088 & 0.0058 \\
\hline sj3Pmausterus & 0.0080 & 0.0058 & 0.0065 & 0.0065 & 0.0080 & 0.0080 & 0.0073 & 0.0066 & 0.0065 & 0.0103 & 0.0073 \\
\hline sj5Pmausterus & 0.0073 & 0.0050 & 0.0058 & 0.0058 & 0.0073 & 0.0072 & 0.0065 & 0.0058 & 0.0058 & 0.0095 & 0.0065 \\
\hline sj6Pmausterus & 0.0073 & 0.0050 & 0.0058 & 0.0058 & 0.0072 & 0.0072 & 0.0065 & 0.0058 & 0.0058 & 0.0095 & 0.0065 \\
\hline sj7Pmausterus & 0.0073 & 0.0050 & 0.0058 & 0.0065 & 0.0072 & 0.0072 & 0.0065 & 0.0058 & 0.0058 & 0.0095 & 0.0065 \\
\hline OSM723Pmman & 0,0430 & 0.0398 & 0.0388 & 0.0415 & 0.0404 & 0.0411 & 0.0434 & 0.0416 & 0.0429 & 0.0452 & 0.0419 \\
\hline OSM726Pmman & 0.0421 & 0.0389 & 0.0380 & 0.0407 & 0.0396 & 0.0402 & 0.0425 & 0.0407 & 0.0421 & 0.0443 & 0.0410 \\
\hline OSM727Pmman & 0.0430 & 0.0398 & 0.0388 & 0.0415 & 0.0404 & 0.0411 & 0.0434 & 0.0407 & 0.0429 & 0.0452 & 0.0419 \\
\hline OSM737Pmman & 0.0421 & 0.0389 & 0.0380 & 0.0407 & 0.0396 & 0.0402 & 0.0425 & 0.0407 & 0.0421 & 0.0443 & 0.0410 \\
\hline U40255PsejugSD & 0.0536 & 0.0485 & 0.0495 & 0.0522 & 0.0492 & 0.0515 & 0.0523 & 0.0525 & 0.0536 & 0.0523 & 0.0507 \\
\hline U40253PsejugSC & 0.0633 & 0.0581 & 0.0591 & 0.0619 & 0.0588 & 0.0594 & 0.0621 & 0.0625 & 0.0635 & 0.0620 & 0.0604 \\
\hline JEB1309APtruei & 0.2449 & 0.2411 & 0.2439 & 0.2452 & 0.2392 & 0.2429 & 0.2440 & 0.2598 & 0.2471 & 0.2440 & 0.2424 \\
\hline AF374578PKEEN & 0.0580 & 0.0508 & 0.0539 & 0.0546 & 0.0556 & 0.0502 & 0.0547 & 0.0538 & 0.0541 & 0.0547 & 0.0531 \\
\hline U40252Pleuc & 0.2455 & 0.2400 & 0.2421 & 0.2374 & 0.2370 & 0.2464 & 0.2392 & 0.2502 & 0.2484 & 0.2476 & 0.2457 \\
\hline JAM160Pleuc & 0.1863 & 0.1911 & 0.1936 & 0.1897 & 0.1893 & 0.1909 & 0.1913 & 0.1974 & 0.1888 & 0.1930 & 0.1952 \\
\hline JAM161Pleuc & 0.2032 & 0.2087 & 0.2111 & 0.2067 & 0.2065 & 0.2092 & 0.2086 & 0.2171 & 0.2068 & 0.2115 & 0.2137 \\
\hline JAM162Pleuc & 0.1944 & 0.2004 & 0.2026 & 0.1974 & 0.1989 & 0.1999 & 0.2002 & 0.2069 & 0.1976 & 0.2031 & 0.2043 \\
\hline JAM163Pleuc & 0.1951 & 0.2014 & 0.2040 & 0.1997 & 0.1996 & 0.2013 & 0.2016 & 0.2082 & 0.1989 & 0.2056 & 0.2057 \\
\hline JAM164Pleuc & 0.2004 & 0.2067 & 0.2095 & 0.2050 & 0.2050 & 0.2067 & 0.2069 & 0.2138 & 0.2041 & 0.2095 & 0.2111 \\
\hline JAM168Pmbairdii & 0.0141 & 0.0148 & 0.0156 & 0.0156 & 0.0140 & 0.0156 & 0.0163 & 0.0159 & 0.0156 & 0.0181 & 0.0164 \\
\hline JAM167Pmbairdii & 0.0148 & 0.0155 & 0.0163 & 0.0163 & 0.0147 & 0.0163 & 0.0171 & 0.0166 & 0.0163 & 0.0188 & 0.0171 \\
\hline JAM159Pmbairdii & 0.0156 & 0.0149 & 0.0157 & 0.0164 & 0.0140 & 0.0172 & 0.0164 & 0.0159 & 0.0172 & 0.0196 & 0.0165 \\
\hline JAM166Pnbairdii & 0.0180 & 0.0188 & 0.0196 & 0.0196 & 0.0180 & 0.0196 & 0.0204 & 0.0199 & 0.0196 & 0.0221 & 0.0204 \\
\hline M.pennsylvanicus & 0.6172 & 0.6267 & 0.6273 & 0.6174 & 0.6170 & 0.6253 & 0.6256 & 0.6284 & 0.6319 & 0.6120 & 0.6282 \\
\hline Oryzomyspalustris & 0.9542 & 0.9313 & 0.9315 & 0.9309 & 0.9376 & 0.9562 & 0.9205 & 0.9581 & 0.9394 & 0.9764 & 0.9280 \\
\hline Sigmodonhispidus & 1.0412 & 1.0384 & 1.0169 & 1.0152 & 1.0307 & 1.0311 & 1.0290 & 1.0412 & 1.0099 & 1.0380 & 1.0344 \\
\hline R.megalotis & 0.5792 & 0.5623 & 0.5376 & 0.5489 & 0.5540 & 0.5572 & 0.5636 & 0.5462 & 0.5623 & 0.5740 & 0.5731 \\
\hline Rattusnorvegicus & 0.7934 & 0.7594 & 0.7437 & 0.7602 & 0.7545 & 0.7577 & 0.7729 & 0.7909 & 0.7576 & 0.7752 & 0.7558 \\
\hline Pmrufinus & 0.0259 & 0.0230 & 0.0237 & 0.0246 & 0.0236 & 0.0223 & 0.0246 & 0.0242 & 0.0239 & 0.0245 & 0.0247 \\
\hline
\end{tabular}




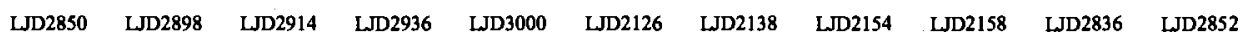

wC7

WC11

WC12

WC13

ljd3256

ljd3304

$1 \mathrm{jd} 3306$

ljd3308

ljd3317

ljd3324

ljd3327

ljd3330

ljd3333

ljd3344

ljd3348

LJD620

LJD774

LJD886

LJD2088

LJD1960

LDD1669

LDD1524

LJD1523

LDD 1122

LJD2522

LJD1637

LJD1638

LJD2161

LJD2651

LJD2737

LJD2834

LJD2850 .

LD2898

LD2914

0.0095

LDD2936

$\begin{array}{ll}0.0029 & 0.0065\end{array}$

LD3000

$\mathbf{0 . 0 0 4 3}$

0.0080

0.0014

LD2126

0.0028

0.0072

$0.0007 \quad 0.0021$

LJD2138

0.0110

0.0118

0.0080

0.0095

$\begin{array}{ll}0.0065 & 0.0102\end{array}$

0.0036

0.0057

0.0087

LD2154

$0.0007 \quad 0.008$

0.0021

0.0036

0.0043

0.0102

LD2158

0.0110

0.0080

0.0102

0.0028

0.0103

0.0057

LD2836

0.0110

0.0118

0.0080

0.0095

0.0087

0.0000

0.0102

0.0103

LD2852

0.0088

0.0110

0.0073

0.0088

$\begin{array}{ll}0.0087 & 0.0000\end{array}$

0.0102

0.0103

0.0000

LD2860

0.0043

0.0080

0.0014

0.0029

0.0021

0.0095

0.0110

0.0095

0.0095

0.0095

LD2865

0.0043

0.0102

0.0036

0.0021

0.0029

0.0117

0.0050

0.0036

$\begin{array}{lll}0.0095 & 0.0095 & 0.0088\end{array}$

125 


\begin{tabular}{|c|c|c|c|c|c|c|c|c|c|c|c|}
\hline LD2890 & 0.0065 & 0.0080 & 0.0043 & 0.0058 & 0.0043 & 0.0095 & 0.0065 & 0.0065 & 0.0095 & 0.0095 & 0.0080 \\
\hline LD2943 & 0.0043 & 0.0080 & 0.0014 & 0.0029 & 0.0021 & 0.0095 & 0.0050 & 0.0036 & 0.0095 & 0.0095 & 0.0088 \\
\hline Jefferson 3 & 0.0057 & 0.0065 & 0.0028 & 0.0014 & 0.0036 & 0.0095 & 0.0072 & 0.0050 & 0.0102 & 0.0095 & 0.0088 \\
\hline ADAMS6 & 0.0065 & 0.0072 & 0.0036 & 0.0050 & 0.0043 & 0.0072 & 0.0079 & 0.0058 & 0.0079 & 0.0073 & 0.0066 \\
\hline clatsop1 & 0.0037 & 0.0068 & 0.0015 & 0.0029 & 0.0015 & 0.0068 & 0.0052 & 0.0037 & 0.0068 & 0.0068 & 0.0053 \\
\hline clatsop2 & 0.0022 & 0.0083 & 0.0015 & 0.0029 & 0.0015 & 0.0098 & 0.0052 & 0.0022 & 0.0098 & 0.0098 & 0.0083 \\
\hline clatsop 3 & 0.0022 & 0.0083 & 0.0015 & 0.0029 & 0.0015 & 0.0098 & 0.0052 & 0.0022 & 0.0098 & 0.0098 & 0.0083 \\
\hline clatsop4 & 0.0022 & 0.0083 & 0.0015 & 0.0029 & 0.0015 & 0.0098 & 0.0052 & 0.0022 & 0.0098 & 0.0098 & 0.0083 \\
\hline LostForest1A & 0.0085 & 0.0081 & 0.0069 & 0.0099 & 0.0084 & 0.0099 & 0.0112 & 0.0069 & 0.0113 & 0.0099 & 0.0130 \\
\hline LostForest2 & 0.0118 & 0.0117 & 0.0094 & 0.0116 & 0.0102 & 0.0147 & 0.0139 & 0.0117 & 0.0139 & 0.0132 & 0.0117 \\
\hline LostForest3 & 0.0073 & 0.0065 & 0.0043 & 0.0050 & 0.0050 & 0.0095 & 0.0087 & 0.0065 & 0.0102 & 0.0095 & 0.0088 \\
\hline LostForest 4 & 0.0118 & 0.0110 & 0.0087 & 0.0102 & 0.0095 & 0.0125 & 0.0132 & 0.0110 & 0.0132 & 0.0125 & 0.0117 \\
\hline sjlPmausterus & 0.0095 & 0.0103 & 0.0065 & 0.0080 & 0.0072 & 0.0058 & 0.0102 & 0.0088 & 0.0058 & 0.0058 & 0.0080 \\
\hline sj2Pmausterus & 0.0095 & 0.0118 & 0.0065 & 0.0080 & 0.0072 & 0.0125 & 0.0102 & 0.0087 & 0.0118 & 0.0118 & 0.0112 \\
\hline sj2aPmausterus & 0.0065 & 0.0087 & 0.0036 & 0.0050 & 0.0036 & 0.0088 & 0.0072 & 0.0058 & 0.0088 & 0.0088 & 0.0081 \\
\hline sj3Pmauslerus & 0.0080 & 0.0103 & 0.0050 & 0.0065 & 0.0050 & 0.0103 & 0.0087 & 0.0073 & 0.0103 & 0.0103 & 0,0096 \\
\hline sj5Pmausterus & 0.0073 & 0.0095 & 0.0043 & 0.0058 & 0.0050 & 0.0095 & 0.0080 & 0.0065 & 0.0095 & 0.0095 & 0.0088 \\
\hline sj6Pmausterus & 0.0072 & 0.0095 & 0.0043 & 0.0058 & 0.0050 & 0.0095 & 0.0080 & 0.0065 & 0.0095 & 0.0095 & 0.0088 \\
\hline sj7Pmauslerus & 0.0072 & 0.0095 & 0.0043 & 0.0058 & 0.0050 & 0.0095 & 0.0080 & 0.0065 & 0.0095 & 0.0095 & 0.0089 \\
\hline OSM723Pmman & 0.0429 & 0.0456 & 0.0391 & 0.0389 & 0.0400 & 0.0474 & 0.0424 & 0.0419 & 0.0482 & 0.0477 & 0.0448 \\
\hline OSM726Pmman & 0.0420 & 0.0447 & 0.0383 & 0.0380 & 0.0391 & 0.0465 & 0.0415 & 0.0410 & 0.0473 & 0.0468 & 0.0439 \\
\hline OSM727Pmman & 0.0421 & 0.0456 & 0.0391 & 0.0389 & 0.0400 & 0.0474 & 0.0424 & 0.0419 & 0.0482 & 0.0477 & 0.0441 \\
\hline OSM737Pmman & 0.0420 & 0.0447 & 0.0383 & 0.0380 & 0.0391 & 0.0465 & 0.0415 & 0.0410 & 0.0473 & 0.0468 & 0.0439 \\
\hline U40255PsejugSD & 0.0517 & 0.0528 & 0.0498 & 0.0494 & 0.0507 & 0.0546 & 0.0503 & 0.0507 & 0.0553 & 0.0548 & 0.0554 \\
\hline U40253PsejugSC & 0.0615 & 0.0626 & 0.0595 & 0.0591 & 0.0604 & 0.0645 & 0.0599 & 0.0604 & 0.0652 & 0.0648 & 0.0635 \\
\hline JEB1309APtruei & 0.2476 & 0.2442 & 0.2425 & 0.2393 & 0.2458 & 0.2348 & 0.2504 & 0.2424 & 0.2354 & 0.2348 & 0.2486 \\
\hline AF374578PKEEN & 0.0531 & 0.0552 & 0.0522 & 0.0539 & 0.0530 & 0.0590 & 0.0526 & 0.0531 & 0.0598 & 0.0593 & 0.0531 \\
\hline U40252Pleuc & 0.2486 & 0.2524 & 0.2387 & 0.2381 & 0.2410 & 0.2517 & 0.2445 & 0.2457 & 0.2526 & 0.2532 & 0.2414 \\
\hline JAM160Pleuc & 0.1967 & 0.1952 & 0.1895 & 0.1902 & 0.1899 & 0.1953 & 0.1943 & 0.1952 & 0.1958 & 0.1963 & 0.1868 \\
\hline JAM161Pleuc & 0.2162 & 0.2139 & 0.2076 & 0.2075 & 0.2095 & 0.2138 & 0.2117 & 0.2137 & 0.2136 & 0.2150 & 0.2053 \\
\hline JAM162Pleuc & 0.2061 & 0.2045 & 0.1983 & 0.1991 & 0.1987 & 0.2048 & 0.2032 & 0.2043 & 0.2050 & 0.2055 & 0.1956 \\
\hline JAM163Pleuc & 0.2073 & 0.2060 & 0.1997 & 0.2005 & 0.2001 & 0.2059 & 0.2046 & 0.2057 & 0.2065 & 0.2070 & 0.1967 \\
\hline JAM164Pleuc & 0.2128 & 0.2116 & 0.2050 & 0.2058 & 0.2054 & 0.2114 & 0.2100 & 0.2111 & 0.2120 & 0.2125 & 0.2020 \\
\hline JAM168Pmbairdii & 0.0172 & 0.0180 & 0.0141 & 0.0156 & 0.0148 & 0.0180 & 0.0170 & 0.0164 & 0.0187 & 0.0180 & 0.0187 \\
\hline JAM167Pmbairdii & 0.0179 & 0.0187 & 0.0148 & 0.0163 & 0.0155 & 0.0187 & 0.0170 & 0.0171 & 0.0187 & 0.0187 & 0.0195 \\
\hline JAM159Pmbairdii & 0.0173 & 0.0196 & 0.0141 & 0.0157 & 0.0149 & 0.0212 & 0.0180 & 0.0165 & 0.0213 & 0.0213 & 0.0204 \\
\hline JAM166Pmbairdii & 0.0212 & 0.0221 & 0.0180 & 0.0196 & 0.0188 & 0.0204 & 0.0204 & 0.0204 & 0.0204 & 0.0205 & 0.0212 \\
\hline M.pennsylvanicus & 0.6288 & 0.6094 & 0.6195 & 0.6103 & 0.6203 & 0.6270 & 0.6191 & 0.6282 & 0.6273 & 0.6270 & 0.6204 \\
\hline Oryzomyspalustris & 0.9286 & 0.9702 & 0.9416 & 0.9255 & 0.9430 & 0.9241 & 0.9768 & 0.9280 & 0.9245 & 0.9239 & 0.9671 \\
\hline Sigmodonhispidus & 1.0352 & 1.0318 & 1.0261 & 1.0407 & 1.0279 & 0.9779 & 1.0103 & 1.0344 & 0.9783 & 0.9776 & 1.0357 \\
\hline R.megalotis & 0.5728 & 0.5754 & 0.5537 & 0.5626 & 0.5537 & 0.5344 & 0.5523 & 0.5731 & 0.5343 & 0.5343 & 0.5616 \\
\hline Rattusnorvegicus & 0.7565 & 0.7893 & 0.7636 & 0.7721 & 0.7679 & 0.7528 & 0.7508 & 0.7558 & 0.7565 & 0.7597 & 0.7714 \\
\hline Pmrufinus & 0.0256 & 0.0247 & 0.0223 & 0.0222 & 0.0231 & 0.0278 & 0.0270 & 0.0247 & 0.0286 & 0.0279 & 0.0256 \\
\hline
\end{tabular}




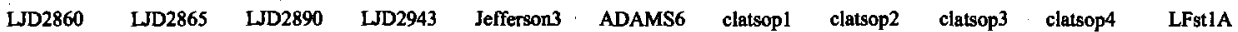

WC7

WC11

WC12

WC13

ljd3256

ljd3304

ljd3306

ljd3308

ljd3317

ljd3324

ljd 3327

ljd3330

ljd3333

ljd3344

ljd 3348

LJD620

LJD774

LD886

LJD2088

LD1960

LD1669

LJD1524

LDD1523

LD1 122

LJD2522

LD1637

LDD1638

LJD2161

LJD2651

LJD2737

LD2834

LJD2850

LJD2898

LD2914

LJD2936

LJD3000

LJD2126

LD2138

LJD2154

LJD2158

LD2836

LJD2852

LD2860

LJD2865 


\begin{tabular}{|c|c|c|c|c|c|c|c|c|c|c|c|}
\hline LJ2890 & 0.0058 & 0,0051 & & & & & & & & & \\
\hline LJ2943 & 0.0000 & 0.0050 & 0.0058 & & & & & & & & \\
\hline Jefferson 3 & 0.0043 & 0.0036 & 0.0058 & 0.0043 & & & & & & & \\
\hline ADAMS6 & 0.0050 & 0.0073 & 0.0051 & 0.0051 & 0.0050 & & & & & ? & \\
\hline clatsop1 & 0.0030 & 0.0029 & 0.0037 & 0.0030 & 0.0037 & 0.0030 & & & & & \\
\hline clatsop 2 & 0.0030 & 0.0029 & 0.0052 & 0.0030 & 0.0052 & 0.0059 & 0.0029 & & & & \\
\hline clatsop3 & 0.0030 & 0.0029 & 0.0052 & 0.0030 & 0.0051 & 0.0059 & 0.0029 & 0.0000 & & & \\
\hline clatsop4 & 0.0030 & 0.0029 & 0.0052 & 0.0030 & 0.0052 & 0.0059 & 0.0029 & 0.0000 & 0.0000 & & \\
\hline LostForest1A & 0.0097 & 0.0133 & 0.0085 & 0.0097 & 0.0084 & 0.0052 & 0.0075 & 0.0090 & 0.0090 & 0.0090 & \\
\hline LostForest2 & 0.0110 & 0.0125 & 0.0103 & 0.0110 & 0.0116 & 0.0108 & 0.0105 & 0.0121 & 0.0120 & 0.0121 & 0.0112 \\
\hline LostForest3 & 0,0058 & 0.0065 & 0.0058 & 0.0058 & 0.0064 & 0.0086 & 0.0090 & 0.0105 & 0.0105 & 0.0105 & 0.0068 \\
\hline LostForest4 & 0.0102 & 0.0125 & 0.0102 & 0.0103 & 0.0086 & 0.0078 & 0.0098 & 0.0113 & 0.0113 & 0.0113 & 0.0113 \\
\hline sj1Pmausterus & 0.0080 & 0.0102 & 0.0081 & 0.0080 & 0.0080 & 0.0043 & 0.0053 & 0.0083 & 0.0083 & 0.0083 & 0.0098 \\
\hline sj2Pmausterus & 0.0080 & 0.0102 & 0.0095 & 0.0080 & 0.0094 & 0.0072 & 0.0052 & 0.0082 & 0.0082 & 0.0082 & 0.0113 \\
\hline sj2aPmausterus & 0.0050 & 0.0065 & 0.0065 & 0.0051 & 0.0050 & 0.0029 & 0.0022 & 0.0052 & 0.0052 & 0.0052 & 0.0083 \\
\hline sj3Pmausterus & 0.0065 & 0.0080 & 0.0080 & 0.0065 & 0.0065 & 0.0043 & 0.0037 & 0.0067 & 0.0067 & 0.0067 & 0.0083 \\
\hline sj5Pmausterus & 0.0058 & 0.0080 & 0.0073 & 0.0058 & 0.0058 & 0.0036 & 0.0030 & 0.0060 & 0.0060 & 0.0060 & 0.0083 \\
\hline sj6Pmaustenus & 0.0058 & 0.0080 & 0.0073 & 0.0058 & 0.0058 & 0.0036 & 0.0030 & 0.0060 & 0.0060 & 0.0060 & 0.0083 \\
\hline sj7Pmausterus & 0.0058 & 0.0080 & 0.0073 & 0.0058 & 0.0058 & 0.0036 & 0.0030 & 0.0059 & 0.0059 & 0.0059 & 0.0083 \\
\hline OSM723Pmman & 0.0410 & 0.0419 & 0.0413 & 0.0411 & 0.0384 & 0.0392 & 0.0395 & 0.0413 & .0 .0412 & 0.0413 & 0.0525 \\
\hline OSM726Pmman & 0.0401 & 0.0410 & 0.0404 & 0.0402 & 0.0376 & 0.0384 & 0.0395 & 0.0412 & 0.0412 & 0.0412 & 0.0509 \\
\hline OSM727Pmman & 0.0410 & 0.0419 & 0.0413 & 0.0411 & 0.0384 & 0.0392 & 0.0395 & 0.0413 & 0.0412 & 0.0413 & 0.0525 \\
\hline OSM737Pmman & 0.0401 & 0.0410 & 0.0404 & 0.0402 & 0.0376 & 0.0384 & 0.0395 & 0.0412 & 0.0412 & 0.0412 & 0.0509 \\
\hline U4025SPsejugSD & 0.0518 & 0.0523 & 0.0518 & 0.0519 & 0.0490 & 0.0519 & 0.0512 & 0.0510 & 0.0509 & 0.0510 & 0.0704 \\
\hline U40253PsejugSC & 0.0615 & 0.0621 & 0.0617 & 0.0617 & 0.0584 & 0.0615 & 0.0612 & 0.0610 & 0.0610 & 0.0610 & 0.0891 \\
\hline JEB1309APtruei & 0.2500 & 0.2522 & 0.2439 & 0.2499 & 0.2391 & 0.2364 & 0.2582 & 0.2613 & 0.2613 & 0.2613 & 0.2454 \\
\hline AF374578PKEEN & 0.0541 & 0.0549 & 0.0485 & 0.0542 & 0.0533 & 0.0505 & 0.0516 & 0.0515 & 0.0514 & 0.0515 & 0.0613 \\
\hline U40252Pleuc & 0.2429 & 0.2449 & 0.2523 & 0.2441 & 0.2403 & 0.2414 & 0.2496 & 0.2481 & 0.2481 & 0.2481 & 0.2071 \\
\hline JAM160Pleuc & 0.1930 & 0.1903 & 0.1936 & 0.1939 & 0.1891 & 0.1862 & 0.1915 & 0.1948 & 0.1948 & 0.1948 & 0.2018 \\
\hline JAM161Pleuc & 0.2113 & 0.2130 & 0.2142 & 0.2123 & 0.2063 & 0.2031 & 0.2110 & 0.2146 & 0.2146 & 0.2146 & 0.2008 \\
\hline JAM162Pleuc & 0.2020 & 0.2007 & 0.2042 & 0.2029 & 0.1988 & 0.1957 & 0.2002 & 0.2037 & 0.2036 & 0.2037 & 0.2097 \\
\hline JAM163Pleuc & 0.2033 & 0.2007 & 0.2042 & 0.2043 & 0.1995 & 0.1963 & 0.2022 & 0.2057 & 0.2057 & 0.2057 & 0.2018 \\
\hline JAM164Pleuc & 0.2087 & 0.2060 & 0.2097 & 0.2097 & 0.2050 & 0.2016 & 0.2076 & 0.2112 & 0.2112 & 0.2112 & 0.1994 \\
\hline JAM168Pmbairdii & 0.0156 & 0.0179 & 0.0157 & 0.0157 & 0.0155 & 0.0133 & 0.0137 & 0.0153 & 0.0153 & 0.0153 & 0.0129 \\
\hline JAM167Pmbairdii & 0.0163 & 0.0186 & 0.0164 & 0.0164 & 0.0163 & 0.0140 & 0.0145 & 0.0160 & 0.0160 & 0.0160 & 0.0130 \\
\hline JAM159Pmbairdii & 0.0157 & 0.0179 & 0.0173 & 0.0157 & 0.0156 & 0.0149 & 0.0137 & 0.0153 & 0.0153 & 0.0153 & 0.0162 \\
\hline JAM166Pmbairdii & 0.0196 & 0.0219 & 0.0197 & 0.0197 & 0.0196 & 0.0172 & 0.0170 & 0.0186 & 0.0186 & 0.0186 & 0.0130 \\
\hline M.pennsylvanicus & 0.6246 & 0.6103 & 0.6115 & 0.6252 & 0.6105 & 0.6206 & 0.6277 & 0.6291 & 0.6292 & 0.6291 & 0.6628 \\
\hline Oryzomyspalustris & 0.9607 & 0.9251 & 0.9359 & 0.9614 & 0.9149 & 0.9314 & 0.9438 & 0.9340 & 0.9344 & 0.9340 & 0.8696 \\
\hline Sigmodonhispidus & 1.0447 & 1.0401 & 1.0212 & 1.0455 & 1.0415 & 1.0235 & 1.0252 & 1.0339 & 1.0344 & 1.0339 & 1.2549 \\
\hline R.megalotis & 0.5489 & 0.5621 & 0.5644 & 0.5486 & 0.5808 & 0.5711 & 0.5621 & 0.5638 & 0.5639 & 0.5638 & 0.7431 \\
\hline Rattusnorvegicus & 0.7773 & 0.7715 & 0.7852 & 0.7852 & 0.7554 & 0.7551 & 0.7479 & 0.7491 & 0.7493 & 0.7491 & 0.8556 \\
\hline Pmrufinus & 0.0239 & 0.0248 & 0.0240 & 0.0239 & 0.0205 & 0.0228 & 0.0238 & 0.0254 & 0.0254 & 0.0254 & 0.0399 \\
\hline
\end{tabular}




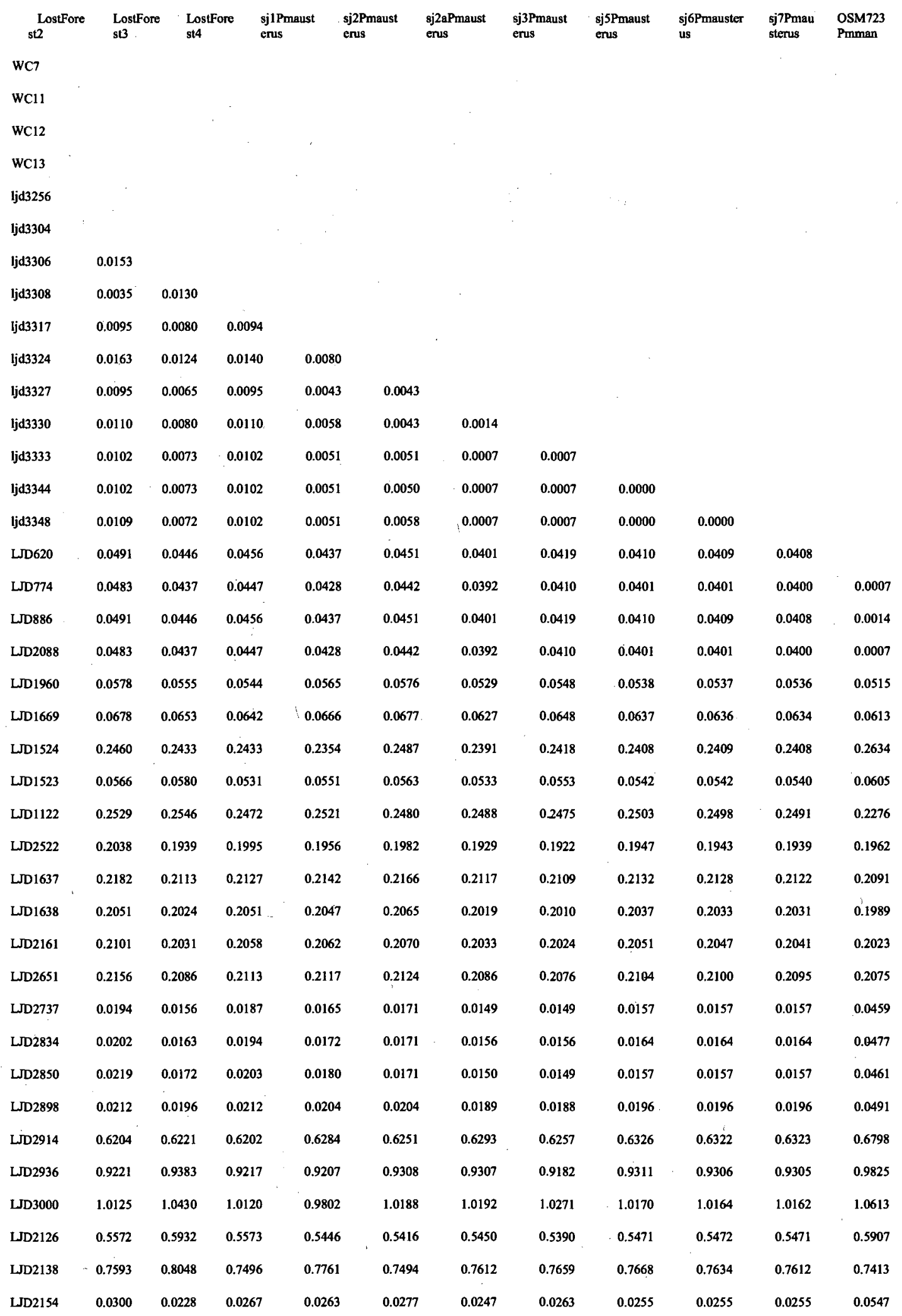




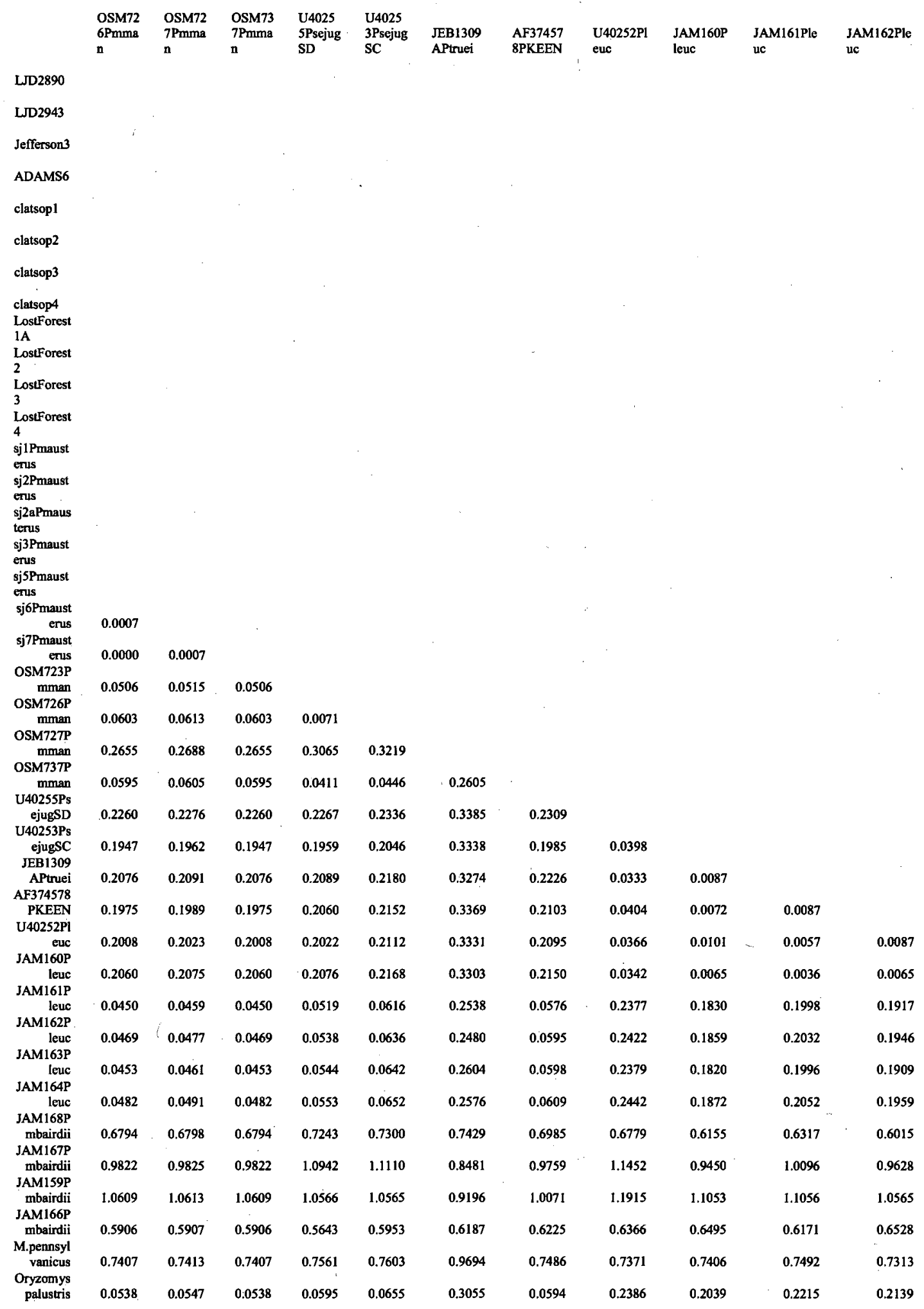




\begin{tabular}{|c|c|c|c|c|c|c|c|c|c|c|}
\hline JAM16 & JAM & $\begin{array}{l}\text { JAM16 } \\
\text { 8Pmbai }\end{array}$ & $\begin{array}{l}\text { JAM16 } \\
\text { 7Pmbai }\end{array}$ & $\begin{array}{l}\text { JAM15 } \\
\text { 9Pmbai }\end{array}$ & $\begin{array}{l}\text { JAM16 } \\
\text { 6Pmbai }\end{array}$ & $\begin{array}{l}\text { Microtusp } \\
\text { ennsylvan- }\end{array}$ & Oryzomys & Sigmodon & $\begin{array}{l}\text { Reithrodont } \\
\text { omysmegalo }\end{array}$ & Rattusnorve \\
\hline
\end{tabular}

\begin{tabular}{|c|c|c|c|c|c|c|c|c|c|c|c|}
\hline $\begin{array}{l}\text { JAM164P } \\
\text { leuc }\end{array}$ & 0.0050 & & & & & & & & & & \\
\hline $\begin{array}{l}\text { JAM168P } \\
\text { mbairdii } \\
\text { JAM167P }\end{array}$ & 0.1930 & 0.1982 & & & & & & & & & \\
\hline $\begin{array}{l}\text { mbairdii } \\
\text { JAM159P }\end{array}$ & 0.1959 & 0.2012 & 0.0028 & & & & & & & & \\
\hline $\begin{array}{l}\text { mbairdii } \\
\text { JAM166P }\end{array}$ & 0.1918 & 0.1970 & 0.0043 & 0.0057 & & & & & & & \\
\hline $\begin{array}{l}\text { mbairdii } \\
\text { M.pennsy }\end{array}$ & 0.1972 & 0.2024 & 0.0050 & 0.0065 & 0.0080 & & & & & & \\
\hline $\begin{array}{l}\text { 1vanicus } \\
\text { Oryzomys }\end{array}$ & 0.6286 & 0.6150 & 0.6340 & 0.6218 & 0.6186 & 0.6294 & & & & & \\
\hline $\begin{array}{l}\text { palustris } \\
\text { Siemodon }\end{array}$ & 0.9927 & 0.9681 & 0.9973 & 0.9941 & 0.9768 & 1.0013 & 0.9124 & & 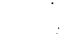 & & \\
\hline $\begin{array}{l}\text { hispidus } \\
\text { R.megalot }\end{array}$ & 1.1198 & 1.0958 & 1.0335 & 1.0591 & 1.0597 & 1.0370 & 1.3044 & 1.0383 & & & \\
\hline $\begin{array}{l}\text { is } \\
\text { Rattusnor }\end{array}$ & 0.6163 & 0.6283 & 0.5736 & 0.5670 & 0.5887 & 0.5836 & 1.0410 & 1.2014 & 1.0315 & & \\
\hline vegicus & 0.7309 & 0.7434 & 0.7605 & 0.7533 & 0.7637 & 0.7285 & 0.7899 & 1.0387 & 1.2793 & 1.0661 & \\
\hline Purrufinus & 0.2144 & 0.2200 & 0.0335 & 0,0336 & 0.0353 & 0.0380 & 0.6000 & 0.9950 & 1.1254 & 0.5850 & 0.8446 \\
\hline
\end{tabular}


Appendix B: Hantavirus Distance Matrix. Pair-wise percent genetic difference (distance) among hantaviruses ( $n=44$ ) based upon $M$ segment $\mathrm{Gl} / \mathrm{G} 2$ glycoprotein precursor gene (613nt).

$$
\begin{array}{llll}
\text { T7 } & \text { T8 } & \text { WC4 } & \text { WCps }
\end{array}
$$

T7

T8

WC4

WCps

WC7

WC12

WC13

LJD1523FP

LJD2126FP

LJD2138FP

LJD2154FP

LJD2493FP

LJD2494FP

LJD2497FP

LJD2499FP

LJD2520FP

LJD2522FP

LJD2737PB

LJD2836FP

LJD2852FP

LJD2860FP

LJD2865FP

LJD2890FP

convict creek

SNVL25783

SNVL37903

NewYorkVirusU36803

BlueRiverAF030552

BayouvirusL 36930

BlackCreekCanalL39950

AndesVirusNC003467

Hu39694virusAF028023

OranVirusAF028024

LechiguanasvirusAF028022

Juquitibalikevinus 168711

MacielvinusAF028027

ElMoroCanyonU26828

ProspectHillX55129

TulavirusNC005228

PuumalavirusNC005223

HantaanvirusDQ371905

DobravavirusNC005234

SeoulVirusNC005237

SangassouvinusDQ268651
0

$0.01689189 \quad 0.01689189$

0.01858108

0.01689189

0.04391892

0.01689189

0.02533784

0.0152027

0.01182432

0.03725455

0.0152027

0.01689189

0.01689189

0.03378378

0.0152027

0.0152027

0.00169827

0.01351351

0.01689189

0.0152027

0.01858108

0.0152027

0.11993244

0.14695945

0.14695945

0.21452703

0.23817568

0.27364865

0.2972973

0.28378379

0.28547296

0.28885135

0.2668919

0.3035008

0.28885135

0.28378379

0.33277026

0.33277026

0.35135135

0.41554055

0.40202704

0.39864865

0.40510133
0.01858108

0.01689189

0.04391892

0.01689189

0.02533784

0.0152027

0.01182432

0.03725455

0.0152027

0.01689189

0.01689189

0.03378378

0.0152027

0.0152027

0.00169827

0.01351351

0.01689189

0.0152027

0.01858108

0.0152027

0.11993244

0.14695945

0.14695945

0.21452703

0.23817568

0.27364865

0.2972973

0.28378379

0.28547296

0.28885135

0.2668919

0.3035008

0.28885135

0.28378379

0.33277026

0.33277026

0.35135135

0.41554055

0.40202704

0.39864865

0.40510133
0.00168919

$0.00675676 \quad 0.00844595$

$\begin{array}{lll}0.03378378 & 0.03547297\end{array}$

$\begin{array}{lll}0.00675676 & 0.00844595\end{array}$

$\begin{array}{lll}0.02533784 & 0.02702703\end{array}$

$0.01182432 \quad 0.01351351$

$\begin{array}{lll}0.00844595 & 0.01013513\end{array}$

$\begin{array}{lll}0.04075247 & 0.04247823\end{array}$

$0.00844595 \quad 0.01013513$

$\begin{array}{ll}0.01013513 & 0.01182432\end{array}$

$\begin{array}{lll}0.01013513 & 0.01182432\end{array}$

$\begin{array}{lll}0.02871622 & 0.03040541\end{array}$

$0.00844595 \quad 0.01013513$

$0.00844595 \quad 0.01013513$

$\begin{array}{lll}0.01520477 & 0.01690304\end{array}$

$0.01013513 \quad 0.01182432$

$\begin{array}{ll}0.01351351 & 0.0152027\end{array}$

$\begin{array}{ll}0.00844595 & 0.01013513\end{array}$

$\begin{array}{ll}0.01182432 & 0.01351351\end{array}$

$\begin{array}{ll}0.00844595 & 0.01013513\end{array}$

$0.1097973 \quad 0.11148649$

0.14189190 .14358108

0.14189190 .14358108

$0.21621622 \quad 0.2179054$

$0.23817568 \quad 0.23986487$

$\begin{array}{lll}0.27533785 & 0.27702704\end{array}$

$0.28885135 \quad 0.29054055$

$0.27702704 \quad 0.27871621$

$0.2804054 \quad 0.2820946$

$0.28378379 \quad 0.28547296$

$0.2652027 \quad 0.2668919$

$0.29921457 \quad 0.29898441$

$0.2804054 \quad 0.2820946$

$0.27702704 \quad 0.27871621$

$0.33277026 \quad 0.33445945$

$0.3277027^{\circ} 0.3293919$

$0.35304055 \quad 0.35472974$

$0.41722974 \quad 0.41891891$

$0.40202704 \quad 0.40371621$

$0.40202704 \quad 0.40371621$

$0.40454447 \quad 0.40432113$ 
T7

T8

WC4

WCps

WC7

WC12

WC13

LJD1523FP

LJD2126FP

LJD2138FP

LJD2154FP

LJD2493FP

LJD2494FP

LJD2497FP

LJD2499FP

LJD2520FP

LJD2522FP

LJD2737PB

LJD2836FP

LJD2852FP

LJD2860FP

LJD2865FP

LJD2890FP

convict creek

SNVL25783

SNVL37903

NewYorkVirusU36803

BlueRiverAF030552

BayouvirusL36930

BlackCreekCanalL39950

AndesVirusNC003467

Hu39694virusAF028023

OranVirusAF028024

LechiguanasvirusAF028022

Juquitibalikevirus 16871 I

MacielvirusAF028027

ElMoroCanyonU26828

ProspectHillX55129

TulavirusNC005228

PuumalavirusNC005223

HantaanvirusDQ371905

DobravavirusNC005234

SeoulVirusNC005237

SangassouvirusDQ268651
wC7

WC12

WC13

LJD1523FP

LJD2126FP

0.03378378

$\begin{array}{ll}0 & 0.03378378\end{array}$

$\begin{array}{llll}0.02533784 & 0.04898649 & 0.02533784\end{array}$

$\begin{array}{lll}0.01182432 & 0.03885135\end{array}$

$\begin{array}{lll}0.00844595 & 0.03547297\end{array}$

0.01182432

0.00844595

0.04075247

0.06115411

0.04075247

$\begin{array}{llll}0.00844595 & 0.03547297 & 0.00844595\end{array}$

$\begin{array}{lll}0.01013513 & 0.03716216\end{array}$

0.01013513

0.01013513

0.03716216

0.01013513

0.03040541

0.03040541

0.00844595

0.05405406

0.00844595

0.03547297

0.00844595

0.00844595

$0.01520477 \quad 0.04227104$

0.01520477

$0.01013513 \quad 0.03716216$

0.01013513

$0.01351351 \quad 0.04054054$

0.01351351

$0.00844595 \quad 0.03547297$

0.00844595

0.01182432

0.03209459

0.01182432

0.00844595

0.03547297

0.00844595

0.10810811

0.12331081

0.10810811

0.1418919

0.1418919

0.1418919

0.1418919

0.1418919

0.1418919

0.21621622

0.21621622

0.21621622

0.23817568

0.23986487

0.23817568

0.27533785

0.2804054

0.27533785

0.29054055

0.28547296

0.29054055

0.2804054

0.27027026

0.2804054

0.2820946

0.2820946

$0.28547296 \quad 0.28716215$

0.2820946

0.28547296

0.2652027

0.2668919

0.2652027

0.30200422

0.30200422

0.29626659

0.2804054

0.2804054

0.27533785

0.27702704

0.27702704

0.27533785

0.33445945

0.33783785

0.33445945

0.3293919

0.32432431

0.3293919

0.35472974

0.3445946

0.35472974

0.41722974

0.40371621

0.39864865

0.4037162

0.39527026

0.40033785

0.40454447
0.02027027

$0.02364865 \quad 0.00337838$

$\begin{array}{ll}0.05596788 & 0.0356652\end{array}$

0.023648650 .00675676

0.02533784

0.02195946

0.04560811

0.02364865

0.02364865

0.02369345

0.02533784

0.02871622

0.02364865

0.02533784

0.02364865

0.125

0.15878378

0.15878378

0.22972973

0.2516892

0.28547296

0.29898649

0.28716215

0.29222974

0.2956081

0.27195945

0.30796087

0.29222974

0.29222974

0.3429054

0.33952704

0.36148649

0.42398649

0.41216215

0.4070946

0.40474871
0.00844595

0.00506757

0.02871622

0.00675676

0.00675676

0.0135065

0.00506757

0.00844595

0.00675676

0.01013513

0.00675676

0.10810811

0.1418919

0.1418919

0.21283785

0.2331081

0.27195945

0.28885135

0.27871621

0.27871621

0.2804054

0.25844595

0.30351657

0.27871621

0.27702704

0.3277027

0.3277027

0.35135135

0.41554055

0.40202704

0.39864865

0.40480602
0.03230368

0.00337838

0.00506757

0.00506757

0.02533784

0.00337838

0.00337838

0.01013055

0.00168919

0.00506757

0.00337838

0.00675676

0.00337838

0.10810811

0.13851352

0.13851352

0.21283785

0.2331081

0.27027026

0.28716215

0.27702704

0.27871621

0.2820946

0.26013514

0.29905257

0.27871621

0.27533785

0.3277027

0.32601351

0.35135135

0.41385135

0.40033785

0.39864865

0.40467227 


\begin{tabular}{|c|c|c|c|c|c|c|}
\hline \multicolumn{7}{|l|}{$\mathrm{T} 7$} \\
\hline T8 & & & & & & \\
\hline WC4 & & - & & & & \\
\hline WCps & & & & & & \\
\hline WC7 & & & & & & \\
\hline WC12 & & & & & & \\
\hline WC13 & & & & & & \\
\hline LJD1523FP & & & & . & & \\
\hline LJD2126FP & & & & & & \\
\hline LJD2138FP & & & & & & \\
\hline LJD2154FP & & & & & & \\
\hline LJD2493FP & 0.0356652 & & & & & \\
\hline LJD2494FP & 0.03736917 & 0.00168919 & & & & \\
\hline LJ2497FP & 0.03736917 & 0.00337838 & 0.00506757 & & & \\
\hline LJ2499FP & 0.0542511 & 0.02533784 & 0.02702703 & 0.02702703 & & \\
\hline LJD2520FP & 0.0356652 & 0.00337838 & 0.00506757 & 0.00506757 & 0.02533784 & \\
\hline LD2522FP & 0.03569596 & 0.00337838 & 0.00506757 & 0.00506757 & 0.02533784 & 0.00337838 \\
\hline LJ2737PB & 0.04062414 & 0.0135065 & 0.01519001 & 0.01519001 & 0.03209038 & 0.0135065 \\
\hline LJD2836FP & 0.03057793 & 0.00506757 & 0.00675676 & 0.00675676 & 0.02702703 & 0.00506757 \\
\hline LJD2852FP & 0.03736861 & 0.00844595 & 0.01013513 & 0.01013513 & 0.03040541 & 0.00844595 \\
\hline LJD2860FP & 0.0356652 & 0.00168919 & 0.00337838 & 0.00168919 & 0.02533784 & 0.00337838 \\
\hline LJD2865FP & 0.03907926 & 0.00506757 & 0.00675676 & 0.00506757 & 0.02871622 & 0.00675676 \\
\hline LJD2890FP & 0.0356652 & 0.00168919 & 0.00337838 & 0.00168919 & 0.02533784 & 0.00337838 \\
\hline convict creek & 0.11374537 & 0.10641892 & 0.10641892 & 0.10304054 & 0.1266892 & 0.10810811 \\
\hline SNVL25783 & 0.14770605 & 0.13851352 & 0.13682432 & 0.13851352 & 0.1570946 & 0.1402027 \\
\hline SNVL37903 & 0.14770605 & 0.13851352 & 0.13682432 & 0.13851352 & 0.1570946 & 0.1402027 \\
\hline NewYorkVirusU36803 & 0.21656698 & 0.21283785 & 0.21283785 & 0.21114865 & 0.22466215 & 0.21452703 \\
\hline BlueRiverAF030552 & 0.22702844 & 0.2331081 & 0.23141892 & 0.22972973 & 0.24662162 & 0.2347973 \\
\hline BayouvirusL36930 & 0.27250528 & 0.27027026 & 0.27195945 & 0.26858109 & 0.27364865 & 0.27195945 \\
\hline BlackCreekCanalL39950 & 0.29472989 & 0.28716215 & 0.28885135 & 0.28547296 & 0.2972973 & 0.28885135 \\
\hline AndesVirusNC003467 & 0.28469363 & 0.27702704 & 0.27702704 & 0.27533785 & 0.28378379 & 0.27871621 \\
\hline Hu39694virusAF028023 & 0.27595124 & 0.27871621 & 0.2804054 & 0.27702704 & 0.29054055 & 0.2804054 \\
\hline OranVirusAF028024 & 0.28090265 & 0.2820946 & 0.28378379 & 0.27871621 & 0.29391891 & 0.28378379 \\
\hline LechiguanasvirusAF028022 & 0.26583359 & 0.26182431 & 0.26182431 & 0.25844595 & 0.27533785 & 0.26351351 \\
\hline Juquitibalikevirus 168711 & 0.29786795 & 0.29902834 & 0.29665294 & 0.29897928 & 0.30965298 & 0.29905257 \\
\hline MacielvirusAF028027 & 0.27945143 & 0.27871621 & 0.2804054 & 0.27533785 & 0.29222974 & 0.2804054 \\
\hline ElMoroCanyonU26828 & 0.28277552 & 0.27364865 & 0.27533785 & 0.27195945 & 0.28885135 & 0.27533785 \\
\hline ProspectHillX55129 & 0.3277843 & 0.3277027 & 0.3277027 & 0.32432431 & 0.33277026 & 0.3293919 \\
\hline TulavirusNC005228 & 0.32489362 & 0.32601351 & 0.32601351 & 0.32432431 & 0.33783785 & 0.3277027 \\
\hline PuumalavirusNC005223 & 0.35704678 & 0.35135135 & 0.35304055 & 0.34797296 & 0.3581081 & 0.35135135 \\
\hline HantaanvirusDQ371905 & 0.41059139 & 0.41216215 & 0.41385135 & 0.41047296 & 0.41216215 & 0.41385135 \\
\hline DobravavirusNC005234 & 0.39590153 & 0.40033785 & 0.40033785 & 0.39864865 & 0.4054054 & 0.40202704 \\
\hline SeoulVirusNC005237 & 0.40626273 & 0.39695945 & 0.39864865 & 0.39358109 & 0.40033785 & 0.39864865 \\
\hline SangassouvirusDQ268651 & 0.41380125 & 0.40467227 & 0.40390712 & 0.40449309 & 0.40497798 & 0.40467227 \\
\hline
\end{tabular}


LJD2522FP LJD2737PB LJD2836FP LJD2852FP LJD2860FP LJD2865FP

T7

T8

WC4

WCps

WC7

WC12

WC13

LJD1523FP

LJD2126FP

LJD2138FP

LJD2154FP

LJD2493FP

LJ2494FP

LJD2497FP

LJD2499FP

LJD2520FP

LJD2522FP

LJD2737PB

LJD2836FP

LJD2852FP

LJD2860FP

LJD2865FP

LJD2890FP

convict creek

SNVL25783

SNVL37903

NewYorkVirusU36803

BlueRiverAF030552

BayouvirusL36930

BlackCreekCanalL39950

AndesVirusNC003467

Hu39694virusAF028023

OranVirusAF028024

LechiguanasvirusAF028022

Juquitibalikevirus 168711

MacielvirusAF028027

ElMoroCanyonU26828

ProspectHillX55129

TulavinusNC005228

PuumalavirusNC005223

HantaanvirusDQ371905

DobravavirusNC005234

SeoulVirusNC005237

SangassouvirusDQ268651
0.01349755

0.00506757

0.00844595

0.00337838

0.00675676

0.00337838

0.10810811

0.1402027

0.1402027

0.21452703

0.2347973

0.27195945

0.28885135

0.27871621

0.2804054

0.28378379

0.26351351

0.30135384

0.2804054

0.27533785

0.3293919

0.3277027

0.35304055

0.41385135

0.40202704

0.39864865

0.40841582
0.01181405

0.01518105

0.0135065

0.01688827

0.0135065

0.11831909

0.14533791

0.14533791

0.21640176

0.23999089

0.27380687

0.29755044

0.28389847

0.28563884

0.28903851

0.2671409

0.30143389

0.28900537

0.28219485

0.331379

0.33300248

0.35167229

0.41745156

0.40388456

0.40057662

0.40530401
0.00675676

$0.00506757 \quad 0.00844595$

$0.00844595 \quad 0.01182432$

$\begin{array}{ll}0.00506757 & 0.00844595\end{array}$

0.10641892

0.11317568

0.14358108

0.1402027

0.1402027

0.14358108

0.21283785

0.23648648

0.27027026

0.28885135

0.2820946

0.2820946

0.28716215

0.26351351

0.30393869

0.2820946

0.27871621

0.33277026

0.33108109

0.35472974

0.41722974

0.40371621

0.40202704

0.40474808

0.39864865
0.00337838

$\begin{array}{ll}0 & 0.00337838\end{array}$

0.104729730 .10810811

$\begin{array}{lll}0.13682432 & 0.13682432\end{array}$

$\begin{array}{lll}0.13682432 & 0.13682432\end{array}$

$0.21114865 \quad 0.21452703$

$0.23141892 \quad 0.2347973$

$0.26858109 \quad 0.27195945$

$0.28547296 \quad 0.28885135$

0.27533785

0.27533785

0.27702704

0.2804054

0.2804054

0.28378379

0.26013514

0.26013514

0.2968154

0.2964232

0.27702704

0.27702704

0.27195945

$0: 27533785$

0.3293919

0.32601351

0.3277027

$0.34966215 \quad 0.34966215$

0.41047296

0.41047296

0.39864865

0.39864865

0.39527026

0.39527026

0.40458268

0.40448716 


\begin{tabular}{|c|c|c|c|c|c|c|}
\hline \multirow{2}{*}{\multicolumn{7}{|c|}{ NewYorkVirus }} \\
\hline & & & & & & \\
\hline T8 & & & & & & \\
\hline WC4 & & & & 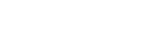 & & \\
\hline WCps & & & & & & . \\
\hline WC7 & & & & & & \\
\hline WC12 & & & & & & : \\
\hline WC13 & & & & & & \\
\hline LJD1523FP & & & & & & \\
\hline LJD2126FP & & & & & & \\
\hline LJD2138FP & & & & & & \\
\hline LJD2154FP & . & & & & & \\
\hline LJD2493FP & & & & & & \\
\hline LJD2494FP & & & & & & \\
\hline LJD2497FP & & & & . & & \\
\hline LJD2499FP & & & & & & \\
\hline LJD2520FP & & & & 1 & & \\
\hline LJD2522FP & & & & & & \\
\hline LJD2737PB & & & & & & \\
\hline LJD2836FP & & & & & & \\
\hline LJD2852FP & & & & & & \\
\hline LJD2860FP & & . & & & & \\
\hline LJD2865FP & & & & & & \\
\hline LJD2890FP & & & & & & \\
\hline convictcreek & 0.10472973 & & & & & \\
\hline SNVL25783 & 0.13682432 & 0.13006757 & & & & \\
\hline SNVL37903 & 0.13682432 & 0.13006757 & 0 & & & \\
\hline NewYorkVirusU36803 & 0.21114865 & 0.21283785 & 0.1891892 & 0.1891892 & & \\
\hline BlueRiverAF030552 & 0.23141892 & 0.21621622 & 0.20101352 & 0.20101352 & 0.19763513 & \\
\hline BayouvirusL36930 & 0.26858109 & 0.28547296 & 0.26013514 & 0.26013514 & 0.24155405 & 0.2804054 \\
\hline BlackCreekCanalL39950 & 0.28547296 & 0.27533785 & 0.2668919 & 0.2668919 & 0.25337839 & 0.27364865 \\
\hline AndesVirusNC003467 & 0.27533785 & 0.27702704 & 0.2516892 & 0.2516892 & 0.26351351 & 0.25 \\
\hline Hu39694virusAF028023 & 0.27702704 & 0.25675675 & 0.2652027 & 0.2652027 & 0.25844595 & 0.2483108 \\
\hline OranVirusAF028024 & 0.2804054 & 0.25844595 & 0.25844595 & 0.25844595 & 0.2516892 & 0.25506756 \\
\hline LechiguanasvirusAF028022 & 0.26013514 & 0.23141892 & 0.25337839 & 0.25337839 & 0.25675675 & 0.27027026 \\
\hline Juquitibalikevirus 168711 & 0.2968154 & 0.29705411 & 0.27894211 & 0.27894211 & 0.29574701 & 0.29207158 \\
\hline MacielvirusAF028027 & 0.27702704 & 0.25675675 & 0.25844595 & 0.25844595 & 0.26858109 & 0.26013514 \\
\hline ElMoroCanyonU26828 & 0.27195945 & 0.25844595 & 0.26858109 & 0.26858109 & 0.2516892 & 0.2516892 \\
\hline ProspectHillX55129 & 0.32601351 & 0.30405405 & 0.30743244 & 0.30743244 & 0.27871621 & 0.31756756 \\
\hline TulavirusNC005228 & 0.32432431 & 0.30405405 & 0.30912161 & 0.30912161 & 0.2956081 & 0.30574325 \\
\hline PuumalavirusNC005223 & 0.34966215 & 0.32432431 & 0.3277027 & 0.3277027 & 0.33277026 & 0.31925675 \\
\hline HantaanvirusDQ371905 & 0.41047296 & 0.39527026 & 0.39864865 & 0.39864865 & 0.38175675 & 0.36824325 \\
\hline DobravavirusNC005234 & 0.39864865 & 0.39864865 & 0.3918919 & 0.3918919 & 0.38175675 & 0.3733108 \\
\hline SeoulVirusNC005237 & 0.39527026 & 0.41216215 & 0.40033785 & 0.40033785 & 0.38682431 & 0.3766892 \\
\hline SangassouvirusDQ268651 & 0.40458268 & 0.41278094 & 0.43484983 & 0.43484983 & 0.40968382 & 0.41773394 \\
\hline
\end{tabular}




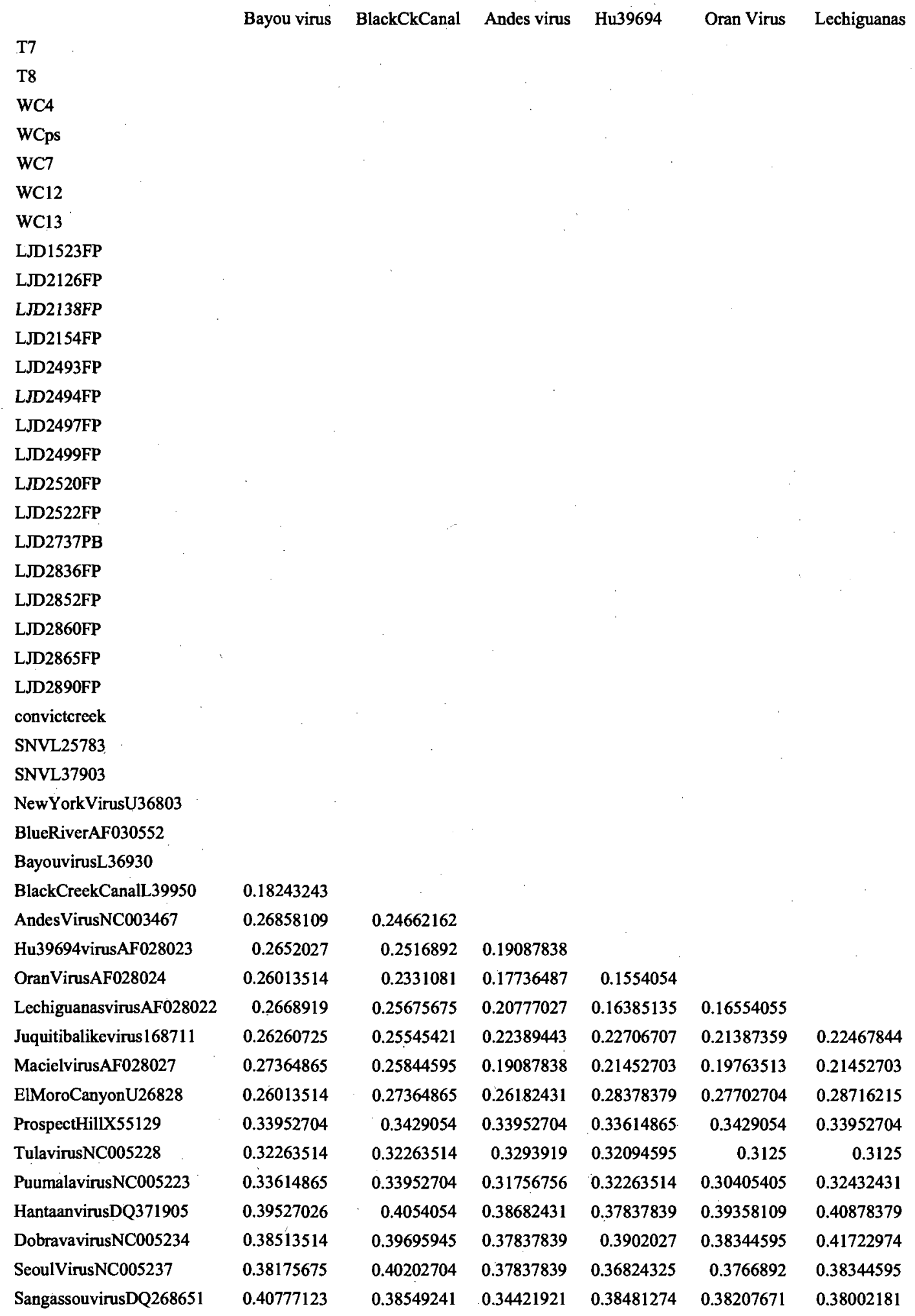


Juquitiba Maciel virus ElMoroCanyon Prospect Hill Tula virus Puumalavirus

T7

T8

WC4

WCps

WC7

WC12

WC13

LJD1523FP

LJD2126FP

LJD2138FP

LJD2154FP

LJD2493FP

LJD2494FP

LJD2497FP

LJD2499FP

LJD2520FP

LJD2522FP

LJD2737PB

LJD2836FP

LJD2852FP

LJD2860FP

LJD2865FP

LJD2890FP

convictcreek

SNVL25783

SNVL37903

NewYorkVirusU36803

BlueRiverAF030552

BayouvirusL36930

BlackCreekCanalL39950

AndesVirusNC003467

Hu39694virusAF028023

OranVirusAF028024

LechiguanasvirusAF028022

Juquitibalikevirus 168711

MacielvirusAF028027

0.21495967

ElMoroCanyonU26828

0.30833328

ProspectHillX55129

0.33723414

0.28547296

TulavinusNC005228

0.3032096

0.34628379

0.32263514

PuumalavirusNC005223

0.33232468

0.31925675

$0.30912161,0.2483108$

HantaanvirusDQ371905

0.38264218

0.31925675

0.33614865

$0.28547296 \quad 0.27871621$

DobravavinusNC005234

0.40304586

0.39695945

0.39527026

$0.41891891 \quad 0.4070946$

$\begin{array}{llll}0.38682431 & 0.42736486 & 0.40878379\end{array}$

0.4054054

SeoulVirusNC005237

0.38056841

0.3766892

0.38344595

$0.41216215 \quad 0.4222973$

0.40202704

SangassouvirusDQ268651

0.40154818

0.40188473

0.40137625

$0.48121792 \quad 0.41895321$

0.39527026

0.42635861 
Hantaan virus Dobrava virus Seoul virus Sangassou virus

T7

T8

WC4

WCps

WC7

WC12

WC13

LJD1523FP

LJD2126FP

LJD2138FP

LJD2154FP

LJD2493FP

LJD2494FP

LJD2497FP

LJD2499FP

LJD2520FP

LJD2522FP

LJD2737PB

LJD2836FP

LJD2852FP

LJD2860FP

LJD2865FP

LJD2890FP

convictcreek

SNVL25783

SNVL37903

NewYorkVirusU36803

BlueRiverAF030552

BayouvirusL36930

BlackCreekCanalL39950

AndesVirusNC003467

Hu39694virusAF028023

OranVirusAF028024

LechiguanasvirusAF028022

Juquitibalikevirus 168711

MacielvirusAF028027

ElMoroCanyonU26828

ProspectHillX55129

TulavirusNC005228

PuumalavirusNC005223

HantaanvirusDQ371905

DobravavirusNC005234

SeoulVirusNC005237

SangassouvirusDQ268651
0.23817568

$0.24155405 \quad 0.25337839$

0.22419307
$0.17467813 \quad 0.28966558$ 
Appendix C: Specimen Capture Data. Small mammal field captures in the Portland, OR region during the period October, 2002 through October, 2005.

$\mathrm{PM}=$ Peromyscus maniculatus

$\mathrm{ST}=$ Sorex trowbridgii

$\mathrm{SV}=$ Sorex vagrans

$\mathrm{NG}=$ Neurotrichus gibsii

$\mathrm{MO}=$ Microtis oregoni

$\mathrm{SB}=$ Sorex bendirii

$\mathrm{PL}=$ Procyon lotor

$\mathrm{MT}=$ Microtus townsendii
$\mathrm{SN}=$ Sciurus niger

$\mathrm{TD}=$ Tamiasciurus douglasii

$\mathrm{TT}=$ Tamias townsendii

$\mathrm{ST}=$ Scapanus townsendii

$\mathrm{DV}=$ Didelphis virginianus

$\mathrm{MM}=$ Mephitis mephitis

$\mathrm{ME}=$ Mustela erminea

$\mathrm{SG}=$ Spilogale gracilis

\begin{tabular}{|l|l|l|l|l|l|}
\hline Specimen \# & Trap & G/S & Sex & Mass & ELISA/Notes \\
\hline Forest Park & & & & & \\
\hline 31 & & PM & & & \\
\hline 70 & & PM & & & \\
\hline 71 & & PM & & & \\
\hline 72 & & PM & & & \\
\hline 73 & & PM & & & trace \\
\hline 74 & & PM & & & positive \\
\hline 76 & & PM & & & \\
\hline 77 & & PM & & & \\
\hline 78 & & PM & & & \\
\hline 79 & & PM & & & \\
\hline 80 & & PM & & & positive \\
\hline 81 & & PM & & & \\
\hline 82 & & PM & & & \\
\hline 83 & & PM & & & \\
\hline 84 & & PM & & & \\
\hline 85 & & PM & & & \\
\hline 86 & & PM & & & positive \\
\hline 87 & & PM & & & \\
\hline 88 & & PM & & & \\
\hline 89 & & PM & & & \\
\hline 90 & & PM & & & \\
\hline 91 & & PM & & & \\
\hline 92 & & PM & & & \\
\hline 93 & & PM & & & \\
\hline 94 & & PM & & & \\
\hline 95 & & PM & & & \\
\hline Tryon Creek & $10 / 02$ & & & & \\
\hline 201 & m108 & PM & F & 18 & \\
\hline 202 & m & PM & F & 18.5 & \\
\hline 203 & m45 & PM & M & 18 & \\
\hline 204 & m & PM & M & 16 & \\
\hline 205 & p28 & PM & M & 14.5 & \\
\hline 206 & p96 & PM & M & 18.5 & \\
\hline 207 & s12 & PM & F & 17 & \\
\hline 208 & p12 & MO & F & 12 & \\
\hline & & & & & \\
\hline
\end{tabular}




\begin{tabular}{|c|c|c|c|c|c|}
\hline 209 & s6 & PM & $F$ & 17.5 & \\
\hline 210 & $\mathrm{p} 48$ & PM & $\mathrm{M}$ & 16 & \\
\hline 211 & s78 & $\mathrm{PM}$ & $\mathbf{M}$ & 16 & \\
\hline 212 & $\mathrm{p} 76$ & PM & $\mathbf{M}$ & 16.5 & \\
\hline 213 & s23 & $\mathrm{PM}$ & $\mathrm{M}$ & 18 & \\
\hline 214 & s24 & PM & $\mathbf{M}$ & 15 & trace \\
\hline 215 & p96 & PM & $\mathrm{F}$ & 21.5 & \\
\hline 216 & s129 & PM & $\mathrm{F}$ & 22.5 & \\
\hline 217 & s125 & PM & $F$ & 17.5 & \\
\hline 218 & s117 & PM & F & 25 & \\
\hline 219 & s116 & PM & $\mathrm{F}$ & 21 & \\
\hline 220 & s114 & PM & $\mathrm{M}$ & 18.5 & \\
\hline 221 & s14 & PM & $\mathrm{F}$ & 16.5 & \\
\hline 222 & $\mathrm{t} 112$ & SN & F & $\sim 600$ & . \\
\hline 223 & s14 & ME & $\mathbf{M}$ & 62 & \\
\hline 224 & $\mathrm{~m} 23$ & SV-NBS & & 5 & \\
\hline 225 & $\mathrm{~s} 128$ & PM & F & 19 & \\
\hline 226 & $\mathrm{~s} 12$ & PM & $\mathrm{M}$ & 17 & \\
\hline 227 & $\mathrm{t} 24$ & $\mathrm{SN}$ & $\mathbf{M}$ & $500+$ & \\
\hline 228 & pl15 & PM & $\mathrm{M}$ & 7 & \\
\hline 229 & p115 & $\overline{\mathrm{PM}}$ & $\mathbf{M}$ & 7.5 &. \\
\hline 230 & $\mathrm{~m} 8$ & PM & $\mathbf{M}$ & 7 & \\
\hline 231 & pl03 & PM & $\mathrm{M}$ & 7.5 & \\
\hline 232 & $\mathrm{~s} 47$ & PM & $F$ & 17 & \\
\hline 233 & s101 & PM & $\mathbf{M}$ & 17 & \\
\hline 234 & $\mathrm{~s} 33$ & PM & $\mathbf{M}$ & 18.5 & \\
\hline 235 & s98 & PM & $F$ & 16 & \\
\hline 236 & s1.01 & PM & F & 14 & \\
\hline 237 & s131 & SV-NBS & & 6 & \\
\hline 238 & s96 & TT & $\mathbf{M}$ & 130 & \\
\hline 239 & t116 & SN & F? & & \\
\hline 240 & p115 & PM-LS & $\mathbf{M}$ & 19.5 & clotted \\
\hline 241 & $\mathrm{p} 48$ & PM & $\mathbf{M}$ & 19 & \\
\hline 242 & p127 & $\mathrm{PM}$ & $\mathrm{M}$ & 6 & \\
\hline 243 & s24 & $\mathrm{TT}$ & $\mathbf{M}$ & 65 & \\
\hline 244 & s89 & PM & $\mathbf{M}$ & 11 & \\
\hline 245 & s129 & PM & $\mathbf{M}$ & 13 & \\
\hline 246 & $?$ & PM-LS & $\mathbf{M}$ & 14 & clotted \\
\hline 247 & s101 & PM & $\mathrm{M}$ & 18 & \\
\hline 248 & s100 & PM & $\mathrm{M}$ & 17 & trace \\
\hline 249 & $\mathrm{~s} 45$ & TT & $\mathbf{M}$ & 68 & \\
\hline 250 & t84 & $\mathrm{SN}$ & $\mathbf{M}$ & & \\
\hline 251 & $\mathrm{p} 40$ & SV-NBS & & 6 & \\
\hline 252 & s44 & SV-NBS & & 6 & \\
\hline Forest & $\begin{array}{l}\text { Park } \\
10 / 02\end{array}$ & & & & \\
\hline 253 & $\mathrm{~m} 108$ & PM & $\mathrm{F}$ & 21 & \\
\hline 254 & $\mathrm{~m} 72$ & PM & $\mathrm{M}$ & 18 & \\
\hline
\end{tabular}




\begin{tabular}{|c|c|c|c|c|c|}
\hline 255 & s95 & PM & $\mathbf{F}$ & 17 & \\
\hline 256 & s74 & PM & $\mathbf{M}$ & 19 & \\
\hline 257 & s127 & PM & $\mathbf{M}$ & 14.5 & \\
\hline 258 & $\mathrm{~m} 54$ & $\mathrm{PM}$ & $F$ & 15.5 & trace \\
\hline 259 & $\mathrm{ml18}$ & PM & $\mathbf{M}$ & 9 & \\
\hline 260 & $\mathrm{~s} 21$ & PM & $\mathbf{M}$ & 18 & positive \\
\hline 261 & $\mathrm{~m} 83$ & PM & $\mathbf{F}$ & 16.5 & \\
\hline 262 & $\mathrm{~s} 83$ & SV-NBS & & 5 & \\
\hline 263 & s64 & SV-NBS & & 5 & \\
\hline 264 & s6 & PM & $\mathbf{M}$ & 18 & \\
\hline 265 & s39 & PM & $\mathbf{M}$ & 12 & \\
\hline 266 & $\mathrm{~s} 130$ & PM & $\mathbf{F}$ & 17 & \\
\hline 267 & s14 & $\mathrm{PM}$ & $\mathbf{M}$ & 14 & trace \\
\hline 268 & s119 & $\mathrm{PM}$ & $\mathbf{M}$ & 20 & positive \\
\hline 269 & s117 & $\mathrm{PM}$ & $\mathbf{F}$ & 18 & \\
\hline 270 & s83 & $\mathrm{PM}$ & $\mathbf{M}$ & 16 & \\
\hline 271 & s95 & PM & $\mathbf{F}$ & 16 & $\therefore$ \\
\hline 272 & s80 & PM & $\mathbf{F}$ & 19 & \\
\hline 273 & p76 & SV-NBS & & 5 & \\
\hline 274 & m74 & PM-NBS & $F$ & 15 & \\
\hline 275 & $\mathrm{~m} 132$ & PM-NBS & $F$ & 50 & \\
\hline 276 & s89 & SV-NBS & & 4.5 & \\
\hline 277 & s76 & SV-NBS & & 5 & \\
\hline 278 & $\mathrm{~s} 45$ & SV-NBS & & 5.5 & \\
\hline 279 & $\mathrm{~m} 72$ & $\mathrm{PM}$ & $\mathbf{F}$ & 24 & positive \\
\hline 280 & s142 & $\mathrm{PM}$ & $\mathbf{M}$ & 20 & \\
\hline 281 & $\mathrm{p} 112$ & PM & $F$ & 20 & \\
\hline 282 & s59 & PM & $\mathbf{M}$ & 11.5 & \\
\hline 283 & s83 & PM & M & 13.5 & \\
\hline 284 & p76 & PM & $\mathbf{F}$ & 16 & \\
\hline 285 & s119 & PM & M & 10 & \\
\hline 286 & s29 & PM & $\mathbf{M}$ & 11.5 & \\
\hline 287 & $\mathrm{~s} 63$ & SV-NBS & & 5 & \\
\hline 288 & $\mathrm{~s} 85$ & SV-NBS & & 4 & \\
\hline 289 & s9 & SV-NBS & & 5 & \\
\hline 290 & $\mathrm{~m} 72$ & PM & $\mathbf{M}$ & 12 & \\
\hline 291 & $\mathrm{~m} 19$ & PM & $\mathbf{M}$ & 12 & \\
\hline 292 & $\mathrm{t} 79$ & TT & $\mathbf{M}$ & 160 & \\
\hline 293 & s23 & PM & $\mathbf{M}$ & 9 & \\
\hline 294 & s130 & PM & $\mathbf{M}$ & 10 & \\
\hline 295 & s127 & PM & $\mathbf{M}$ & 10 & \\
\hline 296 & $\begin{array}{l}\mathrm{s} 80 \\
\end{array}$ & PM & $\mathbf{M}$ & 15 & \\
\hline 297 & s47 & PM & $\mathbf{M}$ & 15 & \\
\hline 298 & $\mathrm{~s} 44$ & PM & $F$ & 11 & \\
\hline 299 & s22 & PM & $\mathbf{M}$ & 14.5 & trace \\
\hline 300 & $\mathrm{~m} 90$ & PM & $\mathbf{F}$ & 9 & \\
\hline 301 & $\mathrm{~m} 118$ & PM & $\mathbf{M}$ & 16 & \\
\hline 302 & $\mathrm{~m}$ & PM & M & 17 & \\
\hline 303 & s119 & PM & $\mathbf{M}$ & 19 & \\
\hline
\end{tabular}




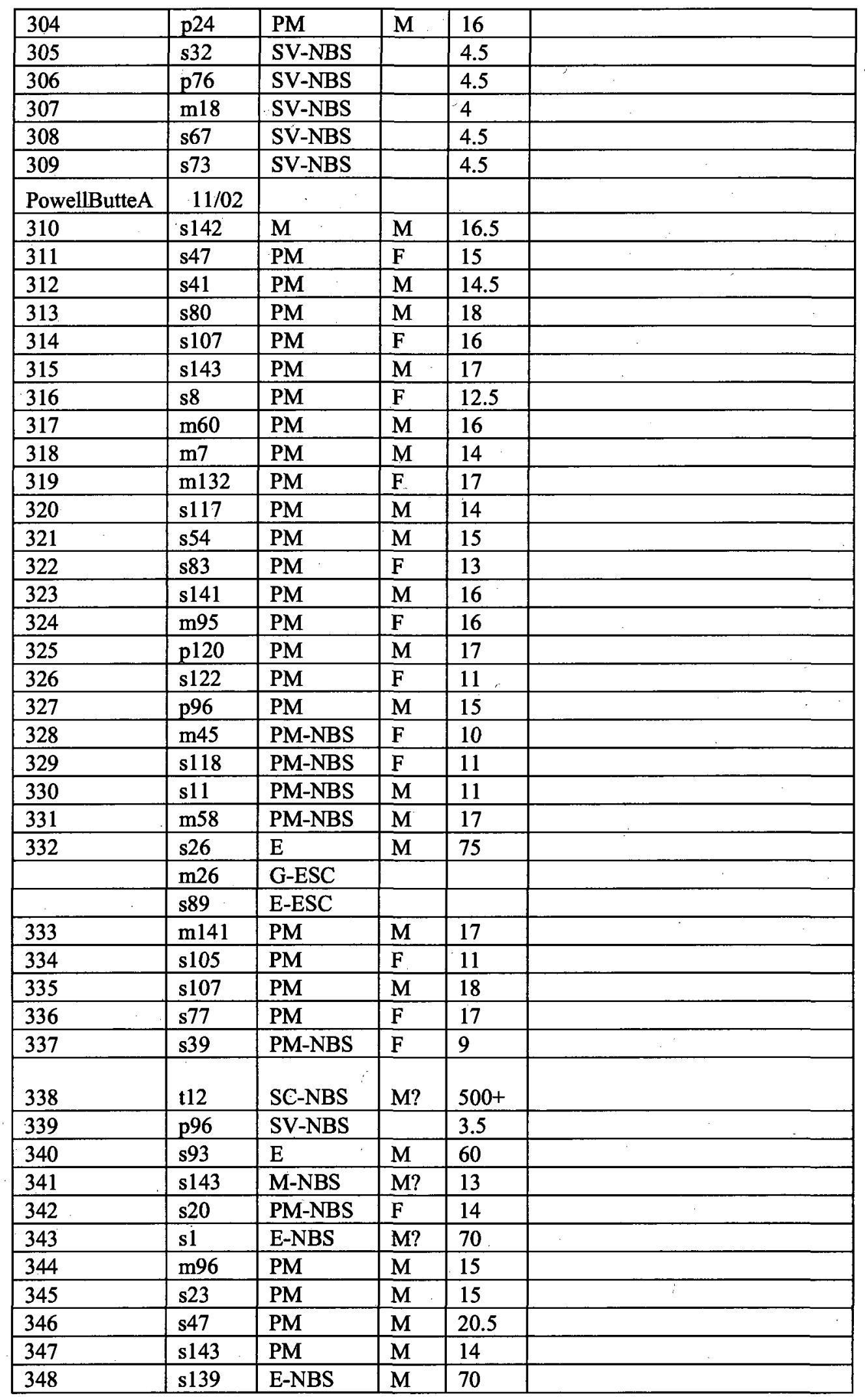




\begin{tabular}{|c|c|c|c|c|c|}
\hline 349 & s94 & SV-NBS & & 4 & \\
\hline PowellButteB & $11 / 02$ & & & & \\
\hline 350 & s6 & PM & $F$ & 14 & \\
\hline 351 & s47 & PM & $F$ & 14 & \\
\hline 352 & s18 & PM & $\mathrm{F}$ & 15 & \\
\hline 353 & $\mathrm{~m} 97$ & $\mathrm{PM}$ & $\mathrm{M}$ & 11.5 & \\
\hline 354 & s104 & PM & $F$ & 10.5 & \\
\hline 355 & s112 & PM & $\mathrm{F}$ & 14 & \\
\hline 356 & s18 & $\mathrm{PM}$ & $\mathrm{M}$ & 19 & \\
\hline 357 & p115 & PM & $\bar{M}$ & 17 & \\
\hline 358 & $\mathrm{~s} 59$ & PM & $F$ & 12 & \\
\hline 359 & s112 & PM & $\mathrm{F}$ & 15 & \\
\hline 360 & $\mathrm{~s} 20$ & PM & $\bar{M}$ & 15 & \\
\hline 361 & $\mathrm{~m} 60$ & PM-NBS & M & 10.5 & \\
\hline 362 & $\mathrm{p} 100$ & PM-NBS & M & 13 & \\
\hline 363 & $\mathrm{s32}$ & PM & $\mathrm{M}$ & 17 & \\
\hline 364 & s73 & PM & $\mathrm{F}$ & 11.5 & \\
\hline 365 & s104 & PM & $\mathrm{M}$ & 14 & \\
\hline 366 & s20 & PM & M & 13 & \\
\hline 367 & s118 & PM. & $\mathrm{F}$ & 22.5 & \\
\hline 368 & s59 & PM & $F$ & 15.5 & \\
\hline 369 & p60 & PM & $\mathrm{F}$ & 14 & \\
\hline 370 & $\mathrm{~m} 60$ & PM & $\mathrm{M}$ & 18 & \\
\hline 371 & 557 & PM & $F$ & 10 & \\
\hline Tryon Creek & $12 / 02$ & & & & \\
\hline 372 & s29 & PM & $\mathrm{M}$ & 19 & \\
\hline 373 & s18 & PM & $\mathrm{F}$ & 12.5 & \\
\hline 374 & s108 & PM & $\mathrm{F}$ & 22 & \\
\hline 375 & s113 & PM & $F$ & 19 & \\
\hline 376 & s117 & PM & $\mathrm{M}$ & 14 & \\
\hline 377 & s55 & PM & $\mathrm{F}$ & 17 & \\
\hline 378 & s57 & $\overline{\mathrm{PM}}$ & $\mathrm{F}$ & 20 & \\
\hline 379 & $\mathrm{~s} 47$ & PM & $\mathrm{F}$ & 14 & \\
\hline 380 & $\mathrm{~s} 45$ & PM & $\mathrm{M}$ & 14 & \\
\hline 381 & $\mathrm{~m} 45$ & PM & $\mathrm{F}$ & 25.5 & \\
\hline 382 & s95 & PM & $\mathrm{M}$ & 18 & \\
\hline 383 & s90 & PM & F & 8 & \\
\hline 384 & s142 & PM & $\mathrm{F}$ & 16.5 & \\
\hline 385 & s141 & PM & $\mathrm{F}$ & 16.5 & \\
\hline 386 & s78 & PM & $\mathrm{M}$ & 11 & \\
\hline 387 & $\mathrm{~s} 55$ & PM & $\mathrm{F}$ & 18 & \\
\hline 388 & $\mathrm{~s} 47$ & PM & $\mathrm{M}$ & 17 & \\
\hline 389 & s11 & PM & $F$ & 20 & \\
\hline 390 & s119 & PM & $M$ & 11 & \\
\hline 391 & s111 & $\overline{\mathrm{PM}}$ & $\mathrm{M}$ & 15.5 & \\
\hline 392 & s108 & PM & $\mathrm{M}$ & 18 & \\
\hline 393 & $\mathrm{~s} 21$ & PM & $\mathrm{M}$ & 15 & \\
\hline 394 & $\mathrm{~m} 103$ & PM & $\mathrm{F}$ & 17.5 & \\
\hline 395 & $\mathrm{t} 120$ & SN & & & \\
\hline
\end{tabular}




\begin{tabular}{|c|c|c|c|c|c|}
\hline 396 & $\mathrm{~m} 90$ & PM-NBS & $\mathrm{F}$ & 16 & \\
\hline 397 & s29 & PM-NBS & F & 11.5 & $i$ \\
\hline & s5 & SV-ESC & & & \\
\hline 398 & s94 & PM & $F$ & 13 & \\
\hline 399 & p55 & PM & $F$ & 7.5 & \\
\hline 400 & $\mathrm{~s} 45$ & PM & $\mathrm{F}$ & 12 & \\
\hline 401 & $s 42$ & $\mathrm{PM}$ & $\mathbf{M}$ & 7.5 & \\
\hline 402 & $s 70$ & PM & $\mathbf{M}$ & 9 & \\
\hline 403 & s91 & PM & $F$ & 13 & trace \\
\hline 404 & t84 & $\mathrm{SN}$ & F & & \\
\hline 405 & $\mathrm{p} 55$ & PM-NBS & $F$ & 6 & \\
\hline 406 & p55 & PM-NBS & $\mathrm{M}$ & 7 & \\
\hline 407 & s1 & PM & $\mathbf{M}$ & 13 & \\
\hline 408 & s7 & PM & $\mathbf{M}$ & 11 & \\
\hline 409 & s90 & PM & $\mathrm{M}$ & 10 & \\
\hline 410 & s94 & PM & $F$ & 10 & \\
\hline 411 & $\mathrm{~m} 67$ & MO & $\mathrm{F}$ & 15 & \\
\hline Oxbow & $12 / 02$ & & & & \\
\hline 412 & s22 & PM & $\mathbf{M}$ & 13 & \\
\hline 413 & s120 & PM & $F$ & 16 & \\
\hline 414 & $s 56$ & PM & $\mathbf{M}$ & 13.5 & \\
\hline 415 & s103 & PM & $F$ & 12 & \\
\hline 416 & s116 & PM & $\mathrm{F}$ & 16.5 & \\
\hline 417 & s68 & PM & $F$ & 16 & \\
\hline 418 & s94 & PM & $\mathbf{F}$ & 13 & \\
\hline 419 & m59 & $\mathrm{PM}$ & $\mathrm{M}$ & 17 & \\
\hline 420 & $\mathrm{~s} 60$ & PM & $F$ & 14 & \\
\hline 421 & s108 & PM & $\mathbf{M}$ & 15 & \\
\hline 422 & s90 & PM & $\mathbf{M}$ & 19 & \\
\hline 423 & $s 80$ & $\overline{\mathrm{PM}}$ & $\mathbf{M}$ & 14 & \\
\hline 424 & s141 & GS & $F$ & 94 & \\
\hline 425 & s137 & GS & $\mathrm{F}$ & 141 & \\
\hline 426 & p144 & S-NBS & & 4 & \\
\hline 427 & s136 & PM-NBS & $\mathrm{M}$ & 12 & \\
\hline 428 & $\mathrm{p} 60$ & S-NBS & & 5 & \\
\hline 429 & $\mathrm{p} 43$ & PM-NBS & $\mathbf{M}$ & 19 & \\
\hline 430 & p120 & S-NBS & & 5 & \\
\hline 431 & p100 & S-NBS & & 4.5 & \\
\hline 432 & $\mathrm{p} 120$ & PM-NBS & $\mathbf{M}$ & 14 & \\
\hline 433 & p120 & PM-NBS & $\mathbf{M}$ & 17.5 & \\
\hline 434 & p112 & S-NBS & & 6 & \\
\hline 435 & p112 & S-NBS & & 5 & \\
\hline 436 & pl12 & S-NBS & & 6 & \\
\hline & $\mathrm{t} 84$ & Sp & & & \\
\hline 437 & $\mathrm{~m} 66$ & PM & $F$ & 15 & \\
\hline 438 & s108 & PM & $\mathrm{M}$ & 12.5 & \\
\hline 439 & $\mathrm{p} 76$ & $\mathrm{PM}$ & $\mathrm{M}$ & 13 & \\
\hline 440 & s142 & PM & $\mathbf{M}$ & 15.5 & \\
\hline 441 & $\mathrm{~s} 82$ & PM & $\mathbf{M}$ & 15 & \\
\hline
\end{tabular}




\begin{tabular}{|c|c|c|c|c|c|}
\hline 442 & s79 & PM & $\mathbf{M}$ & 17 & \\
\hline 443 & p127 & S-NBS & & 5 & \\
\hline 444 & p7 & S-NBS & & 5.5 & \\
\hline 445 & p16 & S-NBS & & 6 & \\
\hline 446 & p16 & S-NBS & & 7 & \\
\hline 447 & s92 & PM-NBS & $\mathbf{F}$ & 11 & \\
\hline 448 & s100 & S-NBS & & 5 & \\
\hline 449 & p100 & S-NBS & & 5 & \\
\hline 450 & p100 & S-NBS & & 6 & \\
\hline 451 & $\mathrm{p} 48$ & S-NBS & & 5 & \\
\hline 452 & p110 & S-NBS & & 4.5 & \\
\hline 453 & p124 & S-NBS & & 5 & \\
\hline 454 & p12 & S-NBS & & 4 & \\
\hline 455 & p12 & S-NBS & & 6 & \\
\hline 456 & $\mathrm{p} 60$ & PM-NBS & $\mathbf{M}$ & 16 & \\
\hline 457 & p60 & S-NBS & & 6 & \\
\hline 458 & p60 & S-NBS & & 5 & \\
\hline \multirow[t]{2}{*}{459} & $\mathrm{p} 96$ & S-NBS & & 6 & \\
\hline & t91 & $\mathrm{Sp}$ & & & \\
\hline 460 & $\mathrm{~m} 60$ & PM & $\mathrm{F}$ & 17 & \\
\hline 461 & s108 & PM & $F$ & 17 & \\
\hline 462 & p64 & S-NBS & & 6 & \\
\hline 463 & p64 & NG & & 11 & \\
\hline 464 & p7 & MO & $F$ & 16 & \\
\hline 465 & p132 & S-NBS & & 5 & \\
\hline 466 & p132 & S-NBS & & 6.5 & \\
\hline 467 & p144 & S-NBS & & 5 & \\
\hline 468 & $\mathrm{p} 28$ & S-NBS & & 5 & \\
\hline 469 & p108 & S-NBS & & 5.5 & \\
\hline 470 & p79 & S-NBS & & 4 & \\
\hline 471 & $\mathrm{p} 79$ & S-NBS & & 6 & \\
\hline 472 & p79 & S-NBS & & 5.5 & \\
\hline \multirow[t]{2}{*}{473} & $\mathrm{p} 60$ & PM-NBS & $\mathbf{M}$ & 14 & \\
\hline & t67 & Sp & & & \\
\hline 474 & s60 & $\mathrm{PM}$ & $\mathbf{M}$ & 16 & \\
\hline 475 & s144 & PM & $\mathrm{M}$ & 12 & trace \\
\hline 476 & $\mathrm{p} 12$ & PM-NBS & $\mathrm{F}$ & 11 & \\
\hline 477 & $\mathrm{p} 12$ & S-NBS & & 5 & \\
\hline 478 & $\mathrm{p} 12$ & S-NBS & & 6 & \\
\hline 479 & p144 & S-NBS & & 6 & \\
\hline 480 & p144 & S-NBS & & 6 & \\
\hline 481 & p127 & S-NBS & & 5 & \\
\hline 482 & p76 & S-NBS & & 5 & \\
\hline 483 & p100 & S-NBS & & 5 & \\
\hline 484 & p96 & S-NBS & & 5 & \\
\hline 485 & p103 & S-NBS & & 5 & \\
\hline 486 & p32 & S-NBS & & 5 & \\
\hline 487 & p108 & S-NBS & & 5.5 & \\
\hline 488 & p108 & S-NBS & & 5 & \\
\hline
\end{tabular}




\begin{tabular}{|c|c|c|c|c|c|}
\hline 489 & s113 & PM-NBS & $?$ & & Half eaten \\
\hline Forest Park & 1.18 .03 & & & & \\
\hline 490 & $\mathrm{s52}$ & PM & $\mathrm{M}$ & 11 & \\
\hline 491 & s60 & PM & $\mathrm{M}$ & 18 & positive \\
\hline 492 & s104 & PM & $\mathrm{M}$ & 15 & \\
\hline 493 & s117 & PM & $\bar{F}$ & 14 & \\
\hline 494 & s18 & PM & $\mathrm{F}$ & 11 & \\
\hline 495 & s33 & PM & $\mathrm{F}$ & 12 & \\
\hline 496 & $\mathrm{s88}$ & PM & $\bar{M}$ & 14 & \\
\hline 497 & s120 & PM & $F$ & 14.5 & \\
\hline 498 & s130 & PM & $F$ & 11 & \\
\hline 499 & s126 & PM & $\mathrm{M}$ & 12 & \\
\hline 500 & $\$ 47$ & PM & $\mathrm{M}$ & 9.5 & \\
\hline 501 & $\mathrm{s33}$ & PM & M & 12 & \\
\hline 502 & $\mathrm{~s} 120$ & PM & M & 11 & \\
\hline 503 & s24 & PM & $\mathrm{M}$ & 14 & \\
\hline 504 & s72 & PM & $\mathrm{F}$ & 14.5 & \\
\hline 505 & s73 & PM & $\mathrm{F}$ & 12 & \\
\hline 506 & s144 & PM & $F$ & 13 & \\
\hline 507 & $\mathbf{s 8 2}$ & PM & M & 9.5 & \\
\hline 508 & s91 & PM & M & 12.5 & \\
\hline 509 & s113 & PM & $\bar{M}$ & 13 & \\
\hline 510 & $\mathrm{~s} 140$ & PM & $F$ & 13 & \\
\hline TRNWR & 2.8.03 & & & & \\
\hline 511 & s84 & PM & $\mathrm{M}$ & 15 & \\
\hline 512 & $\mathrm{~s} 82$ & $\overline{\mathrm{PM}}$ & $\bar{M}$ & 12 & \\
\hline 513 & $\mathrm{~m} 68$ & PM & $\mathrm{F}$ & 14 & \\
\hline 514 & s68 & PM & $\mathrm{M}$ & 15 & \\
\hline 515 & s65 & PM & $\mathrm{M}$ & 12.5 & \\
\hline 516 & p103 & $\mathrm{PM}$ & $F$ & 16 & \\
\hline 517 & $\mathrm{s32}$ & PM & $\bar{M}$ & 16 & \\
\hline 518 & $\mathrm{~s} 45$ & PM & $\bar{M}$ & 17 & \\
\hline 519 & s143 & PM & $\mathrm{M}$ & 17 & \\
\hline 520 & p84 & PM & $F$ & 12.5 & \\
\hline 521 & $\mathrm{~s} 33$ & PM & $F$ & 12 & \\
\hline 522 & s131 & PM & $\mathrm{M}$ & 13 & \\
\hline TRNWR & 2.9 .03 & & & & \\
\hline 523 & $\mathrm{~s} 32$ & PM & $\mathrm{F}$ & 13 & \\
\hline 524 & s46 & PM & $\mathrm{M}$ & 14 & \\
\hline 525 & s143 & $\overline{\mathrm{PM}}$ & $\bar{M}$ & 15 & \\
\hline 526 & s105 & PM & $\mathrm{M}$ & 16 & \\
\hline 527 & s72 & PM & $\bar{F}$ & 16 & \\
\hline 528 & s68 & PM & $\mathbf{F}$ & 14 & \\
\hline TRNWR & 2.10 .03 & & & & \\
\hline 529 & s72 & PM & 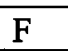 & 13 & \\
\hline 530 & s129 & GS & M? & 82 & \\
\hline TRNWR & 2.11 .03 & & & & \\
\hline 531 & s131 & PM & $\mathrm{F}$ & 12 & \\
\hline 532 & p139 & $\mathbf{S}$ & $?$ & 5 & \\
\hline
\end{tabular}




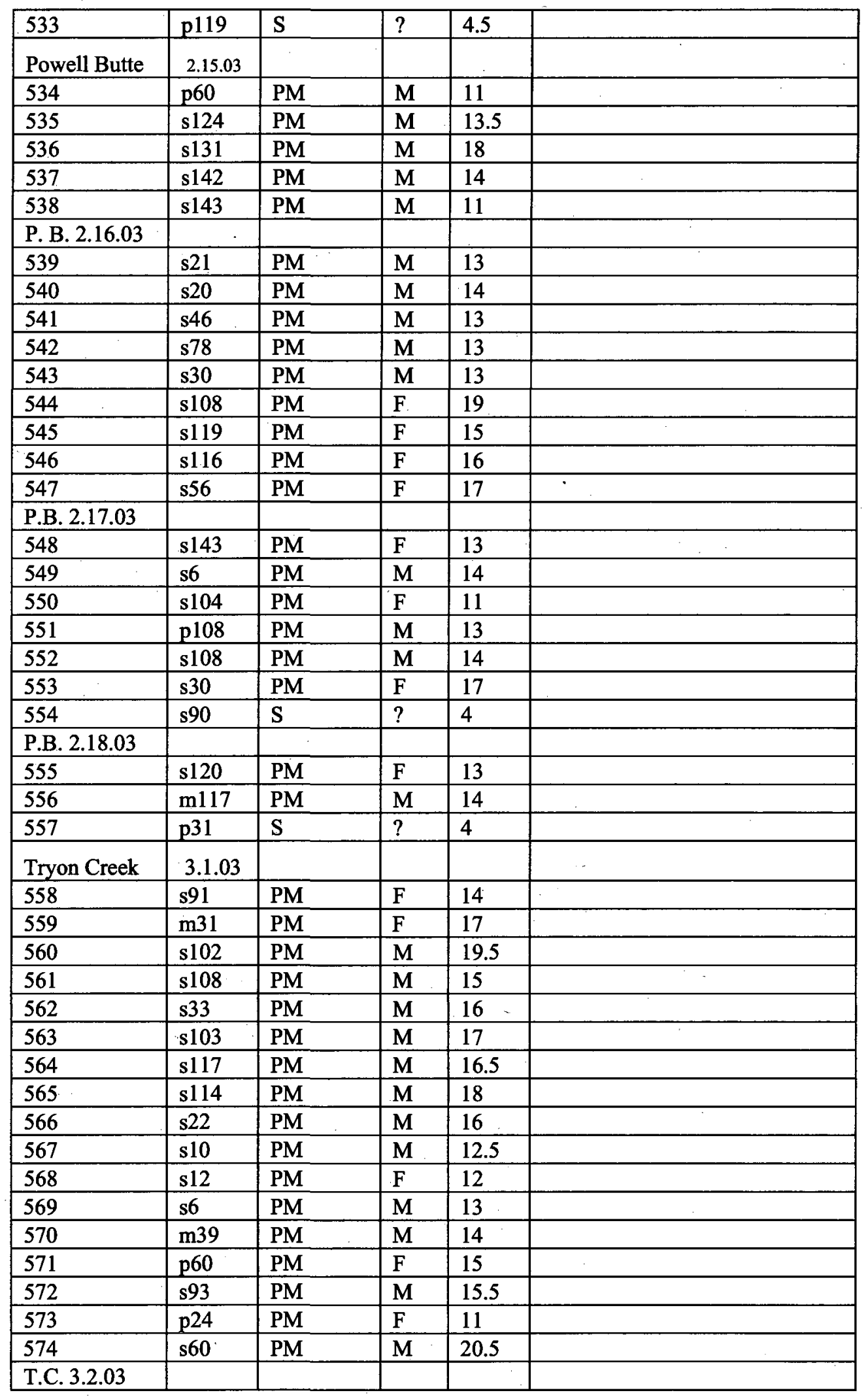




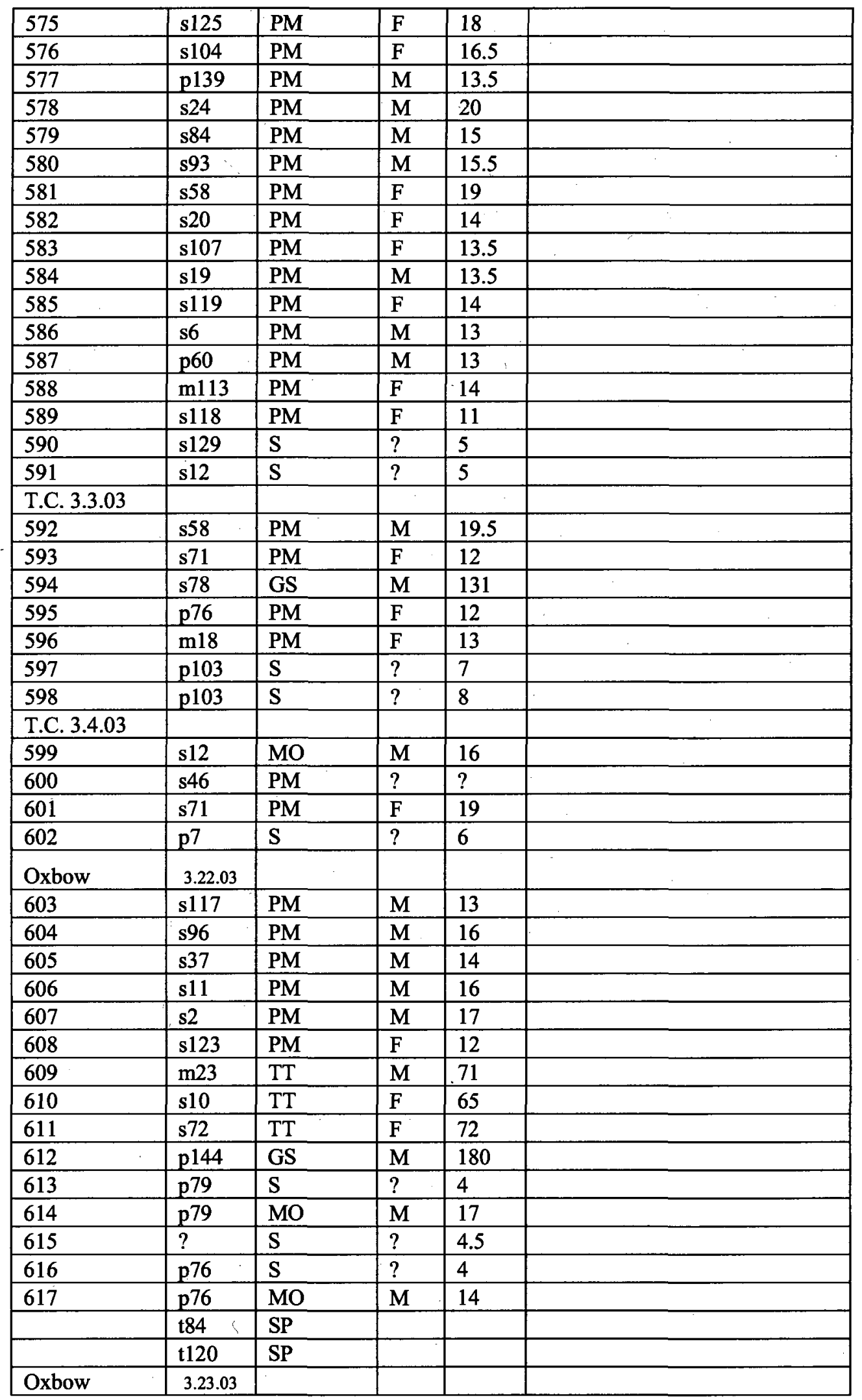




\begin{tabular}{|c|c|c|c|c|c|}
\hline 618 & s11 & $\mathrm{PM}$ & $\mathbf{F}$ & 8 & \\
\hline 619 & s98 & $\mathrm{PM}$ & $\mathbf{M}$ & 15.5 & \\
\hline 620 & s56 & $\mathrm{PM}$ & $F$ & 14 & \\
\hline 621 & $p 12$ & $\mathrm{~S}$ & $?$ & 6.5 & \\
\hline 622 & p67 & S & $?$ & 7 & \\
\hline 623 & p67 & $\mathrm{S}$ & $?$ & 7 & \\
\hline 624 & s35 & PM & $F$ & 14 & \\
\hline 625 & $\mathrm{p} 4$ & MO & $\mathbf{M}$ & 14.5 & \\
\hline 626 & p79 & S & $?$ & 6.5 & \\
\hline \multirow[t]{2}{*}{627} & p24 & PM & $\mathrm{F}$ & 13.5 & \\
\hline & $\mathrm{T} 48$ & SP & & & \\
\hline \multicolumn{6}{|c|}{$\begin{array}{l}\text { Oxbow } \\
3.24 .03\end{array}$} \\
\hline 628 & s72 & PM & $\mathbf{M}$ & 13 & \\
\hline 629 & $\mathrm{p} 24$ & $\mathrm{~S}$ & $?$ & 5.5 & \\
\hline 630 & $\mathrm{~s} 69$ & $\mathrm{TT}$ & $\mathbf{M}$ & 65 & \\
\hline 631 & s38 & TT & $\mathrm{M}$ & 85 & \\
\hline 632 & $\mathrm{p} 28$ & S & $?$ & 5.5 & \\
\hline 633 & p 60 & $\mathrm{~S}$ & $?$ & 5 & \\
\hline 634 & p31 & $\mathrm{S}$ & $?$ & 7 & \\
\hline 635 & s119 & $\mathrm{TT}$ & $\mathbf{M}$ & 68 & \\
\hline \multirow[t]{2}{*}{636} & s96 & S & $?$ & 6.5 & \\
\hline & $\mathrm{t} 103$ & SP & & & \\
\hline \multicolumn{6}{|c|}{$\begin{array}{l}\text { Oxbow } \\
3.25 .03\end{array}$} \\
\hline 637 & s119 & $\mathrm{S}$ & $?$ & 6.5 & \\
\hline 638 & s143 & TT & $\mathbf{M}$ & 69 & \\
\hline 639 & p55 & $\mathrm{S}$ & $?$ & 6 & \\
\hline 640 & $\mathrm{p} 12$ & $S$ & $?$ & 7.5 & \\
\hline 641 & s131 & MO & $\mathbf{M}$ & 13.5 & \\
\hline 642 & p92 & S & $?$ & 6 & \\
\hline 643 & $\mathrm{p} 108$ & $\mathrm{~S}$ & $?$ & 7.5 & \\
\hline 644 & $\mathrm{~s} 65$ & TT & $\mathbf{M}$ & 74 & \\
\hline \multicolumn{6}{|c|}{$\begin{array}{l}\text { Forest Park } \\
4.12 .03\end{array}$} \\
\hline 645 & $\mathrm{~s} 130$ & PM & $\mathbf{M}$ & 22 & \\
\hline 646 & $\mathrm{~s} 82$ & $\overline{\mathrm{PM}}$ & $F$ & 13 & \\
\hline 647 & $\mathrm{~s} 10$ & PM & $\mathrm{M}$ & 20.5 & \\
\hline 648 & p120 & PM & $\mathbf{M}$ & 20.5 & \\
\hline 649 & $\mathrm{~s} 53$ & PM & $\mathbf{M}$ & 19 & \\
\hline 650 & $s 41$ & PM & $\mathbf{F}$ & 13 & \\
\hline 651 & s19 & PM & $\mathbf{M}$ & 17 & \\
\hline 652 & $s 46$ & PM & $\mathbf{M}$ & 20 & \\
\hline 653 & $\mathrm{~s} 21$ & PM & $F$ & 14 & \\
\hline 654 & $\mathrm{p} 36$ & PM & $\mathbf{M}$ & 15 & \\
\hline 655 & $\mathrm{p} 84$ & ST & $?$ & 6 & \\
\hline \multicolumn{6}{|c|}{ F.P. 4.13 .03} \\
\hline 656 & $\mathrm{~m} 84$ & PM & $\mathbf{M}$ & 17 & \\
\hline 657 & s92 & $\overline{\mathrm{PM}}$ & $\mathbf{M}$ & 21 & \\
\hline 658 & s95 & PM & $F$ & 14 & \\
\hline
\end{tabular}




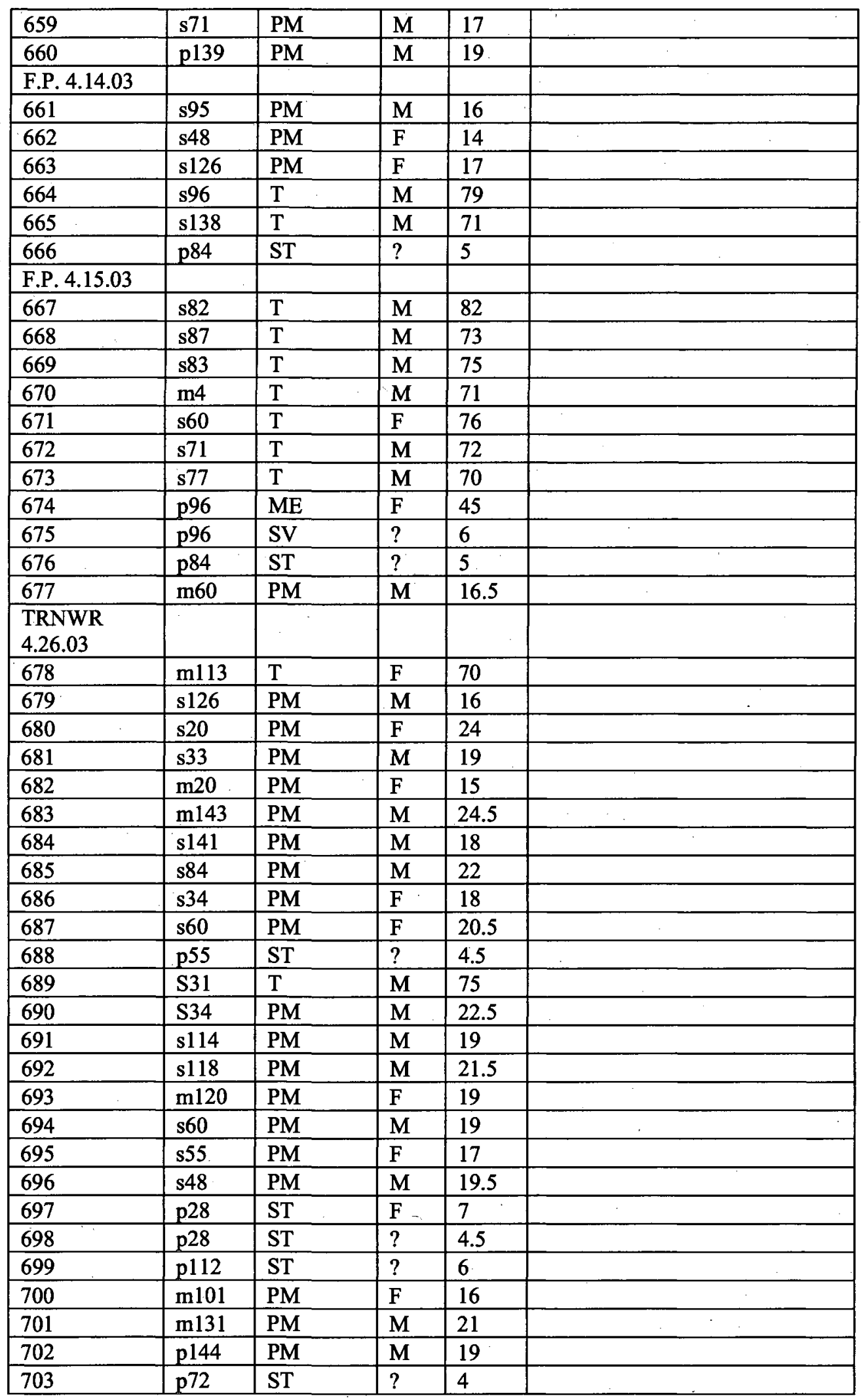




\begin{tabular}{|c|c|c|c|c|c|}
\hline 704 & p112 & ST & $?$ & 8 & \\
\hline 705 & $\mathrm{p} 112$ & ST & $?$ & 8 & \\
\hline 706 & $\mathrm{p} 112$ & ST & $?$ & 7.5 & \\
\hline 707 & $\mathrm{p} 112$ & ST & $?$ & 7.5 & \\
\hline 708 & $\mathrm{p} 112$ & ST & $?$ & 7 & \\
\hline 709 & $\mathrm{p} 112$ & ST & $?$ & 8 & \\
\hline 710 & $\mathrm{p} 112$ & ST & $?$ & 10 & \\
\hline 711 & p112 & ST & $?$ & 5 & \\
\hline 712 & $\mathrm{~m} 30$ & $T$ & $\bar{M}$ & 65 & \\
\hline 713 & s22 & $T$ & $\mathrm{M}$ & 68 & \\
\hline 714 & s142 & $T$ & $\mathbf{M}$ & 75 & \\
\hline 715 & s118 & $T$ & $F$ & 82 & \\
\hline 716 & $\mathrm{s52}$ & $T$ & $\mathbf{M}$ & 71 & \\
\hline 717 & $\mathbf{t 1 1 5}$ & $\mathrm{SN}$ & $\mathrm{M}$ & $>500$ & \\
\hline 718 & p52 & ST & $?$ & 6 & \\
\hline 719 & p112 & ST & $?$ & 7.5 & \\
\hline 720 & $\mathrm{~m} 144$ & PM & $\mathrm{F}$ & 17 & \\
\hline 721 & t60 & $\mathrm{T}$ & $F$ & 68 & \\
\hline 722 & $\mathrm{~s} 52$ & $T$ & $\mathbf{M}$ & 66 & \\
\hline 723 & s117 & $T$ & $\mathbf{M}$ & 63 & \\
\hline 724 & s116 & $\mathrm{T}$ & $F$ & 75 & \\
\hline 725 & s68 & $T$ & $F$ & 76 & \\
\hline 726 & s69 & $T$ & $\mathbf{M}$ & 76 & \\
\hline 727 & s18 & $\mathrm{T}$ & $\mathrm{M}$ & 72 & \\
\hline 728 & $\mathrm{s32}$ & $T$ & $\mathbf{M}$ & 71 & \\
\hline 729 & $\mathrm{~s} 12$ & $T$ & M & 74 & \\
\hline 730 & s115 & PM & $\mathbf{M}$ & 13 & \\
\hline 731 & p144 & $\mathrm{PM}$ & $\mathbf{M}$ & 15 & \\
\hline 732 & $\mathrm{~s} 9$ & PM & $F$ & 25 & \\
\hline 733 & $\mathrm{~s} 20$ & PM & $M$ & 21 & \\
\hline 734 & s106 & PM & $\mathbf{M}$ & 11 & \\
\hline 735 & s48 & PM & $F$ & 19.5 & \\
\hline 736 & $\mathrm{~s} 30$ & ST & $?$ & 4.5 & \\
\hline $\begin{array}{l}737 \\
\end{array}$ & $\mathrm{p} 120$ & ST & $?$ & 4.5 & \\
\hline \multicolumn{6}{|l|}{$\begin{array}{l}\text { Powell Butte } \\
5.10 .03\end{array}$} \\
\hline 738 & s47 & $\mathrm{PM}$ & $\mathbf{M}$ & 16.5 & \\
\hline 739 & 560 & PM & $F$ & 13.5 & \\
\hline 740 & s50 & $\mathrm{PM}$ & $\bar{F}$ & 17.5 & \\
\hline 741 & s47 & PM & M & 16 & \\
\hline 742 & $\mathrm{p} 40$ & ST? & $?$ & 4.5 & \\
\hline \multicolumn{6}{|l|}{ P.B. 5.11.03 } \\
\hline 743 & $\mathrm{~s} 72$ & PM & $F$ & 16 & \\
\hline 744 & s120 & $\mathrm{PM}$ & $F$ & 17 & \\
\hline 745 & $\mathrm{~m} 104$ & $\overline{\mathrm{PM}}$ & $\bar{M}$ & 19.5 & \\
\hline 746 & s119 & PM & M & 23 & \\
\hline 747 & s44 & PM & $M$ & 19.5 & \\
\hline 748 & s128 & $\mathrm{PM}$ & $\mathrm{F}$. & 15.5 & \\
\hline P.B. 5.12.03 & & & & & \\
\hline
\end{tabular}




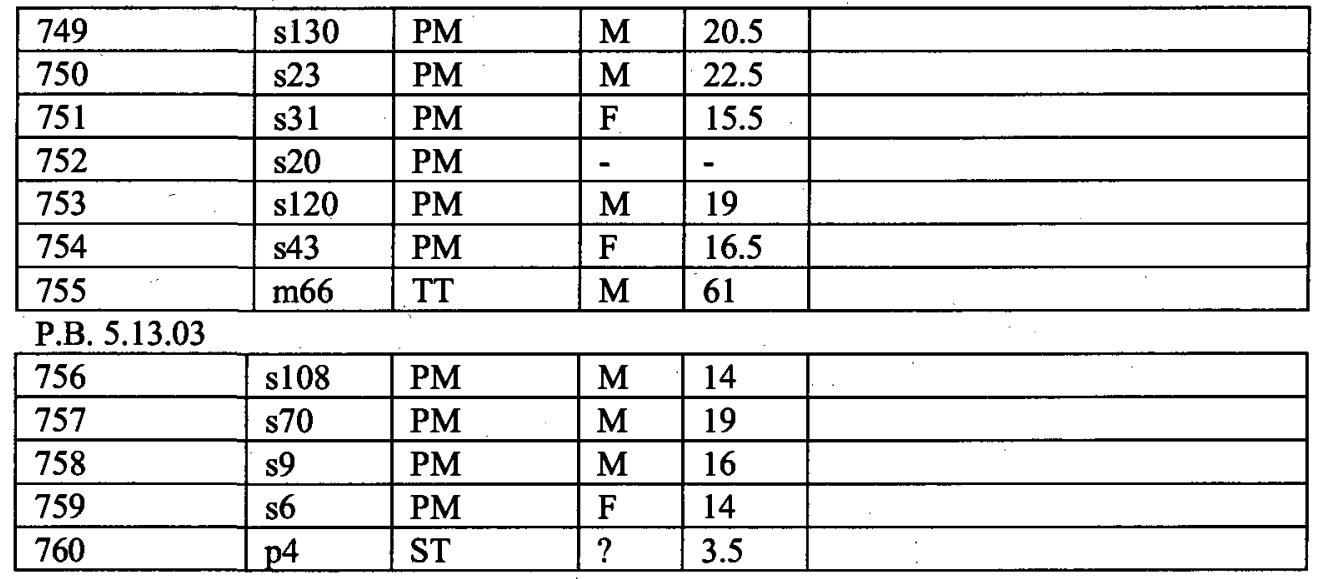

Oxbow 5.17.03

\begin{tabular}{|l|l|l|l|l|l|}
\hline 761 & $\mathrm{~m} 68$ & TT & M & 79 & \\
\hline 762 & s80 & TT & M & 77 & \\
\hline 763 & s97 & TT & M & 88 & \\
\hline 764 & s66 & PM & F & 17.5 & \\
\hline 765 & m102 & PM & F & 19.5 & \\
\hline 766 & p108 & PM & M & 13 & \\
\hline 767 & s127 & PM & M & 17 & \\
\hline 768 & s142 & PM & F & 15.5 & \\
\hline 769 & p4 & ST & $?$ & 4 & \\
\hline 770 & p48 & SB & F & 17.5 & \\
\hline
\end{tabular}

Ox 5.18.03
\begin{tabular}{|l|l|l|l|l|l|}
\hline 771 & s 53 & TT & M & 80 & \\
\hline 772 & s89 & TT & M & 72 & \\
\hline 773 & s27 & TT & M & 78 & \\
\hline 774 & s45 & PM & F & 11 & \\
\hline 775 & p4 & ST & $?$ & 4.5 & \\
\hline 776 & p12 & ST & $?$ & 4.5 & \\
\hline 777 & p112 & ST & $?$ & 4.5 & \\
\hline
\end{tabular}

OX 5.19.03

\begin{tabular}{|l|l|l|l|l|l|}
\hline 778 & p28 & ST & $?$ & 3.5 & \\
\hline 779 & s65 & TT & F & 70 & \\
\hline 780 & s117 & TT & F & 74 & \\
\hline 781 & s74 & TT & M & 79 & \\
\hline 782 & s92 & TT & M & 69 & \\
\hline 783 & s127 & TT & M & 71 & \\
\hline 784 & s131 & TT & F & 73 & \\
\hline 785 & s24 & TT & M & 64 & \\
\hline
\end{tabular}

OX 5.20.03
\begin{tabular}{|l|l|l|l|l|l|}
\hline 786 & $\mathrm{~s} 44$ & TDm & F & 203 & \\
\hline 787 & $\mathrm{~m} 114$ & TT & M & 68 & \\
\hline 788 & $\mathrm{~m} 103$ & TT & M & 82 & \\
\hline 789 & s63 & TT & F & 74 & \\
\hline 790 & s67 & TT & M & 80 & \\
\hline 791 & s141 & GS & M & 98 & \\
\hline 792 & s32 & TT & M & 72 & \\
\hline
\end{tabular}




\begin{tabular}{|c|c|c|c|c|c|}
\hline 793 & s17 & TT & $\mathbf{M}$ & 71 & \\
\hline 794 & s108 & TT & $\mathbf{M}$ & 80 & \\
\hline 795 & s66 & PM & $\mathbf{M}$ & 18 & \\
\hline 796 & $\mathrm{~m} 128$ & TT & $\mathbf{M}$ & 63 & \\
\hline 797 & $\mathrm{~m} 124$ & $\mathrm{TT}$ & $\mathbf{M}$ & 64 & \\
\hline 798 & s8 & ST & $?$ & 5 & \\
\hline 799 & p12 & ST & $?$ & 4 & \\
\hline 800 & p67 & ST & $?$ & 4 & \\
\hline 801 & p64 & ST & $?$ & 4 & \\
\hline 802 & p99 & ST & $?$ & 5 & \\
\hline \multicolumn{6}{|c|}{$\begin{array}{l}\text { TRYON } \\
\text { CREEK } \\
5.24 .03\end{array}$} \\
\hline 803 & $\mathrm{~S} 12$ & $\mathrm{PM}$ & $F$ & 26.5 & \\
\hline 804 & S114 & $\mathbf{P M}$ & $\mathbf{M}$ & 18.5 & \\
\hline 805 & S113 & $\mathrm{PM}$ & $\mathbf{M}$ & 16.5 & \\
\hline 806 & S26 & $\mathrm{PM}$ & $\mathbf{M}$ & 22 & \\
\hline 807 & M36 & PM & $\mathrm{F}$ & 20.5 & \\
\hline 808 & S45 & PM & $\mathrm{F}$ & 19 & \\
\hline 809 & S24 & $\mathrm{PM}$ & $\mathbf{M}$ & 19.5 & \\
\hline 810 & S116 & PM & $\mathbf{M}$ & 24 & \\
\hline 811 & S48 & PM & $\mathbf{M}$ & 20 & \\
\hline 812 & S144 & PM & $\mathbf{M}$ & 23 & \\
\hline 813 & M13 & $\mathrm{PM}$ & $\mathrm{F}$ & 16 & \\
\hline 814 & S129 & PM & $\mathbf{M}$ & 10 & EARS DAMAGED \\
\hline 815 & S? & ST & $?$ & 4.7 & DIT/NBS \\
\hline 816 & $\mathrm{P} 115$ & ST & $?$ & 6.8 & DIT/NBS \\
\hline 817 & $?$ & $\mathrm{ST}$ & $?$ & 5.6 & DIT/NBS not from today \\
\hline 818 & S116 & ST & $?$ & 5.4 & NBS/REL \\
\hline \multicolumn{6}{|c|}{ TC 5.25 .03} \\
\hline 819 & $\mathrm{ml06}$ & $\mathrm{MO}$ & $\mathrm{F}$ & 7.5 & JUV \\
\hline 820 & s108 & PM & $\mathrm{F}$ & 21 & \\
\hline 821 & $\mathrm{~m} 76$ & PM & $\mathbf{M}$ & 21.5 & \\
\hline 822 & p67 & PM & $\mathrm{F}$ & 17.5 & \\
\hline 836 & s137 & $\mathrm{PM}$ & $\mathbf{M}$ & 19.5 & \\
\hline 823 & s23 & PM & $\mathbf{M}$ & 12.5 & JUV \\
\hline 825 & S8 & PM & $F$ & 25 & PREG? \\
\hline 826 & S114 & PM & $\mathrm{F}$ & 21 & \\
\hline 827 & M48 & PM & $\mathbf{M}$ & 17.5 & \\
\hline 828 & S112 & PM & $\mathrm{M}$ & 17 & JUV \\
\hline 829 & $\mathrm{P} 100$. & PM & $\mathbf{M}$ & 14 & \\
\hline 830 & S47 & PM & $\mathbf{M}$ & 19 & \\
\hline 830 & S58 & PM & $\mathrm{F}$ & 21 & \\
\hline 832 & P91 & SCAT & $\mathrm{F}$ ? & 101 & NBS/REL \\
\hline 833 & P84 & ST & $?$ & 4.6 & NBS/REL \\
\hline 834 & P84 & ST & $?$ & 4.0 & DIT/NBS \\
\hline 835 & P103 & SCAT & $?$ & 119 & DIT/NBS \\
\hline 824 & $\mathrm{~S} 118$ & PM & $\mathbf{M}$ & 19 & \\
\hline 837 & P52 & SCAT & $?$ & 100 & DIT/NBS \\
\hline
\end{tabular}




\begin{tabular}{|l|l|l|l|l|l|}
\hline 838 & P124 & MT & $?$ & 70 & DIT/NBS \\
\hline 839 & P136 & MT & $?$ & 61 & DIT/NBS \\
\hline 840 & P96 & PM & M & 19.5 & DIT/NBS \\
\hline 841 & P76 & ST & $?$ & 3.8 & DIT/NBS \\
\hline 842 & P19 & ST & $?$ & 4.4 & DIT/NBS \\
\hline 843 & P72 & ST & $?$ & 4.3 & DIT/NBS \\
\hline 844 & P108 & SCAT & $?$ & 137 & DIT/NBS \\
\hline & P7 & ST & $?$ & $?$ & ESC \\
\hline
\end{tabular}

\section{TC 5.26.03}

\begin{tabular}{|l|l|l|l|r|l|}
\hline 845 & S70 & PM & F & 24 & \\
\hline 846 & P112 & PM & M & 10.5 & JUV \\
\hline 847 & S120 & PM & M & 6.5 & JUV \\
\hline
\end{tabular}

848

849

85

\begin{tabular}{|l}
\hline 852 \\
\hline 853 \\
\hline 854
\end{tabular}

85

\begin{tabular}{|l}
\hline 855 \\
\hline 856 \\
\hline 857
\end{tabular}

\begin{tabular}{|l}
856 \\
\hline 857 \\
\hline 858 \\
\hline
\end{tabular}

858

\begin{tabular}{|l|}
\hline 85 \\
\hline 860 \\
\hline 861 \\
\hline
\end{tabular}

\begin{tabular}{|l|l|l|}
\hline 860 & S101 & ST \\
\hline 861 & P48 & S \\
\hline 862 & P7 & ST \\
\hline 863 & P48 & ST \\
\hline
\end{tabular}

\begin{tabular}{|l|l|l|l|}
\hline 862 & P7 & ST & $?$ \\
\hline 863 & P48 & ST & $?$ \\
\hline 864 & P115 & ST & $?$ \\
\hline 865 & P84 & ST & $?$ \\
\hline 866 & P144 & PM & M \\
\hline 867 & P144 & ST & $?$ \\
\hline TC 5.27.03 & & & \\
\hline 868 & & & F \\
\hline
\end{tabular}

\begin{tabular}{|l|l|l|l|l|l|}
\hline TC 5.27.03 & & & & & \\
\hline 868 & S10 & PM & F & 27.5 & PREG? \\
\hline 869 & S108 & PM & F & 21 & \\
\hline 870 & P144 & PM & F & 17.5 & \\
\hline 871 & S134 & PM & M & 17 & SUBA \\
\hline 872 & S129 & PM & M & 7 & JUV \\
\hline 873 & S101 & PM & M & 8 & JUV \\
\hline 874 & S27 & PM & F & 11.5 & JUV \\
\hline 875 & S70 & TT & M & 61 & \\
\hline 876 & S71 & TT & M & 73 & \\
\hline 877 & S65 & TT & M & 79 & EARTAG R640 \\
\hline 878 & P19 & SV & $?$ & 5.5 & \\
\hline 879 & P115 & ST & $?$ & 4.1 & \\
\hline 880 & P16 & ST & $?$ & 4.0 & \\
\hline 881 & P108 & SCAT & F & 81 & \\
\hline 882 & S91 & TT & M & 63 & DIT/NBS \\
\hline 883 & S61 & ST & F & 11 & DIT/NBS/PREG? \\
\hline
\end{tabular}




\begin{tabular}{|c|c|c|c|c|c|}
\hline 884 & S58 & TT & $\mathbf{M}$ & 69 & DIT/NBS \\
\hline & P91 & SCAT & $?$ & $?$ & ESC \\
\hline \multicolumn{6}{|c|}{$\begin{array}{l}\text { Forest Park } \\
6.6 .03\end{array}$} \\
\hline 885 & s24 & PM & $\mathbf{M}$ & 17.5 & \\
\hline 886 & p96 & PM & $\mathbf{M}$ & 19 & \\
\hline 887 & s80 & PM & $F$ & 17 & \\
\hline 888 & s96 & PM & $F$ & 22 & \\
\hline 889 & s11 & PM & $F$ & 15 & \\
\hline 890 & s132 & PM & $\mathbf{M}$ & 16.5 & \\
\hline 891 & s107 & $\overline{P M}$ & $\mathrm{M}$ & 19.5 & \\
\hline 892 & $s 60$ & PM & $\mathbf{M}$ & 21.5 & \\
\hline 893 & s116 & PM & $F$ & 25 & \\
\hline 894 & $\mathrm{~m} 96$ & $\mathrm{TT}$ & $F$ & 80 & \\
\hline \multicolumn{6}{|c|}{ F.P. 6.7.03 } \\
\hline 895 & s82 & TT & $F$ & 79 & \\
\hline 896 & s106 & TT & $\mathrm{M}$ & 72 & \\
\hline 897 & $\mathrm{~s} 108$ & TT & $\mathbf{M}$ & 62 & \\
\hline 898 & s117 & TT & $\mathrm{M}$ & 60 & \\
\hline 899 & s116 & PM & $\mathbf{M}$ & 17 & \\
\hline 900 & s60 & PM & $F$ & 14.5 & \\
\hline 901 & s71 & PM & $\mathrm{M}$ & 12 & \\
\hline 902 & $\mathrm{p} 60$ & ST & $?$ & 8 & \\
\hline
\end{tabular}

\section{F.P. 6.8 .03}

\begin{tabular}{|l|l|l|l|l|l|}
\hline 903 & $\mathrm{~m} 75$ & TT & M & 71 & \\
\hline 904 & s22 & TT & M & 70 & \\
\hline 905 & s120 & TT & M & 62 & \\
\hline 906 & s88 & TT & M & 72 & \\
\hline 907 & m114 & TT & M & 74 & \\
\hline 908 & s106 & PM & F & 18 & \\
\hline 909 & p60 & ST & $?$ & 4 & \\
\hline 910 & p60 & ST & $?$ & 5 & \\
\hline 911 & p40 & SB & M & 19.5 & \\
\hline
\end{tabular}

F.P. 6.9.03
\begin{tabular}{|l|l|l|l|l|l|}
\hline 912 & s74 & TT & M & 73 & \\
\hline 913 & s83 & TT & M & 68 & \\
\hline 914 & p 60 & ST & $?$ & 4.8 & \\
\hline 915 & p 60 & ST & $?$ & 4.2 & \\
\hline 916 & s20 & ST & $?$ & 7 & \\
\hline 197 & m126 & ST & $?$ & 4 & \\
\hline 918 & m118 & TT & M & 61 & \\
\hline 919 & m63 & TT & F & 66 & \\
\hline 920 & s75 & TT & M & 58 & \\
\hline 921 & s19 & ST & $?$ & 4.4 & \\
\hline
\end{tabular}

TRNWR

6.11 .03

\begin{tabular}{|l|l|l|l|l|l|}
\hline 922 & s82 & PM & M & 17 & \\
\hline 923 & s84 & PM & M & 17.5 & \\
\hline 924 & s32 & PM & M & 19 & \\
\hline 925 & s24 & PM & F & 19.5 & positive \\
\hline
\end{tabular}




\begin{tabular}{|l|l|l|l|l|l|}
\hline 926 & s107 & PM & M & 14 & \\
\hline 927 & m9 & PM & M & 14.5 & \\
\hline 928 & s128 & PM & F & 16 & \\
\hline 929 & s64 & PM & M & 13 & \\
\hline 930 & s12 & PM & M & 18 & \\
\hline 931 & s21 & TT & F & 74 & \\
\hline 932 & s56 & TT & F & 70 & \\
\hline 933 & s92 & TT & M & 75 & \\
\hline 934 & s72 & TT & F & 89 & \\
\hline 935 & s81 & TT & F & 85 & \\
\hline 936 & s118 & TT & M & 62 & \\
\hline 937 & p67 & ST & $?$ & 3.8 & \\
\hline 938 & s67 & ST & $?$ & 5.4 & \\
\hline
\end{tabular}

TRNWR

6.12 .03

\begin{tabular}{|l|l|l|l|l|l|}
\hline 939 & s82 & PM & M & 19.5 & \\
\hline 940 & s12 & PM & F & 16.5 & \\
\hline 941 & s107 & PM & F & 14 & \\
\hline 942 & m9 & PM & M & 18 & \\
\hline 943 & m101 & TT & M & 80 & \\
\hline 944 & s119 & TT & F & 88 & \\
\hline 945 & s91 & TT & F & 84 & \\
\hline 946 & s93 & ST & $?$ & 4.3 & \\
\hline 947 & s100 & ST & $?$ & $?$ & \\
\hline 948 & p4 & ST & $?$ & 3.5 & \\
\hline 949. & p4 & ST & $?$ & 2.8 & \\
\hline 950 & p84 & PM & M & 23 & \\
\hline 951 & p144 & ST & $?$ & 5.4 & \\
\hline 952 & s9 & ST & $?$ & 3.9 & \\
\hline
\end{tabular}

TRNWR

6.13 .03

\begin{tabular}{|l|l|l|l|l|l|}
\hline 953 & s117 & PM & M & 18 & \\
\hline 954 & m34 & TT & F & 80 & \\
\hline 955 & s82 & PM & M & 13.5 & \\
\hline 956 & p88 & ST & $?$ & 4.5 & \\
\hline 957 & p96 & ST & $?$ & 6.2 & \\
\hline 958 & p144 & ST & $?$ & 6 & \\
\hline 959 & s99 & ST & $?$ & 3.6 & \\
\hline 960 & s107 & ST & $?$ & 5.6 & \\
\hline 961 & s72 & TT & F & 82 & \\
\hline 962 & s81 & ST & $?$ & 3.8 & \\
\hline
\end{tabular}

TRNWR

6.14 .03

\begin{tabular}{|l|l|l|l|l|l|}
\hline 963 & S117 & PM & M & 17 & \\
\hline 964 & S80 & ST & $?$ & 3.9 & \\
\hline 965 & S48 & ST & $?$ & 4.2 & \\
\hline 966 & S10 & TT & M & 70 & \\
\hline 967 & S8 & NG & $?$ & 11 & \\
\hline 968 & S120 & ST & $?$ & 4.2 & \\
\hline 969 & S35 & ST & $?$ & 4.2 & \\
\hline
\end{tabular}




\begin{tabular}{|l|l|l|l|l|l|}
\hline 970 & S96 & ST & $?$ & 6 & \\
\hline 971 & S19 & ST & $?$ & 4.3 & \\
\hline 972 & S115 & TT & F & 75 & \\
\hline
\end{tabular}

POWELL BUTTE 6.28.03

\begin{tabular}{|l|l|l|l|l|l|}
\hline 973 & s3 & TT & F & 81 & \\
\hline 974 & s66 & PM & F & 14.5 & \\
\hline 975 & s56 & PM & F & 16 & \\
\hline 976 & s129 & PM & M & 17.5 & \\
\hline 977 & s127 & PM & F & 19 & \\
\hline 978 & s131 & PM & M & 18.5 & \\
\hline 979 & s116 & PM & M & 17.5 & \\
\hline 980 & s8 & PM & M & 15.5 & \\
\hline 981 & s15 & PM & M & 15.5 & \\
\hline 982 & s23 & PM & F & 19.5 & \\
\hline 983 & s68 & PM & M & 15 & \\
\hline 984 & p112 & ST & $?$ & 3.6 & \\
\hline 985 & p16 & ST & $?$ & 3.8 & \\
\hline
\end{tabular}

P.B. 6.29.03
\begin{tabular}{|l|l|l|l|l|l|}
\hline 986 & s17 & GS & F & 156 & \\
\hline 987 & s18 & PM & M & $?$ & \\
\hline 988 & s35 & PM & M & 13 & \\
\hline 989 & s47 & PM & M & 20 & \\
\hline 990 & s3 & ST & $?$ & 5.5 & \\
\hline
\end{tabular}

P.B. 6.30.03
\begin{tabular}{|l|l|l|l|l|l|}
\hline 991 & s 94 & TT & F & 71 & \\
\hline 992 & m57 & PM & M & 15.5 & \\
\hline 993 & s21 & PM & F & 18.5 & \\
\hline 994 & s22 & PM & M & 18.5 & \\
\hline 995 & s125 & ST & $?$ & 2.8 & \\
\hline 996 & p 64 & ST & $?$ & $?$ & \\
\hline 997 & p 64 & ST & $?$ & 3.0 & \\
\hline
\end{tabular}

P.B. 7.1.03

\begin{tabular}{|l|l|l|l|l|l|}
\hline 998 & s90 & TT & M & 31 & \\
\hline 999 & s95 & GS & F & 115 & \\
\hline 1000 & s104 & ST & $?$ & 4.8 & \\
\hline 1001 & s32 & M & F & 20 & \\
\hline 1002 & s76 & ST & $?$ & 6.2 & \\
\hline 1003 & s54 & ST & $?$ & 3.0 & \\
\hline 1004 & s110 & ST & $?$ & 4.7 & \\
\hline 1005 & s66 & ST & $?$ & 5.3 & \\
\hline
\end{tabular}

OXBOW 7.4 .03

\begin{tabular}{|l|l|l|l|l|l|}
\hline 1006 & s71 & PM & F & 19 & \\
\hline 1007 & s72 & PM & F & 16 & \\
\hline 1008 & m104 & PM & F & 26 & positive \\
\hline 1009 & s24 & PM & M & 16.5 & positive \\
\hline 1010 & s143 & PM & F & 8 & \\
\hline 1011 & s23 & PM & F & 18.5 & \\
\hline 1012 & s52 & PM & M & 8 & \\
\hline
\end{tabular}




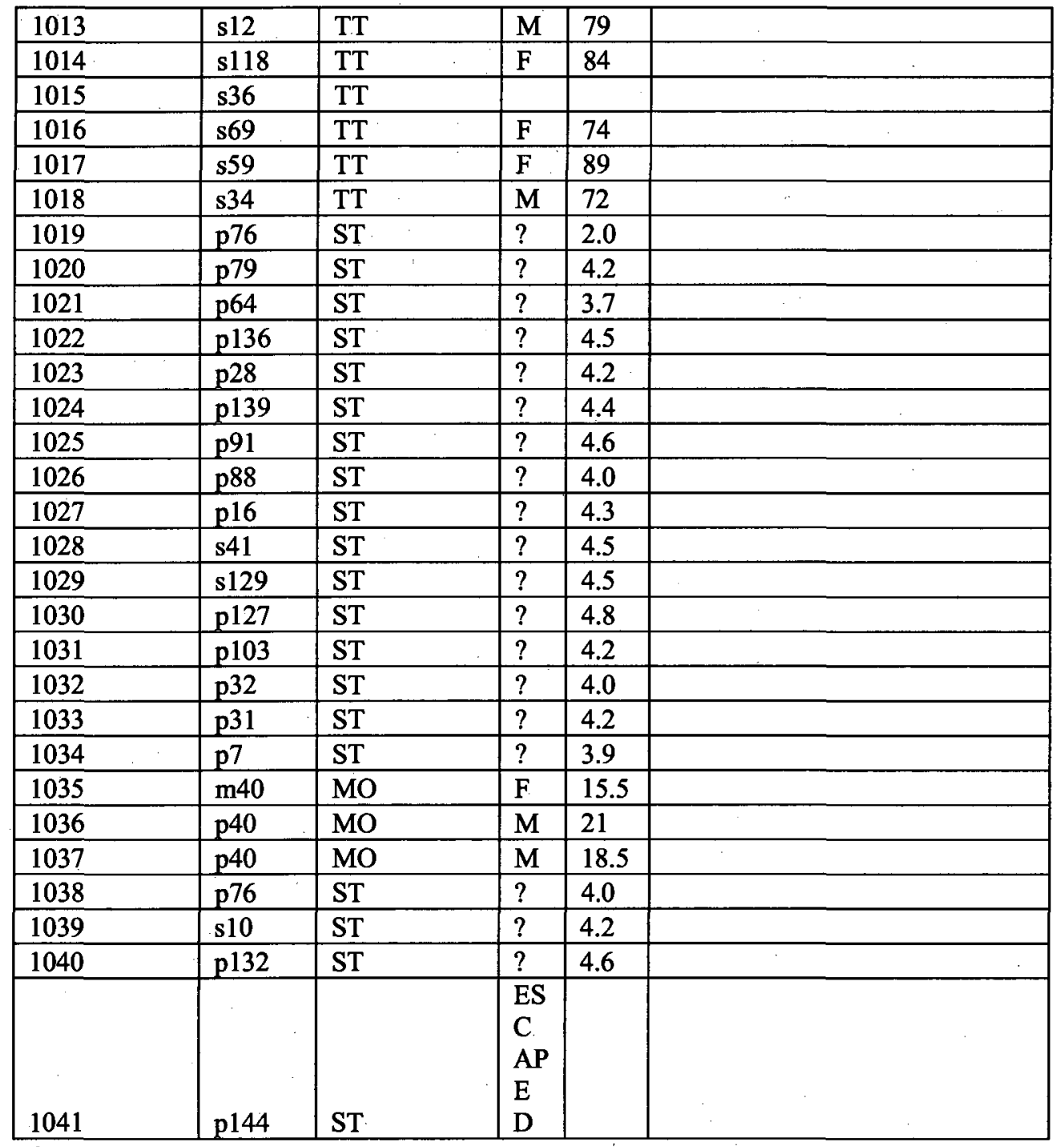

\begin{tabular}{|c|c|c|c|c|c|}
\hline \multicolumn{6}{|c|}{ OX 7.5.03 } \\
\hline 1042 & s68 & TT & $M$ & 62 & \\
\hline 1043 & s124 & TT & $\bar{M}$ & 32 & \\
\hline 1044 & s27 & TT & M & 30 & \\
\hline 1045 & s112 & TT & $\mathrm{M}$ & 68 & \\
\hline 1046 & $\mathrm{~m} 45$ & TT & $\mathrm{M}$ & 72 & \\
\hline 1047 & $\mathrm{~m} 8$ & TT & M & 37 & \\
\hline 1048 & s103 & TT & $\mathrm{M}$ & 61 & \\
\hline 1049 & s115 & $\mathrm{TT}$ & $F$ & 74 & \\
\hline 1050 & $\mathrm{~m} 115$ & MO & $F$ & 13 & \\
\hline 1051 & s79 & PM & $F$ & 17 & \\
\hline 1052 & s81 & PM & $\mathbf{M}$ & 14.5 & \\
\hline 1053 & $s 127$ & PM & $\mathrm{M}$ & 16 & \\
\hline 1054 & s131 & PM & $\mathrm{M}$ & 15 & \\
\hline 1055 & 883 & TT & $\mathbf{M}$ & 70 & \\
\hline 1056 & $\mathrm{p} 115$ & ST & $?$ & 7.2 & \\
\hline 1057 & $\mathrm{p} 120$ & ST & $?$ & 4.5 & \\
\hline
\end{tabular}




\begin{tabular}{|c|c|c|c|c|c|}
\hline 1058 & p132 & ST & $?$ & 3.5 & \\
\hline 1059 & p100 & ST & $?$ & 4.4 & \\
\hline 1060 & p67 & ST & $?$ & 4.3 & \\
\hline 1061 & $\mathrm{p}^{96}$ & $\mathrm{NG}$ & $?$ & 8.1 & \\
\hline 1062 & $\mathrm{~m} 121$ & TT & $\mathrm{M}$ & 31 & \\
\hline \multicolumn{6}{|c|}{ OX 7.6.03 } \\
\hline 1063 & s108 & PM & $\mathrm{F}$ & 23.5 & \\
\hline 1064 & s96 & PM & $\mathrm{F}$ & 18 & \\
\hline 1065 & s1 & PM & $\mathbf{M}$ & 15 & \\
\hline 1066 & s72 & TT & $F$ & 72 & \\
\hline 1067 & s44 & TT & $\mathrm{M}$ & 67 & \\
\hline 1068 & $\mathrm{~s} 18$ & $\mathrm{TT}$ & $\mathbf{M}$ & 70 & \\
\hline 1069 & s47 & TT & F & 83 & \\
\hline 1070 & s15 & TT & $\mathbf{M}$ & 33 & \\
\hline 1071 & $\mathrm{ml10}$ & TT & $F$ & 77 & \\
\hline 1072 & s27 & TT & $\bar{M}$ & 31 & \\
\hline 1073 & s13 & TT & $\mathbf{M}$ & 30 & \\
\hline 1074 & s119 & TT & $\mathrm{F}$ & ESC & \\
\hline 1075 & s61 & TT & $\mathbf{M}$ & 70 & \\
\hline 1076 & $\mathrm{~m} 115$ & $\overline{\mathrm{MO}}$ & $?$ & & \\
\hline 1077 & p67 & SB & $\mathrm{M}$ & 14 & \\
\hline 1078 & s143 & SV & $?$ & ESC & \\
\hline 1079 & p79 & SV & $?$ & 7.1 & \\
\hline 1080 & s144 & ST & $?$ & 4.5 & \\
\hline \multicolumn{6}{|c|}{ OX 7.7.03 } \\
\hline 1081 & p91 & PM & M & 7.5 & \\
\hline 1082 & s19 & TT & $\mathbf{M}$ & 68 & \\
\hline 1083 & $\mathrm{~s} 26$ & TT & $\bar{M}$ & 30 & \\
\hline 1084 & $\mathrm{~s} 120$ & TT & $\bar{F}$ & 80 & \\
\hline 1085 & $\mathrm{~s} 50$ & TT & $\mathbf{M}$ & 30 & \\
\hline 1086 & p144 & NG & $?$ & 7.3 & \\
\hline 1087 & p144 & NG & $?$ & 5.9 . & \\
\hline 1088 & p67 & ST & $?$ & 3.9 & \\
\hline 1089 & p67 & ST & $?$ & 3.3 & \\
\hline 1090 & $s 46$ & SV & $?$ & 5.3 & \\
\hline 1091 & s17 & SV & $?$ & 4.5 & \\
\hline 1092 & p12 & ST & $?$ & 4.1 & \\
\hline 1093 & p108 & ST & $?$ & 4.7 & \\
\hline 1094 & p132 & ST & $?$ & 4.2 & \\
\hline 1095 & p132 & ST & $?$ & 0.6 & \\
\hline 1096 & p37 & ST & $?$ & 3.8 & \\
\hline 1097 & s114 & ST & $?$ & 4.0 & \\
\hline 1098 & $\mathrm{p} 52$ & ST & $?$ & 3.5 & \\
\hline 1099 & p24 & ST & $?$ & 4.3 & \\
\hline 1100 & s139 & $\overline{\mathrm{ME}}$ & $\mathrm{M}$ & 31 & \\
\hline 1101 & $\mathrm{~m} 29$ & TT & $\mathrm{M}$ & 31 & \\
\hline 1102 & s55 & SV & $?$ & 4.6 & \\
\hline 1103 & s56 & TT & $\mathrm{M}$ & 64 & \\
\hline
\end{tabular}

Tryon Creek 7.11.03 (skip 1104-1 109) 


\begin{tabular}{|c|c|c|c|c|c|}
\hline 1110 & s95 & TT & $F$ & 72 & \\
\hline 1111 & s94 & TT & $F$ & 74 & \\
\hline 1112 & $\mathrm{~m} 105$ & TT & $\mathrm{M}$ & 71 & \\
\hline 1113 & s130 & $\mathrm{TT}$ & $\bar{M}$ & 78 & \\
\hline 1114 & $\mathrm{p} 12$ & $\mathrm{PM}$ & $\mathbf{M}$ & 15.5 & \\
\hline 1115 & $\mathrm{~s} 143$ & PM & $\mathrm{M}$ & 20 & \\
\hline 1116 & $\mathrm{~s} 20$ & $\mathrm{PM}$ & $F$ & 7.5 & \\
\hline 1117 & $\mathrm{~m} 58$ & $\mathrm{PM}$ & $\mathbf{M}$ & 19 & \\
\hline 1118 & s59 & $\mathrm{PM}$ & $F$ & 16.5 & \\
\hline 1119 & $\mathrm{~s} 24$ & $\mathrm{PM}$ & $F$ & 20.5 & \\
\hline 1120 & s64 & $\mathrm{PM}$ & $\bar{M}$ & 19.5 & \\
\hline 1121 & s44 & $\mathrm{PM}$ & $\mathbf{M}$ & 20.5 & \\
\hline 1122 & s46 & $\mathrm{PM}$ & $F$ & 19.5 & positive \\
\hline 1123 & s114 & $\mathrm{PM}$ & $\mathrm{M}$ & 13.5 & \\
\hline 1124 & $\mathrm{~s} 108$ & $\mathrm{PM}$ & $\mathrm{M}$ & 16 & \\
\hline 1125 & $\mathrm{~s} 106$ & PM & $\bar{M}$ & 15 & \\
\hline 1126 & $\mathrm{p} 100$ & $\mathrm{PM}$ & $F$ & 15 & \\
\hline 1127 & s107 & $\mathrm{PM}$ & $\bar{F}$ & 18 & \\
\hline 1128 & s11 & PM & $F$ & 15 & \\
\hline 1129 & $\mathrm{p} 48$ & $\mathrm{PM}$ & $F$ & 15 & \\
\hline 1130 & s68 & $\mathrm{PM}$ & $\mathrm{M}$ & 18.5 & \\
\hline 1131 & p19 & ST & $?$ & 4.3 & \\
\hline 1132 & $\mathrm{p} 118$ & ST & $?$ & 4.2 & \\
\hline 1133 & s131 & ST & $?$ & 5 & \\
\hline 1134 & $\mathrm{p} 40$ & ST & $?$ & 4.1 & \\
\hline 1135 & s144 & ST & $?$ & 4 & \\
\hline 1136 & s118 & ST & $?$ & 4.3 & \\
\hline 1137 & $\mathrm{p} 144$ & ST & $?$ & 4.8 & \\
\hline 1138 & $\mathrm{p} 19$ & ST & $?$ & 4.2 & \\
\hline 1139 & $\mathrm{~s} 32$ & SV & $?$ & 9.6 & \\
\hline 1140 & p76 & $\begin{array}{l}\text { ST } \\
\end{array}$ & $?$ & 3.4 & \\
\hline 1141 & s136 & ST & $?$ & 4.1 & \\
\hline 1142 & $\mathrm{p} 124$ & ST & $?$ & 4.2 & \\
\hline 1143 & $\mathrm{p} 127$ & ST & $?$ & 3.9 & \\
\hline 1144 & $\mathrm{p} 88$ & ST & $?$ & 5.1 & \\
\hline 1145 & $\mathrm{p} 88$ & ST & $?$ & 4.3 & \\
\hline 1146 & $\mathbf{t 6 0}$ & TT & $M$ & 69 & \\
\hline
\end{tabular}

T.C. 7.12 .03

\begin{tabular}{|l|l|l|l|l|l|}
\hline 1147 & $\mathrm{~m} 94$ & TT & M & 69 & \\
\hline 1148 & $\mathrm{~s} 96$ & TT & M & 70 & \\
\hline 1149 & $\mathrm{~s} 115$ & TT & F & 71 & \\
\hline 1150 & s94 & TT & F & 68 & \\
\hline 1151 & $\mathrm{~s} 72$ & PM & F & 16 & \\
\hline 1152 & m108 & PM & M & 16 & \\
\hline 1153 & $\mathrm{~s} 71$ & PM & F & 20 & \\
\hline 1154 & s106 & PM & M & 23.5 & \\
\hline 1155 & m113 & PM & F & 22 & \\
\hline 1156 & m132 & PM & M & 14.5 & \\
\hline 1157 & $\mathrm{~s} 24$ & PM & M & 18 & \\
\hline
\end{tabular}




\begin{tabular}{|l|l|l|l|l|l|}
\hline 1158 & s11 & PM & F & 6.5 & \\
\hline 1159 & s33 & PM & M & 14.5 & \\
\hline 1160 & s95 & ST & $?$ & 5 & \\
\hline 1161 & p118 & ST & $?$ & 3.8 & \\
\hline 1162 & p19 & SV & $?$ & 5 & \\
\hline 1163 & p55 & ST & $?$ & 4 & \\
\hline 1164 & s112 & SV & $?$ & 7.9 & \\
\hline 1165 & p24 & ST & $?$ & 3.8 & \\
\hline 1166 & s87 & ST & $?$ & 4.5 & \\
\hline 1167 & p7 & ST & $?$ & 4.5 & \\
\hline 1168 & p7 & NG & $?$ & 8.6 & \\
\hline 1169 & p103 & ST & $?$ & 3.9 & \\
\hline 1170 & s129 & ST & $?$ & 5.2 & \\
\hline 1171 & p119 & ST & $?$ & 4 & \\
\hline 1172 & p119 & ST & $?$ & 3.9 & \\
\hline 1173 & $\mathrm{~s} 143$ & SV & $?$ & 8.5 & \\
\hline 1174 & p144 & ST & $?$ & 4.3 & \\
\hline 1175 & $\mathrm{~s} 69$ & ST & $?$ & 4 & \\
\hline 1176 & $\mathrm{~s} 57$ & ST & $?$ & 4 & \\
\hline 1177 & $\mathrm{~s} 142$ & PM & F & 18 & \\
\hline
\end{tabular}

T.C. 7.13 .03

\begin{tabular}{|l|l|l|l|l|l|}
\hline 1178 & $\mathrm{~m} 107$ & TT & M & 62 & \\
\hline 1179 & $\mathrm{~s} 11$ & PM & F & 18.5 & \\
\hline 1180 & $\mathrm{~s} 107$ & PM & M & 19.5 & \\
\hline 1181 & $\mathrm{~s} 108$ & PM & F & 15 & \\
\hline 1182 & $\mathrm{~s} 33$ & PM & M & 8 & \\
\hline 1183 & $\mathrm{~s} 113$ & PM & M & 16 & \\
\hline 1184 & $\mathrm{~s} 23$ & PM & M & 20 & \\
\hline 1185 & $\mathrm{~s} 58$ & PM & F & 9 & \\
\hline 1186 & $\mathrm{~s} 72$ & PM & M & 17 & \\
\hline 1187 & p40 & SV & $?$ & 7.4 & \\
\hline 1188 & p144 & ST & $?$ & 4.5 & \\
\hline 1189 & $\mathrm{~s} 144$ & ST & $?$ & 4.2 & \\
\hline 1190 & $\mathrm{~s} 124$ & ST & $?$ & 1.6 & \\
\hline 1191 & $\mathrm{~s} 20$ & SV & $?$ & 7.5 & \\
\hline 1192 & $\mathrm{~s} 96$ & TT & F & 61 & \\
\hline
\end{tabular}

T.C. 7.14 .03

\begin{tabular}{|l|l|l|l|l|l|}
\hline 1193 & $\mathrm{p} 60$ & PM & M & 16 & \\
\hline 1194 & $\mathrm{p} 12$ & ST & $?$ & 3.9 & \\
\hline 1195 & $\mathrm{p} 64$ & ST & $?$ & 3.9 & \\
\hline 1196 & $\mathrm{p} 64$ & ST & $?$ & 1.1 & \\
\hline 1197 & $\mathrm{~s} 11$ & ST & $?$ & 4.4 & \\
\hline 1198 & $\mathrm{~s} 140$ & ST & $?$ & 4.3 & \\
\hline 1199 & $\mathrm{p} 144$ & ST & $?$ & $?$ & \\
\hline 1200 & $\mathrm{~s} 108$ & SV & $?$ & 4.8 & \\
\hline 1201 & $\mathrm{~s} 127$ & ST & $?$ & 4.9 & \\
\hline 1202 & $\mathrm{~s} 127$ & ST & $?$ & 5.5 & \\
\hline 1203 & $\mathrm{p} 112$ & ST & $?$ & 4.0 & \\
\hline 1204 & $\mathrm{p} 112$ & ST & $?$ & 4.6 & \\
\hline
\end{tabular}




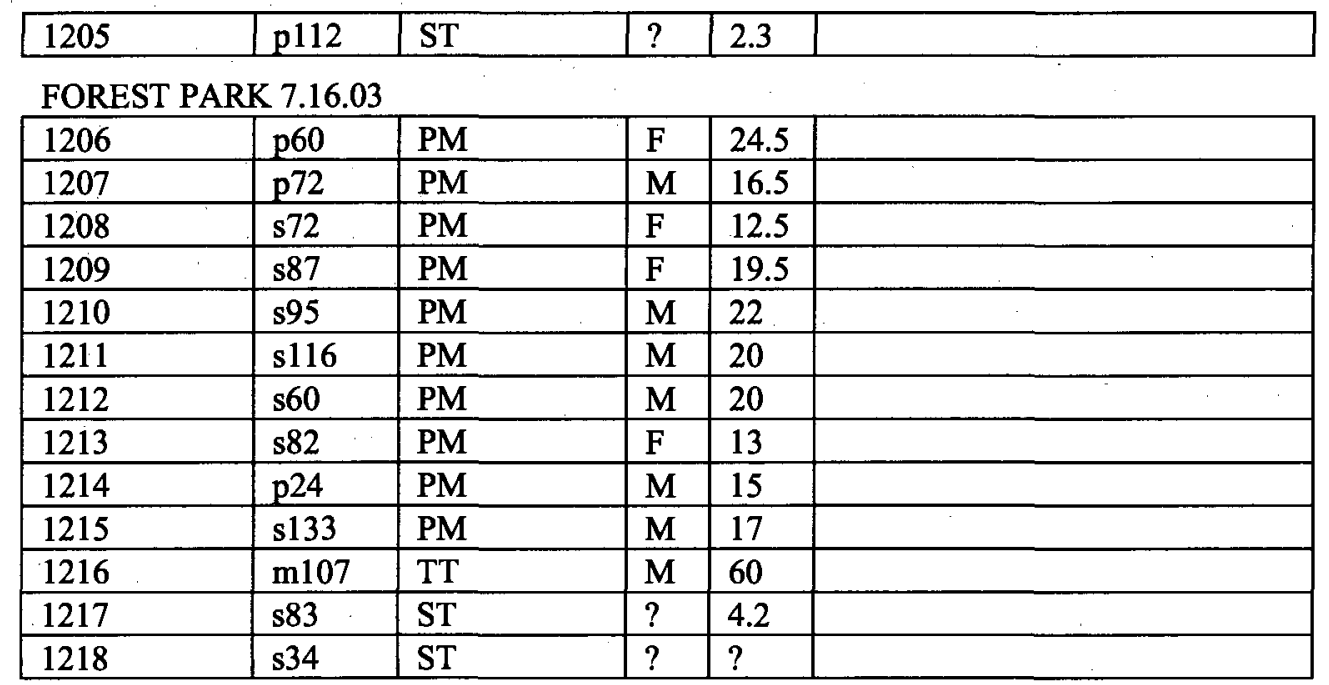

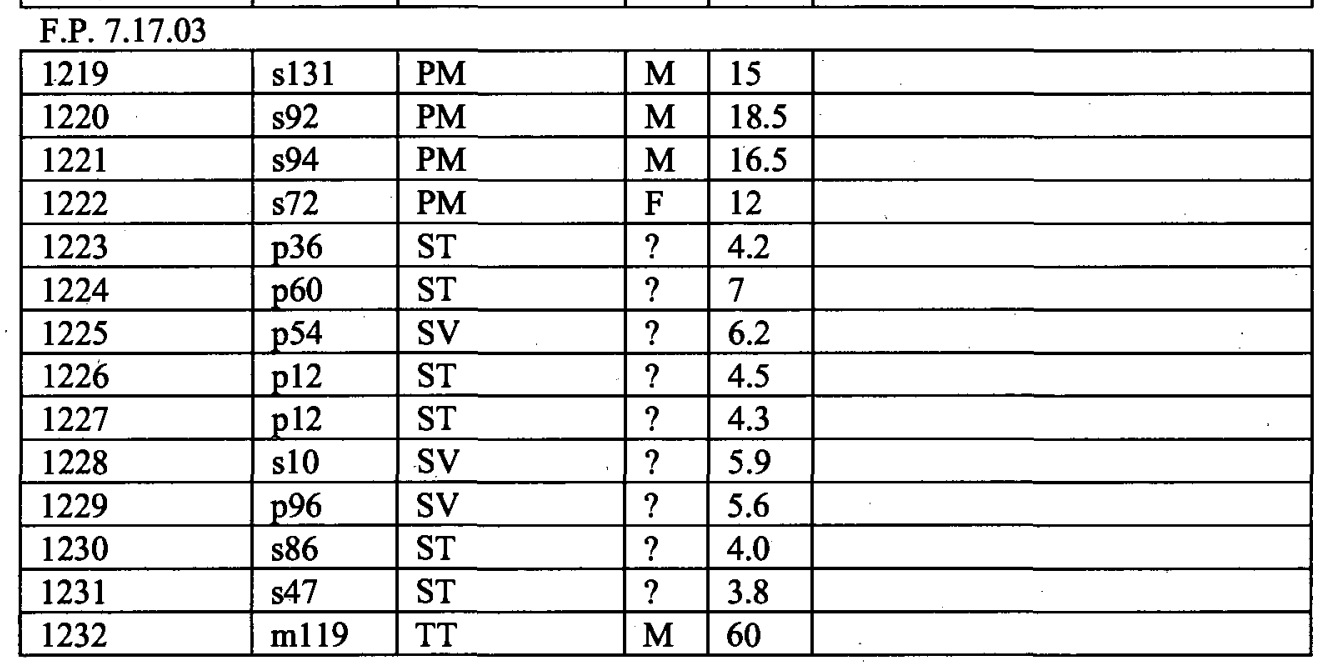

\section{F.P. 7.18.03}

\begin{tabular}{|l|l|l|l|l|l|}
\hline 1233 & s46 & PM & M & 15 & \\
\hline 1234 & s143 & PM & M & 15 & \\
\hline 1235 & s119 & PM & M & 13.5 & \\
\hline 1236 & s82 & PM & M & 18 & \\
\hline 1237 & s72 & PM & F & 9 & \\
\hline 1238 & s44 & PM & F & 17 & \\
\hline 1239 & s108 & TT & F & 79 & \\
\hline 1240 & s83 & TT & M & 60 & \\
\hline 1241 & s144 & ST & $?$ & 4.5 & \\
\hline 1242 & s6 & ST & $?$ & 4 & \\
\hline 1243 & s114 & ST & $?$ & 4.2 & \\
\hline 1244 & p55 & ST & $?$ & 3.9 & \\
\hline 1245 & m73 & TT & M & 71 & \\
\hline
\end{tabular}

\section{F.P. 7.19 .03}

\begin{tabular}{|l|l|l|l|l|l|}
\hline 1246 & $\mathrm{~m} 72$ & PM & M & 16.5 & \\
\hline 1247 & s21 & PM & M & 18 & \\
\hline 1248 & s132 & TT & F & 81 & \\
\hline 1249 & s72 & TT & M & 60 & \\
\hline
\end{tabular}




\begin{tabular}{|l|l|l|l|l|l|}
\hline 1250 & s82 & TT & F & 89 & \\
\hline 1251 & s49 & TT & M & 56 & \\
\hline 1252 & s143 & PM & F & 13 & \\
\hline 1253 & s32 & ST & $?$ & 4.5 & \\
\hline 1254 & s95 & SV & $?$ & 6.1 & \\
\hline 1255 & p96 & S? & $?$ & $?$ & \\
\hline 1256 & s126 & ST & $?$ & 4.5 & \\
\hline 1257 & p96 & ST & $?$ & 3.1 & \\
\hline 1258 & s139 & ST & $?$ & 0.4 & \\
\hline 1259 & s23 & ST & $?$ & 5.2 & \\
\hline 1260 & s18 & SV & $?$ & 4 & \\
\hline 1261 & p31 & ST & $?$ & 4.5 & \\
\hline 1262 & p84 & ST & $?$ & 3.9 & \\
\hline 1263 & s54 & SV & $?$ & 4 & \\
\hline
\end{tabular}

TRNWR 8.5.03 (1264-1289 SKIPPED)

\begin{tabular}{|l|l|l|l|l|l|}
\hline 1290 & s132 & PM & F & 18 & \\
\hline 1291 & s144 & PM & F & 18 & \\
\hline 1292 & s125 & PM & M & 16 & \\
\hline 1293 & s141 & PM & F & 18 & \\
\hline 1294 & s116 & PM & F & 23 & \\
\hline 1295 & s117 & PM & M & 19 & \\
\hline 1296 & s101 & PM & F & 22.5 & \\
\hline 1297 & s92 & PM & F & 20.5 & \\
\hline 1298 & s77 & PM & M & 18 & \\
\hline 1299 & s83 & PM & M & 13 & \\
\hline 1300 & s72 & PM & $?$ & $?$ & \\
\hline 1301 & s65 & PM & F & 21 & \\
\hline 1302 & s53 & PM & M & 14 & \\
\hline 1303 & m12 & TT & M & 61 & \\
\hline 1304 & p67 & ZT & M & 8 & \\
\hline 1305 & t31 & DV & M & 220 & \\
\hline 1306 & p79 & ST & $?$ & 4.6 & \\
\hline 1307 & p79 & ST & $?$ & 4 & \\
\hline 1308 & p79 & ST & $?$ & $?$ & \\
\hline 1309 & p67 & ST & $?$ & 3.4 & \\
\hline 1310 & s140 & ST & $?$ & 4.3 & \\
\hline 1311 & p139 & ST & $?$ & 4.9 & \\
\hline 1312 & s22 & ST & $?$ & 4 & \\
\hline 1313 & s18 & ST & $?$ & 4 & \\
\hline 1314 & m88 & PM & M & 18.5 & \\
\hline 1315 & p28 & PM & F & 24.5 & \\
\hline 1316 & p12 & PM & F & 22 & \\
\hline
\end{tabular}

TRNWR 8.6.03

\begin{tabular}{|l|l|l|l|l|l|}
\hline 1317 & p84 & PM & F & 23 & \\
\hline 1318 & s56 & PM & M & 16 & \\
\hline 1319 & s78 & PM & M & 7 & \\
\hline 1320 & s78 & PM & F & 6.5 & \\
\hline 1321 & s78 & PM & M & 6.5 & \\
\hline 1322 & p72 & PM & M & 10 & \\
\hline
\end{tabular}




\begin{tabular}{|l|l|l|l|l|l|}
\hline 1323 & $\mathrm{~s} 72$ & $\mathrm{PM}$ & $\mathrm{F}$ & 22 & \\
\hline 1324 & $\mathrm{p} 60$ & $\mathrm{ZT}$ & $\mathrm{M}$ & 8 & \\
\hline 1325 & $\mathrm{~s} 82$ & $\mathrm{TT}$ & $\mathrm{F}$ & 62 & \\
\hline 1326 & $\mathrm{~s} 91$ & $\mathrm{TT}$ & $\mathrm{M}$ & 62 & \\
\hline 1327 & $\mathrm{~s} 65$ & NG & $?$ & 8.7 & \\
\hline 1328 & $\mathrm{~s} 119$ & $\mathrm{ST}$ & $?$ & 4 & \\
\hline 1329 & $\mathrm{p} 79$ & $\mathrm{ST}$ & $?$ & 4.5 & \\
\hline 1330 & $\mathrm{p} 84$ & PM & $?$ & $?$ & \\
\hline
\end{tabular}

TRNWR 8.7.03

\begin{tabular}{|l|l|l|l|l|l|}
\hline 1331 & $\mathrm{p} 84$ & $\mathrm{MO}$ & $\mathrm{M}$ & 21.5 & \\
\hline 1332 & $\mathrm{~s} 82$ & $\mathrm{PM}$ & $\mathrm{F}$ & 30 & \\
\hline 1333 & $\mathrm{~s} 67$ & $\mathrm{PM}$ & $\mathrm{F}$ & 15 & \\
\hline 1334 & $\mathrm{~s} 69$ & $\mathrm{PM}$ & $\mathrm{F}$ & 11.5 & \\
\hline 1335 & $\mathrm{~m} 28$ & $\mathrm{PM}$ & $\mathrm{M}$ & 18 & positive \\
\hline 1336 & $\mathrm{~s} 10$ & $\mathrm{PM}$ & $\mathrm{M}$ & 17.5 & \\
\hline 1337 & $\mathrm{~s} 35$ & $\mathrm{PM}$ & $\mathrm{M}$ & 5.5 & \\
\hline 1338 & $\mathrm{~s} 101$ & $\mathrm{PM}$ & $\mathrm{M}$ & 9.5 & \\
\hline 1339 & $\mathrm{~s} 141$ & $\mathrm{PM}$ & $\mathrm{M}$ & 15.5 & \\
\hline 1340 & $\mathrm{p} 79$ & $\mathrm{ST}$ & $?$ & 4.6 & \\
\hline 1341 & $\mathrm{p} 79$ & $\mathrm{ST}$ & $?$ & 4.8 & \\
\hline 1342 & $\mathrm{~m} 24$ & $\mathrm{TT}$ & $\mathrm{M}$ & 62 & \\
\hline 1343 & $\mathrm{~s} 106$ & $\mathrm{NG}$ & $?$ & 8.5 & \\
\hline 1344 & $\mathrm{~s} 116$ & $\mathrm{ST}$ & $?$ & 4.2 & \\
\hline 1345 & $\mathrm{~s} 142$ & $\mathrm{ST}$ & $?$ & 4.5 & \\
\hline 1346 & $\mathrm{~s} 80$ & NG & $?$ & 7.9 & \\
\hline
\end{tabular}

TRNWR 8.8.03

\begin{tabular}{|l|l|l|l|l|l|}
\hline 1347 & $\mathrm{~m} 88$ & PM & M & 13 & \\
\hline 1348 & $\mathrm{~s} 129$ & PM & M & 13.5 & \\
\hline 1349 & $\mathrm{~s} 89$ & PM & M & 17 & \\
\hline 1350 & $\mathrm{~s} 44$ & PM & F & 15.5 & \\
\hline 1351 & $\mathrm{~s} 69$ & PM & M & 16 & positive \\
\hline 1352 & $\mathrm{~s} 128$ & PM & F & $?$ & \\
\hline 1353 & s49 & DV & M & $?$ & \\
\hline 1354 & $\mathrm{p} 88$ & MO & M & 36 & \\
\hline 1355 & $\mathrm{~m} 123$ & MO & M & 28 & \\
\hline 1356 & $\mathrm{p} 36$ & MO & F & 48 & \\
\hline 1357 & $\mathrm{~m} 39$ & ST & $?$ & 5. & \\
\hline 1358 & $\mathrm{~s} 47$ & ST & $?$ & 4 & \\
\hline 1359 & $\mathrm{~s} 58$ & ST & $?$ & 3.9 & \\
\hline 1360 & $\mathrm{~s} 11$ & ST & $?$ & 5.2 & \\
\hline 1361 & $\mathrm{~s} 132$ & ST & $?$ & 3.9 & \\
\hline 1362 & p103 & ST & $?$ & 5. & \\
\hline 1363 & p115 & ST & $?$ & 4.8 & \\
\hline 1364 & p120 & ST & $?$ & 6 & \\
\hline 1365 & p120 & PM & M & 21.5 & \\
\hline 1366 & p88 & PM & F & 11 & \\
\hline 1367 & p100 & ST & $?$ & 6 & \\
\hline
\end{tabular}

POWELL BUTTE 8.19.03

\begin{tabular}{|l|c|c|c|c|}
\hline 1368 & P84 & PM & M & 14.5 \\
\hline \multicolumn{4}{|c}{165} \\
\hline
\end{tabular}




\begin{tabular}{|l|l|l|l|l|l|}
\hline 1369 & S78 & PM & M & 14 & \\
\hline 1370 & P144 & PM & M & 19 & \\
\hline 1371 & S118 & PM & M & 13.5 & \\
\hline 1372 & S98 & SV & $?$ & 3 & \\
\hline 1373 & P72 & ST & $?$ & 4 & \\
\hline
\end{tabular}

POWELL BUTTE 8.20.03

\begin{tabular}{|l|l|l|l|l|l|}
\hline 1374 & S15 & PM & F & 12 & \\
\hline 1375 & T96 & DV & $?$ & $?$ & \\
\hline
\end{tabular}

P.B. 8.21.03
\begin{tabular}{|l|l|l|l|l|l|}
\hline 1376 & S15 & PM & M & 14 & \\
\hline 1377 & S134 & PM & M & 12 & \\
\hline 1378 & S 96 & PM & M & 10.5 & \\
\hline 1379 & P144 & PM & F & 15 & \\
\hline 1380 & P144 & PM & F & 12 & \\
\hline 1381 & S115 & SV & $?$ & 4.7 & \\
\hline 1382 & S126 & SV & $?$ & 3 & \\
\hline
\end{tabular}

\section{P.B. 8.22.03}

\begin{tabular}{|l|l|l|l|l|l|}
\hline 1383 & s144 & PM & M & 16 & \\
\hline 1384 & s139 & PM & M & 12 & \\
\hline 1385 & s127 & PM & F & 15 & \\
\hline 1386 & s83 & MO & F & 15.5 & \\
\hline 1387 & s134 & MO & F & 17.5 & \\
\hline 1388 & s75 & GS & F & 130 & \\
\hline 1389 & s39 & ST & $?$ & 3.2 & \\
\hline
\end{tabular}

TILLY JANE 9.2 .03

\begin{tabular}{|l|l|l|l|l|l|}
\hline 1390 & $\mathrm{~s} 90$ & $\mathrm{PM}$ & $\mathrm{M}$ & 18 & \\
\hline 1391 & $\mathrm{p} 28$ & $\mathrm{MO} ?$ & $\mathrm{~F}$ & 23.5 & \\
\hline
\end{tabular}

TILLY JANE 9.3, 9.4.03 - NA

TJ 9.5.03

\begin{tabular}{|l|l|l|l|l|l|}
\hline 1392 & m36 & PM & M & 10.3 & \\
\hline 1393 & s7 & PM & F & 19.2 & \\
\hline 1394 & s141 & PM & M & 10.0 & \\
\hline 1395 & p139 & PM & F & 13.2 & \\
\hline 1396 & t60 & TA & M & 70 & \\
\hline 1397 & s81 & GS & F & 154 & \\
\hline
\end{tabular}

OXBOW 9.9 .03

\begin{tabular}{|l|l|l|l|l|l|}
\hline 1398 & p12 & PM & M & 6 & \\
\hline 1399 & s12 & PM & F & 9 & \\
\hline 1400 & s115 & PM & M & 11 & \\
\hline 1401 & s28 & PM & F & 6 & \\
\hline 1402 & m108 & PM & F & 10 & \\
\hline 1403 & s93 & PM & F & 7 & \\
\hline 1404 & s71 & PM & F & 10.5 & \\
\hline 1405 & s69 & PM & M & 13 & \\
\hline 1406 & s63 & PM & M & 9 & \\
\hline 1407 & m80 & TT & M & 79 & \\
\hline 1408 & s132 & TT & M & 72 & \\
\hline 1409 & s144 & TT & F & 62 & \\
\hline 1410 & p7 & SV & $?$ & 8 & \\
\hline
\end{tabular}




\begin{tabular}{|l|l|l|l|l|l|}
\hline 1411 & $\mathrm{p} 132$ & ST & $?$ & 4.5 & \\
\hline 1412 & $\mathrm{p} 100$ & SV & $?$ & 5.4 & \\
\hline 1413 & $\mathrm{p} 103$ & ST & $?$ & 4.9 & \\
\hline 1414 & $\mathrm{p} 120$ & PM & F & 6.4 & \\
\hline 1415 & $\mathrm{p} 124$ & ST & $?$ & 5.2 & \\
\hline 1416 & $\mathrm{p} 67$ & ST & $?$ & 3.9 & \\
\hline 1417 & $\mathrm{p} 64$ & ST & $?$ & 3.9 & \\
\hline 1418 & $\mathrm{p} 28$ & NG & $?$ & 6.9 & \\
\hline 1419 & $\mathrm{p} 76$ & SV & $?$ & 7.2 & \\
\hline 1420 & $\mathrm{p} 76$ & SV & $?$ & 5.8 & \\
\hline 1421 & $\mathrm{~m} 33$ & GS & M & 141 & \\
\hline 1422 & $\mathrm{~m} 11$ & TT & M & 71 & \\
\hline 1423 & $\mathrm{~s} 91$ & & & & escaped \\
\hline 1424 & $\mathrm{~s} 127$ & & & & escaped \\
\hline
\end{tabular}

OX 9.10 .03
\begin{tabular}{|l|l|l|l|l|l|}
\hline 1425 & s 94 & PM & M & 8.5 & \\
\hline 1426 & s 84 & PM & M & 6 & \\
\hline 1427 & p108 & PM & F & 9.5 & \\
\hline 1428 & s137 & PM & M & 9 & \\
\hline 1429 & s 38 & TT & M & 81 & \\
\hline 1430 & s 58 & TT & F & 73 & \\
\hline 1431 & s 68 & TT & & & \\
\hline 1432 & s130 & MO & F & 23 & \\
\hline 1433 & p 132 & MO & F & 9.5 & \\
\hline 1434 & s126 & NG & $?$ & 8 & \\
\hline 1435 & p 40 & MO & F & 20.5 & \\
\hline 1436 & s11 & NG & $?$ & 7.5 & \\
\hline 1437 & s 23 & NG & $?$ & 9.5 & \\
\hline 1438 & p 144 & ST & $?$ & 7.5 & \\
\hline 1439 & p 144 & ST & $?$ & 6.5 & \\
\hline OX9.1 & & & & \\
\hline
\end{tabular}

OX 9.11.03

\begin{tabular}{|l|l|l|l|l|l|}
\hline 1440 & $\mathrm{~s} 130$ & MO & F & 10 & \\
\hline 1441 & p 84 & PM & F & 6.5 & \\
\hline 1442 & s84 & PM & F & 13.5 & \\
\hline 1443 & s12 & PM & M & 17.5 & \\
\hline 1444 & s100 & TT & M & 72 & \\
\hline 1445 & s86 & TT & F & 42 & \\
\hline 1446 & s114 & TT & F & 40 & \\
\hline 1447 & s79 & NG & $?$ & 10 & \\
\hline 1448 & s67 & NG & $?$ & 9 & \\
\hline 1449 & p64 & NG & $?$ & 9.5 & \\
\hline 1450 & p 60 & SV & $?$ & 5.6 & \\
\hline 1451 & p67 & ST & $?$ & 4.0 & \\
\hline 1452 & p67 & NG & $?$ & 6.5 & \\
\hline 1453 & s23 & NG & $?$ & 8.2 & \\
\hline 1454 & p 79 & ST & $?$ & 6.7 & \\
\hline 1455 & s 47 & NG & $?$ & 7.6 & \\
\hline 1456 & s118 & NG & $?$ & 9.6 & \\
\hline 1457 & m119 & TT & F & 59 & \\
\hline
\end{tabular}




\begin{tabular}{|l|l|l|l|l|l|}
\hline 1458 & $\mathrm{~m} 91$ & TT & F & 67 & \\
\hline 1459 & m50 & TT & M & 59 & \\
OX 9.12.03 & s106 & TT & M & 79 & \\
\hline 1460 & s80 & TT & F & 78 & \\
\hline 1461 & s24 & MO & F & 19.5 & \\
\hline 1462 & s30 & MO & F & 20.5 & \\
\hline 1463 & p120 & PM & F & 5.5 & \\
\hline 1464 & & & M & & \\
\hline & s72 & NG & $?$ & 7.5 & \\
1465 & s10 & SV & $?$ & 4.3 & \\
\hline 1466 & s118 & NG & $?$ & 8.6 & \\
\hline 1467 & s83 & NG & $?$ & 7.4 & \\
\hline 1468 & s 96 & TT & M & 58 & \\
\hline 1469 & m130 & TT & M & 64 & \\
\hline 1470 & & & & \\
\hline
\end{tabular}

TRYON CREEK 9.16.03

\begin{tabular}{|l|l|l|l|l|l|}
\hline 1471 & s129 & PM & F & 20 & \\
\hline 1472 & s68 & PM & F & $?$ & \\
\hline 1473 & s58 & PM & M & 26 & \\
\hline 1474 & s59 & PM & F & 24.5 & \\
\hline 1475 & s70 & PM & M & 14.5 & \\
\hline 1476 & s89 & PM & M & 18.5 & \\
\hline 1477 & s106 & PM & F & 18 & \\
\hline 1478 & s118 & PM & F & 13.5 & \\
\hline 1479 & s140 & PM & M & 15 & \\
\hline 1480 & s125 & PM & M & 21.5 & \\
\hline 1481 & m120 & PM & M & 16 & \\
\hline 1482 & p28 & ST & $?$ & 5.0 & \\
\hline 1483 & s11 & ST & $?$ & 5.4 & \\
\hline 1484 & s102 & ST & $?$ & 4.4 & \\
\hline 1485 & m117 & PM & M & 17 & \\
\hline 1486 & p79 & ST & $?$ & 5.2 & \\
\hline 1487 & p144 & ST & $?$ & 4.5 & \\
\hline 1488 & s142 & SV & $?$ & 5.2 & \\
\hline
\end{tabular}

TC 9.17 .03

\begin{tabular}{|l|l|l|l|l|l|}
\hline 1489 & s11 & PM & F & 15.5 & \\
\hline 1490 & s130 & PM & M & 14.5 & \\
\hline 1491 & p144 & PM & M & 16.5 & \\
\hline 1492 & m128 & PM & M & 14.5 & \\
\hline 1493 & s140 & PM & M & 16 & \\
\hline 1494 & m47 & PM & M & 18 & \\
\hline 1495 & s26 & PM & F & 17 & \\
\hline 1496 & s106 & PM & M & 14 & \\
\hline 1497 & s107 & PM & F & 17 & \\
\hline 1498 & s96 & PM & F & 26 & \\
\hline 1499 & s70 & PM & F & 19 & \\
\hline 1500 & p19 & ST & $?$ & 4.9 & \\
\hline 1501 & p4 & ST & $?$ & 4.5 & \\
\hline 1502 & s93 & ST & $?$ & 4.2 & \\
\hline
\end{tabular}




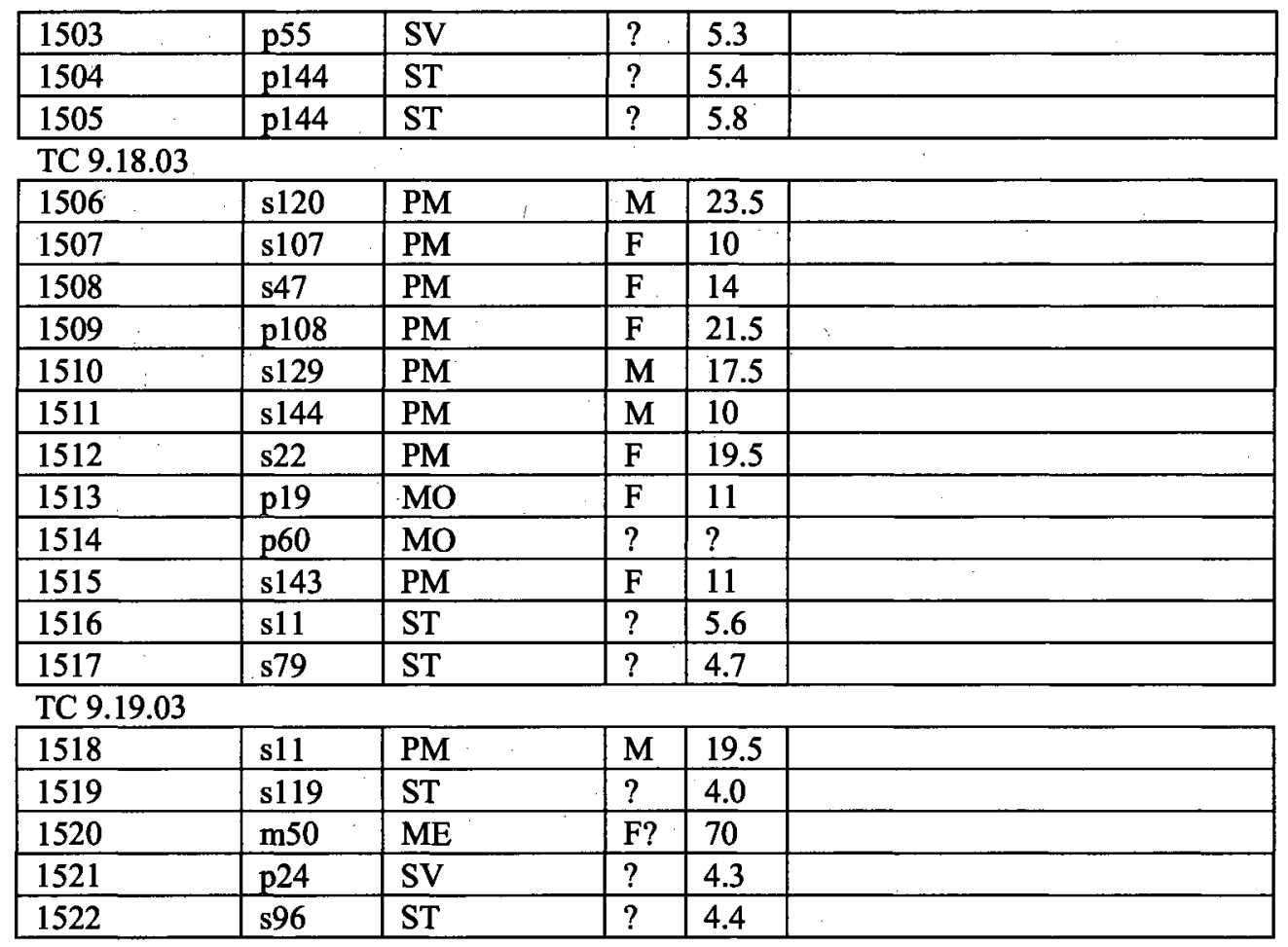

\section{FOREST PARK 9.23.03}

\begin{tabular}{|l|l|l|l|l|l|}
\hline 1523 & p144 & PM & M & 16 & positive \\
\hline 1524 & s142 & PM & M & 17.5 & positive \\
\hline 1525 & s143 & PM & M & 15 & \\
\hline 1526 & p126 & PM & M & 15.5 & \\
\hline 1527 & s112 & PM & F & 16.5 & \\
\hline 1528 & p24 & PM & F & 15.5 & \\
\hline 1529 & p55 & PM & F & 15 & \\
\hline 1530 & m78 & PM & F & 22.5 & \\
\hline
\end{tabular}

\section{FP 9.24.03}

\begin{tabular}{|l|l|l|l|l|l|}
\hline 1531 & s127 & PM & F & 22 & \\
\hline 1532 & m105 & PM & M & 15 & \\
\hline 1533 & m7 & PM & M & 16 & \\
\hline 1534 & s35 & PM & M & 14.5 & \\
\hline 1535 & m60 & PM & F & 18 & \\
\hline 1536 & t43 & TD & M & $?$ & \\
\hline 1537 & m84 & TT & M & 66 & \\
\hline 1538 & t96 & TD & F & 220 & \\
\hline 1539 & p48 & ST & $?$ & 4.2 & \\
\hline 1540 & m22 & ME & F & 34 & \\
\hline 1541 & s43 & SV & $?$ & $?$ & \\
\hline
\end{tabular}

FP 9.25.03

\begin{tabular}{|l|l|l|l|l|l|}
\hline 1542 & $\mathrm{~s} 35$ & PM & M & 15 & \\
\hline 1543 & s118 & PM & M & 16.5 & \\
\hline 1544 & s135 & MO & F & 16 & \\
\hline 1545 & t43 & TD & F & $?$ & \\
\hline
\end{tabular}




\begin{tabular}{|l|l|l|l|l|l|}
\hline 1546 & s96 & PM & M & 17.5 & \\
\hline 1547 & m95 & PM & F & 13.5 & \\
\hline 1548 & s144 & PM & F & 14 & \\
\hline 1549 & s3 & MO & M & 6.5 & \\
\hline 1550 & s143 & PM & M & 16.5 & positive \\
\hline 1551 & s106 & PM & F & 11.5 & \\
\hline 1552 & s115 & PM & M & 13 & \\
\hline 1553 & s107 & PM & M & 18.5 & \\
\hline 1554 & s138 & ST & $?$ & 5.0 & \\
\hline 1555 & s11 & PM & F & 16 & \\
\hline 1556 & s82 & ST & $?$ & 4.4 & \\
\hline 1557 & s5 & MO & M & 5.6 & \\
\hline 1558 & p136 & SV & $?$ & 3.1 & \\
\hline 1559 & s133 & PM & M & 15 & \\
\hline
\end{tabular}

TRNWR 9.30.03

\begin{tabular}{|l|l|l|l|l|l|}
\hline 1560 & s10 & PM & M & 15 & \\
\hline 1561 & s127 & PM & F & 15.5 & \\
\hline 1562 & s131 & PM & M & 11.5 & \\
\hline 1563 & s130 & PM & M & 14.5 & \\
\hline 1564 & s128 & PM & M & 15 & \\
\hline 1565 & p76 & PM & F & 16.5 & \\
\hline 1566 & s82 & PM & F & 19 & \\
\hline 1567 & s92 & PM & M & 19 & \\
\hline 1568 & s45 & PM & M & 16.5 & \\
\hline 1569 & s31 & PM & M & 18.5 & \\
\hline 1570 & s106 & PM & M & 13.5 & \\
\hline 1571 & s23 & PM & F & 17.5 & \\
\hline 1572 & p28 & PM & F & 15 & \\
\hline 1573 & s117 & PM & M & 15.5 & \\
\hline 1574 & s101 & PM & F & 18.5 & \\
\hline 1575 & s118 & PM & F & 21.5 & \\
\hline 1576 & s51 & PM & F & 19 & \\
\hline 1577 & s9 & PM & F & 15.5 & \\
\hline 1578 & p91 & PM & M & 9 & \\
\hline 1579 & s80 & SV & $?$ & 3.5 & \\
\hline 1580 & p144 & ST & $?$ & 4.6 & \\
\hline 1581 & s140 & NG & F & 8.5 & \\
\hline 1582 & s129 & ST & $?$ & 4.5 & \\
\hline 1583 & t120 & MM & $?$ & $?$ & \\
\hline
\end{tabular}

TRNWR 10.1.03

\begin{tabular}{|l|l|l|l|l|l|}
\hline 1584 & s117 & PM & M & 14 & \\
\hline 1585 & s46 & PM & F & 20.5 & \\
\hline 1586 & p76 & PM & F & 6 & \\
\hline 1587 & s113 & PM & M & 17 & \\
\hline 1588 & s48 & PM & M & 15 & \\
\hline 1589 & s131 & PM & F & 13 & \\
\hline 1590 & s45 & PM & F & 19 & \\
\hline 1591 & s35 & PM & F & 15 & \\
\hline 1592 & s68 & PM & M & 19.5 & \\
\hline
\end{tabular}




\begin{tabular}{|c|c|c|c|c|c|}
\hline 1593 & s70 & $\mathrm{PM}$ & $\mathrm{F}$ & 18 & \\
\hline 1594 & $\mathrm{~m} 69$ & PM & $\mathrm{F}$ & 18 & \\
\hline 1595 & $\mathrm{~m} 70$ & PM & M & 20 & \\
\hline 1596 & s105 & ST & $?$ & 4.7 & \\
\hline 1597 & s27 & ST & $?$ & 4.0 & \\
\hline 1598 & s142 & NG & $\begin{array}{l}\mathrm{M} \\
?\end{array}$ & 7.5 & \\
\hline 1599 & s11 & SV & $?$ & 4.6 & \\
\hline 1600 & s32 & $\overline{\mathrm{PM}}$ & F & 13.5 & \\
\hline 1601 & $p^{7}$ & PM & M & 21 & \\
\hline 1602 & p7 & PM & $\mathrm{F}$ & 17.5 & \\
\hline 1603 & p7 & $\overline{\mathrm{PM}}$ & $\mathrm{F}$ & 21.5 & \\
\hline \multicolumn{6}{|c|}{ TRNWR 10.2.03 } \\
\hline 1604 & $\mathrm{~s} 80$ & PM & $\mathbf{F}$ & 18.5 & \\
\hline 1605 & $\mathrm{~s} 21$ & PM & $\mathrm{M}$ & 16.5 & \\
\hline 1606 & s45 & PM & M & 16.5 & \\
\hline 1607 & $\mathrm{~s} 32$ & PM & $\bar{M}$ & 13 & \\
\hline 1608 & s113 & PM & $\mathbf{F}$ & 15 & \\
\hline 1609 & $\mathrm{~m} 23$ & $\overline{\mathrm{PM}}$ & $\mathrm{M}$ & 13.5 & \\
\hline 1610 & $\mathrm{~s} 120$ & $\overline{\mathrm{PM}}$ & $\mathbf{M}$ & 17 & \\
\hline 1611 & $\mathrm{~s} 12$ & PM & $\mathrm{F}$ & 16 & \\
\hline 1612 & $\mathrm{~m} 42$ & PM & $F$ & 17 & \\
\hline 1613 & $\mathrm{p} 72$ & $\overline{\mathrm{PM}}$ & $\mathrm{M}$ & 15.5 & \\
\hline 1614 & p127 & PM & $\bar{F}$ & 14 & \\
\hline 1615 & $\mathrm{~s} 25$ & PM & $\mathrm{M}$ & 14.5 & \\
\hline 1616 & $\mathrm{p} 48$ & PM & $M$ & 13 & \\
\hline 1617 & s74 & $\overline{\mathrm{PM}}$ & $\mathrm{M}$ & 13 & \\
\hline 1618 & s141 & PM & $\bar{M}$ & 11 & \\
\hline 1619 & s144 & PM & $F$ & 6.5 & \\
\hline 1620 & $\mathrm{p} 40$ & PM & $\mathrm{F}$ & 5 & \\
\hline 1621 & $\mathrm{p} 40$ & PM & $\mathrm{M}$ & 5 & \\
\hline 1622 & s96 & SV & $?$ & 4.0 & \\
\hline 1623 & s11 & NG & $\begin{array}{l}\mathrm{M} \\
?\end{array}$ & 9 & \\
\hline 1624 & $\mathrm{p} 43$ & ST & $?$ & 5 & \\
\hline 1625 & $\mathrm{s35}$ & ST & $?$ & 4.6 & \\
\hline 1626 & $\mathrm{~s} 84$ & ST & $?$ & 3.8 & \\
\hline 1627 & $t 72$ & DV & $?$ & $?$ & \\
\hline \multicolumn{5}{|c|}{ TRNWR 10.3 .03} & \\
\hline 1628 & p107 & PM & $\mathrm{F}$ & 11.5 & \\
\hline 1629 & $\mathrm{p} 102$ & PM & $F$ & 19 & \\
\hline 1630 & $\mathrm{~m} 30$ & PM & $\mathbf{M}$ & 12 & \\
\hline 1631 & s141 & PM & $\mathrm{F}$ & 11 & \\
\hline 1632 & s16 & PM & $\mathbf{M}$ & 6 & \\
\hline 1633 & s101 & PM & $\bar{M}$ & 16.5 & \\
\hline 1634 & s103 & PM & $\mathrm{F}$ & 9 & \\
\hline 1635 & s96 & PM & $\mathbf{M}$ & 19 & \\
\hline 1636 & $\mathrm{~s} 33$ & PM & $\mathrm{F}$ & 12 & \\
\hline 1637 & s84 & PM & M & 19.5 & positive \\
\hline 1638 & s116 & PM & $F$ & 18.5 & positive \\
\hline
\end{tabular}




\begin{tabular}{|l|l|l|l|l|l|}
\hline 1639 & s120 & PM & F & 14 & \\
\hline 1640 & s113 & PM & & & \\
\hline 1641 & s115 & PM & F & 16 & \\
\hline 1642 & s87 & PM & F & 7 & \\
\hline 1643 & s35 & ST & & 4.5 & \\
\hline 1644 & s31 & PM & M & 12.5 & \\
\hline 1645 & t7 & TT & M & 62 & \\
\hline
\end{tabular}

Powell Butte 10.14.03

\begin{tabular}{|l|l|l|l|l|l|}
\hline 1646 & s94 & PM & M & 17 & \\
\hline 1647 & m116 & PM & F & 19 & \\
\hline 1648 & s78 & PM & F & 20 & \\
\hline 1649 & s69 & PM & M & 15.5 & \\
\hline 1650 & s8 & PM & F & 23 & \\
\hline 1651 & s106 & MO & F & 15.5 & \\
\hline 1652 & s56 & MO & F & 17 & \\
\hline 1653 & s3 & MO & F & 21 & \\
\hline 1654 & s111 & MO & M & 17 & \\
\hline 1655 & s45 & SV & $?$ & 4.0 & \\
\hline 1656 & p40 & SV & $?$ & 4.2 & \\
\hline 1657 & p120 & ST & $?$ & 5.6 & \\
\hline 1658 & s126 & SV & $?$ & 4.0 & \\
\hline
\end{tabular}

P.B. 10.15.03

\begin{tabular}{|l|l|l|l|l|l|}
\hline 1659 & s129 & PM & F & 21.5 & \\
\hline 1660 & s84 & PM & F & 13.5 & \\
\hline 1661 & s23 & PM & F & 20.5 & \\
\hline 1662 & s104 & MO & F & 13 & \\
\hline 1663 & s2 & MO & M & 18.5 & \\
\hline 1664 & s119 & SV & $?$ & 4.3 & \\
\hline 1665 & s21 & PM & $?$ & $?$ & \\
\hline 1666 & s18 & S? & $?$ & $?$ & \\
\hline
\end{tabular}

P.B. 10.16.03
\begin{tabular}{|l|l|l|l|l|l|}
\hline 1667 & s41 & PM & F & 20 & \\
\hline 1668 & p 28 & PM & F & 12 & \\
\hline 1669 & s28 & PM & M & 18 & positive \\
\hline 1670 & s47 & PM & M & 17 & \\
\hline 1671 & s110 & PM & M & 19 & \\
\hline 1672 & s18 & PM & F & 9 & \\
\hline 1673 & s20 & PM & F & 18 & \\
\hline 1674 & s4 & PM & F & 8 & \\
\hline 1675 & s78 & PM & M & 18 & \\
\hline 1676 & s137 & PM & M & 20 & \\
\hline 1677 & m141 & PM & F & 26 & \\
\hline 1678 & s84 & MO & F & 17 & \\
\hline 1679 & s112 & MO & M & 11 & \\
\hline 1680 & s56 & MO & M & 13 & \\
\hline 1681 & s55 & MO & M & 13 & \\
\hline 1682 & s134 & MO & M & 13 & \\
\hline 1683 & s126 & MO & M & 17.5 & \\
\hline 1684 & s106 & MO & M & 16 & \\
\hline
\end{tabular}




\begin{tabular}{|l|l|l|l|l|l|}
\hline 1685 & s144 & PM & F & 17 & \\
\hline 1686 & s3 & MO & F & 19 & \\
\hline 1687 & s104 & MO & M & 15 & \\
\hline 1688 & p28 & PM & M & 21 & \\
\hline 1689 & p124 & PM & M & 17 & \\
\hline 1690 & t144 & DV & $?$ & $?$ & \\
\hline
\end{tabular}

P.B. 10.17 .03

\begin{tabular}{|l|l|l|l|l|l|}
\hline 1691 & s47 & PM & F & $?$ & \\
\hline 1692 & s44 & PM & M & 14.5 & \\
\hline 1693 & s70 & PM & M & 17 & \\
\hline 1694 & s23 & PM & M & 17 & \\
\hline 1695 & s115 & GS & M & 85 & positive \\
\hline 1696 & p3 & PM & M & 11.5 & \\
\hline 1697 & s7 & PM & M & 22 & \\
\hline 1698 & s118 & PM & M & 16.5 & positive \\
\hline 1699 & p88 & MO & M & 16 & \\
\hline 1700 & p91 & MO & M & 13 & \\
\hline 1701 & p108 & MO & M & 13 & \\
\hline 1702 & s105 & MO & M & 18 & \\
\hline 1703 & s122 & MO & M & 14 & \\
\hline 1704 & s124 & MO & M & 14 & \\
\hline 1705 & s79 & MO & M & 13 & \\
\hline 1706 & s85 & MO & M & 14 & \\
\hline 1707 & s76 & MO & M & 11 & \\
\hline 1708 & p7 & PM & M & 7 & \\
\hline 1709 & s56 & MO & M & 13 & \\
\hline 1710 & s4 & SV & $?$ & 4.5 & \\
\hline
\end{tabular}

OXBOW 10.21.03

\begin{tabular}{|l|l|l|l|l|l|}
\hline 1711 & $\mathrm{~s} 65$ & $\mathrm{PM}$ & $\mathrm{M}$ & 13.5 & \\
\hline 1712 & $\mathrm{~s} 24$ & $\mathrm{PM}$ & $\mathrm{F}$ & 18.5 & \\
\hline 1713 & $\mathrm{p} 36$ & $\mathrm{PM}$ & $\mathrm{M}$ & 13.5 & \\
\hline 1714 & $\mathrm{~s} 7$ & $\mathrm{PM}$ & $\mathrm{M}$ & 15.5 & positive \\
\hline 1715 & $\mathrm{~s} 88$ & $\mathrm{PM}$ & $\mathrm{M}$ & 15 & positive \\
\hline 1716 & $\mathrm{~m} 24$ & $\mathrm{TT}$ & $\mathrm{F}$ & 71 & \\
\hline 1717 & $\mathrm{~m} 75$ & $\mathrm{TT}$ & $\mathrm{M}$ & 70 & \\
\hline 1718 & $\mathrm{~s} 129$ & $\mathrm{TT}$ & $\mathrm{M}$ & 79 & \\
\hline 1719 & $\mathrm{~s} 118$ & $\mathrm{TT}$ & $\mathrm{M}$ & 69 & \\
\hline 1720 & $\mathrm{~s} 4$ & $\mathrm{ST}$ & $?$ & 5.0 & \\
\hline 1721 & $\mathrm{~s} 19$ & $\mathrm{ST}$ & $?$ & 3.8 & \\
\hline
\end{tabular}

OX 10.22.03
\begin{tabular}{|l|l|l|l|l|l|}
\hline 1722 & s59 & PM & F & 16.5 & \\
\hline 1723 & s24 & PM & M & 13 & \\
\hline 1724 & m126 & PM & F & 20 & positive \\
\hline 1725 & s44 & PM & M & 18 & \\
\hline 1726 & s22 & TT & M & 74 & \\
\hline 1727 & s80 & TT & M & 71 & \\
\hline 1728 & s107 & TT & M & 71 & \\
\hline 1729 & s8 & MO & F & 13 & \\
\hline 1730 & s130 & MO & M & 20 & \\
\hline
\end{tabular}




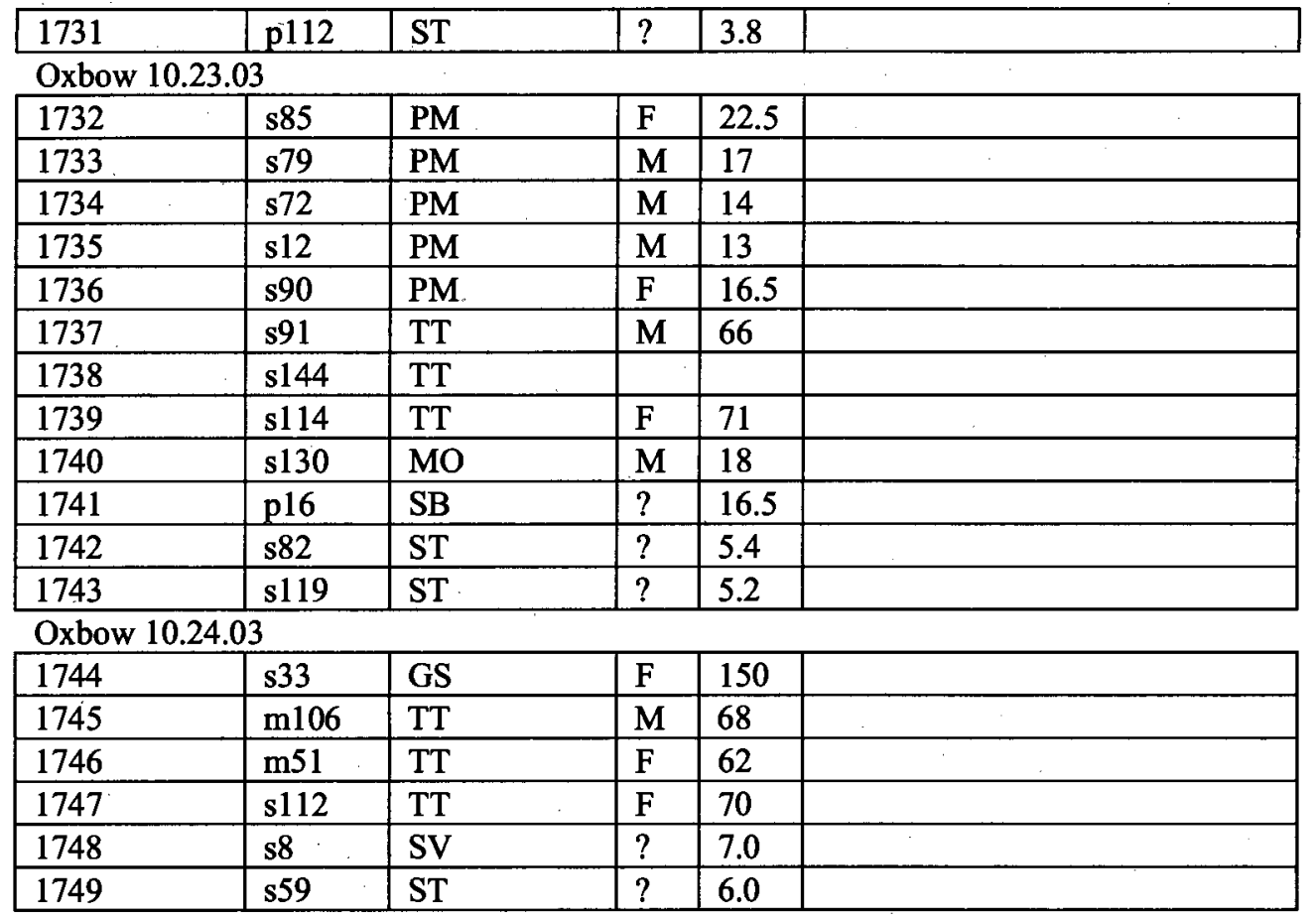

TRYON CREEK 10.28.03

\begin{tabular}{|l|l|l|l|l|l|}
\hline 1750 & s30 & PM & F & 20 & \\
\hline 1751 & s6 & PM & M & 21 & \\
\hline T.C. 10.29 .03 & & & & & \\
\hline 1752 & s60 & PM & F & 18.5 & \\
\hline 1753 & s116 & PM & F & 18 & \\
\hline 1754 & s102 & MO & M & 12.5 & \\
\hline 1755 & s23 & ST & $?$ & 5.2 & \\
\hline 1756 & p14 & ST & $?$ & 6.0 & \\
\hline
\end{tabular}

T.C. 10.30 .03
\begin{tabular}{|l|l|l|l|l|l|}
\hline 1757 & s91 & PM & F & 20 & \\
\hline 1758 & s47 & PM & M & 14 & \\
\hline 1759 & s 99 & PM & F & 16.5 & \\
\hline 1760 & s41 & PM & F & 8.4 & \\
\hline 1761 & s57 & PM & F & 18 & \\
\hline 1762 & s19 & PM & M & 17 & \\
\hline 1763 & s4 & MO & F & 16 & \\
\hline 1764 & s83 & SV & ? & 6 & \\
\hline 1765 & m90 & PM & M & 17 & \\
\hline 1766 & s84 & PM & F & 18 & \\
\hline 1767 & s118 & PM & F & 17 & \\
\hline 1768 & s60 & PM & M & 17.5 & \\
\hline 1769 & s49 & PM & F & 19 & \\
\hline 1770 & s23 & SV & ? & 7 & \\
\hline
\end{tabular}

T.C. 10.31 .03

\begin{tabular}{|l|l|l|l|l|l|}
\hline 1771 & s92 & PM & M & 18.5 & \\
\hline 1772 & s40 & PM & F & 20 & \\
\hline 1773 & s118 & PM & M & 10 & \\
\hline
\end{tabular}




\begin{tabular}{|l|l|l|l|l|l|}
\hline 1774 & p4 & MO & M & 16 & \\
\hline 1775 & s120 & PM & M & 16.5 & \\
\hline 1776 & s75 & ST & $?$ & 5.5 & \\
\hline
\end{tabular}

FOREST PARK 11.4 .03
\begin{tabular}{|l|l|l|l|l|l|}
\hline 1777 & s 71 & PM & M & 20.5 & \\
\hline 1778 & p 24 & ST & $?$ & 3.8 & \\
\hline 1779 & s 119 & ST & $?$ & 4.3 & \\
\hline F.P. 11.5 .03 & s 82 & PM & M & 19 & \\
\hline 1780 & s40 & PM & F & 17.5 & \\
\hline 1781 & s130 & PM & F & 19 & \\
\hline 1782 & m55 & PM & F & 24 & positive \\
\hline 1783 & s 96 & PM & M & 20 & \\
\hline 1784 & s117 & PM & F & 16 & \\
\hline 1785 & s 55 & ST & $?$ & 3.7 & \\
\hline 1786 & p 60 & SV & $?$ & 5.0 & \\
\hline 1787 & p24 & SV & $?$ & 4.6 & \\
\hline 1788 & s22 & ST & $?$ & 4.0 & \\
\hline 1789 & & & & \\
\hline
\end{tabular}

F.P. 11.6.03
\begin{tabular}{|l|l|l|l|l|l|}
\hline 1790 & s119 & PM & M & 19.5 & \\
\hline 1791 & m71 & PM & M & 19 & \\
\hline 1792 & s112 & PM & M & 16 & \\
\hline 1793 & s104 & SV & $?$ & 5.0 & \\
\hline 1794 & s 90 & SV & $?$ & $?$ & \\
\hline
\end{tabular}

F.P. 11.7 .03
\begin{tabular}{|l|l|l|l|l|l|}
\hline 1795 & p 96 & PM & F & 22.5 & \\
\hline 1796 & p 40 & PM & M & 17 & \\
\hline 1797 & m 143 & PM & M & 26 & positive \\
\hline 1798 & s106 & PM & M & 15.5 & \\
\hline 1799 & s112 & PM & M & 21.5 & \\
\hline 1800 & s18 & PM & M & 19.5 & \\
\hline 1801 & p 36 & ST & $?$ & 3.8 & \\
\hline 1802 & s12 & PM & M & 15 & \\
\hline 1803 & s4 & ST & $?$ & 3.9 & \\
\hline 1804 & s140 & ST & $?$ & 4.5 & \\
\hline 1805 & $?$ & ST & $?$ & 4 & \\
\hline
\end{tabular}

TUALATIN RIVER NWR 11.11 .03

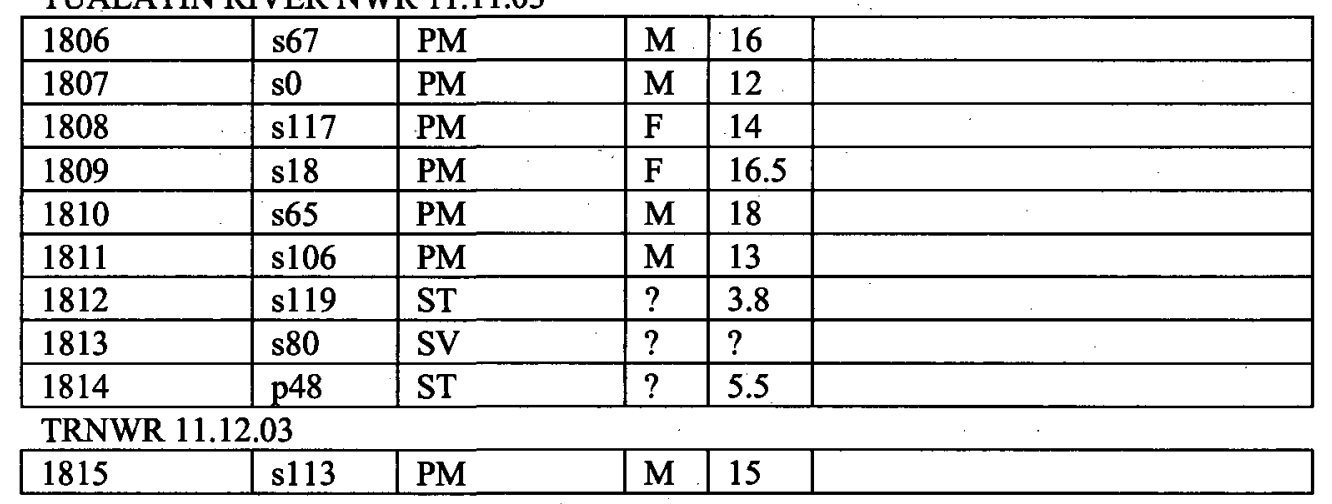




\begin{tabular}{|l|l|l|l|l|l|}
\hline 1816 & s102 & PM & M & 21 & \\
\hline 1817 & s67 & PM & F & 12.5 & \\
\hline 1818 & s66 & PM & M & 15 & \\
\hline 1819 & s99 & PM & M & 13 & \\
\hline 1820 & s18 & PM & M & 14 & \\
\hline 1821 & s60 & ST & $?$ & 4.2 & \\
\hline 1822 & t31 & DV & $?$ & $?$ & \\
\hline
\end{tabular}

TRNWR 11.13.03

\begin{tabular}{|l|l|l|l|l|l|}
\hline 1823 & s32 & PM & M & 15 & \\
\hline 1824 & s83 & PM & M & 18 & \\
\hline 1825 & s23 & PM & M & 15 & \\
\hline 1826 & s102 & PM & F & 11 & \\
\hline 1827 & m53 & PM & F & 16 & \\
\hline 1828 & t31 & DV & M & $?$ & \\
\hline 1829 & s107 & SV & $?$ & 3.5 & \\
\hline 1830 & s96 & SV & $?$ & 3.5 & \\
\hline
\end{tabular}

TRNWR 11.14.03

\begin{tabular}{|l|l|l|l|l|l|}
\hline 1831 & $\mathrm{~m} 81$ & PM & F & 14 & \\
\hline 1832 & m141 & PM & M & 17 & \\
\hline 1833 & s92 & PM & F & 13 & \\
\hline 1834 & s96 & PM & M & 20 & \\
\hline 1835 & s32 & PM & M & 16 & \\
\hline 1836 & s107 & PM & M & 16 & \\
\hline 1837 & s68 & PM & M & 12 & \\
\hline 1838 & s33 & ST & $?$ & 4.8 & \\
\hline
\end{tabular}

POWELL BUTTE 11.18.03

\begin{tabular}{|l|l|l|l|l|l|}
\hline 1839 & s21 & PM & F & 22.5 & \\
\hline 1840 & s105 & PM & M & 18 & \\
\hline 1841 & s31 & PM & F & 18.5 & \\
\hline 1842 & s6 & PM & F & 15 & \\
\hline 1843 & s103 & MO & F & 15 & \\
\hline 1844 & s139 & GS & F & 140 & \\
\hline 1845 & m41 & GS & M & 65 & \\
\hline 1846 & p31 & ST & $?$ & 3.5 & \\
\hline
\end{tabular}

P.B. 11.19.03

\begin{tabular}{|l|l|l|l|l|l|}
\hline 1847 & p92 & PM & F & 14 & \\
\hline 1848 & s102 & MO & F & 14 & \\
\hline 1849 & s111 & GS & F & 132 & \\
\hline
\end{tabular}

P.B. 11.20 .03

\begin{tabular}{|l|l|l|l|l|l|}
\hline 1850 & m108 & PM & F & 22 & \\
\hline 1851 & s92 & GS & M & 132 & \\
\hline 1852 & s0 & GS & M & 74 & \\
\hline
\end{tabular}

P.B. 11.21 .03

\begin{tabular}{|l|l|l|l|l|l|}
\hline 1853 & s83 & GS & M & 92 & \\
\hline 1854 & s110 & SV & $?$ & 3.5 & \\
\hline
\end{tabular}

OXBOW 12.2.03

\begin{tabular}{|l|l|l|l|l|l|}
\hline 1855 & s96 & PM & M & 18 & \\
\hline 1856 & s24 & PM & F & 16 & \\
\hline
\end{tabular}




\begin{tabular}{|l|l|l|l|l|l|}
\hline 1857 & s48 & PM & F & 19 & \\
\hline 1858 & s21 & PM & M & 18 & \\
\hline 1859 & s117 & PM & M & 17 & \\
\hline 1860 & s51 & PM & M & 16 & \\
\hline 1861 & s130 & PM & M & 18 & \\
\hline 1862 & s129 & PM & F & 13 & \\
\hline 1863 & s136 & MO & F & 18 & \\
\hline 1864 & p40 & MO & F & 19 & \\
\hline 1865 & p19 & ST & $?$ & 4.4 & \\
\hline
\end{tabular}

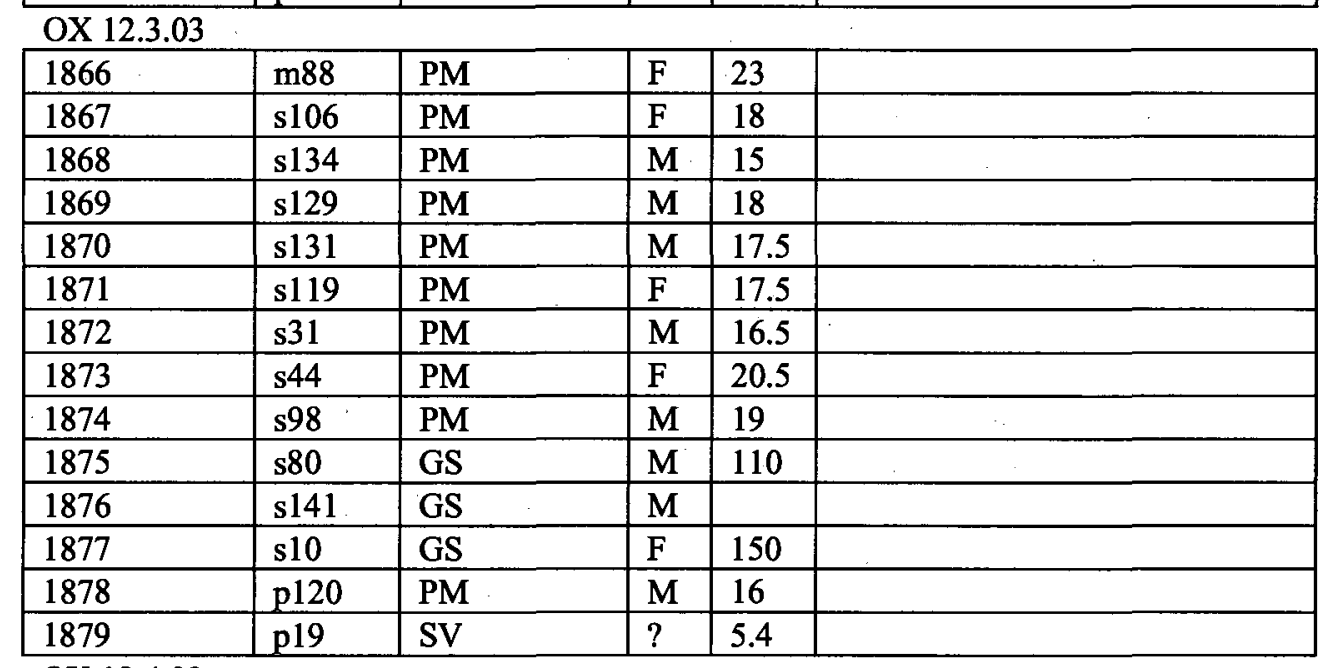

\begin{tabular}{|c|c|c|c|c|c|}
\hline \multicolumn{6}{|c|}{ OX 12.4.03 } \\
\hline 1880 & s93 & PM & $\mathrm{F}$ & 17 & \\
\hline 1881 & s96 & PM & $\mathbf{M}$ & 15.5 & \\
\hline 1882 & s47 & GS & $\mathrm{F}$ & 140 & \\
\hline 1883 & s33 & GS & $\mathrm{F}$ & 110 & \\
\hline 1884 & s44 & MO & $\mathrm{F}$ & 16 & \\
\hline 1885 & s22 & PM & $\mathrm{F}$ & 16 & \\
\hline 1886 & $\mathrm{~s} 28$ & $\overline{P M}$ & $F$ & 11.5 & \\
\hline 1887 & $\mathrm{p} 36$ & PM & $\mathbf{M}$ & 16 & \\
\hline 1888 & p103 & ST & $?$ & 4.6 & \\
\hline 1889 & p79 & SV & $?$ & 5.0 & \\
\hline
\end{tabular}

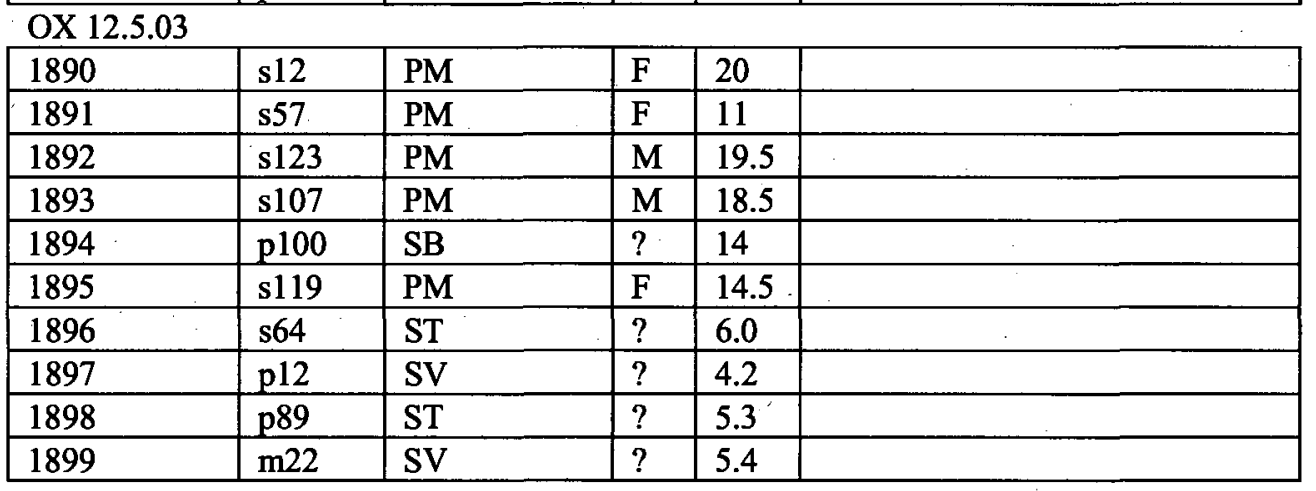

Tryon Creek 12.9.03

\begin{tabular}{|l|l|l|l|l|l|}
\hline 1900 & s78 & PM & F & 19 & \\
\hline 1901 & s83 & PM & F & 17 & \\
\hline
\end{tabular}




\begin{tabular}{|c|c|c|c|c|c|}
\hline 1902 & s105 & PM & $\bar{F}$ & 25 & \\
\hline 1903 & $\mathrm{s42}$ & PM & $\mathbf{M}$ & 21 & \\
\hline 1904 & p91 & PM & $\bar{M}$ & 18 & \\
\hline 1905 & p91 & PM & $\mathrm{M}$ & 20.5 & \\
\hline 1906 & s116 & $\overline{\mathrm{PM}}$ & $\bar{F}$ & 23 & \\
\hline 1907 & s95 & PM & $\mathrm{F}$ & 24.5 & \\
\hline 1908 & $\mathrm{~m} 93$ & PM & $\mathrm{M}$ & 20 & \\
\hline \multicolumn{6}{|c|}{ Tryon Creek 12.10.03 } \\
\hline 1909 & $\mathrm{s83}$ & PM & $\mathrm{F}$ & 23 & \\
\hline 1910 & s91 & PM & $\mathbf{F}$ & 21 & \\
\hline 1911 & s119 & $\mathrm{PM}$ & $\mathrm{F}$ & 21 & \\
\hline 1912 & s5 & $\mathrm{PM}$ & $\bar{M}$ & 18.5 & \\
\hline 1913 & s82 & $\overline{\mathrm{PM}}$ & $\bar{M}$ & 15 & \\
\hline
\end{tabular}

TC 12.11 .03
\begin{tabular}{|l|l|l|l|l|l|}
\hline 1914 & s 15 & PM & F & 19.5 & \\
\hline 1915 & s112 & PM & F & 19 & \\
\hline 1916 & s117 & PM & M & 20 & \\
\hline 1917 & s 5 & PM & M & 21.5 & \\
\hline 1918 & p 91 & PM & F & 17 & \\
\hline 1919 & p 36 & PM & M & 23 & \\
\hline 1920 & m 30 & PM & F & 19 & \\
\hline
\end{tabular}

TC12.12.03
\begin{tabular}{|l|l|l|l|l|l|}
\hline 1921 & s 117 & PM & F & 22.5 & \\
\hline 1922 & m127 & PM & M & 20 & \\
\hline 1923 & s42 & PM & M & 19 & \\
\hline 1924 & s108 & PM & F & 22 & \\
\hline 1925 & s141 & PM & F & 16.5 & \\
\hline 1926 & s102 & PM & M & 22 & \\
\hline 1927 & p 102 & ST & $?$ & 5.5 & \\
\hline 1928 & p 96 & PM & M & 18 & \\
\hline 1929 & s 93 & PM & M & 17 & \\
\hline
\end{tabular}

FOREST PARK 12.16.03

\begin{tabular}{|l|l|l|l|l|l|}
\hline 1930 & s4 & PM & M & 17.5 & \\
\hline 1931 & s116 & PM & M & 20 & \\
\hline 1932 & t55 & TD & F & 179 & \\
\hline 1933 & t132 & PL & $?$ & $?$ & RELEASED \\
\hline
\end{tabular}

FP 12.17 .03
\begin{tabular}{|l|l|l|l|l|l|}
\hline 1934 & s144 & PM & F & 16 & \\
\hline 1935 & s105 & PM & M & 22 & \\
\hline 1936 & s108 & PM & F & 23 & \\
\hline 1937 & s117 & PM & M & 26 & \\
\hline 1938 & s120 & PM & F & 28.5 & \\
\hline 1939 & s19 & PM & M & 20.5 & \\
\hline 1940 & s 96 & PM & F & 20 & \\
\hline 1941 & s42 & PM & F & 17.5 & \\
\hline 1942 & s44 & PM & F & 20 & \\
\hline 1943 & s50 & PM & M & 19 & \\
\hline 1944 & s140 & PM & F & 22.5 & \\
\hline 1945 & p104 & PM & F & 21 & \\
\hline
\end{tabular}




\begin{tabular}{|l|l|l|l|l|l|}
\hline 1946 & p124 & PM & M & 14 & \\
\hline 1947 & p72 & PM & M & 16.5 & \\
\hline 1948 & s91 & SV & $?$ & 7.9 & \\
\hline 1949 & p12 & SV & $?$ & 4.7 & \\
\hline 1950 & s90 & S? & $?$ & $?$ & \\
\hline
\end{tabular}

\begin{tabular}{|l|l|l|l|l|l|}
\hline FP 12.18 .03 & s 98 & PM & M & 23.5 & \\
\hline 1951 & s21 & PM & M & 18 & \\
\hline 1952 & s44 & PM & M & 19 & \\
\hline 1953 & s72 & PM & F & 18.5 & \\
\hline 1954 & p36 & PM & M & 16 & \\
\hline 1955 & & &
\end{tabular}

FP 12.19.03
\begin{tabular}{|l|l|l|l|l|l|}
\hline 1956 & s11 & PM & F & 24.5 & \\
\hline 1957 & s82 & PM & M & 18.5 & \\
\hline 1958 & s139 & PM & M & 22 & \\
\hline 1959 & s106 & PM & M & 18.5 & \\
\hline 1960 & s131 & PM & M & 21.5 & positive \\
\hline 1961 & s104 & PM & M & 21 & \\
\hline 1962 & s72 & PM & F & 22.5 & \\
\hline 1963 & s18 & PM & M & 20 & \\
\hline 1964 & p112 & PM & F & 6.5 & \\
\hline 1965 & t72 & MM & $?$ & $?$ & \\
\hline
\end{tabular}

Tualatin River National Wildlife Refuge 1.13.04

\begin{tabular}{|l|l|l|l|l|l|}
\hline 1966 & s93 & PM & F & 18.5 & \\
\hline 1967 & s33 & PM & M & 14 & \\
\hline 1968 & s104 & PM & M & 16 & \\
\hline 1969 & s118 & PM & M & 16 & \\
\hline 1970 & s53 & PM & F & 13 & \\
\hline 1971 & P91 & PM & F & 14 & \\
\hline 1972 & s24 & PM & M & 13 & \\
\hline 1973 & s54 & PM & M & 15 & \\
\hline 1974 & s103 & PM & F & 14.5 & \\
\hline 1975 & s131 & PM & F & 12.5 & \\
\hline 1976 & s134 & PM & F & 13.5 & \\
\hline 1977 & s117 & PM & F & 10 & \\
\hline
\end{tabular}

TRNWR 1.14.04
\begin{tabular}{|l|l|l|l|l|l|}
\hline 1978 & s132 & PM & M & 16 & \\
\hline 1979 & s131 & PM & M & 15 & \\
\hline 1980 & s11 & PM & F & 13.5 & \\
\hline 1981 & p 67 & PM & F & 13 & \\
\hline 1982 & p 67 & PM & F & 13 & \\
\hline 1983 & s 21 & PM & F & 13.5 & \\
\hline 1984 & s33 & PM & F & 13 & \\
\hline
\end{tabular}

TRNWR 1.15.04

\begin{tabular}{|l|l|l|l|l|l|}
\hline 1985 & s34 & PM & F & 15 & \\
\hline 1986 & s60 & PM & M & 13 & \\
\hline 1987 & s6 & PM & M & 16.5 & \\
\hline 1988 & s78 & PM & F & 12.5 & \\
\hline 1989 & s33 & PM & M & 12 & \\
\hline
\end{tabular}




\begin{tabular}{|l|l|l|l|l|l|l|}
\hline 1990 & s141 & PM & $?$ & $?$ & \\
TRNWR 1.16.04 & s80 & PM & M & 12.5 & \\
\hline 1991 & p 84 & PM & F & 13 & \\
\hline 1992 & s76 & PM & M & 13.5 & \\
\hline 1993 & s122 & PM & M & 15.5 & \\
\hline 1994 & s72 & PM & F & 16 & \\
\hline 1995 & s21 & PM & M & 17 & \\
\hline 1996 & s83 & PM & F & 15 & \\
\hline 1997 & m83 & PM & M & 13 & \\
\hline 1998 & p84 & PM & F & 12 & \\
\hline 1999 & s? & SV & $?$ & 5.2 & \\
\hline 2000 & p16 & PM & $?$ & $?$ & \\
\hline 2001 & s105 & ME & $?$ & $?$ & \\
\hline 2002 & 20.04 & & & \\
\hline
\end{tabular}

Powell Butte 1.20.04

\begin{tabular}{|l|l|l|l|l|l|}
\hline 2003 & m8 & PM & M & 17 & \\
\hline 2004 & s5 & PM & M & 18.5 & \\
\hline 2005 & s144 & PM & M & 16.5 & \\
\hline 2006 & s32 & PM & M & 19 & \\
\hline 2007 & s98 & PM & M & 18.5 & \\
\hline 2008 & s105 & PM & M & 21 & \\
\hline 2009 & p84 & PM & F & 16 & \\
\hline 2010 & s143 & PM & F & 18 & \\
\hline 2011 & s27 & PM & M & 16.5 & \\
\hline 2012 & p72 & PM & F & 19.5 & \\
\hline
\end{tabular}

P.B. 1.21.04

\begin{tabular}{|l|l|l|l|l|l|}
\hline 2013 & s104 & PM & M & 19 & \\
\hline 2014 & s126 & PM & M & 15.5 & \\
\hline 2015 & s32 & PM & F & 13.5 & \\
\hline 2016 & s18 & PM & M & 17 & \\
\hline 2017 & s3 & PM & M & 18.5 & \\
\hline 2018 & m16 & GS & F & 125 & \\
\hline 2019 & s116 & PM & M & 16.5 & \\
\hline 2020 & s105 & PM & F & 17.5 & \\
\hline 2021 & s22 & PM & $?$ & $?$ & escaped \\
\hline
\end{tabular}

P.B. 1.22.04

\begin{tabular}{|l|l|l|l|l|l|}
\hline 2022 & s90 & MO & M & 16 & NBS \\
\hline 2023 & s3 & PM & F & 14 & \\
\hline 2024 & s144 & PM & M & 18 & 1 SAMPLE \\
\hline 2025 & s126 & MO & M & 14.5 & NBS \\
\hline 2026 & s18 & PM & F & 16.5 & \\
\hline 2027 & s5 & PM & M & 15 & \\
\hline 2028 & s132 & PM & M & 18 & \\
\hline 2029 & s22 & PM & M & 15 & \\
\hline 2030 & p84 & PM & F & 12.5 & \\
\hline 2031 & s116 & PM & M & 16 & \\
\hline
\end{tabular}

P.B. 1.23.04

\begin{tabular}{|l|l|l|l|l|l|}
\hline 2032 & s13 & GS & F & 145 & \\
\hline 2033 & p68 & PM & M & 14.8 & \\
\hline
\end{tabular}




\begin{tabular}{|l|l|l|l|l|l|}
\hline 2034 & s132 & PM & M & 15.0 & \\
\hline 2035 & s136 & PM & M & 12.5 & \\
\hline 2036 & s103 & PM & F & 18.5 & \\
\hline 2037 & s78 & PM & M & 17 & \\
\hline 2038 & s26 & PM & M & 13 & \\
\hline 2039 & s3 & PM & F & 16.5 & \\
\hline 2040 & m120 & GS & M & 115 & DIT/NBS \\
\hline 2041 & s116 & PM & M & 10.8 & \\
\hline 2042 & s137 & SV & $?$ & 4.0 & NBS \\
\hline 2043 & s87 & SV & $?$ & 4.5 & NBS \\
\hline
\end{tabular}

Oxbow 2.3.04

\begin{tabular}{|l|l|l|l|l|l|}
\hline 2044 & s70 & PM & M & 18 & \\
\hline 2045 & s53 & PM & M & 17.5 & \\
\hline 2046 & s139 & PM & F & 16 & \\
\hline 2047 & s132 & PM & M & 17 & \\
\hline 2048 & s144 & PM & F & 19.5 & \\
\hline 2049 & m69 & PM & F & 17 & \\
\hline 2050 & m43 & PM & F & 16 & \\
\hline 2051 & s32 & PM & F & 15.5 & \\
\hline 2052 & s43 & MO & F & 17 & \\
\hline 2053 & p64 & ST & F & 4.5 & \\
\hline 2054 & s135 & ST & F & 4.5 & \\
\hline 2055 & s130 & PM & $?$ & $?$ & \\
\hline 2056 & m87 & PM & $?$ & $?$ & \\
\hline
\end{tabular}

OX 2.4.04

\begin{tabular}{|l|l|l|l|l|l|}
\hline 2057 & s94 & PM & F & 21 & \\
\hline 2058 & m90 & PM & F & 18.5 & \\
\hline 2059 & s118 & PM & F & 24 & \\
\hline 2060 & s108 & PM & M & 16.5 & \\
\hline 2061 & p79 & PM & M & 14.5 & \\
\hline 2062 & s126 & PM & F & 22.5 & \\
\hline 2063 & s2 & PM & M & 18.5 & \\
\hline 2064 & s133 & PM & F & 10.5 & \\
\hline 2065 & s123 & PM & M & 23 & \\
\hline 2066 & s32 & PM & M & 17 & \\
\hline 2067 & m69 & PM & M & 18 & \\
\hline 2068 & p24 & PM & M & 15 & \\
\hline 2069 & s23 & PM & F & 19.5 & \\
\hline 2070 & s139 & PM & M & 17.5 & \\
\hline 2071 & s75 & PM & M & 17 & \\
\hline 2072 & s124 & PM & F & 15 & \\
\hline 2073 & p60 & PM & M & 16 & \\
\hline 2074 & s100 & PM & F & 17 & \\
\hline 2075 & s116 & PM & M & 15 & \\
\hline 2076 & p40 & ST & $?$ & 4.4 & \\
\hline 2077 & p64 & SV & $?$ & 5.4 & \\
\hline 2078 & s66 & ST & $?$ & 4.6 & \\
\hline 2079 & p100 & ST & $?$ & 4.3 & \\
\hline 2080 & p100 & PM & $?$ & $?$ & \\
\hline
\end{tabular}




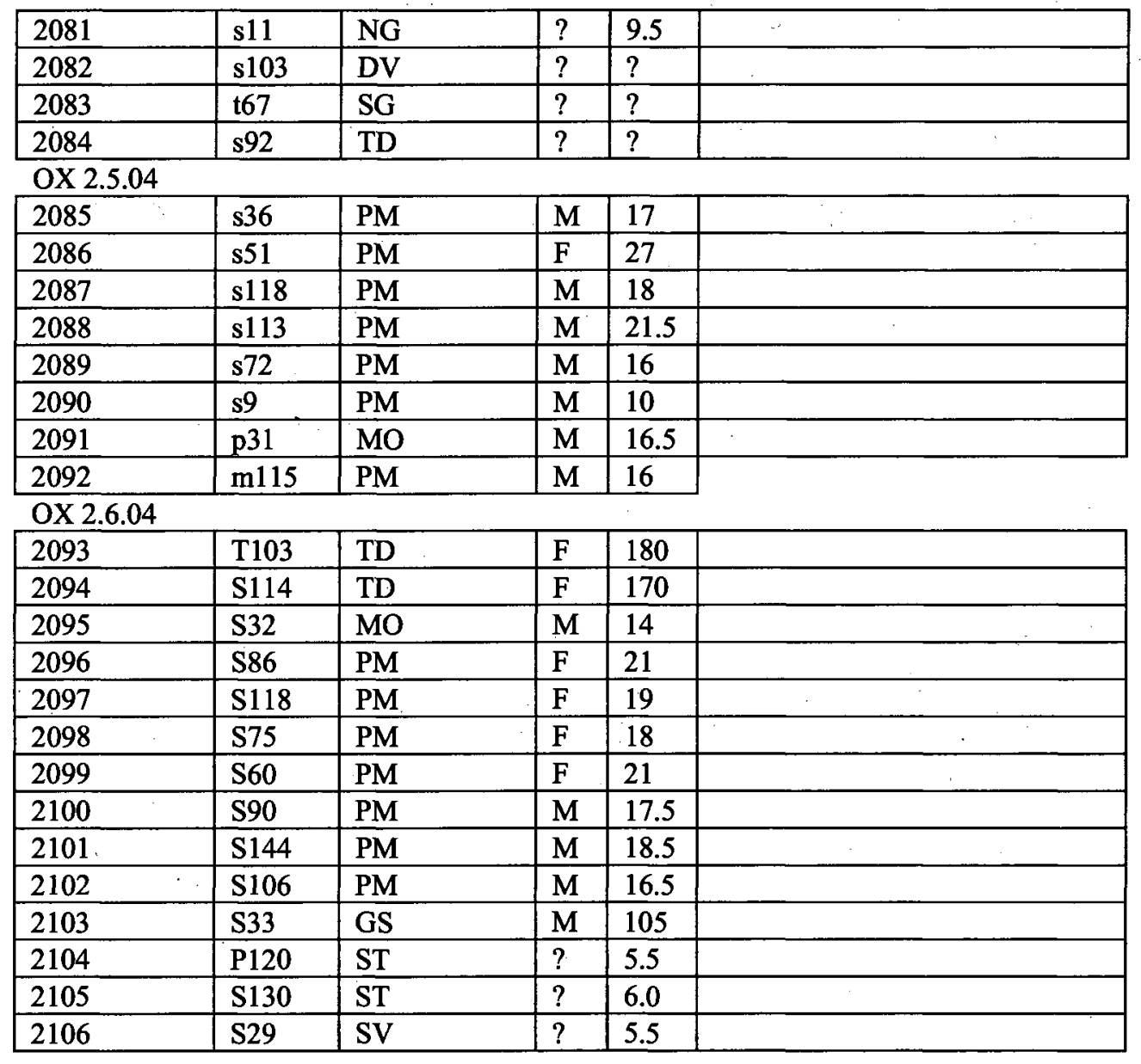

\section{FOREST PARK 2.10.04}

\begin{tabular}{|l|l|l|l|l|l|}
\hline 2107 & s132 & PM & F & 23 & PREG \\
\hline 2108 & s117 & PM & F & 24.5 & PREG \\
\hline 2109 & s45 & PM & F & 27 & PREG \\
\hline 2110 & s118 & PM & M & 18 & \\
\hline 2111 & s120 & PM & F & 20 & PREG \\
\hline 2112 & s71 & PM & F & 19 & PREG \\
\hline 2113 & s60 & PM & F & 26.5 & PREG \\
\hline 2114 & s69 & PM & F & 20 & PREG \\
\hline 2115 & s8 & PM & M & 19 & \\
\hline 2116 & s77 & PM & M & 21 & \\
\hline 2117 & s67 & PM & F & 17 & \\
\hline 2118 & m24 & PM & F & 20.5 & PREG \\
\hline 2119 & s105 & PM & F & 20 & PREG \\
\hline 2120 & s97 & PM & F & 22 & PREG \\
\hline 2121 & s8 & PM & F & 25 & PREG \\
\hline 2122 & p36 & ST & M & 4.8 & \\
\hline 2123 & p4 & SV & F & 5.2 & SUB A \\
\hline 2124 & p19 & ST & $?$ & $?$ & ESCAPED \\
\hline
\end{tabular}

F.P. 2.11.04

\begin{tabular}{|l|l|l|l|l|l|}
\hline 2125 & s64 & PM & F & 23 & \\
\hline
\end{tabular}




\begin{tabular}{|l|l|l|l|l|l|}
\hline 2126 & s3 & PM & M & 18 & \\
\hline 2127 & s58 & PM & F & 21 & \\
\hline 2128 & s67 & PM & M & 20 & \\
\hline 2129 & p127 & PM & F & 21 & \\
\hline 2130 & $\mathrm{~s} 130$ & PM & F & 22 & PREG \\
\hline 2131 & s127 & PM & F & 23 & \\
\hline 2132 & s131 & PM & F & 22.5 & PREG \\
\hline 2133 & s45 & PM & M & 17.5 & \\
\hline 2134 & s95 & PM & F & 20.5 & \\
\hline 2135 & s10 & PM & M & 17.5 & \\
\hline 2136 & m24 & PM & M & 24 & \\
\hline 2137 & s118 & PM & F & 16.5 & \\
\hline 2138 & s97 & PM & M & 21 & \\
\hline 2139 & s60 & PM & F & 29 & PREG \\
\hline 2140 & s77 & PM & M & 19.5 & \\
\hline 2141 & s144 & PM & M & 21 & \\
\hline 2142 & s120 & PM & F & 17.5 & \\
\hline 2143 & p127 & ST & $?$ & $?$ & ESCAPED \\
\hline 2144 & s22 & ST & $?$ & 5.4 & RELEASED \\
\hline 2145 & s33 & PM & F & 19.5 & \\
\hline 2146 & s34 & PM & F & 17 & \\
\hline 2147 & s87 & PM & F & 19 & \\
\hline 2148 & s24 & PM & F & 20.5 & PREG \\
\hline 2149 & $\mathrm{~s} 8$ & PM & F & 29 & PREG \\
\hline 2150 & p88 & SV & $?$ & 4.6 & \\
\hline
\end{tabular}

\section{F.P. 2.12.04}

\begin{tabular}{|l|l|l|l|l|l|}
\hline 2151 & s95 & PM & M & 20.5 & \\
\hline 2152 & s84 & PM & F & 20 & PREG \\
\hline 2153 & s106 & PM & M & 21 & \\
\hline 2154 & p100 & PM & F & 13.5 & SUB A \\
\hline 2155 & p100 & PM & M & 14.5 & SUB A \\
\hline 2156 & s103 & PM & F & 17 & \\
\hline 2157 & s5 & PM & M & 12 & JUV \\
\hline 2158 & m105 & PM & M & 23.5 & \\
\hline 2159 & s127 & PM & M & 20.5 & \\
\hline 2160 & s117 & PM & F & 11 & JUV \\
\hline 2161 & m24 & PM & M & 16 & \\
\hline 2162 & s131 & PM & M & 22 & \\
\hline 2163 & s43 & PM & F & 22 & \\
\hline 2164 & s109 & PM & M & 19 & \\
\hline 2165 & s22 & PM & M & 15.5 & \\
\hline 2166 & s83 & PM & F & 18 & \\
\hline 2167 & s130 & PM & F & 20 & PREG \\
\hline 2168 & s59 & PM & M & 15.5 & \\
\hline 2169 & s139 & PM & F & 24 & \\
\hline 2170 & s3 & PM & F & 15.5 & SUB A \\
\hline 2171 & s60 & PM & M & 22.5 & \\
\hline 2172 & p100 & PM & M & 15 & DIT/NBS \\
\hline 2173 & p100 & PM & M & 12 & DIT/NBS \\
\hline & & & & \\
\hline
\end{tabular}




\begin{tabular}{|l|l|l|l|l|l|}
\hline 2174 & $\mathrm{~s} 19$ & $\mathrm{PM}$ & $\mathrm{F}$ & 20.5 & DIT/NBS \\
\hline 2175 & $\mathrm{~m} 108$ & $\mathrm{PM}$ & $\mathrm{F}$ & 12 & $\mathrm{DIT} / \mathrm{NBS}$ \\
\hline 2176 & $\mathrm{~m} 108$ & $\mathrm{PM}$ & $\mathrm{M}$ & 16 & $\mathrm{DIT} / \mathrm{NBS}$ \\
\hline 2177 & $\mathrm{p} 64$ & $\mathrm{PM}$ & $\mathrm{M}$ & 19 & $\mathrm{DIT} / \mathrm{NBS}$ \\
\hline 2178 & $\mathrm{~s} 33$ & $\mathrm{PM}$ & $\mathrm{M}$ & 17.5 & $\mathrm{DIT} / \mathrm{NBS}$ \\
\hline 2179 & $\mathrm{p} 72$ & $\mathrm{ST}$ & $?$ & 4.6 & $\mathrm{DIT} / \mathrm{NBS}$ \\
\hline 2180 & $\mathrm{p} 19$ & $\mathrm{ST}$ & $?$ & 4.5 & $\mathrm{DIT} / \mathrm{NBS}$ \\
\hline 2181 & $\mathrm{p} 12$ & $\mathrm{ST}$ & $?$ & 4.6 & DIT/NBS \\
\hline 2182 & $\mathrm{p} 24$ & $\mathrm{ST}$ & $?$ & 4.4 & DIT/NBS \\
\hline
\end{tabular}

\begin{tabular}{|c|c|c|c|c|c|}
\hline \multicolumn{6}{|c|}{ F.P. 2.13.04 } \\
\hline 2183 & $\mathrm{~s} 35$ & PM & M & 20 & \\
\hline 2184 & s80 & $\mathrm{PM}$ & $\mathbf{M}$ & 14 & JUV \\
\hline 2185 & s92 & $\overline{\mathrm{PM}}$ & $\mathrm{F}$ & 20 & \\
\hline 2186 & s77 & $\mathrm{PM}$ & $\mathrm{M}$ & 19.5 & \\
\hline 2187 & s117 & PM & $\mathrm{F}$ & 15 & \\
\hline 2188 & s10 & $\mathrm{PM}$ & $\mathrm{M}$ & 20.5 & \\
\hline 2189 & s103 & $\mathrm{PM}$ & $\mathbf{M}$ & 16.5 & \\
\hline 2190 & s130 & $\mathbf{P M}$ & $F$ & 23 & \\
\hline 2191 & $\mathrm{~s} 21$ & $\mathrm{PM}$ & $\mathbf{M}$ & 18 & \\
\hline 2192 & $\mathrm{~m} 8$ & PM & $\mathbf{M}$ & 12.5 & \\
\hline 2193 & $\mathrm{~m} 10$ & PM & $F$ & 21 & \\
\hline 2194 & s22 & PM & $\mathbf{M}$ & 10 & DIT/NBS \\
\hline 2195 & p126 & $\mathrm{PM}$ & $\mathbf{M}$ & 10 & DIT/NBS \\
\hline 2196 & s83 & SV & $?$ & 3.8 & DIT/NBS \\
\hline 2197 & $s 60$ & PM & $\mathrm{F}$ & 27 & DIT/NBS \\
\hline 2198 & s61 & SV & $?$ & 3.2 & DIT/NBS \\
\hline 2199 . & p144 & PM & $F$ & 20 & DIT/NBS \\
\hline 2200 & p96 & ST & $?$ & 5.4 & DIT/NBS \\
\hline
\end{tabular}

TRYON CREEK 2.17.04

\begin{tabular}{|l|l|l|l|l|l|}
\hline 2201 & s94 & PM & M & 18.5 & \\
\hline 2202 & s93 & PM & F & 20 & \\
\hline 2203 & s90 & PM & M & 20.5 & \\
\hline 2204 & s84 & PM & F & 18.5 & \\
\hline 2205 & s82 & PM & F & 26 & \\
\hline 2206 & s48 & PM & F & 22.5 & \\
\hline 2207 & s8 & PM & M & 19 & \\
\hline 2208 & s19 & PM & M & 12.5 & \\
\hline 2209 & s129 & PM & M & 15 & \\
\hline 2210 & s137 & PM & M & 14 & \\
\hline 2211 & s128 & PM & M & 19 & \\
\hline 2212 & s33 & PM & F & 19.5 & \\
\hline 2213 & s28 & PM & M & 17 & \\
\hline 2214 & s72 & PM & F & 22 & \\
\hline 2215 & m108 & PM & F & 22 & \\
\hline 2216 & s26 & PM & F & 22.5 & \\
\hline 2217 & s4 & PM & M & 13 & \\
\hline 2218 & s103 & PM & M & 23 & \\
\hline 2219 & s139 & PM & M & 21.5 & \\
\hline 2220 & s136 & PM & F & 21.5 & DIB/NBS \\
\hline
\end{tabular}




\begin{tabular}{|c|c|c|c|c|c|}
\hline 2221 & $\mathrm{~s} 126$ & PM & $F$ & 27 & DIB/NBS \\
\hline 2222 & p139 & SV & $?$ & 5.6 & DIB/NBS \\
\hline 2223 & s117 & PM & $?$ & $?$ & ESCAPED \\
\hline \multicolumn{6}{|c|}{ T.C. 2.18.04 } \\
\hline 2224 & $\mathrm{p} 43$ & PM & M & 18 & \\
\hline 2225 & p96 & PM & $M$ & 17.5 & \\
\hline 2226 & s118 & PM & $\mathbf{M}$ & 15 & \\
\hline 2227 & $\mathrm{~s} 138$ & PM & $M$ & 14 & \\
\hline 2228 & s92 & PM & $\mathbf{F}$ & 20 & \\
\hline 2229 & s81 & PM & $\mathbf{M}$ & 21 & \\
\hline 2230 & s142 & PM & $F$ & 21 & \\
\hline 2231 & s131 & $\dot{\mathrm{PM}}$ & $\mathrm{F}$ & 18 & \\
\hline 2232 & s137 & PM & $F$ & 18.5 & \\
\hline 2233 & s93 & PM & $\mathrm{F}$ & 14 & \\
\hline 2234 & s79 & PM & $M$ & 20 & \\
\hline 2235 & p10 & PM & $F$ & 13 & \\
\hline 2236 & s11 & PM & $\mathrm{F}$ & 25 & \\
\hline 2237 & $\mathrm{p} 24$ & PM & $\mathrm{M}$ & 15 & \\
\hline 2238 & p108 & PM & M & 14 & DIT/NBS \\
\hline 2239 & p76 & PM & $\mathrm{F}$ & 17 & DIT/NBS \\
\hline 2240 & p24 & PM & $\mathrm{M}$ & 13 & DIT/NBS \\
\hline 2241 & $\mathrm{p} 4$ & $\mathrm{PM}$ & $\mathrm{M}$ & 23.5 & DIT/NBS \\
\hline 2242 & s18 & PM & $\mathrm{F}$ & 15 & DIT/NBS \\
\hline 2243 & p79 & PM & $F$ & 30 & DIT/NBS \\
\hline 2244 & p79 & PM & $F$ & 24.5 & DIT/NBS \\
\hline 2245 & p55 & PM & $M$ & 23.5 & DIT/NBS \\
\hline 2246 & $\mathrm{p} 84$ & PM & $\mathbf{M}$ & 21 & DIT/NBS \\
\hline 2247 & $\mathrm{p} 84$ & ST & $?$ & 7.5 & DIT/NBS \\
\hline 2248 & p84 & ST & $?$ & 7.4 & DIT/NBS \\
\hline 2249 & p84 & ST & $?$ & 7.0 & DIT/NBS \\
\hline 2250 & p67 & SV & $?$ & 6.0 & DIT/NBS \\
\hline
\end{tabular}

T.C. 2.19 .04

\begin{tabular}{|l|l|l|l|l|l|}
\hline 2251 & $\mathrm{p} 12$ & PM & M & 17.5 & \\
\hline 2252 & s19 & PM & M & 18 & \\
\hline 2253 & s10 & PM & M & 20.5 & \\
\hline 2254 & s4 & PM & M & 21 & \\
\hline 2255 & s18 & PM & F & 15.5 & \\
\hline 2256 & p43 & PM & M & 16 & \\
\hline 2257 & s94 & PM & M & 20 & \\
\hline 2258 & s77 & PM & M & 19 & \\
\hline 2259 & s84 & PM & F & 30.5 & \\
\hline 2260 & s83 & PM & M & 22 & \\
\hline 2261 & s139 & PM & F & 16 & \\
\hline 2262 & s138 & PM & F & 23 & \\
\hline 2263 & s24 & PM & M & 19 & \\
\hline 2264 & s43 & PM & M & 22.5 & \\
\hline 2265 & s99 & PM & F & 22.5 & \\
\hline 2266 & s118 & PM & F & 12 & \\
\hline 2267 & p64 & PM & F & 24 & \\
\hline
\end{tabular}




\begin{tabular}{|l|l|l|l|l|l|}
\hline 2268 & $\mathrm{~s} 138$ & PM & M & 21.5 & \\
\hline 2269 & $\mathrm{~s} 127$ & PM & F & 21 & \\
\hline 2270 & $\mathrm{~s} 72$ & PM & M & 20.5 & \\
\hline 2271 & $\mathrm{p} 72$ & PM & F & 19 & DIT/NBS \\
\hline 2272 & $\mathrm{p} 84$ & PM & F & 24.5 & DIT/NBS \\
\hline 2273 & $\mathrm{~s} 6$ & PM & M & 17.5 & DIT/NBS \\
\hline 2274 & $\mathrm{p} 43$ & PM & M & 15.5 & DIT/NBS \\
\hline 2275 & $\mathrm{~s} 65$ & PM & M & 17 & DIT/NBS \\
\hline 2276 & $\mathrm{p} 24$ & PM & F & 17.5 & DIT/NBS \\
\hline 2277 & $\mathrm{p} 91$ & PM & F & 19 & DIT/NBS \\
\hline 2278 & $\mathrm{p} 96$ & PM & M & 21 & DIT/NBS \\
\hline 2279 & $\mathrm{p} 96$ & PM & F & 23.5 & DIT/NBS \\
\hline 2280 & $\mathrm{p} 79$ & PM & F & 21.5 & DIT/NBS \\
\hline 2281 & $\mathrm{p} 79$ & PM & F & 19 & DIT/NBS \\
\hline 2282 & $\mathrm{p} 79$ & ST & $?$ & 7.5 & DIT/NBS \\
\hline 2283 & $\mathrm{p} 115$ & ST & $?$ & 6.8 & DIT/NBS \\
\hline 2284 & $\mathrm{p} 115$ & PM & F & 13 & DIT/NBS \\
\hline 2285 & $\mathrm{~s} 142$ & ST & $?$ & 5.6 & DIT/NBS \\
\hline 2286 & $\mathrm{p} 36$ & ST & $?$ & 6.1 & DIT/NBS \\
\hline 2287 & $\mathrm{p} 36$ & ST & $?$ & 7.6 & DIT/NBS \\
\hline 2288 & $\mathrm{p} 36$ & ST & $?$ & 6.7 & DIT/NBS \\
\hline 2289 & $\mathrm{p} 36$ & ST & $?$ & 6.2 & DIT/NBS \\
\hline 2290 & $\mathrm{p} 36$ & ST & $?$ & 6.9 & DIT/NBS \\
\hline 2291 & $\mathrm{p} 36$ & ST & $?$ & 4.6 & DIT/NBS \\
\hline 2292 & $\mathrm{p} 139$ & ST & $?$ & 5.3 & DIT/NBS \\
\hline
\end{tabular}

T.C. 2.20 .04

\begin{tabular}{|l|l|l|l|l|l|}
\hline 2293 & s69 & PM & M & 18.5 & \\
\hline 2294 & s129 & PM & M & 17.5 & \\
\hline 2295 & s118 & PM & F & 11 & \\
\hline 2296 & s140 & PM & F & 13.5 & \\
\hline 2297 & s105 & PM & M & 17.5 & \\
\hline 2298 & s10 & PM & F & 18 & \\
\hline 2299 & s107 & PM & F & 14 & \\
\hline 2300 & s92 & PM & M & 15 & \\
\hline 2301 & s43 & PM & M & 19 & \\
\hline 2303 & s66 & PM & M & 18 & \\
\hline 2304 & s71 & PM & M & 14 & \\
\hline 2305 & s26 & PM & M & 15.5 & \\
\hline 2306 & s109 & PM & M & 14 & \\
\hline 2307 & s39 & PM & F & 17 & \\
\hline 2308 & s93 & PM & F & 11.5 & \\
\hline 2309 & s79 & PM & M & 21 & \\
\hline 2310 & p36 & PM & F & 15 & DIT/NBS \\
\hline 2311 & p31 & PM & F & 13 & DIT/NBS \\
\hline 2312 & p31 & PM & F & 14.5 & DIT/NBS \\
\hline 2313 & p12 & PM & F & 16 & DIT/NBS \\
\hline 2314 & p115 & PM & F & 13 & DIT/NBS \\
\hline 2315 & s69 & PM & F & 20 & DIT/NBS \\
\hline & m43 & SV & $?$ & 4.8 & DIT/NBS \\
\hline
\end{tabular}




\begin{tabular}{|l|l|l|l|l|l|}
\hline 2316 & s44 & PM & F & 20.5 & DIT/NBS \\
\hline 2317 & s107 & PM & F & 18 & DIT/NBS \\
\hline 2318 & s92 & ST & $?$ & 5.6 & DIT/NBS \\
\hline
\end{tabular}

Tualatin River NWR 2.24.04

\begin{tabular}{|l|l|l|l|l|l|}
\hline 2319 & $\mathrm{~s} 32$ & PM & M & 15 & \\
\hline 2320 & $\mathrm{p} 84$ & PM & F & 13.5 & \\
\hline 2321 & p84 & PM & F & 20.5 & \\
\hline 2322 & s89 & PM & M & 16 & \\
\hline 2323 & s80 & PM & M & 17.5 & \\
\hline 2324 & s72 & PM & F & 15.5 & \\
\hline 2325 & s10 & PM & F & 13 & \\
\hline 2326 & s59 & PM & F & 17 & \\
\hline 2327 & s51 & PM & M & 16 & \\
\hline 2328 & s107 & PM & M & 12.5 & DIT/NBS \\
\hline 2329 & t120 & MM & $?$ & $?$ & RELEASED \\
\hline
\end{tabular}

TRNWR 2.25.04
\begin{tabular}{|l|l|l|l|l|l|}
\hline 2330 & s102 & PM & F & 11.5 & SUB A \\
\hline 2331 & s41 & PM & F & 16 & \\
\hline 2332 & $\mathrm{~s} 31$ & PM & M & 15 & \\
\hline 2333 & s 9 & PM & M & 14.5 & \\
\hline 2334 & $\mathrm{~m} 34$ & PM & F & 16 & \\
\hline 2335 & s 45 & PM & F & 21.5 & \\
\hline 2336 & $\mathrm{~s} 20$ & PM & M & 14.5 & \\
\hline 2337 & $\mathrm{~s} 79$ & ST & F & 4.6 & NBS RELEASED \\
\hline 2338 & s117 & ST & M & 4.4 & NBS RELEASED \\
\hline 2339 & $\mathrm{~s} 51$ & ST & $?$ & $?$ & NBS ESCAPED \\
\hline 2340 & t84 & MM & $?$ & $?$ & NBS RELEASED \\
\hline
\end{tabular}

TRNWR 2.26.04

\begin{tabular}{|l|l|l|l|l|l|}
\hline 2341 & $\mathrm{~s} 144$ & $\mathrm{PM}$ & $\mathrm{F}$ & 16 & \\
\hline 2342 & $\mathrm{~s} 106$ & $\mathrm{PM}$ & $\mathrm{M}$ & 14 & \\
\hline 2343 & $\mathrm{~s} 20$ & $\mathrm{PM}$ & $\mathrm{M}$ & 16 & \\
\hline 2344 & $\mathrm{~s} 82$ & $\mathrm{PM}$ & $\mathrm{M}$ & 20.5 & \\
\hline 2345 & $\mathrm{~s} 129$ & $\mathrm{PM}$ & $\mathrm{F}$ & 16 & \\
\hline 2346 & $\mathrm{p} 132$ & $\mathrm{PM}$ & $\mathrm{M}$ & 13 & \\
\hline 2347 & $\mathrm{~s} 45$ & $\mathrm{PM}$ & $\mathrm{F}$ & 16.5 & \\
\hline 2348 & $\mathrm{p} 28$ & $\mathrm{PM}$ & $\mathrm{F}$ & 14 & SUB A \\
\hline 2349 & $\mathrm{p} 28$ & $\mathrm{PM}$ & $\mathrm{F}$ & 13.5 & SUB A \\
\hline 2350 & $\mathrm{~s} 10$ & $\mathrm{PM}$ & $\mathrm{F}$ & 16 & \\
\hline 2351 & $\mathrm{~s} 59$ & $\mathrm{PM}$ & $\mathrm{M}$ & 15.5 & \\
\hline 2352 & $\mathrm{p} 124$ & $\mathrm{PM}$ & $\mathrm{M}$ & 17 & DIT/NBS \\
\hline 2353 & $\mathrm{p} 48$ & $\mathrm{PM}$ & $\mathrm{F}$ & 17 & DIT/NBS \\
\hline 2354 & $\mathrm{~s} 53$ & $\mathrm{ST}$ & $\mathrm{F}$ & 5.0 & RELEASED \\
\hline 2355 & $\mathrm{t} 139$ & $\mathrm{MM}$ & $?$ & $?$ & RELEASED \\
\hline
\end{tabular}

TRNWR 2.27.04

\begin{tabular}{|l|l|l|l|l|l|}
\hline 2356 & $\mathrm{~s} 134$ & $\mathrm{PM}$ & $\mathrm{F}$ & 13.5 & \\
\hline 2357 & $\mathrm{~s} 140$ & $\mathrm{PM}$ & $\mathrm{F}$ & 16 & \\
\hline 2358 & $\mathrm{~s} 120$ & $\mathrm{PM}$ & $\mathrm{M}$ & 19.5 & \\
\hline 2359 & $\mathrm{p} 43$ & $\mathrm{PM}$ & $\mathrm{F}$ & 17.5 & \\
\hline 2360 & $\mathrm{p} 60$ & $\mathrm{PM}$ & $\mathrm{M}$ & 20.5 & DIT/NBS \\
\hline
\end{tabular}




\begin{tabular}{|l|l|l|l|l|l|}
\hline 2361 & $\mathrm{p} 52$ & $\mathrm{ST}$ & $?$ & 7.7 & DIT/NBS \\
\hline 2362 & $\mathrm{~s} 11$ & $\mathrm{ST}$ & $?$ & 6.0 & DIT/NBS \\
\hline 2363 & $\mathrm{~s} 103$ & $\mathrm{SV}$ & $?$ & 6.2 & DIT/NBS \\
\hline 2364 & $\mathrm{~s} 129$ & $\mathrm{ST}$ & $?$ & 6.0 & DIT/NBS \\
\hline
\end{tabular}

Powell Butte 3.9.04

\begin{tabular}{|c|c|c|c|c|c|}
\hline 2365 & s48 & PM & $\bar{F}$ & 16 & PREG \\
\hline 2366 & s0 & $\mathrm{MO}$ & $\mathbf{F}$ & 15.5 & NBS RELEASED \\
\hline \multicolumn{6}{|c|}{ POWELL BUTTE 3.10 .04} \\
\hline 2367 & $\mathrm{~m} 129$ & PM & $\mathrm{F}$ & 21 & PREG \\
\hline 2368 & s117 & PM & $\mathbf{F}$ & 22 & PREG \\
\hline 2369 & $\mathrm{~m} 101$ & GS & $\mathbf{M}$ & 143 & RELEASED \\
\hline 2370 & s56 & $\overline{\mathrm{PM}}$ & $\mathbf{F}$ & 16.5 & DIT/NBS \\
\hline 2371 & s116 & $\overline{\mathrm{PM}}$ & $\mathbf{M}$ & 16 & DIT/NBS \\
\hline
\end{tabular}

\begin{tabular}{|l|l|l|l|l|l|}
\hline PB 3.11.04 & s105 & GS & M & 123 & RELEASED \\
\hline 2372 & s 32 & PM & F & 20.5 & \\
\hline 2373 & p144 & PM & M & 20.5 & \\
\hline 2374 & s124 & PM & M & 19 & \\
\hline 2375 & s3 & PM & M & 18.5 & \\
\hline 2376 & s83 & PM & F & 20 & \\
\hline 2377 & s 93 & PM & F & 24 & \\
\hline 2378 & s0 & PM & F & 22 & \\
\hline 2379 & s4 & SV & $?$ & 5.5 & DIT/NBS \\
\hline 2380 & & & & \\
\hline
\end{tabular}

PB 3.12.04
\begin{tabular}{|l|l|l|l|l|l|}
\hline 2381 & t120 & TD & M & $?$ & NBS \\
\hline 2382 & s0 & PM & M & 13.5 & \\
\hline 2383 & s32 & PM & F & 19.0 & \\
\hline 2384 & s73 & PM & M & 15.5 & \\
\hline 2385 & s72 & PM & M & 15.0 & ONE BLOOD SAMPLE \\
\hline 2386 & s21 & PM & M & 16.0 & \\
\hline 2387 & s21 & PM & F & 17.5 & PRG. \\
\hline 2388 & s88 & ST & $?$ & 4.2 & \\
\hline 2389 & s0 & SV & $?$ & 6.5 & \\
\hline 2390 & p48 & PM & $?$ & 12 & DIT/NBS \\
\hline 2391 & $?$ & SV & $?$ & 4.3 & \\
\hline
\end{tabular}

Oxbow 3.16.04

\begin{tabular}{|l|l|l|l|l|l|}
\hline 2392 & m106 & TD & M & 170 & \\
\hline 2393 & s75 & TD & $?$ & $?$ & \\
\hline 2394 & s71 & TT & M & 78 & \\
\hline 2395 & p144 & MO & F & 13 & \\
\hline 2396 & s68 & PM & F & 15 & \\
\hline 2397 & s70 & PM & F & 16.5 & \\
\hline 2398 & m129 & PM & F & 12 & \\
\hline 2399 & m94 & PM & M & 15 & \\
\hline 2400 & s113 & PM & M & 18.5 & \\
\hline 2401 & s108 & PM & M & 15 & \\
\hline 2402 & s72 & PM & M & 18 & \\
\hline 2403 & s74 & PM & M & 15.5 & \\
\hline
\end{tabular}




\begin{tabular}{|l|l|l|l|l|l|}
\hline 2404 & $\mathrm{~s} 132$ & PM & M & 16 & \\
\hline 2405 & $\mathrm{~s} 19$ & PM & M & 16 & \\
\hline 2406 & p96 & ST & $?$ & 6.2 & \\
\hline 2407 & p24 & SV & $?$ & 4.7 & \\
\hline 2408 & s129 & ST & M & 5.0 & \\
\hline 2409 & s53 & SV & $?$ & 6.4 & \\
\hline 2410 & $\mathrm{~s} 33$ & ST & $?$ & 4.8 & \\
\hline 2411 & p 96 & ST & $?$ & 4.4 & \\
\hline 2412 & p96 & ST & $?$ & 3.9 & \\
\hline
\end{tabular}

Ox 3.17.04
\begin{tabular}{|l|l|l|l|l|l|}
\hline 2413 & s 118 & TT & M & 72 & Tag G 472 \\
\hline 2414 & p108 & PM & F & 7 & \\
\hline 2415 & m 92 & TD & F & 130 & Tag G701 \\
\hline 2416 & s 52 & PM & M & 15 & \\
\hline 2417 & s88 & PM & M & 12.5 & \\
\hline 2418 & s79 & PM & F & 13.5 & \\
\hline 2419 & s 95 & PM & M & 17 & \\
\hline 2420 & s139 & PM & M & 16.5 & \\
\hline 2421 & s32 & MO & M & 20 & \\
\hline 2422 & s33 & PM & F & 14.5 & \\
\hline 2423 & p 29 & PM & M & 13 & \\
\hline 2424 & s134 & PM & F & 19 & \\
\hline 2425 & p7 & ST & $?$ & 5.0 & \\
\hline 2426 & p 64 & SV & $?$ & 5.0 & \\
\hline 2427 & s128 & ST & F & 5.9 & \\
\hline 2428 & s128 & ST & F & 4.7 & \\
\hline 2429 & s59 & PM & F & 13 & \\
\hline 2430 & p19 & PM & F & 11 & \\
\hline 2431 & p144 & PM & F & 12.5 & \\
\hline 2432 & s 97 & ST & $?$ & 4.0 & \\
\hline 2433 & p 36 & ST & $?$ & 6.1 & \\
\hline 2434 & $?$ & ST & $?$ & 4.9 & \\
\hline 2435 & s 96 & ST & M & 5.8 & \\
\hline $0 \times 3$ & & & & \\
\hline
\end{tabular}

OX 3.18.04

\begin{tabular}{|l|l|l|l|l|l|}
\hline 2436 & s34 & TT & M & 69 & Tag\# G702 \\
\hline 2437 & s71 & PM & M & 15 & \\
\hline 2438 & s96 & PM & M & 15 & \\
\hline 2439 & s120 & PM & M & 13.5 & \\
\hline 2440 & s117 & PM & M & 15 & \\
\hline 2441 & s118 & PM & M & 15.5 & \\
\hline 2442 & s24 & PM & F & 14 & \\
\hline 2443 & s128 & PM & F & 14.5 & \\
\hline 2444 & s108 & PM & M & 18 & \\
\hline 2445 & s94 & PM & M & 17.5 & \\
\hline 2446 & s83 & PM & F & 14.5 & \\
\hline 2447 & p132 & PM & $?$ & $?$ & \\
\hline 2448 & s70 & ST & $?$ & 4.5 & \\
\hline 2449 & s69 & ST & $?$ & 5.0 & \\
\hline
\end{tabular}

OX 3.19 .04 


\begin{tabular}{|l|l|l|l|l|l|}
\hline 2450 & s142 & PM & F & 20 & \\
\hline 2451 & p132 & PM & F & 12 & \\
\hline 2452 & s63 & PM & F & 15 & \\
\hline 2453 & p60 & PM & F & 12 & \\
\hline 2454 & s11 & PM & F & 16.5 & \\
\hline 2455 & s103 & PM & M & 16.5 & \\
\hline 2456 & s36 & PM & F & 11 & \\
\hline 2457 & s70 & PM & F & 20 & \\
\hline 2458 & s33 & PM & M & 19 & \\
\hline 2459 & s106 & PM & F & 16 & \\
\hline 2460 & s117 & PM & F & 15 & \\
\hline 2461 & m89 & PM & F & 17.5 & \\
\hline 2462 & s94 & PM & M & 16 & \\
\hline RECAP & m116 & TT & M & & \\
\hline 2463 & p67 & SV & $?$ & 4.8 & \\
\hline 2464 & s134 & SV & $?$ & 4.9 & \\
\hline 2465 & s96 & ST & $?$ & 5.8 & \\
\hline
\end{tabular}

FOREST PARK 3.30.04

\begin{tabular}{|l|l|l|l|l|r|}
\hline 2466 & s139 & PM & M & 13.5 & \\
\hline 2467 & p55 & PM & F & 16 & \\
\hline 2468 & s137 & PM & M & 18.5 & \\
\hline 2469 & s126 & PM & M & 15.5 & \\
\hline 2470 & s144 & PM & M & 22 & \\
\hline 2471 & s86 & PM & M & 17 & \\
\hline 2472 & s101 & PM & M & 18 & \\
\hline 2473 & m118 & PM & F & 14.5 & \\
\hline 2474 & s102 & PM & M & 12.5 & \\
\hline 2475 & s82 & PM & M & 16 & \\
\hline 2476 & s119 & PM & M & 15.5 & \\
\hline 2477 & s130 & PM & F & 16.5 & \\
\hline 2478 & s21 & PM & F & 17 & \\
\hline 2479 & s45 & PM & F & 17.5 & \\
\hline 2480 & s44 & PM & M & 16 & \\
\hline 2481 & s99 & PM & F & 14.5 & \\
\hline 2482 & s25 & PM & F & 14 & \\
\hline 2483 & s8 & TT & M & 75 & \\
\hline 2484 & s84 & TT & M & 80 & \\
\hline & s119 & TT & M & 80 & \\
\hline 2486 & t144 & TD & M & 225 & G703 \\
\hline 2487 & s86 & ST & F & 5.0 & \\
\hline 2488 & s63 & PM & M & 18.5 & \\
\hline
\end{tabular}

F.P. 3.31.04
\begin{tabular}{|l|l|l|l|l|l|}
\hline 2490 & s137 & PM & F & 14 & \\
\hline 2491 & s44 & PM & M & 20 & \\
\hline 2492 & s119 & PM & M & 20.5 & \\
\hline 2493 & s45 & PM & F & 17.5 & \\
\hline 2494 & m18 & PM & F & 21.5 & \\
\hline 2495 & p120 & PM & F & 24 & \\
\hline 2496 & s117 & PM & M & 20.5 & \\
\hline
\end{tabular}




\begin{tabular}{|l|l|l|l|l|l|}
\hline 2497 & s130 & PM & M & 20.5 & \\
\hline 2498 & s86 & PM & M & 16.5 & \\
\hline 2499 & s144 & PM & F & 17.5 & \\
\hline 2500 & s80 & PM & M & 19 & \\
\hline 2501 & s64 & PM & F & 15.5 & \\
\hline 2502 & s118 & PM & F & 12.5 & \\
\hline 2503 & p120 & PM & F & 7 & \\
\hline 2504 & s55 & PM & M & 8 & \\
\hline 2505 & p55 & PM & F & 7 & \\
\hline 2506 & p55 & PM & M & 7.5 & \\
\hline 2507 & s104 & ST & $?$ & 5.2 & \\
\hline 2508 & s102 & PM & $?$ & $?$ & \\
\hline
\end{tabular}

\begin{tabular}{|c|c|c|c|c|c|}
\hline 2510 & s79 & TT & $\mathrm{M}$ & 79 & G706 \\
\hline 2511 & $\mathrm{~s} 12$ & $\mathrm{PM}$ & $\overline{\mathrm{M}}$ & 22.5 & \\
\hline 2512 & s119 & $\mathrm{PM}$ & $\bar{M}$ & 14.5 & \\
\hline 2513 & $\mathrm{~s} 21$ & PM & $\mathbf{M}$ & 19.5 & \\
\hline 2514 & s117 & $\mathrm{PM}$ & $\mathrm{F}$ & 21.5 & \\
\hline 2515 & s102 & PM & $\mathrm{M}$ & 15 & \\
\hline 2516 & s103 & PM & $\overline{\mathbf{M}}$ & 13.5 & \\
\hline 2517 & s104 & $\overline{\mathrm{PM}}$ & $\mathbf{M}$ & 13.5 & \\
\hline 2518 & $\mathrm{~s} 120$ & PM & $F$ & 7 & \\
\hline 2519 & s5 & $\overline{P M}$ & $\overline{\mathbf{M}}$ & 19 & \\
\hline 2520 & $\mathrm{~m} 10$ & PM & $\mathrm{F}$ & 26.5 & \\
\hline 2521 & s137 & PM & $\mathbf{M}$ & 16 & \\
\hline 2522 & $\mathrm{~s} 31$ & PM & $\bar{M}$ & 17.5 & \\
\hline 2523 & s83 & PM & $\mathbf{M}$ & 20 & \\
\hline 2524 & s75 & PM & F & 15.5 & \\
\hline 2525 & $\mathrm{~s} 70$ & PM & $F$ & 19.5 & \\
\hline 2526 & $\mathrm{~s} 40$ & PM & $F$ & 16 & \\
\hline 2527 & $\mathrm{p} 120$ & PM & $\bar{M}$ & 6.5 & \\
\hline 2528 & $\mathrm{~s} 142$ & PM & $\mathbf{M}$ & 19.5 & \\
\hline 2529 & p24 & PM & $\bar{M}$ & 18.5 & \\
\hline 2530 & s101 & SV & $\bar{F}$ & 4.7 & \\
\hline 2531 & $\mathrm{p} 12$ & ST & $?$ & 5.9 & 3 \\
\hline
\end{tabular}

\section{F.P. 4.2.04}

\begin{tabular}{|l|l|l|l|l|l|}
\hline 2532 & p120 & PM & M & 8.5 & \\
\hline 2533 & p120 & PM & M & 7.5 & \\
\hline 2534 & s7 & PM & M & 16 & \\
\hline 2535 & s97 & PM & F & 19 & \\
\hline 2536 & s69 & PM & M & 11 & \\
\hline 2537 & s122 & PM & M & 19 & \\
\hline 2538 & p91 & PM & M & 13 & \\
\hline 2539 & p19 & PM & M & 20 & \\
\hline 2540 & s32 & PM & F & 14 & \\
\hline 2541 & s80 & PM & F & 13 & \\
\hline 2542 & s89 & PM & M & 13 & \\
\hline 2543 & s113 & PM & M & 16 & \\
\hline 2544 & p120 & PM & M & 11.5 & \\
\hline
\end{tabular}




\begin{tabular}{|l|l|l|l|l|l|}
\hline 2546 & s118 & PM & F & 12.5 & \\
\hline 2547 & s117 & SV & $?$ & 6.9 & \\
\hline 2548 & s73 & ST & M & 5.6 & \\
\hline
\end{tabular}

Tryon Creek 4.6.04

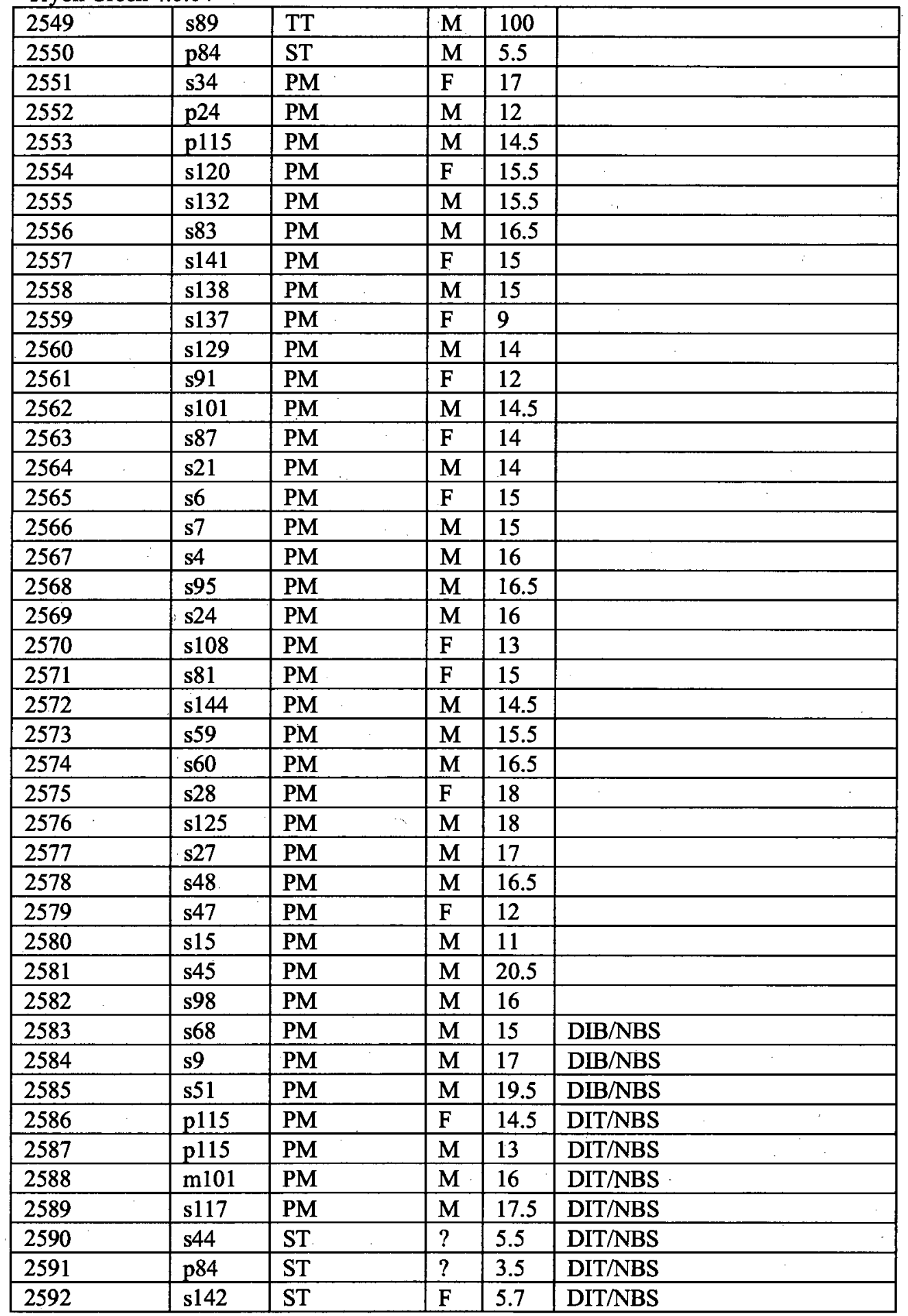

T.C. 4.7.04 


\begin{tabular}{|c|c|c|c|c|c|}
\hline 2593 & s9 & PM & $\bar{M}$ & 18.5 & \\
\hline 2594 & $\mathrm{s88}$ & PM & $\mathbf{M}$ & 21.5 & \\
\hline 2595 & s96 & PM & $F$ & 19.5 & \\
\hline 2596 & $\mathrm{s80}$ & PM & $\mathbf{M}$ & 17.5 & BROKEN TAIL \\
\hline 2597 & s72 & PM & $\mathrm{F}$ & 19 & \\
\hline 2598 & s4 & PM & $\mathrm{F}$ & 17 & \\
\hline 2599 & s144 & PM & $\mathrm{F}$ & 11.5 & JUVY \\
\hline 2600 & s101 & PM & $\mathrm{M}$ & 14 & SUB A \\
\hline 2601 & s51 & PM & $\bar{F}$ & 22.5 & PREG \\
\hline 2602 & s117 & PM & $\bar{M}$ & 13 & SUB A \\
\hline 2603 & s103 & $\mathbf{P M}$ & $\mathrm{M}$ & 21 & \\
\hline 2604 & s115 & PM & $\bar{F}$ & 20 & \\
\hline 2605 & s107 & PM & $\mathrm{M}$ & 17.5 & \\
\hline 2606 & s118 & PM & $F$ & 13.5 & SUB A \\
\hline 2607 & $\mathbf{s 1 3 0}$ & PM & $\bar{F}$ & 14.5 & SUB A \\
\hline 2608 & s128 & PM & $F$ & 18.5 & \\
\hline 2609 & s142 & PM & F & 13 & JUVY \\
\hline 2610 & s33 & PM & $F$ & 14 & \\
\hline 2611 & s132 & PM & $F$ & 19 & \\
\hline 2612 & $\mathrm{p} 48$ & $\mathbf{P M}$ & $\bar{F}$ & 17 & \\
\hline 2613 & s48 & PM & $\mathrm{M}$ & 21 & DIB/NBS \\
\hline 2614 & s44 & PM & $\mathrm{F}$ & 14 & DIB/NBS \\
\hline 2615 & $s 42$ & PM & $\mathrm{F}$ & 19.5 & DIB/NBS \\
\hline 2616 & s12 & PM & $\mathbf{M}$ & 16 & DIT/NBS \\
\hline 2617 & s141 & PM & $\mathrm{F}$ & 12 & DIT/NBS \\
\hline 2618 & s135 & PM & $F$ & 10 & DIT/NBS \\
\hline 2619 & $\mathrm{~s} 22$ & $\mathrm{MO}$ & $\mathrm{F}$ & 20 & DIT/NBS \\
\hline 2620 & s81 & ST & $?$ & 6 & DIT/NBS \\
\hline 2621 & s35 & PM & $?$ & $?$ & ESCAPED \\
\hline
\end{tabular}

\section{T.C. 4.8 .04}

\begin{tabular}{|l|l|l|l|l|l|}
\hline 2622 & $\mathrm{~s} 105$ & TT & M & 75 & RELEASED \\
\hline 2623 & $\mathrm{~m} 105$ & TT & & & RELEASED \\
\hline 2624 & $\mathrm{~m} 113$ & TT & M & 80 & RELEASED \\
\hline 2625 & $\mathrm{~s} 142$ & PM & M & 11.5 & \\
\hline 2626 & $\mathrm{~s} 36$ & PM & F & 15.5 & \\
\hline 2627 & $\mathrm{~s} 36$ & PM & M & 11.5 & \\
\hline 2628 & $\mathrm{~s} 130$ & PM & M & 14.5 & \\
\hline 2629 & $\mathrm{~s} 64$ & PM & M & 13.5 & \\
\hline 2630 & $\mathrm{~s} 26$ & PM & M & 18 & \\
\hline 2631 & $\mathrm{~s} 33$ & PM & M & 16.5 & \\
\hline 2632 & $\mathrm{~m} 119$ & PM & M & 13.5 & \\
\hline 2633 & $\mathrm{~s} 11$ & PM & M & 13.5 & \\
\hline 2634 & $\mathrm{~s} 132$ & PM & M & 14 & \\
\hline 2635 & $\mathrm{~s} 109$ & PM & F & 14 & \\
\hline 2636 & $\mathrm{~s} 120$ & PM & F & 11 & \\
\hline 2637 & $\mathrm{~s} 12$ & PM & F & 11 & \\
\hline 2638 & $\mathrm{~s} 34$ & PM & M & 13.5 & \\
\hline 2639 & $\mathrm{~s} 113$ & PM & M & 11.5 & DIT/NBS \\
\hline 2640 & $\mathrm{~s} 117$ & PM & F & 20 & DIB/NBS \\
\hline
\end{tabular}




\begin{tabular}{|c|c|c|c|c|c|}
\hline 2641 & s57 & PM & $\mathbf{M}$ & 16 & DIB/NBS \\
\hline 2642 & s107 & TT & $\mathbf{M}$ & 70 & DIT/NBS \\
\hline 2643 & p79 & ST & $F$ & 4.9 & DIT/NBS \\
\hline 2644 & $\mathrm{p} 31$ & PM & $\mathbf{M}$ & 6.5 & DIT/NBS \\
\hline 2645 & s137 & ST & $?$ & 5.6 & DIT/NBS \\
\hline \multicolumn{6}{|c|}{ T.C. 4.9 .04} \\
\hline 2646 & $\mathrm{~s} 125$ & TT & $\mathbf{M}$ & 78 & \\
\hline 2647 & s129 & PM & $\mathbf{M}$ & 16 & \\
\hline 2648 & $\mathrm{~s} 22$ & $\mathrm{PM}$ & $F$ & 14 & \\
\hline 2649 & $\mathrm{~s} 132$ & $\mathrm{PM}$ & $\mathbf{M}$ & 14.5 & \\
\hline 2650 & $\mathrm{~s} 47$ & PM & $F$ & 13 & \\
\hline 2651 & $\mathrm{~s} 12$ & PM & $\mathbf{M}$ & 17 & \\
\hline 2652 & s11 & PM & $\mathbf{M}$ & 14 & \\
\hline 2653 & s57 & PM & $\mathbf{F}$ & 23 & PREG \\
\hline 2654 & $\mathrm{~s} 121$ & PM & $F$ & 12 & \\
\hline 2655 & s45 & $\mathrm{PM}$ & $\mathbf{M}$ & 20 & \\
\hline 2656 & s? & PM & $\mathbf{M}$ & 21 & \\
\hline 2657 & s137 & ST & $?$ & 4.5 & DIB/NBS \\
\hline 2658 & p132 & NG & $?$ & 7.6 & DIB/NBS \\
\hline 2659 & p96 & ST & $?$ & 4.6 & DIT/NBS \\
\hline \multirow[t]{2}{*}{2660} & $\mathrm{~s} 88$ & TT & - & - & DIT/NBS \\
\hline & s105 & TT & - & - & RELEASED \\
\hline
\end{tabular}

TRNWR 4.20.04

\begin{tabular}{|l|l|l|l|l|l|}
\hline 2661 & s36 & PM & F & 15 & \\
\hline 2662 & s18 & PM & F & 16 & \\
\hline 2663 & s14 & PM & F & 17 & \\
\hline 2664 & s124 & PM & M & 16 & \\
\hline 2665 & s126 & PM & M & 19.5 & \\
\hline 2666 & s120 & PM & M & 28 & \\
\hline 2667 & p127 & PM & M & 16 & \\
\hline 2668 & s105 & PM & F & 21 & PREG \\
\hline 2669 & s67 & PM & M & 18 & \\
\hline 2670 & s53 & PM & M & 18.5 & \\
\hline 2671 & s131 & PM & M & 18 & \\
\hline 2672 & p132 & PM & F & 16.5 & \\
\hline 2673 & s23 & PM & F & 17.5 & \\
\hline 2675 & s45 & PM & F & 18 & \\
\hline 2676 & s44 & PM & M & 17 & \\
\hline 2677 & s48 & PM & F & 19.5 & \\
\hline 2678 & s32 & PM & M & 14.5 & \\
\hline 2679 & s34 & ST & F & 6.2 & RECAP \\
\hline 2680 & t19 & SN & F & 550 & TAGGED G711 RELEASED \\
\hline 2681 & p91 & ST & F & 4.6 & DIT \\
\hline 2682 & p88 & SV & F & 3.6 & DIT \\
\hline 2683 & p64 & SV & $?$ & 4.2 & DIT \\
\hline 2684 & p64 & ST & F & 5.9 & DIT \\
\hline 2685 & p64 & SV & $?$ & 4.8 & DIT \\
\hline 2686 & p127 & PM & F & 12 & DIT \\
\hline & s84 & ME & M & 75 & DIT \\
\hline
\end{tabular}


TRNWR 4.21.04

\begin{tabular}{|l|l|l|l|l|l|}
\hline 2687 & s113 & PM & F & 14.5 & \\
\hline 2688 & s131 & PM & F & 13 & \\
\hline 2689 & s80 & PM & M & 13.5 & \\
\hline 2690 & s23 & PM & M & 17.5 & \\
\hline 2691 & s142 & PM & M & 17.5 & \\
\hline 2692 & p48 & PM & M & 17.5 & DIT/NBS \\
\hline 2693 & s83 & SV & F & 5.7 & DIT/NBS \\
\hline 2694 & s117 & SV & M & 6.2 & RELEASED/NBS \\
\hline
\end{tabular}

TRNWR 4.22.04

\begin{tabular}{|l|l|l|l|l|l|}
\hline 2695 & s17 & PM & M & 17.5 & \\
\hline 2696 & s97 & SV & M & 6.2 & DIT/NBS \\
\hline 2697 & s16 & ST & F & 6.1 & RELEASED \\
\hline
\end{tabular}

TRNWR 4.23.04

\begin{tabular}{|l|l|l|l|l|l|}
\hline 2698 & s116 & PM & F & 18 & \\
\hline 2699 & s107 & SV & $?$ & 3.8 & DIT/NBS \\
\hline 2700 & s105 & SV & M & 6.4 & DIT/NBS \\
\hline 2701 & $?$ & ST & F & 5.8 & DIT/NBS \\
\hline
\end{tabular}

Powell Butte 4.27.04

\begin{tabular}{|l|l|l|l|l|l|}
\hline 2702 & p144 & PM & F & 13.5 & \\
\hline 2703 & s6 & PM & M & 16.5 & \\
\hline 2704 & s126 & PM & M & 15 & \\
\hline 2705 & s56 & PM & M & 16.5 & \\
\hline 2706 & s79 & PM & F & 15 & \\
\hline 2707 & s82 & PM & M & 19 & \\
\hline 2708 & s116 & PM & M & 14.5 & \\
\hline 2709 & s84 & PM & M & & \\
\hline 2710 & s43 & PM & F & 13 & \\
\hline 2711 & t24 & SN & F & 700 & TAG G712 \\
\hline 2712 & p136 & SV & $?$ & & \\
\hline 2713 & p136 & SV & $?$ & 3.2 & \\
\hline
\end{tabular}

\section{P.B. 4.28.04}

\begin{tabular}{|l|l|l|l|l|l|}
\hline 2714 & m122 & PM & F & 15 & \\
\hline 2715 & s134 & PM & M & 18 & \\
\hline 2716 & s139 & PM & F & 13.5 & \\
\hline 2717 & s130 & PM & M & 15.5 & \\
\hline 2718 & s5 & PM & M & 15 & \\
\hline 2719 & p144 & PM & M & 15 & \\
\hline 2720 & p144 & PM & M & 16 & \\
\hline 2721 & s117 & PM & F & 9 & \\
\hline 2722 & s85 & PM & M & 16 & \\
\hline 2723 & p84 & PM & F & 14.5 & \\
\hline 2724 & s56 & PM & M & 15.5 & \\
\hline 2725 & s6 & PM & F & 13 & \\
\hline 2726 & s126 & MO & F & 17 & \\
\hline 2728 & p139 & SV & M & 4.4 & \\
\hline 2729 & s136 & SV & M & 6.0 & \\
\hline 2730 & p136 & MO & F & 17.5 & \\
\hline 2731 & p57 & MO & M & 24 & \\
\hline
\end{tabular}




\begin{tabular}{|c|c|c|c|c|c|}
\hline 2732 & p139 & SV & $\mathbf{M}$ & 5.0 & \\
\hline 2733 & p139 & SV & $?$ & 6.0 & \\
\hline \multicolumn{6}{|c|}{ P.B. 4.29 .04} \\
\hline 2734 & S133 & PM & $\mathbf{M}$ & 16 & \\
\hline 2735 & S11 & $\mathrm{PM}$ & $\mathbf{M}$ & 17 & \\
\hline 2736 & S8 & $\mathrm{PM}$ & $\mathrm{M}$ & 16 & \\
\hline 2737 & P20 & $\overline{\mathrm{PM}}$ & $\bar{F}$ & 15.5 & \\
\hline 2738 & S17 & PM & M & 14 & \\
\hline 2739 & S80 & PM & $M$ & 16 & \\
\hline 2740 & S11 & $\mathrm{PM}$ & $\bar{M}$ & 15 & \\
\hline 2741 & S79 & $\mathrm{PM}$ & $\mathrm{F}$ & 12 & \\
\hline 2742 & S44 & $\mathrm{PM}$ & $\mathrm{M}$ & 17 & \\
\hline 2743 & P31 & PM & $\mathbf{M}$ & 17 & \\
\hline 2744 & S118 & PM & $\mathbf{M}$ & 16.5 & \\
\hline 2745 & $\mathrm{P} 4$ & ST & $?$ & 3.5 & \\
\hline 2746 & S108 & PM & $?$ & & \\
\hline 2747 & P108 & SV & $?$ & & \\
\hline 2748 & $\mathrm{P} 20$ & SV & ? & 3.5 & \\
\hline 2750 & T144 & Sba & $?$ & & \\
\hline
\end{tabular}

P.B. 4.30 .04

\begin{tabular}{|l|l|l|l|l|l|}
\hline 2751 & $\mathrm{p} 96$ & $\mathrm{PM}$ & $\mathrm{M}$ & 16.5 & \\
\hline 2752 & $\mathrm{~s} 36$ & $\mathrm{PM}$ & $?$ & $?$ & \\
\hline 2753 & $\mathrm{~s} 35$ & $\mathrm{PM}$ & $\mathrm{M}$ & 14 & \\
\hline 2754 & $\mathrm{~s} 128$ & $\mathrm{PM}$ & $\mathrm{M}$ & 14.5 & \\
\hline 2755 & $\mathrm{p} 144$ & $\mathrm{PM}$ & $\mathrm{F}$ & 13 & \\
\hline 2756 & $\mathrm{~s} 80$ & $\mathrm{PM}$ & $\mathrm{M}$ & 17.5 & \\
\hline 2757 & $\mathrm{p} 14$ & $\mathrm{PM}$ & $\mathrm{F}$ & 13.5 & \\
\hline 2758 & $\mathrm{p} 115$ & $\mathrm{PM}$ & $\mathrm{M}$ & 14.5 & \\
\hline 2759 & $\mathrm{~s} 7$ & $\mathrm{PM}$ & $\mathrm{F}$ & 17.5 & \\
\hline 2760 & $\mathrm{~s} 39$ & $\mathrm{PM}$ & $\mathrm{F}$ & 18 & \\
\hline 2761 & $\mathrm{~s} 77$ & $\mathrm{PM}$ & $\mathrm{F}$ & 12 & \\
\hline 2762 & $\mathrm{p} 76$ & $\mathrm{SV}$ & $?$ & 4.5 & \\
\hline 2763 & $\mathrm{p} 139$ & $\mathrm{SV}$ & $\mathrm{M}$ & 6.0 & \\
\hline 2764 & $\mathrm{p} 20$ & $\mathrm{SV}$ & $?$ & 3.4 & \\
\hline 2765 & $\mathrm{~s} 2$ & $\mathrm{ST}$ & $\mathrm{F}$ & 5.3 & \\
\hline 2766 & $\mathrm{t} 72$ & $\mathrm{DV}$ & $\mathrm{M}$ & $?$ & \\
\hline
\end{tabular}

OXBOW 5.11 .04

\begin{tabular}{|l|l|l|l|l|l|}
\hline 2767 & s72 & TT & M & 80 & TAG G722 RELEASED \\
\hline 2768 & m141 & GS & F & 118 & TAG G726 RELEASED \\
\hline 2769 & t84 & NF & M & 100 & TAG G723 \\
\hline 2770 & m17 & TD & F & 228 & TAG G719 \\
\hline 2771 & s107 & PM & F & 19.5 & \\
\hline 2772 & s68 & PM & M & 14.5 & \\
\hline 2773 & s71 & PM & M & 14 & \\
\hline 2774 & s116 & PM & M & 16.5 & \\
\hline 2775 & s118 & PM & M & 17 & \\
\hline 2776 & s60 & PM & M & 16 & \\
\hline 2777 & s19 & PM & F & 15.5 & \\
\hline 2778 & p109 & PM & M & 18 & \multicolumn{1}{|l|}{} \\
\hline
\end{tabular}




\begin{tabular}{|l|l|l|l|l|l|}
\hline 2779 & s27 & PM & M & 18 & \\
\hline 2780 & s119 & PM & M & 17 & \\
\hline 2781 & s144 & PM & F & 14.5 & \\
\hline 2782 & s22 & PM & M & 19 & \\
\hline 2783 & s84 & PM & M & 14.5 & \\
\hline 2784 & s82 & PM & F & 15 & \\
\hline 2785 & p64 & PM & F & 15.5 & \\
\hline 2786 & p91 & PM & M & 13.5 & \\
\hline 2787 & s93 & PM & M & 12 & \\
\hline 2788 & s55 & ST & $?$ & 4.0 & \\
\hline 2789 & p60 & SV & F & 5.5 & \\
\hline 2790 & s28 & ST & $?$ & 4.6 & \\
\hline 2791 & p126 & ST & F & 5.5 & \\
\hline 2792 & p1332 & ST & F & 6 & \\
\hline 2793 & p96 & ST & F & 6.2 & \\
\hline 2794 & p96 & ST & M & 4.6 & \\
\hline 2795 & p64 & NG & $?$ & 11 & \\
\hline 2796 & p40 & SB & M & 21 & \\
\hline 2797 & s81 & SV & M & 7.5 & \\
\hline
\end{tabular}

OXBOW 5.12.04

\begin{tabular}{|l|l|l|l|l|l|}
\hline 2798 & S66 & TT & M & 85 & TAG G728 \\
\hline 2799 & S31 & TT & M & 70 & TAG G720 \\
\hline 2800 & S115 & GS & M & 130 & TAG G727 \\
\hline 2801 & S95 & GS & M & 175 & TAG G729 \\
\hline 2802 & S12 & TT & F & 71 & TAG G 730 \\
\hline 2803 & S70 & PM & F & 16.5 & \\
\hline 2804 & S107 & PM & M & 17 & \\
\hline 2805 & S69 & PM & M & 20 & \\
\hline 2806 & S141 & PM & M & 16 & \\
\hline 2807 & S23 & PM & F & 15 & \\
\hline 2808 & S8 & PM & F & 17 & \\
\hline 2809 & P52 & ST & M & 4.5 & \\
\hline 2810 & P24 & ST & $?$ & 4.0 & \\
\hline 2811 & P96 & ST & $?$ & 4.5 & \\
\hline
\end{tabular}

OX 5.13.04

\begin{tabular}{|l|l|l|l|l|l|}
\hline 2812 & s14 & GS & F & 13.5 & TAG G731 \\
\hline 2813 & s107 & PM & M & 18.5 & \\
\hline 2814 & s106 & PM & M & 16 & \\
\hline 2815 & s24 & PM & M & 18.5 & \\
\hline 2816 & s53 & SV & M & 7.6 & \\
\hline 2817 & p84 & ST & F & 4.6 & \\
\hline 2818 & p112 & ST & M & 4.7 & \\
\hline 2819 & p115 & ST & $?$ & 4.0 & \\
\hline 2820 & p127 & ST & F & 4.6 & \\
\hline 2821 & p88 & ST & F & 3.8 & \\
\hline 2822 & p28 & MO & F & 23.5 & \\
\hline
\end{tabular}

\section{OX 5.14.04}

\begin{tabular}{|l|l|l|l|l|l|}
\hline 2823 & M110 & GS & F & 150 & TAG G732 \\
\hline 2824 & s67 & TT & M & 70 & TAG G733 \\
\hline
\end{tabular}




\begin{tabular}{|l|l|l|l|l|l|}
\hline 2826 & t48 & TD & M & 190 & \\
\hline 2827 & s5 & PM & F & 17 & \\
\hline 2828 & s48 & PM & M & 17 & \\
\hline 2829 & & TT & M & 70 & \\
\hline 2830 & p64 & ST & $?$ & 4 & \\
\hline 2831 & p127 & NG & $?$ & 7 & \\
\hline 2832 & s48 & MO & M & 10 & \\
\hline
\end{tabular}

FOREST PARK 5.18.04

\begin{tabular}{|l|l|l|l|l|l|}
\hline 2833 & s79 & TT & m & 63 & TAG G734 RELEASED \\
\hline 2834 & s5 & PM & m & 19.5 & \\
\hline 2835 & m12 & PM & m & 16.5 & \\
\hline 2836 & s90 & PM & f & 15 & \\
\hline 2837 & s83 & PM & m & 21 & \\
\hline 2838 & m83 & PM & f & 16.5 & \\
\hline 2839 & s91 & PM & f & 15 & \\
\hline 2840 & s81 & PM & f & 19.5 & \\
\hline 2841 & m131 & PM & f & 16 & \\
\hline 2842 & s23 & PM & m & 13 & \\
\hline 2843 & s80 & PM & m & 15.5 & \\
\hline 2844 & s84 & PM & m & 15 & \\
\hline 2845 & s96 & PM & m & 14.5 & \\
\hline 2846 & s24 & PM & f & 13.5 & \\
\hline 2847 & s70 & PM & m & 16 & \\
\hline 2848 & s19 & PM & m & 17.5 & \\
\hline 2849 & s125 & PM & m & 19.5 & \\
\hline 2850 & s58 & PM & f & 14 & \\
\hline 2851 & s53 & PM & f & 12 & \\
\hline 2852 & s71 & PM & m & 20 & \\
\hline 2853 & s44 & PM & m & 16 & \\
\hline 2854 & s46 & PM & f & 16.5 & \\
\hline 2855 & s95 & PM & m & 14.5 & \\
\hline 2856 & p84 & PM & f & 14.5 & \\
\hline 2857 & s34 & PM & f & 17 & \\
\hline 2858 & s103 & PM & f & 18.5 & \\
\hline 2859 & p120 & PM & m & 18.5 & \\
\hline 2860 & s78 & PM & m & 17.5 & \\
\hline 2861 & s107 & PM & m & 15 & \\
\hline 2862 & s115 & PM & m & 14.5 & \\
\hline 2863 & s119 & PM & m & 17.5 & \\
\hline 2864 & s141 & PM & f & 14.5 & \\
\hline 2865 & s144 & PM & m & 17.5 & \\
\hline 2866 & p115 & PM & m & 14.5 & \\
\hline 2867 & p120 & PM & m & 15.5 & \\
\hline 2868 & m115 & PM & f & 16 & \\
\hline 2869 & p120 & PM & m & 17.5 & \\
\hline 2870 & s & m & m & 14.5 & \\
\hline 2871 & s & & & \\
\hline 2872 & & m & 15.5 & \\
\hline 2873 & & & & \\
\hline
\end{tabular}




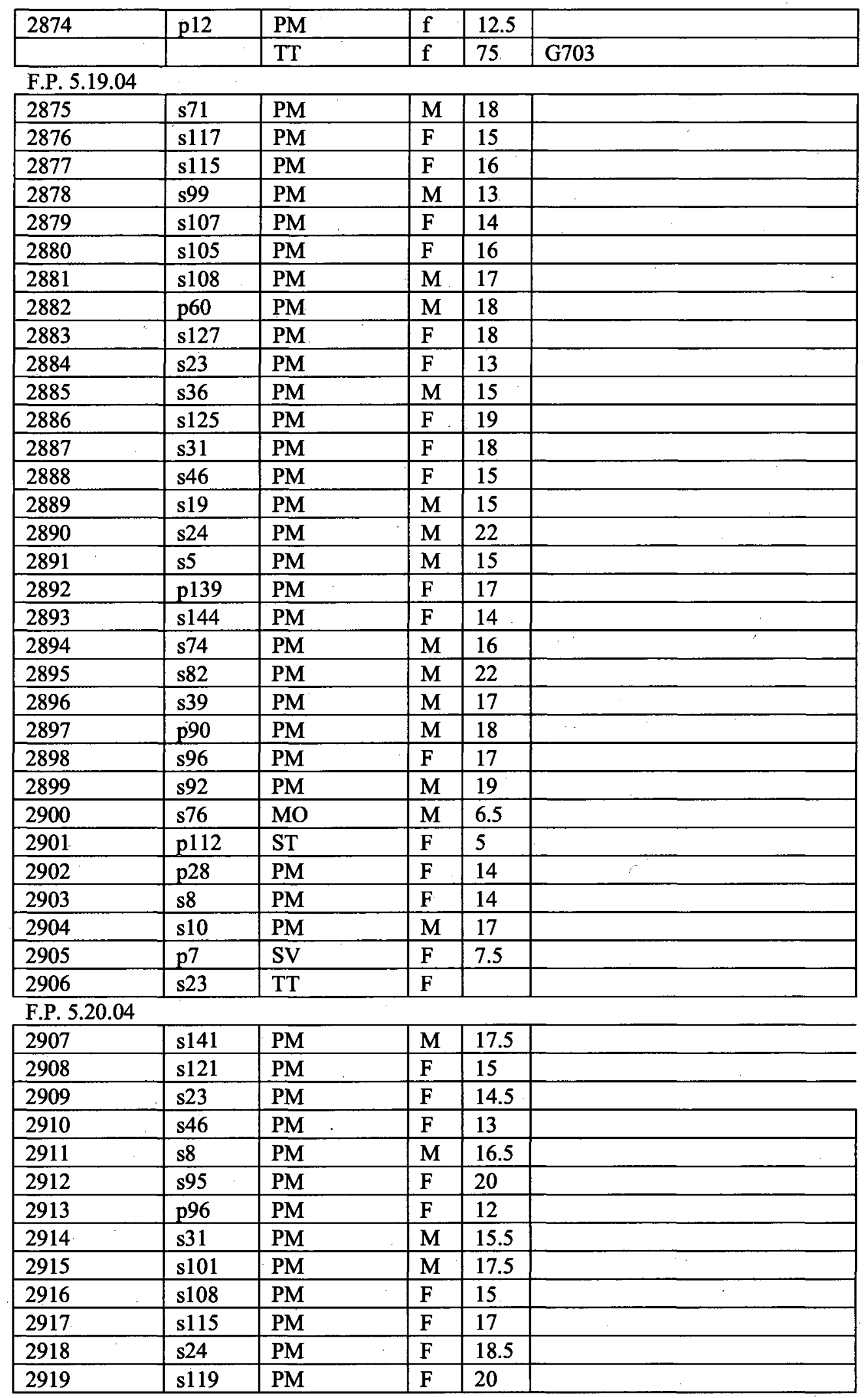




\begin{tabular}{|l|l|l|l|l|l|}
\hline 2920 & $\mathrm{~s} 79$ & PM & F & 20 & \\
\hline 2921 & m80 & PM & F & 18 & \\
\hline 2922 & $\mathrm{~s} 36$ & PM & M & 16.5 & \\
\hline 2923 & $\mathrm{~s} 45$ & PM & M & 17 & \\
\hline 2924 & p64 & PM & M & 13 & \\
\hline 2925 & p60 & PM & F & 13 & \\
\hline 2926 & s71 & PM & F & 15 & \\
\hline 2927 & p55 & PM & F & 15 & \\
\hline 2928 & s80 & PM & M & 17.5 & \\
\hline 2929 & s78 & ST & $?$ & 5.4 & \\
\hline 2930 & s76 & MO & $?$ & 6.5 & \\
\hline 2931 & p19 & ST & $?$ & 4.5 & \\
\hline 2932 & p31 & SV & $?$ & 4.9 & \\
\hline 2933 & p43 & SV & $?$ & 6.7 & \\
\hline 2934 & $\mathrm{~s} 131$ & PM & F & 15.5 & \\
\hline
\end{tabular}

\section{F.P. 5.21.04}

\begin{tabular}{|l|l|l|l|l|l|}
\hline 2935 & s58 & PM & M & 15 & \\
\hline 2936 & s24 & PM & F & 14.5 & \\
\hline 2937 & s104 & PM & F & 13.5 & \\
\hline 2938 & s108 & PM & M & 16.5 & \\
\hline 2939 & s74 & PM & F & 19.5 & \\
\hline 2940 & s51 & PM & F & 14 & \\
\hline 2941 & s35 & PM & F & 13.5 & \\
\hline 2942 & s135 & PM & F & 12 & \\
\hline 2943 & s8 & PM & M & 17.5 & \\
\hline 2944 & s132 & PM & M & 18 & \\
\hline 2945 & s124 & ST & $?$ & 4.4 & \\
\hline 2946 & p115 & SV & F & 7.9 & \\
\hline
\end{tabular}

6.8.04 TRYON CREEK

\begin{tabular}{|l|l|l|l|l|l|}
\hline 2947 & $\mathrm{~m} 82$ & PM & M & 19 & WET \\
\hline 2948 & $\mathrm{~m} 127$ & PM & M & 23.5 & WET \\
\hline 2949 & p72 & PM & F & 17.5 & \\
\hline 2950 & s69 & PM & F & 18.5 & \\
\hline 2951 & s88 & PM & M & 19.5 & \\
\hline 2952 & s96 & PM & M & 20.5 & \\
\hline 2953 & s77 & PM & M & 24 & \\
\hline 2954 & s39 & PM & M & 20.5 & \\
\hline 2955 & s6 & PM & M & 16.5 & \\
\hline 2956 & s48 & PM & F & 15 & \\
\hline 2957 & s24 & PM & M & 23 & \\
\hline 2958 & s23 & PM & F & 17.5 & \\
\hline 2959 & m118 & PM & F & 20 & \\
\hline 2960 & s132 & PM & M & 20 & \\
\hline 2961 & s130 & PM & M & 19 & \\
\hline 2962 & m12 & PM & M & 20.5 & \\
\hline 2963 & s107 & TT & F & 92 & TAGGED G735 \\
\hline 2964 & s76 & GS & M & 135 & TAGGED G736 \\
\hline 2965 & s59 & ST & $?$ & 6.4 & DIT/NBS \\
\hline 2966 & p136 & SV & $?$ & 6.6 & DIT/NBS \\
\hline
\end{tabular}




\begin{tabular}{|c|c|c|c|c|c|}
\hline 2967. & p132 & PM & $\mathbf{M}$ & 23 & DIT/NBS \\
\hline 2968 & $\mathrm{~m} 47$ & PM & $\mathbf{M}$ & 21 & DIT/NBS \\
\hline 2969 & $\mathrm{~m} 113$ & TT & $\mathbf{M}$ & 82 & DIT/NBS \\
\hline 2970 & $\mathrm{p} 144$ & ScT & $?$ & 110 & DIT/NBS \\
\hline \multicolumn{6}{|c|}{ T.C. 6.9 .04} \\
\hline 2971 & $\mathrm{~S} 12$ & $\mathrm{PM}$ & $\mathbf{M}$ & 19 & \\
\hline 2972 & S18 & $\mathrm{PM}$ & $\mathbf{M}$ & 18.5 & \\
\hline 2973 & S66 & PM & $\mathrm{M}$ & 20 & \\
\hline 2974 & M59 & PM & M & 18 & \\
\hline 2975 & S70 & $\mathrm{PM}$ & $\mathbf{M}$ & 15 & \\
\hline 2976 & S117 & $\mathrm{PM}$ & $\mathrm{M}$ & 16.5 & \\
\hline 2977 & $\mathbf{S 1 0 6}$ & $\mathrm{PM}$ & $\mathrm{F}$ & 21 & \\
\hline 2978 & S139 & PM & $\mathrm{F}$ & 14.5 & \\
\hline 2979 & S143 & $\mathrm{PM}$ & $\mathrm{M}$ & 24 & \\
\hline 2980 & S142 & $\mathrm{PM}$ & $\mathrm{M}$ & 20 & \\
\hline 2981 & S107 & PM & $\mathbf{M}$ & 22 & \\
\hline 2982 & S118 & $\mathrm{PM}$ & $\mathrm{M}$ & 19 & \\
\hline 2983 & S47 & $\mathrm{PM}$ & $\mathrm{F}$ & 13 & \\
\hline 2984 & S32 & PM & $F$ & 15 & \\
\hline 2985 & P137 & MO & $\mathbf{M}$ & 26 & DIT/NBS \\
\hline 2986 & P96 & PM & $\mathrm{M}$ & 24 & DIT/NBS \\
\hline 2987 & P31 & ST & $?$ & 5.2 & DIT/NBS \\
\hline 2988 & P31 & ST & $?$ & 5.4 & DIT/NBS \\
\hline 2989 & P124 & SV & $?$ & 6.5 & DIT/NBS \\
\hline 2990 & P127 & ST & $?$ & 5.3 & DIT/NBS \\
\hline 2991 & P48 & ST & $?$ & 4.7 & DIT/NBS \\
\hline 2992 & P48 & ST & $?$ & 6.5 & DIT/NBS \\
\hline 2993 & $\mathrm{P} 48$ & ST & $?$ & 6.2 & DIT/NBS \\
\hline : & S108 & TT & - & & RECAP G735 \\
\hline
\end{tabular}

T.C. 6.10 .04
\begin{tabular}{|l|l|l|l|l|l|}
\hline 2994 & p 132 & PM & M & 18 & \\
\hline 2995 & m58 & PM & F & 17 & \\
\hline 2996 & s106 & PM & F & 18 & \\
\hline 2997 & s114 & PM & F & 24.5 & PREG \\
\hline 2998 & s 45 & PM & F & 22.5 & \\
\hline 2999 & s4 & PM & F & 16.5 & \\
\hline 3000 & s 24 & PM & M & 19 & \\
\hline 3001 & s129 & PM & F & 15 & \\
\hline 3002 & s 75 & PM & F & 20.5 & \\
\hline 3003 & s107 & TT & M & 43 & TAGGED G737 \\
\hline 3004 & t72 & SN & F & 570 & TAGGED G739 \\
\hline 3005 & s 80 & SV & $?$ & 6.1 & RELEASED \\
\hline 3006 & s108 & MT & M & 28.5 & DIT/NBS \\
\hline 3007 & s33 & PM & M & 19 & DIT/NBS \\
\hline 3008 & p100 & NG & $?$ & 13.5 & DIT/NBS \\
\hline 3009 & p100 & NG & $?$ & 12.5 & DIT/NBS \\
\hline 3010 & p100 & ST & $?$ & 6.6 & DIT/NBS \\
\hline
\end{tabular}

T.C. 6.11 .04

\begin{tabular}{|l|l|l|l|l|}
\hline 3011 & s22 & PM & M & 18.5 \\
\hline
\end{tabular}




\begin{tabular}{|l|l|l|l|l|l|}
\hline 3012 & $\mathrm{~s} 80$ & $\mathrm{PM}$ & $\mathrm{M}$ & 18 & \\
\hline 3013 & $\mathrm{~s} 56$ & $\mathrm{PM}$ & $\mathrm{M}$ & 18.5 & \\
\hline 3014 & $\mathrm{~s} 92$ & $\mathrm{PM}$ & $\mathrm{M}$ & 16.5 & \\
\hline 3015 & $\mathrm{p} 96$ & $\mathrm{PM}$ & $\mathrm{M}$ & 19.5 & \\
\hline 3016 & $\mathrm{~s} 45$ & $\mathrm{PM}$ & $\mathrm{M}$ & 17.5 & \\
\hline 3017 & $\mathrm{~s} 14$ & $\mathrm{PM}$ & $\mathrm{M}$ & 18 & \\
\hline 3018 & $\mathrm{~s} 132$ & $\mathrm{PM}$ & $\mathrm{M}$ & 19.5 & DIT/NBS \\
\hline 3019 & $\mathrm{p} 144$ & $\mathrm{ST}$ & $?$ & 5.4 & DIT/NBS \\
\hline 3020 & $\mathrm{p} 100$ & $\mathrm{ST}$ & $?$ & 5.0 & DIT/NBS \\
\hline 3021 & $\mathrm{p} 91$ & $\mathrm{ST}$ & $?$ & 4.5 & DIT/NBS \\
\hline
\end{tabular}

Tualatin River National Wildlife Refuge 6.22.04

\begin{tabular}{|l|l|l|l|l|l|}
\hline 3022 & s82 & TT & M & 62 & TAG G751 \\
\hline 3023 & s77 & PM & M & 13.5 & \\
\hline 3024 & s80 & PM & M & 25 & \\
\hline 3025 & p143 & PM & M & 14 & \\
\hline 3026 & s32 & PM & F & 23.5 & \\
\hline 3027 & s136 & PM & F & 15.5 & \\
\hline 3028 & m24 & PM & M & 14.5 & \\
\hline 3029 & s118 & PM & M & 16 & \\
\hline 3030 & s4 & PM & M & 14 & \\
\hline 3031 & p139 & PM & M & 19 & \\
\hline 3032 & s24 & ST & $?$ & 5.0 & \\
\hline 3033 & p84 & PM & F & 20 & \\
\hline 3034 & p76 & ST & $?$ & 5.3 & \\
\hline 3035 & p67 & ST & $?$ & 5.4 & \\
\hline 3036 & p124 & ST & $?$ & 5.9 & \\
\hline
\end{tabular}

TRNWR 6.23.04

\begin{tabular}{|l|l|l|l|l|l|}
\hline 3037 & s114 & TT & F & 68 & TAG G749 \\
\hline 3038 & p40 & MT & M & 45 & \\
\hline 3039 & s80 & PM & F & 15 & \\
\hline 3040 & s60 & TT & M & 60 & \\
\hline & & & & $23 .$. & \\
\hline 3041 & s20 & PM & F & 5 & \\
\hline 3042 & s95 & PM & M & 16 & \\
\hline 3043 & s87 & PM & M & 14 & \\
\hline 3044 & s140 & PM & M & 24 & \\
\hline 3045 & s116 & PM & M & 23.5 & \\
\hline 3046 & s36 & PM & F & 20 & \\
\hline 3047 & s9 & PM & F & 23.5 & \\
\hline 3048 & s95 & ME & M & 73 & \\
\hline 3049 & s32 & ME & M & 70 & \\
\hline 3050 & s117 & ME & M & 72 & TAG G740 \\
\hline 3051 & p120 & PM & F & 10.5 & \\
\hline
\end{tabular}

TRNWR 6.24.04

\begin{tabular}{|l|l|l|l|l|l|}
\hline 3052 & s103 & PM & F & 13.5 & \\
\hline 3053 & s88 & PM & M & 19 & \\
\hline 3054 & s118 & PM & F & 23 & \\
\hline 3055 & s47 & PM & M & 17 & \\
\hline 3056 & s87 & PM & F & 17 & \\
\hline
\end{tabular}




\begin{tabular}{|l|l|l|l|l|l|}
\hline 3057 & s78 & PM & F & 21 & \\
\hline 3058 & s20 & PM & M & 10 & \\
\hline 3059 & p79 & SV & $?$ & 8.6 & \\
\hline 3060 & p76 & ST & $?$ & 4.3 & \\
\hline & t36 & PL & $?$ & $?$ & \\
\hline
\end{tabular}

TRNWR 6.25.04
\begin{tabular}{|l|l|l|l|l|l|}
\hline 3061 & m29 & PM & M & 15 & \\
\hline 3062 & s141 & PM & M & 18 & \\
\hline 3063 & s80 & SV & $?$ & 4 & \\
\hline 3064 & p 79 & ST & $?$ & 5.5 & \\
\hline 3065 & p 79 & SV & $?$ & 3.5 & \\
\hline 3066 & 77 & PM & M & 4.5 & \\
\hline 3067 & s116 & ME & M & 65 & \\
\hline \multirow{5}{*}{} & t120 & DV & F & & \\
\cline { 2 - 7 } & t48 & DV & M & & \\
\hline
\end{tabular}

Powell Butte 6.29.04

\begin{tabular}{|l|l|l|l|l|l|}
\hline 3068 & $\mathrm{~s} 46$ & MO & F & 16.5 & \\
\hline 3069 & p 64 & PM & F & 11.5 & \\
\hline 3070 & p119 & PM & M & 15 & \\
\hline 3071 & s69 & PM & M & 15.5 & \\
\hline 3072 & $\mathrm{~s} 60$ & PM & F & 19 & \\
\hline 3073 & s68 & PM & F & 17 & \\
\hline 3074 & $?$ & PM & M & 19.5 & \\
\hline 3075 & s48 & PM & M & 16.5 & \\
\hline 3076 & s84 & PM & M & 12 & \\
\hline 3077 & s89 & PM & M & 21 & \\
\hline 3078 & s21 & PM & F & 18 & \\
\hline 3079 & m117 & PM & F & 14 & \\
\hline 3080 & p28 & PM & F & 17 & \\
\hline 3081 & s28 & PM & F & 17.5 & \\
\hline 3082 & s142 & PM & M & 20 & \\
\hline 3083 & s63 & PM & M & 17 & \\
\hline 3084 & s129 & PM & F & 15 & \\
\hline 3085 & s128 & PM & F & 16 & \\
\hline 3086 & p64 & NG & M & 8.5 & \\
\hline 3087 & p4 & SV & $?$ & 3.5 & \\
\hline 3088 & p124 & SV & $?$ & 3.6 & \\
\hline 3089 & p64 & SV & $?$ & 4.1 & \\
\hline 3090 & $\mathrm{~s} 33$ & PM & M & 18.5 & \\
\hline 3091 & p7 & MO & M & 6.9 & \\
\hline 3092 & p20 & SV & F & 4.7 & \\
\hline & m5 & SV & $?$ & & \\
\hline
\end{tabular}

P.B. 6.30 .04
\begin{tabular}{|l|l|l|l|l|l|}
\hline 3093 & p144 & PM & M & 19 & \\
\hline 3094 & s144 & PM & F & 16.5 & \\
\hline 3095 & m129 & PM & F & 14.5 & \\
\hline 3096 & s129 & PM & M & 17.5 & \\
\hline 3097 & s128 & PM & F & 10 & \multicolumn{4}{|c|}{203} \\
\hline 3098 & s 57 & PM & M & 20.5 & \\
\hline
\end{tabular}




\begin{tabular}{|l|l|l|l|l|l|}
\hline 3099 & s72 & PM & M & 16 & \\
\hline 3100 & s24 & PM & M & 18.5 & \\
\hline 3101 & s21 & PM & F & 10 & \\
\hline 3102 & s78 & PM & F & 11 & \\
\hline 3103 & p84 & PM & M & 13.5 & \\
\hline 3104 & p89 & PM & M & 16 & \\
\hline 3105 & s35 & PM & M & 19 & \\
\hline 3106 & s0 & PM & F & 18 & \\
\hline 3107 & p52 & SV & ? & 4.2 & \\
\hline 3108 & p24 & MO & M & 13.5 & \\
\hline 3109 & s117 & PM & M & 132 & \\
\hline 3110 & s127 & PM & F & 8 & \\
\hline 3111 & s31 & SV & $?$ & 5.2 & \\
\hline
\end{tabular}

P.B. 7.1.04

\begin{tabular}{|l|l|l|l|l|l|}
\hline 3112 & p67 & MT & M & 34 & \\
\hline 3113 & s143 & PM & M & 17 & \\
\hline 3114 & s144 & PM & M & 18 & \\
\hline 3115 & s46 & PM & M & 15.5 & \\
\hline 3116 & s22 & PM & F & 15.5 & \\
\hline 3117 & s131 & PM & M & 18 & \\
\hline 3118 & s31 & PM & F & 15 & \\
\hline 3119 & p72 & PM & M & 18.5 & \\
\hline 3120 & s118 & ST & F & 4.6 & \\
\hline 3121 & p108 & ST & M & 4.3 & \\
\hline 3122 & s72 & PM & F & 16 & \\
\hline 3123 & s14 & PM & M & 18.5 & \\
\hline 3124 & s21 & PM & M & 11 & \\
\hline 3125 & p92 & ST & F & 5.2 & \\
\hline 3126 & s135 & SV & F & 3.5 & \\
\hline
\end{tabular}

\section{P.B. 7.2.04}

\begin{tabular}{|l|l|l|l|l|l|}
\hline 3127 & s21 & PM & M & 17 & \\
\hline 3128 & s68 & PM & M & 17 & \\
\hline 3129 & s85 & PM & M & 19 & \\
\hline 3130 & s140 & PM & M & 18.5 & \\
\hline 3131 & s23 & PM & M & 9.5 & \\
\hline 3132 & s143 & PM & M & 13 & \\
\hline 3133 & s82 & PM & M & 19 & \\
\hline 3134 & s68 & PM & F & 11 & \\
\hline 3135 & s22 & PM & F & 16 & \\
\hline 3136 & p7 & ST & $?$ & 3 & \\
\hline 3137 & s4 & SV & $?$ & 5 & \\
\hline 3138 & s34 & SV & $?$ & 3.3 & \\
\hline 3139 & p36 & SV & F & 5.4 & \\
\hline 3140 & s2 & ST & $?$ & 3.5 & \\
\hline 3141 & s14 & SV & $?$ & 5 & \\
\hline 3142 & p92 & ST & $?$ & 3 & \\
\hline 3143 & p84 & ST & $?$ & 4.3 & \\
\hline 3144 & p84 & ST & $?$ & 4.7 & \\
\hline 3145 & s41 & SV & $?$ & 4.21 & \\
\hline
\end{tabular}


OXBOW 7.6.04

\begin{tabular}{|c|c|c|c|c|c|}
\hline 3146 & $\mathrm{~S} 81$ & MO & $\mathrm{F}$ & 20.5 & . \\
\hline 3147 & S86 & MO & $\mathbf{M}$ & 20 & \\
\hline 3148 & S93 & TT & $\mathrm{F}$ & 73 & TAG G724 \\
\hline 3149 & S72 & TT & $\mathrm{M}$ & 70 & G738 \\
\hline 3150 & S21 & TT & $\mathrm{F}$ & 84 & G740 \\
\hline 3151 & M129 & TT & M & 60 & G741 \\
\hline 3152 & S92 & NG & $\mathrm{M}$ & 7.4 & \\
\hline 3153 & P132 & PM & $\mathrm{F}$ & 18 & \\
\hline 3154 & S18 & PM & $\mathrm{M}$ & 14 & \\
\hline 3155 & S96 & PM & $\mathrm{F}$ & 15 & \\
\hline 3156 & S94 & PM & $\mathbf{M}$ & 15 & \\
\hline 3157 & S136 & PM & $\bar{M}$ & 18 & \\
\hline 3158 & S47 & PM & $\mathrm{F}$ & 19.5 & \\
\hline 3159 & S105 & PM & $\mathbf{M}$ & 16 & \\
\hline 3160 & s119 & $\mathrm{PM}$ & $\mathrm{F}$ & 20.5 & \\
\hline 3161 & p52 & PM & $F$ & 14.5 & \\
\hline 3162 & s59 & PM & $\mathbf{M}$ & 16 & \\
\hline 3163 & $\mathrm{p} 40$ & ST & $\mathbf{F}$ & 4.1 & \\
\hline 3164 & $\mathrm{p} 48$ & $\mathrm{ST}$ & $\mathbf{F}$ & 4.5 & \\
\hline 3165 & s144 & ST & $\mathrm{M}$ & 4.6 & \\
\hline 3166 & s130 & ST & $\mathbf{M}$ & 4.6 & \\
\hline 3167 & p24 & ST & $\mathbf{F}$ & 3.8 & \\
\hline 3168 & $\mathrm{p} 4$ & ST & $\mathrm{F}$ & 5.4 & \\
\hline 3169 & $\mathrm{p} 7$ & ST & $F$ & 4.0 & \\
\hline 3170 & p12 & ST & $\mathrm{M}$ & 4.3 & \\
\hline 3171 & p79 & SV & $\mathrm{M}$ & 5.0 & \\
\hline 3172 & $\mathrm{p} 88$ & ST & $\mathbf{F}$ & 3.9 & \\
\hline 3173 & p91 & NG & $?$ & 7.9 & \\
\hline 3174 & p115 & ST & $\mathbf{M}$ & 3.9 & \\
\hline 3175 & p67 & ST & $?$ & 4.2 & \\
\hline 3176 & p132 & ST & $\mathbf{M}$ & 4.5 & \\
\hline \multirow[t]{4}{*}{3177} & p132 & ST & $\mathbf{M}$ & 4.6 & \\
\hline & p144 & ST & $?$ & $?$ & \\
\hline & $\mathrm{p} 60$ & ST & $?$ & $?$ & \\
\hline & p131 & ST & $?$ & $?$ & \\
\hline
\end{tabular}

\section{OXBOW 7.7.04}

\begin{tabular}{|l|l|l|l|l|r|}
\hline 3178 & $\mathrm{~m} 72$ & TT & F & 95 & TAG G742 \\
\hline 3179 & s54 & TT & M & 50 & G743 \\
\hline 3180 & s144 & TT & F & 90 & G744 \\
\hline 3181 & s33 & TT & M & 64 & G745 \\
\hline 3182 & s95 & GS & F & 155 & G754 \\
\hline 3183 & s59 & PM & F & 16.5 & \\
\hline 3184 & s115 & PM & M & 21 & \\
\hline 3185 & s84 & PM & M & 17 & \\
\hline 3186 & s17 & PM & M & 14 & \\
\hline 3187 & s141 & PM & F & 13 & \\
\hline 3188 & p43 & SCAT & M & 48 & \\
\hline 3189 & s72 & ME & M & 60 & \\
\end{tabular}




\begin{tabular}{|l|l|l|l|l|l|}
\hline 3190 & $\mathrm{p} 128$ & SV & M & 4.2 & \\
\hline 3191 & $\mathrm{p} 28$ & SV & M & 5.0 & \\
\hline 3192 & $\mathrm{p} 12$ & SV & F & 5.3 & \\
\hline 3193 & $\mathrm{~s} 36$ & TT & F & 69 & \\
\hline 3194 & $\mathrm{~s} 80$ & SV & $?$ & 4.5 & \\
\hline 3195 & $\mathrm{p} 112$ & ST & $?$ & 4.5 & \\
\hline 3196 & $\mathrm{p} 88$ & ST & $?$ & 4.3 & \\
\hline 3197 & $\mathrm{p} 91$ & ST & $?$ & 3.9 & \\
\hline 3198 & $\mathrm{p} 96$ & ST & $?$ & 4.6 & \\
\hline 3199 & p128 & ST & $?$ & 3.5 & \\
\hline
\end{tabular}

\section{Oxbow 7.8.04}

\begin{tabular}{|l|l|l|l|l|r|}
\hline 3200 & s96 & TT & M & 68 & TAG G757 \\
\hline 3201 & s81 & MO & M & 15.5 & \\
\hline 3202 & s83 & MO & M & 18.5 & G750 \\
\hline 3203 & m21 & TT & F & 74 & G729 \\
\hline 3204 & s4 & GS & M & 125 & G759 \\
\hline 3205 & s32 & TT & M & 74 & G732 \\
\hline 3206 & p124 & TT & M & 41 & \\
\hline 3207 & m132 & TT & M & 50 & \\
\hline 3208 & s1112 & TT & M & $?$ & \\
\hline 3209 & s100 & GS & F & 130 & \\
\hline 3211 & p67 & PM & F & 15.5 & \\
\hline 3212 & s143 & PM & F & 13 & \\
\hline 3213 & s117 & PM & F & 20 & \\
\hline 3214 & s119 & PM & M & 15 & \\
\hline 3215 & s66 & PM & F & 19 & \\
\hline 3216 & p144 & ST & $?$ & 4.2 & \\
\hline 3217 & p79 & ST & $?$ & 4.4 & \\
\hline 3218 & p96 & SV & $?$ & 5.0 & \\
\hline 3219 & p24 & ST & F & 4.4 & \\
\hline 3220 & p36 & ST & $?$ & 4.0 & \\
\hline 3221 & s48 & SV & $?$ & 7.1 & \\
\hline $0 \times 1$ & & & & & \\
\hline
\end{tabular}

OXBOW 7.9.04

\begin{tabular}{|l|l|l|l|l|l|}
\hline 3222 & $\mathrm{~s} 42$ & TT & M & 49 & TAG G755 \\
\hline 3223 & $\mathrm{~s} 45$ & TT & M & 60 & G756 \\
\hline 3225 & $\mathrm{~s} 81$ & MO & F & 19 & \\
\hline 3226 & m132 & GS & F & 130 & G760 \\
\hline 3227 & s117 & MO & F & 15 & \\
\hline 3228 & s137 & TT & M & 54 & G761 \\
\hline 3229 & s108 & TT & M & 71 & G762 \\
\hline 3230 & s72 & PM & M & 18 & \\
\hline 3231 & s49 & PM & F & 16 & \\
\hline 3232 & p84 & ST & M & 4.2 & \\
\hline 3234 & s116 & MO & M & 15 & \\
\hline 3235 & s104 & TT & M & 51 & \\
\hline 3236 & m104 & TT & F & 62 & \\
\hline 3237 & p31 & ST & $?$ & 4.6 & \\
\hline 3238 & p103 & ST & $?$ & 5.0 & \\
\hline 3239 & $\mathrm{~s} 79$ & SV & M & 7.1 & \\
\hline
\end{tabular}




\begin{tabular}{|l|l|l|l|l|l|}
\hline 3240 & p79 & NG & $?$ & 8 & \\
\hline 3241 & $\mathrm{~s} 128$ & ST & $?$ & 4.0 & \\
\hline & P84 & SV & & & \\
\hline
\end{tabular}

\section{FOREST PARK 7.13.04}

\begin{tabular}{|c|c|c|c|c|c|}
\hline 3242 & s75 & $\mathrm{MO}$ & $\mathbf{M}$ & 11 & \\
\hline 3243 & p67 & MT & F & 28.5 & \\
\hline 3244 & s108 & TT & $\mathbf{F}$ & 34 & TAG G763 \\
\hline 3245 & s84 & $\mathrm{TT}$ & $\mathbf{M}$ & 65 & G764 \\
\hline 3246 & s24 & PM & $F$ & 15 & \\
\hline 3247 & $\mathrm{~m} 46$ & $\mathrm{PM}$ & $F$ & 18 & \\
\hline 3248 & s89 & $\mathrm{PM}$ & $\mathbf{M}$ & 18.5 & \\
\hline 3249 & s56 & PM & F & 14 & \\
\hline 3250 & s66 & PM & $\mathbf{M}$ & 18 & \\
\hline 3251 & p36 & PM & $\mathrm{F}$ & 17.5 & \\
\hline 3252 & p36 & $\mathrm{PM}$ & $\mathbf{M}$ & 17 & \\
\hline 3253 & $\mathrm{ml15}$ & PM & $\mathbf{M}$ & 19.5 & \\
\hline 3254 & $\mathrm{~s} 11$ & PM & $\mathbf{M}$ & 23 & \\
\hline 3255 & s115 & $\mathrm{PM}$ & $\mathrm{F}$ & 19 & \\
\hline 3256 & s12 & PM & $\mathbf{M}$ & 18.5 & \\
\hline 3257 & s10 & PM & $\mathbf{M}$ & 15 & \\
\hline 3258 & $\mathrm{~s} 33$ & $\mathrm{PM}$ & $\mathrm{F}$ & 21.5 & \\
\hline 3259 & p19 & PM & $\mathrm{F}$ & 17 & \\
\hline 3260 & s131 & PM & $\mathrm{F}$ & 23 & \\
\hline 3261 & s96 & PM & $F$ & 18 & \\
\hline 3262 & s79 & PM & $\mathbf{M}$ & 18.5 & \\
\hline 3263 & s118 & PM & $\mathrm{F}$ & 17 & \\
\hline 3264 & $s 42$ & PM & $\mathbf{M}$ & 17.5 & \\
\hline 3265 & s144 & PM & $\mathrm{F}$ & 15 & \\
\hline 3266 & s130 & PM & $\mathrm{F}$ & 16 & \\
\hline 3267 & s119 & PM & $\mathbf{M}$ & 21 & \\
\hline 3268 & s82 & PM & $\mathrm{F}$ & 15.5 & \\
\hline 3269 & s118 & PM & $\mathbf{M}$ & 16 & \\
\hline 3270 & s29 & $\mathbf{P M}$ & $\mathbf{M}$ & 16.5 & \\
\hline 3271 & s54 & PM & $\mathrm{F}$ & 15 & \\
\hline 3272 & s64 & PM & $\mathbf{M}$ & 15.5 & \\
\hline 3273 & s72 & PM & $\mathbf{M}$ & 17.5 & \\
\hline 3274 & $\mathrm{~m} 131$ & PM & $\mathbf{M}$ & 18 & \\
\hline 3275 & s36 & PM & $\mathbf{M}$ & 19.5 & \\
\hline 3276 & $\mathrm{~m} 143$ & PM & $\mathbf{M}$ & 19 & \\
\hline 3277 & s92 & $\mathrm{PM}$ & $\mathbf{M}$ & 17 & \\
\hline 3278 & s71 & PM & $\mathbf{M}$ & 18.5 & $\vdots$ \\
\hline 3279 & $\mathrm{~m} 19$ & $\mathrm{PM}$ & $\mathrm{F}$ & 23 & \\
\hline 3280 & s139 & PM & $\mathbf{M}$ & 20. & \\
\hline 3281 & $\mathrm{p} 72$ & PM & $\mathrm{F}$ & 19 & \\
\hline 3282 & $\mathrm{p} 72$ & $\mathrm{PM}$ & $\mathrm{F}$ & 14 & \\
\hline 3283 & s127 & PM & $\mathrm{F}$ & 16 & \\
\hline 3284 & $\mathrm{~m} 102$ & PM & $\mathbf{M}$ & 18.5 & \\
\hline 3285 & $\mathrm{~s} 107$ & PM & $\mathbf{M}$ & 17.5 & \\
\hline 3286 & $\mathrm{~s} 81$ & PM & $F$ & 13 & \\
\hline
\end{tabular}




\begin{tabular}{|l|l|l|l|l|l|}
\hline 3287 & s126 & PM & M & 20.5 & \\
\hline 3288 & s143 & PM & M & 17.5 & \\
\hline 3289 & s125 & PM & F & 15 & \\
\hline 3290 & s46 & PM & M & 20.5 & \\
\hline 3291 & s60 & PM & M & 19 & \\
\hline 3292 & s103 & PM & F & 15.5 & \\
\hline 3293 & s9 & PM & F & 22 & \\
\hline 3294 & s116 & ST & $?$ & 4.5 & \\
\hline 3295 & p36 & ST & M & 4.1 & \\
\hline 3296 & p28 & ST & $?$ & 4.8 & \\
\hline
\end{tabular}

\section{FOREST PARK 7.14:04}

\begin{tabular}{|l|l|l|l|l|l|}
\hline 3297 & s22 & TT & M & 65 & TAG G766 \\
\hline 3298 & s84 & TT & M & 60 & \\
\hline 3299 & s140 & PM & M & 14 & \\
\hline 3300 & s143 & PM & M & 16 & \\
\hline 3301 & s92 & PM & F & 17.5 & \\
\hline 3302 & p132 & PM & M & 21.5 & \\
\hline 3303 & s12 & PM & M & 18 & \\
\hline 3304 & s132 & PM & M & 16.5 & \\
\hline 3305 & s130 & PM & F & 15 & \\
\hline 3306 & s89 & PM & F & 17 & \\
\hline 3307 & s24 & PM & F & 14 & \\
\hline 3308 & s60 & PM & F & 16 & \\
\hline 3309 & p82 & PM & F & 12.5 & \\
\hline 3310 & s33 & PM & F & 17 & \\
\hline 3311 & s96 & PM & M & 17 & \\
\hline 3312 & s120 & PM & F & 15 & \\
\hline 3313 & s107 & PM & M & 16 & \\
\hline 3314 & s118 & PM & M & 19 & \\
\hline 3315 & s102 & PM & F & 20.5 & \\
\hline 3316 & s108 & PM & F & 16 & \\
\hline 3317 & s115 & PM & M & 16 & \\
\hline 3318 & p28 & SV & F & 4.1 & \\
\hline 3319 & p115 & SV & $?$ & $?$ & \\
\hline 3320 & p76 & ST & $?$ & 3.8 & \\
\hline & p91 & SCAT & $?$ & $?$ & \\
\hline
\end{tabular}

\section{FOREST PARK 7.15.04}

\begin{tabular}{|l|l|l|l|l|l|}
\hline 3321 & s81 & PM & M & 18 & \\
\hline 3322 & s104 & PM & M & 15.5 & \\
\hline 3323 & s33 & PM & M & 17 & \\
\hline 3324 & m60 & PM & F & 16.5 & \\
\hline 3325 & s107 & PM & F & 16 & \\
\hline 3326 & s60 & PM & M & 16.5 & \\
\hline 3327 & s59 & PM & F & 17 & \\
\hline 3328 & s19 & PM & M & 16 & \\
\hline 3329 & s143 & PM & F & 26 & \\
\hline 3330 & s120 & PM & M & 17.5 & \\
\hline 3331 & s48 & PM & M & 17 & \\
\hline 3332 & s108 & PM & M & 18 & \\
\hline
\end{tabular}




\begin{tabular}{|l|l|l|l|l|l|}
\hline 3333 & s24 & PM & M & 19 & \\
\hline 3334 & p78 & PM & M & 15 & \\
\hline 3335 & p91 & SCAT & F & 86 & \\
\hline 3336 & s82 & ST & $?$ & 4.5 & \\
\hline 3337 & s101 & SV & F & 5.2 & \\
\hline 3338 & s9 & ST & F & 4.2 & \\
\hline 3339 & s91 & SV & $?$ & 5.6 & \\
\hline
\end{tabular}

FOREST PARK 7.16.04

\begin{tabular}{|l|l|l|l|l|l|}
\hline 3340 & $\mathrm{~s} 109$ & $\mathrm{MO}$ & $\mathrm{F}$ & 17 & \\
\hline 3341 & $\mathrm{~s} 35$ & $\mathrm{PM}$ & $\mathrm{M}$ & 14 & \\
\hline 3342 & $\mathrm{~s} 69$ & $\mathrm{PM}$ & $\mathrm{M}$ & 15 & \\
\hline 3343 & $\mathrm{~s} 66$ & $\mathrm{PM}$ & $\mathrm{M}$ & 16 & \\
\hline 3344 & $\mathrm{~s} 72$ & $\mathrm{PM}$ & $\mathrm{M}$ & 19 & \\
\hline 3345 & $\mathrm{~s} 42$ & $\mathrm{PM}$ & $\mathrm{M}$ & 17 & \\
\hline 3346 & $\mathrm{~s} 120$ & $\mathrm{PM}$ & $\mathrm{F}$ & 15.5 & \\
\hline 3347 & $\mathrm{p} 48$ & $\mathrm{PM}$ & $\mathrm{F}$ & 13.5 & \\
\hline 3348 & $\mathrm{~s} 81$ & $\mathrm{PM}$ & $\mathrm{M}$ & 15 & \\
\hline 3349 & $\mathrm{p} 91$ & $\mathrm{MT}$ & $\mathrm{M}$ & 28 & \\
\hline 3350 & $\mathrm{~s} 117$ & $\mathrm{ST}$ & $?$ & 4.3 & \\
\hline 3351 & $\mathrm{~s} 8$ & $\mathrm{MO}$ & $\mathrm{F}$ & 17.5 & \\
\hline 3352 & $\mathrm{~s} 139$ & ST & $?$ & 4.0 & \\
\hline 3353 & $\mathrm{~s} 140$ & ST & $?$ & 4.8 & \\
\hline 3354 & $\mathrm{~s} 9$ & ST & $?$ & 3.7 & \\
\hline 3355 & $\mathrm{~s} 103$ & ST & $?$ & 4.7 & \\
\hline 3356 & $\mathrm{~s} 89$ & ST & $?$ & 4.7 & \\
\hline 3357 & $\mathrm{~s} 18$ & MO & M & 8 & \\
\hline 3358 & $\mathrm{p} 28$ & SV & M & 4.0 & \\
\hline
\end{tabular}

TRYON CREEK 7.20.04

\begin{tabular}{|l|l|l|l|l|l|}
\hline 3359 & s117 & TT & F & 75 & G73? \\
\hline 3360 & s24 & PM & M & 21.5 & \\
\hline 3361 & s41 & PM & F & 12 & JUVY \\
\hline 3362 & s12 & PM & F & 16.5 & \\
\hline 3363 & s44 & PM & M & 17 & \\
\hline 3364 & p36 & PM & M & 14 & \\
\hline 3365 & s125 & PM & M & 17 & \\
\hline 3366 & s73 & PM & M & 16.5 & \\
\hline 3367 & p91 & PM & F & 19 & PREG \\
\hline 3368 & p96 & PM & F & 18 & \\
\hline 3369 & m54 & PM & F & 15.5 & \\
\hline 3370 & s23 & PM & M & 17 & \\
\hline 3371 & m24 & PM & F & 20 & PREG \\
\hline 3372 & s118 & PM & F & 15 & \\
\hline 3373 & $?$ & PM & M & 21 & \\
\hline 3374 & s132 & PM & M & 19 & \\
\hline 3375 & s124 & PM & F & 16 & \\
\hline 3376 & $\mathrm{~s} 57$ & PM & F & 20.5 & PREG \\
\hline 3377 & s108 & PM & M & 19 & \\
\hline 3378 & s72 & PM & F & 15.5 & DIB/NBS \\
\hline 3379 & s32 & PM & M & 21 & DIB/NBS \\
\hline & & & & 209 & \\
\hline
\end{tabular}




\begin{tabular}{|c|c|c|c|c|c|}
\hline 3380 & $\mathrm{p} 43$ & ST & $?$ & 5.2 & DIT/NBS \\
\hline 3381 & s140 & ST & $?$ & 4.5 & RELEASED \\
\hline \multicolumn{6}{|c|}{ TRYON CREEK 7.21 .04} \\
\hline 3382 & s5 & MO & $\mathrm{F}$ & 20 & RELEASED \\
\hline 3383 & s77 & $\overline{\mathrm{PM}}$ & $\mathrm{F}$ & 16.5 & \\
\hline 3384 & $\mathrm{p} 43$ & PM & $\mathbf{M}$ & 19.5 & \\
\hline 3385 & s117 & PM & $\mathrm{F}$ & 18 & \\
\hline 3386 & m59 & PM & $\overline{\mathrm{M}}$ & 16 & \\
\hline 3387 & s108 & PM & M & 19 & \\
\hline 3388 & s78 & PM & $\mathbf{M}$ & 19 & \\
\hline 3389 & s32 & PM & $F$ & 12.5 & JUVY \\
\hline 3390 & s57 & PM & $\mathrm{M}$ & 19.5 & \\
\hline 3391 & s144 & $\overline{\mathrm{PM}}$ & $\mathrm{F}$ & 19 & \\
\hline 3392 & $\mathrm{~s} 130$ & PM & $\mathrm{F}$ & 18.5 & \\
\hline 3393 & $\mathrm{~s} 45$ & PM & $\mathrm{F}$ & 16 & \\
\hline 3394 & s47 & $\overline{P M}$ & $\mathrm{~F}$ & 13 & JUVY \\
\hline 3395 & s117 & $\overline{P M}$ & $\mathrm{~F}$ & 18 & \\
\hline 3396 & $\mathrm{~s} 24$ & PM & $\bar{F}$ & 17.5 & \\
\hline 3397 & s113 & SV & $F$ & 8.5 & RELEASED \\
\hline 3398 & $\mathrm{p} 24$ & ST. & $?$ & 4.6 & DIT/NBS \\
\hline 3399 & s93 & ST & $?$ & 3.8 & DIT/NBS \\
\hline 3400 & s114 & ST & $?$ & 4.6 & DIT/NBS \\
\hline \multirow[t]{2}{*}{3401} & $\mathrm{~s} 100$ & ST & $?$ & 4.8 & DIT/NBS \\
\hline & s118 & SV & $?$ & $?$ & ESCAPED \\
\hline
\end{tabular}

\begin{tabular}{|c|c|c|c|c|c|}
\hline \multicolumn{6}{|c|}{ TC 7.22.04 } \\
\hline 3402 & S66 & PM & $\mathbf{M}$ & 18.5 & \\
\hline 3403 & S83 & PM & $\mathbf{M}$ & 16.5 & SUBA \\
\hline 3404 & S75 & PM & $F$ & 16.5 & \\
\hline 3405 & M10 & PM & $F$ & 15.5 & \\
\hline 3406 & S108 & PM & F & 14.5 & \\
\hline 3407 & S45 & PM & $\mathrm{M}$ & 19.5 & \\
\hline 3408 & S47 & PM & $\mathbf{M}$ & 15 & \\
\hline 3409 & $\mathrm{~T} 84$ & $\mathrm{SN}$ & $\mathrm{F}$ & 520 & TAGGED G777 RELEASED \\
\hline 3410 & P91 & ST & $?$ & 4.7 & DIT/NBS \\
\hline 3411 & S116 & ST & $?$ & 4.9 & DIT/NBS \\
\hline 3412 & S93 & SV & $?$ & 5.1 & DIT/NBS \\
\hline 3413 & S86 & ST & $?$ & 4.2 & DIT/NBS \\
\hline 3414 & P88 & ST & $?$ & 4.3 & \\
\hline 3415 & S19 & ST & $?$ & 5.0 & \\
\hline
\end{tabular}

TC 7.23.04

\begin{tabular}{|l|l|l|l|l|l|}
\hline 3416 & S4 & MO & F & 15.5 & DIT/NBS \\
\hline 3417 & S142 & PM & M & 17 & \\
\hline 3418 & S105 & PM & F & 21.5 & \\
\hline 3419 & S45 & PM & F & 15.5 & \\
\hline 3420 & S60 & PM & M & 15.5 & \\
\hline 3421 & S22 & NG & F & 9 & NBS/RELEASED \\
\hline 3422 & S104 & SV & $?$ & 4.6 & DIT/NBS \\
\hline 3423 & P127 & ST & $?$ & 4.2 & DIT/NBS \\
\hline 3424 & P72 & SV & $?$ & 4.7 & DIT/NBS \\
\hline
\end{tabular}




\begin{tabular}{|l|l|l|l|l|l|}
\hline 3425 & S5 & MO & M & 23 & DIT/NBS \\
\hline 3426 & P12 & ST & $?$ & 4.2 & DIT/NBS \\
\hline 3427 & S128 & ST & $?$ & 3.8 & DIT/NBS \\
\hline 3428 & S127 & SV & $?$ & 5.5 & DIT/NBS \\
\hline
\end{tabular}

TUALATIN RIVER NWR 7.27.04

\begin{tabular}{|l|l|l|l|l|l|}
\hline 3429 & M51 & PM & M & 16.5 & \\
\hline 3430 & s84 & PM & F & 20 & PREG \\
\hline 3431 & p115 & PM & M & 14 & SUBA \\
\hline 3432 & s125 & PM & M & 15 & \\
\hline 3433 & s89 & PM & F & 17 & PREG \\
\hline 3434 & s82 & PM & M & 14 & \\
\hline 3435 & p76 & ST & M & 4.8 & DIT/NBS \\
\hline 3436 & S142 & ST & $?$ & 4.4 & DIT/NBS \\
\hline 3437 & P12 & SV & F & 4.0 & DIT/NBS \\
\hline 3438 & S26 & PM & M & 14 & DIT/NBS \\
\hline 3439 & S91 & SV & $?$ & 3.7 & RELEASED \\
\hline
\end{tabular}

TRNWR 7.28.04

\begin{tabular}{|c|c|c|c|c|c|}
\hline 3440 & s24 & PM & $\mathrm{F}$ & 20 & \\
\hline 3441 & $\begin{array}{l}\text { S141 } \\
\end{array}$ & PM & $\overline{\mathbf{M}}$ & 17 & \\
\hline 3442 & s9 & $\mathrm{PM}$ & $\mathbf{M}$ & 14 & SUBA/A \\
\hline 3443 & $\mathrm{~S} 40$ & PM & $\bar{M}$ & 12.5 & SUBA \\
\hline 3444 & $\mathrm{P} 103$ & PM & $\mathrm{F}$ & 15.5 & $\begin{array}{l}\text { GROWTH ON POST } \\
\text { VENTER/1 BS }\end{array}$ \\
\hline 3445 & S10 & ME & $\mathbf{M}$ & 73 & G771 RELEASED \\
\hline 3446 & S142 & ST & $\mathrm{F}$ & 4.0 & \\
\hline 3447 & s81 & SV & $?$ & 3.8 & RELEASED \\
\hline 3448 & T48 & MM & $?$ & $?$ & RELEASED \\
\hline
\end{tabular}

TRNWR 7.29.04

\begin{tabular}{|l|l|l|l|l|l|}
\hline 3449 & S142 & TT & M & 73 & G774 REL \\
\hline 3450 & S34 & PM & F & 20 & \\
\hline 3451 & S141 & PM & F & 19.5 & PREG \\
\hline 3452 & S51 & ST & $?$ & 5.5 & DIT/NBS \\
\hline 3453 & S108 & SV & F & 4.2 & RELEASED \\
\hline & S127 & ME & & & G771 RECAP/DIT \\
\hline & T72 & MM & $?$ & & RELEASED \\
\hline & T144 & DV & $?$ & & RELEASED \\
\hline
\end{tabular}

TRNWR 7.30.04
\begin{tabular}{|l|l|l|l|l|l|}
\hline 3454 & S141 & PM & M & 14.5 & \\
\hline 3455 & S105 & PM & F & 19 & PREG \\
\hline 3456 & M36 & PM & F & 10.5 & JUVY \\
\hline 3457 & s114 & SN & F & 600 & G770 REL \\
\hline 3458 & S80 & SV & $?$ & 4 & RELEASED \\
\hline
\end{tabular}

Powell Butte 8.24.04

\begin{tabular}{|l|l|l|l|l|l|}
\hline 3459 & m47 & PM & M & 17.5 & G768 \\
\hline 3460 & s120 & PM & F & 25 & G713 \\
\hline 3461 & p31 & PM & M & 12.5 & G714 \\
\hline 3462 & s108 & PM & M & 17 & G716 \\
\hline 3463 & s24 & PM & F & 21.5 & G717 \\
\hline 3464 & s11 & PM & F & 12.5 & G718 \\
\hline
\end{tabular}




\begin{tabular}{|c|c|c|c|c|c|}
\hline 3465 & $\mathrm{~s} 66$ & PM & $\mathbf{M}$ & 15 & G779 \\
\hline 3466 & s58 & PM & $\mathbf{M}$ & 18.5 & G775 \\
\hline 3467 & s71 & PM & $\mathrm{F}$ & 20 & G776 \\
\hline 3468 & $\mathrm{~s} 50$ & PM & $\mathbf{M}$ & 13 & G773 \\
\hline 3469 & s94 & $\mathrm{PM}$ & $F$ & 27.5 & G772 \\
\hline 3470 & s141 & PM & $\mathbf{M}$ & 15 & G769 \\
\hline 3471 & s32 & $\overline{\mathrm{PM}}$ & $\mathrm{F}$ & 13 & G790 \\
\hline 3472 & s34 & PM & M & 20 & G791 \\
\hline 3473 & $\mathrm{s36}$ & $\mathrm{PM}$ & $\mathbf{F}$ & 22 & G792 \\
\hline 3474 & $\mathrm{~s} 40$ & $\mathrm{PM}$ & $\mathbf{M}$ & 15 & G793 \\
\hline 3475 & s139 & PM & $\mathbf{M}$ & 14.5 & G794 \\
\hline 3476 & s136 & PM & $\mathrm{F}$ & 14.5 & G795 \\
\hline 3477 & s131 & $\mathrm{PM}$ & $\mathbf{M}$ & 16 & G796 \\
\hline 3478 & s128 & PM & $F$ & 17 & G797 \\
\hline 3479 & $\mathrm{~s} 102$ & SV & $\mathbf{M}$ & 4.1 & DIT/NBS \\
\hline 3480 & p112 & SV & $?$ & $?$ & ESCAPED \\
\hline 3481 & p79 & SV & $?$ & 3.8 & DIT/NBS \\
\hline 3482 & p84 & MO & $\mathbf{M}$ & 7.1 & DIT/NBS \\
\hline 3483 & p64 & $\mathrm{MO}$ & $\mathrm{F}$ & 16 & DIT/NBS \\
\hline 3484 & p64 & $\mathrm{MO}$ & $\mathrm{M}$ & 8.9 & RELEASED \\
\hline 3485 & p64 & $\mathrm{MO}$ & $\mathrm{M}$ & 8.6 & RELEASED \\
\hline
\end{tabular}

P.B. 8.25.04

\begin{tabular}{|l|l|l|l|l|l|}
\hline 3486 & $\mathrm{p} 40$ & $\mathrm{MO}$ & $\mathrm{F}$ & 14.5 & \\
\hline 3487 & $\mathrm{p} 64$ & $\mathrm{MO}$ & $\mathrm{F}$ & 13.5 & \\
\hline 3488 & $\mathrm{~s} 110$ & $\mathrm{MO}$ & $\mathrm{M}$ & 13 & \\
\hline 3489 & $\mathrm{~s} 58$ & $\mathrm{PM}$ & $\mathrm{M}$ & 22.5 & TAG G798 \\
\hline 3490 & $\mathrm{~s} 141$ & $\mathrm{PM}$ & $\mathrm{M}$ & 15.5 & TAG G799 \\
\hline 3491 & $\mathrm{~s} 96$ & $\mathrm{PM}$ & $\mathrm{M}$ & 16.5 & TAG G800 \\
\hline 3492 & $\mathrm{~s} 35$ & $\mathrm{PM}$ & $\mathrm{M}$ & 16 & TAG G601 \\
\hline 3493 & $\mathrm{~s} 23$ & $\mathrm{PM}$ & $\mathrm{F}$ & 12 & TAG G602 \\
\hline 3494 & $\mathrm{~s} 84$ & $\mathrm{PM}$ & $\mathrm{M}$ & 21 & TAG G603 \\
\hline 3495 & $\mathrm{~s} 2$ & $\mathrm{PM}$ & $\mathrm{M}$ & 16 & TAG G604 \\
\hline 3496 & $\mathrm{p} 84$ & SV & $?$ & 4.2 & \\
\hline 3497 & $\mathrm{p} 115$ & SV & $?$ & $?$ & \\
\hline 3498 & $\mathrm{~s} 22$ & PM & $\mathrm{M}$ & 18 & \\
\hline 3499 & $\mathrm{p} 136$ & SV & $?$ & 4.2 & \\
\hline
\end{tabular}

\section{P.B. 8.26.04}

\begin{tabular}{|l|l|l|l|l|l|}
\hline 3500 & s59 & MO & M & 15 & TAG G605 \\
\hline 3501 & s97 & MO & F & 13 & TAG G607 \\
\hline 3502 & s66 & MO & M & 14 & TAG G608 \\
\hline 3503 & s118 & PM & M & 16 & TAG G609 \\
\hline 3504 & s10 & PM & M & 15 & TAG G610 \\
\hline 3505 & s42 & SV & $?$ & 3.5 & \\
\hline 3506 & s123 & MO & F & 16 & \\
\hline 3507 & p36 & SV & $?$ & 4.7 & \\
\hline 3508 & p144 & PM & F & 16.5 & \\
\hline 3509 & p7 & SV & $?$ & 4.9 & \\
\hline 3510 & p7 & SV & $?$ & 4.5 & \\
\hline 3511 & p7 & SV & $?$ & $?$ & \\
\hline
\end{tabular}




\begin{tabular}{l}
\begin{tabular}{|l|l|l|l|l|l|}
\hline 3512 & s78 & MO & M & 10.0 & \\
\hline 3513 & p84 & ST & $?$ & 5.1 & \\
\hline 3514 & p84 & ST & $?$ & 5.3 & \\
P.B. 8.27.04 & S59 & MO & M & 11 & TAG G611 \\
\hline 3515 & S25 & MO & F & 14 & TAG G612 \\
\hline 3516 & S127 & MO & M & 7 & G613 \\
\hline 3517 & S11 & MO & M & 10 & G614 \\
\hline 3518 & S129 & PM & M & 17 & G615 \\
\hline 3519 & S108 & PM & M & 18 & G616 \\
\hline 3520 & S119 & PM & F & 17.5 & G617 \\
\hline 3521 & S11 & PM & F & 19 & G618 \\
\hline 3522 & S84 & PM & M & 16 & G619 \\
\hline 3523 & P84 & MO & M & 15 & \\
\hline 3524 & P64 & PM & F & 19 & \\
\hline 3525 & P28 & PM & M & 19 & \\
\hline 3526 & & &
\end{tabular} \\
\hline
\end{tabular}

OXBOW 8.31.04

\begin{tabular}{|c|c|c|c|c|c|}
\hline 3527 & T115 & TT & $\mathbf{M}$ & 64 & G620 \\
\hline 3528 & M82 & $\mathrm{TT}$ & $\mathbf{M}$ & 80 & G621 \\
\hline 3529 & S111 & TT & $F$ & 70 & G622 \\
\hline 3530 & S107 & TT & $\mathbf{M}$ & 68 & G623 \\
\hline 3531 & M17 & TT & $\bar{M}$ & 62 & G624 \\
\hline 3532 & M94 & $\mathrm{TT}$ & $\mathbf{M}$ & 64 & G626 \\
\hline 3533 & M15 & TT & $F$ & 63 & G627 \\
\hline 3534 & S24 & TT & $\mathrm{F}$ & 73 & G628 \\
\hline 3535 & S142 & TT & $\mathbf{M}$ & 74 & G629 \\
\hline 3536 & S44 & TT & $\mathbf{M}$ & 79 & G756 \\
\hline 3537 & M23 & TT & $F$ & 83 & G740 \\
\hline 3538 & S84 & $\mathrm{PM}$ & $\mathrm{F}$ & 14.5 & G630 \\
\hline 3539 & S7 & PM & $\mathbf{M}$ & 13.5 & G632 \\
\hline 3540 & S88 & PM & $\mathbf{M}$ & 14.5 & G633 \\
\hline 3541 & S65 & PM & $\mathbf{M}$ & 13.5 & G634 \\
\hline 3542 & $\mathbf{S 5 8}$ & PM & $\mathbf{M}$ & 15 & G635 \\
\hline 3543 & S52 & PM & $\mathbf{M}$ & 13 & G636 \\
\hline 3544 & S83 & PM & $\mathbf{M}$ & 15 & G637 \\
\hline 3545 & S60 & $\mathrm{PM}$ & $\mathrm{M}$ & 13.5 & G638 \\
\hline 3546 & $\mathrm{~S} 101$ & PM & $F$ & 16 & G639 \\
\hline 3547 & $\mathbf{S 1 2 5}$ & PM & $\mathbf{M}$ & 11.5 & G640 \\
\hline 3548 & S49 & PM & $\mathbf{M}$ & 14 & G641 \\
\hline 3549 & S23 & PM & $\mathbf{M}$ & 14 & G642 \\
\hline 3550 & S140 & PM & $F$ & 18 & G643 \\
\hline 3551 & S103 & PM & $\mathbf{M}$ & 16.5 & G644 \\
\hline 3552 & S129 & PM & $F$ & 15 & G645 \\
\hline 3553 & S17 & PM & $\mathrm{M}$ & 13 & G646 \\
\hline 3554 & S110 & PM & $F$ & 18 & G647 \\
\hline 3555 & S106 & S? & $?$ & 5.7 & \\
\hline 3556 & P136 & S? & $?$ & 5.4 & \\
\hline 3557 & P84 & S? & $?$ & 3.8 & \\
\hline 3558 & P24 & S? & $?$ & 5.2 & \\
\hline
\end{tabular}




\begin{tabular}{|c|c|c|c|c|c|}
\hline 3559 & P136 & S? & $?$ & 3.9 & \\
\hline 3560 & T67 & TD & $\mathbf{M}$ & 172 & \\
\hline \multicolumn{6}{|c|}{ OX 9.1.04 } \\
\hline 3561 & $\mathrm{~m} 47$ & TT & $\mathbf{M}$ & 68 & G648 \\
\hline 3562 & s72 & TT & $\mathbf{M}$ & 61 & G649 \\
\hline 3563 & s55 & TT & $F$ & 73 & G743 \\
\hline 3564 & s48 & TT & $M$ & 61 & G650 \\
\hline 3565 & s10 & TT & $\vec{M}$ & 70 & G651 \\
\hline 3566 & s93 & TT & $\mathbf{M}$ & 79 & G621 \\
\hline 3567 & s70 & TT & $\mathbf{M}$ & 60 & G653 \\
\hline 3568 & s139 & TT & $\mathbf{M}$ & 69 & G654 \\
\hline 3569 & s144 & TT & $\mathbf{M}$ & 72 & G655 \\
\hline 3570 & $\mathrm{~m} 23$ & TT & $\mathbf{M}$ & 54 & G656 \\
\hline 3571 & $\mathrm{~m} 116$ & TT & $?$ & $?$ & \\
\hline 3572 & s80 & PM & $\overline{\mathrm{M}}$ & 15 & G657 \\
\hline 3573 & $\mathrm{~s} 132$ & PM & $\mathrm{F}$ & 10 & G658 \\
\hline 3574 & s36 & PM & $\bar{F}$ & 14 & G659 \\
\hline 3575 & s71 & PM & $F$ & 12.5 & G660 \\
\hline 3576 & p7 & PM & $\mathbf{M}$ & 12.5 & G661 \\
\hline 3577 & $\mathrm{p} 24$ & PM & $\mathrm{M}$ & 8.5 & G662 \\
\hline 3578 & $\mathrm{~s} 21$ & PM & $\mathrm{M}$ & 15 & G663 \\
\hline 3579 & s41 & PM & $F$ & 11 & G664 \\
\hline 3580 & s120 & PM & $\mathbf{M}$ & 18.5 & G665 \\
\hline 3581 & $\mathrm{p} 88$ & S? & $?$ & $?$ & \\
\hline 3582 & p115 & PM & $\mathrm{M}$ & 14 & \\
\hline 3583 & m53 & TT & $F$ & 59 & G627 \\
\hline \multicolumn{6}{|c|}{ OX 9.2.04 } \\
\hline 3584 & S132 & TT & $\mathbf{M}$ & 72 & G667 \\
\hline 3585 & S34 & TT & $\mathbf{M}$ & 70 & G668 \\
\hline 3586 & S58 & TT & $F$ & 69 & G669 \\
\hline 3587 & S120 & TT & $\mathbf{M}$ & 78 & G670 \\
\hline 3588 & S46 & TT & $\mathbf{M}$ & 60 & G671 \\
\hline 3589 & S116 & TT & $\mathbf{M}$ & 73 & G672 \\
\hline 3590 & s91 & $\mathrm{TT}$ & $\mathbf{M}$ & 71 & G673 \\
\hline 3591 & $\mathrm{~m} 87$ & TT & $\bar{M}$ & 79 & G674 \\
\hline 3592 & s141 & PM & $F$ & 6.5 & G675 \\
\hline 3593 & $\mathrm{~m} 116$ & PM & $\mathrm{F}$ & 15.5 & G676 \\
\hline 3594 & s6 & PM & $\mathrm{F}$ & 12 & G677 \\
\hline 3595 & s130 & PM & $F$ & 13.5 & G678 \\
\hline 3596 & s89 & PM & $\mathrm{M}$ & 14 & G679 \\
\hline 3597 & p52 & SV & $?$ & 4.5 & \\
\hline 3598 & $\mathrm{~s} 87$ & SV & $?$ & 6.1 & \\
\hline 3599 & $\mathrm{~s} 81$ & SV & $?$ & 6.6 & \\
\hline 3600 & s16 & $\mathrm{ME}$ & $\mathbf{M}$ & 54 & G666 \\
\hline 3601 & s87 & SB & $?$ & 17 & \\
\hline \multicolumn{6}{|c|}{ OX 9.3.04 } \\
\hline 3602 & S81 & SV & $\mathbf{M}$ & 5.8 & \\
\hline 3603 & S42 & TT & $\mathbf{M}$ & 69 & G680 \\
\hline 3604 & s69 & TT & $\mathbf{M}$ & 73 & G681 \\
\hline
\end{tabular}




\begin{tabular}{|l|l|l|l|l|l|}
\hline 3605 & s47 & PM & M & 15.5 & G682 \\
\hline 3606 & m47 & PM & F & 10 & \\
\hline 3607 & s127 & PM & F & 16.5 & G683 \\
\hline 3608 & s15 & PM & M & 17.5 & G684 \\
\hline 3609 & s116 & PM & M & 18 & \\
\hline 3610 & s89 & MO & F & 15.5 & \\
\hline 3611 & s122 & TT & M & 68 & \\
\hline 3612 & s56 & MO & F & 18 & \\
\hline 3613 & t60 & TD & M & 191 & \\
\hline 3614 & s117 & MO & F & 14.5 & G685 \\
\hline
\end{tabular}

FOREST PARK 9.14.04

\begin{tabular}{|l|l|l|l|l|l|}
\hline 3615 & s77 & PM & M & 15.5 & G686 \\
\hline 3616 & s90 & PM & M & 18 & G687 \\
\hline 3617 & s118 & PM & M & 18.5 & G688 \\
\hline 3618 & s105 & PM & M & 20 & G689 \\
\hline 3619 & s108 & PM & F & 20 & G690 \\
\hline 3620 & s105 & PM & M & 20.5 & G691 \\
\hline 3621 & s81 & PM & F & 16 & G692 \\
\hline 3622 & s144 & PM & M & 21 & G693 \\
\hline 3623 & s140 & PM & M & 18.5 & G694 \\
\hline 3624 & s130 & PM & F & 17 & G695 \\
\hline 3625 & s83 & PM & M & 18 & G783 \\
\hline 3626 & s127 & PM & F & 19 & G785 \\
\hline 3627 & s84 & PM & F & 16.5 & G780 \\
\hline 3628 & s92 & PM & $?$ & $?$ & \\
\hline 3629 & s51 & PM & M & 15 & G696 \\
\hline 3630 & s35 & PM & F & 18 & G697 \\
\hline 3631 & s36 & PM & M & 16 & G698 \\
\hline 3632 & s72 & PM & M & 16.5 & G699 \\
\hline 3633 & s66 & PM & F & 22.5 & G700 \\
\hline 3634 & s69 & PM & F & 17 & TAG 1 \\
\hline 3635 & s31 & PM & M & 20 & \\
\hline 3636 & s47 & PM & F & 10.5 & \\
\hline 3637 & s45 & PM & F & 20.5 & \\
\hline 3638 & s12 & PM & M & 20.5 & \\
\hline 3639 & s11 & PM & F & 18 & \\
\hline 3640 & s17 & PM & F & 18.5 & \\
\hline 3641 & s143 & PM & F & 15.5 & \\
\hline 3642 & s8 & SV & $?$ & 5.5 & \\
\hline
\end{tabular}

F.P. 9.15.04

\begin{tabular}{|l|l|l|l|l|l|}
\hline 3643 & s72 & PM & F & 21.5 & TAG 8 \\
\hline 3644 & s70 & PM & M & 17 & 9 \\
\hline 3645 & s43 & PM & M & 15 & 10 \\
\hline 3646 & s60 & PM & F & 24 & 11 \\
\hline 3647 & m23 & PM & M & 19 & 12 \\
\hline 3648 & s48 & PM & M & 18 & 13 \\
\hline 3649 & s32 & PM & F & 7.5 & 14 \\
\hline 3650 & s36 & PM & M & 18.5 & 15 \\
\hline 3651 & s132 & PM & M & 19.5 & 16 \\
\hline
\end{tabular}




\begin{tabular}{|l|l|l|l|l|r|}
\hline 3652 & s134 & PM & F & 11 & 17 \\
\hline 3653 & s144 & PM & F & 19.5 & 18 \\
\hline 3654 & s115 & PM & M & 19 & 19 \\
\hline 3655 & s102 & PM & F & 19.5 & 20 \\
\hline 3656 & s118 & PM & F & 22 & 21 \\
\hline 3657 & s107 & PM & M & 19 & 22 \\
\hline 3658 & s82 & PM & M & 16 & 23 \\
\hline 3659 & s75 & PM & M & 20.5 & 24 \\
\hline 3660 & s77 & PM & F & 15 & 25 \\
\hline 3661 & s140 & PM & F & 18 & 26 \\
\hline 3662 & s130 & PM & M & 14.5 & 27 \\
\hline 3663 & s90 & MO & M & 10 & \\
\hline 3664 & s8 & SV & $?$ & 7.8 & \\
\hline
\end{tabular}

\section{F.P. 9.16.04}

\begin{tabular}{|l|l|l|l|l|r|}
\hline 3665 & $\mathrm{p} 72$ & PM & M & 15 & TAG 28 \\
\hline 3666 & s54 & PM & M & 18 & 29 \\
\hline 3667 & s60 & PM & F & 21 & 30 \\
\hline 3668 & s144 & PM & F & 18 & 31 \\
\hline 3670 & s130 & PM & F & 19 & 32 \\
\hline 3671 & s139 & PM & M & 19 & G707? \\
\hline 3672 & s140 & SV & $?$ & 5.1 & \\
\hline 3674 & $\mathrm{t} 132$ & TD & M & 141 & \\
\hline 3675 & $\mathrm{t} 12$ & MM & $?$ & $?$ & \\
\hline
\end{tabular}

F.P. 9.17.04

\begin{tabular}{|l|l|l|l|l|r|}
\hline 3676 & p96 & PM & F & 15 & TAG 33 \\
\hline 3677 & s103 & PM & M & 14 & 34 \\
\hline 3678 & s98 & PM & M & 12.5 & 35 \\
\hline 3679 & s109 & PM & M & 21 & 36 \\
\hline 3680 & s144 & PM & M & 7 & 37 \\
\hline 3681 & s144 & PM & F & 6.5 & 38 \\
\hline 3682 & s42 & PM & F & 9 & 39 \\
\hline 3683 & s65 & PM & F & 19 & 40 \\
\hline 3684 & t120 & TD & F & 196 & \\
\hline 3685 & p67 & SV & ? & 3.5 & \\
\hline 3686 & s123 & SV & M & 8.2 & \\
\hline 3687 & s81 & PM & F & 14 & \\
\hline
\end{tabular}

TRNWR 9.22.04

\begin{tabular}{|l|l|l|l|l|ll|}
\hline 3688 & s44 & PM & F & 17.5 & TAG 41 & 43 \\
\hline 3689 & m138 & PM & M & 19 & 44 \\
\hline 3690 & s79 & PM & F & 16 & 45 \\
\hline 3691 & s87 & PM & M & 17 & 46 \\
\hline 3692 & s118 & PM & F & 19.5 & 47 \\
\hline 3693 & s68 & PM & F & 18 & 48 \\
\hline 3694 & s77 & PM & F & 14.5 & 49 \\
\hline 3695 & s82 & PM & M & 14.5 & & \\
\hline 3696 & p127 & PM & M & 14.5 & DIT/NBS & \\
\hline 3697 & s43 & PM & M & 14 & DIT/NBS & \\
\hline 3698 & s11 & SV & $?$ & 4 & DIT/NBS & \\
\hline 3699 & s7 & SV & F? & 4.9 & DIT/NBS & \\
\hline
\end{tabular}




\begin{tabular}{|c|c|c|c|c|c|}
\hline 3700 & s127 & ST & $?$ & 4.3 & DIT/NBS \\
\hline 3701 & S101 & SV & $?$ & 3.7 & DIT/NBS \\
\hline \multirow[t]{2}{*}{3702} & s117 & SV & $?$ & 4.0 & DIT/NBS \\
\hline & t72 & DV & $\mathrm{M}$ & & \\
\hline TRNWR & \multicolumn{5}{|c|}{9.23 .04} \\
\hline 3703 & s9 & PM & $F$ & 16 & TAG 51 \\
\hline 3704 & s12 & PM & $F$ & 18 & 52 \\
\hline 3705 & s118 & PM & $\mathbf{M}$ & 12 & 53 \\
\hline 3706 & $\mathrm{~m} 23$ & PM & $\mathbf{M}$ & 17 & 54 \\
\hline 3707 & s38 & PM & $F$ & 16.5 & 55 \\
\hline 3708 & s113 & PM & $F$ & 14 & 56 \\
\hline 3709 & s130 & PM & $F$ & 15 & 57 \\
\hline 3710 & s95 & PM & $F$ & 20 & 58 \\
\hline 3711 & s93 & PM & F & 18.5 & 59 \\
\hline 3712 & 568 & PM & $\mathbf{M}$ & 15.5 & 60 \\
\hline 3713 & s77 & PM & $F$ & 12.5 & 61 \\
\hline 3714 & $\mathrm{ml} 38$ & PM & $\mathbf{M}$ & 14 & 62 \\
\hline 3715 & p7 & ST & $\mathbf{F}$ & 4.5 & \\
\hline 3716 & s33. & ST & $?$ & 4.7 & \\
\hline & $\mathrm{t} 132$ & DV & & & \\
\hline
\end{tabular}

\begin{tabular}{|l|l|l|l|l|ll|}
\hline \multicolumn{3}{|l}{9.24 .04} & \multicolumn{1}{l|}{} & \\
\hline 3717 & S21 & PM & M & 8.5 & TAG 63 & 64 \\
\hline 3718 & S7 & PM & M & 15 & & 65 \\
\hline 3719 & S127 & PM & M & 8 & & 66 \\
\hline 3720 & s113 & PM & M & 15 & & 67 \\
\hline 3721 & S30 & PM & M & 13.5 & & 68 \\
\hline 3722 & S70 & PM & M & 16 & & 69 \\
\hline 3723 & S81 & PM & F & 21 & & 74 \\
\hline 3724 & S106 & PM & M & 15 & & 70 \\
\hline 3725 & M125 & PM & F & 22 & & \\
\hline 3726 & S 89 & ST & F & 3.7 & DIT/NBS & \\
\hline 3727 & S8 & ST & $?$ & 4 & DIT/NBS & \\
\hline 3728 & P139 & ST & $?$ & 5 & DIT/NBS & \\
\hline
\end{tabular}

TRNWR 9.25.04

\begin{tabular}{|l|l|l|l|r|rr|}
\hline 3729 & s106 & PM & F & 13 & TAG 71 & 72 \\
\hline 3730 & s12 & PM & M & 15 & & 73 \\
\hline 3731 & s69 & PM & F & 18.5 & 75 \\
\hline 3732 & s53 & PM & M & 12.5 & \\
\hline 3733 & t24 & MM & $?$ & & & \\
\hline
\end{tabular}

\section{TRYON CREEK 9.28.04}

\begin{tabular}{|l|l|l|l|l|ll|}
\hline 3734 & s54 & PM & F & 19 & TAG 76 & 77 \\
\hline 3735 & s82 & PM & M & 16.5 & 78 \\
\hline 3736 & s117 & PM & F & 20 & 79 \\
\hline 3737 & s42 & PM & M & 16.5 & 80 \\
\hline 3738 & m106 & PM & F & 19.5 & 82 \\
\hline 3739 & s6 & PM & M & 18 & 83 \\
\hline 3740 & s142 & PM & F & 17 & 84 \\
\hline 3741 & s125 & PM & M & 17 & \\
\hline 3742 & s118 & PM & M & 17.5 & DIT/NBS & \\
\hline
\end{tabular}




\begin{tabular}{l|l|l|l|l|l|l|}
\hline 3743 & p 64 & ST & $?$ & 6 & \\
\hline TC 9.29 .04 & p 67 & PM & M & 13 & TAG 86 & 87 \\
\hline 3744 & s114 & PM & M & 21 & & 88 \\
\hline 3745 & s108 & PM & F & 15 & 89 \\
\hline 3746 & s14 & PM & F & 17 & 90 \\
\hline 3747 & s118 & PM & F & 13 & 91 \\
\hline 3748 & s126 & PM & F & 15.5 & 92 \\
\hline 3749 & s58 & PM & M & 21 & & 9 \\
\hline 3750 & &
\end{tabular}

\begin{tabular}{l|l|l|l|l|l|ll|}
\hline TC 9.30 .04 & s79 & PM & M & 17 & TAG 93 & 94 \\
\hline 3751 & s33 & PM & F & 27 & & 95 \\
\hline 3752 & s9 & PM & F & 13 & & 96 \\
\hline 3753 & s142 & PM & M & 20 & & 97 \\
\hline 3754 & s108 & PM & M & 21 & & 92 \\
\hline 3755 &
\end{tabular}

TC 10.1.04
\begin{tabular}{|l|l|l|l|l|lr|}
\hline 3756 & s2 & PM & F & 16 & TAG 98 & 99 \\
\hline 3757 & s106 & PM & M & 15.5 & & 100 \\
\hline 3758 & s108 & PM & M & 19 & & 101 \\
\hline 3759 & s100 & PM & F & 15 & & \\
\hline 3760 & p 72 & ST & $?$ & 5.3 & DIT/NBS \\
\hline 3761 & p 40 & PM & & & Esc/NBS & \\
\hline
\end{tabular}

POWELL BUTTE 10.5.04

\begin{tabular}{|l|l|l|l|l|r|}
\cline { 3 - 6 } \multicolumn{1}{l}{3762} & s58 & MO & F & 17 & TAG 102 \\
\hline 3763 & s56 & PM & M & 15 & 103 \\
\hline & s5 & PM & F & 15.5 & G795 \\
\hline 3765 & s127 & PM & M & 17 & 104 \\
\hline 3766 & s68 & PM & M & 20.5 & 105 \\
\hline 3767 & s130 & PM & F & 15 & 106 \\
\hline 3768 & s29 & PM & M & 16.5 & 107 \\
\hline 3769 & s110 & MO & F & 10.5 & G797 \\
\hline & s128 & PM & F & 17.5 & 108 \\
\hline 3771 & s21 & PM & F & 17.5 & 109 \\
\hline 3772 & s144 & PM & F & 16 & G768 \\
\hline & s33 & PM & M & 15 & 110 \\
\hline 3774 & s46 & PM & F & 15.5 & 111 \\
\hline 3775 & m60 & PM & M & 17 & 112 \\
\hline 3776 & s84 & PM & F & 16 & G619 \\
\hline 3777 & s69 & ST & $?$ & 4.2 & \\
\hline 3778 & s84 & PM & M & 15.5 & \\
\hline
\end{tabular}

\begin{tabular}{|l|l|l|l|l|r|r|}
\hline PB 10.06.04 & s14 & PM & F & 17 & TAG 113 \\
\hline 3779 & s22 & PM & F & 20.5 & 114 \\
\hline 3780 & s23 & PM & M & 14 & 115 \\
\hline 3781 & s33 & PM & M & 15.5 & 116 \\
\hline 3782 & s63 & PM & F & 8 & 117 \\
\hline 3783 & s68 & PM & F & 14 & 118 \\
\hline 3784 & s103 & PM & M & 16 & 119 \\
\hline 3785 & s105 & PM & M & 16.5 & 120 \\
\hline 3786 & \multicolumn{7}{|c|}{218} & \\
\hline
\end{tabular}




\begin{tabular}{|l|l|l|l|l|r|}
\hline 3787 & s101 & MO & F & 13 & 122 \\
\hline 3788 & s108 & PM & M & 12 & 123 \\
\hline 3789 & p100 & PM & F & 13 & 124 \\
\hline 3790 & s29 & SV & $?$ & 4.9 & \\
\hline
\end{tabular}

PB 10.7.04
\begin{tabular}{|l|l|l|l|l|l|}
\hline 3791 & s 58 & PM & F & 20 & TAG 125 \\
\hline 3792 & s104 & PM & F & 14.5 & 126 \\
\hline 3793 & s130 & PM & F & 19 & 127 \\
\hline 3794 & s32 & PM & M & 14 & 128 \\
\hline 3795 & s39 & PM & F & 14.5 & 129 \\
\hline 3796 & s114 & PM & M & 13.5 & 130 \\
\hline 3797 & s109 & PM & F & 8 & 131 \\
\hline 3798 & s84 & PM & M & 15 & 132 \\
\hline 3799 & p 96 & PM & F & 19 & 133 \\
\hline 3800 & m96 & PM & F & 13 & 134 \\
\hline 3801 & s 67 & MO & F & 14 & \\
\hline 3802 & s66 & ST & $?$ & 5.0 & \\
\hline 3803 & p120 & MO & M & 18 & \\
\hline
\end{tabular}

\section{PB 10.8.04}

\begin{tabular}{|l|l|l|l|l|r|}
\hline 3804 & m96 & PM & F & 12.5 & TAG 135 \\
\hline & & & & 15.3 & 136 \\
3805 & s58 & PM & M & 5 & 137 \\
\hline 3806 & s144 & PM & M & 16 & \\
\hline & t72 & DV & & & \\
\hline
\end{tabular}

OXBOW 10.12.04

\begin{tabular}{|c|c|c|c|c|c|}
\hline 3807 & s95 & TT & $\mathrm{M}$ & 80 & TAG 138 \\
\hline 3808 & s61 & $\mathrm{TT}$ & $F$ & 72 & 139 \\
\hline \multirow[t]{3}{*}{3809} & s96 & PM & $\mathbf{M}$ & 16 & 140 \\
\hline & s131 & PM & $F$ & 19 & G678 \\
\hline & s126 & PM & $F$ & 17.5 & G683 \\
\hline 3812 & $\mathrm{~s} 142$ & $\mathrm{PM}$ & $\mathbf{M}$ & 14 & 141 \\
\hline 3813 & $\mathrm{~s} 130$ & $\mathrm{PM}$ & $\mathbf{M}$ & 15 & 143 \\
\hline 3814 & s83 & PM & M & $?$ & \\
\hline 3815 & s113 & PM & $\mathbf{M}$ & 14 & 144 \\
\hline \multirow[t]{2}{*}{3816} & s1 19 & PM & $\mathbf{M}$ & 16 & 145 \\
\hline & s59 & PM & $\mathbf{M}$ & 13 & G638 \\
\hline 3818 & $s 60$ & PM & $F$ & 17.5 & 146 \\
\hline \multirow[t]{2}{*}{3819} & s46 & PM & $\mathbf{F}$ & 11 & 147 \\
\hline & s55 & PM & $\mathbf{M}$ & 18 & G684 \\
\hline 3821 & $\mathrm{~s} 44$ & PM & $F$ & 17 & 148 \\
\hline 3822 & s41 & $\mathrm{PM}$ & $F$ & 15 & G664 \\
\hline \multirow[t]{2}{*}{3823} & s7 & $\mathrm{PM}$ & $F$ & 15.5 & 149 \\
\hline & s23 & PM & $\mathbf{F}$ & 13.5 & 150 \\
\hline 3825 & $\mathrm{~s} 22$ & PM & $F$ & 19.5 & 151 \\
\hline 3826 & s119 & SV & $\mathbf{M}$ & 5.2 & \\
\hline 3827 & $\mathrm{p} 7$ & ST & $?$ & 3.5 & \\
\hline 3828 & s70 & $\mathrm{PM}$ & $F$ & 19 & \\
\hline 3829 & $t 24$ & TD & $\mathbf{M}$ & 192 & \\
\hline
\end{tabular}

Ox 10.13.04 


\begin{tabular}{|c|c|c|c|c|c|}
\hline 3830 & $\mathrm{~s} 62$ & TT & $\mathbf{M}$ & 78 & TAG 152 \\
\hline 3831 & s116 & PM & $\mathbf{M}$ & 9.5 & 153 \\
\hline 3832 & $\mathrm{~m} 116$ & PM & $F$ & 20 & 154 \\
\hline 3833 & m68 & $\mathrm{PM}$ & $\mathbf{M}$ & 17 & 155 \\
\hline 3834 & $\mathrm{~s} 41$ & PM & $\mathrm{M}$ & 7.5 & 156 \\
\hline 3835 & $\mathrm{~s} 69$ & PM & $\mathrm{M}$ & 16 & 157 \\
\hline 3836 & s35 & PM & $\mathrm{F}$ & 16 & 158 \\
\hline 3837 & $s 49$ & PM & $\mathrm{M}$ & 16 & 159 \\
\hline 3838 & $\mathrm{~s} 143$ & PM & $\mathrm{F}$ & 13.5 & 160 \\
\hline 3839 & $\mathrm{~s} 23$ & PM & $\mathrm{M}$ & 17.5 & 161 \\
\hline 3840 & $\mathrm{~s} 26$ & PM & $\overline{\mathrm{M}}$ & 14.5 & 162 \\
\hline 3841 & s24 & PM & $\mathbf{M}$ & 17 & 163 \\
\hline 3842 & s5 & PM & $\mathrm{M}$ & 13.5 & 164 \\
\hline 3843 & $\mathrm{~m} 21$ & PM & $\mathbf{M}$ & 16 & 166 \\
\hline 3844 & $\mathrm{p} 40$ & ST & $\mathrm{F}$ & 4.4 & \\
\hline 3845 & s66 & SV & $\mathrm{M}$ & 7.5 & \\
\hline 3846 & $\mathrm{~s} 108$ & $\mathrm{PM}$ & $\mathbf{M}$ & 11 & \\
\hline 3847 & $\mathrm{~m} 100$ & PM & $\mathrm{F}$ & 17 & \\
\hline 3848 & t84 & TD & $\mathrm{F}$ & 176 & \\
\hline 3849 & $\mathrm{p} 84$ & ST & $\mathrm{F}$ & 4.5 & \\
\hline \multicolumn{6}{|c|}{ OX 10.14.04 } \\
\hline 3850 & $\mathrm{p} 95$ & PM & $F$ & 8.5 & TAG 167 \\
\hline 3851 & s90 & TT & $\mathbf{M}$ & $?$ & \\
\hline 3852 & s130 & $\mathrm{TT}$ & $F$ & 69 & 168 \\
\hline 3853 & s93 & TT & $\mathrm{F}$ & 68 & 169 \\
\hline 3854 & s49 & $\mathrm{ME}^{\prime}$ & $\mathrm{M}$ & 64 & 170 \\
\hline 3856 & +24 & TD & F & 139 & \\
\hline \multicolumn{6}{|c|}{ OX 10.15 .04} \\
\hline 3857 & $\mathrm{~s} 68$ & TT & $\mathbf{M}$ & 70 & TAG 171 \\
\hline $38 \overline{58}$ & s71 & $\mathrm{PM}$ & $\mathrm{M}$ & 10.5 & 172 \\
\hline 3859 & s84 & TT & $\mathrm{F}$ & 72 & 173 \\
\hline 3860 & $\mathrm{~s} 66$ & PM & $\bar{F}$ & 11 & 174 \\
\hline 3861 & $\mathrm{~s} 46$ & TT & $\bar{M}$ & 75 & 176 \\
\hline 3862 & s27 & PM & $\mathbf{M}$ & 11 & 177 \\
\hline $38 \overline{63}$ & $\mathrm{~s} 30$ & PM & $F$ & 13.5 & 178 \\
\hline 3864 & s119 & PM & F & 14 & \\
\hline 3865 & s125 & TT & $F$ & 70 & \\
\hline 3866 & p139 & PM & $\mathbf{M}$ & 13.5 & \\
\hline 3867 & p139 & $\mathrm{PM}$ & $\mathrm{F}$ & 9.5 & \\
\hline 3868 & p7 & ST & $\bar{F}$ & 4 & \\
\hline 3869 & p28 & ST & $\mathrm{F}$ & 3.5 & \\
\hline 3870 & p31 & ST & F & 2.5 & \\
\hline 3871 & $\mathrm{p} 40$ & ST & $?$ & 4 & \\
\hline 3872 & t84 & $\mathrm{TD}$ & $F$ & 122 & \\
\hline 3873 & $\mathrm{t} 144$ & TD & $\mathbf{M}$ & 150 & \\
\hline 3874 & $\mathrm{p} 4$ & ST & $\mathrm{F}$ & 4.25 & \\
\hline
\end{tabular}

Forest Park 10.26.04

\begin{tabular}{|l|l|l|l|l|l|}
\hline 3874 & s46 & TT & F & 75 & TAG 179 \\
\hline \multicolumn{5}{|c|}{220} \\
\hline
\end{tabular}




\begin{tabular}{|c|c|c|c|c|c|}
\hline 3875 & $\mathrm{~s} 43$ & $\mathrm{PM}$ & $\mathrm{F}$ & 14.5 & 180 \\
\hline 3876 & $\mathrm{s50}$ & PM & $\bar{F}$ & 14.5 & 181 \\
\hline 3877 & $\mathrm{~s} 102$ & PM & M & 14 & 182 \\
\hline 3879 & s38 & PM & $\mathrm{M}$ & 19.5 & 183 \\
\hline 3880 & s95 & PM & $\mathbf{M}$ & 19 & 184 \\
\hline 3883 & s59 & PM & $F$ & 22 & 185 \\
\hline 3884 & s72 & PM & $F$ & 16.5 & 186 \\
\hline 3885 & s67 & PM & $\mathrm{F}$ & 12 & 187 \\
\hline 3886 & s119 & PM & $\bar{F}$ & 19 & 188 \\
\hline 3893 & $\mathrm{~s} 120$ & $\mathrm{PM}$ & $F$ & 18.5 & \\
\hline 3894 & s69 & ST & $?$ & 4.8 & \\
\hline 3895 & s144 & PM & $\mathrm{F}$ & 15.5 & \\
\hline 3896 & s84 & PM & $\mathbf{M}$ & 16.5 & \\
\hline 3897 & p144 & PM & $F$ & 16.5 & \\
\hline 3898 & p144 & PM & $\mathbf{M}$ & 15 & \\
\hline 3666 & s65 & PM & $\mathbf{M}$ & 18 & 29 \\
\hline 3646 & s60 & PM & $F$ & 21.5 & 11 \\
\hline 3656 & $\mathrm{~m} 120$ & PM & F & 20 & 21 \\
\hline 3629 & s61 & $\overline{\mathrm{PM}}$ & $\mathbf{M}$ & 16.5 & 696 \\
\hline 3639 & s70 & PM & $\mathrm{M}$ & 16 & 6 \\
\hline 3650 & s9 & PM & $\mathbf{M}$ & 16.5 & 15 \\
\hline 3670 & $\mathrm{~s} 132$ & PM & $\mathrm{F}$ & 17 & 32 \\
\hline 3659 & s91 & PM & $\mathbf{M}$ & 17.5 & 24 \\
\hline 3679 & s89 & PM & $\mathbf{M}$ & 17 & 36 \\
\hline
\end{tabular}

\begin{tabular}{|c|c|c|c|c|c|}
\hline \multicolumn{6}{|c|}{ FP 10.27 .04} \\
\hline 3899 & $s 69$ & PM & F & 14.5 & TAG 190 \\
\hline 3900 & s44 & TT & $F$ & 80 & 191 \\
\hline 3901 & $\mathrm{~s} 22$ & $\mathrm{PM}$ & $F$ & 13 & 192 \\
\hline 3902 & s24 & $\mathrm{PM}$ & $F$ & 14 & 193 \\
\hline 3903 & s95 & PM & F & 19.5 & 194 \\
\hline 3904 & s96 & PM & $\mathrm{F}$ & 18 & 195 \\
\hline 3905 & $\mathrm{~s} 85$ & PM & $F$ & 17.5 & 197 \\
\hline 3906 & $\mathrm{~s} 12$ & PM & $\mathbf{M}$ & 18 & \\
\hline 3907 & s96s & PM & $F$ & 10.5 & \\
\hline 3645 & $\mathrm{~s} 32$ & PM & $\mathbf{M}$ & 15 & 10 \\
\hline 3671 & $\mathrm{~s} 138$ & PM & $\mathrm{M}$ & 18 & 787 \\
\hline 3660 & s78 & PM & $F$ & 15.5 & 25 \\
\hline
\end{tabular}

\begin{tabular}{|c|c|c|c|c|c|}
\hline \multicolumn{6}{|c|}{ FP 10.28 .04} \\
\hline 3908 & p55 & PM & $\mathrm{F}$ & 16 & TAG 198 \\
\hline 3909 & s105 & PM & $\mathrm{F}$ & 19.5 & 200 \\
\hline 3910 & s71 & PM & $\bar{M}$ & 17.5 & 201 \\
\hline 3911 & s90 & PM & M & 13. & 202 \\
\hline 3913 & $s 46$ & PM & $\mathbf{M}$ & 18.5 & 203 \\
\hline 3914 & $\mathrm{~s} 47$ & PM & $\bar{M}$ & 13 & 204 \\
\hline 3915 & s55 & $\mathrm{PM}$ & $\bar{M}$ & 15 & 205 \\
\hline 3916 & $\mathrm{~m} 117$ & TT & $\mathrm{F}$ & 71 & \\
\hline 3626 & s139 & PM & F & 15.5 & 785 \\
\hline 3617 & p96 & PM & $\mathbf{M}$ & 16.5 & 688 \\
\hline
\end{tabular}

FP 10.29.04 


\begin{tabular}{|l|l|l|l|l|ll|}
\hline 3917 & s72 & PM & F & 17.5 & TAG 206 & 207 \\
\hline 3918 & s47 & PM & M & 20 & & 208 \\
\hline 3919 & s0 & PM & M & 14 & & 209 \\
\hline 3920 & s46 & PM & M & 18 & & 210 \\
\hline 3921 & s78 & PM & M & 17 & & \\
\hline 3922 & s9 & PM & M & 10 & & \\
\hline 3923 & t7 & TD & M & 180 & & \\
\hline 3615 & s78 & PM & M & 17 & G686 & \\
\hline 3621 & s90 & PM & F & 15 & G692 & \\
\hline 3618 & s105 & PM & M & 17 & G689 & \\
\hline
\end{tabular}

TRNWR 11.2.04

\begin{tabular}{|l|l|l|l|l|r|}
\hline 3924 & s10 & PM & M & 13 & TAG 210 \\
\hline 3925 & s83 & PM & M & 17 & 211 \\
\hline 3926 & s11 & PM & M & 14 & 212 \\
\hline 3928 & s23 & PM & F & 13 & 213 \\
\hline 3929 & s140 & PM & M & 15.5 & 214 \\
\hline 3930 & s102 & PM & M & 15 & 215 \\
\hline 3931 & s24 & PM & F & 15 & 217 \\
\hline 3932 & s30 & PM & F & 11.5 & 218 \\
\hline 3933 & s46 & PM & M & 14 & 219 \\
\hline 3934 & m8 & PM & F & 18 & 220 \\
\hline 3935 & m81 & PM & F & 13 & 221 \\
\hline 3936 & p84 & PM & F & 14 & 222 \\
\hline 3937 & p31 & PM & M & 14.5 & 223 \\
\hline 3938 & p48 & PM & M & 13 & 224 \\
\hline 3940 & p43 & PM & F & 13.5 & 225 \\
\hline 3941 & p84 & PM & F & 18 & 226 \\
\hline 3942 & p48 & PM & F & 15.5 & \\
\hline 3943 & $?$ & PM & F & 15 & 70 \\
\hline 3725 & s126 & PM & M & 15.5 & 57 \\
\hline 3709 & s128 & PM & F & 14.5 & 45 \\
\hline 3691 & s123 & PM & M & 16 & 54 \\
\hline 3706 & s33 & PM & M & 18 & 53 \\
\hline 3705 & s118 & PM & M & 14 & \\
\hline
\end{tabular}

\begin{tabular}{|c|c|c|c|c|c|}
\hline \multicolumn{6}{|c|}{ TRNWR 11.3.04 } \\
\hline 3944 & s131 & PM & M & 11.5 & TAG 227 \\
\hline 3945 & $\mathrm{~s} 125$ & PM & $\mathrm{F}$ & 13 & 228 \\
\hline 3946 & s47 & PM & $F$ & 20.5 & 229 \\
\hline 3947 & s83 & PM & $\mathbf{M}$ & 16 & 230 \\
\hline 3948 & s118 & PM & $\mathrm{F}$ & 11.5 & 231 \\
\hline 3949 & s4 & PM & $\mathrm{F}$ & 15 & 232 \\
\hline 3950 & s45 & PM & $?$ & 5.7 & \\
\hline 3951 & s32 & PM & $\mathbf{M}$ & 54 & \\
\hline 3690 & s7 & $\mathrm{PM}$ & $\mathrm{F}$ & 16 & 44 \\
\hline
\end{tabular}

TRNWR 11.4.04

\begin{tabular}{|l|l|l|l|l|ll|}
\hline 3952 & p48 & PM & M & 16 & TAG 233 & 234 \\
\hline 3953 & s144 & PM & F & 17 & 235 \\
\hline 3954 & s128 & PM & M & 17 & 236 \\
\hline 3955 & s7 & PM & F & 15 & & 20 \\
\hline
\end{tabular}




\begin{tabular}{|l|l|l|l|l|l|}
\hline 3956 & s46 & PM & M & 12 & \\
\hline 3957 & s96 & PM & M & 17 & \\
\hline 3958 & s103 & PM & F & 21 & \\
\hline 3959 & s83 & MT & F & 38 & \\
\hline
\end{tabular}

TRNWR 11.5.04

\begin{tabular}{|l|l|l|l|l|ll|}
\hline 3960 & s33 & PM & F & 11.5 & TAG 238 & 239 \\
\hline 3961 & s134 & PM & M & 17.5 & 240 \\
\hline 3962 & s128 & PM & F & 12.5 & & 24 \\
\hline
\end{tabular}

TRYON CREEK 11.9.04

\begin{tabular}{|l|l|l|l|l|r|}
\hline 3963 & s68 & PM & M & 16.5 & TAG 242 \\
\hline 3964 & s55 & PM & F & 15.5 & 243 \\
\hline 3965 & s83 & PM & M & 16 & 245 \\
\hline 3966 & s108 & PM & M & 20 & 244 \\
\hline 3967 & s14 & PM & F & 21.5 & 246 \\
\hline 3968 & s128 & PM & F & 18 & 247 \\
\hline 3969 & s119 & PM & F & 15.5 & 248 \\
\hline 3970 & s96 & PM & M & 18 & 249 \\
\hline 3971 & s102 & PM & F & 18.5 & 250 \\
\hline 3972 & m140 & PM & F & 28.5 & 251 \\
\hline 3973 & p91 & PM & F & 14 & \\
\hline 3974 & p67 & PM & F & 4.4 & 78 \\
\hline 3975 & p115 & PM & F & 5 & 98 \\
\hline 3736 & s116 & PM & F & 19.5 & 87 \\
\hline 3756 & s97 & PM & F & 17.5 & \\
\hline 3745 & m112 & PM & M & 17.5 & \\
\hline
\end{tabular}

TC 11.10 .04
\begin{tabular}{|l|l|l|l|l|ll|}
\hline 3976 & s89 & PM & F & 18 & TAG 252 & 253 \\
\hline 3977 & s 92 & PM & F & 21 & & 254 \\
\hline 3978 & s114 & PM & F & 18 & 255 \\
\hline 3979 & s 99 & PM & M & 17 & & \\
\hline 3980 & s137 & PM & M & & & 100 \\
\hline 3981 & s43 & TT & F & 68 & & \\
\hline 3982 & p 40 & PM & M & 11 & & \\
\hline 3758 & S108 & PM & M & 18 & & \\
\hline
\end{tabular}

\begin{tabular}{|c|c|c|c|c|c|}
\hline \multicolumn{6}{|c|}{ TC 11.11.04 } \\
\hline 3983 & $\mathrm{~s} 45$ & PM & $\mathrm{F}$ & 24 & TAG 256 \\
\hline 3984 & s137 & PM & $\mathbf{M}$ & 20.5 & 257 \\
\hline 3985 & $\mathrm{~s} 58$ & $\overline{\mathrm{PM}}$ & $\mathrm{M}$ & 22 & 258 \\
\hline 3986 & $\mathrm{~s} 48$ & PM & $F$ & 22 & 259 \\
\hline 3987 & s116 & PM & $\mathbf{M}$ & 10 & 260 \\
\hline 3988 & s59 & $\mathrm{PM}$ & $F$ & 17.5 & 261 \\
\hline 3989 & $\mathrm{p} 79$ & $\mathrm{PM}$ & $\mathrm{F}$ & 17.5 & 262 \\
\hline 3990 & $\mathrm{~s} 81$ & PM & $\mathrm{F}$ & 13 & \\
\hline 3991 & $\mathrm{~s} 102$ & SV & $\mathbf{M}$ & 8.5 & \\
\hline 3753 & s8 & $\mathrm{PM}$ & $F$ & 20 & 95 \\
\hline 3754 & $\mathrm{~s} 128$ & PM & $\mathbf{M}$ & 19 & 96 \\
\hline 3746 & s108 & $\mathrm{PM}$ & $\mathrm{F}$ & 13 & 88 \\
\hline & 560 & PM & $?$ & $?$ & \\
\hline
\end{tabular}

TC 11.12.04 


\begin{tabular}{|l|l|l|l|l|r|}
\hline 3992 & P115 & PM & M & 12 & TAG 263 \\
\hline 3993 & S66 & PM & F & 22.5 & 264 \\
\hline 3994 & S9 & PM & M & 17.5 & 265 \\
\hline 3995 & S8 & PM & F & 14.5 & 267 \\
\hline 3996 & S20 & PM & F & 19 & 268 \\
\hline 3997 & S60 & PM & M & 13.5 & 269 \\
\hline 3998 & P60 & PM & M & 19 & 270 \\
\hline 3999 & P60 & PM & M & 15 & 271 \\
\hline 4000 & S21 & PM & F & 12 & 272 \\
\hline 4001 & P24 & SV & M & 8.5 & \\
\hline 4002 & S132 & PM & F & 17 & \\
\hline 4003 & S89 & PM & M & 16 & \\
\hline 4004 & P76 & ST & $?$ & 5.2 & \\
\hline 4005 & T36 & SN & M & & \\
\hline 3739 & S16 & PM & F & & \\
\hline
\end{tabular}

POWELL BUTTE 11.16.04

\begin{tabular}{|l|l|l|l|l|r|}
\hline 4007 & s142 & PM & F & 16 & TAG 273 \\
\hline 4008 & s143 & PM & F & 18 & 274 \\
\hline 4009 & s116 & PM & M & 17 & 275 \\
\hline 4010 & s45 & PM & M & 15 & 276 \\
\hline 4011 & s3 & PM & F & 17.5 & 277 \\
\hline 3765 & s127 & PM & & 15.5 & 104 \\
\hline 3478 & s128 & PM & & 15.5 & 797 \\
\hline 3796 & s123 & PM & & 15 & 130 \\
\hline 3784 & s56 & PM & & 14 & 118 \\
\hline 3466 & s60 & PM & & 21.5 & 775 \\
\hline 3782 & s33 & PM & & 16.5 & 116 \\
\hline 3797 & s26 & PM & & 13.5 & 131 \\
\hline
\end{tabular}

PB 11.17.04

\begin{tabular}{|l|l|l|l|l|r|}
\hline 4012 & s25 & PM & F & 13.5 & TAG 279 \\
\hline 4013 & s111 & PM & M & 15 & 280 \\
\hline 4014 & s104 & PM & M & 16 & 281 \\
\hline 4015 & s57 & PM & M & 14 & 282 \\
\hline 4016 & s5 & PM & M & 13 & 283 \\
\hline 4017 & s33 & PM & M & 12.5 & 284 \\
\hline 4018 & s48 & PM & M & 17 & 285 \\
\hline 4019 & p108 & PM & F & 13 & \\
\hline 4020 & p144 & PM & F & 14 & 800 \\
\hline 3491 & m96 & PM & & 20 & 601 \\
\hline 3492 & s36 & PM & & 16 & \\
\hline
\end{tabular}

PB 11.18.04
\begin{tabular}{|l|l|l|l|l|r|}
\hline 4021 & s69 & MO & F & 15 & TAG 286 \\
\hline 4022 & s142 & PM & M & 16 & 287 \\
\hline 4023 & p84 & PM & M & 14 & 288 \\
\hline 4024 & s77 & PM & M & 13.5 & 289 \\
\hline 4025 & m77 & PM & M & 16.5 & 290 \\
\hline
\end{tabular}

PB 11.19.04

\begin{tabular}{|l|l|l|l|l|r|}
\hline 4026 & s95 & GS & F & 120 & TAG 291 \\
\hline 4027 & s142 & PM & M & 13 & 292 \\
\hline
\end{tabular}




\begin{tabular}{|l|l|l|l|l|r|}
\hline 4028 & s5 & PM & M & 13 & 293 \\
\hline 4029 & s81 & PM & M & 17.5 & 294 \\
\hline 4030 & s7 & PM & F & 15 & \\
\hline 4031 & s139 & PM & F & 12 & \\
\hline 4032 & s108 & PM & F & 18.5 & \\
\hline
\end{tabular}

OXBOW 11.30 .04

\begin{tabular}{|c|c|c|c|c|c|}
\hline 4033 & s78 & PM & $\mathbf{M}$ & 16.5 & TAG 295 \\
\hline 4034 & s77 & PM & $\mathrm{F}$ & 14 & 296 \\
\hline 4035 & S108 & PM & $\mathrm{F}$ & 13 & 297 \\
\hline 4036 & s128 & PM & $F$ & 18 & 298 \\
\hline 4037 & s134 & TT & $\mathrm{M}$ & 82 & 300 \\
\hline 4038 & $\mathrm{~s} 28$ & TT & $\mathbf{M}$ & 78 & 301 \\
\hline 4039 & $\mathrm{~s} 32$ & $\overline{\mathrm{PM}}$ & $F$ & 15 & 302 \\
\hline 4040 & s59 & PM & $\mathrm{M}$ & 13.5 & 303 \\
\hline 4041 & s33 & PM & $\mathbf{M}$ & 14 & 304 \\
\hline 4042 & $\mathrm{p} 55$ & PM & $\mathbf{M}$ & 13 & 305 \\
\hline 4043 & s59 & ST & $F$ & 4.2 & \\
\hline 4044 & $\mathrm{p} 48$ & ST & $\mathrm{F}$ & 5.0 & \\
\hline 4045 & p100 & ST & $\mathrm{F}$ & 6.3 & \\
\hline 4046 & s119 & ST & $\mathrm{F}$ & 5.5 & \\
\hline 4047 & p144 & ST & $?$ & 4.3 & \\
\hline 4048 & p60 & ST & $?$ & 3.8 & \\
\hline 4049 & $\mathrm{~m} 114$ & $\overline{\mathrm{MO}}$ & $\bar{F}$ & 17.5 & \\
\hline 4050 & $\mathrm{~s} 68$ & PM & $\mathbf{M}$ & 14 & \\
\hline 4051 & $\mathrm{p} 84$ & SV & $?$ & & \\
\hline 4052 & p112 & ST & $?$ & & \\
\hline 3859 . & s105 & TT & $F$ & 72 & 173 \\
\hline 3592 & s143 & PM & $F$ & 14.5 & 675 \\
\hline 3843 & s72 & $\mathrm{PM}$ & $\mathbf{M}$ & 15 & 166 \\
\hline 3835 & s69 & $\overline{P M}$ & $\overline{\mathrm{M}}$ & 16 & 157 \\
\hline 3840 & $s 66$ & PM & $\mathbf{M}$ & 16 & 162 \\
\hline 3580 & $\mathrm{~s} 120$ & PM & $\mathbf{M}$ & 14 & 665 \\
\hline
\end{tabular}

\begin{tabular}{|c|c|c|c|c|c|}
\hline \multicolumn{6}{|c|}{ OX 12.1.04 } \\
\hline 4054 & s14 & PM & F & 17 & 306 \\
\hline 4055 & $\mathrm{~m} 127$ & PM & $F$ & 12 & 307 \\
\hline 4056 & p120 & PM & F & 11 & 308 \\
\hline 4057 & $\mathrm{s59}$ & PM & $F$ & 13 & 309 \\
\hline 4058 & $\mathrm{~s} 126$ & PM & $F$ & 13 & 310 \\
\hline 4059 & s98 & PM & $\mathbf{M}$ & 14 & 311 \\
\hline 4060 & s49 & $\overline{\mathbf{P M}}$ & $\mathbf{M}$ & 12 & \\
\hline 4061 & p64 & SV & $?$ & 8.4 & \\
\hline 4062 & s41 & ST & $?$ & 4.8 & \\
\hline 4063 & $\mathrm{p} 48$ & ST & $?$ & 4.4 & \\
\hline 4064 & p31 & ST & $?$ & 5.7 & \\
\hline 2768 & s1 & GS & $F$ & 120 & G726 \\
\hline 3816 & $\mathrm{~m} 115$ & $\overline{P M}$ & $\mathbf{M}$ & 17 & 145 \\
\hline 3545 & s58 & PM & $\mathbf{M}$ & 11.5 & G638 \\
\hline 3579 & $\mathrm{~m} 65$ & PM & $\mathrm{F}$ & 12 & G664 \\
\hline 3819 & $\mathrm{~s} 45$ & PM & $\mathrm{F}$ & 11 & 147 \\
\hline
\end{tabular}




\begin{tabular}{|c|c|c|c|c|c|}
\hline 3841 & s24 & PM & $\mathrm{M}$ & 14 & 163 \\
\hline \multicolumn{6}{|c|}{ OX 12.2.04 } \\
\hline 4065 & S95 & GS & $\mathbf{M}$ & $?$ & ESC \\
\hline 4066 & S118 & GS & $F$ & 134 & 312 \\
\hline 4067 & S114 & PM & $F$ & 15.5 & 313 \\
\hline 4068 & $\mathrm{P} 120$ & PM & $\mathrm{F}$ & 10 & 314 \\
\hline 4069 . & P96 & $\overline{P M}$ & $\bar{F}$ & 14.5 & 315 \\
\hline 4070 & P55 & SV & $\mathbf{M}$ & 8.5 & \\
\hline 4071 & T144 & DV & $?$ & $?$ & \\
\hline 3839 & s23 & PM & $\mathbf{M}$ & & \\
\hline 3825 & s33 & $\mathrm{PM}$ & $F$ & & \\
\hline \multicolumn{6}{|c|}{ OX 12.3.04 } \\
\hline 4072 & t60 & TD & $?$ & $?$ & $\mathrm{ESC}$ \\
\hline 4073 & s126 & TT & $\mathbf{M}$ & 102 & 316 \\
\hline 4074 & s96 & PM & $\mathbf{M}$ & 14 & 317 \\
\hline 4075 & s95 & $\mathrm{PM}$ & $\mathbf{M}$ & 16.5 & 318 \\
\hline 4076 & $\mathrm{~s} 23$ & $\overline{P M}$ & $F$ & 12 & \\
\hline 4077 & s1 & PM & $F$ & 10 & 319 \\
\hline 4078 & s134 & $\overline{\mathrm{PM}}$ & $F$ & 8 & 320 \\
\hline 4079 & s58 & $\mathrm{PM}$ & $F$ & 10 & 321 \\
\hline 4080 & p76 & SV & $?$ & 6.2 & \\
\hline 4081 & $s 96$ & SV & $?$ & 5.4 & \\
\hline 4082 & p67 & SV & $?$ & 5.2 & \\
\hline 4083 & $\mathrm{p} 60$ & MO & $F$ & 12.5 & \\
\hline
\end{tabular}

\section{FOREST PARK 12.7.04}

\begin{tabular}{|c|c|c|c|c|c|}
\hline 4084 & $\mathrm{~s} 12$ & PM & $\mathbf{M}$ & 14.5 & TAG 323 \\
\hline 4085 & s120 & PM & $\mathbf{M}$ & 15 & 324 \\
\hline 4086 & s144 & PM & $\mathbf{M}$ & 13 & 325 \\
\hline 4087 & s107 & PM & $\mathbf{M}$ & 16.5 & 326 \\
\hline 4088 & s115 & PM & $\mathrm{M}$ & 15 & 327 \\
\hline 4089 & $\mathrm{~m} 95$ & $\overline{P M}$ & $F$ & 11 & 328 \\
\hline 4090 & s119 & $\overline{\mathbf{P M}}$ & F & 13.5 & 329 \\
\hline 4091 & s97 & PM & $\mathrm{F}$ & 12 & 330 \\
\hline 4092 & p136 & PM & $F$ & 11 & 331 \\
\hline 4093 & s102 & PM & $\mathbf{M}$ & 15 & 332 \\
\hline 4094 & p115 & PM & $F$ & 16 & \\
\hline 3615 & $\mathrm{~s} 82$ & PM & $\mathbf{M}$ & 16 & G686 \\
\hline 3618 & s116 & PM & $\mathrm{M}$ & 15 & G689 \\
\hline 3633 & s84 & PM & $\mathrm{F}$ & 15.5 & G700 \\
\hline 3874 & $\mathrm{s35}$ & TT & $\mathrm{F}$ & 73 & 179 \\
\hline 3920 & $\mathrm{~m} 46$ & PM & $\mathrm{M}$ & 16 & 209 \\
\hline 3883 & s46 & PM & $\mathrm{F}$ & 16.5 & 185 \\
\hline 3915 & s54 & PM & $\mathrm{M}$ & 15 & 205 \\
\hline 3666 & $\mathrm{~s} 56$ & PM & $\mathrm{M}$ & 16.5 & 29 \\
\hline 3639 & s47 & $\overline{\mathbf{P M}}$ & $\mathrm{M}$ & 17 & 6 \\
\hline 3647 & s23 & $\mathrm{PM}$ & $\mathrm{M}$ & 17.5 & 12 \\
\hline 3879 & s34 & PM & $\mathbf{M}$ & 17.5 & 183 \\
\hline \multicolumn{6}{|c|}{ FP 12.8.04 } \\
\hline 4095 & p120 & PM & $\mathbf{M}$ & 13 & 333 \\
\hline
\end{tabular}




\begin{tabular}{|l|l|l|l|l|r|}
\hline 4096 & s51 & PM & M & 15 & 335 \\
\hline 4097 & s70 & PM & F & 13 & 336 \\
\hline 4098 & s5 & PM & F & 13 & 337 \\
\hline 4099 & p100 & PM & F & 12.5 & 338 \\
\hline 4100 & p72 & PM & F & 19 & 14 \\
\hline 3649 & p36 & PM & F & 12.5 & 22 \\
\hline 3657 & s89 & PM & M & 20 & \\
\hline
\end{tabular}

FP 12.9.04
\begin{tabular}{|l|l|l|l|l|r|}
\hline 4101 & s42 & PM & F & 14 & 339 \\
\hline 4102 & s70 & PM & F & 12.5 & 340 \\
\hline 4103 & p144 & PM & M & 18 & \\
\hline 4104 & p 115 & PM & F & 17.5 & 203 \\
\hline 3913 & s46 & PM & M & 17.5 & 180 \\
\hline 3875 & s44 & PM & F & 13.5 & \\
\hline
\end{tabular}

\begin{tabular}{|c|c|c|c|c|c|}
\hline \multicolumn{6}{|c|}{ FP 12.10 .04} \\
\hline 4105 & s41 & PM & $F$ & 18 & 341 \\
\hline 4106 & s101 & PM & $\mathbf{F}$ & 14 & 342 \\
\hline 4107 & s59 & PM & $F$ & 15 & 343 \\
\hline 4108 & p79 & SV & $\mathrm{F}$ ? & 5.6 & \\
\hline 4109 & p28 & ST & $?$ & 5 & \\
\hline 4110 & p127 & $\mathrm{ME}$ & $\bar{M}$ & 80 & . \\
\hline 4111 & p55 & PM & $\mathrm{F}$ & 11 & \\
\hline 3671 & $?$ & PM & $\mathbf{M}$ & & G787 \\
\hline
\end{tabular}

TRNWR 12.14.04

\begin{tabular}{|c|c|c|c|c|c|}
\hline 4112 & s72 & PM & F & 17.5 & 344 \\
\hline 4113 & s23 & PM & $\mathbf{M}$ & 17.0 & 345 \\
\hline 4114 & s118 & $\overline{P M}$ & $\mathrm{~F}$ & 15.0 & 347 \\
\hline 4115 & s71 & PM & $\mathbf{M}$ & 13.5 & 348 \\
\hline 4116 & s93 & PM & $\mathrm{F}$ & 13.5 & 349 \\
\hline 4117 & s141 & PM & $\mathbf{M}$ & 16.0 & 351 \\
\hline 4118 & $\mathrm{~s} 82$ & PM & $\mathrm{F}$ & 13.0 & 352 \\
\hline 4119 & s112 & PM & $\bar{F}$ & 15.5 & 353 \\
\hline 4120 & s9 & PM & $\mathbf{M}$ & 16.5 & 354 \\
\hline 3706 & $\mathrm{~s} 20$ & PM & $\mathbf{M}$ & 16.5 & 54 \\
\hline 3725 & $\mathrm{~s} 128$ & $\overline{P M}$ & $\mathrm{~F}$ & 17.5 & 70 \\
\hline 3931 & s23 & PM & $\mathrm{F}$ & 21.0 & 217 \\
\hline 3694 & s26 & PM & $\mathrm{F}$. & 16.0 & 48 \\
\hline 3713 & s49 & PM & $\mathrm{F}$ & 12.5 & 61 \\
\hline 3707 & s35 & PM & $\mathrm{F}$ & 14.5 & 55 \\
\hline 3932 & s29 & PM & $\mathrm{F}$ & 14.5 & 218 \\
\hline 3730 & s24 & PM & $\mathbf{M}$ & 15.0 & 72 \\
\hline \multirow[t]{3}{*}{3937} & s32 & PM & $\mathrm{F}$ & 14.0 & 223 \\
\hline & s119 & PM & & & \\
\hline & $\mathrm{m} 80$ & PM & & & \\
\hline \multicolumn{6}{|c|}{ TRNWR 12.15.04 } \\
\hline 4121 & $\mathrm{~s} 130$ & PM & $\mathrm{F}$ & 17.5 & 355 \\
\hline 4122 & s131 & PM & $\mathbf{M}$ & 15.0 & 356 \\
\hline 4123 & $\mathrm{~s} 47$ & PM & $F$ & 16.0 & $357 ?$ \\
\hline
\end{tabular}




\begin{tabular}{|c|c|c|c|c|c|}
\hline 3941 & s21 & PM & $F$ & 13.0 & 226 \\
\hline 3955 & $\mathrm{~s} 126$ & PM & $\mathbf{M}$ & 13.5 & 236 \\
\hline 3928 & $\mathrm{~s} 21$ & PM & $\mathrm{F}$ & 13.0 & 213 \\
\hline \multicolumn{6}{|c|}{ TRNWR 12.16.04 } \\
\hline 4124 & s132 & PM & $\mathbf{M}$ & 13.5 & 359 \\
\hline 4125 & s9 & PM & $\mathrm{F}$ & 13.5 & 360 \\
\hline 3705 & $\mathrm{~s} 128$ & PM & $\mathrm{M}$ & 13.5 & 53 \\
\hline 3719 & $\mathrm{~s} 12$ & PM & $\mathbf{M}$ & 16.5 & 65 \\
\hline 3707 & s36 & PM & $\mathrm{F}$ & 9.0 & 55 \\
\hline 3729 & s102 & PM & $\mathrm{F}$ & 13.0 & 71 \\
\hline 3712 & $\mathrm{~m} 68$ & PM & $\mathbf{M}$ & 16.0 & 60 \\
\hline
\end{tabular}

TRNWR 12.17.04

\begin{tabular}{|l|l|l|l|l|r|}
\hline 4126 & s68 & PM & M & 15 & 361 \\
\hline 4127 & p141 & PM & M & 11 & 362 \\
\hline 4128 & s53 & PM & F & 13.5 & 363 \\
\hline 4129 & s7 & GS & F & 148 & 364 \\
\hline 4130 & s25 & NG & F & 13 & \\
\hline 4131 & p139 & ST & F & 5.8 & 239 \\
\hline 3961 & s143 & PM & M & 17 & \\
\hline
\end{tabular}

\section{TRYON CREEK 1.11.05}

\begin{tabular}{|l|l|l|l|l|r|}
\hline 4132 & S44 & PM & M & 19 & 366 \\
\hline 4134 & S93 & PM & M & 16.0 & 367 \\
\hline 4135 & S91 & PM & M & 17 & 368 \\
\hline 4136 & S89 & PM & F & 14 & 369 \\
\hline 4137 & S106 & PM & M & 18 & 370 \\
\hline 4138 & S87 & PM & F & 20 & 371 \\
\hline 4139 & S114 & PM & M & 18 & 372 \\
\hline 4140 & S60 & PM & F & 14 & 373 \\
\hline 4141 & S115 & PM & F & 16 & 374 \\
\hline 4142 & S32 & ST & $?$ & 5 & \\
\hline 4143 & S44 & PM & F & 15 & \\
\hline 4144 & S117 & PM & F & 19 & \\
\hline 4145 & S43 & PM & F & 18 & \\
\hline 4146 & S9 & ST & $?$ & 6 & \\
\hline 3994 & S17 & PM & M & 21 & \\
\hline 3971 & S90 & PM & F & 18 & \\
\hline
\end{tabular}

TC 1.12 .05

\begin{tabular}{|l|l|l|l|l|r|}
\hline 4147 & S6 & PM & M & 16 & 376 \\
\hline 4148 & S24 & PM & M & 18.5 & 377 \\
\hline 4149 & S116 & ST & F? & 5 & \\
\hline 4150 & S8 & ST & F & 5.5 & \\
\hline 4151 & S20 & PM & F & 14 & \\
\hline 4152 & S67 & PM & M & 17 & \\
\hline 4153 & S12 & PM & F & 18 & \\
\hline 4154 & S35 & PM & F & 14 & \\
\hline 4155 & P28 & ST & $?$ & 5.5 & \\
\hline 4156 & S130 & ST & F & 5.3 & \\
\hline & T127 & SN & M & & \\
\hline 3744 & S68 & PM & M & 20 & \\
\hline
\end{tabular}


TC 1.13.05

\begin{tabular}{|l|l|l|l|l|ll|}
\hline 4157 & S95 & PM & F & 20 & 378 \\
\hline 4158 & S44 & PM & M & $?$ & ESC/NBS & 379 \\
\hline 4159 & S70 & PM & M & 16 & 381 \\
\hline 4160 & S89 & PM & M & 17 & \\
\hline 4161 & P120 & PM & M & 14.5 & \\
\hline 4162 & S105 & PM & $?$ & 6.0 & \\
\hline & T139 & SN & F & & & \\
\hline
\end{tabular}

TC 1.14 .05
\begin{tabular}{|l|l|l|l|l|l|}
\hline 4163 & S32 & PM & M & 17 & \\
\hline 4164 & S47 & ST & $?$ & 5.4 & \\
\hline 4165 & S 96 & ST & $?$ & 6.0 & \\
\hline 4166 & S119 & PM & F & 14 & \\
\hline 4167 & S66 & ST & $?$ & 4.4 & \\
\hline 4168 & P40 & ST & $?$ & 4.5 & \\
\hline 4169 & P40 & ST & $?$ & 4.7 & \\
\hline 3993 & S65 & PM & F & 22 & \\
\hline
\end{tabular}

POWELL BUTTE 1.18.05

\begin{tabular}{|l|l|l|l|l|r|}
\hline 4170 & S105 & PM & M & 13 & 383 \\
\hline 4171 & S11 & PM & F & 14 & 384 \\
\hline 4172 & S29 & PM & M & 15 & 385 \\
\hline 4173 & S4 & PM & F & 14 & 386 \\
\hline 4174 & S56 & ST & $?$ & 5.0 & 126 \\
\hline 3792 & P120 & PM & M & 12.5 & 285 \\
\hline 4018 & S46 & PM & M & 17.5 & 275 \\
\hline 4009 & S117 & PM & M & 18 & 112 \\
\hline 3776 & S84 & PM & F & 16 & 130 \\
\hline 3796 & S115 & PM & M & 15.5 & 276 \\
\hline 4010 & S58 & PM & M & 16.5 & 287 \\
\hline 4022 & S143 & PM & M & 15 & 387 \\
\hline PB 1.19.05 & \multicolumn{7}{|l|}{} & M & 16 & 388 \\
\hline 4175 & S10 & PM & F & 15.5 & 389 \\
\hline 4176 & S58 & PM & F & 118 \\
\hline 4177 & S84 & PM & M & 16 & 16 \\
\hline 3784 & S65 & PM & F & 14.5 & \\
\hline
\end{tabular}

\begin{tabular}{|l|l|l|l|l|r|}
\hline PB 1.20.05 & S31 & PM & M & 11 & 390 \\
\hline 4179 & S47 & PM & M & 16.0 & 391 \\
\hline 4180 & S2 & PM & F & 13.5 & 131 \\
\hline
\end{tabular}

PB 1.21.05
\begin{tabular}{|l|l|l|l|l|r|}
\hline 4181 & S22 & PM & M & $\begin{array}{l}151 \\
6\end{array}$ & 392 \\
\hline 3796 & S85 & PM & M & & 130 \\
\hline
\end{tabular}

OXBOW 1.25 .05

\begin{tabular}{|l|l|l|l|l|r|}
\hline 4182 & S36 & PM & M & 17 & 393 \\
\hline 4183 & S54 & PM & M & 13.5 & 395 \\
\hline 4184 & S57 & PM & F & 15 & 396 \\
\hline 4185 & S143 & PM & M & 15 & 397 \\
\hline 4186 & S3 & SV & $?$ & 7 & \\
\hline
\end{tabular}




\begin{tabular}{|c|c|c|c|c|c|c|}
\hline 4186.5 & S87 & PM & $F$ & 12 & DIT/NBS & 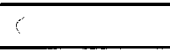 \\
\hline 4187 & S125 & SV & $?$ & 7.4 & DIT/NBS & \\
\hline 4041 & S43 & PM & $\mathbf{M}$ & 16.5 & & 304 \\
\hline 4033 & S62 & PM & $\mathbf{M}$ & $?$ & & 295 \\
\hline 3809 & S106 & PM & $\mathbf{M}$ & $?$ & & 140 \\
\hline 3824 & S24 & PM & $F$ & 16.5 & & 150 \\
\hline 3840 & S59 & PM & $\mathbf{M}$ & 15 & & 162 \\
\hline 3816 & S117 & PM & $\mathbf{M}$ & 15 & & 145 \\
\hline \multicolumn{7}{|c|}{ OX 1.26 .05} \\
\hline 4188 & $\mathrm{P} 120$ & PM & $\mathbf{M}$ & 13 & & 398 \\
\hline 4189 & S81 & PM & $\mathrm{F}$ & 15.5 & & 400 \\
\hline 4190 & S15 & GS & F & 150 & & 402 \\
\hline 4191 & S24 & PM & $\mathbf{M}$ & 15.5 & & 394 \\
\hline 4192 & S117 & SV & $F$ & 5.7 & & \\
\hline 4193 & S9 & SV & F & 6.5 & & \\
\hline 4194 & S60 & SV & $?$ & 5.1 & & \\
\hline 4195 & S47 & SV & $?$ & 5.3 & & \\
\hline \multirow[t]{2}{*}{4196} & S62 & MO & $\mathbf{M}$ & 14 & & \\
\hline & $\mathrm{P} 120$ & PM & & & & \\
\hline 3578 & S36 & $\overline{\mathrm{PM}}$ & $\mathrm{M}$ & 15.5 & & \\
\hline 4033 & S87 & PM & $\mathbf{M}$ & 16.5 & & \\
\hline 4059 & S111. & PM & $\mathbf{M}$ & 15 & & \\
\hline 4069 & S106 & $\mathrm{PM}$ & $\mathrm{F}$ & 14.5 & & \\
\hline \multicolumn{7}{|c|}{ OX 1.27 .05} \\
\hline 4197 & S5 & PM & F & 12 & & \\
\hline 4198 & S128 & ST & $F$ & 4.5 & & \\
\hline 4199 & S80 & PM & $\mathbf{M}$ & 16 & & 403 \\
\hline 4200 & P84 & ST & $\mathrm{F}$ & 4.4 & & \\
\hline 4048 & S93 & $\mathrm{PM}$ & $\mathbf{M}$ & 14.0 & & 303 \\
\hline 4056 & S128. & PM & $F$ & 15.0 & & 308 \\
\hline \multicolumn{7}{|c|}{ OX 1.28 .05} \\
\hline 4201 & S22 & ST & $F$ & 5.3 & & \\
\hline 4202 & S41 & ST & $\mathbf{M}$ & 5.7 & & \\
\hline 4203 & S29 & ST & $?$ & 5.5 & & \\
\hline 4204 & S59 & $\mathrm{PM}$ & $\mathbf{M}$ & 14.5 & & \\
\hline 4205 & S102 & $\mathrm{PM}$ & $\mathrm{F}$ & 14.0 & & \\
\hline 3862 & P60 & $\overline{P M}$ & $\mathbf{M}$ & 14.5 & & 177 \\
\hline
\end{tabular}

FOREST PARK 2.1.05

\begin{tabular}{|l|l|l|l|l|r|}
\hline 4206 & S132 & PM & M & 14.5 & 404 \\
\hline 4207 & S75 & PM & F & 15.5 & 405 \\
\hline 4208 & S20 & PM & M & 14 & 406 \\
\hline 4209 & S99 & PM & F & 6.0 & \\
\hline 4210 & T103 & SN & M & 510 & 328 \\
\hline 4089 & S95 & PM & F & 12.5 & 324 \\
\hline 4085 & S120 & PM & M & 16.0 & 342 \\
\hline 4106 & S117 & PM & F & 16.0 & 329 \\
\hline 4090 & S119 & PM & M & 13.5 & 407 \\
\hline FP 2.2.05 & S133 & PM & M & 15 & \\
\hline 4211 &
\end{tabular}




\begin{tabular}{|c|c|c|c|c|c|}
\hline 4212 & S108 & PM & $\mathbf{M}$ & 19 & 408 \\
\hline 4213 & S130 & PM & $F$ & 4.5 & \\
\hline 4214 & S119 & PM & $\mathbf{F}$ & 11 & \\
\hline 4215 & S127 & PM & $\mathbf{M}$ & 12 & \\
\hline 4216 & P31 & ST & F? & 4.0 & \\
\hline 4217 & S82 & SV & F? & 4.5 & \\
\hline 4218 & P4 & ST & $?$ & 4.5 & \\
\hline 4219 & $\mathrm{P} 48$ & ST & $?$ & 4.5 & \\
\hline 3626 & S125 & PM & $\mathrm{M}$ & 18 & 785 \\
\hline 3909 & S105 & $\mathrm{PM}$ & F & 17 & 200 \\
\hline 4088 & S117 & $\mathrm{PM}$ & $\mathbf{M}$ & 13 & 327 \\
\hline \multicolumn{6}{|c|}{ FP 2.3.05 } \\
\hline 4220 & S80 & PM & $\mathbf{M}$ & 15 & 410 \\
\hline 4221 & S24 & PM & $\mathbf{F}$ & 13 & 411 \\
\hline 4222 & S132 & ST & $?$ & 4.5 & \\
\hline 4223 & S140 & ST & $?$ & 4.5 & \\
\hline 4224 & P31 & ST & $?$ & 4.5 & \\
\hline \multicolumn{6}{|c|}{ FP 2.4.05 } \\
\hline 4225 & P72 & PM & $F$ & 13.5 & 421 \\
\hline 4226 & P55 & PM & $\mathbf{M}$ & 16 & 414 \\
\hline 4227 & P55 & ST & $\mathbf{F}$ & 3.4 & \\
\hline 4228 & P124 & ST & $\mathbf{M}$ & 5.0 & \\
\hline 4229 & P84 & ST & $?$ & 4.7 & \\
\hline 4230 & S133 & ST & $\begin{array}{l}\mathrm{M} \\
?\end{array}$ & 5.1 & \\
\hline 3908 & S140 & PM & $F$ & 15 & 198 \\
\hline
\end{tabular}

\section{TRNWR 2.8.05}

\begin{tabular}{|l|l|l|l|l|r|}
\hline 4231 & S46 & PM & M & 15 & 417 \\
\hline 4232 & S47 & PM & M & 17 & 416 \\
\hline 4233 & S103 & PM & F & 13 & 415 \\
\hline 4234 & S35 & ST & F? & 5.5 & \\
\hline 3929 & S140 & PM & M & & \\
\hline 3725 & S126 & PM & F & 16 & \\
\hline 3705 & S128 & PM & M & 16.5 & \\
\hline 3713 & S53 & PM & F & 15.5 & \\
\hline 4115 & S69 & PM & M & 16 & \\
\hline 3718 & S21 & PM & M & 16 & \\
\hline 4120 & S9 & PM & F & 17 & \\
\hline 4112 & S72 & PM & F & 12.5 & \\
\hline & S143 & PM & & & \\
\hline & S119 & PM & & & \\
\hline
\end{tabular}

TRNWR 2.9.05

\begin{tabular}{|c|c|c|c|c|c|}
\hline 4235 & S95 & PM & $\mathbf{M}$ & 16.5 & 418 \\
\hline 4236 & S86 & ST & $\mathbf{F}$ & 4.5 & \\
\hline 4237 & S140 & ST & F? & 4.8 & \\
\hline 4238 & P48 & ST & $F$ & 11.5 & \\
\hline 3706 & S31 & PM & $\mathbf{M}$ & 16.5 & 54 \\
\hline 4126 & S105 & PM & $\bar{M}$ & 15 & 361 \\
\hline 3929 & S37 & PM & $\mathbf{M}$ & 17.5 & 214 \\
\hline
\end{tabular}




\begin{tabular}{|l|l|l|l|l|l|}
\hline 3961 & S122 & PM & M & 14.5 & 239 \\
\hline & S128 & PM & & & \\
\hline & S48 & MM & & & \\
\hline
\end{tabular}

TRNWR 2.10.05

\begin{tabular}{|c|c|c|c|c|c|}
\hline 4239 & $\mathrm{~S} 72$ & PM & $\mathbf{M}$ & 14 & 419 \\
\hline & & & & & \\
\hline 4241 & S118 & ST & $\begin{array}{l}\mathrm{M} \\
?\end{array}$ & 5.5 & \\
\hline 4242 & S8 & ST & $F$ & 4.8 & \\
\hline 4243 & P76 & ST & $\begin{array}{l}\mathrm{M} \\
?\end{array}$ & 6.1 & \\
\hline 4244 & S144 & ST & $F$ & 5.5 & \\
\hline 4245 & S29 & ST & $\mathbf{M}$ & 5.5 & \\
\hline 4246 & S25 & ST & $\mathbf{M}$ & 12 & \\
\hline & T96 & SYB & & & \\
\hline 3948 & $\mathrm{~S} 130$ & PM & & & 231 \\
\hline 3932 & S18 & PM & $\mathrm{M}$ & 13 & 218 \\
\hline
\end{tabular}

TRNWR 2.11.05

\begin{tabular}{|l|l|l|l|l|l|}
\hline 4247 & S78 & ST & $?$ & 5.7 & \\
\hline 4248 & S81 & PM & M & 14.5 & \\
\hline & T31 & DV & & & \\
\hline
\end{tabular}

TRYON CREEK 2.15.05

\begin{tabular}{|c|c|c|c|c|c|}
\hline 4249 & S32 & PM & $\mathbf{F}$ & 21 & 421 \\
\hline 4250 & S35 & PM & $F$ & 21 & 422 \\
\hline 4251 & S31 & PM & $F$ & 19 & 423 \\
\hline 4252 & S7 & PM & M & 17 & 424 \\
\hline 4253 & S22 & PM & $\mathbf{M}$ & 19 & 425 \\
\hline 4254 & S91 & PM & $\mathbf{M}$ & 17 & 426 \\
\hline 4255 & $\mathrm{~S} 72$ & PM & F & 15 & 427 \\
\hline 4256 & S92 & PM & $F$ & 14 & 428 \\
\hline 4257 & S90 & PM & $\mathrm{M}$ & 17 & 429 \\
\hline 4258 & S106 & PM & $\mathbf{F}$ & 17 & 430 \\
\hline 4259 & S117 & PM & $\mathbf{M}$ & 15 & 431 \\
\hline 4260 & P84 & ST & $?$ & 7.0 & \\
\hline 4261 & S119. & TT & $\mathbf{F}$ & 75 & 432 \\
\hline 4262 & S61 & ST & $?$ & 6.3 & \\
\hline 4263 & S75 & ST & $\begin{array}{l}\mathrm{M} \\
?\end{array}$ & 5.2 & \\
\hline 4264 & S118 & ST & $\begin{array}{l}\mathrm{M} \\
?\end{array}$ & 5.2 & \\
\hline 4265 & $\mathrm{~S} 40$ & ST & $\mathrm{M} /$ & 6.0 & \\
\hline 4266 & S142 & PM & $\mathbf{M}$ & 18 & \\
\hline 4267 & S67 & ST & F? & 6.5 & \\
\hline 4268 & S71 & PM & $\mathbf{M}$ & 17 & \\
\hline 3744 & S77 & PM & $\mathbf{M}$ & 20 & 86 \\
\hline 3983 & S68 & PM & $\mathbf{F}$ & 24 & 256 \\
\hline 3994 & S5 & PM & $\mathbf{M}$ & 21 & 265 \\
\hline \multicolumn{6}{|c|}{ TC 2.16 .05} \\
\hline 4269 & $\mathrm{~S} 17$ & PM & $\mathbf{M}$ & 8.5 & 433 \\
\hline
\end{tabular}




\begin{tabular}{|l|l|l|l|l|r|}
\hline 4270 & S8 & PM & F & 14.5 & 434 \\
\hline 4271 & S96 & PM & M & 18 & 435 \\
\hline 4272 & S36 & PM & M & 20 & 436 \\
\hline 4273 & S47 & PM & F & 14.5 & 437 \\
\hline 4274 & S21 & PM & F & 15.5 & 438 \\
\hline 4275 & P4 & PM & M & 8.5 & \\
\hline 4276 & P120 & PM & M & 13.5 & \\
\hline 4277 & P72 & ST & $?$ & 5.2 & \\
\hline 4278 & S116 & ST & F & 6.2 & \\
\hline 4279 & P108 & ST & M & 5.8 & \\
\hline 4280 & S103 & ST & M & 5.6 & \\
\hline
\end{tabular}

\begin{tabular}{|c|c|c|c|c|c|}
\hline \multicolumn{6}{|c|}{ ТC 2.17.05 } \\
\hline 4281 & S10 & PM & M & 17 & 439 \\
\hline 4282 & $\begin{array}{l}\mathrm{P} 68 \\
\end{array}$ & PM & $F$ & 16 & 440 \\
\hline 4283 & S101 & PM & $\mathbf{M}$ & 19 & 441 \\
\hline 4284 & S94 & PM & $F$ & 15 & 442 \\
\hline 4285 & S107 & PM & $\mathrm{M}$ & $?$ & \\
\hline 4286 & S127 & PM & $\mathbf{M}$ & 16.5 & 443 \\
\hline 4287 & S104 & ST & $\begin{array}{l}\mathrm{M} \\
?\end{array}$ & 5.5 & \\
\hline 4288 & P144 & ST & $?$ & 4.5 & \\
\hline 4289 & S90 & ST & $\mathbf{M}$ & 5.5 & \\
\hline 4290 & S72 & ST & M/ & 5.0 & \\
\hline 4291 & S120 & ST & $\mathbf{M}$ & 5.0 & \\
\hline 4292 & S118 & ST & $?$ & 5.0 & \\
\hline 4293 & P84 & ST & $\mathbf{M}$ & 4.5 & \\
\hline 4294 & S32 & PM & $\mathrm{M}$ & 13 & \\
\hline 4295 & S84 & PM & $\mathrm{F}$ & 17 & \\
\hline 4296 & S137 & PM & $\mathbf{M}$ & 16 & \\
\hline 4297 & S15 & PM & $\mathrm{M}$ & 10 & \\
\hline & P31 & ST & $?$ & & \\
\hline & P31 & ST & $?$ & & \\
\hline & $\overline{\text { P31 }}$ & ST & $?$ & & \\
\hline & P31 & ST & $?$ & & \\
\hline & P31 & ST & ? & & \\
\hline
\end{tabular}

\begin{tabular}{|l|l|l|l|l|r|}
\hline TC 2.18 .05 & \multicolumn{1}{|c|}{} & \\
\hline 4298 & S0 & PM & M & 16 & 444 \\
\hline 4299 & S 90 & PM & M & 17 & 445 \\
\hline 4300 & S77 & PM & F & 13.5 & 446 \\
\hline 4301 & S130 & PM & F & 13.5 & 447 \\
\hline 4302 & S120 & PM & M & 17 & 448 \\
\hline 3969 & S107 & PM & F & 19 & 248 \\
\hline 4157 & S 94 & PM & F & 15.5 & 378 \\
\hline & P4 & ST & & & \\
\hline
\end{tabular}

POWELL BUTTE 3.1 .05
\begin{tabular}{|l|l|l|l|l|r|}
\hline 4303 & S23 & PM & F & 13 & 449 \\
\hline 4304 & S116 & PM & M & 15 & 450 \\
\hline 4305 & S104 & ST & F & & \\
\hline & T12 & SYB & & & \\
\hline
\end{tabular}




\begin{tabular}{|c|c|c|c|c|c|}
\hline 4176 & S72 & PM & $F$ & 17 & 388 \\
\hline 4173 & S3 & PM & $F$ & 18 & 386 \\
\hline 3478 & S129 & PM & $\mathbf{F}$ & 18 & 797 \\
\hline 4175 & S22 & $\overline{\mathbf{P M}}$ & $\mathbf{M}$ & 15 & 387 \\
\hline 3792 & S108 & PM & $\mathrm{F}$ & 16 & 126 \\
\hline 3796 & S114 & $\mathbf{P M}$ & $\mathbf{M}$ & 20 & 130 \\
\hline 3784 & S56 & PM & $\mathrm{F}$ & 13 & 118 \\
\hline \multicolumn{6}{|c|}{ PB 3.2.05 } \\
\hline 4306 & S56 & PM & $\mathrm{F}$ & 14 & 451 \\
\hline 4307 & S104 & ST & $?$ & 5.5 & 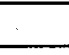 \\
\hline 4170 & S91 & $\mathbf{P M}$ & $\mathbf{M}$ & 15 & 383 \\
\hline 4172 & $\mathrm{~S} 25$ & PM & $\mathbf{M}$ & 17 & 385 \\
\hline \multicolumn{6}{|c|}{ PB 3.3.05 } \\
\hline 4308 & P108 & ST & $?$ & 4.9 & \\
\hline \multicolumn{6}{|c|}{ PB 3.04 .05} \\
\hline 4309 & S128 & SV & $?$ & 4.7 & \\
\hline 4310 & S84 & PM & $\mathbf{M}$ & 15 & 452 \\
\hline
\end{tabular}

OXBOW 3.8.05

\begin{tabular}{|l|l|l|l|l|r|}
\hline 4311 & S144 & PM & M & 14.5 & 453 \\
\hline 4312 & S72 & PM & F & 22 & 454 \\
\hline 4313 & S82 & PM & M & 18 & 456 \\
\hline 4314 & S88 & PM & M & 16.5 & 457 \\
\hline 4315 & S107 & PM & M & 13.5 & 458 \\
\hline 4316 & S24 & PM & M & 16.5 & 459 \\
\hline & & & M & & \\
4317 & P19 & SV & $?$ & 7.0 & \\
\hline 4318 & P96 & ST & F? & 4.5 & \\
\hline 4319 & P96 & ST & F? & 5.0 & 310 \\
\hline 4058 & S115 & PM & F & 16 & 321 \\
\hline 4079 & S87 & PM & F & 13.5 & 296 \\
\hline 4034 & S59 & PM & F & 20 & 726 \\
\hline 2768 & M41 & GS & F & 105 & 400 \\
\hline 4189 & S95 & PM & F & 14.5 & \\
\hline
\end{tabular}

OX 3.9.05

\begin{tabular}{|l|l|l|l|l|r|}
\hline 4320 & P60 & PM & M & 15.5 & 460 \\
\hline 4321 & S106 & PM & M & 16 & 461 \\
\hline 4322 & S23 & PM & F & 14.5 & 462 \\
\hline 4323 & S96 & GS & M & & \\
\hline 4324 & S128 & ST & M & 4.8 & \\
\hline 4325 & P96 & ST & M & 5.4 & \\
\hline 4326 & S115 & ST & F & 5.3 & \\
\hline 4327 & S35 & ST & M & 6.4 & \\
\hline 4328 & P36 & SV & F & 5.2 & \\
\hline 4057 & M58 & PM & F & 13.5 & \\
\hline
\end{tabular}

\begin{tabular}{|l|l|l|l|l|l|}
\hline OX 3.10 .05 & S 94 & ST & F & 6.0 & \\
\hline 4329 & S46 & ST & F & 5.0 & \\
\hline 4330 & P144 & ST & M & 4.6 & \\
\hline 4331 & S5 & SV & M & 5.5 & \\
\hline
\end{tabular}


OX 3.11 .05

\begin{tabular}{|l|l|l|l|l|l|}
\hline 4333 & S60 & TT & M & 52 & 463 \\
\hline 4334 & S54 & SV & M & 6.4 & \\
\hline 4335 & S43 & SV & F & 5.8 & \\
\hline 4336 & P120 & SV & F & 6.3 & \\
\hline 4337 & P108 & ST & F & 6.2 & \\
\hline 4338 & P80 & ST & F & 6.5 & \\
\hline 4339 & S55 & ST & M & 4.5 & \\
\hline 4034 & S66 & PM & F & 15 & \\
\hline
\end{tabular}

FOREST PARK 3.15.05

\begin{tabular}{|l|l|l|l|l|r|}
\hline 4340 & S72 & PM & F & 13.5 & 464 \\
\hline 4341 & S99 & PM & M & 17 & 465 \\
\hline 4342 & S83 & PM & M & 13.5 & 466 \\
\hline 4343 & M132 & PM & M & 13 & 467 \\
\hline 4344 & S116 & PM & M & 16 & 468 \\
\hline 4345 & M96 & PM & M & 12 & 209 \\
\hline 3920 & S46 & PM & M & 17 & 324 \\
\hline 4085 & S120 & PM & M & 16.5 & 405 \\
\hline 4207 & S90 & PM & F & 15.5 & \\
\hline
\end{tabular}

FP 3.17.05
\begin{tabular}{|l|l|l|l|l|r|}
\hline 4351 & S96 & PM & M & 16 & 472 \\
\hline 4352 & S120 & PM & M & 14 & 473 \\
\hline 4353 & S58 & ST & F & 4.3 & \\
\hline 4354 & S131 & ST & F & 4.5 & \\
\hline 4220 & S82 & PM & M & 17 & \\
\hline 4089 & S 92 & PM & F & 13 & \\
\hline
\end{tabular}

FP 3.18.05

\begin{tabular}{|l|l|l|l|l|l|}
\hline 4355 & S131 & PM & M & & \\
\hline
\end{tabular}

Tualatin River NWR 4.5.05

\begin{tabular}{|l|l|l|l|l|r|}
\hline 4356 & S12 & PM & F & 22 & 475 \\
\hline 4357 & S82 & PM & F & 14 & 476 \\
\hline 4358 & S83 & PM & M & 15 & 477 \\
\hline 4359 & S139 & PM & M & 20 & 478 \\
\hline 4360 & S84 & ST & F & 6.6 & \\
\hline 4361 & P127 & ST & F & 5.6 & \\
\hline 4362 & P127 & ST & F & 5.6 & 218 \\
\hline 3932 & S20 & PM & F & 23 & 54 \\
\hline 3706 & S9 & PM & M & 20 & 56 \\
\hline 3708 & S127 & PM & M & 17 & 61 \\
\hline 3713 & S114 & PM & F & 17 & 231 \\
\hline 3948 & S118 & PM & F & 17 & \\
\hline
\end{tabular}

TRNWR 4.6.05 


\begin{tabular}{|c|c|c|c|c|c|}
\hline 4363 & S35 & PM & $\bar{F}$ & 80 & 479 \\
\hline 4364 & S53 & ST & $\mathrm{F}$ & 7.0 & \multirow{3}{*}{$\begin{array}{l}\text { DIT/NBS } \\
\text { DIT/NBS } \\
\text { DIT/NBS }\end{array}$} \\
\hline 4365 & S66 & ST & $F$ & 5.4 & \\
\hline \multirow[t]{2}{*}{4366} & S87 & ST & $\mathrm{F}$ & 6.2 & \\
\hline & $S 80$ & PM & & & \\
\hline 3705 & S65 & $\overline{\mathbf{P M}}$ & $\mathbf{M}$ & 18.5 & 53 \\
\hline \multicolumn{6}{|c|}{ TRNWR 4.7.05 } \\
\hline 4367 & S23 & PM & $\mathbf{M}$ & 15 & 480 \\
\hline 4368 & S92 & $\overline{\mathrm{PM}}$ & $\bar{M}$ & 13 & 481 \\
\hline 4369 & S46 & ST & $\mathrm{F}$ & 6.0 & \\
\hline 4370 & 580 & SV & $\mathrm{F}$ & 6.5 & \\
\hline & T96 & DV & & & \\
\hline
\end{tabular}

TRNWR 4.8.05

\begin{tabular}{|l|l|l|l|l|l|}
\hline $4371^{-}$ & S117 & PM & M & & \\
\hline & & & M & & \\
\hline 4372 & S128 & ST & $?$ & & \\
\hline 4373 & S141 & ST & F & & \\
\hline & T120 & MM & & & REL \\
\hline
\end{tabular}

TRYON CREEK STATE PARK 4.12.05

\begin{tabular}{|c|c|c|c|c|c|}
\hline 4374 & S127 & PM & $\mathbf{M}$ & 19 & 483 \\
\hline 4375 & S23 & PM & $\mathrm{F}$ & 12 & 484 \\
\hline 4376 & S58 & PM & $\mathrm{F}$ & 20 & 486 \\
\hline 4377 & S55 & PM & $\mathbf{M}$ & 17 & 487 \\
\hline 4378 & S59 & PM & $\mathbf{M}$ & 17 & \\
\hline 4379 & S48 & PM & $\mathrm{F}$ & 15 & 488 \\
\hline 4380 & S104 & $\mathrm{PM}$ & $\mathbf{M}$ & 19 & 489 \\
\hline 4381 & S107 & $\mathrm{PM}$ & $\mathrm{F}$ & 21 & 490 \\
\hline 4382 & $\mathrm{~S} 120$ & TT & $\mathbf{M}$ & 75 & 491 \\
\hline 4383 & S72 & TT & $F$ & 80 & 492 \\
\hline 4384 & $\begin{array}{l}\text { S68 } \\
\end{array}$ & TT & $\mathbf{M}$ & 70 & 493 \\
\hline 4386 & S45 & $\mathrm{PM}$ & $\mathbf{M}$ & 19 & 494 \\
\hline 4387 & S48 & $\mathrm{PM}$ & $\mathbf{M}$ & 18 & 495 \\
\hline 4388 & $\mathrm{~S} 122$ & PM & $\mathbf{M}$ & 17 & 496 \\
\hline 4389 & S128 & $\mathrm{PM}$ & $\mathbf{M}$ & 18 & 497 \\
\hline 4390 & S132 & $\mathrm{PM}$ & $\mathbf{M}$ & 22 & 498 \\
\hline 4391 & S20 & $\mathrm{PM}$ & $F$ & 15 & 499 \\
\hline 4392 & S30 & $\overline{\mathrm{PM}}$ & $F$ & 19 & 500 \\
\hline 4393 & S46 & PM & $\mathbf{M}$ & 19 & 501 \\
\hline 4394 & S24 & PM & $\mathbf{M}$ & 18 & 502 \\
\hline 4395 & S36 & ST & $\mathrm{F}$ & 7.5 & \\
\hline 4396 & P124 & ST & $\mathrm{F}$ & 5.5 & \\
\hline 4301 & $\mathrm{~S} 129$ & $\mathrm{PM}$ & $F$ & 22 & 447 \\
\hline 4300 & S62 & $\mathrm{PM}$ & $\mathrm{F}$ & 24 & 446 \\
\hline 3477 & S19 & PM & $\mathbf{M}$ & 18 & 86 \\
\hline 4141 & S115 & PM & $\mathrm{F}$ & 25 & 374 \\
\hline 4299 & $\mathrm{~S} 102$ & $\mathrm{PM}$ & $\mathbf{M}$ & 19 & 445 \\
\hline 4284 & S91 & $\overline{P M}$ & $\mathrm{~F}$ & 17 & 442 \\
\hline 3969 & S106 & PM & $\mathrm{F}$ & 26 & 248 \\
\hline 4270 & S6. & $\mathrm{PM}$ & $F$ & 16 & 434 \\
\hline
\end{tabular}




\begin{tabular}{|c|c|c|c|c|c|}
\hline 4272 & S33 & PM & $\mathbf{M}$ & 17 & 436 \\
\hline 4253 & S10 & $\mathrm{PM}$ & $\mathrm{M}$ & 17 & 425 \\
\hline 4274 & S22 & PM & $\mathrm{F}$ & 17 & 438 \\
\hline 4147 & $\mathrm{~S} 11$ & $\mathrm{PM}$ & $\mathbf{M}$ & 18 & 376 \\
\hline 4271 & M105 & PM & $\mathrm{M}$ & 18 & 435 \\
\hline \multicolumn{6}{|c|}{ TC 4.13.05 } \\
\hline 4397 & S104 & PM & $\mathrm{F}$ & 17.5 & 504 \\
\hline 4398 & S108 & PM & $\mathbf{M}$ & 22.5 & 506 \\
\hline 4399 & S129 & $\mathrm{PM}$ & $F$ & 24.5 & 507 \\
\hline 4400 & S126 & PM & $\mathrm{F}$ & 27 & 508 \\
\hline 4401 & S7 & $\mathrm{PM}$ & $\mathbf{M}$ & 18.5 & 511 \\
\hline 4402 & S144 & PM & $\mathbf{M}$ & 20.5 & 512 \\
\hline 4403 & S140 & $\mathrm{PM}$ & $F$ & 20 & 513 \\
\hline 4404 & S143 & $\mathrm{PM}$ & $F$ & 16 & 514 \\
\hline 4405 & S118 & $\mathrm{PM}$ & $\mathbf{M}$ & 21.5 & 515 \\
\hline 4406 & S79 & $\mathrm{PM}$ & $\mathbf{M}$ & 15 & 516 \\
\hline 4407 & S77 & PM & $\mathbf{M}$ & 18 & 517 \\
\hline 4408 & S33 & PM & $\mathbf{M}$ & 19.5 & 518 \\
\hline 4409 & S54 & PM & $\mathbf{M}$ & 19 & 519 \\
\hline 4410 & S63 & PM & $\mathbf{M}$ & 20 & 520 \\
\hline 4411 & P36 & $\mathrm{PM}$ & $\mathbf{M}$ & 18 & 521 \\
\hline 4412 & P96 & ST & $F$ & 3.9 & \\
\hline 4413 & $\mathrm{~S} 48$ & PM & $\mathbf{M}$ & 13.5 & \\
\hline 4414 & P64 & SCAT & $F$ & 99 & \\
\hline 4415 & P108 & ST & $\mathbf{M}$ & 10 & \\
\hline 4416 & P108 & ST & $\mathbf{M}$ & 12.5 & \\
\hline \multirow[t]{4}{*}{4287} & S89 & PM & $\mathrm{M}$ & 20.5 & 429 \\
\hline & S130 & PM & & & \\
\hline & S6 & PM & & & \\
\hline & S29 & PM & & & \\
\hline
\end{tabular}

TC 4.14.05
\begin{tabular}{|l|l|l|l|l|r|}
\hline 4417 & S141 & PM & F & 17 & 521 \\
\hline 4418 & S140 & PM & F & 15 & 522 \\
\hline 4419 & S114 & PM & M & 19.5 & 523 \\
\hline 4420 & S132 & PM & F & 16.5 & 524 \\
\hline 4421 & S117 & PM & M & 20 & 525 \\
\hline 4422 & S37 & PM & F & 20 & 526 \\
\hline 4423 & S 69 & PM & F & 16.5 & 527 \\
\hline 4424 & S23 & PM & F & 16 & 528 \\
\hline 4425 & S2 & PM & F & 19 & 529 \\
\hline 4426 & S15 & PM & F & 15.5 & 530 \\
\hline 4427 & S22 & PM & F & 17.5 & 531 \\
\hline 4428 & M 93 & PM & F & 13.5 & 532 \\
\hline 4429 & P84 & ST & F & 4.3 & 533 \\
\hline 4430 & P36 & PM & M & 19.5 & \\
\hline 4431 & S81 & PM & M & 16 & \\
\hline 4432 & P 72 & ST & F & 4.0 & \\
\hline 4433 & S36 & PM & M & 17 & \\
\hline 4434 & P40 & ST & F & 5.3 & \\
\hline
\end{tabular}




\begin{tabular}{|l|l|l|l|l|r|}
\hline 4435 & S31 & PM & F & 18.5 & \\
\hline 4257 & S89 & PM & M & 20.5 & 429 \\
\hline 4255 & S72 & PM & F & 16 & 427 \\
\hline & P4 & ST & $?$ & 5.4 & \\
\hline & P28 & ST & $?$ & 5.8 & \\
\hline
\end{tabular}

\begin{tabular}{|c|c|c|c|c|c|}
\hline \multicolumn{6}{|c|}{ TC 4.15.05 } \\
\hline 4436 & S94 & PM & $\mathbf{M}$ & 20 & 535 \\
\hline 4437 & S114 & PM & $\mathbf{M}$ & 17 & 536 \\
\hline 4438 & S81 & PM & $F$ & 17.5 & 537 \\
\hline 4439 & S106 & PM & $\mathbf{M}$ & 15 & 538 \\
\hline 4440 & S92 & ST & $F$ & 3.7 & \\
\hline 4441 & S84 & SV & $F$ & 9.3 & \\
\hline 4442 & $\mathbf{S} 43$ & ST & $F$ & 4.5 & \\
\hline 4443 & P67 & ST & $\mathrm{M}$ & 4.3 & \\
\hline 4444 & P67 & ST & $F$ & 4.6 & \\
\hline 4445 & S46 & ST & $F$ & 4.6 & \\
\hline$\overline{4446}$ & S57 & PM & $\mathbf{M}$ & 18 & \\
\hline & S72 & PM & & & \\
\hline
\end{tabular}

POWELL BUTTE 4.26.05

\begin{tabular}{|l|l|l|l|l|r|}
\hline 4447 & P16 & MT & M & 40 & 539 \\
\hline 4448 & S20 & PM & M & 20 & 540 \\
\hline 4449 & S144 & PM & M & 20 & 542 \\
\hline 4450 & S23 & PM & F & 20 & 543 \\
\hline 4451 & M130 & PM & F & 18 & 544 \\
\hline 4452 & S11 & PM & F & 20 & 545 \\
\hline 4454 & S29 & PM & M & 20 & 546 \\
\hline 4455 & S101 & PM & M & 20 & 549 \\
\hline 4456 & S119 & PM & M & 20 & 550 \\
\hline 4457 & S56 & PM & M & 19 & 551 \\
\hline 4458 & S42 & PM & F & 16 & 552 \\
\hline 3792 & M88 & PM & F & 19 & 126 \\
\hline 3796 & S114 & PM & M & 17 & 130 \\
\hline
\end{tabular}

PB 4.27.05
\begin{tabular}{|l|l|l|l|l|r|}
\hline 4459 & S56 & PM & M & 22.5 & 554 \\
\hline 4460 & S33 & PM & M & 19.5 & 555 \\
\hline 4461 & S22 & PM & F & 20.5 & 556 \\
\hline 4462 & P72 & PM & M & 18.5 & 558 \\
\hline 4463 & S91 & MO & M & 17.5 & \\
\hline
\end{tabular}

PB 4.28.05

\begin{tabular}{|l|l|l|l|l|r|}
\hline 4464 & P72 & PM & F & 15.5 & 559 \\
\hline 4465 & P72 & PM & F & 14.0 & 560 \\
\hline 4466 & S25 & PM & M & 17 & 561 \\
\hline 4467 & S69 & PM & F & 17 & 562 \\
\hline 4468 & P124 & ST & M & 5.5 & \\
\hline 4469 & P55 & ST & F & 5.7 & \\
\hline & S141 & PM & & & \\
\hline
\end{tabular}

PB 4.29.05
\begin{tabular}{|l|l|l|l|l|r|}
\hline 4469 & P43 & MO & M & 21 & 563 \\
\hline 4470 & P84 & PM & F & 14 & 564 \\
\hline
\end{tabular}




\begin{tabular}{|l|l|l|l|l|r|}
\hline 4471 & M56 & PM & F & 21 & 565 \\
\hline 4472 & S10 & PM & M & 21 & 566 \\
\hline 4473 & S51 & PM & M & 20 & 567 \\
\hline 4474 & P115 & MT & F & 26 & \\
\hline 4475 & S111 & SV & F & 6 & \\
\hline 4476 & P48 & MT & M & 21 & \\
\hline & S1 & PM & & & \\
\hline
\end{tabular}

OXBOW 5.3.05

\begin{tabular}{|l|l|l|l|l|r|}
\hline 4477 & M89 & TT & M & 75 & 568 \\
\hline 4478 & S18 & TT & M & 80 & 569 \\
\hline 4479 & S93 & TT & F & 85 & 570 \\
\hline 4480 & S21 & TT & F & 85 & 571 \\
\hline 4481 & S106 & TT & M & 85 & 572 \\
\hline 4482 & S111 & TT & M & 95 & 573 \\
\hline 4483 & S108 & TT & M & 85 & 574 \\
\hline 4484 & S117 & TT & F & 100 & 575 \\
\hline 4485 & S104 & TT & M & 90 & 576 \\
\hline 4486 & S60 & PM & M & 19 & 577 \\
\hline 4487 & S69 & PM & M & 16 & 578 \\
\hline 4488 & S61 & PM & M & 14 & 579 \\
\hline 4489 & S115 & PM & M & 20 & 580 \\
\hline 4490 & P52 & ST & F & 4.4 & \\
\hline 4491 & P84 & ST & F & 4.4 & F \\
\hline 4492 & P127 & ST & F & 4.4 & \\
\hline 4493 & P31 & SV & F & 7.9 & M \\
\hline 4494 & P136 & ST & M & 4.2 & 316 \\
\hline 4495 & P136 & SV & F & 4.0 & 310 \\
\hline 3859 & S116 & TT & F & 90 & \\
\hline 4058 & S131 & TT & F & 85 & F \\
\hline 4073 & S127 & PM & F & 19 & \\
\hline
\end{tabular}

OXBOW 5.4.05
\begin{tabular}{|l|l|l|l|l|r|}
\hline 4496 & P108 & PM & F & 17.5 & 581 \\
\hline 4497 & S83 & TT & F & 70 & 582 \\
\hline 4498 & S65 & PM & M & 18.5 & 583 \\
\hline 4499 & S106 & TT & M & 78 & 584 \\
\hline 4500 & S 90 & PM & M & 19.5 & 585 \\
\hline 4501 & S36 & TT & M & 85 & 586 \\
\hline 4502 & S68 & PM & F & 14 & 587 \\
\hline 4503 & S128 & TT & M & 78 & 588 \\
\hline 4504 & S131 & PM & M & 17.5 & 589 \\
\hline 4505 & S142 & PM & F & 23 & 590 \\
\hline 4506 & S21 & PM & F & 15 & 591 \\
\hline 4507 & S60 & PM & F & 22 & 592 \\
\hline 4508 & S24 & TT & F & 72 & 593 \\
\hline 4509 & P64 & ST & F & 4.2 & \\
\hline 4510 & P 64 & NG & $?$ & $?$ & \\
\hline 4511 & S31 & SV & F & 7.2 & \\
\hline 4512 & P24 & ST & F & 3.8 & \\
\hline 4513 & P96 & SV & F & 8.5 & \\
\hline
\end{tabular}




\begin{tabular}{|l|l|l|l|l|r|}
\hline 4514 & P144 & ST & F & 4.3 & \\
\hline 4515 & S126 & NG & F & 9.0 & \\
\hline 4516 & P136 & ST & F & 4.0 & \\
\hline 4517 & P7 & ST & F & 3.7 & 402 \\
\hline 4190 & S140 & GS & F & 130 & 454 \\
\hline 4312 & S80 & PM & F & 17 & 463 \\
\hline 4333 & M129 & TT & M & 82 & \\
\hline
\end{tabular}

OXBOW 5.5.05
\begin{tabular}{|l|l|l|l|l|r|}
\hline 4518 & M 65 & TT & F & 90 & 594 \\
\hline 4519 & S19 & PM & M & 17 & 595 \\
\hline 4520 & P36 & ST & M & 5.5 & \\
\hline 4521 & S125 & SV & F & 5.5 & \\
\hline & S72 & SV & & & \\
\hline
\end{tabular}

OXBOW 5.6.05

\begin{tabular}{|l|l|l|l|l|r|}
\hline 4522 & P64 & NG & F & 8 & \\
\hline 4523 & S35 & PM & M & 22 & 596 \\
\hline 4524 & S82 & TT & F & 70 & 597 \\
\hline 4525 & S72 & ST & M & 5.6 & \\
\hline 4526 & P144 & ST & F & 4.5 & \\
\hline 4527 & S119 & MO & F & 19 & \\
\hline 4528 & S32 & SV & M & 8.9 & \\
\hline 4311 & M7 & PM & M & 18.0 & 453 \\
\hline
\end{tabular}

FOREST PARK 5.17.05

\begin{tabular}{|l|l|l|l|l|r|}
\hline 4529 & M82 & PM & F & 21 & 598 \\
\hline 4530 & S71 & PM & M & 22 & 599 \\
\hline 4531 & S83 & PM & M & 23.5 & 601 \\
\hline 4532 & S141 & PM & M & 18.5 & 602 \\
\hline 4533 & S96 & PM & M & 18.5 & 603 \\
\hline 4534 & S119 & PM & F & 16 & 604 \\
\hline 4535 & S107 & PM & F & 18.5 & 605 \\
\hline 4536 & S116 & PM & F & 16 & 606 \\
\hline 4537 & P139 & ST & F & 5.6 & \\
\hline 4086 & S143 & PM & M & 19.5 & 325 \\
\hline 4220 & S25 & PM & M & 20 & 410 \\
\hline 4089 & S95 & PM & F & 15 & 328 \\
\hline 4208 & S137 & PM & M & 19 & 406 \\
\hline
\end{tabular}

\begin{tabular}{|c|c|c|c|c|c|}
\hline \multicolumn{6}{|c|}{ FP 5.18.05 } \\
\hline 4538 & S96 & PM & $\mathrm{F}$ & 16 & 608 \\
\hline 4539 & S117 & PM & $\mathbf{M}$ & 18.5 & 609 \\
\hline 4540 & S34 & ST & $F$ & 6.6 & \\
\hline 4541 & P84 & ST? & $F$ & 4.2 & \\
\hline 4542 & P60 & SV & $\mathbf{M}$ & 9.5 & \\
\hline 4543 & $\mathrm{P} 12$ & ST & $\mathrm{F}$ & 6.8 & \\
\hline 4544 & T127 & TD & $F$ & 204 & \\
\hline 4085 & S107 & $\mathrm{PM}$ & $\mathbf{M}$ & 24.5 & 324 \\
\hline
\end{tabular}

FP 5.19.05
\begin{tabular}{|l|l|l|l|l|r|}
\hline 4545 & S30 & PM & M & 18.5 & 610 \\
\hline 4546 & S36 & PM & F & 19 & 611 \\
\hline 4547 & S46 & PM & M & 24 & 612 \\
\hline
\end{tabular}




\begin{tabular}{|l|l|l|l|l|r|}
\hline 4548 & S7 & PM & F & 18 & 614 \\
\hline 4549 & S83 & PM & M & 20 & 615 \\
\hline 4550 & S127 & PM & F & 17.5 & 616 \\
\hline 4551 & T127 & TD & M & 220 & 617 \\
\hline 4552 & P7 & PM & M & 21.5 & \\
\hline 4553 & P120 & SV? & F & 7.5 & 618 \\
\hline FP 5.20.05 & P60 & PM & F & 19.5 & 619 \\
\hline 4554 & S107 & PM & F & 14 & 621 \\
\hline 4555 & P84 & PM & M & 22.5 & 622 \\
\hline 4556 & S30 & PM & F & 17 & \\
\hline 4557 & S141 & PM & F & 16 & \\
\hline 4558 & P36 & ST & F & 4.0 & \\
\hline 4559 & S58 & ST & F & 3.7 & \\
\hline 4560 & STA & PARK & & & 6 \\
\hline
\end{tabular}

TRYON CREEK STATE PARK 5.24.05

\begin{tabular}{|c|c|c|c|c|c|}
\hline 4561 & $\mathrm{~S} 113$ & PM & $\mathbf{M}$ & 16 & 600 \\
\hline 4562 & S115 & PM & $\mathbf{M}$ & 17 & 626 \\
\hline 4563 & S141 & PM & $\mathbf{M}$ & 18 & 627 \\
\hline 4564 & S138 & TT & $\mathbf{M}$ & 100 & 628 \\
\hline 4565 & S143 & PM & F & 16 & 629 \\
\hline 4566 & $\mathrm{~S} 140$ & PM & $\mathbf{M}$ & 17 & 630 \\
\hline 4567 & S131 & PM & $F$ & 20 & 632 \\
\hline 4568 & S83 & PM & $\mathbf{M}$ & 21 & 633 \\
\hline 4569 & S57 & PM & F & 26 & 634 \\
\hline 4570 & S36 & PM & F & 22 & 635 \\
\hline 4571 & $\mathrm{~S} 19$ & PM & $\mathbf{M}$ & 20 & 636 \\
\hline 4572 & M30 & $\mathrm{PM}$ & $\mathbf{M}$ & 15 & 637 \\
\hline 4573 & S31 & PM & $\mathrm{F}$ & 16 & 638 \\
\hline 4574 & $\mathrm{~S} 12$ & PM & $\mathbf{M}$ & 20 & 639 \\
\hline 4575 & $\mathrm{~S} 20$ & PM & $\mathbf{M}$ & 18 & 640 \\
\hline 4576 & $\mathrm{S33}$ & PM & $\mathbf{M}$ & 22 & 641 \\
\hline 4577 & S21 & SV & $\mathrm{F}$ & 9 & \\
\hline 4578 & P84 & ST & $?$ & 4 & \\
\hline 4579 & P67 & ST & $\mathrm{F}$ & 4.4 & \\
\hline 4580 & P4 & ST & $\mathrm{F}$ ? & 3.8 & \\
\hline 4581 & P84 & ST & & & \\
\hline 4582 & P67 & ST & & & \\
\hline 4583 & P55 & ST & F? & 6.0 & \\
\hline 4584 & P110 & ST & & 4.4 & \\
\hline 4585 & P124 & ST & & 4.8 & \\
\hline 4586 & P19 & SV & & 4.4 & \\
\hline 4390 & S132 & PM & $\mathbf{M}$ & 21 & 498 \\
\hline 4588 & $\mathrm{P96}$ & ST & & & 2 \\
\hline 4589 & P96 & ST & & 4.9 & \\
\hline 4300 & S97 & PM & $\mathrm{F}$ & 28 & 446 \\
\hline 4418 & $\mathrm{~S} 142$ & PM & $\mathrm{F}$ & 15 & 522 \\
\hline 4388 & S134 & PM & $\mathbf{M}$ & 21 & 496 \\
\hline 4399 & $\mathbf{S} 128$ & PM & $F$ & 27 & 507 \\
\hline 4427 & S80 & $\mathrm{PM}$ & $\mathrm{F}$ & 21 & 531 \\
\hline
\end{tabular}




\begin{tabular}{|l|l|l|l|l|r|}
\hline 3744 & S77 & PM & M & 21 & 86 \\
\hline 4299 & S112 & PM & M & 20 & 445 \\
\hline 4407 & S67 & PM & M & 21 & 517 \\
\hline 4250 & S35 & PM & F & 22 & 422 \\
\hline 3969 & S95 & PM & F & 28 & 248 \\
\hline 4425 & S17 & PM & F & 20 & 529 \\
\hline 4394 & M36 & PM & M & 19 & 502 \\
\hline & T60 & SN & F & & \\
\hline
\end{tabular}

\begin{tabular}{|l|l|l|l|l|r|}
\hline \multicolumn{9}{|c|}{ TC 5.25.05 } & \multicolumn{1}{|c|}{ S58 } & PM & F & 18.5 & 624 \\
\hline 4590 & S 99 & PM & F & 22.5 & 642 \\
\hline 4591 & S54 & PM & F & 21.5 & 643 \\
\hline 4592 & S45 & PM & M & 21 & 644 \\
\hline 4594 & S24 & PM & M & 22 & 645 \\
\hline 4595 & S89 & PM & & & \\
\hline 4596 & P84 & ST & & 4.1 & \\
\hline 4596.1 & P76 & SV & F & 4.4 & \\
\hline 4597 & P64 & ST & F & 4.6 & \\
\hline 4598 & P79 & SV & F & 3.9 & \\
\hline 4598.1 & P12 & ST & & 5.0 & \\
\hline 4599 & S8 & SV & M & 6.0 & \\
\hline 4600 & S109 & ST & M & 4.7 & \\
\hline 4601 & S107 & TT & & 70 & \\
\hline 4602 & P4 & ST & F & 4.8 & \\
\hline 4602.1 & P7 & SV & F & 6.1 & \\
\hline 4603 & P16 & ST & F & 4.4 & \\
\hline 4604 & P16 & ST & F & 5.8 & \\
\hline 4605 & S53 & TT & M & 75 & \\
\hline 4605 & & & & & 482 \\
\hline
\end{tabular}

\begin{tabular}{|c|c|c|c|c|c|}
\hline \multicolumn{6}{|c|}{ TC 5.26.05 } \\
\hline 4606 & $\mathrm{P} 40$ & PM & $\mathbf{F}$ & 26 & 646 \\
\hline 4607 & S120 & PM & $\mathbf{F}$ & 18.5 & 647 \\
\hline 4608 & S93 & $\overline{P M}$ & $\mathbf{M}$ & 22 & 648 \\
\hline 4609 & S33 & PM & $\mathrm{M}$ & 19 & 650 \\
\hline 4610 & S26 & PM & $\mathrm{F}$ & 17 & 651 \\
\hline 4611 & S80 & $\overline{\mathrm{PM}}$ & $\mathbf{M}$ & 20 & 652 \\
\hline 4612 & S0 & PM & $\mathrm{F}$ & 21.5 & 653 \\
\hline 4613 & S48 & PM & F & 19.5 & 654 \\
\hline 4614 & P24 & SV & F? & 4.4 & \\
\hline 4615 & P139 & ST & F? & 5.1 & \\
\hline 4616 & P12 & $\overline{\text { ST }}$ & $F$ & 5.5 & \\
\hline 4617 & $\mathrm{P} 12$ & ST & $\mathbf{F}$ & 4.7 & \\
\hline 4618 & S117 & PM & $\mathbf{F}$ & 25.5 & \\
\hline 4619 & P115 & ST & $F$ & 5.8 & \\
\hline 4620 & P115 & ST & $\mathbf{F}$ & 6 & \\
\hline 4621 & $\overline{\mathrm{P} 4}$ & SV? & $\mathbf{F}$ & 4 & \\
\hline 4622 & S20 & ST & $\mathbf{F}$ & 4.4 & \\
\hline 4623 & P55 & SV & F & 4.6 & \\
\hline 4624 & P96 & ST & $\mathrm{F}$ & 4.9 & \\
\hline 4625 & P72 & SCAT & F? & 88 & \\
\hline
\end{tabular}




\begin{tabular}{l|l|l|l|l|r|r|}
\hline & P72 & SCAT & F? & & \\
\hline 189 & S105 & PM & M & 20 & \\
\hline TC 5.27.05 & S47 & PM & M & 18 & 655 \\
\hline 4626 & S41 & PM & M & 21.5 & 656 \\
\hline 4627 & S81 & PM & F & 13.5 & 657 \\
\hline 4928 & S121 & PM & M & 20 & 658 \\
\hline 4629 & S116 & PM & F & 19.5 & 659 \\
\hline 4630 & S0 & PM & F & 20 & 660 \\
\hline 4631 & S59 & PM & M & 20.5 & 661 \\
\hline 4632 & P96 & ST & F? & 4.5 & \\
\hline 4633 & P96 & ST & F & 5.8 & \\
\hline 4634 & P55 & SV & & 3.9 & \\
\hline 4635 & P124 & SV & & 4.3 & \\
\hline 4637 & S14 & SV & M & 8 & \\
\hline 4638 & S15 & PM & F & 13.5 & \\
\hline 4639 & P136 & SV & F & 5 & \\
\hline 4640 & P31 & SV & & 3.5 & \\
\hline 4641 & S23 & ST & F & 4.2 & \\
\hline 4642 & P48 & SV & & 4.6 & \\
\hline 4643 & T79 & SN & F & 425 & \\
\hline 4644 & SN & & & \\
\hline
\end{tabular}

Tualatin River NWR 5.31.05

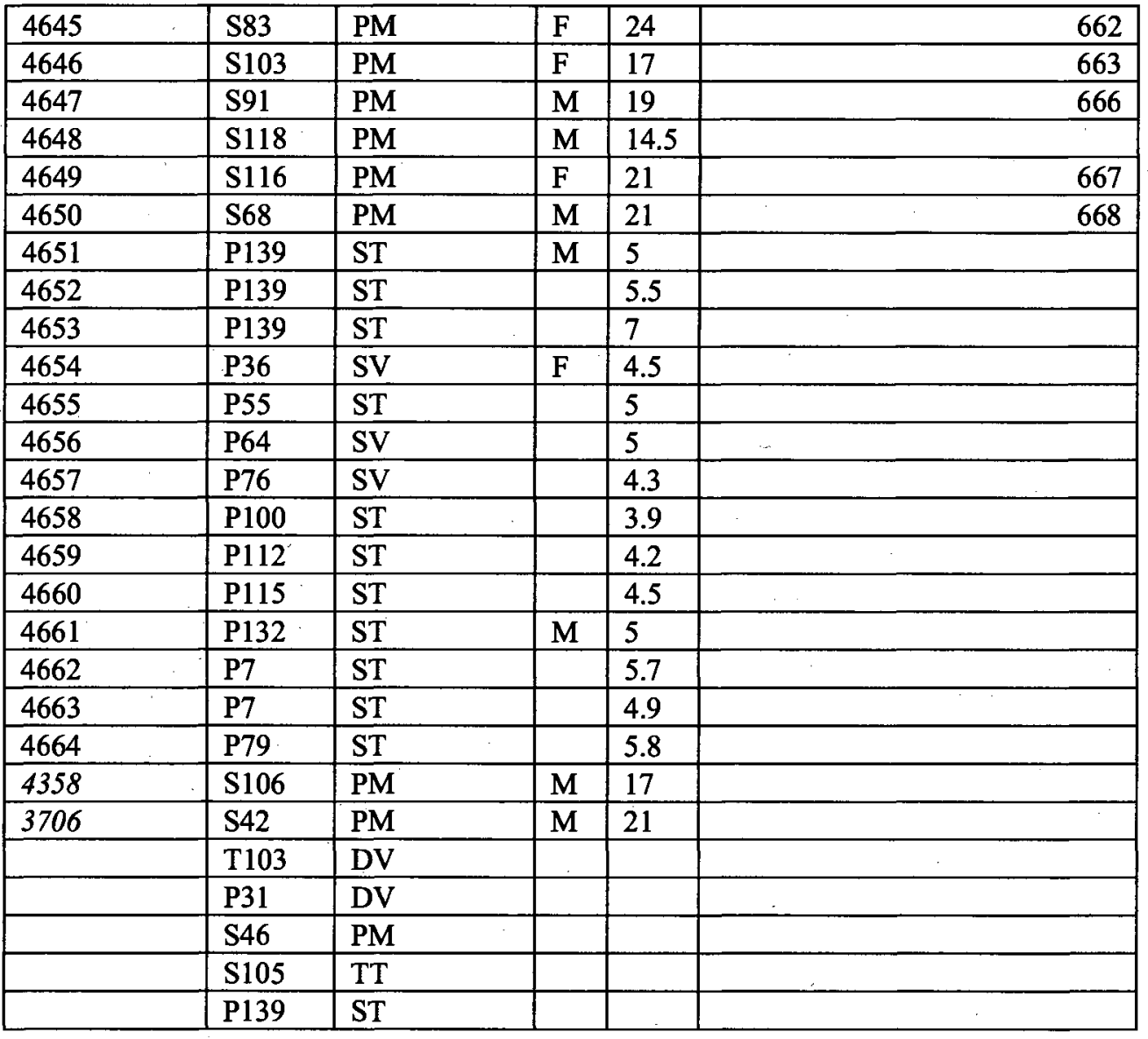




\begin{tabular}{|l|l|l|l|l|l|}
\hline & P139 & ST & & & \\
\hline & P55 & ST & & & \\
\hline & P79 & ST & & & \\
\hline
\end{tabular}

TRNWR 6.1.05
\begin{tabular}{|l|l|l|l|l|l|}
\hline 4665 & S117 & TT & M & 70 & 675 DIED \\
\hline 4666 & S114 & TT & M & 75 & \\
\hline 4667 & S115 & PM & M & 24 & \\
\hline 4668 & P48 & PM & F & 18.5 & \\
\hline 4669 & P36 & MT & F & 40 & \\
\hline 4670 & P36 & MT & F & 35 & \\
\hline 4671 & P36 & MT & M & 50 & \\
\hline 4672 & P 28 & MT & F & 40 & \\
\hline 4673 & P64 & ST & F & 4.1 & \\
\hline 4674 & P7 & ST & F & 5.3 & \\
\hline 4675 & P40 & ST & F & 4.5 & \\
\hline 4676 & S144 & ST & F & 5.6 & \\
\hline 4677 & P36 & MT & M & 37 & \\
\hline 4678 & P36 & MT & M & 46 & \\
\hline & S 92 & TT & F & & \\
\hline & T96 & DV & & & \\
\hline & P79 & SV & & & \\
\hline
\end{tabular}

\begin{tabular}{|c|c|c|c|c|c|}
\hline \multicolumn{6}{|c|}{ TRNWR 6.2.05 } \\
\hline 4679 & S128 & PM & M & 19.5 & 625 \\
\hline 4680 & S106 & TT & $\mathrm{F}$ & 75 & 655 \\
\hline 4681 & S6 & TT & $\mathbf{M}$ & 85 & 631 \\
\hline 4682 & $\mathbf{S 8 7}$ & TT & & 85 & \\
\hline 4683 & $\mathbf{S 9 2}$ & TT & $\mathrm{F}$ & 80 & \\
\hline 4684 & P136 & ST & $\mathbf{M}$ & 6.5 & \\
\hline 4685 & S31 & MT & F? & 40 & \\
\hline 4686 & P36 & ST & & 5.2 & \\
\hline 4687 & S140 & PM & $\mathrm{F}$ & 21 & \\
\hline 4371 & S102 & PM & $\mathbf{M}$ & 21.5 & 482 \\
\hline & 580 & DV & & & \\
\hline & P115 & SCAT & & & \\
\hline & S43 & TT & & & \\
\hline
\end{tabular}

\section{TRNWR 6.3.05}

\begin{tabular}{|c|c|c|c|c|c|}
\hline 4688 & $\mathbf{S 2}$ & PM & M & 17 & 674 \\
\hline 4689 & S141 & PM & $F$ & 17 & 675 \\
\hline 4690 & S115 & TT & $\mathrm{F}$ & 52.5 & 676 \\
\hline 4691 & S69 & TT & $F$ & 56.5 & DIT/NBS \\
\hline 4692 & S78 & ST & M & 4.6 & DIT/NBS \\
\hline 4693 & P112 & ST & $F$ & 4.1 & DIT/NBS \\
\hline \multirow[t]{4}{*}{4694} & S96 & ST & $\mathrm{F}$ & 6.8 & DIT/NBS \\
\hline & $\mathrm{T} 12$ & SV & & & REL/NBS \\
\hline & $\mathrm{T} 48$ & DV & & & REL/NBS \\
\hline & T108 & MM & & & REL/NBS \\
\hline
\end{tabular}

POWELL BUTTE 6.7.05

\begin{tabular}{|l|l|l|l|l|l|}
\hline 4695 & S117 & PM & M & 21 & 677 \\
\hline 4696 & S50 & PM & M & 15 & 678 \\
\hline
\end{tabular}




\begin{tabular}{|c|c|c|c|c|c|}
\hline 4697 & S48 & PM & $\mathbf{M}$ & 20 & 679 \\
\hline 4698 & S12 & PM & $\mathbf{M}$ & 22 & 680 \\
\hline 4699 & M120 & PM & $\mathrm{F}$ & 27 & 681 \\
\hline 4700 & $\mathrm{P} 60$ & NG & $?$ & 9 & \\
\hline 4701 & P144 & ST & $?$ & 4.4 & \\
\hline 4702 & P12 & SV & $?$ & 4.4 & \\
\hline \multirow[t]{2}{*}{4703} & P52 & MO & $F$ & 20 & \\
\hline & T108 & DV & $?$ & & \\
\hline 4457 & S71 & PM & $\mathbf{M}$ & 20 & 551 \\
\hline 4464 & S95 & PM & $F$ & 20 & 559 \\
\hline 4473 & S143 & PM & $\mathbf{M}$ & 18 & 567 \\
\hline \multicolumn{6}{|c|}{ PB 6.8.05 } \\
\hline 4704 & S46 & PM & $\mathbf{M}$ & 15 & 682 \\
\hline 4705 & S128 & PM & $\mathrm{F}$ & 17.5 & 683 \\
\hline 4706 & P144 & PM & $\mathrm{M}$ & 19 & 684 \\
\hline 4707 & P48 & SV & & 3.4 & \\
\hline 4708 & P108 & ST & & 3.7 & \\
\hline 4709 & P76 & SV & & 3.6 & \\
\hline 4710 & M31 & $\mathrm{TT}$ & $\mathrm{F}$ & 75 & \\
\hline 4711 & P31 & SV & & 3.6 & \\
\hline 4712 & P52 & MO & $\mathbf{M}$ & 20 & \\
\hline 4713 & P60 & ST & $\mathrm{F}$ & 7.9 & \\
\hline 4714 & P60 & SV & & 5.3 & \\
\hline 4715 & P64 & SV & $\mathbf{M}$ & 4.1 & \\
\hline \multicolumn{6}{|c|}{ PB 6.9.05 } \\
\hline 4717 & S143 & PM & $\mathrm{F}$ & 17.5 & 685 \\
\hline 4718 & S56 & PM & $F$ & 18 & 686 \\
\hline 4719 & P64 & SV & F & 3.8 & \\
\hline 4720 & P52 & SV & $\mathrm{F}$ & 6 & \\
\hline 4721 & P24 & SV & $\mathrm{F}$ & 6.7 & \\
\hline 4722 & P19 & SV & $\mathrm{F}$ & 6.5 & \\
\hline 4456 & S118 & PM & $\mathbf{M}$ & 20.5 & 550 \\
\hline \multicolumn{6}{|c|}{ PB 6.10.05 } \\
\hline 4723 & S71 & PM & $\mathrm{F}$ & 16.5 & 687 \\
\hline 4724 & S7 & PM & $\mathbf{M}$ & 15.5 & 688 \\
\hline 4725 & P60 & MO & & 20 & \\
\hline 4726 & S100 & SV & $\bar{F}$ & 7.3 & \\
\hline 4727 & P120 & PM & $\mathrm{F}$ & 18 & \\
\hline 4728 & P31 & ST & F? & 4.0 & \\
\hline 4729 & P40 & SV & $F$ & 6.4 & \\
\hline \multirow[t]{3}{*}{4730} & P126 & ST & F? & 4.3 & \\
\hline & P144 & ST & & & \\
\hline & P124 & SV & & & \\
\hline
\end{tabular}

OXBOW 7.05.05

\begin{tabular}{|l|l|l|l|l|r|}
\hline 4731 & S18 & ME & F & 52 & 689 \\
\hline 4732 & S84 & TT & M & 72 & 690 \\
\hline 4733 & S29 & TT & F & 100 & 691 \\
\hline 4734 & S33 & PM & M & 20 & 692 \\
\hline 4735 & S30 & TT & F & 90 & 693 \\
\hline
\end{tabular}




\begin{tabular}{|l|l|l|l|l|l|}
\hline 4736 & S42 & PM & M & 15.5 & 694 \\
\hline 4737 & S131 & ST & F & 5.4 & \\
\hline 4738 & P52 & NG & M & 9.5 & 69 \\
\hline 4739 & P12 & PM & F & 10.5 & \\
\hline 4740 & S104 & SV & M & 9.0 & \\
\hline 4741 & P7 & ST & $?$ & 4.4 & \\
\hline 4742 & P55 & ST & $?$ & 4.5 & \\
\hline 4743 & S130 & ST & $?$ & 4.9 & \\
\hline 4744 & T144 & SB & $?$ & $?$ & \\
\hline 4519 & S127 & PM & M & 16.5 & 595 \\
\hline 4190 & S132 & GS & F & 96 & 402 \\
\hline 4504 & S120 & PM & M & 21 & 589 \\
\hline 4312 & P67 & PM & F & 22 & 454 \\
\hline
\end{tabular}

OXBOW 7.06.05

\begin{tabular}{|l|l|l|l|l|r|}
\hline 4746 & S120 & TT & M & 75 & 696 \\
\hline 4747 & S125 & TT & M & $?$ & 697 \\
\hline 4748 & S66 & PM & F & 20.5 & 698 \\
\hline 4749 & S103 & PM & F & 10 & 699 \\
\hline 4750 & S105 & PM & M & 16.5 & 700 \\
\hline 4751 & S93 & PM & M & 15 & 701 \\
\hline 4752 & S130 & PM & M & 9.5 & 702 \\
\hline 4753 & P67 & PM & M & 6.5 & \\
\hline 4754 & P36 & ST & F & 4.6 & \\
\hline 4755 & P144 & NG & F & 9.5 & \\
\hline 4756 & P88 & ST & F & 4.4 & \\
\hline 4757 & P52 & SV & M & 7.7 & \\
\hline 4758 & P126 & ST & F & 4.0 & \\
\hline 4759 & P84 & ST & F & 3.8 & \\
\hline 4760 & P96 & ST & F & 4.0 & 593 \\
\hline 4761 & P4 & ST & M & 5.0 & \\
\hline 4508 & S23 & TT & M & 72 & \\
\hline 4500 & S105 & PM & M & 20.5 & \\
\hline 0
\end{tabular}

OXBOW 7.07.05

\begin{tabular}{|l|l|l|l|l|r|}
\hline 4762 & S120 & PM & F & 22 & 703 \\
\hline 4763 & S91 & PM & M & 17 & 704 \\
\hline 4764 & P16 & SV & $?$ & 7 & \\
\hline 4765 & S85 & SV & $?$ & 7.5 & \\
\hline 4766 & S118 & SV & M & 5 & \\
\hline 4767 & P76 & ST & M & 5.5 & \\
\hline 4768 & S117 & SV & M & 9.5 & \\
\hline 4499 & S106 & TT & M & 76 & 584 \\
\hline 4506 & P12 & PM & F & 18 & 591 \\
\hline & S111 & PM & & & \\
\hline
\end{tabular}

OXBOW 7.08.05

\begin{tabular}{|l|l|l|l|l|r|}
\hline 4769 & S114 & TT & F & 95 & 705 \\
\hline 4770 & S135 & PM & F & 9 & 708 \\
\hline 4771 & S117 & PM & M & 20 & 710 \\
\hline 4772 & S59 & PM & M & 17.5 & 711 \\
\hline 4773 & S27 & PM & M & 15 & 712 \\
\hline
\end{tabular}




\begin{tabular}{|l|l|l|l|l|l|}
\hline 4774 & S108 & PM & F & 17 & \\
\hline 4775 & S14 & SV & F & 7 & \\
\hline 4776 & S13 & SV & F & 6 & \\
\hline 4777 & P52 & MO & M & 19 & \\
\hline 4778 & P76 & ST & $?$ & 4.3 & \\
\hline 4779 & P132 & SV & F & 4.8 & \\
\hline 4780 & P144 & SV & M & 7.7 & \\
\hline 4781 & S107 & ST & $?$ & 4.5 & \\
\hline
\end{tabular}

FOREST PARK 7:13.05

\begin{tabular}{|l|l|l|l|l|r|}
\hline 4782 & S83 & PM & M & 20 & 715 \\
\hline 4783 & S30 & PM & M & 20.5 & 716 \\
\hline 4784 & S59 & PM & F & 19.5 & 717 \\
\hline 4785 & S46 & PM & M & 15.5 & 718 \\
\hline 4786 & P36 & SV & $?$ & 3.1 & 602 \\
\hline 4532 & S139 & PM & M & 18 & 606 \\
\hline 4536 & S127 & PM & F & 22 & 616 \\
\hline 4550 & S116 & PM & F & 20 & 612 \\
\hline 4547 & S47 & PM & M & 21 & 599 \\
\hline 4530 & S69 & PM & M & 24 & 614 \\
\hline 4548 & S24 & PM & F & 20.5 & 598 \\
\hline 4529 & S107 & PM & F & 20.5 & 603 \\
\hline 4533 & S81 & PM & M & 19.5 & 324 \\
\hline 4085 & S118 & PM & M & 23.5 & \\
\hline & S19 & PM & & & \\
\hline
\end{tabular}

FOREST PARK 7.14.05

\begin{tabular}{|l|l|l|l|l|r|}
\hline 4787 & M70 & PM & F & 21 & 719 \\
\hline 4788 & S120 & PM & M & 25 & 720 \\
\hline 4789 & S0 & PM & M & 18 & 721 \\
\hline 4790 & S24 & PM & M & 19 & 722 \\
\hline 4791 & S139 & PM & F & 21 & 723 \\
\hline 4792 & S47 & PM & F & 16 & 724 \\
\hline 4793 & P96 & SV & F & 4.8 & \\
\hline 4794 & S90 & ST & F & 4.3 & 621 \\
\hline 4556 & S99 & PM & M & 23 & \\
\hline
\end{tabular}

FOREST PARK 7.15 .05
\begin{tabular}{|l|l|l|l|l|r|}
\hline 4795 & S 34 & PM & M & 17 & 725 \\
\hline 4796 & S 39 & PM & M & 15.5 & 726 \\
\hline 4797 & S2 & PM & F & 18 & 727 \\
\hline 4798 & S104 & ST & & 4 & \\
\hline 4799 & P7 & SV & & 4.5 & \\
\hline 4801 & S143 & ST & & 4 & \\
\hline 4802 & S 86 & SV & F & 4.5 & \\
\hline 3632 & S 69 & PM & M & 18 & \\
\hline
\end{tabular}

FOREST PARK 7.16.05

\begin{tabular}{|l|l|l|l|l|l|}
\hline 4803 & S29 & ST & & 4.0 & \\
\hline 4804 & S44 & PM & M & 14.5 & 728 \\
\hline 4805 & P72 & ST & F & 4.5 & \\
\hline 4806 & P40 & ST & F & 5.0 & \\
\hline 4807 & S107 & SV & M & 3.5 & \\
\hline
\end{tabular}




\begin{tabular}{|c|c|c|c|c|c|}
\hline 4808 & P7 & SV & $\mathrm{F}$ & 5.0 & \\
\hline 4531 & 583 & $\mathrm{PM}$ & $\mathbf{M}$ & 21.5 & 601 \\
\hline \multicolumn{6}{|c|}{ TRNWR 7.19.05 } \\
\hline 4809 & S143 & $\mathrm{PM}$ & $\mathrm{F}$ & 16 & 729 \\
\hline 4810 & S118 & TT & $\mathbf{M}$ & 65 & 730 \\
\hline 4811 & S24 & TT & & & \\
\hline 4812 & P139 & ST & F & 4.6 & \\
\hline 4813 & S19 & ST & $\mathrm{F}$ & 4.1 & \\
\hline 4814 & S119 & ST & F & 4.3 & \\
\hline 4815 & P48 & ST & F & 3.7 & \\
\hline 4816 & P48 & ST & $\mathbf{F}$ & 4.3 & \\
\hline 4817 & T144 & DV & & & \\
\hline 4647 & S90 & PM & $\mathbf{M}$ & 17 & 666 \\
\hline 4667 & $\mathrm{~S} 30$ & PM & $\mathbf{M}$ & 20 & 671 \\
\hline 4359 & S9 & PM & $\mathbf{M}$ & 21.5 & 478 \\
\hline 4688 & S29 & PM & $\bar{M}$ & 18.5 & 674 \\
\hline
\end{tabular}

TRNWR 7.20.05

\begin{tabular}{|l|l|l|l|l|l|}
\hline 4818 & S31 & PM & F & 23.5 & \\
\hline 4819 & P36 & ST & F & 4.4 & \\
\hline 4820 & P88 & ST & M & 5.0 & \\
\hline 4821 & P76 & ST & F & 5.1 & \\
\hline 4822 & P79 & ST & M & 5.3 & \\
\hline 4679 & S131 & PM & M & 20 & 625 \\
\hline
\end{tabular}

TRNWR 7.21.05
\begin{tabular}{|l|l|l|l|l|r|}
\hline 4823 & S 89 & PM & M & 15 & \\
\hline 4824 & S 33 & TT & M & 65 & 732 \\
\hline 4825 & S7 & ST & F & 3.9 & \\
\hline 4826 & P55 & SV & & 4.0 & \\
\hline 4827 & S10 & ST & & 3.7 & \\
\hline & P48 & ST & & & \\
\hline
\end{tabular}

TRNWR 7.22.05

\begin{tabular}{|l|l|l|l|l|r|}
\hline 4823 & P42 & PM & M & 17 & 734 \\
\hline 4824 & P79 & MT & M & 35 & 735 \\
\hline 4825 & P79 & MT & F & 17 & 736 \\
\hline 4826 & P36 & MT & & 47 & \\
\hline 4827 & P28 & ST & F & 5.3 & \\
\hline 4528 & P136 & ST & F & 6.2 & \\
\hline 4829 & S3 & NG & & 14 & \\
\hline 48300 & S141 & ST & F & 4.4 & \\
\hline 4831 & S3 & ST & F & 4.8 & \\
\hline & T60 & MM & & & \\
\hline
\end{tabular}

TRYON CREEK 8.16.05

\begin{tabular}{|l|l|l|l|l|r|}
\hline 4832 & S82 & PM & M & 22.5 & 738 \\
\hline 4833 & S36 & PM & F & 24 & 739 \\
\hline 4834 & S33 & PM & M & 19. & 740 \\
\hline & & & & 520. & 741 \\
\hline 4835 & S10 & PM & F & 5 & 742 \\
\hline 4836 & S142 & PM & M & 19 & 743 \\
\hline 4837 & M142 & PM & M & 20.5 & \\
\hline
\end{tabular}




\begin{tabular}{|l|l|l|l|l|r|}
\hline 4838 & S115 & PM & F & 16 & 744 \\
\hline & & & & $19 .$. & 745 \\
\hline 4839 & S118 & PM & M & 5 & 746 \\
\hline 4840 & S84 & PM & M & 23 & 747 \\
\hline 4841 & S117 & PM & F & 20 & 748 \\
\hline 4842 & S97 & PM & M & 22 & \\
\hline 4843 & S102 & ST & F & 4.4 & \\
\hline 4844 & P100 & ST & M & 5.4 & \\
\hline 4845 & S83 & ST & F & 4.4 & \\
\hline 4846 & S82 & ST & F & 4.5 & \\
\hline & P67 & SV & & & 634 \\
\hline 4569 & S43 & PM & F & 27 & 529 \\
\hline 4425 & S41 & PM & F & 22.5 & 489 \\
\hline 4380 & S105 & PM & M & 20.5 & 528 \\
\hline 4424 & S23 & PM & F & 19 & 636 \\
\hline 4571 & S7 & PM & M & 20.5 & 537 \\
\hline 4572 & M7 & PM & M & 18 & 632 \\
\hline 4403 & S139 & PM & F & 23 & 660 \\
\hline 4567 & S129 & PM & F & 19.5 & 519 \\
\hline 4631 & S66 & PM & F & 19.5 & 527 \\
\hline 4409 & S65 & PM & M & 20 & 647 \\
\hline 4423 & S69 & PM & F & 18 & 526 \\
\hline 4607 & S119 & PM & F & 17 & \\
\hline 4422 & S117 & PM & F & 17.5 &. \\
\hline 4438 & S81 & PM & F & 20 & \\
\hline TC 817705 & & & & &
\end{tabular}

TC 8.18.05
\begin{tabular}{|l|l|l|l|l|r|}
\hline 4861 & S23 & PM & F & 13.5 & 753 \\
\hline 4862 & S108 & PM & F & 24 & 754 \\
\hline 4863 & S103 & PM & M & 15.5 & 755 \\
\hline 4864 & S 77 & PM & F & 11.5 & 756 \\
\hline 4865 & S 59 & PM & M & 18.5 & 758 \\
\hline 4866 & S123 & PM & F & 22 & 759 \\
\hline 4867 & S 93 & PM & F & 22 & 760 \\
\hline
\end{tabular}




\begin{tabular}{|l|l|l|l|l|r|}
\hline 4868 & S70 & PM & F & 22.5 & 761 \\
\hline 4869 & S47 & PM & M & 23 & 762 \\
\hline 4870 & S129 & PM & F & 16 & 763 \\
\hline 4871 & S143 & PM & M & 17 & 764 \\
\hline 4872 & P124 & ST & F & 4.1 & \\
\hline 4873 & S140 & SV & F & 8.7 & \\
\hline 4874 & S32 & SV & F & 5.7 & \\
\hline 4875 & P40 & SV & F & 5.4 & \\
\hline TC 8.19.05 & \multicolumn{7}{|l|}{} & M & 14 & \\
\hline 4876 & P136 & PM & M & 14.5 & 765 \\
\hline 4877 & S85 & PM & M & 14 \\
\hline 4878 & S53 & PM & M & 16 & 767 \\
\hline 4879 & S98 & PM & M & 19 & 768 \\
\hline 4880 & S103 & PM & & & \\
\hline 4881 & P96 & ST & F & 4.2 & \\
\hline 4883 & S93 & ST & F & 4.3 & \\
\hline 4885 & S12 & PM & F & 16.5 & \\
\hline 3984 & P7 & PM & M & 19 & \\
\hline 4887 & S84 & PM & F & 11.5 & \\
\hline 4888 & P24 & SV & F & 3.3 & \\
\hline 4889 & P115 & SV & & 4.1 & \\
\hline 4890 & P120 & SV & F & 3.9 & \\
\hline 4611 & S72 & PM & M & 19.5 & \\
\hline
\end{tabular}

POWELL BUTTE 8.30.05

\begin{tabular}{|c|c|c|c|c|c|}
\hline 4891 & S24 & TT & $\mathbf{M}$ & 80 & \\
\hline 4892. & S34 & PM & $\mathbf{M}$ & 19 & 769 \\
\hline 4893 & S29 & PM & $F$ & 16 & 770 \\
\hline 4894 & S140 & PM & $\mathbf{M}$ & 19.5 & 771 \\
\hline 4895 & S11 & PM & $\mathrm{M}$ & 20 & 772 \\
\hline 4896 & S8 & PM & $\mathbf{M}$ & 17.5 & 773 \\
\hline 4897 & $\mathrm{P} 48$ & PM & $\mathrm{M}$ & 15 & 774 \\
\hline 4898 & P60 & $\mathrm{MO}$ & $M$ & 19.5 & \\
\hline $4899^{\circ}$ & P60 & ST & $\mathbf{M}$ & 4.9 & \\
\hline 4900 & P60 & ST & $F$ & 5.3 & \\
\hline 4901 & P144 & ST & $F$ & 4.0 & \\
\hline 4902 & S139 & ST & $F$ & 4.3 & \\
\hline 4903 & P36 & MO & $\mathbf{M}$ & 16.5 & \\
\hline \multirow[t]{2}{*}{4904} & P36 & ST & $\mathrm{F}$ & 5.2 & \\
\hline & T60 & DV & & & \\
\hline \multirow[t]{2}{*}{4697} & $\mathrm{~S} 48$ & PM & $\mathbf{M}$ & 26.5 & 679 \\
\hline & S39 & PM & $\mathbf{M}$ & 22.5 & 210 \\
\hline \multirow[t]{3}{*}{4696} & S6 & $\overline{\mathbf{P M}}$ & $\mathbf{M}$ & 22.5 & 678 \\
\hline & S10 & PM & $\mathrm{F}$ & 26.5 & 225 \\
\hline & M93 & PM & $F$ & 24.5 & 94 \\
\hline 4699 & S119 & $\mathrm{PM}$ & $F$ & 23.5 & 681 \\
\hline 4695 & S116 & PM & $\mathrm{M}$ & 26 & 677 \\
\hline \multicolumn{6}{|c|}{ PB 8.31.05 } \\
\hline 4905 & S34 & PM & $\mathrm{F}$ & 14 & 775 \\
\hline 4906 & S104 & PM & $\mathbf{F}$ & 13 & 776 \\
\hline
\end{tabular}




\begin{tabular}{|c|c|c|c|c|c|}
\hline 4907 & P72 & $\mathrm{PM}$ & $F$ & 16 & 777 \\
\hline 4908 & S27 & PM & $\mathbf{M}$ & 16 & 778 \\
\hline 4909 & S91 & TT & F & 70 & 779 \\
\hline 4910 & S47 & PM & $\mathbf{M}$ & 15.5 & 780 \\
\hline 4911 & P12 & ST & $F$ & 3.8 & \\
\hline 4912 & P100 & SV & $\mathrm{F}$ & 3.4 & \\
\hline 4913 & P108 & ST & F & 3.7 & \\
\hline \multirow[t]{2}{*}{4914} & S55 & TT & $\mathbf{M}$ & 60 & \\
\hline & $\mathrm{S} 23$ & PM & $\mathbf{M}$ & 25.5 & 92 \\
\hline 4473 & S143 & $\overline{\mathrm{PM}}$ & $\overline{\mathbf{M}}$ & 21.5 & 567 \\
\hline \multicolumn{6}{|c|}{ PB 9.1.05 } \\
\hline 4915 & $\mathrm{P} 100$ & MO & $\mathbf{M}$ & 35 & \\
\hline 4916 & S66 & PM & $\mathrm{F}$ & 19.5 & 782 \\
\hline 4917 & S68 & MO & $F$ & 14.5 & 783 \\
\hline 4918 & S108 & ST & $\bar{M}$ & 4.7 & \\
\hline 4919 & S105 & ST & $F$ & 4.6 & \\
\hline 4920 & S69 & TT & $\mathbf{M}$ & 68 & \\
\hline 4921 & S67 & ST & $F$ & 3.7 & \\
\hline 4922 & S20 & PM & & & \\
\hline \multirow[t]{4}{*}{4923} & T48 & DV & & & \\
\hline & P127 & ST & $\mathrm{F}$ & 4.5 & \\
\hline & P84 & MO & $\mathbf{M}$ & 20.5 & \\
\hline & $\mathbf{S} 23$ & PM & $M$ & 23 & 217 \\
\hline
\end{tabular}

PB 9.2.05
\begin{tabular}{|l|l|l|l|l|r|}
\hline 4925 & S 77 & MO & M & 15 & 784 \\
\hline 4926 & S 55 & PM & M & 13.5 & 785 \\
\hline 4947 & S16 & PM & M & 17 & 786 \\
\hline 4928 & S108 & MO & M & 17 & 787 \\
\hline 4929 & S117 & PM & M & 14 & 788 \\
\hline 4930 & S139 & PM & F & 26 & 789 \\
\hline 4931 & S144 & PM & F & 21.5 & 790 \\
\hline 4932 & S130 & MO & & 13 & \\
\hline 4933 & S 95 & ST & & 2.5 & \\
\hline
\end{tabular}

\section{OXBOW 10.11.05}

\begin{tabular}{|l|l|l|l|l|r|}
\hline 4934 & S23 & PM & M & 19 & 791 \\
\hline 4935 & S74 & PM & F & 12 & 792 \\
\hline 4936 & S120 & PM & F & 13 & 793 \\
\hline 4937 & S20 & PM & F & 19 & 794 \\
\hline 4938 & S67 & PM & M & 15 & 795 \\
\hline 4939 & S34 & PM & F & 16.5 & 796 \\
\hline 4940 & S9 & PM & F & 17 & 797 \\
\hline 4941 & S84 & TT & M & 66 & 798 \\
\hline 4942 & S144 & TT & M & 82 & 799 \\
\hline 4943 & S108 & TT & F & 72 & 800 \\
\hline 4944 & S129 & TT & F & 60 & 801 \\
\hline 4945 & P120 & SB & M & 13 & \\
\hline 4946 & P120 & SB & M & 15 & \\
\hline 4947 & P108 & ST & F & 4.7 & \\
\hline 4948 & P31 & ST & $?$ & 5.0 & \\
\hline
\end{tabular}




\begin{tabular}{|l|l|l|l|l|l|}
\hline 4949 & P40 & MO & F & 12 & \\
\hline 4950 & P52 & MO & M & 15 & \\
\hline 4951 & P52 & MO & M & 13 & \\
\hline 4952 & P84 & ST & $?$ & 4.4 & \\
\hline 3831 & P96 & PM & M & 17.5 & \\
\hline 4058 & S107 & PM & F & 19.5 & \\
\hline 4312 & S92 & PM & F & 20 & \\
\hline 4333 & M47 & TT & M & 79 & \\
\hline & P108 & SV & & & \\
\hline & P76 & ST & & & \\
\hline
\end{tabular}

OX 10.12 .05
\begin{tabular}{|l|l|l|l|l|r|}
\hline 4953 & S117 & PM & M & 14 & 801 \\
\hline 4954 & S 92 & PM & M & 12 & 803 \\
\hline 4955 & S66 & PM & F & 12 & 804 \\
\hline 4956 & P52 & MO & M & 13 & \\
\hline & S126 & SV & & & \\
\hline & S17 & SV & & & \\
\hline & P28 & ST & & & \\
\hline & P36 & ST & & & \\
\hline & P64 & SV & & & \\
\hline
\end{tabular}

OX 10.13.05
\begin{tabular}{|l|l|l|l|l|r|}
\hline 4957 & P91 & PM & M & 14 & 805 \\
\hline 4958 & S55 & PM & F & 16 & 806 \\
\hline 4959 & P76 & ST & F & 6.8 & \\
\hline 4960 & P96 & SV & F & 6.6 & \\
\hline 4961 & S96 & SV & M & 5.5 & \\
\hline & S79 & SV & & & \\
\hline & S139 & SV & & & \\
\hline & S141 & ST & & & \\
\hline & S132 & MO & & & \\
\hline
\end{tabular}

OX 10.14.05
\begin{tabular}{|l|l|l|l|l|r|}
\hline 4962 & S111 & TT & M & 61 & 807 \\
\hline 4963 & S117 & TT & M & 86 & 808 \\
\hline 4964 & M16 & TT & M & 70 & 809 \\
\hline 4965 & P136 & ST & M & 4.9 & \\
\hline & P108 & SV & & & \\
\hline & S 90 & SV & & & \\
\hline & S6 & SV & & & \\
\hline & S11 & ST & & & \\
\hline
\end{tabular}

\section{TRNWR 10.18.05}

\begin{tabular}{|l|l|l|l|l|r|}
\hline 4966 & S142 & TT & M & 69 & 810 \\
\hline 4967 & S10 & TT & M & 72 & 811 \\
\hline 4968 & S10 & PM & M & 13 & 812 \\
\hline 4969 & S127 & PM & M & 14 & 813 \\
\hline 4970 & S118 & PM & F & 13 & 814 \\
\hline 4971 & S32 & PM & F & 13 & 815 \\
\hline 4972 & P103 & PM & M & 10 & 816 \\
\hline 4973 & P40 & PM & F & 15 & 817 \\
\hline 4979 & S130 & PM & M & 20 & 625 \\
\hline
\end{tabular}




\begin{tabular}{|c|c|c|c|c|c|}
\hline 4667 & $\mathrm{~S} 30$ & $\mathrm{PM}$ & $\mathbf{M}$ & 18 & 671 \\
\hline \multicolumn{6}{|c|}{ TRNWR 10.19.05 } \\
\hline 4974 & S138 & PM & $\mathbf{M}$ & 18 & 818 \\
\hline 4975 & $\mathrm{~S} 43$ & PM & $\mathrm{M}$ & 16 & 819 \\
\hline 4976 & S8 & $\mathrm{PM}$ & $F$ & 16 & 820 \\
\hline 4977 & S31 & $\mathrm{PM}$ & $\mathbf{M}$ & 18 & 821 \\
\hline 4978 & $\mathrm{~S} 40$ & PM & $\mathrm{M}$ & 16 & 822 \\
\hline 4979 & P103 & PM & F & 12 & 823 \\
\hline 4980 & $\mathrm{~S} 102$ & PM & $F$ & 14 & 824 \\
\hline 4981 & P139 & ST & & & \\
\hline
\end{tabular}

TRNWR 10.20.05

\begin{tabular}{|l|l|l|l|l|l|}
\hline 4982 & S114 & PM & M & 15 & NO TAG \\
\hline 4983 & S106 & PM & M & 18 & NO TAG \\
\hline 4984 & S142 & PM & F & 18 & NO TAG \\
\hline 4985 & S19 & PM & M & 20 & NO TAG \\
\hline 4986 & S95 & PM & F & 18 & NO TAG \\
\hline 4987 & S84 & PM & M & 18 & NO TAG \\
\hline 4988 & P48 & PM & M & 18 & NO TAG \\
\hline 4989 & S95 & PM & M & 20 & NO TAG \\
\hline 4990 & T12 & DV & & & NO TAG \\
\hline
\end{tabular}

TRNWR 10.21.05

\begin{tabular}{|l|l|l|l|l|l|}
\hline 4991 & S127 & PM & M & 12 & NO TAG \\
\hline 4992 & S8 & PM & M & 18 & NO TAG \\
\hline 4993 & S142 & PM & F & 15 & NO TAG \\
\hline 4994 & S33 & PM & M & 14 & NO TAG \\
\hline 4995 & S86 & PM & M & 83 & NO TAG \\
\hline 4996 & S113 & PM & M & 13 & NO TAG \\
\hline 4997 & S31 & PM & M & 10 & NO TAG \\
\hline 4998 & T24 & DV & & & \\
\hline 4999 & S143 & ST & & 6.6 & \\
\hline
\end{tabular}


Appendix D: Hantavirus Antibody-Positive Specimens. Small mammals found infected with Hantavirus during the period October, 2002 through October, 2005. The positive Glaucomys sabrinus (likely due to a spill-over event) was unable to be confirmed after release of the specimen.

\begin{tabular}{lc} 
Specimen \# & Species \\
260 & Peromyscus maniculatus \\
268 & P. maniculatus \\
279 & P. maniculatus \\
491 & P. maniculatus \\
925 & P. maniculatus \\
1008 & P. maniculatus \\
1009 & P. maniculatus \\
1122 & P. maniculatus \\
1335 & P. maniculatus \\
1351 & P. maniculatus \\
1523 & P. maniculatus \\
1524 & P. maniculatus \\
1550 & P. maniculatus \\
1637 & P. maniculatus \\
1638 & P. maniculatus \\
1669 & P. maniculatus \\
1695 & Glaucomys sabrinus \\
1698 & P. maniculatus \\
1714 & P. maniculatus \\
1715 & P. maniculatus \\
1724 & P. maniculatus \\
1783 & P. maniculatus \\
1797 & P. maniculatus \\
1960 & P. maniculatus \\
2059 & P. maniculatus \\
2063 & P. maniculatus \\
2067 & P. maniculatus \\
2126 & P. maniculatus \\
2138 & P. maniculatus \\
2154 & P. maniculatus \\
2161 & P. maniculatus \\
2293 & P. maniculatus \\
2468 & P. maniculatus \\
2470 & P. maniculatus \\
2471 & P. maniculatus \\
2472 & P. maniculatus \\
2479 & P. maniculatus \\
2491 & P. maniculatus \\
2493 & P. maniculatus \\
2497 & P. maniculatus \\
2499 & Paniculatus \\
& \\
\hline & Paniculatus \\
&
\end{tabular}




$\begin{array}{ll}2511 & \text { P. maniculatus } \\ 2520 & \text { P. maniculatus } \\ 2522 & \text { P. maniculatus } \\ 2525 & \text { P. maniculatus } \\ 2630 & \text { P. maniculatus } \\ 2651 & \text { P. maniculatus } \\ 2722 & \text { P. maniculatus } \\ 2737 & \text { P. maniculatus } \\ 2834 & \text { P. maniculatus } \\ 2836 & \text { P. maniculatus } \\ 2850 & \text { P. maniculatus } \\ 2852 & \text { P. maniculatus } \\ 2860 & \text { P. maniculatus } \\ 2865 & \text { P. maniculatus } \\ 2890 & \text { P. maniculatus } \\ 2891 & \text { P. maniculatus } \\ 2892 & \text { P. maniculatus } \\ 2898 & \text { P. maniculatus } \\ 2900 & \text { Microtus oregoni } \\ 2914 & \text { P. maniculatus } \\ 2936 & \text { P. maniculatus } \\ 2940 & \text { P. maniculatus } \\ 2943 & \text { P. maniculatus } \\ 2947 & \text { P. maniculatus } \\ 3000 & \text { P. maniculatus } \\ \text { LAR } 9 & \text { P. maniculatus } \\ \text { LAR 10 } & \text { P. maniculatus } \\ 3254 & \text { P. maniculatus } \\ 3268 & \text { P. maniculatus } \\ 3277 & \text { P. maniculatus } \\ 3278 & \text { P. maniculatus } \\ 3279 & \text { P. maniculatus } \\ 3304 & \text { P. maniculatus } \\ 3306 & \text { P. maniculatus } \\ 3308 & \text { P. maniculatus } \\ 3314 & \text { P. maniculatus } \\ 3317 & \text { P. maniculatus } \\ 3321 & \text { P. maniculatus } \\ 3323 & \text { P. maniculatus } \\ 3324 & \text { P. maniculatus } \\ 3327 & \text { P. maniculatus } \\ 3330 & \text { P. maniculatus } \\ 3333 & \text { P. maniculatus } \\ 3344 & \text { P. maniculatus } \\ \text { MT HOOD Holatus } \\ \end{array}$




\begin{tabular}{|c|c|}
\hline 3617 & P. maniculatus \\
\hline 3621 & P. maniculatus \\
\hline 3622 & P. maniculatus \\
\hline 3626 & P. maniculatus \\
\hline 3627 & P. maniculatus \\
\hline 3631 & P. maniculatus \\
\hline 3644 & P. maniculatus \\
\hline 3645 & P. maniculatus \\
\hline 3651 & P. maniculatus \\
\hline 3654 & P. maniculatus \\
\hline 3656 & P. maniculatus \\
\hline 3662 & P. maniculatus \\
\hline 3665 & P. maniculatus \\
\hline 3666 & P. maniculatus \\
\hline 3671 & P. maniculatus \\
\hline 3676 & P. maniculatus \\
\hline 3679 & P. maniculatus \\
\hline 3782 & P. maniculatus \\
\hline 3822 & P. maniculatus \\
\hline 3834 & P. maniculatus \\
\hline 3877 & P. maniculatus \\
\hline 3879 & P. maniculatus \\
\hline 3659 & P. maniculatus \\
\hline 3629 & P. maniculatus \\
\hline 3639 & P. maniculatus \\
\hline 3666 & P. maniculatus \\
\hline 3656 & P. maniculatus \\
\hline 3902 & P. maniculatus \\
\hline 3903 & P. maniculatus \\
\hline 3905 & P. maniculatus \\
\hline 3909 & P. maniculatus \\
\hline 3910 & P. maniculatus \\
\hline 3911 & P. maniculatus \\
\hline 3645 & P. maniculatus \\
\hline 3621 & P. maniculatus \\
\hline 3639 & P. maniculatus \\
\hline 3666 & P. maniculatus \\
\hline 3879 & P. maniculatus \\
\hline 3883 & P. maniculatus \\
\hline 3913 & P. maniculatus \\
\hline 3920 & P. maniculatus \\
\hline 4087 & P. maniculatus \\
\hline 4091 & P. maniculatus \\
\hline 4096 & P. maniculatus \\
\hline 4148 & P. maniculatus \\
\hline 4207 & P. maniculatus \\
\hline 3909 & P. maniculatus \\
\hline
\end{tabular}




\begin{tabular}{|c|c|}
\hline 4250 & P. maniculatus \\
\hline 4298 & P. maniculatus \\
\hline 4306 & P. maniculatus \\
\hline 3920 & P. maniculatus \\
\hline 4207 & P. maniculatus \\
\hline 4341 & P. maniculatus \\
\hline 4449 & P. maniculatus \\
\hline 4451 & P. maniculatus \\
\hline 4549 & P. maniculatus \\
\hline 4086 & P. maniculatus \\
\hline 4539 & P. maniculatus \\
\hline 4570 & P. maniculatus \\
\hline 4576 & P. maniculatus \\
\hline 422 & P. maniculatus \\
\hline 4627 & P. maniculatus \\
\hline 4631 & P. maniculatus \\
\hline 4667 & P. maniculatus \\
\hline 4688 & P. maniculatus \\
\hline 4717 & P. maniculatus \\
\hline 589 & P. maniculatus \\
\hline 4782 & P. maniculatus \\
\hline 4783 & P. maniculatus \\
\hline 4784 & P. maniculatus \\
\hline 602 & P. maniculatus \\
\hline 612 & P. maniculatus \\
\hline 621 & P. maniculatus \\
\hline 4788 & P. maniculatus \\
\hline 4789 & P. maniculatus \\
\hline MH 353 & P. maniculatus \\
\hline 671 & P. maniculatus \\
\hline 674 & P. maniculatus \\
\hline 4818 & P. maniculatus \\
\hline MH346 & P. maniculatus \\
\hline 660 & P. maniculatus \\
\hline 652 & P. maniculatus \\
\hline 4851 & P. maniculatus \\
\hline 4861 & P. maniculatus \\
\hline 4870 & P. maniculatus \\
\hline 217 & P. maniculatus \\
\hline 4907 & P. maniculatus \\
\hline 4992 & P. manicu \\
\hline
\end{tabular}


Appendix E: Rodent Specimen mtDNA Sequences. Resolved sequences of mitochondrial ND3, ND4, ND4L, and arginine tRNA genes (1,463bp). $\mathrm{N}=88$.

$\underline{\mathrm{WC} 7}$

ACAGCTGACTTCCAATCAGTTAGATCTAGACCTAACCTAGAAGAAAGTAA TAAATATACTAACAGCCCTATTAGTAAATATTACGCTATCAATACTCCTAA TCATCATTGCCTTCTGACTACCCCAACTTAATTTATACACTGAAAAAGCAA ACCCGTATGAGTGCGGATTTGACCCCATAGGCTCTGCTCGCCTCCCATTCT CAATAAAATTTTTCCTAGTAGCAATCACTTTCTTACTATTTGACCTAGAAAT TGCACTTCTACTTCCACTACCATGAGCTATCCAAATATATAATATTAACAT CATAATATTAACAGCTTTCATCCTAGTCTCCGTATTAGCATTAGGCTTAGC CTACGAGTGACTACAAAAAGGACTAGAATGAACTGAA-

TAACTGGTAATTAGTTTAATTAAAACAAATGATTTCGACTCATTAGATTAT GATATACATTATAATTACCAAAA----

ATGTCATCTGTAACCTCCAATATTATATTAGCATTCTCATTCTCATTTCTAG GAACACTAGTATTTCGATCCCACCTAATATCAACTCTCCTATGCCTTGAAG GAATAATACTATCACTGTTTATTATAACCACAATTACATCCCTTAATTCCC ACTCAATAATTATATACCCTCTACCCATTGTTATTCTAGTATTCGCAGCATG TGAAGCAGCCATTGGCTTAGCCCTATTAGCAAAACTATCAAACTCCTACGG AACAGATTATGTGCAAAATCTAAACCTACTTCAATGT-

TAAAAATTATTTTTCCCTCTATCATATTACTCCCACTCACCTGATTATCAAA CAAAAAAAACCTATGAGTCAATGTTACCTCCTACAGCTTTATAATCAGCTT GACTTCAGCTATGTTTTTATGACAAAACGACATAAATAACCTAAACTTCTC ACTACTATTCTCAACTGACTCCCTATCCTCCCCTCTTATCATTCTAACAACA TGACTTCTACCACTCATACTGCTAGCCAGCCAAAACCACATAAAAAAGGA AACAGAGTCAAACAAAAAAACATACATTTCAATACTAGTCTTACTACAAA TTCTTCTAATCATAACATTTTCTGCAAACGAACTAATTATATTTTACATCTT ATTTGAAGCCACCCTAATCCCCACCCTTATTATCATCACCCGATGGGGTAA CCAAACAGAACGACTAAATGCAGGACTCTACTTTCTATTTTATACCCTAAT TGGATCAATCCCACTACTAATCGCCCTTATCCACATTCAAAATATAAAAGG AACATTAAACTTCATACTATTCCCACTCACCTTTACACCCCTAGACCAAAC ATGGTCTAATAATATTCTATGATTAGCATGTATAATGGCATTTATGATTAA AATACCACTATACGGAGTCCACTTATGACTCCCTAAAGCCCAC

WC11

ACCGAACTAGTACAGCTGACTTCCAATCAGTTAGATCTAGACCTAACCTAG AAGAAAGTAATAAATATACTAACAGCCCTATTAGTAAATATTACGCTATC AATACTCCTAATCATCATTGCCTTCTGACTACCCCAACTTAATTTATATACT GAAAAAGCAAACCCGTATGAGTGCGGATTTGACCCCATAGGCTCTGCTCG CCTCCCATTCTCAATAAAATTTTTCCTAGTAGCAATCACTTTCTTACTATTT GACCTAGAAATTGCACTTCTACTTCCACTACCATGAGCTATCCAAATATAT AATATTAACATCATAATATTAACAGCTTTCATCCTAGTCTCCGTATTAGCA TTAGGCTTAGCCTACGAGTGACTACAAAAAGGACTAGAATGAACTGAATAACTGGTAATTAGTTTAATTAAAACAAATGATTTCGACTCATTAGATTAT 
GATATACATTATAATTACCAAAA----

ATGTCATCTGTAACCTCCAATATTATATTAGCATTCTCATTCTCATTTCTAG GAACACTAGTATTTCGATCCCACCTAATATCAACTCTCCTATGCCTTGAAG GAATAATACTATCACTGTTTATTATAACCACAATTACATCCCTTAATTCCC ACTCAATAATTATATACCCTCTACCCATTGTTATTCTAGTATTCGCAGCATG TGAAGCAGCCATTGGCTTAGCCCTATTAGCAAAACTATCAAACTCCTACGG AACAGATTATGTGCAAAATCTAAACCTACTTCAATGT-

TAAAAATTATTTTTCCCTCTATCATATTACTCCCACTCACCTGATTATCAAA CAAAAAAAACCTATGAGTCAATGTTACCTCCTACAGCTTTATAATCAGCTT GACTTCAGCTATGTTTTTATGACAAAACGACATAAATAACCTAAACTTCTC ACTACTATTCTCAACTGACTCCCTATCCTCCCCTCTTATTATTCTAACAACA TGACTTCTACCACTCATACTGCTAGCCAGCCAAAACCACATAAAAAAGGA AACAGAGTCAAACAAAAAAACATACATTTCAATACTAGTCTTACTACAAA TTCTTCTAATCATAACATTTTCTGCAAACGAACTAATTATATTTTACATCTT ATTTGAAGCCACCCTAATCCCCACCCTTATTATCATCACCCGATGGGGTAA CCAAACAGAACGACTAAATGCAGGACTCTACTTTCTATTTTATACCCTAAT TGGATCAATCCCACTACTAATCGCCCTTATCCACATTCAAAATATAAAAGG AACATTAAACTTCATACTATTCCCACTCACCTTTACACCCCTAGACCAAAC ATGGTCTAATAATATTCTATGATTAGCATGTATAATGGCATTTATGATTAA AATACCACTATACGGAGTCCACTTATGACTCCCTAAAGCCCACGAGAAAG CTCCAAA

\section{WC12}

ACCGAACTAGTACAGCTGACTTCCAATCAGTTAGATCTAGACCTAACCTAG AAGAAAGTAATAAATATACTAACAGCCCTATTAGTAAATATTACGCTATC AATACTCCTAATCATCATTGCCTTCTGACTACCCCAACTTAATTTATACACT GAAAAAGCAAACCCGTATGAGTGCGGATTTGACCCCATAGGCTCTGCTCG CCTCCCATTCTCAATAAAATTTTTCCTAGTAGCAATCACTTTCTTACTATTT GACCTAGAAATTGCACTTCTACTTCCACTACCATGAGCTATCCAAATATAT AATATTAACATCATAATATTAACAGCTTTCATCCTAGTCTCCGTATTAGCA TTAGGCTTAGCCTACGAGTGACTACAAAAAGGACTAGAATGAACTGAATAACTGGTAATTAGTTTAATTAAAACAAATGATTTCGACTCATTAGATTAT GATATACATTATAATTACCAAAA----

ATGTCATCTGTAACCTCCAATATTATATTAGCATTCTCATTCTCATTTCTAG GAACACTAGTATTTCGATCCCACCTAATATCAACTCTCCTATGCCTTGAAG GAATAATACTATCACTGTTTATTATAACCACAATTACATCCCTTAATTCCC ACTCAATAATTATATACCCTCTACCCATTGTTATTCTAGTATTCGCAGCATG TGAAGCAGCCATTGGCTTAGCCCTATTAGCAAAACTATCAAACTCCTACGG AACAGATTATGTGCAAAATCTAAACCTACTTCAATGT-

TAAAAATTATTTTTCCCTCTATCATATTACTCCCACTCACCTGATTATCAAA CAAAAAAAACCTATGAGTCAATGTTACCTCCTACAGCTTTATAATCAGCTT GACTTCAGCTATGTTTTTATGACAAAACGACATAAATAACCTAAACTTCTC ACTACTATTCTCAACTGACTCCCTATCCTCCCCTCTTATCATTCTAACAACA TGACTTCTACCACTCATACTGCTAGCCAGCCAAAACCACATAAAAAAGGA AACAGAGTCAAACAAAAAAACATACATTTCAATACTAGTCTTACTACAAA 
TTCTTCTAATCATAACATTTTCTGCAAACGAACTAATTATATTTTACATCTT ATTTGAAGCCACCCTAATCCCCACCCTTATTATCATCACCCGATGGGGTAA CCAAACAGAACGACTAAATGCAGGACTCTACTTTCTATTTTATACCCTAAT TGGATCAATCCCACTACTAATCGCCCTTATCCACATTCAAAATATAAAAGG AACATTAAACTTCATACTATTCCCACTCACCTTTACACCCCTAGACCAAAC ATGGTCTAATAATATTCTATGATTAGCATGTATAATGGCATTTATGATTAA AATACCACTATACGGAGTCCACTTATGACTCCCTAAAGCCC-

$\underline{\mathrm{WC13}}$

ACCGAACTAGTACAGCTGACTTCCAATCAGTTAGATCTAGACCTAACCTAG AAGAAAGTAATAAATATACTAACAGCCCTATTAGTAAATATTACGCTATC AATACTCCTAATCATCATTGCCTTCTGACTACCCCAACTTAATTTATACACT GAAAAAGCAAACCCGTATGAGTGCGGATTTGACCCCATAGGCTCTGCTCG CCTCCCATTCTCAATAAAATTTTTCCTAGTAGCAATCACTTTCTTACTATTT GACCTAGAAATTGCACTTCTACTTCCACTACCATGAGCTATCCAAATATAT AATATTAACATCATAATATTAACAGCTTTCATCCTAGTCTCCGTATTAGCA TTAGGCTTAGCCTACGAGTGACTACAAAAAGGACTAGAATGAACTGAATAACTGGTAATTAGTTTAATTAAAACAAATGATTTCGACTCATTAGATTAT GATATACATTATAATTACCAAAA----

ATGTCATCTGTAACCTCCAATATTATATTAGCATTCTCATTCTCATTTCTAG GAACACTAGTATTTCGATCCCACCTAATATCAACTCTCCTATGCCTTGAAG GAATAATACTATCACTGTTTATTATAACCACAATTACATCCCTTAATTCCC ACTCAATAATTATATACCCTCTACCCATTGTTATTCTAGTATTCGCAGCATG TGAAGCAGCCATTGGCTTAGCCCTATTAGCAAAACTATCAAACTCCTACGG AACAGATTATGTGCAAAATCTAAACCTACTTCAATGT-

TAAAAATTATTTTTCCCTCTATCATATTACTCCCACTCACCTGATTATCAAA CAAAAAAAACCTATGAGTCAATGTTACCTCCTACAGCTTTATAATCAGCTT GACTTCAGCTATGTTTTTATGACAAAACGACATAAATAACCTAAACTTCTC ACTACTATTCTCAACTGACTCCCTATCCTCCCCTCTTATCATTCTAACAACA TGACTTCTACCACTCATACTGCTAGCCAGCCAAAACCACATAAAAAAGGA AACAGAGTCAAACAAAAAAACATACATTTCAATACTAGTCTTACTACAAA TTCTTCTAATCATAACATTTTCTGCAAACGAACTAATTATATTTTACATCTT ATTTGAAGCCACCCTAATCCCCACCCTTATTATCATCACCCGATGGGGTAA CCAAACAGAACGACTAAATGCAGGACTCTACTTTCTATTTTATACCCTAAT TGGATCAATCCCACTACTAATCGCCCTTATCCACATTCAAAATATAAAAGG AACATTAAACTTCATACTATTCCCACTCACCTTTACACCCCTAGACCAAAC ATGGTCTAATAATATTCTATGATTAGCATGTATAATGGCATTTATGATTAA AATACCACTATACGGAGTCCACTTATGACTCCCTAAAGCCCACG-

$\underline{\operatorname{lid} 3256}$ CGAACTAGTACAGCTGACTTCCAATCAGTTAGATCTAGACCTAACCTAGAA GAAAGTAATAAATATACTAACAGCCCTATTAGTAAATATTACGCTATCAAT ACTCCTAATCATCATTGCCTTCTGACTACCCCAACTTAATTTATATACTGAA AAAGCAAACCCGTATGAGTGCGGATTTGACCCCATAGGCTCTGCTCGCCTC CCATTCTCAATAAAATTTTTCCTAGTAGCAATCACTTTCTTACTATTTGACC 
TAGAAATTGCACTTCTACTTCCACTACCATGAGCTATCCAAATATATAATA TTAACATCATAATATTAACAGCTTTCATCCTAGTCTCCGTATTAGCATTAG GCTTAGCCTACGAGTGACTACAAAAAGGACTAGAATGAACTGAA-

TAACTGGTAATTAGTTTAATTAAAACAAATGATTTCGACTCATTAGATTAT GATATACATTATAATTACCAAAA----

ATGTCATCTGTAACCTCCAATATTATATTAGCATTCTCATTCTCATTTCTAG GAACACTAGTATTTCGATCCCACCTAATATCAACTCTCTTATGCCTTGAAG GAATAATACTATCACTGTTTATTATAACCACAATTACATCCCTTAATTCCC ACTCAATAATTATATACCCTCTACCCATTGTTATTCTAGTATTCGCAGCATG TGAAGCAGCCATTGGCTTAGCCCTATTAGCAAAACTATCAAACTCCTACGG AACAGATTATGTGCAAAACCTAAACTTACTTCAATGT-

TAAAAATTATTTTTCCCTCTATCATATTACTCCCACTCACCTGATTATCAAA CAAAAAAAACCTATGAGTCAATGTTACCTCCTACAGCTTTATAATCAGCTT GACTTCAGCTATGTTTTTATGACAAAACGACATAAATAACCTAAACTTCTC ACTACTATTCTCAACTGACTCCCTATCCTCCCCTCTTATTATTCTAACAACA TGACTTCTACCACTCATACTGCTAGCCAGCCAAAACCACATAAAAAAGGA AACAGAGTCAAACAAAAAAACATATATTTCAATACTAGTCTTACTACAAA TTCTTCTAATCATAACATTTTCTGCAAACGAACTAATTATATTTTACATCTT ATTTGAAGCCACCCTAATCCCCACCCTTATTATCATCACCCGATGGGGTAA CCAAACAGAACGACTAAATGCAGGACTCTACTTTCTATTTTATACCCTAAT TGGATCAATCCCACTACTAATCGCCCTTATCCACATTCAAAATATAAAAGG AACATTAAACTTCATACTATTCCCACTCACCTTTACACCCCTAGACCAAAC ATGGTCTAATAATATTCTATGATTAGCATGTATAATGGCATTTATGATTAA AATACCACTATACGGAGTCCACTTATGACTCCCTAAAGCCCACG-

$\underline{\operatorname{ljd} 3304}$

CGAACTAGTACAGCTGACTTCCAATCAGTTAGATCTAGACCTAACCTAGAA GAAAGTAATAAATATACTAACAGCCCTATTAGTAAATATTACGCTATCAAT ACTCCTAATCATCATTGCCTTCTGACTACCCCAACTTAATTTATATACTGAA AAAGCAAACCCATATGAGTGCGGATTTGACCCCATAGGCTCTGCTCGCCTC CCATTCTCAATAAAATTTTTCCTAGTAGCAATCACTTTCTTACTATTTGACC TAGAAATTGCACTTCTACTTCCACTACCATGAGCTATCCAAATATATAATA TTAACATCATAATATTAACAGCTTTCATCCTAGTCTCCGTATTAGCATTAG GCTTAGCCTACGAGTGACTACAAAAAGGACTAGAATGAACTGAATAACTGGTAATTAGTTTAATTAAAACAAATGATTTCGACTCATTAGATTAT GATATACATTATAATTACCAAAA----

ATGTCATCTGTAACCTCCAATATTATATTAGCATTCTCATTCTCATTTCTAG GAACACTAGTATTTCGATCCCACCTAATATCAACTCTCCTATGCCTTGAAG GAATAATACTATCACTGTTTATTATAACCACAATTACATCCCTTAATTCCC ACTCAATAATTATATACCCTCTACCCATTGTTATTCTAGTATTCGCAGCATG TGAAGCAGCCATTGGCTTAGCCCTATTAGCAAAACTATCAAACTCCTACGG AACAGATTATGTGCAAAATCTAAACCTACTTCAATGT-

TAAAAATTATTTTTCCCTCTATCATATTACTCCCACTCACCTGATTATCAAA CAAAAAAAACCTATGAGTCAATGTTACCTCCTACAGCTTTATAATCAGCTT GACTTCAGCTATGTTTTTATGACAAAACGACATAAATAGCCTAAACTTCTC 
ACTACTATTCTCAACTGACTCCCTATCCTCCCCTCTTATTATTCTAACAACA TGACTTCTACCACTCATACTGCTAGCCAGCCAAAACCACATAAAAAAGGA AACAGAGTCAAACAAAAAAACATACATTTCAATACTAGTCTTACTACAAA TTCTTCTAATCATAACATTTTCTGCAAATGAACTAATTATATTTTACATCTT ATTTGAAGCCACCCTAATCCCCACCCTTATTATCATCACCCGATGGGGTAA CCAAACAGAACGACTAAATGCAGGACTCTACTTTCTATTTTATACCCTAAT TGGATCAATCCCACTACTAATCGCCCTTATCCACATTCAAAATATAAAAGG AACATTAAACTTCATACTATTTCCACTCACCTTTACACCCCTAGACCAAAC ATGGTCTAATAATATTCTATGATTAGCATGTATAATGGCATTTATGATTAA AATACCACTATACGGAGTCCACTTATGACTCCCTAAAGCCCACG

$\underline{\text { ljd3306 }}$

AGTACAGCTGACTTCCAATCAGTTAGATCTAGACCTAACCTAGAAGAAAG TAATAAATATACTAACAGCCCTATTAGTAAATATTACGCTATCAATACTCC TAATCATCATTGCCTTCTGACTACCCCAACTTAATTTATATACTGAAAAAG CAAACCCGTATGAGTGCGGATTTGACCCCATAGGCTCTGCTCGCCTCCCAT TCTCAATAAAATTTTTCCTAGTAGCAATCACTTTCTTACTATTTGACCTAGA AATTGCACTTCTACTTCCACTACCATGAGCTATCCAAATATATAATATTAA CATCATAATATTAACAGCTTTCATCCTAGTCTCCGTATTAGCATTAGGCTTA GCCTACGAGTGACTACAAAAAGGACTAGAATGAACTGAA-

TAACTGGTAATTAGTTTAATTAAAACAAATGATTTCGACTCATTAGATTAT GATATACATTATAATTACCAAAA----

ATGTCATCTGTAACCTCCAATATTATATTAGCATTCTCATTCTCATTTCTAG GAACACTAGTATTTCGATCCCACCTAATATCAACTCTCCTATGCCTTGAAG GAATAATACTATCACTGTTTATTATAACCACAATTACATCCCTTAATTCCC ACTCAATAATTATATACCCTCTACCCATTGTTATTCTAGTATTCGCAGCATG TGAAGCAGCCATTGGCTTAGCCCTATTAGCAAAACTATCAAACTCCTACGG AACAGATTATGTGCAAAATCTAAACCTACTTCAATGT-

TAAAAATTATTTTTCCCTCTATCATATTACTCCCACTCACCTGATTATCAAA CAAAAAAAACCTATGAGTCAATGTTACCTCCTACAGCTTTATAATCAGCTT GACTTCAGCTATGTTTTTATGACAAAACGACATAAATAGCCTAAACTTCTC ACTACTATTCTCAACTGACTCCCTATCCTCCCCTCTTATTATTCTAACAACA TGACTTCTACCACTCATACTGCTAGCCAGCCAAAACCACATAAAAAAGGA AACAGAGTCAAACAAAAAAACATACATTTCAATACTAGTCTTACTACAAA TTCTTCTAATCATAACATTTTCTGCAAATGAACTAATTATATTTTACATCTT ATTTGAAGCCACCCTAATCCCCACCCTTATTATCATCACCCGATGGGGTAA CCAAACAGAACGACTAAATGCAGGACTCTACTTTCTATTTTATACCCTAAT TGGATCAATCCCACTACTAATCGCCCTTATCCACATTCAAAATATAAAAGG AACATTAAACTTCATACTATTTCCACTCACCTTTACACCCCTAGACCAAAC ATGGTCTAATAATATTCTATGATTAGCATGTATAATGGCATTTATGATTAA AATACCACTATACGGAGTCCACTTATGACTCCCTAAAGCCCACG-

$\underline{\operatorname{lid} 3308}$

AGCTGACTTCCAATCAGTTAGATCTAGACCTAACCTAGAAGAAAGTAATA AATATACTAACAGCCCTATTAGTAAATATTACGCTATCAATACTCCTAATC 
ATCATTGCCTTCTGACTACCCCAACTTAATTTATATACTGAAAAAGCAAAC CCGTATGAGTGCGGATTTGACCCCATAGGCTCTGCTCGCCTCCCATTCTCA ATAAAATTTTTCCTAGTAGCAATCACTTTCTTACTATTTGACCTAGAAATTG CACTTCTACTTCCACTACCATGAGCTATCCAAATATATAATATTAACATCA TAATATTAACAGCTTTCATCCTAGTCTCCGTATTAGCATTAGGCTTAGCCTA CGAGTGACTACAAAAAGGACTAGAATGAACTGAA-

TAACTGGTAATTAGTTTAATTAAAACAAATGATTTCGACTCATTAGATTAT GATATACATTATAATTACCAAAA----

ATGTCATCTGTAACCTCCAATATTATATTAGCATTCTCATTCTCATTTCTAG GAACACTAGTATTTCGATCCCACCTAATATCAACTCTCCTATGCCTTGAAG GAATAATACTATCACTGTTTATTATAACCACAATTACATCCCTTAATTCCC ACTCAATAATTATATACCCTCTACCCATTGTTATTCTAGTATTCGCAGCATG TGAAGCAGCCATTGGCTTAGCCCTATTAGCAAAACTATCAAACTCCTACGG AACAGATTATGTGCAAAATCTAAACCTACTTCAATGT-

TAAAAATTATTTTTCCCTCTATCATATTACTCCCACTCACCTGATTATCAAA CAAAAAAAACCTATGAGTCAATGTTACCTCCTACAGCTTTATAATCAGCTT GACTTCAGCTATGTTTTTATGACAAAACGACATAAATAATCTAAACTTCTC ACTACTATTCTCAACTGACTCCCTATCCTCCCCTCTTATTATTCTAACAACA TGACTTCTACCACTCATACTGCTAGCCAGCCAAAACCACATAAAAAAGGA AACAGAGTCAAACAAAAAAACATACATTTCAATACTAGTCTTACTACAAA TTCTTCTAATCATAACATTTTCTGCAAACGAACTAATTATATTTTACATCTT ATTTGAAGCTACCCTAATCCCCACCCTTATTATCATCACCCGATGGGGTAA CCAAACAGAACGACTAAATGCAGGACTCTACTTTCTATTTTATACCCTAAT TGGATCAATCCCACTACTAATCGCCCTTATCCACATTCAAAATATAAAAGG AACATTAAACTTCATACTATTCCCACTCACCTTTACACCCCTAGACCAAAC ATGGTCTAATAATATTCTATGATTAGCATGTATAATGGCATTTATGATTAA AATACCACTATACGGAGTCCACTTATGACTCCCTAAAGCCCACG-

\section{$\underline{\operatorname{ljd} 3317}$}

CCGAACTAGTACAGCTGACTTCCAATCAGTTAGATCTAGACCTAACCTAGA AGAAAGTAATAAATATACTAACAGCCCTATTAGTAAATATTACGCTATCA ATACTCCTAATCATCATTGCCTTCTGACTACCCCAACTTAATTTATATACTG AAAAAGCAAACCCGTATGAGTGCGGATTTGACCCCATAGGCTCTGCTCGC CTCCCATTCTCAATAAAATTTTTCCTAGTAGCAATCACTTTCTTACTATTTG ACCTAGAAATTGCACTTCTACTTCCACTACCATGAGCTATCCAAATATATA ATATTAACATCATAATATTAACAGCTTTCATCCTAGTCTCCGTATTAGCATT AGGCTTAGCCTACGAGTGACTACAAAAAGGACTAGAATGAACTGAA-

TAACTGGTAATTAGTTTAATTAAAACAAATGATTTCGACTCATTAGATTAT GATATACATTATAATTACCAAAA----

ATGTCATCTGTAACCTCCAATATTATATTAGCATTCTCATTCTCATTTCTAG GAACACTAGTATTTCGATCCCACCTAATATCAACTCTCCTATGCCTTGAAG GAATAATACTATCACTGTTTATTATAACCACAATTACATCCCTTAATTCCC ACTCAATAATTATATACCCTCTACCCATTGTTATTCTAGTATTCGCAGCATG TGAAGCAGCCATTGGCTTAGCCCTATTAGCAAAACTATCAAACTCCTACGG AACAGATTATGTGCAAAATCTAAACCTACTTCAATGT- 
TAAAAATTATTTTTCCCTCTATCATATTACTCCCACTCACCTGATTATCAAA CAAAAAAAACCTATGAGTCAATGTTACCTCCTACAGCTTTATAATCAGCTT GACTTCAGCTATGTTTTTATGACAAAACGACATAAATAACCTAAACTTCTC ACTACTATTCTCAACTGACTCCCTATCCTCCCCTCTTATTATTCTAACAACA TGACTTCTACCACTCATACTGCTAGCCAGCCAAAACCACATAAAAAAGGA AACAGAGTCAAACAAAAAAACATACATTTCAATACTAGTCTTACTACAAA TTCTTCTAATCATAACATTTTCTGCAAACGAACTAATTATATTTTACATCTT ATTTGAAGCCACCCTAATCCCCACCCTTATTATCATCACCCGATGGGGTAA CCAAACAGAACGACTAAATGCAGGACTCTACTTTCTATTTTATACCCTAAT TGGATCAATCCCACTACTAATCGCCCTTATCCACATTCAAAATATAAAAGG AACATTAAACTTCATACTATTCCCACTCACCTTTACACCCCTAGACCAAAC ATGGTCTAATAATATTCTATGATTAGCATGTATAATGGCATTTATGATTAA AATACCACTATACGGAGTCCACTTATGACTCCCTAAAGCCCACGTAGAAG CTCCCA-

$\underline{\operatorname{ljd} 3324}$

CCGAACTAGTACAGCTGACTTCCAATCAGTTAGATCTAGACCTAACCTAGA AGAAAGTAATAAATATACTAACAGCCCTATTAGTAAATATTACGCTATCA ATACTCCTAATCATCATTGCCTTCTGACTACCCCAACTTAATTTATATACTG AAAAAGCAAACCCATATGAGTGCGGATTTGACCCCATAGGCTCTGCTCGC CTCCCATTCTCAATAAAATTTTTCCTAGTAGCAATCACTTTCTTACTATTTG ACCTAGAAATTGCACTTCTACTTCCACTACCATGAGCTATCCAAATATATA ATATTAACATCATAATATTAACAGCTTTCATCCTAGTCTCCGTATTAGCATT AGGCTTAGCCTACGAGTGACTACAAAAAGGACTAGAATGAACTGAATAACTGGTAATTAGTTTAATTAAAACAAATGATTTCGACTCATTAGATTAT GATATACATTATAATTACCAAAA----

ATGTCATCTGTAACCTCCAATATTATATTAGCATTCTCATTCTCATTTCTAG GAACACTAGTATTTCGATCCCACCTAATATCAACTCTCCTATGCCTTGAAG GAATAATACTATCACTGTTTATTATAACCACAATTACATCCCTTAATTCCC ACTCAATAATTATATACCCTCTACCCATTGTTATTCTAGTATTCGCAGCATG TGAAGCAGCCATTGGCTTAGCCCTATTAGCAAAACTATCAAACTCCTACGG AACAGATTATGTGCAAAATCTAAACCTACTTCAATGT-

TAAAAATTATTTTTCCCTCTATCATATTACTCCCACTCACCTGATTATCAAA CAAAAAAAACCTATGAGTCAATGTTACCTCCTACAGCTTTATAATCAGCTT GACTTCAGCTATGTTTTTATGACAAAACGACATAAATAACCTAAACTTCTC ACTACTATTCTCAACTGACTCCCTATCCTCCCCTCTTATTATTCTAACAACA TGACTTCTACCACTCATACTGCTAGCCAGCCAAAACCACATAAAAAAGGA AACAGAGTCAAACAAAAAAACATATATTTCAATACTAGTCTTACTACAAA TTCTTCTAATCATAACATTTTCTGCAAACGAACTAATTATATTTTACATCTT ATTTGAAGCCACCCTAATCCCCACCCTTATTATCATCACCCGATGGGGTAA CCAAACAGAACGACTAAATGCAGGACTCTACTTTCTATTTTATACCCTAAT TGGATCAATCCCACTACTAATCGCCCTTATCCACATTCAAAATATAAAAGG AACATTAAACTTCATACTATTCCCACTCACCTTTACACCCCTAGACCAAAC ATGGTCTAATAATATTCTATGATTAGCATGTATAATGGCATTTATGATTAA AATACCACTATACGGAGTCCACTTATGACTCCCTAAAGCCCA---:------ 
$\underline{\text { lid} 3327}$

AGCTGACTTCCAATCAGTTAGATCTAGACCTAACCTAGAAGAAAGTAATA AATATACTAACAGCCCTATTAGTAAATATTACGCTATCAATACTCCTAATC ATCATTGCCTTCTGACTACCCCAACTTAATTTATATACTGAAAAAGCAAAC CCGTATGAGTGCGGATTTGACCCCATAGGCTCTGCTCGCCTCCCATTCTCA ATAAAATTTTTCCTAGTAGCAATCACTTTCTTACTATTTGACCTAGAAATTG CACTTCTACTTCCACTACCATGAGCTATCCAAATATATAATATTAACATCA TAATATTAACAGCTTTCATCCTAGTCTCCGTATTAGCATTAGGCTTAGCCTA CGAGTGACTACAAAAAGGACTAGAATGAACTGAA-

TAACTGGTAATTAGTTTAATTAAAACAAATGATTTCGACTCATTAGATTAT GATATACATTATAATTACCAAAA---

ATGTCATCTGTAACCTCCAATATTATATTAGCATTCTCATTCTCATTTCTAG GAACACTAGTATTTCGATCCCACCTAATATCAACTCTCCTATGCCTTGAAG GAATAATACTATCACTGTTTATTATAACCACAATTACATCCCTTAATTCCC ACTCAATAATTATATACCCTCTACCCATTGTTATTCTAGTATTCGCAGCATG TGAAGCAGCCATTGGCTTAGCCCTATTAGCAAAACTATCAAACTCCTACGG AACAGATTATGTGCAAAATCTAAACCTACTTCAATGT-

TAAAAATTATTTTTCCCTCTATCATATTACTCCCACTCACCTGATTATCAAA CAAAAAAAACCTATGAGTCAATGTTACCTCCTACAGCTTTATAATCAGCTT GACTTCAGCTATGTTTTTATGACAAAACGACATAAATAATCTAAACTTCTC ACTACTATTCTCAACTGACTCCCTATCCTCCCCTCTTATTATTCTAACAACA TGACTTCTACCACTCATACTGCTAGCCAGCCAAAACCACATAAAAAAGGA AACAGAGTCAAACAAAAAAACATACATTTCAATACTAGTCTTACTACAAA TTCTTCTAATCATAACATTTTCTGCAAACGAACTAGTTATATTTTACATCTT ATTTGAAGCTACCCTAATCCCCACCCTTATTATCATCACCCGATGGGGTAA CCAAACAGAACGACTAAATGCAGGACTCTACTTTCTATTTTATACCCTAAT TGGATCAATCCCACTACTAATCGCCCTTATCCACATTCAAAATATAAAAGG AACATTAAACTTCATACTATTCCCACTCACCTTTACACCCCTAGACCAAAC ATGGTCTAATAATATTCTATGATTAGCATGTATAATGGCATTTATGATTAA AATACCACTATACGGAGTCCACTTATGACTCCCTAAAGCCCACGTA---:--.-$-$

$\underline{\operatorname{ljd} 3330}$

CAGCTGACTTCCAATCAGTTAGATCTAGACCTAACCTAGAAGAAAGTAAT AAATATACTAACAGCCCTATTAGTAAATATTACGCTATCAATACTCCTAAT CATCATTGCCTTCTGACTACCCCAACTTAATTTATATACTGAAAAAGCAAA CCCGTATGAGTGCGGATTTGACCCCATAGGCTCTGCTCGCCTCCCATTCTC AATAAAATTTTTCCTAGTAGCAATCACTTTCTTACTATTTGACCTAGAAATT GCACTTCTACTTCCACTACCATGAGCTATCCAAATATATAATATTAACATC ATAATATTAACAGCTTTCATCCTAGTCTCCGTATTAGCATTAGGCTTAGCCT ACGAGTGACTACAAAAAGGACTAGAATGAACTGAG-

TAACTGGTAATTAGTTTAATTAAAACAAATGATTTCGACTCATTAGATTAT GATATACATTATAATTACCAAAA----

ATGTCATCTGTAACCTCCAATATTATACTAGCATTCTCATTCTCATTTCTAG GAACACTAGTATTTCGATCCCACCTAATATCAACTCTCCTATGCCTTGAAG 
GAATAATACTATCACTGTTTATTATAACCACAATTACATCCCTTAATTCCC ACTCAATAATTATATACCCCCTACCCATTGTTATTCTAGTATTCGCAGCATG TGAAGCAGCCATTGGCTTAGCCCTATTAGCAAAACTATCAAACTCCTACGG AACAGATTATGTGCAAAATCTAAACCTACTTCAATGT-

TAAAAATTATTTTTCCCTCTATCATATTACTCCCACTCACCTGATTATCAAA CAAAAAAAACCTATGAGTCAATGTTACCTCCTACAGCTTTATAATCAGCTT GACTTCAGCTATGTTTTTATGACAAAACGACATAAATAGCCTAAACTTCTC ACTACTATTCTCAACTGACTCCCTATCCTCCCCTCTTATTATTCTAACAACA TGACTTCTACCACTCATACTGCTAGCCAGCCAAAACCACATAAAAAAGGA AACAGAGTCAAACAAAAAAACATACATTTCAATACTAGTCTTACTACAAA TTCTTCTAATCATAACATTTTCTGCAAATGAACTAATTATATTTTACATCTT ATTTGAAGCCACCCTAATCCCCACCCTTATTATCATCACCCGATGGGGTAA CCAAACAGAACGACTAAATGCAGGACTCTACTTTCTATTTTATACCCTAAT TGGATCAATCCCACTACTAATCGCCCTTATCCACATTCAAAATATAAAAGG AACATTAAACTTCATACTATTTCCACTCACCTTTACACCCCTAGACCAAAC ATGGTCTAATAATATTCTATGATTAGCATGTATAATGGCATTTATGATTAA AATACCACTATACGGAGTCCACTTATGACTCCCTAAAGCCCACGT-

lid3333

CAGCTGACTTCCAATCAGTTAGATCTAGACCTAACCTAGAAGAAAGTAAT AAATATACTAACAGCCCTATTAGTAAATATTACGCTATCAATACTCCTAAT CATCATTGCCTTCTGACTACCCCAACTTAATTTATATACTGAAAAAGCAAA CCCGTATGAGTGCGGATTTGACCCCATAGGCTCTGCTCGCCTCCCATTCTC AATAAAATTTTTCCTAGTAGCAATCACTTTCTTACTATTTGACCTAGAAATT GCACTTCTACTTCCACTACCATGAGCTATCCAAATATATAATATTAACATC ATAATATTAACAGCTTTCATCCTAGTCTCCGTATTAGCATTAGGCTTAGCCT ACGAGTGACTACAAAAAGGACTAGAATGAACTGAA-

TAACTGGTAATTAGTTTAATTAAAACAAATGATTTCGACTCATTAGATTAT GATATACATTATAATTACCAAAA----

ATGTCATCTGTAACCTCCAATATTATATTAGCATTCTCATTCTCATTTCTAG GAACACTAGTATTTCGATCCCACCTAATATCAACTCTCCTATGCCTTGAAG GAATAATACTATCACTGTTTATTATAACCACAATTACATCCCTTAATTCCC ACTCAATAATTATATACCCTCTACCCATTGTTATTCTAGTATTCGCAGCATG TGAAGCAGCCATTGGCTTAGCCCTATTAGCAAAACTATCAAACTCCTACGG AACAGATTATGTGCAAAATCTAAACCTACTTCAATGT-

TAAAAATTATTTTTCCCTCTATCATATTACTCCCACTCACCTGATTATCAAA CAAAAAAAACCTATGAGTCAATGTTACCTCCTACAGCTTTATAATCAGCTT GACTTCAGCTATGTTTTTATGACAAAACGACATAAATAGCCTAAACTTCTC ACTACTATTCTCAACTGACTCCCTATCCTCCCCTCTTATTATTCTAACAACA TGACTTCTACCACTCATACTGCTAGCCAGCCAAAACCACATAAAAAAGGA AACAGAGTCAAACAAAAAAACATACATTTCAATACTAGTCTTACTACAAA TTCTTCTAATCATAACATTTTCTGCAAATGAACTAATTATATTTTACATCTT ATTTGAAGCCACCCTAATCCCCACCCTTATTATCATCACCCGATGGGGTAA CCAAACAGAACGACTAAATGCAGGACTCTACTTTCTATTTTATACCCTAAT TGGATCAATCCCACTACTAATCGCCCTTATCCACATTCAAAATATAAAAGG 266 
AACATTAAACTTCATACTATTTCCACTCACCTTTACACCCCTAGACCAAAC ATGGTCTAATAATATTCTATGATTAGCATGTATAATGGCATTTATGATTAA AATACCACTATACGGAGTCCACTTATGACTCCCTAAAGCCCAC-

ljd3344

CGAACTAGTACAGCTGACTTCCAATCAGTTAGATCTAGACCTAACCTAGAA GAAAGTAATAAATATACTAACAGCCCTATTAGTAAATATTACGCTATCAAT ACTCCTAATCATCATTGCCTTCTGACTACCCCAACTTAATTTATATACTGAA AAAGCAAACCCGTATGAGTGCGGATTTGACCCCATAGGCTCTGCTCGCCTC CCATTCTCAATAAAATTTTTCCTAGTAGCAATCACTTTCTTACTATTTGACC TAGAAATTGCACTTCTACTTCCACTACCATGAGCTATCCAAATATATAATA TTAACATCATAATATTAACAGCTTTCATCCTAGTCTCCGTATTAGCATTAG GCTTAGCCTACGAGTGACTACAAAAAGGACTAGAATGAACTGAA-

TAACTGGTAATTAGTTTAATTAAAACAAATGATTTCGACTCATTAGATTAT GATATACATTATAATTACCAAAA----

ATGTCATCTGTAACCTCCAATATTATATTAGCATTCTCATTCTCATTTCTAG GAACACTAGTATTTCGATCCCACCTAATATCAACTCTCCTATGCCTTGAAG GAATAATACTATCACTGTTTATTATAACCACAATTACATCCCTTAATTCCC ACTCAATAATTATATACCCTCTACCCATTGTTATTCTAGTATTCGCAGCATG TGAAGCAGCCATTGGCTTAGCCCTATTAGCAAAACTATCAAACTCCTACGG AACAGATTATGTGCAAAATCTAAACCTACTTCAATGT-

TAAAAATTATTTTTCCCTCTATCATATTACTCCCACTCACCTGATTATCAAA CAAAAAAAACCTATGAGTCAATGTTACCTCCTACAGCTTTATAATCAGCTT GACTTCAGCTATGTTTTTATGACAAAACGACATAAATAGCCTAAACTTCTC ACTACTATTCTCAACTGACTCCCTATCCTCCCCTCTTATTATTCTAACAACA TGACTTCTACCACTCATACTGCTAGCCAGCCAAAACCACATAAAAAAGGA AACAGAGTCAAACAAAAAAACATACATTTCAATACTAGTCTTACTACAAA TTCTTCTAATCATAACATTTTCTGCAAATGAACTAATTATATTTTACATCTT ATTTGAAGCCACCCTAATCCCCACCCTTATTATCATCACCCGATGGGGTAA CCAAACAGAACGACTAAATGCAGGACTCTACTTTCTATTTTATACCCTAAT TGGATCAATCCCACTACTAATCGCCCTTATCCACATTCAAAATATAAAAGG AACATTAAACTTCATACTATTTCCACTCACCTTTACACCCCTAGACCAAAC ATGGTCTAATAATATTCTATGATTAGCATGTATAATGGCATTTATGATTAA AATACCACTATACGGAGTCCACTTATGACTCCCTAAAGCCCACGT-

$\underline{\operatorname{lid} 3348}$

CGAACTAGTACAGCTGACTTCCAATCAGTTAGATCTAGACCTAACCTAGAA GAAAGTAATAAATATACTAACAGCCCTATTAGTAAATATTACGCTATCAAT ACTCCTAATCATCATTGCCTTCTGACTACCCCAACTTAATTTATATACTGAA AAAGCAAACCCGTATGAGTGCGGATTTGACCCCATAAGCTCTGCTCGCCTC CCATTCTCAATAAAATTTTTCCTAGTAGCAATCACTTTCTTACTATTTGACC TAGAAATTGCACTTCTACTTCCACTACCATGAGCTATCCAAATATATAATA TTAACATCATAATATTAACAGCTTTCATCCTAGTCTCCGTATTAGCATTAG GCTTAGCCTACGAGTGACTACAAAAAGGACTAGAATGAACTGAG-

TAACTGGTAATTAGTTTAATTAAAACAAATGATTTCGACTCATTAGATTAT 
GATATACATTATAATTACCAAAA----

ATGTCATCTGTAACCTCCAATATTATATTAGCATTCTCATTCTCATTTCTAG GAACACTAGTATTCCGATCCCACCTAATATCAACTCTCCTATGCCTTGAAG GAATAATACTGTCACTGTTTATTATAACCACAATTACATCCCTTAATTCCC ACTCAATAATTATATACCCTCTACCCATTGTTATTCTAGTATTCGCAGCATG TGAAGCAGCCATTGGCTTAGCCCTATTAGCAAAACTATCAAACTCCTACGG AACAGATTATGTACAAAATCTAAACCTACTTCAATGT-

TAAAAATTATTTTTCCCTCTATCATATTACTCCCACTCACCTGATTATCAAA CAAAAAAAACCTATGAATTAATGTTACCTCCTACAGCTTTATAATCAGCTT GACTTCAGCTATGTTTTTATGACAAAACGACATAAATAATCTAAACTTCTC ACTACTATTCTCAACCGACTCCCTATCCTCCCCTCTTATTATTCTAACAACA TGACTTCTGCCACTCATACTGCTAGCCAGCCAAAACCACATAAAAAAGGA AACAGAGTTAAACAAAAAAACATACATTTCAATACTAGTCTTACTACAAA TTCTTCTAATCATAACATTTTCTGCAAACGAACTAATTATATTTTACATCTT ATTTGAAGCCACCCTAATCCCCACCCTTATTATCATCACCCGATGGGGTAA CCAAACAGAACGACTAAATGCAGGACTCTACTTTCTATTTTATACCCTAAT TGGATCAATCCCACTACTAATCGCCCTTATCCACATTCAAAATATAAAAGG AACATTAAACTTCATACTATTCCCACTCACCTTTACACCCCTAGACCAAAC ATGGTCTAATAATATTCTATGATTAGCATGTATAATGGCATTTATGATTAA AATACCACTATACGGAGTCCACTTATGACTCCCTAAAGCCCA-

\section{$\underline{\mathrm{LJD} 620}$}

CGAACTAGTACAGCTGACTTCCAATCAGTTAGATCTAGACCTAACCTAGAA GAAAGTAATAAATATACTAACAGCCCTATTAGTAAATATTACGCTATCAAC ACTCCTAATCATCATTGCCTTCTGACTACCCCAACTTAATTTATATACTGAA AAAGCAAACCCGTATGAGTGCGGATTTGACCCCATAGGCTCTGCTCGCCTC CCATTCTCAATAAAATTTTTCCTAGTAGCAATCACTTTCTTACTATTTGACC TAGAAATTGCACTTCTACTTCCACTACCATGAGCTATCCAAATATATAATA TTAACATCATAATATTAACAGCTTTCATCCTAGTCTCCGTATTAGCATTAG GCTTAGCCTACGAGTGACTACAAAAAGGACTAGAATGAACTGAA-

TAACTGGTAATTAGTTTAATTAAAACAAATGATTTCGACTCATTAGATTAT GATATACATTATAATTACCAAAA----

ATGTCATCTGTAACCTCCAATATTATATTAGCATTCTCATTCTCATTTCTAG GAACACTAGTATTTCGATCCCACCTAATATCAACTCTCCTATGCCTTGAAG GAATAATACTATCACTGTTTATTATAACCACAATTACATCCCTTAATTCCC ACTCAATAATTATATACCCTCTGCCCATTGTTATTCTAGTATTCGCAGCATG TGAAGCAGCCATTGGCTTAGCCCTATTAGCAAAACTATCAAACTCCTACGG AACAGATTATGTGCAAAATCTAAACCTACTTCAATGT-

TAAAAATTATTTTTCCCTCTATCATATTACTCCCACTCACCTGATTATCAAA CAAAAAAAACCTATGAGTCAATGTTACCTCCTACAGCTTTATAATCAGCTT GATTTCAGCTATGTTTTTATGACAAAACGACATAAATAATCTAAACTTCTC ACTACTATTCTCAACTGACTCCCTATCCTCCCCTCTTATTATTCTAACAACA TGACTTCTACCACTCATACTGCTAGCCAGCCAAAACCACATAAAAAAGGA AACAGAGTCAAACAAAAAAACATACATTTCAATACTAGTCTTACTACAAA TTCTTCTAATCATAACATTTTCTGCAAACGAACTAATTATATTTTACATCTT 
ATTTGAAGCCACCCTAATCCCCACCCTTATTATCATCACCCGATGGGGTAA CCAAACAGAACGACTAAATGCAGGACTCTACTTTCTATTTTACACCCTAAT TGGATCAATCCCACTACTAATCGCCCTTATCCACATTCAAAATATAAAAGG AACATTAAACTTCATACTATTCCCACTCACCTTTACACCCCTAGACCAAAC ATGGTCTAATAATATTCTATGACTAGCATGTATAATGGCATTTATGATTAA AATACCACTATACGGAGTCCACTTATGACTCCCTAAAGCCCACGTAGAAG CTCCA--

$\underline{\text { LJD774 }}$ CGAACTAGTACAGCTGACTTCCAATCAGTTAGATCTAGACCTAACCTAGAA GAAAGTAATAAATATACTAACAGCCCTATTAGTAAATATTACGCTATCAAT ACTCCTAATCATCATTGCCTTCTGACTACCCCAACTTAATTTATATACTGAA AAAGCAAACCCGTATGAGTGCGGATTTGACCCCATAGGCTCTGCTCGCCTC CCATTCTCAATAAAATTTTTCCTAGTAGCAATCACTTTCTTACTATTTGACC TAGAAATTGCACTTCTACTTCCACTACCATGAGCTATCCAAATATATAATA TTAACATCATAATATTAACAGCTTTCATCCTAGTCTCCGTATTAGCATTAG GCTTAGCCTACGAGTGACTACAAAAAGGACTAGAATGAACTGAG-

TAATTGGTAATTAGTTTAATTAAAACAAATGATTTCGACTCATTAGATTAT GATATACATTATAATTACCAAAA----

ATGTCATCTGTAACCTCCAATATTATATTAGCATTCTCATTCTCATTTCTAG GAACACTAGTATTTCGATCCCACCTAATATCAACTCTCCTATGCCTTGAAG GAATAATACTATCACTGTTTATTATAACCACAATTACATCCCTTAATTCCC ACTCAATAATTATATACCCTCTACCCATTGTTATTCTAGTATTCGCAGCATG TGAAGCAGCCATTGGCTTAGCCCTATTAGCAAAACTATCAAACTCCTACGG AACAGATTATGTGCAAAATCTAAACCTACTTCAATGT-

TAAAAATTATTTTTCCCTCTATCATATTACTCCCACTCACCTGATTATCAAA CAAAAAAAACCTATGAGTCAATGTTACCTCCTACAGCTTTATAATCAGCTT GATTTCAGCTATGTTTTTATGACAAAACGACATAAATAATCTAAACTTCTC ACTACTATTCTCAACTGACTCCCTATCCTCCCCTCTTATTATTCTAACAACA TGACTTCTACCACTCATACTGCTAGCCAGCCAAAACCACATAAAAAAGGA AACAGAGTCAAACAAAAAAACATATATTTCAATACTAGTCTTACTACAAA TTCTTCTAATCATAACATTTTCTGCAAACGAACTAATTATATTTTACATCTT ATTTGAAGCCACCCTAATCCCCACCCTTATTATCATCACCCGATGGGGTAA CCAAACAGAACGACTAAATGCAGGACTCTACTTTCTATTTTATACCCTAAT TGGATCAATCCCACTACTAATCGCCCTTATCCACATTCAAAATATAAAAGG AACATTAAACTTCATACTATTCCCACTCACCTTTACACCCCTAGACCAAAC ATGGTCTAATAATATTCTATGATTAGCATGTATAATGGCATTTATGATTAA AATACCACTATACGGAGTCCACTTATGACTCCCTAAAGCCCACGTAGAAG CTCCA--

$\underline{\text { LJD886 }}$

CGAACTAGTACAGCTGACTTCCAATCAGTTAGATCTAGACCTAACCTAGAA GAAAGTAATAAATATACTAACAGCCCTATTAGTAAATATTACGCTATCAAT ACTCCTAATCATCATTGCCTTCTGACTACCCCAACTTAATTTATATACTGAA AAAGCAAACCCATATGAGTGCGGATTTGACCCCATAGGCTCTGCTCGCCTC 
CCATTCTCAATAAAATTTTTCCTAGTAGCAATCACTTTCTTACTATTTGACC TAGAAATTGCACTTCTACTTCCACTACCATGAGCTATCCAAATATATAATA TTAACATCATAATATTAACAGCTTTCATCCTAGTCTCCGTATTAGCATTAG GCTTAGCCTACGAGTGACTACAAAAAGGACTAGAATGAACTGAA-

TAACTGGTAATTAGTTTAATTAAAACAAATGATTTCGACTCATTAGATTAT GATATACATTATAATTACCAAAA----

ATGTCATCTGTAACCTCCAATATTATATTAGCATTCTCATTCTCATTTCTAG GAACACTAGTATTTCGATCCCACCTAATATCAACTCTCCTATGCCTTGAAG GAATAATACTATCACTGTTTATTATAACCACAATTACATCCCTTAATTCCC ACTCAATAATTATATACCCTCTACCCATTGTTATTCTAGTATTCGCAGCATG TGAAGCAGCCATTGGCTTAGCCCTATTAGCAAAACTATCAAACTCCTACGG AACAGATTATGTGCAAAATCTAAACCTACTTCAATGT-

TAAAAATTATTTTTCCCTCTATCATATTACTCCCACTCACCTGATTATCAAA CAAAAAAAACCTATGAGTCAATGTTACCTCCTACAGCTTTATAATCAGCTT GACTTCAGCTATGTTTTTATGACAAAACGACATAAATAACCTAAACTTCTC ACTACTATTCTCAACTGACTCCCTATCCTCCCCTCTTATTATTCTAACAACA TGACTTCTACCACTCATACTGCTAGCCAGCCAAAACCACATAAAAAAGGA AACAGAGTCAAACAAAAAAACATATATTTCAATACTAGTCTTACTACAAA TTCTTCTAATCATAACATTTTCTGCAAACGAACTAATTATATTTTACATCTT ATTTGAAGCCACCCTAATCCCCACCCTTATTATCATCACCCGATGGGGTAA CCAAACAGAACGACTAAATGCAGGACTCTACTTTCTATTTTATACCCTAAT TGGATCAATCCCACTACTAATCGCCCTTATCCACATTCAAAATATAAAAGG AACATTAAACTTCATACTATTCCCACTCACCTTTACACCCCTAGACCAAAC ATGGTCTAATAATATTCTATGATTAGCATGTATAATGGCATTTATGATTAA AATACCACTATACGGAGTCCACTTATGACTCCCTAAAGCCCACGTAGAAG CTCCA--

$\underline{\text { LJD2088 }}$

GAACTAGTACAGCTGACTTCCAATCAGTTAGATCTAGACCTAACCTAGAA GAAAGTAATAAATATACTAACAGCCCTATTAGTAAATATTACGCTATCAAT ACTCCTAATCACCATTGCCTTCTGACTACCCCAACTTAATTTATATACTGAA AAAGCAAACCCGTATGAGTGCGGATTTGACCCCATAGGCTCTGCTCGCCTC CCATTCTCAATAAAATTTTTCCTAGTAGCAATCACTTTCTTACTATTTGACC TAGAAATTGCACTTCTACTTCCACTACCATGAGCTATCCAAATATATAATA TTAACATCATAATATTAACAGCTTTCATCCTAGTCTCCGTATTAGCATTAG GCTTAGCCTACGAGTGACTACAAAAAGGACTAGAATGAACTGAG-

TAACTGGTAATTAGTTTAATTAAAACAAATGATTTCGACTCATTAGATTAT GATATACATTATAATTACCAAAA----

ATGTCATCTGTAACCTCCAATATTATATTAGCATTCTCATTCTCATTTCTAG GAACACTAGTATTTCGATCCCACCTAATATCAACTCTCCTATGCCTTGAAG GAATAATACTATCACTGTTTATTATAACCACAATTACATCCCTTAATTCCC ACTCAATAATTATATACCCTCTACCCATTGTTATTTTAGTATTCGCAGCATG TGAAGCAGCCATTGGCTTAGCCCTATTAGCAAAACTATCAAACTCCTACGG AACAGACTATGTACAAAACCTAAACCTACTTCAATGT-

TAAAAATTATTTTTCCCTCTATCATATTACTCCCACTCACCTGATTATCAAA 
CAAAAAAAACCTATGAGTCAATGTTACCTCCTACAGCTTTATAATCAGCTT GACTTCAGCTATGTTTTTATGACAAAACGACATAAATAATCTAAACTTCTC ACTACTATTCTCAACTGACTCCCTATCCTCCCCTCTTATTATTCTAACAACA TGACTTCTACCACTCATACTGCTAGCCAGCCAAAACCACATAAAAAAGGA AACAGAGTCAAACAAAAAAACATACATTTCAATACTAATCTTACTACAAA TTCTTCTAATCATAACATTTTCTGCAAACGAACTAATTATATTTTACATCTT ATTTGAAGCCACCCTAATCCCCACCCTTATTATCATCACCCGATGGGGTAA CCAAACAGAACGACTAAATGCAGGACTCTACTTTCTATTTTATACCCTAAT TGGATCAATCCCACTACTAATCGCCCTTATCCATATTCAAAATATAAAAGG AACATTAAACTTCATACTATTCCCACTCACCTTTACACCCCTAGACCAAAC ATGGTCTAATAATATTCTATGATTAGCATGTATAATGGCATTTATGATTAA AATACCACTATACGGAGTCCACTTATGACTCCCTAAAGCCCACGTA-.-.---. $-$

\section{$\underline{\text { LJD1960 }}$}

TACAGCTGACTTCCAATCAGTTAGATCTAGACCTAACCTAGAAGAAAGTA ATAAATATACTAACAGCCCTATTAGTAAATATCACGCTATCAATACTCCTA ATCATCATTGCCTTCTGACTACCCCAACTTAATTTATATACTGAAAAAGCA AACCCGTATGAGTGCGGATTTGACCCCATAGGCTCTGCTCGCCTCCCATTC TCAATAAAATTTTTCCTAGTAGCAATCACTTTCTTACTATTTGACCTAGAAA TTGCACTTCTACTTCCACTACCATGAGCTATCCAAATATATAATATTAACA TCATAATATTAACAGCTTTCATCCTAGTCTCCGTATTAGCATTAGGCTTAGC CTACGAGTGACTACAAAAAGGACTAGAATGAACTGAA-

TAACTGGTAATTAGTTTAATTAAAACAAATGATTTCGACTCATTAGATTAT GATATACATTATAATTACCAAAA----

ATGTCATCTGTAACCTCCAATATTATATTAGCATTCTCATTCTCATTTCTAG GAACACTAGTATTTCGATCCCACCTAATATCAACTCTCCTATGCCTTGAAG GAATAATACTATCACTGTTTATTATAACCACAATTACATCCCTTAATTCCC ACTCAATAATTATATACCCTCTACCCATTGTTATTCTAGTATTCGCAGCATG TGAAGCAGCCATTGGCTTAGCCCTATTAGCAAAACTATCAAACTCCTACGG AACAGATTATGTGCAAAATCTAAACCTACTTCAATGT-

TAAAAATTATTTTTCCCTCTATCATATTACTCCCACTCACCTGATTATCAAA CAAAAAAAACCTATGAGTCAATGTTACCTCCTACAGCTTTATAATCAGCTT GACTTCAGCTATGTTTTTATGACAAAACGACATAAATAATCTAAACTTCTC ACTACTATTCTCAACTGACTCCCTATCCTCCCCTCTTATTATTCTAACAACA TGACTTCTACCACTCATACTGCTAGCCAGCCAAAACCACATAAAAAAGGA AACAGAGTCAAACAAAAAAACATATATTTCAATACTAGTCTTACTACAAA TTCTTCTAATCATAACATTTTCTGCAAACGAACTAATTATATTTTACATCTT ATTTGAAGCCACCCTAATCCCCACCCTTATTATCATCACCCGATGGGGTAA CCAAACAGAACGACTAAATGCAGGACTCTACTTTCTATTTTATACCCTAAT TGGATCAATCCCACTACTAATCGCCCTTATCCACATTCAAAATATAAAAGG AACATTAAACTTCATACTATTCCCACTCACCTTTACACCCCTAGACCAAAC ATGGTCTAATAATATTCTATGATTAGCATGTATAATGGCATTTATAATTAA AATACCACTATACGGAGTCCACTTATGACTCCCTAAAGCCCACGTAGAAA GCTCCA- 
$\underline{\text { LJD1669 }}$

TAGTACAGCTGACTTCCAATCAGTTAGATCTAGACCTAACCTAGAAGAAA GTAATAAATATACTAACAGCCCTATTAGTAAATATCACGCTATCAATACTC CTAATCATCATTGCCTTCTGACTACCCCAACTTAATTTATATACTGAAAAA GCAAACCCGTATGAGTGCGGATTTGACCCCATAGGCTCTGCTCGCCTCCCA TTCTCAATAAAATTTTTCCTAGTAGCAATCACTTTCTTACTATTTGACCTAG AAATTGCACTTCTACTTCCACTACCATGAGCTATCCAAATATATAATATTA ACATCATAATATTAACAGCTTTCATCCTAGTCTCCGTATTAGCATTAGGCTT AGCCTACGAGTGACTACAAAAAGGACTAGAATGAACTGAG-

TAACTGGTAATTAGTTTAATTAAAACAAATGATTTCGACTCATTAGATTAT GATATACATTATAATTACCAAAA----

ATGTCATCTGTAACCTCCAATATTATATTAGCATTCTCATTCTCATTTCTAG GAACACTAGTATTTCGATCCCACCTAATATCAACTCTCCTATGCCTTGAAG GAATAATACTGTCACTGTTTATTATAACCACAATTACATCCCTTAATTCCC ACTCAATAATTATATACCCTCTACCCATTGTTATTCTAGTATTCGCAGCATG TGAAGCAGCCATTGGCTTAGCCCTATTAGCAAAACTATCAAACTCCTACGG AACAGATTATGTGCAAAATCTAAACCTACTTCAATGT-

TAAAAATTATTTTTCCCTCTATCATATTACTCCCACTCACCTGATTATCAAA CAAAAAAAACCTATGAGTCAATGTTACCTCCTACAGCTTTATAATCAGCTT GACTTCAGCTATGTTTTTATGACAAAACGACATAAATAATCTAAACTTCTC ACTACTATTCTCAACTGACTCCCTATCCTCCCCTCTTATTATTCTAACAACA TGACTTCTACCACTCATACTGCTAGCCAGCCAAAACCACATAAAAAAGGA AACAGAGTCAAACAAAAAAACATATATTTCAATACTAGTCTTACTACAAA TTCTTCTAATCATAACATTTTCTGCAAACGAACTAATTATATTTTACATCTT ATTTGAAGCCACCCTAATCCCCACCCTTATTATCATCACCCGATGGGGTAA CCAAACAGAACGACTAAATGCAGGACTCTACTTTCTATTTTATACCCTAAT TGGATCAATCCCACTACTAÄTCGCCCTTATCCACATTCAAAATATAAAAGG AACATTAAACTTCATACTATTCCCACTCACCTTTACACCCCTAGACCAAAC ATGGTCTAATAATATTCTATGATTAGCATGTATAATGGCATTTATAATTAA AATACCACTATACGGAGTCCACTTATGACTCCCTAAAGCCCACGTAGAAA GCTCCA-

LJD1524

AGTACAGCTGACTTCCAATCAGTTAGATCTAGACCTAACCTAGAAGAAAG TAATAAATATACTAACAGCCCTATTAGTAAATATTACGCTATCAATACTCC TAATCATCATTGCCTTCTGACTACCCCAACTTAATTTATATACTGAAAAAG CAAACCCGTATGAGTGCGGATTTGACCCCATAGGCTCTGCTCGCCTCCCAT TCTCAATAAAATTTTTCCTAGTAGCAATCACTTTCTTACTATTTGACCTAGA AATTGCACTTCTACTTCCACTACCATGAGCTATCCAAATATATAATATTAA CATCATAATATTAACAGCTTTCATCCTAGTCTCCGTATTAGCATTAGGCTTA GCCTACGAGTGACTACAAAAAGGACTAGAATGAACTGAA-

TAACTGGTAATTAGTTTAATTAAAACAAATGATTTCGACTCATTAGATTAT GATATACATTATAATTACCAAAA----

ATGTCATCTGTAACCTCCAATATTATATTAGCATTCTCATTCTCATTTCTAG GAACACTAGTATTTCGATCCCACCTAATATCAACTCTCCTATGCCTTGAAG 
GAATAATACTATCACTGTTTATTATAACCACAATTACATCCCTTAATTCCC ACTCAATAATTATATACCCTCTACCCATTGTTATTCTAGTATTCGCAGCATG TGAAGCAGCCATTGGCTTAGCCCTATTAGCAAAACTATCAAACTCCTACGG AACAGATTATGTGCAAAATCTAAACCTACTTCAATGT-

TAAAAATTATTTTTCCCTCTATCATATTACTCCCACTCACCTGATTATCAAA CAAAAAAAACCTATGAGTCAATGTTACCTCCTACAGCTTTATAATCAGCTT GACTTCAGCTATGTTTTTATGACAAAACGACATAAATAACCTAAACTTCTC ACTACTATTCTCAACTGACTCCCTATCCTCCCCTCTTATTATTCTAACAACA TGACTTCTACCACTCATACTGCTAGCCAGCCAAAACCACATAAAAAAGGA AACAGAGTCAAACAAAAAAACATACATTTCAATACTAGTCTTACTACAAA TTCTTCTAATCATAACATTTTCTGCAAATGAACTAATTATATTTTACATCTT ATTTGAAGCCACCCTAATCCCCACCCTTATTATCATCACCCGATGGGGTAA CCAAACAGAACGACTAAATGCAGGACTCTACTTTCTATTTTATACCCTAAT TGGATCAATCCCACTACTAATCGCCCTTATCCACATTCAAAATATAAAAGG AACATTAAACTTCATACTATTCCCACTCACCTTTACACCCCTAGACCAAAC ATGGTCTAATAATATTCTATGATTAGCATGTATAATGGCATTTATGATTAA AATACCACTATACGGAGTCCACTTATGACTCCCTAAAGCCCACGTA----:---$-$

LJD1523 GAAAGTAATAAATATACTAACAGCCCTATTAGTAAATATTACGCTATCAAT ACTCCTAATCATCATTGCCTTCTGACTACCCCAACTTAATTTATACACTGAA AAAGCAAACCCGTATGAGTGCGGATTTGACCCCATAGGCTCTGCTCGCCTC CCATTCTCAATAAAATTTTTCCTAGTAGCAATCACTTTCTTACTATTTGACC TAGAAATTGCACTTCTACTTCCACTACCATGAGCTATCCAAATATATAATA TTAACATCATAATATTAACAGCTTTCATCCTAGTCTCCGTATTAGCATTAG GCTTAGCCTACGAGTGACTACAAAAAGGACTAGAATGAACTGAA-

TAACTGGTAATTAGTTTAATTAAAACAAATGATTTCGACTCATTAGATTAT GATATACATTATAATTACCAAAA----

ATGTCATCTGTAACCTCCAATATTATATTAGCATTCTCATTCTCATTTCTAG GAACACTAGTATTTCGATCCCACCTAATATCAACTCTCCTATGCCTTGAAG GAATAATACTATCACTGTTTATTATAACCACAATTACATCCCTTAATTCCC ACTCAATAATTATATACCCTCTACCCATTGTTATTCTAGTATTCGCAGCATG TGAAGCAGCCATTGGCTTAGCCCTATTAGCAAAACTATCAAACTCCTACGG AACAGATTATGTGCAAAATCTAAACCTACTTCAATGT-

TAAAAATTATTTTTCCCTCTATCATATTACTCCCACTCACCTGATTATCAAA CAAAAAAAACCTATGAGTCAATGTTACCTCCTACAGCTTTATAATCAGCTT GACTTCAGCTATGTTTTTATGACAAAACGACATAAATAACCTAAACTTCTC ACTACTATTCTCAACTGACTCCCTATCCTCCCCTCTTATCATTCTAACAACA TGACTTCTACCACTCATACTGCTAGCCAGCCAAAACCACATAAAAAAGGA AACAGAGTCAAACAAAAAAACATACATTTCAATACTAGTCTTACTACAAA TTCTTCTAATCATAACATTTTCTGCAAACGAACTAATTATATTTTACATCTT ATTTGAAGCCACCCTAATCCCCACCCTTATTATCATCACCCGATGGGGTAA CCAAACAGAACGACTAAATGCAGGACTCTACTTTCTATTTTATACCCTAAT 
TGGATCAATCCCACTACTAATCGCCCTTATCCACATTCAAAATATAAAAGG AACATTAAACTTCATACTATTCCCACTCACCTTTACACCCCTAGACCAAAC ATGGTCTAATAATATTCTATGATTAGCATGTATAATGGCATTTATGATTAA AATACCACTATACGGAGTCCACTTATGACTCCCTAAAGCCCAC

$\underline{\mathrm{LJD} 1122}$

TAGTACAGCTGACTTCCAATCAGTTAGATCTAGACCTAACCTAGAAGAAA GTAATAAATATACTAACAGCCCTATTAGTAAATATTACGCTATCAATACTC CTAATCATCATTGCCTTCTGACTACCCCAACTTAATTTATATACTGAAAAA GCAAACCCGTATGAGTGCGGATTTGACCCCATAGGCTCTGCTCGCCTCCCA TTCTCAATAAAATTTTTCCTAGTAGCAATCACTTTCTTACTATTTGACCTAG AAATTGCACTTCTACTTCCACTACCATGAGCTATCCAAATATATAATATTA ACATCATAATATTAACAGCTTTTATCCTAGTCTCCGTATTAGCATTAGGCTT AGCCTACGAGTGACTACAAAAAGGACTAGAATGAACTGAA-

TAACTGGTAATTAGTTTAATTAAAACAAATGATTTCGACTCATTAGATTAT GATATACATTATAATTACCAAAA----

ATGTCATCTGTAACCTCCAATATTATATTAGCATTCTCATTCTCATTTCTAG GAACACTAGTATTTCGATCCCACCTAATATCAACTCTCCTATGCCTTGAAG GAATAATACTATCACTGTTTATTATAACCACAATTACATCCCTTAATTCCC ACTCAATAATTATATACCCTCTACCCATTGTTATTCTAGTATTCGCAGCATG TGAAGCAGCCATTGGCTTAGCCCTATTAGCAAAACTATCAAACTCCTACGG AACAGATTATGTGCAAAATCTAAACCTACTTCAATGT-

TAAAAATTATTTTTCCCTCTATCATATTACTCCCACTCACCTGATTATCAAA CAAAAAAAACCTATGAGTCAATGTTACCTCCTACAGCTTTATAATCAGCTT GACTTCAGCTATGTTTTTATGACAAAACGACATAAATAGCCTAAACTTCTC ACTACTATTCTCAACTGACTCCCTATCCTCCCCTCTTATTATTCTAACAACA TGACTTCTACCACTCATACTGCTAGCCAGCCAAAACCACATAAAAAAGGA AACAGAGTCAAACAAAAAAACATACATTTCAATACTAGTCTTACTACAAA TTCTTCTAATCATAACATTTTCTGCAAACGAACTAATTATATTTTACATCTT ATTTGAAGCCACCCTAATCCCCACCCTTATTATCATCACCCGATGGGGTAA CCAAACAGAACGACTAAATGCAGGACTCTACTTTCTATTTTATACCCTAAT TGGATCAATCCCACTACTAATCGCCCTTATCCACATTCAAAATATAAAAGG AACATTAAACTTCATACTATTCCCACTCACCTTTACACCCCTAGACCAAAC ATGGTCTAATAATATTCTATGATTAGCATGTATAATGGCATTTATGATTAA AATACCACTATACGGAGTCCACTTATGACTCCCTAAAGCCCACGA

LJD2522

CGAACTAGTACAGCTGACTTCCAATCAGTTAGATCTAGACCTAACCTAGAA GAAAGTAATAAATATACTAACAGCCCTATTAGTAAATATTACGCTATCAAT ACTCCTAATCATCATTGCCTTCTGACTACCCCAACTTAATTTATATACTGAA AAAGCAAACCCATATGAGTGCGGATTTGACCCCATAGGCTCTGCTCGCCTC CCATTCTCAATAAAATTTTTCCTAGTAGCAATCACTTTCTTACTATTTGACC TAGAAATTGCACTTCTACTTCCACTACCATGAGCTATCCAAATATATAATA TTAACATCATAATATTAACAGCTTTCATCCTAGTCTCCGTATTAGCATTAG GCTTAGCCTACGAGTGACTACAAAAAGGACTAGAATGAACTGAA- 
TAACTGGTAATTAGTTTAATTAAAACAAATGATTTCGACTCATTAGATTAT GATATACATTATAATTACCAAAA----

ATGTCATCTGTAACCTCCAATATTATATTAGCATTCTCATTCTCATTTCTAG GAACACTAGTATTTCGATCCCACCTAATATCAACTCTCCTATGCCTTGAAG GAATAATACTATCACTGTTTATTATAACCACAATTACATCCCTTAATTCCC ACTCAATAATTATATACCCTCTACCCATTGTTATTCTAGTATTCGCAGCATG TGAAGCAGCCATTGGCTTAGCCCTATTAGCAAAACTATCAAACTCCTACGG AACAGATTATGTGCAAAACCTAAACCTACTTCAATGT-

TAAAAATTATTTTTCCCTCTATCATATTACTCCCACTCACCTGATTATCAAA CAAAAAAAACCTATGAGTCAATGTTACCTCCTACAGCTTTATAATCAGCTT GACTTCAGCTATGTTTTTATGACAAAACGACATAAATAACCTAAACTTCTC ACTACTATTCTCAACTGACTCCCTATCCTCCCCTCTTATTATTCTAACAACA TGACTTCTACCACTCATACTGCTAGCCAGCCAAAACCACATAAAAAAGGA AACAGAGTCAAACAAAAAAACATATATTTCAATACTAGTCTTACTACAAA TTCTTCTAATCATAACATTTTCTGCAAACGAACTAATTATATTTTACATCTT ATTTGAAGCCACCCTAATCCCCACCCTTATTATCATCACCCGATGGGGTAA CCAAACAGAACGACTAAATGCAGGACTCTACTTTCTATTTTATACCCTAAT TGGATCAATCCCACTACTAATCGCCCTTATCCACATTCAAAATATAAAAGG AACATTAAACTTCATACTATTCCCACTCACCTTTACACCCCTAGACCAAAC ATGGTCTAATAATATTCTATGATTAGCATGTATAATGGCATTTATGATTAA AATACCACTATACGGAGTCCACTTATGACTTCCTAAAGCCCACGTAGAA----

\section{LJD1637}

ACAGCTGACTTCCAATCAGTTAGATCTAGACCTAACCTAGAAGAAAGTAA TAAATATACTAACAGCCCTATTAGTAAATATTACGCTATCAATACTCCTAA TCATCATTGCCTTCTGACTACCCCAACTTAATTTATATACTGAAAAAGCAA ACCCGTATGAGTGCGGATTTGACCCCATAGGCTCTGCTCGCCTCCCATTCT CAATAAAATTTTTCCTAGTAGCAATCACTTTCTTACTATTTGACCTAGAAAT TGCACTTCTACTTCCACTACCATGAGCTATCCAAATATATAATATTAACAT CATAATATTAACAGCTTTCATCCTAGTCTCCGTATTAGCATTAGGCTTAGC CTACGAGTGACTACAAAAAGGACTAGAATGAACTGAG-

TAACTGGTAATTAGTTTAATTAAAACAAATGATTTCGACTCATTAGATTAT GATATACATTATAATTACCAAAA----

ATGTCATCTGTAACCTCCAATATTATATTAGCATTCTCATTCTCATTTCTAG GAACACTAGTATTTCGATCCCACCTAATATCAACTCTCCTATGCCTTGAAG GAATAATACTATCACTGTTTATTATAACCACAATTACATCCCTTAATTCCC ACTCAATAATTATATACCCCCTACCCATTGTTATTCTAGTATTCGCAGCATG TGAAGCAGCCATTGGCTTAGCCCTATTAGCAAAACTATCAAACACCTACG GAACAGATTATGTGCAAAATCTAAACCTACTTCAATGT-

TAAAAATTATTTTTCCCTCTATCATATTACTCCCACTCACCTGATTATCAAA CAAAAAAAACCTATGAGTCAATGTTACCTCCTACAGCTTTATAATCAGCTT GACTTCAGCTATGTTTTTATGACAAAACGACATAAATAATCTAAACTTCTC ACTACTATTCTCAACTGACTCCCTATCCTCCCCTCTTATCATTCTAACAACA TGACTTCTACCACTCATACTGCTAGCCAGCCAAAACCACATAAAAAAGGA 
AACAGAGTCAAACAAAAAAACATACATTTCAATACTAGTCTTACTACAAA TTCTTCTAATCATAACATTTTCTGCAAACGAACTAATTATATTTTACATCTT ATTTGAAGCCACCCTAATCCCCACCCTTATTATCATCACCCGATGAGGTAA CCAAACAGAACGACTAAATGCAGGACTCTACTTTCTATTTTATACCCTAAT TGGATCAATCCCACTACTAATCGCCCTTATCCACATTCAAAATATAAAAGG AACATTAAACTTCATACTATTCCCACTCACCTTTACACCCCTAGACCAAAC ATGGTCTAATAATATTCTATGATTAGCATGTATAATGGCATTTATGATTAA AATACCACTATACGGAGTCCACTTATGACTCCCTAAAGCC-

\section{$\underline{\text { LJD1638 }}$}

CGAAACTAGTACAGCTGACTTCCAATCAGTTAGATCTAGACCTAACCTAGA AGAAAGTAATAAATATACTAACAGCCCTATTAGCAAATATTACGCTATCA ATACTCCTAATCATCATTGCCTTCTGACTACCCCAACTTAATTTATATACTG AAAAAGCAAACCCATATGAGTGCGGATTTGACCCCATAGGCTCTGCTCGC CTCCCATTCTCAATAAAATTTTTCCTAGTAGCAATCACTTTCTTACTATTTG ACCTAGAAATTGCACTTCTACTTCCACTACCATGAGCTATCCAAATATATA ATATTAACATCATAATATTAACAGCTTTCATCCTAGTCTCCGTATTAGCATT AGGCTTAGCCTACGAGTGACTACAAAAAGGACTAGAATGAACTGAA-

TAACTGGTAATTAGTTTAATTAAAACAAATGATTTCGACTCATTAGATTAT GATATACATTATAATTACCAAAA----

ATGTCATCTGTAACCTCCAATATTATATTAGCATTCTCATTCTCATTTCTAG GAACACTAGTATTTCGATCCCACCTAATATCAACTCTCCTATGCCTTGAAG GAATAATACTATCACTGTTTATTATAACCACAATTACATCCCTTAATTCCC ACTCAATAATTATATACCCTCTACCCATTGTTATTCTAGTATTCGCAGCATG TGAAGCAGCCATTGGCTTAGCCCTATTAGCAAAACTATCAAACTCCTACGG AACAGATTATGTGCAAAATCTAAACCTACTTCAATGT-

TAAAAATTATTTTTCCCTCTATCATATTACTCCCACTCACCTGATTATCAAA CAAAAAAAACCTATGAGTCAATGTTACCTCCTACAGCTTTATAATCAGCTT GACTTCAGCTATGTTTTTATGACAAAACGACATAAATAACCTAAACTTCTC ACTACTATTCTCAACTGACTCCCTATCCTCCCCTCTTATTATTCTAACAACA TGACTTCTACCACTCATACTGCTAGCCAGCCAAAACCACATAAAAAAGGA AACAGAGTCAAACAAAAAAACATACATTTCAATACTAGTCTTACTACAAA TTCTTCTAATCATAACATTTTCTGCAAACGAACTAATTATATTTTACATCTT ATTTGAAGCCACCCTAATCCCCACCCTTATTATCATCACCCGATGGGGTAA CCAAACAGAACGACTAAATGCAGGACTCTACTTTCTATTTTATACCCTAAT TGGATCAATCCCACTACTAATCGCCCTTATCCACATTCAAAATATGAAAGG AACATTAAACTTCATACTATTCCCACTCACCTTTACACCCCTAGACCAAAC ATGGTCTAATAATATTCTATGATTAGCATGTATAATGGCATTTATGATTAA AATACCACTATACGGAGTCCACTTATGACTCCCTAAAGCCCACG

\section{LJD2161}

ACAGTTAGATCTAGACCTAACCTAGAAGAAAGTAATAAATATACTAACAG CCCTATTAGTAAATATTACGCTATCAATACTCCTAATCATCATTGCCTTCTG ACTACCCCAACTTAATTTATATACTGAAAAAGCAAACCCGTATGAGTGCG GATTTGACCCCATAGGCTCTGCTCGCCTCCCATTCTCAATAAAATTTTTCCT 
AGTAGCAATCACTTTCTTACTATTTGACCTAGAAATTGCACTTCTACTTCCA CTACCATGAGCTATCCAAATATATAATATTAACATCATAATATTAACAGCT TTCATCCTAGTCTCCGTATTAGCATTAGGCTTAGCCTACGAGTGACTACAA AAAGGACTAGAATGAACTGAA-

TAACTGGTAATTAGTTTAATTAAAACAAATGATTTCGACTCATTAGATTAT GATATACATTATAATTACCAAAA----

ATGTCATCTGTAACCTCCAATATTATATTAGCATTCTCATTCTCATTTCTAG GAACACTAGTATTTCGATCCCACCTAATATCAACTCTCTTATGCCTTGAAG GAATAATACTATCACTGTTTATTATAACCACAATTACATCCCTTAATTCCC ACTCAATAATTATATACCCTCTACCCATTGTTATTCTAGTATTCGCAGCATG TGAAGCAGCCATTGGCTTAGCCCTATTAGCAAAACTATCAAACTCCTACGG AACAGATTATGTGCAAAATCTAAACCTACTTCAATGT-

TAAAAATTATTTTTCCCTCTATCATATTACTCCCACTCACCTGATTATCAAA CAAAAAAAACCTATGAGTCAATGTTACCTCCTACAGCTTTATAATCAGCTT GACTTCAGCTATGTTTTTATGACAAAACGACATAAATAACCTAAACTTCTC ACTACTATTCTCAACTGACTCCCTATCCTCCCCTCTTATTATTCTAACAACA TGACTTCTACCACTCATACTGCTAGCCAGCCAAAACCACATAAAAAAGGA AACAGAGTCAAACAAAAAAACATACATTTCAATACTAGTCTTACTACAAA TTCTTCTAATCATAACATTTTCTGCAAACGAACTAATTATATTTTACATCTT ATTTGAAGCCACCCTAATCCCCACCCTTATTATCATCACCCGATGGGGTAA CCAAACAGAACGACTAAATGCAGGACTCTACTTTCTATTTTATACCCTAAT TGGATCAATCCCACTACTAATCGCCCTTATCCACATTCAAAATATAAAAGG AACATTAAACTTCATACTATTCCCACTCACCTTTACACCCCTAGACCAAAC ATGGTCTAATAATATTCTATGATTAGCATGTATAATGGCATTTATGATTAA AATACCACTATACGGAGTCCACTTATGACTCCCTAAAG-

\section{LJD2651}

ACAGCTGACTTCCAATCAGTTAGATCTAGACCTAACCTAGAAGAAAGTAA TAAATATACTAACAGCCCTATTAGTAAATATTACGCTATCAATACTCCTAA TCATCATTGCCTTCTGACTACCCCAACTTAATTTATATACTGAAAAAGCAA ACCCGTATGAGTGCGGATTTGACCCCATAGGCTCTGCTCGCCTCCCATTCT CAATAAAATTTTTCCTAGTAGCAATCACTTTCTTACTATTTGACCTAGAAAT TGCACTTCTACTTCCACTACCATGAGCTATCCAAATATATAATATTAACAT CATAATATTAACAGCTTTCATCCTAGTCTCCGTATTAGCATTAGGCTTAGC CTACGAGTGACTACAAAAAGGACTAGAATGAACTGAG-

TAATTGGTAATTAGTTTAATTAAAACAAATGATTTCGACTCATTAGATTAT GATATACATTATAATTACCAAAA----

ATGTCATCTGTAACCTCCAATATTATATTAGCATTCTCATTCTCATTTCTAG GAACACTAGTATTTCGATCCCACCTAATATCAACTCTCCTATGCCTTGAAG GAATAATACTGTCACTGTTTATTATAACCACAATTACATCCCTTAATTCCC ACTCAATAATTATATACCCTCTACCCATTGTTATTCTAGTATTCGCAGCATG TGAAGCAGCCATTGGCTTAGCCCTATTAGCAAAACTATCAAACTCCTACGG AACAGATTATGTGCAAAATCTAAACCTACTTCAATGT-

TAAAAATTATTTTTCCCTCTATCATATTACTCCCACTCACCTGATTATCAAA CAAAAAAAACCTATGAGTCAATGTTACCTCCTACAGCTTTATAATCAGCTT 
GACTTCAGCTATGTTTTTATGACAAAACGACATAAATAATCTAAACTTCTC ACTACTATTCTCAACCGACTCCCTATCCTCCCCTCTTATTATTCTAACAACA TGACTTCTACCACTCATACTGCTAGCCAGCCAAAACCACATAAAAAAGGA AACAGAGTCAAACAAAAAAACATACATTTCAATACTAGTCTTACTACAAA TTCTTCTAATCATAACATTTTCTGCAAACGAACTAATTATATTTTACATCTT ATTTGAAGCCACCCTAATCCCCACCCTTATTATCATCACCCGATGGGGTAA CCAAACAGAACGACTAAATGCAGGACTCTACTTTCTATTTTATACCCTAAT TGGATCAATCCCACTACTAATCGCCCTTATCCACATTCAAAATATAAAAGG AACATTAAACTTCATACTATTCCCACTCACCTTTACACCCCTAGACCAAAC ATGGTCTAATAATATTCTATGATTAGCATGTATAATAGCATTTATGATTAA AATACCACTATACGGAGTCCACTTATGACTCCCTAAAGCCCAC-

$\underline{\mathrm{LJD} 2737}$

ACAGCTGACTTCCAATCAGTTAGATCTAGACCTAACCTAGAAGAAAGTAA TAAATATACTAACAGCCCTATTAGTAAATATTACGCTATCAATACTCCTAA TCATCATTGCCTTCTGACTACCCCAACTTAATTTATATACTGAAAAAGCAA ACCCGTATGAGTGCGGATTTGACCCCATAGGCTCTGCTCGCCTCCCATTCT CAATAAAATTTTTCCTAGTAGCAATCACTTTCTTACTATTTGACCTAGAAAT TGCACTTCTACTTCCATTACCATGAGCTATCCAAATATATAATATTAACAT CATAATATTAACAGCTTTCATCCTAGTCTCCGTATTAGCATTAGGCTTAGC CTACGAGTGACTACAAAAAGGACTAGAATGAACTGAG-

TAACTGGTAATTAGTTTAATTAAAACAAATGATTTCGACTCATTAGATTAT GATATACATTATAATTACCAAAA----

ATGTCATCTGTAACCTCCAATATTATATTAGCATTCTCATTCTCATTTCTAG GAACACTAGTATTTCGATCCCACCTAATATCGACTCTCCTATGCCTTGAAG GAATAATACTATCACTGTTTATTATAACCACAATTACATCCCTTAATTCCC ACTCAATAATTATATACCCTCTACCCATTGTTATTCTAGTATTCGCAGCATG TGAAGCAGCCATTGGCTTAGCCCTATTAGCAAAACTATCAAACTCCTACGG AACAGATTATGTGCAAAATCTAAACCTACTTCAATGT-

TAAAAATTATTTTTCCCTCTATCATATTACTCCCACTCACCTGNTTATCAAA CAAAAAAAACCTATGAGTCAATGTTACCTCNTACAGCTTTATAATCAGCTT GATTTCAGCTATGTTTTTATGACAAAACGACATAAATAATCTAAACTTCTC ACTACTATTCTCAACTGACCCCCTATCCTCCCCTCTTATTATTCTAACAACA TGACTTCTACCACTCATACTGCTAGCCAGCCAAAACCACATAAAAAAGGA AACAGAGTCAAACAAAAAAACATACATTTCAATACTAGTCTTACTACAAA TTCTTCTAATCATAACATTTTCTGCAAACGAACTAATTATATTTTACATCTT ATTTGAAGCCACCCTAATCCCCACCCTTATTATCATCACCCGATGGGGTAA CCAAACAGAACGACTAAATGCAGGACTCTACTTTCTATTTTATACCTTAAT TGGATCAATCCCACTACTAATCGCCCTTATCCACATTCAAAATATAAAAGG AACATTAAACTTCATACTATTCCCACTCACCTTTACACCCCTAGACCAAAC ATGGTCTAATAATATCCTATGATTAGCATGTATAATGGCATTTATGATTAA AATACCACTATACGGGGTCCACTTATGACTCCCTAAAGCCCACGTAGAAG CTCCAA- 
$\underline{\mathrm{LJD} 2834}$

ACAGCTGACTTCCAATCAGTTAGATCTAGACCTAACCTAGAAGAAAGTAA TAAATATACTAACAGCCCTATTAGTAAATATTACGCTATCAATACTCCTAA TCATCATTGCCTTCTGACTACCCCAACTTAATTTATATACTGAAAAAGCAA ACCCGTATGAGTGCGGATTTGACCCCATAGGCTCTGCTCGCCTCCCATTCT CAATAAAATTTTTCCTAGTAGCAATCACTTTCTTACTATTTGACCTAGAAAT TGCACTTCTACTTCCACTACCATGAGCTATCCAAATATATAATATTAACAT CATAATATTAACAGCTTTCATCCTAGTCTCCGTATTAGCATTAGGCTTAGC CTACGAGTGACTACAAAAAGGACTAGAATGAACTGAA-

TAACTGGTAATTAGTTTAATTAAAACAAATGATTTCGACTCATTAGATTAT GATATACATTATAATTACCAAAA----

ATGTCATCTGTAACCTCCAATATTATATTAGCATTCTCATTCTCATTTCTAG GAACACTAGTATTTCGATCCCACCTAATATCAACTCTCCTATGCCTTGAAG GAATAATACTATCACTGTTTATTATAACCACAATTACATCCCTTAATTCCC ACTCAATAATTATATACCCTCTACCCATTGTTATTCTAGTATTCGCAGCATG TGAAGCAGCCATTGGCTTAGCCCTATTAGCAAAACTATCAAACTCCTACGG AACAGATTATGTGCAAAATCTAAACCTACTTCAATGT-

TAAAAATTATTTTTCCCTCTATCATATTACTCCCACTCACCTGATTATCAAA CAAAAAAAACCTATGAGTCAATGTTACCTCCTACAGCTTTATAATCAGCTT GACTTCAGCTATGTTTTTATGACAAAACGACATAAATAGCCTAAACTTCTC ACTACTATTCTCAACTGACTCCCTATCCTCCCCTCTTATTATTCTAACAACA TGACTTCTACCACTCATACTGCTAGCCAGCCAAAACCACATAAAAAAGGA AACAGAGTCAAACAAAAAAACATACATTTCAATACTAGTCTTACTACAAA TTCTTCTAATCATAACATTTTCTGCAAATGAACTAATTATATTTTACATCTT ATTTGAAGCCACCCTAATCCCCACCCTTATTATCATCACCCGATGGGGTAA CCAAACAGAACGACTAAATGCAGGACTCTACTTTCTATTTTATACCCTAAT TGGATCAATCCCACTACTAATCGCCCTTATCCACATTCAAAATATAAAAGG AACATTAAACTTCATACTATTTCCACTCACCTTTACACCCCTAGACCAAAC ATGGTCTAATAATATTCTATGATTAGCATGTATAATGGCATTTATGATTAA AATACCACTATACGGAGTCCACTTATGACTCCCTAAAGCCCAC

$\underline{\mathrm{LJD} 2850}$

ATGACTTCCAATCAGTTAGATCTAGACCTAACCTAGAAGAAAGTAATAAA TATACTAACAGCCCTATTAGTAAATATTACGCTATCAATACTCCTAATCAT CATTGCCTTCTGACTACCCCAACTTAATTTATATACTGAAAAAGCAAACCC GTATGAGTGCGGATTTGACCCCATAGGCTCTGCTCGCCTCCCATTCTCAAT AAAATTTTTCCTAGTAGCAATCACTTTCTTACTATTTGACCTAGAAATTGCA CTTCTACTTCCACTACCATGAGCTATCCAAATATATAATATTAACATCATA ATATTAACAGCTTTCATCCTAGTCTCCGTATTAGCATTAGGCTTAGCCTAC GAGTGACTACAAAAAGGACTAGAATGAACTGAA-

TAACTGGTAATTAGTTTAATTAAAACAAATGATTTCGACTCATTAGATTAT GATATACATTATAATTACCAAAA----

ATGTCATCTGTAACCTCCAATATTATATTAGCATTCTCATTCTCATTTCTAG GAACACTAGTATTTCGATCCCACCTAATATCAACTCTCCTATGCCTTGAAG GAATAATACTATCACTGTTTATTATAACCACAATTACATCCCTTAATTCCC 
ACTCAATAATTATATACCCTCTACCCATTGTTATTCTAGTATTCGCAGCATG TGAAGCAGCCATTGGCTTAGCCCTATTAGCAAAACTATCAAACTCCTACGG AACAGATTATGTGCAAAATCTAAACCTACTTCAATGT-

TAAAAATTATTTTTCCCTCTATCATATTACTCCCACTCACCTGATTATCAAA CAAAAAAAACCTATGAGTCAATGTTACCTCCTACAGCTTTATAATCAGCTT GACTTCAGCTATGTTTTTATGACAAAACGACATAAATAGCCTAAACTTCTC ACTACTATTCTCAACTGACTCCCTATCCTCCCCTCTTATTATTCTAACAACA TGACTTCTACCACTCATACTGCTAGCCAGCCAAAACCACATAAAAAAGGA AACAGAGTCAAACAAAAAAACATACATTTCAATACTAGTCTTACTACAAA TTCTTCTAATCATAACATTTTCTGCAAATGAACTAATTATATTTTACATCTT ATTTGAAGCCACCCTAATCCCCACCCTTATTATCATCACCCGATGGGGTAA CCAAACAGAACGACTAAATGCAGGACTCTACTTTCTATTTTATACCCTAAT TGGATCAATCCCACTACTAATCGCCCTTATCCACATTCAAAATATAAAAGG AACATTAAACTTCATACTATTTCCACTCACCTTTACACCCCTAGACCAAAC ATGGTCTAATAATATTCTATGATTAGCATGTATAATGGCATTTATGATTAA AATACCACTATACGGAGTCCACTTATGACTCCCTAAAGCCCAC

\section{$\underline{\mathrm{LJD} 2898}$}

ACAGCTGACTTCCAATCAGTTAGATCTAGACCTAACCTAGAAGAAAGTAA TAAATATACTAACAGCCCTATTAGTAAATATTACGCTATCAATACTCCTAA TCATCATTGCCTTCTGACTACCCCAACTTAATTTATATACTGAAAAAGCAA ACCCGTATGAGTGCGGATTTGACCCCATAGGCTCTGCTCGCCTCCCATTCT CAATAAAATTTTTCCTAGTAGCAATCACTTTCTTACTATTTGACCTAGAAAT TGCACTTCTACTTCCATTACCATGAGCTATCCAAATATATAATATTAACAT CATAATATTAACAGCTTTCATCCTAGTCTCCGTATTAGCATTAGGCTTAGC CTACGAGTGACTACAAAAAGGACTAGAATGAACTGAG-

TAACTGGTAATTAGTTTAATTAAAACAAATGATTTCGACTCATTAGATTAT GATATACATTATAATTACCAAAA----

ATGTCATCTGTAACCTCCAATATTATATTAGCATTCTCATTCTCATTTCTAG GAACACTAGTATTTCGATCCCACCTAATATCGACTCTCCTATGCCTTGAAG GAATAATACTATCACTGTTTATTATAACCACAATTACATCCCTTAATTCCC ACTCAATAATTATATACCCTCTACCCATTGTTATTCTAGTATTCGCAGCATG TGAAGCAGCCATTGGCTTAGCCCTATTAGCAAAACTATCAAACTCCTACGG AACAGATTATGTGCAAAATCTAAACCTACTTCAATGT-

TAAAAATTATTTTTCCCTCTATCATATTACTCCCACTCACCTGATTATCAAA CAAAAAAAACCTATGAGTCAATGTTACCTCCTACAGCTTTATAATCAGCTT GATTTCAGCTATGTTTTTATGACAAAACGACATAAATAATCTAAACTTCTC ACTACTATTCTCAACTGACCCCCTATCCTCCCCTCTTATTATTCTAACAACA TGACTTCTACCACTCATACTGCTAGCCAGCCAAAACCACATAAAAAAGGA AACAGAGTCAAACAAAAAAACATACATTTCAATACTAGTCTTACTACAAA TTCTTCTAATCATAACATTTTCTGCAAACGAACTAATTATATTTTACATCTT ATTTGAAGCCACCCTAATCCCCACCCTTATTATCATCACCCGATGGGGTAA CCAAACAGAACGACTAAATGCAGGACTCTACTTTCTATTTTATACCTTAAT TGGATCAATCCCACTACTAATCGCCCTTATCCACATTCAAAATATAAAAGG AACATTAAACTTCATACTATTCCCACTCACCTTTACACCCCTAGACCAAAC 
ATGGTCTAATAATATCCTATGATTAGCATGTATAATGGCATTTATGATTAA AATACCACTATACGGGGTCCACTTATGACTCCCTAAAGCCCAC-

LJD2914

ACAGCTGACTTCCAATCAGTTAGATCTAGACCTAACCTAGAAGAAAGTAA TAAATATACTAACAGCCCTATTAGTAAATATTACGCTATCAATACTCCTAA TCATCATTGCCTTCTGACTACCCCAACTTAATTTATATACTGAAAAAGCAA ACCCGTATGAGTGCGGATTTGACCCCATAGGCTCTGCTCGCCTCCCATTCT CAATAAAATTTTTCCTAGTAGCAATCACTTTCTTACTATTTGACCTAGAAAT TGCACTTCTACTTCCACTACCATGAGCTATCCAAATATATAATATTAACAT CATAATATTAACAGCTTTCATCCTAGTCTCCGTATTAGCATTAGGCTTAGC CTACGAGTGACTACAAAAAGGACTAGAATGAACTGAA-

TAACTGGTAATTAGTTTAATTAAAACAAATGATTTCGACTCATTAGATTAT GATATACATTATAATTACCAAAA----

ATGTCATCTGTAACCTCCAATATTATATTAGCATTCTCATTCTCATTTCTAG GAACACTAGTATTTCGATCCCACCTAATATCAACTCTCCTATGCCTTGAAG GAATAATACTATCACTGTTTATTATAACCACAATTACATCCCTTAATTCCC ACTCAATAATTATATACCCTCTACCCATTGTTATTCTAGTATTCGCAGCATG TGAAGCAGCCATTGGCTTAGCCCTATTAGCAAAACTATCAAACTCCTACGG AACAGATTATGTGCAAAATCTAAACCTACTTCAATGT-

TAAAAATTATTTTTCCCTCTATCATATTACTCCCACTCACCTGATTATCAAA CAAAAAAAACCTATGAGTCAATGTTACCTCCTACAGCTTTATAATCAGCTT GACTTCAGCTATGTTTTTATGACAAAACGACATAAATAACCTAAACTTCTC ACTACTATTCTCAACTGACTCCCTATCCTCCCCTCTTATTATTCTAACAACA TGACTTCTACCACTCATACTGCTAGCCAGCCAAAACCACATAAAAAAGGA AACAGAGTCAAACAAAAAAACATACATTTCAATACTAGTCTTACTACAAA TTCTTCTAATCATAACATTTTCTGCAAACGAACTAATTATATTTTACATCTT ATTTGAAGCCACCCTAATCCCCACCCTTATTATCATCACCCGATGGGGTAA CCAAACAGAACGACTAAATGCAGGACTCTACTTTCTATTTTATACCCTAAT TGGATCAATCCCACTACTAATCGCCCTTATCCACATTCAAAATATAAAAGG AACATTAAACTTCATACTATTCCCACTCACCTTTACACCCCTAGACCAAAC ATGGTCTAATAATATTCTATGATTAGCATGTATAATGGCATTTATGATTAA AATACCACTATACGGAGTCCACTTATGACTCCCTAAAGCCCAC-

$\underline{\text { LJD2936 }}$

ACGAACTAGTACAGCTGACTTCCAATCAGTTAGATCTAGACCTAACCTAGA AGAAAGTAATAAATATACTAACAGCCCTATTAGTAAATATTACGCTATCA ATACTCCTAATCATCATTGCCTTCTGACTACCCCAACTTAATTTATATACTG AAAAAGCAAACCCATATGAGTGCGGATTTGACCCCATAGGCTCTGCTCGC CTCCCATTCTCAATAAAATTTTTCCTAGTAGCAATCACTTTCTTACTATTTG ACCTAGAAATTGCACTTCTACTTCCACTACCATGAGCTATCCAAATATATA ATATTAACATCATAATATTAACAGCTTTCATCCTAGTCTCCGTATTAGCATT AGGCTTAGCCTACGAGTGACTACAAAAAGGACTAGAATGAACTGAATAACTGGTAATTAGTTTAATTAAAACAAATGATTTCGACTCATTAGATTAT GATATACATTATAATTACCAAAA---- 
ATGTCATCTGTAACCTCCAATATTATATTAGCATTCTCATTCTCATTTCTAG GAACACTAGTATTTCGATCCCACCTAATATCAACTCTCCTATGCCTTGAAG GAATAATACTATCACTGTTTATTATAACCACAATTACATCCCTTAATTCCC ACTCAATAATTATATACCCTCTACCCATTGTTATTCTAGTATTCGCAGCATG TGAAGCAGCCATTGGCTTAGCCCTATTAGCAAAACTATCAAACTCCTACGG AACAGATTATGTGCAAAATCTAAACCTACTTCAATGT-

TAAAAATTATTTTTCCCTCTATCATATTACTCCCACTCACCTGATTATCAAA CAAAAAAAACCTATGAGTCAATGTTACCTCCTACAGCTTTATAATCAGCTT GACTTCAGCTATGTTTTTATGACAAAACGACATAAATAACCTAAACTTCTC ACTACTATTCTCAACTGACTCCCTATCCTCCCCTCTTATTATTCTAACAACA TGACTTCTACCACTCATACTGCTAGCCAGCCAAAACCACATAAAAAAGGA AACAGAGTCAAACAAAAAAACATATATTTCAATACTAGTCTTACTACAAA TTCTTCTAATCATAACATTTTCTGCAAACGAACTAATTATATTTTACATCTT ATTTGAAGCCACCCTAATCCCCACCCTTATTATCATCACCCGATGGGGTAA CCAAACAGAACGACTAAATGCAGGACTCTACTTTCTATTTTATACCCTAAT TGGATCAATCCCACTACTAATCGCCCTTATCCACATTCAAAATATAAAAGG AACATTAAACTTCATACTATTCCCACTCACCTTTACACCCCTAGACCAAAC ATGGTCTAATAATATTCTATGATTAGCATGTATAATGGCATTTATGATTAA AATACCACTATACGGAGTCCACTTATGACTCCCTAAAGCCCAC-

\section{LJD3000}

AAAGCTGACTTCCAATCAGTTAGATCTAGACCTAACCTAGAAGAAAGTAA TAAATATACTAACAGCCCTATTAGTAAATATTACGCTATCAATACTCCTAA TCATCATTGCCTTCTGACTACCCCAACTTAATTTATATACTGAAAAAGCAA ACCCGTATGAGTGCGGATTTGACCCCATAGGCTCTGCTCGCCTCCCATTCT CAATAAAATTTTTCCTAGTAGCAATCACTTTCTTACTATTTGACCTAGAAAT TGCACTTCTACTTCCACTACCATGAGCTATCCAAATATATAATATTAACAT CATAATATTAACAGCTTTCATCCTAGTCTCCGTATTAGCATTAGGCTTAGC CTACGAGTGACTACAAAAAGGACTAGAATGAACTGAA-

TAACTGGTAATTAGTTTAATTAAAACAAATGATTTCGACTCATTAGATTAT GATATACATTATAATTACCAAAA----

ATGTCATCTGTAACCTCCAATATTATATTAGCATTCTCATTCTCATTTCTAG GAACACTAGTATTTCGATCCCACCTAATATCAACTCTCCTATGCCTTGAAG GAATAATACTATCACTGTTTATTATAACCACAATTACATCCCTTAATTCCC ACTCAATAATTATATACCCTCTACCCATTGTTATTCTAGTATTCGCAGCATG TGAAGCAGCCATTGGCTTAGCCCTATTAGCAAAACTATCAAACTCCTACGG AACAGATTATGTGCAAAATCTAAACCTACTTCAATGT-

TAAAAATTATTTTTCCCTCTATCATATTACTCCCACTCACCTGATTATCAAA CAAAAAAAACCTATGAGTCAATGTTACCTCCTACAGCTTTATAATCAGCTT GACTTCAGCTATGTTTTTATGACAAAACGACATAAATAACCTAAACTTCTC ACTACTATTCTCAACTGACTCCCTATCCTCCCCTCTTATTATTCTAACAACA TGACTTCTACCACTCATACTGCTAGCCAGCCAAAACCACATAAAAAAGGA AACAGAGTCAAACAAAAAAACATACATTTCAATACTAGTCTTACTACAAA TTCTTCTAATCATAACATTTTCTGCAAACGAACTAATTATATTTTACATCTT ATTTGAAGCCACCCTAATCCCCACCCTTATTATCATCACCCGATGGGGTAA 
CCAAACAGAACGACTAAATGCAGGACTCTACTTTCTATTTTATACCCTAAT TGGATCAATCCCACTACTAATCGCCCTTATCCACATTCAAAATATAAAAGG AACATTAAACTTCATACTATTCCCACTCACCTTTACACCCCTAGACCAAAC ATGGTCTAATAATATTCTATGATTAGCATGTATAATGGCATTTATGATTAA AATACCACTATACGGAGTCCACTTATGACTCCCTAAAGCCCA-----:-----

\section{LJD2126}

ACAGCTGACTTCCAATCAGTTAGATCTAGACCTAACCTAGAAGAAAGTAA TAAATATACTAACAGCCCTATTAGTAAATATTACGCTATCAATACTCCTAA TCATCATTGCCTTCTGACTACCCCAACTTAATTTATATACTGAAAAAGCAA ACCCGTATGAGTGCGGATTTGACCCCATAAGCTCTGCTCGCCTCCCATTCT CAATAAAATTTTTCCTAGTAGCAATCACTTTCTTACTATTTGACCTAGAAAT TGCACTTCTACTTCCACTACCATGAGCTATCCAAATATATAATATTAACAT CATAATATTAACAGCTTTCATCCTAGTCTCCGTATTAGCATTAGGCTTAGC CTACGAGTGACTACAAAAAGGACTAGAATGAACTGAG-

TAACTGGTAATTAGTTTAATTAAAACAAATGATTTCGACTCATTAGATTAT GATATACATTATAATTACCAAAA----

ATGTCATCTGTAACCTCCAATATTATATTAGCATTCTCATTCTCATTTCTAG GAACACTAGTATTCCGATCCCACCTAATATCAACTCTCCTATGCCTTGAAG GAATAATACTGTCACTGTTTATTATAACCACAATTACATCCCTTAATTCCC ACTCAATAATTATATACCCTCTACCCATTGTTATTCTAGTATTCGCAGCATG TGAAGCAGCCATTGGCTTAGCCCTATTAGCAAAACTATCAAACTCCTACGG AACAGATTATGTGCAAAATCTAAACCTACTTCAATGT-

TAAAAATTATTTTTCCCTCTATCATATTACTCCCACTCACCTGATTATCAAA CAAAAAAAACCTATGAGTTAATGTTACCTCCTACAGCTTTATAATCAGCTT GACTTCAGCTATGTTTCTATGACAAAACGACATAAATAATCTAAACTTCTC ACTACTATTCTCAACCGACTCCCTATCCTCCCCTCTTATTATTCTAACAACA TGACTTCTGCCACTCATACTGCTAGCCAGCCAAAACCACATAAAAAAGGA AACAGAGTTAAACAAAAAAACATACATTTCAATACTAGTCTTACTACAAA TTCTTCTAATCATAACATTTTCTGCAAACGAACTAATTATATTTTACATCTT ATTTGAAGCCACCCTAATCCCCACCCTTATTATCATCACCCGATGGGGTAA CCAAACAGAACGACTAAATGCAGGACTCTACTTTCTATTTTATACCCTAAT TGGATCAATCCCACTACTAATCGCCCTTATCCACATTCAAAATATAAAAGG AACATTAAACTTCATACTATTCCCACTCACCTTTACACCCCTAGACCAAAC ATGGTCTAATAATATTCTATGATTAGCATGTATAATGGCATTTATGATTAA AATACCATTATACGGAGTCCACTTATGACTCCCTAAAGCCCACGTA--:---$-$

LJD2138

ATAGTACAGCTGACTTCCAATCAGTTAGATCTAGACCTAACCTAGAAGAA AGTAATAAATATACTAACAGCCCTATTAGTAAATATTACGCTATCAATACT CCTAATCATCATTGCCTTCTGACTACCCCAACTTAATTTATATACTGAAAA AGCAAACCCGTATGAGTGCGGATTTGACCCCATAGGCTCTGCTCGCCTCCC ATTCTCAATAAAATTTTTCCTAGTAGCAATCACTTTCTTACTATTTGACCTA GAAATTGCACTTCTACTTCCACTTCCATGAGCTATCCAAATATATAATATT 
AACATCATAATATTAACAGCTTTCATCCTAGTCTCCGTATTAGCATTAGGC TTAGCCTACGAGTGACTACAAAAAGGACTAGAATGAACTGAA-

TAACTGGTAATTAGTTTAATTAAAACAAATGATTTCGACTCATTAGATTAT GATATACATTATAATTACCAAAA----

ATGTCATCTGTAACCTCCAATATTATATTAGCATTCTCATTCTCATTTCTAG GAACACTAGTATTTCGATCCCACCTAATATCAACTCTCCTATGCCTTGAAG GAATAATACTATCACTGTTTATTATAACCACAATTACATCCCTTAATTCCC ACTCAATAATTATATACCCTCTACCCATTGTTATTCTAGTATTCGCAGCATG TGAAGCAGCCATTGGCTTAGCCTTATTAGCAAAACTATCAAACTCCTACGG AACAGATTATGTGCAAAATCTAAACCTACTTCAATGT-

TAAAAATTATTTTTCCCTCTATCATATTACTCCCACTCACCTGATTATCAAA CAAAAAAAACCTATGAGTCAATGTTACCTCCTACAGCTTTATAATCAGCTT GACTTCAGCTATGTTTTTGTGACAAAACGACATAAATAACCTAAACTTCTC ACTACTATTCTCAACTGACTCCCTATCCTCCCCTCTTATTATTCTAACAACA TGACTTCTACCACTCATACTGCTAGCCAGCCAAAACCACATAAAAAAGGA AACAGAGTTAAACAAAAAAACATACATTTCAATACTAGTCTTACTACAAA TTCTTCTAATCATAACATTTTCTGCAAACGAACTAATTATATTTTACATCTT ATTTGAAGCCACCCTAATCCCCACCCTTATTATCATCACCCGATGGGGTAA CCAAACAGAACGACTAAATGCAGGACTCTACTTTCTATTTTATACCCTAAT TGGATCAATCCCACTACTAATCGCCCTTATCCATATTCAAAATATAAAAGG AACATTAAACTTCATACTATTCCCACTCACCTTTACACCCCTAGACCAAAC ATGGTCTAATAATATTCTATGATTAGCATGTATAATGGCATTTATGATTAA AATACCACTATACGGAGTCCACTTATGACTCCCTAAAGCCCAC

$\underline{\mathrm{LJD}} 2154$

ACAGCTGACTTCCAATCAGTTAGATCTAGACCTAACCTAGAAGAAAGTAA TAAATATACTAACAGCCCTATTAGTAAATATTACGCTATCAATACTCCTAA TCATCATTGCCTTCTGACTACCCCAACTTAATTTATATACTGAAAAAGCAA ACCCGTATGAGTGCGGATTTGACCCCATAGGCTCTGCTCGCCTCCCATTCT CAATAAAATTTTTCCTAGTAGCAATCACTTTCTTACTATTTGACCTAGAAAT TGCACTTCTACTTCCACTACCATGAGCTATCCAAATATATAATATTAACAT CATAATATTAACAGCTTTCATCCTAGTCTCCGTATTAGCATTAGGCTTAGC CTACGAGTGACTACAAAAAGGACTAGAATGAACTGAA-

TAACTGGTAATTAGTTTAATTAAAACAAATGATTTCGACTCATTAGATTAT GATATACATTATAATTACCAAAA----

ATGTCATCTGTAACCTCCAATATTATATTAGCATTCTCATTCTCATTTCTAG GAACACTAGTATTTCGATCCCACCTAATATCAACTCTCCTATGCCTTGAAG GAATAATACTATCACTGTTTATTATAACCACAATTACATCCCTTAATTCCC ACTCAATAATTATATACCCTCTACCCATTGTTATTCTAGTATTCGCAGCATG TGAAGCAGCCATTGGCTTAGCCCTATTAGCAAAACTATCAAACTCCTACGG AACAGATTATGTGCAAAATCTAAACCTACTTCAATGT-

TAAAAATTATTTTTCCCTCTATCATATTACTCCCACTCACCTGATTATCAAA CAAAAAAAACCTATGAGTCAATGTTACCTCCTACAGCTTTATAATCAGCTT GACTTCAGCTATGTTTTTATGACAAAACGACATAAATAGCCTAAACTTCTC ACTACTATTCTCAACTGACTCCCTATCCTCCCCTCTTATTATTCTAACAACA 
TGACTTCTACCACTCATACTGCTAGCCAGCCAAAACCACATAAAAAAGGA AACAGAGTCAAACAAAAAAACATACATTTCAATACTAGTCTTACTACAAA TTCTTCTAATCATAACATTTTCTGCAAATGAACTAATTATATTTTACATCTT ATTTGAAGCCACCCTAATCCCCACCCTTATTATCATCACCCGATGGGGTAA CCAAACAGAACGACTAAATGCAGGACTCTACTTTCTATTTTATACCCTAAT TGGATCAATCCCACTACTAATCGCCCTTATCCACATTCAAAATATAAAAGG AACATTAAACTTCATACTATTTCCACTCACCTTTACACCCCTAGACCAAAC ATGGTCTAATAATATTCTATGATTAGCATGTATAATGGCATTTATGATTAA AATACCACTATACGGAGTCCACTTATGACTCCCTAAAGCCCAC-

\section{LJD2158}

ATAGTACAGCTGACTTCCAATCAGTTAGATCTAGACCTAACCTAGAAGAA AGTAATAAATATACTAACAGCCCTATTAGTAAATATTACGCTATCAATACT CCTAATCATCATTGCCTTCTGACTACCCCAACTTAATTTATATACTGAAAA AGCAAACCCGTATGAGTGCGGATTTGACCCCATAAGCTCTGCTCGCCTCCC ATTCTCAATAAAATTTTTCCTAGTAGCAATCACTTTCTTACTATTTGACCTA GAAATTGCACTTCTACTTCCACTACCATGAGCTATCCAAATATATAATATT AACATCATAATATTAACAGCTTTCATCCTAGTCTCCGTATTAGCATTAGGC TTAGCCTACGAGTGACTACAAAAAGGACTAGAATGAACTGAG-

TAACTGGTAATTAGTTTAATTAAAACAAATGATTTCGACTCATTAGATTAT GATATACATTATAATTACCAAAA----

ATGTCATCTGTAACCTCCAATATTATATTAGCATTCTCATTCTCATTTCTAG GAACACTAGTATTCCGATCCCACCTAATATCAACTCTCCTATGCCTTGAAG GAATAATACTGTCACTGTTTATTATAACCACAATTACATCCCTTAATTCCC ACTCAATAATTATATACCCTCTACCCATTGTTATTCTAGTATTCGCAGCATG TGAAGCAGCCATTGGCTTAGCCCTATTAGCAAAACTATCAAACTCCTACGG AACAGATTATGTGCAAAATCTAAACCTACTTCAATGT-

TAAAAATTATTTTTCCCTCTATCATATTACTCCCACTCACCTGATTATCAAA CAAAAAAAACCTATGAGTTAATGTTACCTCCTACAGCTTTATAATCAGCTT GACTTCAGCTATGTTTCTATGACAAAACGACATAAATAATCTAAACTTCTC ACTACTATTCTCAACCGACTCCCTATCCTCCCCTCTTATTATTCTAACAACA TGACTTCTGCCACTCATACTGCTAGCCAGCCAAAACCACATAAAAAAGGA AACAGAGTTAAACAAAAAAACATACATTTCAATACTAGTCTTACTACAAA TTCTTCTAATCATAACATTTTCTGCAAACGAACTAATTATATTTTACATCTT ATTTGAAGCCACCCTAATCCCCACCCTTATTATCATCACCCGATGGGGTAA CCAAACAGAACGACTAAATGCAGGACTCTACTTTCTATTTTATACCCTAAT TGGATCAATCCCACTACTAATCGCCCTTATCCACATTCAAAATATAAAAGG AACATTAAACTTCATACTATTCCCACTCACCTTTACACCCCTAGACCAAAC ATGGTCTAATAATATTCTATGATTAGCATGTATAATGGCATTTATGATTAA AATACCATTATACGGAGTCCACTTATGACTCCCTAAAGCCCAC LJD2836

ACAGCTGACTTCCAATCAGTTAGATCTAGACCTAACCTAGAAGAAAGTAA TAAATATACTAACAGCCCTATTAGTAAATATTACGCTATCAATACTCCTAA TCATCATTGCCTTCTGACTACCCCAACTTAATTTATATACTGAAAAAGCAA ACCCGTATGAGTGCGGATTTGACCCCATAAGCTCTGCTCGCCTCCCATTCT 
CAATAAAATTTTTCCTAGTAGCAATCACTTTCTTACTATTTGACCTAGAAAT TGCACTTCTACTTCCACTACCATGAGCTATCCAAATATATAATATTAACAT CATAATATTAACAGCTTTCATCCTAGTCTCCGTATTAGCATTAGGCTTAGC CTACGAGTGACTACAAAAAGGACTAGAATGAACTGAG-

TAACTGGTAATTAGTTTAATTAAAACAAATGATTTCGACTCATTAGATTAT GATATACATTATAATTACCAAAA----

ATGTCATCTGTAACCTCCAATATTATATTAGCATTCTCATTCTCATTTCTAG GAACACTAGTATTCCGATCCCACCTAATATCAACTCTCCTATGCCTTGAAG GAATAATACTGTCACTGTTTATTATAACCACAATTACATCCCTTAATTCCC ACTCAATAATTATATACCCTCTACCCATTGTTATTCTAGTATTCGCAGCATG TGAAGCAGCCATTGGCTTAGCCCTATTAGCAAAACTATCAAACTCCTACGG AACAGATTATGTGCAAAATCTAAACCTACTTCAATGT-

TAAAAATTATTTTTCCCTCTATCATATTACTCCCACTCACCTGATTATCAAA CAAAAAAAACCTATGAGTTAATGTTACCTCCTACAGCTTTATAATCAGCTT GACTTCAGCTATGTTTCTATGACAAAACGACATAAATAATCTAAACTTCTC ACTACTATTCTCAACCGACTCCCTATCCTCCCCTCTTATTATTCTAACAACA TGACTTCTGCCACTCATACTGCTAGCCAGCCAAAACCACATAAAAAAGGA AACAGAGTTAAACAAAAAAACATACATTTCAATACTAGTCTTACTACAAA TTCTTCTAATCATAACATTTTCTGCAAACGAACTAATTATATTTTACATCTT ATTTGAAGCCACCCTAATCCCCACCCTTATTATCATCACCCGATGGGGTAA CCAAACAGAACGACTAAATGCAGGACTCTACTTTCTATTTTATACCCTAAT TGGATCAATCCCACTACTAATCGCCCTTATCCACATTCAAAATATAAAAGG AACATTAAACTTCATACTATTCCCACTCACCTTTACACCCCTAGACCAAAC ATGGTCTAATAATATTCTATGATTAGCATGTATAATGGCATTTATGATTAA AATACCATTATACGGAGTCCACTTATGACTCCCTAAAGCCC

LJD2852

ATGACTTCCAATCAGTTAGATCTAGACCTAACCTAGAAGAAAGTAATAAA TATACTAACAGCCCTATTAGTAAATATTACGCTATCAATACTCCTAATCAT CATTGCCTTCTGACTACCCCAACTCAATTTATATACTGAAAAAGCAAACCC GTATGAGTGCGGATTTGACCCCATAGGCTCTGCTCGCCTCCCATTCTCAAT AAAATTTTTCCTAGTAGCAATCACTTTCTTACTATTTGACCTAGAAATTGCA CTTCTACTTCCACTACCATGAGCTATCCAAATATATAATATTAACATCATA ATATTAACAGCTTTCATCCTAGTCTCCGTATTAGCATTAGGCTTAGCCTAC GAATGACTACAAAAAGGACTAGAATGAACTGAG-

TAACTGGTAATTAGTTTAATTAAAACAAATGATTTCGACTCATTAGATTAT GATATACATTATAATTACCAAAA----

ATGTCATCTGTAACCTCCAATATTATATTAGCATTCTCATTCTCATTTCTAG GAACACTAGTATTTCGATCACACCTAATATCAACTCTCCTATGCCTTGAAG GAATAATACTGTCACTGTTTATTATAACCACAATTACATCCCTTAATTCCC ACTCAATAATTATATACCCTCTACCCATTGTTATTCTAGTATTCGCAGCATG TGAAGCAGCCATTGGCTTAGCCCTATTAGCAAAACTATCAAACTCCTACGG AACAGATTATGTGCAAAATCTAAACCTACTTCAATGT-

TAAAAATTATTTTTCCCTCTATCATATTACTCCCACTCACCTGATTATCAAA CAAAAAAAACCTATGAGTCAATGTTACCTCCTACAGCTTTATAATCAGCTT 
AACTTCAGCTATGTTTTTATGACAAAACGACATAAATAATCTAAACTTCTC ACTACTATTCTCAACCGACTCCCTATCCTCCCCTCTTATTATTCTAACAACA TGACTTCTACCACTCATACTGCTAGCCAGCCAAAACCACATAAAAAAGGA AACAGAGTCAAACAAAAAAACATACATTTCAATACTAGTCTTACTACAAA TTCTTCTAATCATAACATTTTCTGCAAACGAACTAATTATATTTTACATCTT ATTTGAAGCCACCCTAATCCCCACCCTTATTATCATCACCCGATGAGGTAA CCAAACAGAACGACTAAATGCAGGACTCTACTTTCTATTTTATACCCTAAT TGGATCAATCCCACTACTAATCGCCCTTATCCACATTCAAAATATAAAAGG AACATTAAACTTCATACTATTCCCACTCACCTTTACACCCCTAGACCAAAC ATGGTCTAATAATATTCTATGATTAGCATGTATAATGGCATTTATGATTAA AATACCACTATACGGAGTCCACTTATGACTCCCTAAAGCCCAC

\section{$\underline{\mathrm{LJD} 2860}$}

ACAGCTGACTTCCAATCAGTTAGATCTAGACCTAACCTAGAAGAAAGTAA TAAATATACTAACAGCCCTATTAGTAAATATTACGCTATCAATACTCCTAA TCATCATTGCCTTCTGACTACCCCAGCTTAATTTATATACTGAAAAAGCAA ACCCGTATGAGTGCGGATTTGACCCCATAGGCTCTGCTCGCCTCCCATTCT CAATAAAATTTTTCCTAGTAGCAATCACTTTCTTACTATTTGACCTAGAAAT TGCACTTCTACTTCCACTACCATGAGCTATCCAAATATATAATATTAACAT CATAATATTAACAGCTTTCATCCTAGTCTCCGTATTAGCATTAGGCTTAGC CTACGAGTGACTACAAAAAGGACTAGAATGAACTGAA-

TAACTGGTAATTAGTTTAATTAAAACAAATGATTTCGACTCATTAGATTAT GATATACATTATAATTACCAAAA---

ATGTCATCTGTAACCTCCAATATTATATTAGCATTCTCATTCTCATTTCTAG GAACACTAGTATTTCGATCCCACCTAATATCAACTCTCTTATGCCTTGAAG GAATAATACTATCACTGTTTATTATAACCACAATTACATCCCTTAATTCCC ACTCAATAATTATATACCCTCTACCCATTGTTATTCTAGTATTCGCAGCATG TGAAGCAGCCATTGGCTTAGCCCTATTAGCAAAACTATCAAACTCCTACGG AACAGATTATGTGCAAAATCTAAACCTACTTCAATGT-

TAAAAATTATTTTTCCCTCTATCATATTACTCCCACTCACCTGATTATCAAA CAAAAAAAACCTATGAGTCAATGTTACCTCCTACAGCTTTATAATCAGCTT GACTTCAGCTATGTTTTTATGACAAAACGACATAAATAACCTAAACTTCTC ACTACTATTCTCAACTGACTCCCTATCCTCCCCTCTTATTATTCTAACAACA TGACTTCTACCACTCATACTGCTAGCCAGCCAAAACCACATAAAAAAGGA AACAGAGTCAAACAAAAAAACATACATTTCAATACTAGTCTTACTACAAA TTCTTCTAATCATAACATTTTCTGCAAACGAACTAATTATATTTTACATCTT ATTTGAAGCCACCCTAATCCCCACCCTTATTATCATCACCCGATGGGGTAA CCAAACAGAACGACTAAATGCAGGACTCTACTTTCTATTTTATACCCTAAT TGGATCAATCCCACTACTAATCGCCCTTATCCACATTCAAAATATAAAAGG AACATTAAACTTCATACTATTCCCACTCACCTTTACACCCCTAGACCAAAC ATGGTCTAATAATATTCTATGATTAGCATGTATAATGGCATTTATGATTAA AATACCACTATACGGAGTCCACTTATGACTCCCTAAAGCCCAC

LJD2865

ACACTGACTTCCAATCAGTTAGATCTAGACCTAACCTAGAAGAAAGTAAT 
AAATATACTAACAGCCCTATTAGTAAATATTACGCTATCAATACTCCTAAT CATCATTGCCTTCTGACTACCCCAACTTAATTTATATACTGAAAAAGCAAA CCCATATGAGTGCGGATTTGACCCCATAGGCTCTGCTCGCCTCCCATTCTC AATAAAATTTTTCCTAGTAGCAATCACTTTCTTACTATTTGACCTAGAAATT GCACTTCTACTTCCACTACCATGAGCTATCCAAATATATAATATTAACATC ATAATATTAACAGCTTTCATCCTAGTCTCCGTATTAGCATTAGGCTTAGCCT ACGAGTGACTACAAAAAGGACTAGAATGAACTGAA-

TAACTGGTAATTAGTTTAATTAAAACAAATGATTTCGACTCATTAGATTAT GATATACATTATAATTACCAAAA----

ATGTCATCTGTAACCTCCAATATTATATTAGCATTCTCATTCTCATTTCTAG GAACACTAGTATTTCGATCCCACCTAATATCAACTCTCCTATGCCTTGAAG GAATAATACTATCACTGTTTATTATAACCACAATTACATCCCTTAATTCCC ACTCAATAATTATATACCCTCTACCCATTGTTATTCTAGTATTCGCAGCATG TGAAGCAGCCATTGGCTTAGCCCTATTAGCAAAACTATCAAACTCCTACGG AACAGATTATGTGCAAAATCTAAACCTACTTCAATGT-

TAAAAATTATTTTTCCCTCTATCATATTACTCCCACTCACCTGATTATCAAA CAAAAAAAACCTATGAGTCAATGTTACCTCCTACAGCTTTATAATCAGCTT GACTTCAGCTATGTTTTTATGACAAAACGACATAAATAACCTAAACTTCTC ACTACTATTCTCAACTGACTCCCTATCCTCCCCTCTTATTATTCTAACAACA TGACTTCTACCACTCATACTGCTAGCCAGCCAAAACCACATAAAAAAGGA AACAGAGTCAAACAAAAAAACATATATTTCAATACTAGTCTTACTACAAA TTCTTCTAATCATAACATTTTCTGCAAACGAACTAATTATATTTTACATCTT ATTTGAAGCCACCCTAATCCCCACCCTTATTATCATCACCCGATGGGGTAA CCAAACAGAACGACTAAATGCAGGACTCTACTTTCTATTTTATACCCTAAT TGGATCAATCCCACTACTAATCGCCCTTATCCACATTCAAAATATAAAAGG AACATTAAACTTCATACTATTCCCACTCACCTTTACACCCCTAGACCAAAC ATGGTCTAATAATATTCTATGATTAGCATGTATAATGGCATTTATGATTAA AATACCACTATACGGAGTCCACTTATGACTCCCTAAAGCCCAC-

\section{LJD2890}

ACTGACTTCCAATCAGTTAGATCTAGACCTAACCTAGAAGAAAGTAATAA ATATACTAACAGCCCTATTAGTAAATATTACGCTATCAATACTCCTAATCA TCATTGCCTTCTGACTACCCCAACTTAATTTATATACTGAAAAAGCAAACC CGTATGAGTGCGGATTTGACCCCATAGGCTCTGCTCGCCTCCCATTCTCAA TAAAATTTTTCCTAGTAGCAATCACTTTCTTACTATTTGACCTAGAAATTGC ACTTCTACTTCCACTACCATGAGCTATCCAAATATATAATATTAACATCAT AATATTAACAGCTTTCATCCTAGTCTCCGTATTAGCATTAGGCTTAGCCTA CGAGTGACTACAAAAAGGACTAGAATGAACTGAG-

TAACTGGTAATTAGTTTAATTAAAACAAATGATTTCGACTCATTAGATTAT GATATACATTATAATTACCAAAA----

ATGTCATCTGTAACCTCCAATATTATATTAGCATTCTCATTCTCATTTCTAG GAACACTAGTATTTCGATCCCACCTAATATCAACTCTCCTATGCCTTGAAG GAATAATACTATCACTGTTTATTATAACCACAATTACATCCCTTAATTCCC ACTCAATAATTATATACCCCCTACCCATTGTTATTCTAGTATTCGCAGCATG TGAAGCAGCCATTGGCTTAGCCCTATTAGCAAAACTATCAAACTCCTACGG 
AACAGATTATGTGCAAAATCTAAACCTACTTCAATGT-

TAAAAATTATTTTTCCCTCTATCATATTACTCCCACTCACCTGATTATCAAA CAAAAAAAACCTATGAGTCAATGTTACCTCCTACAGCTTTATAATCAGCTT GACTTCAGCTATGTTTTTATGACAAAACGACATAAATAATCTAAACTTCTC ACCACTATTCTCAACTGACTCCCTATCCTCCCCTCTTATTATTCTAACAACA TGACTTCTACCACTCATACTGCTAGCCAGCCAAAACCACATAAAAAAGGA AACAGAGTCAAACAAAAAAACATACATTTCAATACTAGTCTTACTACAAA TTCTTCTAATCATAACATTTTCTGCAAACGAACTAATTATATTTTACATCTT ATTTGAAGCCACCCTAATCCCCACCCTTATTATCATCACCCGATGGGGTAA CCAAACAGAACGACTAAATGCAGGACTCTACTTTCTATTTTATACCCTAAT TGGATCAATCCCACTACTAATCGCCCTTATCCATATTCAAAATATAAAAGG AACATTAAACTTCATACTATTCCCACTCACCTTTACACCCCTAGACCAAAC ATGGTCTAATAATATTCTATGATTAGCATGTATAATGGCATTTATGATTAA AATACCACTATACGGAGTCCACTTATGACTCCCTAAAGC-

\section{LJD2943}

ACAGCTGACTTCCAATCAGTTAGATCTAGACCTAACCTAGAAGAAAGTAA TAAATATACTAACAGCCCTATTAGTAAATATTACGCTATCAATACTCCTAA TCATCATTGCCTTCTGACTACCCCAGCTTAATTTATATACTGAAAAAGCAA ACCCGTATGAGTGCGGATTTGACCCCATAGGCTCTGCTCGCCTCCCATTCT CAATAAAATTTTTCCTAGTAGCAATCACTTTCTTACTATTTGACCTAGAAAT TGCACTTCTACTTCCACTACCATGAGCTATCCAAATATATAATATTAACAT CATAATATTAACAGCTTTCATCCTAGTCTCCGTATTAGCATTAGGCTTAGC CTACGAGTGACTACAAAAAGGACTAGAATGAACTGAATAACTGGTAATTAGTTTAATTAAAACAAATGATTTCGACTCATTAGATTAT GATATACATTATAATTACCAAAA----

ATGTCATCTGTAACCTCCAATATTATATTAGCATTCTCATTCTCATTTCTAG GAACACTAGTATTTCGATCCCACCTAATATCAACTCTCTTATGCCTTGAAG GAATAATACTATCACTGTTTATTATAACCACAATTACATCCCTTAATTCCC ACTCAATAATTATATACCCTCTACCCATTGTTATTCTAGTATTCGCAGCATG TGAAGCAGCCATTGGCTTAGCCCTATTAGCAAAACTATCAAACTCCTACGG AACAGATTATGTGCAAAATCTAAACCTACTTCAATGT-

TAAAAATTATTTTTCCCTCTATCATATTACTCCCACTCACCTGATTATCAAA CAAAAAAAACCTATGAGTCAATGTTACCTCCTACAGCTTTATAATCAGCTT GACTTCAGCTATGTTTTTATGACAAAACGACATAAATAACCTAAACTTCTC ACTACTATTCTCAACTGACTCCCTATCCTCCCCTCTTATTATTCTAACAACA TGACTTCTACCACTCATACTGCTAGCCAGCCAAAACCACATAAAAAAGGA AACAGAGTCAAACAAAAAAACATACATTTCAATACTAGTCTTACTACAAA TTCTTCTAATCATAACATTTTCTGCAAACGAACTAATTATATTTTACATCTT ATTTGAAGCCACCCTAATCCCCACCCTTATTATCATCACCCGATGGGGTAA CCAAACAGAACGACTAAATGCAGGACTCTACTTTCTATTTTATACCCTAAT TGGATCAATCCCACTACTAATCGCCCTTATCCACATTCAAAATATAAAAGG AACATTAAACTTCATACTATTCCCACTCACCTTTACACCCCTAGACCAAAC ATGGTCTAATAATATTCTATGATTAGCATGTATAATGGCATTTATGATTAA AATACCACTATACGGAGTCCACTTATGACTCCCTAAAGCC- 
Jefferson3

CGAACTAGTACAGCTGACTTCCAATCAGTTAGATCTAGACCTAACCTAGAA GAAAGTAATAAATATACTAACAGCCCTATTAGTAAATATTACGCTATCAAT ACTCCTAATCATCATTGCCTTCTGACTACCCCAACTTAATTTATATACTGAA AAAGCAAACCCATATGAGTGCGGATTTGACCCCATAGGCTCTGCTCGCCTC CCATTCTCAATAAAATTTTTCCTAGTAGCAATCACTTTCTTACTATTTGACC TAGAAATTGCACTTCTACTTCCACTACCATGAGCTATCCAAATATATAATA TTAACATCATAATATTAACAGCTTTCATCCTAGTCTCCGTATTAGCATTAG GCTTAGCCTACGAGTGACTACAAAAAGGACTAGAATGAACTGAATAACTGGTAATTAGTTTAATTAAAACAAATGATTTCGACTCATTAGATTAT GATATACATTATAATTACCAAAA----

ATGTCATCTGTAACCTCCAATATTATATTAGCATTCTCATTCTCATTTCTAG GAACACTAGTATTTCGATCCCACCTAATATCAACTCTCCTATGCCTTGAAG GAATAATACTATCACTGTTTATTATAACCACAATTACATCCCTTAATTCCC ACTCAATAATTATATACCCTCTACCCATTGTTATTCTAGTATTCGCAGCATG TGAAGCAGCCATTGGCTTAGCCCTATTAGCAAAACTATCAAACTCCTACGG AACAGATTATGTGCAAAATCTAAACCTACTTCAATGT-

TAAAAATTATTTTTCCCTCTATCATATTACTCCCACTCACCTGATTATCAAA CAAAAAAAACCTATGAGTCAATGTTACCTCCTACAGCTTTATAATCAGCTT GATTTCAGCTATGTTTTTATGACAAAACGACATAAATAATCTAAACTTCTC ACTACTATTCTCAACTGACTCCCTATCCTCCCCTCTTATTATTCTAACAACA TGACTTCTACCACTCATACTGCTAGCCAGCCAAAACCACATAAAAAAGGA AACAGAGTCAAACAAAAAAACATATATTTCAATACTAGTCTTACTACAAA TTCTTCTAATCATAACATTTTCTGCAAACGAACTAATTATATTTTACATCTT ATTTGAAGCCACCCTAATCCCCACCCTTATTATCATCACCCGATGGGGTAA CCAAACAGAACGACTAAATGCAGGACTCTACTTTCTATTTTATACCCTAAT TGGATCAATCCCACTACTAATCGCCCTTATCCACATTCAAAATATAAAAGG AACATTAAACTTCATACTATTCCCACTCACCTTTACACCCCTAGACCAAAC ATGGTCTAATAATATTCTATGATTAGCATGTATAATGGCATTTATGATTAA AATACCACTATACGGAGTCCACTTATGACTCCCTAAAGCCCACGTAGAAG CTCCA--

\footnotetext{
$\underline{\text { ADAMS6 }}$

CGAACTAGTACAGCTGACTTCCAATCAGTTAGATCTAGACCTAACCTAGAA GAAAGTAATAAATATACTAACAGCCCTATTAGTAAATATTACGCTATCAAT ACTCCTAATCATCATTGCCTTCTGACTACCCCAACTTAATTTATATACTGAA AAAGCAAACCCGTATGAGTGCGGATTTGACCCCATAGGCTCTGCTCGCCTC CCATTCTCAATAAAATTTTTCCTAGTAGCAATCACTTTCTTACTATTTGACC TAGAAATTGCACTTCTACTTCCACTACCATGAGCTATCCAAATATATAATA TTAACATCATAATATTAACAGCTTTCATCCTAGTCTCCGTATTAGCATTAG GCTTAGCCTACGAGTGACTACAAAAAGGACTAGAATGAACTGAG-

TAACTGGTAATTAGTTTAATTAAAACAAATGATTTCGACTCATTAGATTAT GATATACATTATAATTACCAAAA----

ATGTCATCTGTAACCTCCAATATTATATTAGCATTCTCATTCTCATTTCTAG GAACACTAGTATTTCGATCCCACCTAATATCAACTCTCCTATGCCTTGAAG 
GAATAATACTATCACTGTTTATTATAACCACAATTACATCCCTTAATTCCC ACTCAATAATTATATACCCTCTACCCATTGTTATTCTAGTATTCGCAGCATG TGAAGCAGCCATTGGCTTAGCCCTATTAGCAAAACTATCAAACTCCTACGG AACAGATTATGTGCAAAATCTAAACCTACTTCAATGT-

TAAAAATTATTTTTCCCTCTATCATATTACTCCCACTCACCTGATTATCAAA CAAAAAAAACCTATGAATCAATGTTACCTCCTACAGCTTTATAATCAGCTT GACTTCAGCTATGTTTTTATGACAAAACGACATAAATAATCTAAACTTCTC ACTACTATTCTCAACCGACTCCCTATCCTCCCCTCTTATTATTCTAACAACA TGACTTCTACCACTCATACTGCTAGCCAGCCAAAACCACATAAAAAAGGA AACAGAGTCAAACAAAAAAACATACATTTCAATACTAGTCTTACTACAAA TTCTTCTAATCATAACATTTTCTGCAAACGAACTAATTATATTTTACATCTT ATTTGAAGCCACCCTAATCCCCACCCTTATTATCATCACCCGATGGGGTAA CCAAACAGAACGACTAAATGCAGGACTCTACTTTCTATTTTACACCCTAAT TGGATCAATCCCACTACTAATCGCCCTTATCCACATTCAAAATATAAAAGG AACATTAAACTTCATACTATTCCCACTCACCTTTACACCCCTAGACCAAAC ATGGTCTAATAATATTCTATGATTAGCATGTATAATGGCATTTATGATTAA AATACCACTATACGGAGTCCACTTATGACTCCCTAAAGCCCACGTAGAAG CTCCA--

clatsopl

AATAAATATACTAACAGCCCTATTAGTAAATATTACGCTATCAATACTCCT AATCATCATTGCCTTCTGACTACCCCAACTTAATTTATATACTGAAAAAGC AAACCCGTATGAGTGCGGATTTGACCCCATAGGCTCTGCTCGCCTCCCATT CTCAATAAAATTTTTCCTAGTAGCAATCACTTTCTTACTATTTGACCTAGAA ATTGCACTTCTACTTCCACTACCATGAGCTATCCAAATATATAATATTAAC ATCATAATATTAACAGCTTTCATCCTAGTCTCCGTATTAGCATTAGGCTTA GCCTACGAGTGACTACAAAAAGGACTAGAATGAACTGAA-

TAACTGGTAATTAGTTTAATTAAAACAAATGATTTCGACTCATTAGATTAT GATATACATTATAATTACCAAAA---:

ATGTCATCTGTAACCTCCAATATTATATTAGCATTCTCATTCTCATTTCTAG GAACACTAGTATTTCGATCCCACCTAATATCAACTCTCCTATGCCTTGAAG GAATAATACTATCACTGTTTATTATAACCACAATTACATCCCTTAATTCCC ACTCAATAATTATATACCCTCTACCCATTGTTATTCTAGTATTCGCAGCATG TGAAGCAGCCATTGGCTTAGCCCTATTAGCAAAACTATCAAACTCCTACGG AACAGATTATGTGCAAAATCTAAACCTACTTCAATGT-

TAAAAATTATTTTTCCCTCTATCATATTACTCCCACTCACCTGATTATCAAA CAAAAAAAACCTATGAGTCAATGTTACCTCCTACAGCTTTATAATCAGCTT GACTTCAGCTATGTTTTTATGACAAAACGACATAAATAATCTAAACTTCTC ACTACTATTCTCAACCGACTCCCTATCCTCCССTCTTATTATTCTAACAACA TGACTTCTACCACTCATACTGCTAGCCAGCCAAAACCACATAAAAAAGGA AACAGAGTCAAACAAAAAAACATACATTTCAATACTAGTCTTACTACAAA TTCTTCTAATCATAACATTTTCTGCAAACGAACTAATTATATTTTACATCTT ATTTGAAGCCACCCTAATCCCCACCCTTATTATCATCACCCGATGGGGTAA CCAAACAGAACGACTAAATGCAGGACTCTACTTTCTATTTTATACCCTAAT TGGATCAATCCCACTACTAATCGCCCTTATCCACATTCAAAATATAAAAGG 
AACATTAAACTTCATACTATTCCCACTCACCTTTACACCCCTAGACCAAAC ATGGTCTAATAATATTCTATGATTAGCATGTATAATGGCATTTATGATTAA AATACCACTATACGGAGTCCACTTATGACTCCCTAAAGCCCACGTAAAAG CTCCA--

clatsop2

AATAAATATACTAACAGCCCTATTAGTAAATATTACGCTATCAATACTCCT AATCATCATTGCCTTCTGACTACCCCAACTTAATTTATATACTGAAAAAGC AAACCCGTATGAGTGCGGATTTGACCCCATAGGCTCTGCTCGCCTCCCATT CTCAATAAAATTTTTCCTAGTAGCAATCACTTTCTTACTATTTGACCTAGAA ATTGCACTTCTACTTCCACTACCATGAGCTATCCAAATATATAATATTAAC ATCATAATATTAACAGCTTTCATCCTAGTCTCCGTATTAGCATTAGGCTTA GCCTACGAGTGACTACAAAAAGGACTAGAATGAACTGAA-

TAACTGGTAATTAGTTTAATTAAAACAAATGATTTCGACTCATTAGATTAT GATATACATTATAATTACCAAAA----

ATGTCATCTGTAACCTCCAATATTATATTAGCATTCTCATTCTCATTTCTAG GAACACTAGTATTTCGATCCCACCTAATATCAACTCTCCTATGCCTTGAAG GAATAATACTATCACTGTTTATTATAACCACAATTACATCCCTTAATTCCC ACTCAATAATTATATACCCTCTACCCATTGTTATTCTAGTATTCGCAGCATG TGAAGCAGCCATTGGCTTAGCCCTATTTGCAAAACTATCAAACTCCTACGG AACAGATTATGTGCAAAATCTAAACCTACTTCAATGT-

TAAAAATTATTTTTCCCTCTATCATATTACTCCCACTCACCTGATTATCAAA CAAAAAAAACCTATGAGTCAATGTTACCTCCTACAGCTTTATAATCAGCTT GACTTCAGCTATGTTTTTATGACAAAACGACATAAATAACCTAAACTTCTC ACTACTATTCTCAACTGACTCCCTATCCTCCCCTCTTATTATTCTAACAACA TGACTTCTACCACTCATACTGCTAGCCAGCCAAAACCACATAAAAAAGGA AACAGAGTCAAACAAAAAAACATACATTTCAATACTAGTCTTACTACAAA TTCTTCTAATCATAACATTTTCTGCAAATGAACTAATTATATTTTACATCTT ATTTGAAGCCACCCTAATCCCCACCCTTATTATCATCACCCGATGGGGTAA CCAAACAGAACGACTAAATGCAGGACTCTACTTTCTATTTTATACCCTAAT TGGATCAATCCCACTACTAATCGCCCTTATCCACATTCAAAATATAAAAGG AACATTAAACTTCATACTATTCCCACTCACCTTTACACCCCTAGACCAAAC ATGGTCTAATAATATTCTATGATTAGCATGTATAATGGCATTTATGATTAA AATACCACTATACGGAGTCCACTTATGACTCCCTAAAGCCCACGTAAAAG CTCCA--

clatsop3

AATAAATATACTAACAGCCCTATTAGTAAATATTACGCTATCAATACTCCT AATCATCATTGCCTTCTGACTACCCCAACTTAATTTATATACTGAAAAAGC AAACCCGTATGAGTGCGGATTTGACCCCATAGGCTCTGCTCGCCTCCCATT CTCAATAAAATTTTTCCTAGTAGCAATCACTTTCTTACTATTTGACCTAGAA ATTGCACTTCTACTTCCACTACCATGAGCTATCCAAATATATAATATTAAC ATCATAATATTAACAGCTTTCATCCTAGTCTCCGTATTAGCATTAGGCTTA GCCTACGAGTGACTACAAAAAGGACTAGAATGAACTGAA-

TAACTGGTAATTAGTTTAATTAAAACAAATGATTTCGACTCATTAGATTAT 
GATATACATTATAATTACCAAAA----

ATGTCATCTGTAACCTCCAATATTATATTAGCATTCTCATTCTCATTTCTAG GAACACTAGTATTTCGATCCCACCTAATATCAACTCTCCTATGCCTTGAAG GAATAATACTATCACTGTTTATTATAACCACAATTACATCCCTTAATTCCC ACTCAATAATTATATACCCTCTACCCATTGTTATTCTAGTATTCGCAGCATG TGAAGCAGCCATTGGCTTAGCCCTATTTGCAAAACTATCAAACTCCTACGG AACAGATTATGTGCAAAATCTAAACCTACTTCAATGT-

TAAAAATTATTTTTCCCTCTATCATATTACTCCCACTCACCTGATTATCAAA CAAAAAAAACCTATGAGTCAATGTTACCTCCTACAGCTTTATAATCAGCTT GACTTCAGCTATGTTTTTATGACAAAACGACATAAATAACCTAAACTTCTC ACTACTATTCTCAACTGACTCCCTATCCTCCCCTCTTATTATTCTAACAACA TGACTTCTACCACTCATACTGCTAGCCAGCCAAAACCACATAAAAAAGGA AACAGAGTCAAACAAAAAAACATACATTTCAATACTAGTCTTACTACAAA TTCTTCTAATCATAACATTTTCTGCAAATGAACTAATTATATTTTACATCTT ATTTGAAGCCACCCTAATCCCCACCCTTATTATCATCACCCGATGGGGTAA CCAAACAGAACGACTAAATGCAGGACTCTACTTTCTATTTTATACCCTAAT TGGATCAATCCCACTACTAATCGCCCTTATCCACATTCAAAATATAAAAGG AACATTAAACTTCATACTATTCCCACTCACCTTTACACCCCTAGACCAAAC ATGGTCTAATAATATTCTATGATTAGCATGTATAATGGCATTTATGATTAA AATACCACTATACGGAGTCCACTTATGACTCCCTAAAGCCCACGTAAAAG CTCCAA-

clatsop4

AATAAATATACTAACAGCCCTATTAGTAAATATTACGCTATCAATACTCCT AATCATCATTGCCTTCTGACTACCCCAACTTAATTTATATACTGAAAAAGC AAACCCGTATGAGTGCGGATTTGACCCCATAGGCTCTGCTCGCCTCCCATT CTCAATAAAATTTTTCCTAGTAGCAATCACTTTCTTACTATTTGACCTAGAA ATTGCACTTCTACTTCCACTACCATGAGCTATCCAAATATATAATATTAAC ATCATAATATTAACAGCTTTCATCCTAGTCTCCGTATTAGCATTAGGCTTA GCCTACGAGTGACTACAAAAAGGACTAGAATGAACTGAA-

TAACTGGTAATTAGTTTAATTAAAACAAATGATTTCGACTCATTAGATTAT GATATACATTATAATTACCAAAA----

ATGTCATCTGTAACCTCCAATATTATATTAGCATTCTCATTCTCATTTCTAG GAACACTAGTATTTCGATCCCACCTAATATCAACTCTCCTATGCCTTGAAG GAATAATACTATCACTGTTTATTATAACCACAATTACATCCCTTAATTCCC ACTCAATAATTATATACCCTCTACCCATTGTTATTCTAGTATTCGCAGCATG TGAAGCAGCCATTGGCTTAGCCCTATTTGCAAAACTATCAAACTCCTACGG AACAGATTATGTGCAAAATCTAAACCTACTTCAATGT-

TAAAAATTATTTTTCCCTCTATCATATTACTCCCACTCACCTGATTATCAAA CAAAAAAAACCTATGAGTCAATGTTACCTCCTACAGCTTTATAATCAGCTT GACTTCAGCTATGTTTTTATGACAAAACGACATAAATAACCTAAACTTCTC ACTACTATTCTCAACTGACTCCCTATCCTCCCCTCTTATTATTCTAACAACA TGACTTCTACCACTCATACTGCTAGCCAGCCAAAACCACATAAAAAAGGA AACAGAGTCAAACAAAAAAACATACATTTCAATACTAGTCTTACTACAAA TTCTTCTAATCATAACATTTTCTGCAAATGAACTAATTATATTTTACATCTT 
ATTTGAAGCCACCCTAATCCCCACCCTTATTATCATCACCCGATGGGGTAA CCAAACAGAACGACTAAATGCAGGACTCTACTTTCTATTTTATACCCTAAT TGGATCAATCCCACTACTAATCGCCCTTATCCACATTCAAAATATAAAAGG AACATTAAACTTCATACTATTCCCACTCACCTTTACACCCCTAGACCAAAC ATGGTCTAATAATATTCTATGATTAGCATGTATAATGGCATTTATGATTAA AATACCACTATACGGAGTCCACTTATGACTCCCTAAAGCCCACGTAAAAG CTCCA--

LostForest1A

ATCGAACTAGTACAGCTGACTTCCAATCAGTTAGATCTAGACCTAACCTAG AAGAAAGTAATAAATATACTAACAGCCCTATTAGTAAATATTACTCTATCA ATACTCCTAATCACCATTGCCTTCTGACTACCCCAACTTAATTTATATACTG AAAAAGCAAACCCGTATGAGTGCGGATTTGACCCCATAGGCTCTGCTCGC CTCCCATTCTCAATAAAATTTTTCCTAGTAGCAATCACTTTCTTACTATTTG ACCTAGAAATTGCACTTCTACTTCCACTACCATGAGCTATCCAAATATACA ATATTAACATCATAATATTAACAGCTTTCATCCTAGTCTCCGTATTAGCATT AGGCTTAGCCTACGAGTGACTACAAAAAGGACTAGAATGAACTGAG-

TAACTGGTAATTAGTTTAATTAAAACAAATGATTTCGACTCATTAGATTAT GATATACATTATAATTACCAAAA----

ATGTCATCTGTAACCTCCAATATTATATTAGCATTCTCATTCTCATTTCTAG GAACACTAGTATTTCGATCCCACCTAATATCAACTCTCCTATGCCTTGAAG GAATAATACTATCACTATTTATTATAACCACAATTACATCCCTTAATTCCC ACTCAATAATTATATACCCTCTACCCATTGTTATTCTAGTATTCGCAGCATG TGAAGCAGCCATTGGCTTAGCCCTATTA-

LostForest2

ATCGAACTAGTTCAGCTGACTTCCAATCAGTTAGATCTAGACCTAACCTAG AAGAAAGTAATAAATATACTAACAGCCCTATTAGTAAATATTACGCTATC AACACTCCTAATCATCATTGCCTTCTGACTACCCCAACTTAATTTATATACT GAAAAAGCAAACCCGTATGAGTGCGGATTTGACCCCATAAGCTCTGCTCG CCTCCCATTCTCAATAAAATTTTTCCTAGTAGCAATCACTTTCTTATTATTT GACCTAGAAATTGCACTTCTACTTCCACTACCATGAGCTATCCAAATATAT AATATTAACATCATAATATTAACAGCTTTCATCCTAGTCTCCGTATTAGCA TTAGGCTTAGCCTACGAGTGACTACAAAAAGGACTAGAATGAACTGAGTAACTGGTAATTAGTTTAATTAAAACAAATGATTTCGACTCATTAGATTAT GATATACATTATAATTACCAAAA----

ATGTCATCTGTAACCTCCAATATTATATTAGCATTCTCATTCTCATTTCTAG GAACACTAGTATTTCGATCCCACCTAATATCAACTCTCCTATGCCTTGAAG 
GAATAATACTATCACTGTTTATTATAACCACAATTACATCCCTTAATTCCC ACTCAATAATTATATACCCTCTACCCATTGTTATTCTAGTATTCGCAGCATG TGAAGCAGCCATTGGCTTAGCCCTATTAGCAAAACTATCAAACTCCTACGG AACAGATTATGTGCAAAATCTAAACCTACTTCAATGT-

TAAAAATTATTTTTCCCTCTATCATATTACTCCCACTCACCTGATTATCAAA CAAAAAAAACCTATGAATCAATGTTACCTCCTACAGCTTTATTATCAGCTT AATTTCAGCTATGTTTTTATGACAAAACGACATAAATAATCTAAACTTCTC ACTACTATTCTCAACTGACTCCCTATCCTCCCCTCTTATTATTCTAACAACA TGACTTCTACCACTCATACTGCTAGCTAGCCAAAACCACATAAAAAAGGA AACAGAGTCAAACAAAAAAACATACATTTCAATACTAGTCTTACTACAAA TTCTTCTAATCATAACATTTTCTGCAAACGAACTAATTATATTTTACATCTT ATTTGAAGCCACCCTAATCCCCACCCTTATTATCATCACCCGATGGGGTAA CCAAACAGAACGACTAAATGCAGGACTCTACTTTCTATTTTATACCCTAAT TGGATCAATCCCACTACTAATCGCCCTTATTCACATTCAAAATATAAAAGG GACATTAAACTTCATACTATTCCCACTCACCTTTACACCCCTAGACCAAAC ATGGTCTAATAATATTCTATGATTAGCATGTATAATGGCATTTATGATTAA AATACCACTATACGGAGTCCACTTATGACTCCCTAAAGCCCACGAGAAAG CTCCAA-

\section{LostForest3}

ATCGAACTAGTACAGCTGACTTCCAATCAGTTAGATCTAGACCTAACCTAG AAGAAAGTAATAAATATACTAACAGCCCTATTAGTAAATATTACGCTATC AATACTCCTAATCATCATTGCCTTCTGACTACCCCAACTTAATTTATATACT GAAAAAGCAAACCCGTATGAGTGCGGATTTGACCCCATAGGCTCTGCTCG CCTCCCATTCTCAATAAAATTTTTCCTAGTAGCAATCACTTTCTTACTATTT GACCTAGAAATTGCACTTCTACTTCCACTACCATGAGCTATCCAAATATAT AATATTAACATCATAATATTAACAGCTTTCATCCTAGTCTCCGTATTAGCA TTAGGCTTAGCCCACGAGTGACTACAAAAAGGACTAGAATGAACTGAGTAACTGGTAATTAGTTTAATTAAAACAAATGATTTCGACTCATTAGATTAT GATATACATTATAATTACCAAAA----

ATGTCATCTGTAACCTCCAATATTATATTAGCATTCTCATTCTCATTTCTAG GAACACTAGTATTTCGATCCCACCTAATATCAACTCTCCTATGCCTTGAAG GAATAATACTATCACTGTTTATTATAACCACAATTACATCCCTTAATTCCC ACTCAATAATTATATACCCTCTACCCATTGTTATTCTAGTATTCGCAGCATG TGAAGCAGCCATTGGCTTAGCCCTATTAGCAAAACTGTCAAACTCCTACGG AACAGATTATGTGCAAAATCTAAACCTACTTCAATGT-

TAAAAATTATTTTTCCCTCTATCATATTACTCCCACTCACCTGATTATCAAA CAAAAAAAACCTATGAGTCAATGTTACCTCCTACAGCTTTATAATCAGCTT GATTTCAGCTATGTTTTTATGACAAAACGACATAAATAATCTAAACTTCTC ACTACTATTCTCAACTGACTCCCTATCCTCCCCTCTTATTATTCTAACAACA TGACTTCTACCACTCATACTGCTAGCCAGCCAAAACCACATAAAAAAGGA AACAGAGTCAAACAAAAAAACATATATTTCAATACTAGTCTTACTACAAA TTCTTCTAATCATAACATTTTCTGCAAACGAACTAATTATATTTTACATCTT ATTTGAAGCCACCCTAATCCCCACCCTTATTATCATCACCCGATGGGGTAA CCAAACAGAACGACTAAATGCAGGACTCTACTTTCTATTTTATACCCTAAT 
TGGATCAATCCCACTACTAATCGCCCTTATCCACATTCAAAATATAAAAGG AACATTAAACTTCATACTATTCCCACTCACCTTTACACCCCTAGACCAAAC ATGGTCTAATAATATTCTATGATTAGCATGTATAATGGCATTTATGATTAA AATACCACTATACGGAGTCCACTTATGACTCCCTAAAGCCCACGTAGAAA GCTCCAA

LostForest4 ACGAACTAGTACAGCTGACTTCCAATCAGTTAGATCTAGACCTAACCTAGA AGAAAGTAATAAATATACTAACAGCCCTATTAGTAAATATTACGCTATCA ACACTCCTAATCATCATTGCCTTCTGACTACCCCAACTTAATTTATATACTG AAAAAGCAAACCCGTATGAGTGCGGATTTGACCCCATAAGCTCTGCTCGC CTCCCATTCTCAATAAAATTTTTCCTAGTAGCAATCACTTTCTTATTATTTG ACCTAGAAATTGCACTTCTACTTCCACTACCATGAGCTATCCAAATATATA ATATTAACATCATAATATTAACAGCTTTCATCCTAGTCTCCGTATTAGCATT AGGCTTAGCCTACGAGTGACTACAAAAAGGACTAGAATGAACTGAGTAACTGGTAATTAGTTTAATTAAAACAAATGATTTCGACTCATTAGATTAT GATATACATTATAATTACCAAAA----

ATGTCATCTGTAACCTCCAATATTATATTAGCATTCTCATTCTCATTTCTAG GAACACTAGTATTTCGATCCCACCTAATATCAACTCTCCTATGCCTTGAAG GAATAATACTATCACTGTTTATTATAACCACAATTACATCCCTTAATTCCC ACTCAATAATTATATACCCTCTACCCATTGTTATTCTAGTATTCGCAGCATG TGAAGCAGCCATTGGCTTAGCCCTATTAGCAAAACTATCAAACTCCTACGG AACAGATTATGTGCAAAATCTAAACCTACTTCAATGT-

TAAAAATTATTTTTCCCTCTATCATATTACTCCCACTCACCTGATTATCAAA CAAAAAAAACCTATGAATCAATGTTACCTCCTACAGCTTTATTATCAGCTT AATTTCAGCTATGTTTTTATGACAAAACGACATAAATAATCTAAACTTCTC ACTACTATTCTCAACTGACTCCCTATCCTCCCCTCTTATTATTCTAACAACA TGACTTCTACCACTCATACTGCTAGCTAGCCAAAACCACATAAAAAAGGA AACAGAGTCAAACAAAAAAACATACATTTCAATACTAGTCTTACTACAAA TTCTTCTAATCATAACATTTTCTGCAAACGAACTAATTATATTTTACATCTT ATTTGAAGCCACCCTAATCCCCACCCTTATTATCATCACCCGATGGGGTAA CCAAACAGAACGACTAAATGCAGGACTCTACTTTCTATTTTATACCCTAAT TGGATCAATCCCACTACTAATCGCCCTTATTCACATTCAAAATATAAAAGG GACATTAAACTTCATACTATTCCCACTCACCTTTACACCCCTAGACCAAAC ATGGTCTAATAATATTCTATGATTAGCATGTATAATGGCATTTATGATTAA AATACCACTATACGGAGTCCACTTATGACTCCCTAAAGCCCACGTAGAAG CTCCA--

sj1Pmausterus

CAGCTGACTTCCAATCAGTTAGATCTAGACCTAACCTAGAAGAAAGTAAT AAATATACTAACAGCCCTATTAGTAAATATTACGCTATCAATACTCCTAAT CATCATTGCCTTCTGACTACCCCAACTTAATTTATATACTGAAAAAGCAAA CCCGTATGAGTGCGGATTTGACCCCATAAGCTCTGCTCGCCTCCCATTCTC AATAAAATTTTTCCTAGTAGCAATCACTTTCTTACTATTTGACCTAGAAATT GCACTTCTACTTCCACTACCATGAGCTATCCAAATATATAATATTAACATC 
ATAATATTAACAGCTTTCATCCTAGTCTCCGTATTAGCATTAGGCTTAGCCT ACGAGTGACTACAAAAAGGACTAGAATGAACTGAG-

TAACTGGTAATTAGTTTAATTAAAACAAATGATTTCGACTCATTAGATTAT GATATACATTATAATTACCAAAA----

ATGTCATCTGTAACCTCCAATATTATATTAGCATTCTCATTCTCATTTCTAG GAACACTAGTATTCCGATCCCACCTAATATCAACTCTCCTATGCCTTGAAG GAATAATACTGTCACTGTTTATTATAACCACAATTACATCCCTTAATTCCC ACTCAATAATTATATACCCTCTACCCATTGTTATTCTAGTATTCGCAGCATG TGAAGCAGCCATTGGCTTAGCCCTATTAGCAAAACTATCAAACTCCTACGG AACAGATTATGTGCAAAATCTAAACCTACTCCAATGT-

TAAAAATTATTTTTCCCTCTATCATATTACTCCCACTCACCTGATTATCAAA CAAAAAAAACCTATGAATCAATGTTACCTCCTACAGCTTTATAATCAGCTT GACTTCAGCTATGTTTTTATGACAAAACGACATAAATAATCTAAACTTCTC ACTACTATTCTCAACCGACTCCCTATCCTCCCCTCTCATTATTCTAACAACA TGACTTCTACCACTCATACTGCTAGCCAGCCAAAACCACATAAAAAAGGA AACAGAGTCAAACAAAAAAACATACATTTCAATACTAGTCTTACTACAAA TTCTTCTAATCATAACATTTTCTGCAAACGAACTAATTATATTTTACATCTT ATTTGAAGCCACCCTAATCCCCACCCTTATTATCATCACCCGATGGGGTAA CCAAACAGAACGACTAAATGCAGGACTCTACTTTCTATTTTATACCCTAAT TGGATCAATCCCACTACTAATCGCCCTTATCCACATTCAAAATATAAAAGG AACATTAAACTTCATACTATTCCCACTCACCTTTACACCCCTAGACCAAAC ATGGTCTAATAATATTCTATGATTAGCATGTATAATGGCATTTATGATTAA AATACCACTATACGGAGTCCACTTATGACTCCCTAAAGCCCACG-

sj2Pmausterus

CAGCTGACTTCCAATCAGTTAGATCTTAGCCTAACCTAGAAGAAAGTAATA AATATACTAACAGCCCTATTAGTAAATATTACGCTATCAATACTCCTAATC ATCATTGCCTTCTGACTACCCCAACTTAATTTATATACTGAAAAAGCAAAC CCGTATGAGTGCGGATTTGACCCCATAGGCTCTGCTCGCCTCCCATTCTCA ATAAAATTTTTCCTAGTAGCAATCACTTTCTTACTATTTGACCTAGAAATTG CACTTCTACTTCCACTACCATGAGCTATCCAAATATATAATATTAACATCA TAATATTAACAGCTTTCATCCTAGTCTCCGTATTAGCATTAGGCTTAGCCTA CGAGTGACTACAAAAAGGACTAGAATGAACTGAA-

TAACTGGTAATTAGTTTAATTAAAACAAATGATTTCGACTCATTAGATTAT GATATACATTATAATTACCAAAA---

ATGTCATCTGTAACCTCCAATATTATATTAGCATTCTCATTCTCATTTCTAG GAACACTAGTATTTCGATCCCACCTAATATCAACTCTCCTATGCCTTGAAG GAATAATACTATCACTGTTTATTATAACCACAATTACATCCCTTAATTCCC ACTCAATAATTATATACCCTCTACCCATTGTTATTCTAGTATTCGCAGCATG TGAAGCAGCCATTGGCTTAGCCCTATTAGCAAAACTATCAAACTCCTACGG AACAGATTATGTACAAAATCTAAACCTACTTCAATGT-

TAAAAATTATTTTTCCCTCTATCATATTACTCCCACTCACCTGATTATCAAA CAAAAAAAACCTATGAATCAATGTTACCTCCTACAGCTTTATAATCAGCTT GACTTCAGCTATGTTTTTATGACAAAACGACATAAATAATCTAAACTTCTC ACTACTATTCTCAACCGACTCCCTATCCTCCCCTCTCATTATTCTAACAACA 
TGACTTCTACCACTCATACTGCTAGCCAGCCAAAACCACATAAAAAAGGA AACAGAGTCAAACAAAAAAACATACATTTCAATACTAGTCTTACTACAAA TTCTTCTAATCATAACATTTTCTGCAAACGAACTAATTATATTTTACATCTT ATTTGAAGCCACCCTAATCCCCACCCTTATTATCATCACCCGATGGGGTAA CCAAACAGAACGACTAAATGCAGGACTCTACTTTCTATTTTATACCCTAAT TGGATCAATCCCACTACTAATCGCCCTTATCCACATTCAAAATATAAAAGG AACATTAAACTTCATACTATTCCCACTCACCTTCACACCCCTAGACCAAAC ATGGTCTAATAATATTCTATGATTAGCATGTATAATGGCATTTATGATTAA AATACCACTATACGGAGTCCACTTATGACTCCCTAAAGCCCACTTAGAAAC TCC---

sj2aPmausterus AGCTGACTTCCAATCAGTTAGATCTAGACCTAACCTAGAAGAAAGTAATA AATATACTAACAGCCCTATTAGTAAATATTACGCTATCAATACTCCTAATC ATCATTGCCTTCTGACTACCCCAACTTAATTTATATACTGAAAAAGCAAAC CCGTATGAGTGCGGATTTGACCCTATAGGCTCTGCTCGCCTCCCATTCTCA ATAAAATTTTTCCTAGTAGCAATCACTTTCTTACTATTTGACCTAGAAATTG CACTTCTACTTCCACTACCATGAGCTATCCAAATATATAATATTAACATCA TAATATTAACAGCTTTCATCCTAGTCTCCGTATTAGCATTAGGCTTAGCCTA CGAGTGACTACAAAAAGGACTAGAATGAACTGAA-

TAACTGGTAATTAGTTTAATTAAAACAAATGATTTCGACTCATTAGATTAT GATATACATTATAATTACCAAAA----

ATGTCATCTGTAACCTCCAATATTATATTAGCATTCTCATTCTCATTTCTAG GAACACTAGTATTTCGATCCCACCTAATATCAACTCTCCTATGCCTTGAAG GAATAATACTATCACTGTTTATTATAACCACAATTACATCCCTTAATTCCC ACTCAATAATTATATACCCTCTACCCATTGTTATTCTAGTATTCGCAGCATG TGAAGCAGCCATTGGCTTAGCCCTATTAGCAAAACTATCAAACTCCTACGG AACAGATTATGTGCAAAATCTAAACCTACTTCAATGT-

TAAAAATTATTTTTCCCTCTATCATATTACTCCCACTCACCTGATTATCAAA CAAAAAAAACCTATGAATCAATGTTACCTCCTACAGCTTTATAATCAGCTT GACTTCAGCTATGTTTTTATGACAAAACGACATAAATAATCTAAACTTCTC ACTACTATTCTCAACCGACTCCCTATCCTCCCCTCTCATTATTCTAACAACA TGACTTCTACCACTCATACTGCTAGCCAGCCAAAACCACATAAAAAAGGA AACAGAGTCAAACAAAAAAACATACATTTCAATACTAGTCTTACTACAAA TTCTTCTAATCATAACATTTTCTGCAAACGAACTAATTATATTTTACATCTT ATTTGAAGCCACCCTAATCCCCACCCTTATTATCATCACCCGATGGGGTAA CCAAACAGAACGACTAAATGCAGGACTCTACTTTCTATTTTATACCCTAAT TGGATCAATCCCACTACTAATCGCCCTTATCCACATTCAAAATATAAAAGG AACATTAAACTTCATACTATTCCCACTCACCTTTACACCCCTAGACCAAAC ATGGTCTAATAATATTCTATGATTAGCATGTATAATGGCATTTATGATTAA AATACCACTATACGGAGTCCACTTATGACTCCCTAAAGCCCAC

sj3Pmausterus AGCTGACTTCCAATCAGTTAGATCTAGACCTAACCTAGAAGAAAGTAATA AATATACTAACAGCCCTATTAGTAAATATTACGCTATCAATACTCCTAATC 
ATCATTGCCTTCTGACTACCCCAACTTAATTTATATACTGAAAAAGCAAAC CCGTATGAGTGCGGATTTGACCCTATAGGCTCTGCTCGCCTCCCATTCTCA ATAAAATTTTTCCTAGTAGCAATCACTTTCTTACTATTTGACCTAGAAATTG CACTTCTACTTCCACTACCATGAGCTATCCAAATATATAATATTAACATCA TAATATTAACAGCTTTCATCCTAGTCTCCGTATTAGCATTAGGCTTAGCCTA CGAGTGACTACAAAAAGGACTAGAATGAACTGAA-

TAACTGGTAATTAGTTTAATTAAAACAAATGATTTCGACTCATTAGATTAT GATATACATTATAATTACCAAAA----

ATGTCATCTGTAACCTCCAATATTATATTAGCATTCTCATTCTCATTTCTAG GAACACTAGTATTTCGATCCCACCTAATATCAACTCTCCTATGCCTTGAAG GAATAATACTATCACTGTTTATTATAACCACAATTACATCCCTTAATTCCC ACTCAATAATTATATACCCTCTACCCATTGTTATTCTAGTATTCGCAGCATG TGAAGCAGCCATTGGCTTAGCCCTATTAGCAAAACTATCGAACTCCTACGG AACAGATTATGTGCAAAATCTAAACCTACTTCAATGT-

TAAAAATTATTTTTCCCTCTATCATATTACTCCCACTCACCTGATTATCAAA CAAAAAAAACCTATGAATCAATGTTACCTCCTACAGCTTTATAATCAGCTT GACTTCAGCTATGTTTTTATGACAAAACGACATAAATAATCTAAACTTCTC ACTACTATTCTCAACCGACTCCCTATCCTCCCCTCTCATTATTCTAACAACA TGACTTCTACCACTCATACTGCTAGCCAGCCAAAACCACATAAAAAAGGA AACAGAGTCAAACAAAAAAACATACATTTCAATACTAGTCTTACTACAAA TTCTTCTAATCATAACATTTTCTGCAAACGAACTAATTATATTTTACATCTT ATTTGAAGCCACCCTAATCCCCACCCTTATTATCATCACCCGATGGGGTAA CCAAACAGAACGACTAAATGCAGGACTCTACTTTCTATTTTATACCCTAAT TGGATCAATCCCACTACTAATCGCCCTTATCCACATTCAAAATATAAAAGG AACATTAAACTTCATACTATTCCCACTCACCTTCACACCCCTAGACCAAAC ATGGTCTAATAATATTCTATGATTAGCATGTATAATGGCATTTATGATTAA AATACCACTATACGGAGTCCACTTATGACTCCCTAAAGCCCAC

si5Pmausterus

CAGCTGACTTCCAATCAGTTAGATCTAGACCTAACCTAGAAGAAAGTAAT AAATATACTAACAGCCCTATTAGTAAATATTACGCTATCAATACTCCTAAT CATCATTGCCTTCTGACTACCCCAACTTAATTTATATACTGAAAAAGCAAA CCCGTATGAGTGCGGATTTGACCCTATAGGCTCTGCTCGCCTCCCATTCTC AATAAAATTTTTCCTAGTAGCAATCACTTTCTTACTATTTGACCTAGAAATT GCACTTCTACTTCCACTACCATGAGCTATCCAAATATATAATATTAACATC ATAATATTAACAGCTTTCATCCTAGTCTCCGTATTAGCATTAGGCTTAGCCT ACGAGTGACTACAAAAAGGACTAGAATGAACTGAA-

TAACTGGTAATTAGTTTAATTAAAACAAATGATTTCGACTCATTAGATTAT GATATACATTATAATTACCAAAA----

ATGTCATCTGTAACCTCCAATATTATATTAGCATTCTCATTCTCATTTCTAG GAACACTAGTATTTCGATCCCACCTAATATCAACTCTCCTATGCCTTGAAG GAATAATACTATCACTGTTTATTATAACCACAATTACATCCCTTAATTCCC ACTCAATAATTATATACCCTCTACCCATTGTTATTCTAGTATTCGCAGCATG TGAAGCAGCCATTGGCTTAGCCCTATTAGCAAAACTATCGAACTCCTACGG AACAGATTATGTGCAAAATCTAAACCTACTTCAATGT- 
TAAAAATTATTTTTCCCTCTATCATATTACTCCCACTCACCTGATTATCAAA CAAAAAAAACCTATGAATCAATGTTACCTCCTACAGCTTTATAATCAGCTT GACTTCAGCTATGTTTTTATGACAAAACGACATAAATAATCTAAACTTCTC ACTACTATTCTCAACCGACTCCCTATCCTCCCCTCTCATTATTCTAACAACA TGACTTCTACCACTCATACTGCTAGCCAGCCAAAACCACATAAAAAAGGA AACAGAGTCAAACAAAAAAACATACATTTCAATACTAGTCTTACTACAAA TTCTTCTAATCATAACATTTTCTGCAAACGAACTAATTATATTTTACATCTT ATTTGAAGCCACCCTAATCCCCACCCTTATTATCATCACCCGATGGGGTAA CCAAACAGAACGACTAAATGCAGGACTCTACTTTCTATTTTATACCCTAAT TGGATCAATCCCACTACTAATCGCCCTTATCCACATTCAAAATATAAAAGG AACATTAAACTTCATACTATTCCCACTCACCTTTACACCCCTAGACCAAAC ATGGTCTAATAATATTCTATGATTAGCATGTATAATGGCATTTATGATTAA AATACCACTATACGGAGTCCACTTATGACTCCCTAAAGCCCA---_-_-_--

sj6Pmausterus

CAGCTGACTTCCAATCAGTTAGATCTAGACCTAACCTAGAAGAAAGTAAT AAATATACTAACAGCCCTATTAGTAAATATTACGCTATCAATACTCCTAAT CATCATTGCCTTCTGACTACCCCAACTTAATTTATATACTGAAAAAGCAAA CCCGTATGAGTGCGGATTTGACCCTATAGGCTCTGCTCGCCTCCCATTCTC AATAAAATTTTTCCTAGTAGCAATCACTTTCTTACTATTTGACCTAGAAATT GCACTTCTACTTCCACTACCATGAGCTATCCAAATATATAATATTAACATC ATAATATTAACAGCTTTCATCCTAGTCTCCGTATTAGCATTAGGCTTAGCCT ACGAGTGACTACAAAAAGGACTAGAATGAACTGAA-

TAACTGGTAATTAGTTTAATTAAAACAAATGATTTCGACTCATTAGATTAT GATATACATTATAATTACCAAAA----

ATGTCATCTGTAACCTCCAATATTATATTAGCATTCTCATTCTCATTTCTAG GAACACTAGTATTTCGATCCCACCTAATATCAACTCTCCTATGCCTTGAAG GAATAATACTATCACTGTTTATTATAACCACAATTACATCCCTTAATTCCC ACTCAATAATTATATACCCTCTACCCATTGTTATTCTAGTATTCGCAGCATG TGAAGCAGCCATTGGCTTAGCCCTATTAGCAAAACTATCGAACTCCTACGG AACAGATTATGTGCAAAATCTAAACCTACTTCAATGT-

TAAAAATTATTTTTCCCTCTATCATATTACTCCCACTCACCTGATTATCAAA CAAAAAAAACCTATGAATCAATGTTACCTCCTACAGCTTTATAATCAGCTT GACTTCAGCTATGTTTTTATGACAAAACGACATAAATAATCTAAACTTCTC ACTACTATTCTCAACCGACTCCCTATCCTCCCCTCTCATTATTCTAACAACA TGACTTCTACCACTCATACTGCTAGCCAGCCAAAACCACATAAAAAAGGA AACAGAGTCAAACAAAAAAACATACATTTCAATACTAGTCTTACTACAAA TTCTTCTAATCATAACATTTTCTGCAAACGAACTAATTATATTTTACATCTT ATTTGAAGCCACCCTAATCCCCACCCTTATTATCATCACCCGATGGGGTAA CCAAACAGAACGACTAAATGCAGGACTCTACTTTCTATTTTATACCCTAAT TGGATCAATCCCACTACTAATCGCCCTTATCCACATTCAAAATATAAAAGG AACATTAAACTTCATACTATTCCCACTCACCTTTACACCCCTAGACCAAAC ATGGTCTAATAATATTCTATGATTAGCATGTATAATGGCATTTATGATTAA AATACCACTATACGGAGTCCACTTATGACTCCCTAAAGCCCAC 
sj7Pmausterus

CAGCTGACTTCCAATCAGTTAGATCTAGACCTAACCTAGAAGAAAGTAAT AAATATACTAACAGCCCTATTAGTAAATATTACGCTATCAATACTCCTAAT CATCATTGCCTTCTGACTACCCCAACTTAATTTATATACTGAAAAAGCAAA CCCGTATGAGTGCGGATTTGACCCTATAGGCTCTGCTCGCCTCCCATTCTC AATAAAATTTTTCCTAGTAGCAATCACTTTCTTACTATTTGACCTAGAAATT GCACTTCTACTTCCACTACCATGAGCTATCCAAATATATAATATTAACATC ATAATATTAACAGCTTTCATCCTAGTCTCCGTATTAGCATTAGGCTTAGCCT ACGAGTGACTACAAAAAGGACTAGAATGAACTGAA-

TAACTGGTAATTAGTTTAATTAAAACAAATGATTTCGACTCATTAGATTAT GATATACATTATAATTACCAAAA----

ATGTCATCTGTAACCTCCAATATTATATTAGCATTCTCATTCTCATTTCTAG GAACACTAGTATTTCGATCCCACCTAATATCAACTCTCCTATGCCTTGAAG GAATAATACTATCACTGTTTATTATAACCACAATTACATCCCTTAATTCCC ACTCAATAATTATATACCCTCTACCCATTGTTATTCTAGTATTCGCAGCATG TGAAGCAGCCATTGGCTTAGCCCTATTAGCAAAACTATCGAACTCCTACGG AACAGATTATGTGCAAAATCTAAACCTACTTCAATGT-

TAAAAATTATTTTTCCCTCTATCATATTACTCCCACTCACCTGATTATCAAA CAAAAAAAACCTATGAATCAATGTTACCTCCTACAGCTTTATAATCAGCTT GACTTCAGCTATGTTTTTATGACAAAACGACATAAATAATCTAAACTTCTC ACTACTATTCTCAACCGACTCCCTATCCTCCССТCTCATTATTCTAACAACA TGACTTCTACCACTCATACTGCTAGCCAGCCAAAACCACATAAAAAAGGA AACAGAGTCAAACAAAAAAACATACATTTCAATACTAGTCTTACTACAAA TTCTTCTAATCATAACATTTTCTGCAAACGAACTAATTATATTTTACATCTT ATTTGAAGCCACCCTAATCCCCACCCTTATTATCATCACCCGATGGGGTAA CCAAACAGAACGACTAAATGCAGGACTCTACTTTCTATTTTATACCCTAAT TGGATCAATCCCACTACTAATCGCCCTTATCCACATTCAAAATATAAAAGG AACATTAAACTTCATACTATTCCCACTCACCTTTACACCCCTAGACCAAAC ATGGTCTAATAATATTCTATGATTAGCATGTATAATGGCATTTATGATTAA AATACCACTATACGGAGTCCACTTATGACTCCCTAAAGCCCACGT

OSM723Pmman

CGAACTAGTACAGCTGACTTCCAATCAGTTAGATCTAGACCTAAGCTAGG AGAAAGTAATAAATATACTAACAGCCCTATTAGTAAATATTACACTATCA ATACTCCTAATCATCATTGCCTTCTGACTACCACAACTTAATTTATACACTG AAAAAGCAAACCCGTACGAATGCGGGTTTGACCCCATAGGCTCTGCTCGC CTCCCATTCTCAATAAAGTTTTTCCTAGTAGCAATCACTTTCTTACTATTTG ACCTAGAAATTGCACTTCTACTTCCACTACCATGAGCTATCCAAATATATA ATATTAACACCATAATATTAACAGCTTTCATCCTAATCTCCGTATTAGCATT GAGCCTAGCCTACGAATGACTACAAAAAGGACTAGAATGAACTGAATAACTGGTAATTAGTTTAACTAAAACAAATGATTTCGACTCATTAGATTAT AATAAATATTATAATTACCAAAA----

ATGCCATCTGTAACCTCCAATATTATATTAGCATTCTCGTTCTCATTCCTAG GAACACTAGTATTTCGATCCCACCTAATATCAACTCTCCTGTGCCTTGAAG GAATAATACTATCACTGTTTATTATAACCACAATTACATCCCTTAATTCCC 
ACTCAATAATTATATACCCCCTACCCATTGTTATTCTAGTATTCGCAGCATG TGAAGCAGCTATTGGCTTAGCCTTGCTAGCAAAACTATCAAACTCCTACGG AACAGATTATGTGCAAAATCTAAACCTACTTCAATGC-

TAAAAATTATTCTCCCCTCTATCATATTACTCCCACTCACCTGATTATCAAA CAAAAAAAACCTATGAATCAATGTTACCTCCTACAGCTTTATAATCAGCTT GGCTTCAGCTATGCTTTTATGACAAAACGACATAAATAATTTAAACTTCTC ATTACTATTCTCAACTGACTCCCTATCCTCCCCTCTTATTATTTTAACAACA TGACTACTACCACTCATACTGCTAGCCAGCCAAAACCACATAAAAGAGGA AACAGAGTCAAACAAAAAAACCTATATTTCAATACTAGTCTTACTACAAA TTCTTCTAATCATAACATTTTCTGCAAACGAACTAATTATATTCTACATCTT ATTTGAAGCCACCCTGATCCCCACCCTTATTATCATCACCCGATGGGGTAA CCAAACAGAACGACTAAATGCAGGACTCTACTTTCTATTTTATACCCTAAT TGGATCAATCCCTCTACTAATTGCCCTTATCTACATTCAAAATATAAAAGG AACATTAAACTTCATACTATTCCCACTCACCTTTACACCCCTAGACCAAAC ATGGTCTAATAATATTCTATGATTAGCATGCATAATGGCATTTATAATTAA AATACCACTATACGGAGTCCACTTATGACTCCCTAAAGCCCACGTAGAAG CTCCA--

OSM726Pmman

CGAACTAGTACAGCTGACTTCCAATCAGTTAGATCTAGACCTAACCTAGGA GAAAGTAATAAATATACTAACAGCCCTATTAGTAAATATTACACTATCAAT ACTCCTAATCATCATTGCCTTCTGACTACCACAACTTAATTTATACACTGA AAAAGCAAACCCGTACGAATGCGGGTTTGACCCCATAGGCTCTGCTCGCC TCCCATTCTCAATAAAGTTTTTCCTAGTAGCAATCACTTTCTTACTATTTGA CCTAGAAATTGCACTTCTACTTCCACTACCATGAGCTATCCAAATATATAA TATTAACACCATAATATTAACAGCTTTCATCCTAATCTCCGTATTAGCATTG AGCCTAGCCTACGAATGACTACAAAAAGGACTAGAATGAACTGAATAACTGGTAATTAGTTTAACTAAAACAAATGATTTCGACTCATTAGATTAT AATAAATATTATAATTACCAAAA----

ATGCCATCTGTAACCTCCAATATTATATTAGCATTCTCGTTCTCATTCCTAG GAACACTAGTATTTCGATCCCACCTAATATCAACTCTCCTGTGCCTTGAAG GAATAATACTATCACTGTTTATTATAACCACAATTACATCCCTTAATTCCC ACTCAATAATTATATACCCCCTACCCATTGTTATTCTAGTATTCGCAGCATG TGAAGCAGCTATTGGCTTAGCCTTGCTAGCAAAACTATCAAACTCCTACGG AACAGATTATGTGCAAAATCTAAACCTACTTCAATGC-

TAAAAATTATTCTCCCCTCTATCATATTACTCCCACTCACCTGATTATCAAA CAAAAAAAACCTATGAATCAATGTTACCTCCTACAGCTTTATAATCAGCTT GGCTTCAGCTATGCTTTTATGACAAAACGACATAAATAATTTAAACTTCTC ATTACTATTCTCAACTGACTCCCTATCCTCCCCTCTTATTATTTTAACAACA TGACTACTACCACTCATACTGCTAGCCAGCCAAAACCACATAAAAGAGGA AACAGAGTCAAACAAAAAAACCTATATTTCAATACTAGTCTTACTACAAA TTCTTCTAATCATAACATTTTCTGCAAACGAACTAATTATATTCTACATCTT ATTTGAAGCCACCCTGATCCCCACCCTTATTATCATCACCCGATGGGGTAA CCAAACAGAACGACTAAATGCAGGACTCTACTTTCTATTTTATACCCTAAT TGGATCAATCCCTCTACTAATTGCCCTTATCTACATTCAAAATATAAAAGG 
AACATTAAACTTCATACTATTCCCACTCACCTTTACACCCCTAGACCAAAC ATGGTCTAATAATATTCTATGATTAGCATGCATAATGGCATTTATAATTAA AATACCACTATACGGAGTCCACTTATGACTCCCTAAAGCCCACGTAGAAG CTCCA--

OSM727Pmman

CGAACTAGTACAGGTGACTTCCAATCAGTTAGATCTAGACCTAACCTAGG AGAAAGTAATAAATATACTAACAGCCCTATTAGTAAATATTACACTATCA ATACTCCTAATCATCATTGCCTTCTGACTACCACAACTTAATTTATACACTG AAAAAGCAAACCCGTACGAATGCGGGTTTGACCCCATAGGCTCTGCTCGC CTCCCATTCTCAATAAAGTTTTTCCTAGTAGCAATCACTTTCTTACTATTTG ACCTAGAAATTGCACTTCTACTTCCACTACCATGAGCTATCCAAATATATA ATATTAACACCATAATATTAACAGCTTTCATCCTAATCTCCGTATTAGCATT GAGCCTAGCCTACGAATGACTACAAAAAGGACTAGAATGAACTGAA-

TAACTGGTAATTAGTTTAACTAAAACAAATGATTTCGACTCATTAGATTAT AATAAATATTATAATTACCAAAA----

ATGCCATCTGTAACCTCCAATATTATATTAGCATTCTCGTTCTCATTCCTAG GAACACTAGTATTTCGATCCCACCTAATATCAACTCTCCTGTGCCTTGAAG GAATAATACTATCACTGTTTATTATAACCACAATTACATCCCTTAATTCCC ACTCAATAATTATATACCCCCTACCCATTGTTATTCTAGTATTCGCAGCATG TGAAGCAGCTATTGGCTTAGCCTTGCTAGCAAAACTATCAAACTCCTACGG AACAGATTATGTGCAAAATCTAAACCTACTTCAATGC-

TAAAAATTATTCTCCCCTCTATCATATTACTCCCACTCACCTGATTATCAAA CAAAAAAAACCTATGAATCAATGTTACCTCCTACAGCTTTATAATCAGCTT GGCTTCAGCTATGCTTTTATGACAAAACGACATAAATAATTTAAACTTCTC ATTACTATTCTCAACTGACTCCCTATCCTCCCCTCTTATTATTTTAACAACA TGACTACTACCACTCATACTGCTAGCCAGCCAAAACCACATAAAAGAGGA AACAGAGTCAAACAAAAAAACCTATATTTCAATACTAGTCTTACTACAAA TTCTTCTAATCATAACATTTTCTGCAAACGAACTAATTATATTCTACATCTT ATTTGAAGCCACCCTGATCCCCACCCTTATTATCATCACCCGATGGGGTAA CCAAACAGAACGACTAAATGCAGGACTCTACTTTCTATTTTATACCCTAAT TGGATCAATCCCTCTACTAATTGCCCTTATCTACATTCAAAATATAAAAGG AACATTAAACTTCATACTATTCCCACTCACCTTTACACCCCTAGACCAAAC ATGGTCTAATAATATTCTATGATTAGCATGCATAATGGCATTTATAATTAA AATACCACTATACGGAGTCCACTTATGACTCCCTAAAGCCCACGTAGAAG CTCCA--

OSM737Pmman

CGAACTAGTACAGCTGACTTCCAATCAGTTAGATCTAGACCTAACCTAGGA GAAAGTAATAAATATACTAACAGCCCTATTAGTAAATATTACACTATCAAT ACTCCTAATCATCATTGCCTTCTGACTACCACAACTTAATTTATACACTGA AAAAGCAAACCCGTACGAATGCGGGTTTGACCCCATAGGCTCTGCTCGCC TCCCATTCTCAATAAAGTTTTTCCTAGTAGCAATCACTTTCTTACTATTTGA CCTAGAAATTGCACTTCTACTTCCACTACCATGAGCTATCCAAATATATAA TATTAACACCATAATATTAACAGCTTTCATCCTAATCTCCGTATTAGCATTG 
AGCCTAGCCTACGAATGACTACAAAAAGGACTAGAATGAACTGAATAACTGGTAATTAGTTTAACTAAAACAAATGATTTCGACTCATTAGATTAT AATAAATATTATAATTACCAAAA----

ATGCCATCTGTAACCTCCAATATTATATTAGCATTCTCGTTCTCATTCCTAG GAACACTAGTATTTCGATCCCACCTAATATCAACTCTCCTGTGCCTTGAAG GAATAATACTATCACTGTTTATTATAACCACAATTACATCCCTTAATTCCC ACTCAATAATTATATACCCCCTACCCATTGTTATTCTAGTATTCGCAGCATG TGAAGCAGCTATTGGCTTAGCCTTGCTAGCAAAACTATCAAACTCCTACGG AACAGATTATGTGCAAAATCTAAACCTACTTCAATGC-

TAAAAATTATTCTCCCCTCTATCATATTACTCCCACTCACCTGATTATCAAA CAAAAAAAACCTATGAATCAATGTTACCTCCTACAGCTTTATAATCAGCTT GGCTTCAGCTATGCTTTTATGACAAAACGACATAAATAATTTAAACTTCTC ATTACTATTCTCAACTGACTCCCTATCCTCCCCTCTTATTATTTTAACAACA TGACTACTACCACTCATACTGCTAGCCAGCCAAAACCACATAAAAGAGGA AACAGAGTCAAACAAAAAAACCTATATTTCAATACTAGTCTTACTACAAA TTCTTCTAATCATAACATTTTCTGCAAACGAACTAATTATATTCTACATCTT ATTTGAAGCCACCCTGATCCCCACCCTTATTATCATCACCCGATGGGGTAA CCAAACAGAACGACTAAATGCAGGACTCTACTTTCTATTTTATACCCTAAT TGGATCAATCCCTCTACTAATTGCCCTTATCTACATTCAAAATATAAAAGG AACATTAAACTTCATACTATTCCCACTCACCTTTACACCCCTAGACCAAAC ATGGTCTAATAATATTCTATGATTAGCATGCATAATGGCATTTATAATTAA AATACCACTATACGGAGTCCACTTATGACTCCCTAAAGCCCACGTAGAAG CTCCA--

U40255PsejugSD (GenBank) AACTAGTACAGCTGACTTCCAATCAGTTAGATCTAGACTTAACCTAGAAGA AAGTAATAAATATACTAACAGCTTTATTAGTAAATATTACGCTATCAATAC TCCTAATCATCGTTGCTTTCTGATTTTTCCAACTTAATTTATACACTGAAAA AGCAAATCCGTATGAATGCGGATTTGACCCCATAGGCTCTGCCCGTCTCCC ATTCTCAATAAAATTCTTTCTAGTAGCAATCACTTTCTTATTATTTGACCTA GAAATTGCACTTCTACTTCCACTGCCATGAGCTATCCAAATATATAATACT AACATCATAATATTAACAGCTTTCATCCTAATCTCCGTATTAGCACTAGGC CTAGCCTACGAATGACTACAAAAAGGACTAGAATGAACTGAA-

TAACTGGTAATTAGTTTAATTAAAACAAATGATTTCGACTCATTAGATTAT AATAAATATTATAATTACCAAAA----

ATGCCGTCTGTAACCTCCAATATTATATTAGCATTCTCATTTTCATTTCTAG GAACACTAGTATTTCGAGCCCACCTAATATCGACTCTCCTATGCCTTGAAG GAATAATATTATCACTGTTTATTATAACCACAATTACATCCCTTAATTCCCA CTCAATAATTATATACCCCCTACCCATTGTTATTCTAGTATTCGCAGCATGT GAAGCAGCTATTGGCTTAGCCTTGTTAGCAAAAGTATCAAACTCCTACGGA ACAGATTATGTGCAAAACCTAAACCTACTTCAATGT-

TAAAAATTATTTTTCCCTCTATCATATTACTTCCACTCACCTGATTATCAAA CAAAAAAAACCTATGAGTCAATGTTACCTCCTACAGCTTTATAATCAGCTT GACTTCAGCTATGTTTTTATGACAAAACGACATAAATAATTTAAACTTCTC ACTACTATTCTCAACTGACCCCCTATCCTCCCCTCTTATTATTTTAACAACA 304 
TGACTACTGCCACTCATACTGCTAGCCAGCCAAAACCACATAAAAAAGGA AACAGAGTTAAACAAAAAAACCTATATTTCAATACTAGTATTACTACAAA TTCTTCTAATCATAACATTCTCTGCAAATGAACTAATTATATTTTATATCTT ATTTGAAGCTACCCTAATCCCCACCCTTATTATCATCACCCGATGGGGTAA CCAAACAGAACGACTAAATGCAGGACTCTACTTTCTATTTTATACTCTAAT TGGATCAATCCCACTACTAATTGCCCTTATTTACATTCAAAATATAAAAGG AACATTAAACTTCATACTATTCCCACTCACCTTTACACCCCTAGACCAAAC ATGGTCTAATAATATTCTATGATTAGCATGCATAATGGCATTTATAATTAA AATGCCACTATACGGAGTCCACTTATGACTCCCTAAAGCCCACGTAGAAG CTCCA--

U40253PsejugSC (GenBank)

AACTAGTACAGCTGACTTCCAATCAGTTAGATCTAGACTTAACCTAGAAGA AAGTAATAAATATACTAACAGCTTTATTAGTAAATATTACGCTATCAATAC TCCTAATCATCGTTGCTTTCTGATTTTTCCAACTTAATTTATACACTGAAAA AGCAAATCCGTATGAATGCGGATTTGACCCCATAGGCTCTGCCCGTCTCCC ATTCTCAATAAAATTCTTTCTAGTAGCAATCACTTTCTTATTATTTGACCTA GAAATTGCACTTCTACTTCCACTGCCATGAGCTATCCAAATATATAATACT AACATCATAATATTAACAGCTTTCATCCTAATCTCCGTATTAGCACTAGGC CTAGCCTACGAATGACTACAAAAAGGACTAGAATGAACTGAATAACTGGTAATTAGTTTAATTAAAACAAATGATTTCGACTCATTAGATTAT AATAAATATTATAATTACCAAAA----

ATGCCGTCTGTAACCTCCAATATTATATTAGCATTCTCATTTTCATTTCTAG GAACACTAGTATTTCGAGCCCACCTAATATCGACTCTCCTATGCCTTGAAG GAATAATATTATCACTGTTTATTATAACCACAATTACGGTCCTTGACCCCC ACTCAATAATTATATGCGCCCTACCTATTGTTATTCTAGTATTCGCAGCATG TGAAGCAGCTATTGGCTTAGCCTTGTTAGCAAAAGTATCAAACTCCTACGG AACAGATTATGTGCAAAACCTAAACCTACTTCAATGT-

TAAAAATTATTTTTCCCTCTATCATATTACTTCCACTCACCTGATTATCAAA CAAAAAAAACCTATGAGTCAATGTTACCTCCTACAGCTTTATAATCAGCTT GACTTCAGCTATGTTTTTATGACAAAACGACATAAATAATTTAAACTTCTC ACTACTATTCTCAACTGACCCCCTATCCTCCCCTCTTATTATTTTAACAACA TGACTACTGCCACTCATACTGCTAGCCAGCCAAAACCACATAAAAAAGGA AACAGAGTTAAACAAAAAAACCTATATTTCAATACTAGTATTACTACAAA TTCTTCTAATCATAACATTCTCTGCAAATGAACTAATTATATTTTATATCTT ATTTGAAGCTACCCTAATCCCCACCCTTATTATCATCACCCGATGAGGTAA CCAAACAGAACGACTAAATGCAGGACTCTACTTTCTATTTTATACTCTAAT TGGATCAATCCCACTACTAATTGCCCTTATTTACATTCAAAATATAAAAGG AACATTAAACTTCATACTATTCCCACTCACCTTTACACCCCTAGACCAAAC ATGGTCTAATAATATTCTATGATTAGCATGCATAATGGCATTTATAATTAA AATGCCACTATACGGAGTCCACTTATGACTCCCTAAAGCCCACGTAGAAG CTCCA--

JEB1309APtruei (GenBank) AACTAGTACAGCTGACTTCCAATCAGTTAGATCTAGCCATAATCTAGAAGA 305 
AAGTAATAAACATACTTTTAGTCCTATCAGTAAACATTACTTTATCCTTAT GCCTAATTTTAATTGCCTTCTGACTACCACAACTAAATCTTTATACTGAAA AAGCAAACCCTTATGAATGTGGATTTGACCCTATAAGCTCGGCTCGCCTCC CCTTCTCCATAAAATTCTTCCTAGTAGCTATTACTTTCCTACTATTTGACCT AGAAATTGCACTACTACTACCACTACCCTGAGCTATTCAAATATACAACAT TAACACAACTATATTTGTATCTTTCATCCTAATCTCTATCCTAGCTCTAGGG CTTGCCTACGAATGAGTACAAAAAGGATTAGAATGAACTGAG-

TAGCTGGTAATTAGTTTAATAAAAACAAATGATTTCGACTCATTAAATTAT GGGTTACCCCATAATTACCAAA----

ATGCTATCTGTAACTTTCAACATTATATTAGCATTTATATTTTCATTTTTAG GAACACTAGTATTCCGATCCCACCTTATGTCTACACTACTATGCCTAGAGG GAATAATATTATCACTGTTTATTATAACCACAATTACATCCCTAAACTCCC ACTCAATAAATATATACCCGATTCCTATTGTTATTTTAGTGTTTGCAGCATG CGAAGCGGCCATTGGCCTAGCTCTGCTAGCAAAACTATCAAACTCCTAC??-

AF374578PKEEN (GenBank)

AACTAGTACAACTGACTTCCAATCAGTTAAATCTAGACTTAACCTAGAAGA AAGTAATAAACATACTAACAGCTTTATTAGTAAATATTACACTATCAATAC TCCTAATCATCGTTGCTTTCTGATTACCCCAACTTAATTTATATACTGAAAA AGCAAACCCGTATGAATGCGGATTTGACCCCATAGGCTCTGCTCGCCTCCC ATTCTCAATAAAATTTTTGCTAGTAGCAATCACTTTCTTATTATTTGACCTA GAAATTGCACTTCTACTTCCACTGCCATGAGCTATCCAAATATGTAATATT AACATCATAATATTAACAGCTTTCATCCTAGTCTCCGTATTAGCACTAGGC CTAGCCTACGAATGACTACAAAAAGGATTAGAATGAACTGAG-

TAACTGGTAATTAGTTTAATTAAAACAAATGATTTCGACTCATTAGATTAT GATAAATATTATAATTACCAAAA----

ATGCCGTCTGTAACCTCCAATATTATATTAGCATTCTCATTTTCATTTCTAG GAACACTAGTATTTCGATCCCACCTAATATCGACTCTCCTATGCCTTGAAG GAATAATATTATCACTGTTTATTATAACCACAATTACATCCCTTACCTCCTA CTCAATAATTATATGCCCCCTACCCATTGTTATTCTAGTATTCGCAGCATGT GAAGCAGCCATTGGCCTGGCCTTATTAGCAAAAGTATCAAACTCCTACGG AACAGATTATGTACAAAATCTAAACCTACTTCAATGT-

TAAAAATTATTTTCCCCTCTATCATATTACTCCCACTCACCTGATTATCAAA CAAAAAAAACCTATGAATCAATGTTACCTCCTACAGCTTTATAATCAGCTT GACTTCAGCTATGTTTTTATGACAAAACGACATAAATAATTTAAACTCCTC ACTACTATTTTCAACTGACTCCCTATCCTCCCCTCTTATTATTTTAACAACA TGACTACTACCACTCATACTGCTAGCCAGCCAAAACCACATAAAAAAGGA AACAGAGTCAAACAAAAAAACCTACATTTCAATACTAGTCTTATTACAAA 
TTCTTCTAATCATAACATTCTCTGCAAATGAGCTAATTATATTTTATATCTT ATTTGAAGCCACCCTGATTCCTACCCTGATTATCATCACCCGATGAGGTAA CCAAACAGAACGACTAAATGCAGGACTCTACTTTCTATTTTATACTCTAAT TGGATCAATCCCACTACTAATTGCCCTTATTTATATTCAAAATATAAAAGG AACATTAAACTTCATACTATTCCCACTCACCTTTACACCCCTAGACCAAAC ATGGTCTAATTATATTCTATGATTAGCATGCATAATGGCATTTATGATTAA AATACCACTATACGGAGTCCACTTATGACTCCCTAAAGCCCACGTAGAAG CTCCA--

U40252Pleuc (GenBank)

AACTAGTACAGCTGACTTCCAATCAGTTAGATCTAGACTCAACCTAGAAG ATAGTAATAAACATACTGATAGCTCTAATAGTAAACATCACACTATCAATC CTCCTAATCACAGTTGCCTTCTGACTACCCCAACTCAATCTATATACTGAA AAAGCAAATCCATACGAATGCGGGTTTGATCCAATAAGCTCCGCCCGCCT CCCATTTTCAATAAAATTTTTTCTAGTAGCAATCACTTTTTTACTATTTGAC CTAGAAATTGCACTACTACTACCACTACCATGAGCCATCCAAATATATAAC ACCAACACCATAATATTAACAGCCTTTATTCTAGTCTCTGTGTTAGCCCTA GGCCTAGCCTACGAATGAATACAAAAGGGGTTAGAATGAACTGAG-

TAGCTGGTAATTAGTTTAATTAACACAAATGATTTCGACTCATTAGATTAT AATAAATATTATAATTACCAAAA----

ATGCCATCTGTTACCTCCAATATTATCCTAGCATTCTCATTTTCATTCTTAG GAACTCTAGTGTTTCGCTCTCACCTCATATCCACCTTACTATGCCTAGAAG GGATAATGTTATCCCTATTCATTATAACAACAATTACTTCCCTCAACTCCC ACTCAATAATCATATGCCCTATGCCCATTGTTATCTTAGTATTCGCAGCAT GTGAAGCAGCTATTGGCCTAGCCCTGCTAGCAAAAGTATCAAATTCATATG GAACAGACTATGTACAAAACCTAAACCTACTTCAATGC-

TAAAAATCATTTTTCCTTCAATTATACTACTCCCGCTCACCTGACTATCAAA CAACAAAAGTCTGTGAGCCAATGTCACTTCATACAGCTTTTTAATCAGCTT GGCCTCAGCCATATTTTTATGACAAAATGATATAAACAACTTAAACTTCTC ATTACTATTCTCAACTGACTCACTATCCTCCCCCCTTATTATCCTAACAACA TCATTATTACCACTAATATTACTAGCTAGTCAGAACCACATAAAAAAAGA AACCGAACTAAACAATAAAATCTACATCTCAATACTAACTCTCCTACAAAT CCTCCTAATCATAACTTTTTCTGCAAACGAACTAATTATATTTTATATCTTA TTCGAAGCTACATTAATTCCTACCCTTATTATTATCACCCGCTGAGGTAATC AAACAGAACGACTAAATGCAGGACTCTACTTTCTATTCTATACCCTAATTG GATCAATCCCGCTACTAATTGCTCTTATCTTCATCCAAAACTCAAAAGGAA CACTAAACTTCCTTCTATTCCCACTAACTTTCACACCCCTAAATCAAACAT GATCTAACAACATTCTATGATTAGCGTGCATAATGGCATTCATAATTAAAA TACCACTATACGGGGTCCACCTATGACTCCCCAAAGCCCACGTAGAAGCTC CA--

JAM160Pleuc

CTGACTTCCAATCAGTTAGATCTAGATTCAACCTAGAAGATAGTAATAAAC ATACTGATAGCCCTAATAGTAAACATCACACTATCAATCCTCCTAATCACA GTTGCCTTCTGACTACCCCAACTCAATCTATATACTGAAAAAGCAAATCCA 
TACGAATGCGGGTTTGATCCAATAAGCTCCGCCCGCCTCCCATTTTCAATA AAATTTTTTCTAGTAGCAATCACCTTTTTACTATTTGACCTAGAAATTGCAC TACTTCTACCACTACCATGAGCCATCCAAATATATAACACCAACACTATAA TATTAACAGCTTTTATTCTAGTCTCCGTGTTAGCCCTAGGCCTAGCCTACGA ATGAATACAAAAGGGGTTAGAATGAACTGAG-

TAGTTGGTAATTAGTTTAATTAAAACAAATGATTTCGACTCATTAGATTAT GATAAATATTATAATTACCAAAA----

ATGCCATCTGTGACCTCCAATATTATACTAGCATTCTCATTTTCATTCTTAG GGACTCTAGTGTTTCGCTCTCACCTCATATCCACCTTACTATGCCTAGAAG GGATAATGCTATCCCTATTCATTATAACAACAATTACTTCCCTCAACTCCC ACTCAATAATCATATACCCTATCCCCATTGTTATCTTAGTATTCGCAGCATG TGAAGCAGCCATTGGCCTAGCCCTGCTAGCAAAACTATCAAACTCCTACG GAACAGATTATGTGCAAAACCTAAACCTACTTCAATGT-

TAAAAATCATTTTTCCTTCAATCATATTACTTCCGCTCACCTGATTATCAAA CAACAAAAGCCTGTGAGCCAATGTCACTTCATACAGCTTTTTAATCAGCTT GGCCTCAGCCATATTTTTATGACAAAACGACATAAACAATTTAAACTTCTC ACTACTATTCTCAACTGACTCCCTATCCTCCCCCCTTATTATTCTAACAACA TGATTACTACCACTAATACTACTAGCCAGTCAAAACCACATAAAAAAAGA AACCGAACTAAACAAAAAAATCTACATCTCAATACTAACTCTCCTACAAA TCCTCCTAATCATAACTTTTTCTGCAAACGAGCTAATTATATTTTATATCTT ATTTGAAGCCACATTAATCCCTACCCTTATTATTATTACCCGCTGAGGTAA CCAAACAGAACGACTTAATGCAGGACTCTACTTTCTATTCTACACCCTAAT TGGATCAATCCCACTACTAATTGCTCTTATCTTCATCCAAAACTCAAAAGG AACACTAAACTTCCTTCTATTCCCACTAACTTTCACACCCCTAAATCAAAC ATGATCTAACAACATTCTATGATTAGCGTGCATAATGGCATTCATAATTAA AATACCACTATACGGGGTCCACCTATGACTCCCCAAAGCCCACGTAGAAG-

JAM161Pleuc

TAGTACAGCTGACTTCCAATCAGTTAGATCTAGATTCAACCTAGAAGATAG TAATAAACATACTGATAGCCCTAATAGTAAACATCACACTATCAATCCTCC TAATCACAGTTGCCTTCTGACTACCCCAACTCAATCTÄTATACTGAAAAAG CAAATCCATACGAATGCGGGTTTGATCCAATAAGCTCCGCCCGCCTCCCAT TTTCAATAAAATTTTTTCTAGTAGCAATCACCTTTTTACTATTTGACCTAGA AATTGCACTACTTCTACCACTACCATGAGCCATCCAAATATATAACACCAA CACTATAATATTAACAGCTTTTATTCTAGTCTCCGTGTTAGCCCTAGGCCTA GCCTACGAATGAATACAAAAGGGGTTAGAATGAACTGAG-

TAGTTGGTAATTAGTTTAATTAAAACAAATGATTTCGACTCATTAGATTAT GATAAATATTATAATTACCAAAG----

ATGCCATCTGTGACCTCCAATATTATACTAGCATTCTCATTTTCATTCTTAG GGACTCTAGTGTTTCGCTCTCACCTCATATCCACCTTACTATGCCTAGAAG GGATAATGCTATCCCTATTCATTATAACAACAATTACTTCCCTCAACTCCC ACTCAATAATCATATACCCTATCCCCATTGTTATCTTAGTATTCGCAGCATG TGAAGCAGCCATTGGCCTAGCCCTGCTAGCAAAACTATCAAACTCCTACG GAACAGATTATGTGCAAAACCTAAACCTACTTCAATGT- 
TAAAAATCATTTTTCCTTCAATCATACTACTTCCGCTCACCTGACTATCAAA CAACAAAAGCCTGTGAGCCAATGTCACTTCATACAGCTTTTTAATCAGCTT GGCCTCAGCCATATTTTTATGACAAAACGATATAAACAATTTAAACTTCTC ATTACTATTCTCAACTGACTCACTATCCTCCCCCCTTATTATTCTAACAACA TGATTACTACCACTAATACTACTAGCTAGTCAGAACCACATAAAAAAAGA AACCGAACTAAACAAGAAAATCTACATCTCAATACTAACTCTCCTACAAA TCCTCCTAATCATAACTTTTTCTGCAAACGAGCTAATTATATTTTATATCTT ATTTGAAGCTACATTAATCCCCACCCTTATTATTATTACCCGCTGAGGTAA TCAAACAGAACGACTTAATGCAGGACTCTACTTTCTATTCTACACCCTAAT TGGATCAATCCCACTACTAATTGCTCTTATCTTCATCCAAAACTCAAAAGG AACACTAAACTTCCTTCTATTCCCACTAACTTTCACACCCCTAAATCAAAC ATGATCTAACAACATTCTATGATTAGCGTGCATAATGGCATTCATAATTAA AATACCACTATACGGGGTCCACCTATGACTCCCCAAAGCCCACGTAGAAG-

JAM162Pleuc

GCTGACTTCCAATCAGTTAGATCTAGATTCAACCTAGAAGATAGTAATAAA CATACTGATAGCCCTAATAGTAAACATCACACTATCAATCCTCCTAATCAC AGTTGCCTTCTGACTACCACAACTCAATCTATATACTGAAAAAGCAAATCC ATACGAATGCGGGTTTGATCCAATAAGCTCCGCCCGCCTCCCATTTTCAAT AAAATTTTTTCTAGTAGCAATCACCTTTTTACTATTTGACCTAGAAATTGCA CTACTTCTACCACTACCATGAGCCATCCAAATATATAACACCAACACTATA ATATTAACAGCTTTTATTCTAGTCTCCGTGTTAGCCCTAGGCCTAGCCTACG AATGAATACAAAAGGGGTTAGAATGAACTGAG-

TAGTTGGTAATTAGTTTAATTAAAACAAATGATTTCGACTCATTAGATTAT GATAAATATTATAATTACCAAAA---

ATGCCATCTGTGACCTCCAATATTATACTAGCATTCTCATTTTCATTCTTAG GGACTCTAGTGTTTCGCTCTCACCTCATATCCACCTTACTATGCCTAGAAG GGATAATGCTATCCCTATTCATTATAACAACAATCACTTCCCTCAACTCCC ACTCAATAATCATATACCCTATCCCCATTGTTATCTTAGTATTCGCAGCATG TGAAGCAGCCATTGGCCTAGCCCTGCTAGCAAAACTATCAAACTCCTACG GAACAGATTATGTGCAAAACCTAAACCTACTTCAATGT-

TAAAAATCATTTTTCCCTCTATCATACTACTTCCGCTCACCTGATTATCAAA CAACAAAAGCCTGTGAGCCAATGTCACTTCATACAGCTTTTTAATCAGCTT GGCCTCAGCCATATTTTTATGACAAAACGACATAAACAATTTAAACTTCTC ATTACTATTCTCAACTGACTCCCTATCCTCCCCCCTTATTATTCTAACAACA TGATTACTACCACTAATACTACTAGCTAGTCAAAACCACATAAAAAAAGA AACCGAACTAAACAAGAAAATCTACATCTCAATACTAACTCTCCTACAAA TCCTCCTAATCATAACTTTTTCTGCAAACGAGCTAATTATATTTTATATCTT ATTTGAAGCCACATTAATCCCTACCCTTATTATTATTACCCGATGAGGTAA TCAAACAGAACGACTTAATGCAGGACTCTACTTTCTATTCTACACCCTAAT TGGATCAATCCCACTACTAATTGCTCTTATCTTCATCCAAAACTCAAAAGG AACACTAAACTTCCTTCTATTCCCACTAACTTTCACACCCCTAAATCAAAC ATGATCTAACAACATTCTATGATTAGCGTGCATAATGGCATTCATAATTAA AATACCACTATACGGGGTCCACCTATGACTCCCCAAAGCCCACG- 
JAM163Pleuc

CTGACTTCCAATCAGTTAGATCTAGATTCAACCTAGAAGATAGTAATAAAC ATACTGATAGCCCTAATAGTAAACATCACACTATCAATCCTCCTAATCACA GTTGCCTTCTGACTACCCCAACTCAATCTATATACTGAAAAAGCAAATCCA TACGAATGCGGGTTTGATCCAATAAGCTCCGCCCGCCTCCCATTTTCAATA AAATTTTTTCTAGTAGCAATCACCTTTTTACTATTTGACCTAGAAATTGCAC TACTTCTACCACTACCATGAGCCATCCAAATATATAACACCAACACTATAA TATTAACAGCTTTTATTCTAGTCTCCGTGTTAGCCCTAGGCCTAGCCTACGA ATGAATACAAAAGGGGTTAGAATGAACTGAG-

TAGTTGGTAATTAGTTTAATTAAAACAAATGATTTCGACTCATTAGATTAT GATAAATATTATAATTACCAAAA----

ATGCCATCTGTGACCTCCAATATTATACTAGCATTCTCATTTTCATTCTTAG GGACTCTAGTGTTTCGCTCTCACCTCATATCCACCTTACTATGCCTAGAAG GGATAATGCTATCCCTATTCATTATAACAACAATTACTTCCCTCAACTCCC ACTCAATAATCATATACCCTATCCCCATTGTTATCTTAGTATTCGCAGCATG TGAAGCAGCCATTGGCCTAGCCCTGCTAGCAAAACTATCAAACTCCTACG GAACAGATTATGTGCAAAACCTAAACCTACTTCAATGT-

TAAAAATCATTTTTCCTTCTATCATACTACTTCCGCTCACCTGACTATCAAA CAACAAAAGCCTGTGAGCCAATGTCACTTCCTACAGCTTTTTAATCAGCTT GGCCTCAGCCATATTTTTATGACAAAACGATATAAATAATTTAAACTTCTC ATTACTATTCTCAACTGACTCCCTATCCTCCCCCCTTATTATTCTAACAACA TGATTACTACCACTAATACTGCTAGCTAGTCAGAACCACATAAAAAAAGA AACCGAACTAAACAAGAAAATCTACATCTCAATACTAACTCTCCTACAAA TCCTCCTAATCATAACTTTTTCTGCAAACGAGCTAATTATATTTTATATCTT ATTTGAAGCTACCTTAATCCCTACCCTTATTATTATTACCCGCTGAGGTAAT CAAACAGAACGACTTAATGCAGGACTCTACTTTCTATTCTACACCCTAATT GGATCAATCCCACTACTAATTGCTCTTATCTTCATCCAAAACTCAAAAGGA ACACTAAACTTCCTTCTATTCCCACTAACTTTCACACCCCTAAATCAAACA TGATCTAACAACATTCTATGATTAGCGTGCATAATGGCATTCATAATTAAA ATACCACTATACGGGGTCCACCTATGACTCCCCAAAGCCCACGTAGAA---$---$

JAM164Pleuc

CTGACTTCCAATCAGTTAGATCTAGATTCAACCTAGAAGATAGTAATAAAC ATACTGATAGCCCTAATAGTAAACATCACACTATCAATCCTCCTAATCACA GTTGCCTTCTGACTACCCCAACTCAATCTATATACTGAAAAAGCAAATCCA TACGAATGCGGGTTTGATCCAATAAGCTCCGCCCGCCTCCCATTTTCAATA AAATTTTTTCTAGTAGCAATCACCTTTTTACTATTTGACCTAGAAATTGCAC TACTTCTACCACTACCATGAGCCATCCAAATATATAACACCAACACTATAA TATTAACAGCTTTTATTCTAGTCTCCGTGTTAGCCCTAGGCCTAGCCTACGA ATGAATACAAAAAGGGTTAGAATGAACTGAG-

TAGTTGGTAATTAGTTTAATTAAAACAAATGATTTCGACTCATTAGATTAT GATAAATATTATAATTACCAAAA----

ATGCCATCTGTGACCTCCAATATTATACTAGCATTCTCATTTTCATTCTTAG GGACTCTAGTGTTTCGCTCTCACCTCATATCCACCTTACTATGCCTAGAAG 
GGATAATGCTATCCCTATTCATTATAACAACAATTACTTCCCTCAACTCCC ACTCAATAATCATATACCCTATCCCCATTGTTATCTTAGTATTCGCAGCATG TGAAGCAGCCATTGGCCTAGCCCTGCTAGCAAAACTATCAAACTCCTACG GAACAGATTATGTGCAAAACCTAAACCTACTTCAATGT-

TAAAAATCATTTTTCCTTCAATCATACTACTTCCGCTCACCTGACTATCAAA CAACAAAAGCCTGTGAGCCAATGTCACTTCATACAGCTTTTTAATCAGCTT GGCCTCAGCCATATTTTTATGACAAAACGATATAAACAATTTAAACTTCTC ATTACTATTCTCAACTGACTCCCTATCCTCCCCCCTTATTATTCTAACAACA TGATTACTACCACTAATACTACTAGCTAGTCAAAACCACATAAAAAAAGA AACCGAACTAAACAAGAAAATCTACATCTCAATACTAACTCTCCTACAAA TCCTCCTAATCATAACTTTTTCTGCAAACGAGCTAATTATATTTTATATCTT ATTTGAAGCTACATTAATCCCTACCCTTATTATTATTACCCGCTGAGGTAAT CAAACAGAACGACTTAATGCAGGACTCTACTTTCTATTCTACACCCTAATT GGATCAATCCCACTACTAATTGCTCTTATCTTCATCCAAAACTCAAAAGGA ACACTAAACTTCCTTCTATTCCCACTAACTTTCACACCCCTAAATCAAACA TGATCTAACAACATTCTATGATTAGCGTGCATAATGGCATTCATAATTAAA ATACCACTATACGGGGTCCACCTATGACTCCCCAAAGCCCACGTAGA------$-$

\section{JAM168Pmbairdii}

ACTAGTACAGCTGACTTCCAATCAGTTAGATCTAGACTTAACCTAGAAGAA AGTAATAAATATACTAACAGCCCTACTAGTAAATACTACTCTATCAATTCT CCTAATCACCATTGCCTTCTGACTACCCCAACTTAATTTATATACTGAAAA AGCAAACCCGTATGAGTGCGGATTTGACCCCATAGGCTCTGCTCGCCTCCC ATTCTCAATAAAATTCTTCCTAGTAGCAATCACTTTCTTACTATTTGACCTA GAAATTGCACTTCTACTTCCACTACCATGAGCTATCCAAATATATAATATT AACATCATAATATTAACAGCTTTCATCCTAGTCTCCGTATTAGCATTAGGC TTAGCCTACGAGTGACTACAAAAAGGACTAGAATGAACTGAG-

TAACTGGTAATTAGTTTAATTAAAACAAATGATTTCGACTCATTAGATTAT GATATATATTATAATTACCAAAA---

ATGTCATCTGTAACCTCCAATATTATATTAGCATTCTCATTCTCATTTCTAG GGACACTAGTATTTCGATCCCACCTAATATCAACTCTCCTATGCCTTGAAG GAATAATACTATCACTGTTTATTATAACCACAATTACATCCCTTAATTCCC ACTCAATAATTATATACCCTCTACCCATTGTTATTCTAGTATTCGCAGCATG TGAAGCAGCCATTGGCTTAGCCCTATTAGCAAAACTATCAAACTCCTACGG AACAGATTATGTGCAAAACCTAAACCTACTTCAATGT-

TAAAAATTATTTTTCCCTCTATCATATTACTCCCACTCACCTGATTATCAAA CAAAAAAAACCTATGAATCAATGTTACCTCCTACAGCTTTATAATCAGCTT GACTTCAGCTATGTTTTTATGACAAAACGACATAAATAATCTAAACTTCTC ACTACTATTCTCAACTGACTCCCTATCCTCCCCTCTTATTATTTTAACAACA TGACTTCTACCACTCATACTGCTAGCCAGCCAAAACCACATAAAAAAGGA AACAGAGTTAAACAAAAAAACATACATTTCAATACTAGTCTTACTACAAA TTCTTCTAATCATAACATTTTCTGCAAACGAACTAATTATATTTTACATCTT ATTTGAAGCCACCCTAATCCCCACCCTTATTATCATCACCCGATGGGGTAA CCAAACAGAACGACTAAATGCAGGACTCTACTTTCTATTTTATACCCTAAT 
TGGATCAATCCCACTACTAATCGCCCTTATCCACATTCAAAATATAAAAGG AACATTAAACTTCATACTATTCCCACTCACCTTCACACCCCTAGACCAAAC ATGGTCCAATAATATTCTATGATTAGCATGTATAATGGCATTTATAATTAA AATACCACTATACGGAGTCCACTTATGACTTCCTAAAGCCCACG-

JAM167Pmbairdii

GTACAGCTGACTTCCAATCAGTTAGATCTAGACTTAACCTAGAAGAAAGT AATAAATATACTAACAGCCCTACTAGTAAATATTACTCTATCAATTATCCT AATCACCATTGCCTTCTGACTACCCCAACTTAATTTATATACTGAAAAAGC AAACCCGTATGAGTGCGGATTTGACCCCATAGGCTCTGCTCGCCTCCCATT CTCAATAAAATTCTTCCTAGTAGCAATCACTTTCTTACTATTTGACCTAGAA ATTGCACTTCTACTTCCACTACCATGAGCTATCCAAATATATAATATTAAC ATCATAATATTAACAGCTTTCATCCTAGTCTCCGTATTAGCATTAGGCTTA GCCTACGAGTGACTACAAAAAGGACTAGAATGAACTGAG-

TAACTGGTAATTAGTTTAATTAAAACAAATGATTTCGACTCATTAGATTAT GATATAAATTATAATTACCAAAA---

ATGTCATCTGTAACCTCCAATATTATATTAGCATTCTCATTCTCATTTCTAG GGACACTAGTATTTCGATCCCACCTAATATCAACTCTCCTATGCCTTGAAG GAATAATACTATCACTGTTTATTATAACCACAATTACATCCCTTAATTCCC ACTCAATAATTATATACCCTCTACCCATTGTTATTCTAGTATTCGCAGCATG TGAAGCAGCCATTGGCTTAGCCCTATTAGCAAAACTATCAAACTCCTACGG AACAGATTATGTGCAAAACCTAAACCTACTTCAATGT-

TAAAAATTATTTTTCCCTCTATCATATTACTCCCACTCACCTGATTATCAAA CAAAAAAAACCTATGAATCAATGTTACCTCCTACAGCTTTATAATCAGCTT GACTTCAGCTATGTTTTTATGACAAAACGACATAAATAATCTAAACTTCTC ACTACTATTCTCAACTGACTCCCTATCCTCCCCTCTTATTATTTTAACAACA TGACTTCTACCACTCATACTGCTAGCCAGCCAAAACCACATAAAAAAGGA AACAGAGTTAAACAAAAAAACATACATTTCAATACTAGTCTTACTACAAA TTCTTCTAATCATAACATTTTCTGCAAACGAACTAATTATATTTTACATCTT ATTTGAAGCCACCCTAATCCCCACCCTTATTATCATCACCCGATGGGGTAA CCAAACAGAACGACTAAATGCAGGACTCTACTTTCTATTTTATACCCTAAT CGGATCAATCCCACTACTAATCGCCCTTATCCACATTCAAAATATAAAAGG AACATTAAACTTCATACTATTCCCACTCACCTTCACACCCCTAGACCAAAC ATGGTCCAATAATATTCTATGATTAGCATGTATAATGGCATTTATAATTAA AATACCACTATACGGAGTCCACTTATGACTTCCTAAAGCCCAC-

JAM159Pmbairdii

CAGCTGACTTCCAATCAGTTAGATCTAGACTTAACCTAGAAGAAAGTAAT AAATATACTAACAGCCCTACTAGTAAATATTACTCTATCAATTCTCCTAAT CACCATTGCCTTCTGACTACCCCAACTTAATTTATATACTGAAAAAGCAAA CCCGTATGAGTGCGGATTTGACCCCATAGGCTCTGCTCGCCTCCCATTTTC AATAAAATTCTTCCTAGTAGCAATCACTTTCTTACTATTTGACCTAGAAATT GCACTTCTACTTCCACTACCATGAGCTATCCAAATATATAATATTAACATC ATAATATTAACAGCTTTCATCCTAGTCTCCGTATTAGCATTAGGCTTAGCCT ACGAGTGACTACAAAAAGGACTAGAATGAACTGAA- 
TAACTGGTAATTAGTTTAATTAAAACAAATGATTTCGACTCATTAGATTAT GATATATATTATAATTACCAAAA----

ATGTCATCTGTAACCTCCAATATTATATTAGCATTCTCATTCTCATTTCTAG GGACACTAGTATTTCGATCCCACCTAATATCAACCCTCCTATGCCTTGAAG GAATAATACTATCACTGTTTATTATAACCACAATTACATCCCTTAATTCCC ACTCAATAATTATATACCCTCTACCCATTGTTATTCTAGTATTCGCAGCATG TGAAGCAGCCATTGGCTTAGCCCTATTAGCAAAACTATCAAACTCCTACGG AACAGATTATGTGCAAAACCTAAACCTACTTCAATGT-

TAAAAATTATTTTTCCCTCTATCATATTACTCCCACTCACCTGATTATCAAA CAAAAAAAACCTATGAATCAATGTTACCTCCTACAGCTTCATAATCAGCTT GACTTCAGCTATGTTTTTATGACAAAACGACATAAATAATCTAAACTTCTC ACTACTATTCTCAACTGACTCCCTATCCTCCCCTCTTATTATTTTAACAACA TGACTTCTACCACTCATACTGCTAGCCAGCCAAAACCACATAAAAAAGGA AACAGAGTCAAACAAAAAAACATACATTTCAATACTAGTCTTACTACAAA TTCTTCTAATCATAACATTTTCTGCAAACGAACTAATTATATTTTACATCTT ATTTGAAGCCACCCTAATCCCCACCCTTATTATCATCACCCGATGGGGTAA CCAAACAGAACGACTAAATGCAGGACTCTACTTTCTATTTTATACCCTAAT TGGATCAATCCCACTACTAATCGCCCTTATCCACATTCAAAATATAAAAGG AACATTAAACTTCATACTATTCCCACTCACCTTCACACCCCTAGACCAAAC ATGGTCCAATAATATTCTATGATTAGCATGTATAATGGCATTTATAATTAA AATACCACTATACGGAGTCCACTTATGACTTCCTAAAGCCCACGTA-

$-$

JAM166Pmbairdii

CAGCTGACTTCCAATCAGTTAGATCTAGACTCAACCTAGAAGAAAGTAAT AAATATACTAACAGCCCTACTAGTAAATATTACTCTATCAATTCTCCTAAT CACCATTGCCTTCTGACTACCCCAACTTAATTTATATACTGAAAAAGCAAA CCCGTATGAGTGCGGATTTGACCCCATAGGCTCTGCTCGCCTCCCATTCTC AATAAAATTCTTCCTAGTAGCAATCACTTTCTTACTATTTGACCTAGAAATT GCACTTCTACTTCCACTACCATGAGCTATCCAAATATATAATATTAACATC ATAATATTAACAGCTTTCATCCTAGTCTCCGTATTAGCATTAGGCTTAGCCT ACGAGTGACTACAAAAAGGACTAGAATGAACTGAG-

TAACTGGTAATTAGTTTAATTAAAACAAATGATTTCGACTCATTAGATTAT GATATATATTATAATTACCAAAA---

ATGTCATCTGTAACCTCCAATATTATATTAGCATTCTCATTCTCATTTCTAG GGACACTAGTATTTCGATCCCACCTAATATCAACTCTCCTATGCCTTGAAG GAATAATACTATCACTGTTTATTATAACCACAATTACATCCCTTAATTCCC ACTCAATAATTATATACCCTCTACCCATTGTTATTCTAGTATTCGCAGCATG TGAAGCAGCCATTGGCTTAGCCCTATTAGCAAAACTATCAAACTCCTACGG AACAGATTATGTGCAAAACCTAAACCTACTTCAATGT-

TAAAAATTATTTTCCCCTCTATCATATTACTCCCACTCACCTGATTATCAAA CAAAAAAAACCTATGAATCAATGTTACCTCCTACAGCTTTATAATCAGCTT AACTTCAGCTATGTTTTTATGACAAAACGACATAAATAATCTAAACTTCTC ACTACTATTCTCAACTGACTCCCTGTCCTCCCCTCTTATTATTTTAACAACA TGACTTCTGCCACTCATACTGCTAGCCAGCCAAAACCACATAAAAAAGGA 
AACAGAGTTAAACAAAAAAACATACATTTCAATACTAGTCTTACTACAAA TTCTTCTAATCATAACATTTTCTGCAAACGAACTAATTATATTTTACATCTT ATTTGAAGCCACCCTAATCCCCACCCTTATTATCATCACCCGATGGGGTAA CCAAACAGAACGACTAAATGCAGGACTCTACTTCCTATTTTATACCCTAAT TGGATCAATCCCACTACTAATCGCCCTTATCCACATTCAAAATATAAAAGG AACATTAAACTTCATACTATTCCCACTCACCTTCACACCCCTAGACCAAAC ATGGTCCAATAATATTCTATGATTAGCATGTATAATGGCATTTATAATTAA AATACCACTATACGGAGTCCACTTATGACTTCCTAAAGCCCAC-

\section{Microtus pennsylvanicus (GenBank)}

ATTAACATAATACTTATTCTACTAATAAACATCGCCCTAGCTGCCATCCTA ATTTCTCTAGCCTTCTGGCTCCCACACCTCAACGTCTATACTGAAAAAGCC AACCCATATGAATGCGGATTTGATCCAATAAGCTCCGCCCGATTACCATTC TCAATAAAATTTTTCCTAGTAGCAATCACCTTCCTACTCTTCGATCTTGAAA TCGCACTACTCCTTCCACTACCCTGAGCAATACAATTTCCTAACATAAACA TACTAATAACCATAGCATTTATCCTTATCACAATTTTAGCCCTAGGCTTAG CCTACGAATGAACACAAAAAGGCTTAGAATGAACAGAA-

TAATTGGTAATTAGTTTAATTAAAATTAATGATTTCGACTCATTAGATTAT GATAATATCATAATTACCAAAAA----

ATGACACCTGCCGTATTTAACATCACCCTAGCCTTCTCTTTCTCTCTTCTAG GGACTCTCATATTCCGATCACACCTTATATCCACCCTCCTCTGCCTAGAAG GCATAATATTATCTCCCTTTATCCTAGCTACTATCACACCCTTAAACACAC ACTCAATAATTATATTTCCCATCCCTATCGTTATCTTAGTATTCGCTGCCTG TGAAGCAGCCGTTGGATTGGCCCTACTAGCAAAAATCTCAAACACTTATG GCTCTGATTTCGTCCATAATTTAAACCTTCTACAATGC-

TAAAAATTATTTTTCCATCATTTATACTTCTACCTCTAACCTGACTTTCAAG CAATAAAAAAGTATGGATCAACGTAACAACCTACAGCCTCCTAATTAACC TAATCTCCATTAACCTCCTGTGACAAAACAACGAAAACAACCTCAGTTTTT CCACCATGTTCTCCGCAGACCCCTTATCAGCTCCACTTCTAGTCCTAACAA CCTGACTGCTACCCCTAATACTCTTAGCCAGCCAAAACCACATCAAAAAA GAATCCGAACACAATAAAAAACTATATATTTCCCTATTAGTTTGCCTCCAA ACCCTTCTTATCATGACGTTCTCTGCAAACGAACTTATCATATTTTACATCC TATTTGAAGCCACTCTCATCCCAACCCTCATTATCATTACACGATGGGGTA ATCAAACAGAACGACTTAACGCAGGAATTTACTTCCTGTTTTACACCTTAG TAGGATCAATCCCACTACTAATTGCCCTAATTTATATTCAAAATTGAACAG GAACACTAAACCTAATCCTAATAACACTAGACTCCTCACCAATTAACCAA ACATGATCTAACAATATCCTCTGATTAGCCTGTATTATGGCTTTCATAGTTA AAATACCT

Oryzomys palustris (GenBank)

ATCAATATACTCCTAATTATATTAACTAACATTGTACTATCATCACTTCTCA TTTCCGTAGCTTTCTGACTACCACAACCCAACTCTTACGTATTAAAAGCCA GCCCATATGAATGCGGCTTTGACCCTATAAGCTCAGCTTGCCTACCCTTCT 
CAATAAAATTTTTCCTTGTAGGCATTACATTTCTCCTTTTTGATCTTGAAAT TGCTCTCCTTCTCCCCATCCCCTGAGCTATACAATATACAGACACACACTT AACCATAGCTGCCTCTTTTATTCTTGTCTCTATCTTAACTCTAGGCCTAGCT TATGAGTGACTAAATAAAGGGTTAGAATGAACTGAA-

TAATTGGCAGTTAGTTTAAACAAAATAAATGATTTCAACTCATTAGATAAT GAGATGAACCATGACTACCAAAA-----

TGACTCCCATTACTATCAACTTTATAATAGCATTTTCCTTCTCCCTCCTAGA AAATATGATATTCCGCTCCCACCTTATATCTACCCTCCTATGCCTTGAAGGT ATGATGCTATCATTATTTATTCTAATTATCACTTTAGCCCTTAACACACAGT CAATAATCACATATGTAATCCCAATTATTATACTAGTATTTGCAGCATGTG AAGCAGCCATTGGCCTTGCTCTCCTAGTTATCATTTCTAATACTTACTGCAC AGACTATGTCCAAAATTTAAACCTATTCCAATAT-

TAAAAACTATTATACCACCCATCATACTACTACCACTTACTTGATTATCCA ACAACAAAAATATATGAATTCACACAACCTCCTACAGCTTTCTCATCAGCT TTGTTACCCTGACTTTACTATGACAAAACAATGAAAGC---

CTGGGGTTTTCTCCCATATTCTTCACAGACTACTTATCCTCCCCCTTACTAA TTCTGACAACCTGACTGCTCCCCCTTATACTTATTGCAAGCCAAAACTATA TAAAAAAAGAAACAAAAACTAACAAAAAAATTTATACCTCCATACTAATT AATCTCCAAATTCTCCATACCGTTACATTCTCTGTGAATGAATTAATTCTAT TCTACATCTTATTTGAAGCCACTCTAATTCCTACACTCATTATTATTACTCG ATGGGGAAACCAAACAGAACGCCTTGATGCAGGACTATATTTTTTATTCTA CACCCTTGTAGGATCAATTCCCCTACTTATTGCCCTGATTTGACTACAAAA CTACCTAGGA.AACCTAAATATTATGATGATATCAATTGACTCCGAAACAAT AACTCTATCTTGATCAAATGATATTCTATGACTAGCATGCATATTAGCCTTT CTTGTGAAAATACCC

Sigmodon hispidus (GenBank)

TTAATCTCTTTGTTGCCCTCTTCATCAACGCCTCCTTATCCTTCATCCTAATC TCAGTCCTTTCTGACTCCCTCAACTTAATATCTACACAGAAAAAGCAAGCC CCTACGAATGCGGATTTGACCCCCTAAGCTCCGCTCGACTCCCTTTCTCCA TAAAATTTTTCCTCGTAGCTATTACCTTCCTTCTTTTCGACCTAGAAATTGC ACTCCTCCTCCCCCTTCCATGAGCCATTCAAATCACCAAGCTTTCCGCGAT AATAATCACATCCTTTATCTTAATTTCAATCCTAGCCCTAGGCCTAATCTAC GAATGGATAAATAAAGGCTTAGAGTGAACCGAG-

TAATGGTAATTAGTTTAATTAAAATTAATGATTTCGGCTCATTAGATTATG GTTCTATCCCATAATTGCCAGAG----

TGATTTCCTCAACTACTAACATCATCCTAGCCTTCATATTTTCCCTTCTAGG AACCTTTATATTCCGATCCCACCTCATGTCTACCCTCCTATGCCTCGAAGG AATAATGTTATCCTTATTTATTTTAACCGCCTTCTCGTCACTCAGCTCTCAA TCCATAATCATATATTCTCTTCCTATTGTTATTTTAGTATTTGCAGCATGTG AAGCAGCTATTGGTCTAGCTCTCCTGGCTATAATTTCCAGCACATACGGAA CTGACTACGTTCAAAACCTAAACTTACTCCAATGT-

TAAAAATTATCTTCCCCTCCCTCATACTCCTGCCCCTCACCTGATTCTCTAA TAACAAAAAAATTTGAATTCACACAACCTCCTACAGCTTTATCATCAGCAT 
CATTTCCCTCATTTCCCTTTCCCTACACACTGATGGCATCATCAGCTTTTCC CCTATTTTCTTCACCGACAATTTATCCACCCCCCTCTTAATTCTCACAACCT GACTTCTCCCCCTTATACTCCTTGCTAGTCAAAATCATCTGCATAAAGAGT CTGGCTTAAGTAAAAAAACTTACATCTCTCTTCTTATTAGCCTTCAAATTCT CCTCACTATCACATTCTCTGCTAACGAGCTAATTATATTCTATATCTTATTT GAAGCCACATTGATCCCAACGCTTATTGTTATTACCCGATGAGGAAATCAA ACTGAACGATTAAATGCAGGACTTTACTTTCTATTTTATACATTAATTGGA TCCATCCCCCTCCTAATTGCCTTAATTTCTCTTCATAAAAATTTAGGCACCC TAAACATAATATTAATATCTCTCCGCTCTCACCCTATAGACCCCTCTTGATC AAATAATATTCTATGACTAGCGTGCATAATAGCATTTCTTATCAAAATGCC C-

Reithrodontomys megalotis (GenBank)

ATAAATATATTTATCGTTTTATTAGTAAATATTTCCCTAGCATCCTGTCTTA TTCTTATTGCTTTCTGATTACCACAACTTAATATCTACACCGAAAAAGCAA ATCCTTACGAGTGTGGATTTGACCCTATAAGCTCAGCTCGCCTCCCATTCT CTATAAAATTTTTCCTAGTAGCAATCACCTTCCTCCTATTTGACCTAGAAAT TGCACTCCTATTACCACTACCATGAGCCATTCAAATATCAAACATTAAAGC AACAATATTAACATCATTTATTCTAGTATCAGTACTAGCCATAGGACTAGC CTACGAATGACTACAAAAGGGTCTAGAATGAACTGAA-

TAAAATGGTAATTAGTTTAATAAAAATAAATGATTTCGACTCATTAGATTA TAACTTATAATTATAATTACCAAAAA-

ATGTCATCTATTACTCTCAACATCATACTAGCATTTATTTTTTCCTTTCTAG GAACATTAATATTCCGCTCTCATCTTATATCAACATTATTATGCCTAGAGG GTATAATACTTTCATTATTTATTATTATTACAATTACATCACTAAATTCTCA TTCAATAATTATATATACAATTCCAATTATCATTTTAGTATTTGCAGCATGT GAAGCAGCTATTGGTCTAGCTCTATTAGCAAAAGTATCAAATTCATACGGC ACAGACTATGTACAAAACTTAAATCTACTACAATGT-

TAAAAATTATTTTTCCATCTATTATATTACTTCCACTCACCTGACTATCAAA CAATAAAAATCTATGAATTAATACTACCTCATACAGTTTTATAATTAGCCT ATCTTCAATGGCACTCCTATGACAAAACGATGTTAATAACCTAAACTTTTC ACTACTATTTTCAACAGATCCTCTATCCTCCCCCCTCATCATTTTAACAACC TGATTATTACCATTAATACTACTAGCTAGCCAAAATCAT---

AAAAAAGAAACTGAATTAAACAAAAAAATTTACATCTCAATATTAGTAAC ACTCCAAATTTTATTAATTATGACATTTTCTGCAAACGAACTAATTATATTT TACATCTTATTTGAAGCTACCCTAATCCCCACACTTATTATTATTACCCGAT GAGGTAATCAAACAGAACGATTAAACGCAGGAATTTATTTTCTCTTCTATA CTTTAATTGGATCAATTCCACTCCTCATTGCTCTCATTTATATTCAAAATCT ATTAGGAACACTAAACTTCTTACTATTCCCCCTCACCTTCACTCAACTTAAT CAAACATGATCTAATAACATTTTATGACTAGCCTGCATGATAGCATTCCTA ATTAAAATACCT 
Rattus norvegicus (GenBank)

ATTAACCTACCCATTATCATCACAATTAACATCACCTTATCCTTTATCCTCA TTTCAATTGCATTCTGATTGCCTCAAATAAACTTATACTCCGAAAAAGCAA ACCCATATGAATGTGGCTTCGACCCAACAAGTTCTGCACGCCTTCCTTTTT CAATAAAATTTTTCTTAGTAGCCATTACATTTCTACTATTCGACCTAGAAAT CGCCTTACTACTTCCCCTCCCATGAGCGATTCAAACAACCAATACCACTAC AATAATAGCAACTGCCTTTATTTTAGTCACTATTTTGTCTCTTGGCCTAGCC TACGAATGAACACAAAAAGGACTAGAATGAACAGAA-

TAATTGGTAATTAGTTTAAATAAAATTAATGATTTCGACTCATTAGATTAT GATAATAATCATAATTACCAACA----

ATGACATCTGCTTTCCTAAATTTAACTATAGCCTTTACATTATCTCTACTAG GTACTTTTATATTTCGCTCCCACCTAATATCCACTCTCCTCTGCCTAGAAGG AATAATACTATCACTATTTGTCATAACTTCAACATCCACATTAAACTCCAA CTCCATAATCTCCATAACCATCCCAATTACCATTCTAGTTTTTGCAGCCTGC GAAGCAGCAGTAGGTTTAGCCTTACTAGTAAAAATTTCAAATACTTACGG AACAGACTACGTACAAAACCTCAACCTTCTACAATGT-

TAAAAATTATTTTCCCATCTATCATACTCCTCCCACTAACATGACTCTCAGC CAACAAAAAAATCTGAACCAATGTCACCTCCTACAGCTTTCTAGTGAGCCT ATTAAGCTTATCACTCCTATGACAAAATGACGAAAATTACCTAAATTTCTC AGTTATATTCTCCTCCGATCCATTATCCACCCCACTAATCATTCTAACAACT TGACTCCTCCCACTAATAATGCTCGCTAGCCAAAATCACATAAAAAAAGA AAATATAATGCATCAAAAACTTTACATCTCAATACTTATTAGCCTCCAAAT TTTACTCATCATAACATTCTCCGCAACAGAACTAATTTTATTTTATATCCTG TTCGAGGCCACTCTAATCCCAACACTAATTATCATTACACGATGAGGCAAC CAAACAGAACGCTTAAATGCAGGAATTTATTTCCTGTTTTATACACTAATT GGCTCCATCCCACTCTTAATTGCCCTCATTTCAATCCAAAACTCAATAGGA ACACTCAACTTCCTAATCCTTTCCCTCACAACACACCCCCTACCCTCAACA TGATCCAACACCATTCTATGACTAGCATGTATAATAGCATTTATAATCAAA ATACCATTATACGGAGTCCATCTATGATTACCAAAAGCCCACGTAGAAGCT CCA--

$\underline{\text { Pmrufinus }}$

AACTAGTACAGCTGACTTCCAATCAGTTAGATCTAGACCTAACCTAGAAG AAAGTAATAAATATACTAACAGCTCTATTAGTAAATATTACGCTATCAATA CTCCTAATCATCATTGCCTTCTGACTACCCCAACTTAATTTATATACTGAAA AAGCAAACCCGTATGAGTGCGGATTTGAACCCATAGGCTCTGCTCGCCTCC CATTCTCAATAAAATTTTTTCTAGTAGCAATCACTTTCTTACTATTTGACCT AGAAATTGCACTTCTACTTCCACTACCATGAGCTATCCAAATATATAATAT TAACATCATAATATTAACAGCTTTCATCCTAGTCTCCGTATTAGCATTAGG CTTAGCCCACGAGTGACTACAAAAAGGACTAGAATGAACTGAG-

TAACTGGTAATTAGTTTAATTAAAACAAATGATTTCGACTCATTAGATTAT ATAAACATTATAATTACCAAAAA-----

TGTCATCTGTAACCTCCAATATTATATTAGCATTCTCATTCTCATTTCTAGG AACGCTAGTATTTCGATCCCACCTAATATCAACTCTCCTATGCCTTGAAGG 
AATAATACTATCACTGTTTATTATAACCACAATTACATCCCTTAATTCCCAC TCAATAATTATATGACCTCTACCCATTGTTATCCTAGTATTCGCAGCATGTG AAGCAGCCATTGGCTTAGCCCTATTAGCAAAAGTATCAAACTCCTACGGA ACAGATTATGTACAAAATCTAAACCTACTTCAATGT-

TAAAAATTATTTTTCCCTCTATCATATTACTCCCACTCACCTGATTATCAAA CAAAAAAAACCTATGAGTCAATGTTACCTCCTACAGCTTTATAATCAGCTT GATTTCAGCTATGTTTTTATGACAAAACGACATAAATAATCTAAACTTCTC ACTACTATTCTCAACTGACTCCCTATCCTCCCCTCTTATTATTCTAACAACA TGACTTCTACCACTCATACTGCTAGCCAGCCAAAACCACATAAAAAAGGA AACAGAGTCAAACAAAAAAACATATATTTCAATACTAGTCTTACTACAAA TTCTTCTAATCATAACATTTTCTGCAAACGAACTAATTATATTTTACATCTT ATTTGAAGCCACCCTAATCCCCACCCTTATTATCATCACCCGATGAGGTAA CCAAACAGAACGACTAAATGCAGGACTCTACTTTCTATTTTATACCCTAAT TGGATCAATCCCACTACTAATCGCCCTTATCCACATTCAAAATATAAAAGG AACATTAAACTTCATACTATTCCCACTCACCTTTACACCCCTAGACCAAAC ATGGTCTAATAATATTCTATGATTAGCATGCATAATGGCATTTATGATTAA AATACCACTATACGGAGTCCACTTATGACTCCCTAAAGCCCACGTAGAAG CTCCA-- 
Appendix F: Hantavirus Specimen Sequences. Resolved sequences M segment G1/G2 glycoprotein precursor gene (617nt). $\mathrm{N}=44$.

$\underline{\mathrm{T} 7}$

ATGAGTGGGAGACAGCAAAAGAACTCTGGACACATAAGAAAAGTT GTCCGGAAGGTCAATGTCCATGCTGCATGACAATATCTGAATCAACTGAG AGCGCGCTGCAAGCTCATTTTTCAATCTGTAAGCTAACAAACAGGTTCCAG GAAAATCTAAAAAAATCACTAAAACGCCCAGAAGTACGGAAAGGTTGCTA CAGGACATTAGGAGTTTTCCGGTACAAAAGTAGGTGTTATGTTGGCTTAGT ATGGGGAATTCTCTTGACAACGGAGCTGATTATATGGGCCGCCAGTGCCG AA-

TTGTGAGTATCAAGGGAAGACAGTGTCTGGATTCCAGCGCATGATGGCAA CTCGAGATTCTTTTCAATCATTCAATGTTACAGAACCACACATTACCAGTA ATCGACTTGAGTGGATTGATCCAGATAGTAGCATTAAAGACCATATCAAC ATGGTTTTAAATAGAGATGTTTCCTTTCAAGATCTAAGTGATAACCCATGT AAGGTTGACTTGCATACACAATCTATTGACGGCGCCTGGGGATCAGGAGT GGGCTTCACATTAGTATGTACGGAGGCGCTCATAGCACATC

$\underline{T 8}$

ATGAGTGGGAGACAGCAAAAGAACTCTGGACACATAAGAAAAGTT GTCCGGAAGGTCAATGTCCATGCTGCATGACAATATCTGAATCAACTGAG AGCGCGCTGCAAGCTCATTTTTCAATCTGTAAGCTAACAAACAGGTTCCAG GAAAATCTAAAAAAATCACTAAAACGCCCAGAAGTACGGAAAGGTTGCTA CAGGACATTAGGAGTTTTCCGGTACAAAAGTAGGTGTTATGTTGGCTTAGT ATGGGGAATTCTCTTGACAACGGAGCTGATTATATGGGCCGCCAGTGCCG AA-

TTGTGAGTATCAAGGGAAGACAGTGTCTGGATTCCAGCGCATGATGGCAA CTCGAGATTCTTTTCAATCATTCAATGTTACAGAACCACACATTACCAGTA ATCGACTTGAGTGGATTGATCCAGATAGTAGCATTAAAGACCATATCAAC ATGGTTTTAAATAGAGATGTTTCCTTTCAAGATCTAAGTGATAACCCATGT AAGGTTGACTTGCATACACAATCTATTGACGGCGCCTGGGGATCAGGAGT GGGCTTCACATTAGTATGTACGGAGGCGCTCATAGCACATC

$\underline{\mathrm{WC}} 4$

ATGAGTGGGAGACAGCAAAAGAACTCGGAACACATAAGAAAAGTT GTCCGGAAGGTCAATGTCCATACTGCATGACAATAACTGAATCAACTGAG AGCGCATTGCAAGCTCATTTTTCAATCTGTAAGATAACAAACAGGTTCCAG GAAAATCTCAAAAAATCATTAAAACGCCCAGAAGTACGGAAAGGTTGCTA CAGGACATTAGGAGTTTTCCGGTACAAAAGTAGGTGTTATGTTGGCTTAGT ATGGGGAATTCTCTTGACAACGGAGCTGATTATATGGGCCGCCAGTGCCG AA-

TTGTGAGTATCAAGGGAAGACAGTGTCTGGATTCCAGCGCATGATGGCAA CTCGAGATTCTTTTCAATCATTCAATGTTACAGAACCACACATTACCAGCA ATCGACTTGAGTGGATTGATCCAGATAGTAGCATTAAAGACCATATCAAC ATGGTTTTAAATAGAGATGTTTCCTTTCAAGATCTAAGTGATAACCCATGT 
AAGGTTGACTTGCATACACAATCTATTGACGGCGCCTGGGGATCAGGAGT GGGCTTCACATTAGTATGTACGGAGGCGCTCATAGCACATC

$\underline{\text { WCps }}$

ATGAGTGGGAGACAGCAAAAGAACTCGGAACACATAAGAAAAGTT GTCCGGAAGGTCAATGTCAATACTGCATGACAATAACTGAATCAACTGAG AGCGCATTGCAAGCTCATTTTTCAATCTGTAAGATAACAAACAGGTTCCAG GAAAATCTCAAAAAATCATTAAAACGCCCAGAAGTACGGAAAGGTTGCTA CAGGACATTAGGAGTTTTCCGGTACAAAAGTAGGTGTTATGTTGGCTTAGT ATGGGGAATTCTCTTGACAACGGAGCTGATTATATGGGCCGCCAGTGCCG AA-

TTGTGAGTATCAAGGGAAGACAGTGTCTGGATTCCAGCGCATGATGGCAA CTCGAGATTCTTTTCAATCATTCAATGTTACAGAACCACACATTACCAGCA ATCGACTTGAGTGGATTGATCCAGATAGTAGCATTAAAGACCATATCAAC ATGGTTTTAAATAGAGATGTTTCCTTTCAAGATCTAAGTGATAACCCATGT AAGGTTGACTTGCATACACAATCTATTGACGGCGCCTGGGGATCAGGAGT GGGCTTCACATTAGTATGTACGGAGGCGCTCATAGCACATC

$\underline{\text { WC7 }}$

ATGAGTGGGAGACAGCAAAAGAACTCGGAACACATAAGAAAAGTT GTCCGGAAGGTCAATGTCCATACTGCATGACAATAACTGAATCAACTGAG AGCGCATTGCAAGCTCATTTTTCAATCTGTAAGCTAACCAACAGGTTCCAG GAAAATCTAAAAAAATCATTAAAACGCCCAGAAGTACGGAAAGGTTGCTA CAGGACATTAGGAGTTTTCCGGTACAAAAGTAGGTGTTATGTTGGCTTAGT ATGGGGAATTCTCTTGACAACGGAGCTGATTATATGGGACGCCAGTGCCG AA

TTGTGAGTATCAAGGGAAGACAGTGTCTGGATTCCAGCGCATGATGGCAA CTCGAGATTCTTTTCAATCATTCAATGTTACAGAACCACACATTACCAGCA ATCGACTTGAGTGGATTGATCCAGATAGTAGCATTAAAGACCATATCAAC ATGGTTTTAAATAGAGATGTTTCCTTTCAAGATCTAAGTGATAACCCATGT AAGGTTGACTTGCATACACAATCTATTGACGGCGCCTGGGGATCAGGAGT GGGCTTCACATTAGTATGTACGGAGGCGCTCATAGCACATC

$\underline{\text { WC12 }}$

ATGAGTGGGAGACAGCAAAAGAACTCGGAACACATAAGAAAAGCT GTCCAGAAGGTCAATGTCCATATGGTATGACAATAACTGAATCTACTGAA AGTGCATTGCAAGCCCATTTTTCAATTTGTAAGCTAACAAACAGGTTTCAG GAAAATCTAAAAAAATCATTAAAACGTCCAGAGGTACGGAAAGGTTGCTA CAGAACATTAGGGGTTTTCCGGTATAAAAGTAGGTGTTATGTTGGCTTAGT ATGGGGAATTCTCTTGTCAACGGAGCTGATTATATGGGCGGCCAGTGCCG AA---------------------

TTGTGAGTATCAAGGGAAGACAGTGTCTGGATTCCAGCGCATGATGGCAA CTCGAGATTCTTTTCAATCATTCAATGTTACAGAACCACACATTACCAGCA ATCGACTTGAGTGGATTGATCCAGATAGTAGCATTAAAGACCATATCAAC ATGGTTTTAAATAGAGATGTTTCCTTTCAAGATCTAAGTGATAACCCATGT 
AAGGTTGACTTGCATACACAATCTATTGACGGCGCCTGGGGATCAGGAGT GGGCTTCACATTAGTATGTACGGAGGCGCTCATAGCACATC

$\underline{\mathrm{WC} 13}$

ATGAGTGGGAGACAGCAAAAGAACTCGGAACACATAAGAAAAGTT GTCCGGAAGGTCAATGTCCATACTGCATGACAATAACTGAATCAACTGAG AGCGCATTGCAAGCTCATTTTTCAATCTGTAAGCTAACCAACAGGTTCCAG GAAAATCTAAAAAAATCATTAAAACGCCCAGAAGTACGGAAAGGTTGCTA CAGGACATTAGGAGTTTTCCGGTACAAAAGTAGGTGTTATGTTGGCTTAGT ATGGGGAATTCTCTTGACAACGGAGCTGATTATATGGGACGCCAGTGCCG AA-

TTGTGAGTATCAAGGGAAGACAGTGTCTGGATTCCAGCGCATGATGGCAA CTCGAGATTCTTTTCAATCATTCAATGTTACAGAACCACACATTACCAGCA ATCGACTTGAGTGGATTGATCCAGATAGTAGCATTAAAGACCATATCAAC ATGGTTTTAAATAGAGATGTTTCCTTTCAAGATCTAAGTGATAACCCATGT AAGGTTGACTTGCATACACAATCTATTGACGGCGCCTGGGGATCAGGAGT GGGCTTCACATTAGTATGTACGGAGGCGCTCATAGCACATC

\section{LJD1523FP}

ATGAGTGGGAGACAGCAAAAGAACTCTGGACACATAAGAAAAGTT GTCCGGAAGGTCAATGTCCATGGTGCCTGACAATAACTGAATCAACTGAG AGCGCATTGCAAGCTCCTTTTTCAATATGTAAGCTAACAAGCAGGTTCCAG GAAAATCTAAAAAAATCATTAAAACGCCCAGAAGTACGGAAAGGTTGCTA CAGGAAATTAGGAGTTTTCCGGTACAAAAGTAGGTGTTATGTTGGCTTAGT ATGGGGAATTCTCTTGACAACGCAGATGATTATATGGGCCGCCAGTGCCG AA-

TTGTGAGTATCAAGGGAAGACAGTGTCTGGATTCCAGCGCATGATGGCAA CTCGAGATTCTTTTCAATCATTCAATGTTACAGAACCACACATTACCAGCA ATCGACTTGAGTGGATTGATCCAGATAGTAGCATTAAAGACCATATCAAC ATGGTTTTAAATAGAGATGTTTCCTTTCAAGATCTAAGTGATAACCCATGT AAGGTTGACTTGCATACACAATCTATTGACGGCGCCTGGGGATCAGGAGT GGGCTTCACATTAGTATGTACGGAGGCTCTCATCGCACATC

\section{LJD2126FP}

ATGAGTGGGAGACAGCAAAAGAACTCGAGACACATAAGAAAAGTT GTCCGGAAGGTCAATGTCCATACTGCATGACAATAACTGAATCAACTGAG AGCGCGTTGCAAGCTCATTTTTCAATCTGTAAGCTAACAAACAGGTTCCAG GAAAATCTAAAAAAATCATTAAAACGCCCAGAAGTACGGAAAGGTTGCTA CAGGACATTAGGAGTTTTCCGGTACAAAAGTAGGTGTTATGTTGGCTTAGT ATGGGGAATTCTCTTGACAACGGAGCTGATTATATGGGCCGCCAGTGCCG

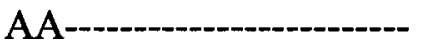

TTGTGAGTATCAAGGGAAGACAGTGTCTGGATTCCAGCGCATGATGGCAA CTCGAGATTCTTTTCAATCATTCAATGTTACAGAACCACACATTACCAGCA ATCGACTTGAGTGGATTGATCCAGATAGTAGCATTAAAGACCATATCAAC ATGGTTTTAAATAGAGATGTTTCCTTTCAAGATCTAAGTGATAACCCATGT 
AAGGTTGACTTGCATACACAATCTATTGACGGCGCCTGGGGATCAGGAGT GGGCTTCACATTAGTATGTACGGAGGCTCTCATCGCACATC

\section{LJD2138FP}

ATGAGTGGGAGACAGCAAAAGAACTCGAGACACATAAGAAAAGTT GTCCGGAAGGTCAATGTCCATACTGCATGACAATAACTGAATCAACTGAG AGCGCGTTGCAAGCTCATTTTTCAATCTGTAAGCTAACAAACAGGTTCCAG GAAAATCTAAAAAAATCATTAAAACGCCCAGAAGTACGGAAAGGTTGCTA CAGGACATTAGGAGTTTTCCGGTACAAAAGTAGGTGTTATGTTGGCTTAGT ATGGGGAATTCTCTTGACAACGGAGCTGATTATATGGGCCGCCAGTGCCG AATTGTGAGTATCAAGGGAAGACAGTGTCTGGATTCCAGCGCATGATGGCAA CTCGAGATTCTTTTCAATCATTCAATGTTACAGAACCACACATTACCAGCA ATCGACTTGAGTGGATTGATCCAGATAGTAGCATTAAAGACCATATCAAC ATGGTTTTAAATAGAGATGTTTCCTTTCAAGATCTAAGTGATAACCCATGT AAGGTTGACTTGCATACACAATCTATTGACGGCGCCTGGGGATCAGGAGT GGGCTTCACATTAGTATGTACGGAGGCGCTCATAGCACATC

\section{$\underline{\text { LJD2154FP }}$}

ATGAGTGGGAGACAGCAAAAGAACTTGAGACACATAAAAAAAGTT GCCCGGAAGGTCAATGTCCATACTGCATGACAATAACTGAATCAACTGAG AGTGCGCTGCAAGCTCATTTTTCAATCTGCAAGCTAACAAACAGGTTCCAG GAAAATCTAAAGAAATCATTAAAACGCCCAGAAGTACGGAAAGGTTGCTA TAGGACATTAGGAGTTTTCCGGTATAAGAGTAGGTGTTATGTTGGCTTAGT ATGGGGAATTCTCTTGACAACGGAGCTGATTATATGGGCCGCCAGTGCCG AA-

CAAGATGTGCTATGGCGCCTCCGTTTGTGAGTATCAAGGGAAGACAGTAT CTGGATTCCAGCGCATGATGGCAACTCGAGATTCTTTTCAATCATTCAACG TGACAGAACCACATATTACCAGTAATCGACTTGAGTGGATTGATCCAGAC AGTAGTATTAAAGACCATATCAACATGGTTTTGAATAGAGATGTTTCCTTC CAAGATCTAAGTGATAACCCATGTAAGGTTGACTTGCATACACAATCTATT GACGGCGCCTGGGGATCAGGAGTGGGCTTCACATTAGTATGTACGGAGGC GCTCATAGCACATC

\section{LJD2493FP}

ATGAGTGGGAGACAGCAAAAGAACTCGAGACACATAAGAAAAGTT GTCCGGAAGGTCAATGTCCATACTGCATGACAATAACTGAATCAACTGAG AGCGCATTGCAAGCTCATTTTTCAATCTGTAAGCTAACAAACAGGTTCCAG GAAAATCTAAAAAAATCATTAAAACGCCCAGAAGTACGGAAAGGTTGCTA CAGGACATTAGGAGTTTTCCGGTACAAAAGTAGGTGTTATGTTGGCTTAGT ATGGGGAATTCTCTTGACAACGGAGCTGATTATATGGGCCGCCAGTGCCG AATTGTGAGTATCAAGGGAAGACAGTGTCTGGATTCCAGCGCATGATGGCAA CTCGAGATTCTTTTCAATCATTCAATGTTACAGAACCACACATTACCAGCA ATCGACTTGAGTGGATTGATCCAGATAGTAGCATTAAAGACCATATCAAC 
ATGGTTTTAAATAGAGATGTTTCCTTTCAAGATCTAAGTGATAACCCATGT AAGGTTGACTTGCATACACAATCTATTGACGGCGCCTGGGGATCAGGAGT GGGCTTCACATTAGTATGTACGGGGGCGCTCATAGCACATC

\section{LJD2494FP}

ATGAGTGGGAGACAGCAAAAGAACTCGAGACACATAAGAAAAGTT GTCCGGAAGGTCAATGTCCATACTGCATGACAATAACTGAATCAACTGAG AGCGCATTGCAAGCTCATTTTTCAATCTGTAAGCTAACAAACAGGTTCCAG GAAAATCTAAAAAAATCATTAAAACGCCCAGAAGTACGGAAAGGTTGCTA CAGGACATTAGGAGTTTTCCGGTACAAAAGTAGGTGTTATGTTGGCTTAGT ATGGGGAATTCTCTTGACAACGGAGCTGATTATATGGGCCGCCAGTGCCG AA---------------------

TTGTGAGTATCAAGGCAAGACAGTGTCTGGATTCCAGCGCATGATGGCAA CTCGAGATTCTTTTCAATCATTCAATGTTACAGAACCACACATTACCAGCA ATCGACTTGAGTGGATTGATCCAGATAGTAGCATTAAAGACCATATCAAC ATGGTTTTAAATAGAGATGTTTCCTTTCAAGATCTAAGTGATAACCCATGT AAGGTTGACTTGCATACACAATCTATTGACGGCGCCTGGGGATCAGGAGT GGGCTTCACATTAGTATGTACGGGGGCGCTCATAGCACATC

\section{LJD2497FP}

ATGAGTGGGAGACAGCAAAAGAACTCGAGACACATAAGAAAAGTT GTCCGGAAGGTCAATGTCCATACTGCATGACAATAACTGAATCAACTGAG AGCGCATTGCAAGCTCATTTTTCAATCTGTAAGCTAACAAACAGGTTCCAG GAAAATCTAAAAAAATCATTAAAACGCCCAGAAGTACGGAAAGGTTGCTA CAGGACATTAGGAGTTTTCCGGTACAAAAGTAGGTGTTATGTTGGCTTAGT ATGGGGAATTCTCTTGACAACGGAGCTGATTATATGGGCCGCCAGTGCCG AA-

TTGTGAGTATCAAGGGAAGACAGTGTCTGGATTCCAGCGCATGATGGCAA CTCGAGATTCTTTTCAATCATTCAATGTTACAGAACCACACATTACCAGCA ATCGACTTGAGTGGATTGATCCAGATAGTAGCATTAAAGACCATATCAAC ATGGTTTTAAATAGAGATGTTTCCTTTCAAGATCTAAGTGATAACCCATGT AAGGTTGACTTGCATACACAATCTATTGACGGCGCCTGGGGATCAGGAGT GGGCTTCACATTAGTATGTACGGTGGCTCTCATAGCACATC

\section{LJD2499FP}

ATGAGTGGGAGACAGCAAAAGAACTCGAGACACATAAGAAAAGTT GTCCGGAAGGTCAATGTCCATACTGCATGACAATAACTGAATCAACTGAG AGTTCATTGCAAGCTCATTTTTCATTCTGTCACGTAACAAAGAGGTTCCAG GAAAATCTAAGAAAATCACATCAACTCCCAGAAGTACGGAAAGGTTGCTA CAGGACATTACGAGTTTTCCGGTACAAAAGTAGGTGTTATGTTGGCTTAGT ATGGGGAATTCTCTTGACAACGGAGCTGATTATATGGGCCGCCAGTGCCG AA

TTGTGAGTATCAAGGGAAGACAGTGTCTGGATTCCAGCGCATGATGGCAA CTCGAGATTCTTTTCAATCATTCAATGTTACAGAACCACACATTACCAGCA ATCGACTTGAGTGGATTGATCCAGATAGTAGCATTAAAGACCATATCAAC 
ATGGTTTTAAATAGAGATGTTTCCTTTCAAGATCTAAGTGATAACCCATGT AAGGTTGACTTGCATACACAATCTATTGACGGCGCCTGGGGATCAGGAGT GGGCTTCACATTAGTATGTACGGAGGCGCTCATAGCACATC

\section{$\underline{\text { LJD2520FP }}$}

ATGAGTGGGAGACAGCAAAAGAACTCGAGACACATAAGAAAAGTT GTCCGGAAGGTCAATGTCCATACTGCATGACAATAACTGAATCAACTGGG AGCGCATTGCAAGCTCATTTTTCAATCTGTAAGCTAACAAACAGGTTCCAG GAAAATCTAAAAAAATCATTAAAACGCCCAGAAGTACGGAAAGGTTGCTA CAGGACATTAGGAGTTTTCCGGTACAAAAGTAGGTGTTATGTTGGCTTAGT ATGGGGAATTCTCTTGACAACGGAGCTGATTATATGGGCCGCCAGTGCCG AA

TTGTGAGTATCAAGGGAAGACAGTGTCTGGATTCCAGCGCATGATGGCAA CTCGAGATTCTTTTCAATCATTCAATGTTACAGAACCACACATTACCAGCA ATCGACTTGAGTGGATTGATCCAGATAGTAGCATTAAAGACCATATCAAC ATGGTTTTAAATAGAGATGTTTCCTTTCAAGATCTAAGTGATAACCCATGT AAGGTTGACTTGCATACACAATCTATTGACGGCGCCTGGGGATCAGGAGT GGGCTTCACATTAGTATGTACGGAGGCGCTCATAGCACATC

\section{LJD2522FP}

ATGAGTGGGAGACAGCAAAAGAACTCGAGACACATAAGAAAAGTT GTCCGGAAGGTCAATGTCCATACTGCATGACAATAACTGAATCAACTGAG AGCGCATTGCAAGCTCATTTTTCAATCTGTAAGCTAACAAACAGGTTCCAG GAAAATCTAAAAAAATCATTAAAACGCCCAGAAGTACGGAAAGGTTGCTA CAGGACATTAGGAGTTTTCCGGTACAAAAGTAGGTGTTATGTTGGCTTAGT ATGGGGAATTCTCTTGACAACGGAGCTGATTATATGGGCCGCCAGTGCCG AA----------------

TTGTGAGTATCAAGGGAAGACAGTGTCTGGATTCCAGCGCATGATGGCAA CTCGAGATTCTTTTCAATCATTCAATGTTACAGAACCACACATTACCAGCA ATCGACTTGAGTGGATTGATCCAGATAGTAGCATTAAAGACCATATCAAC ATGGTTTTAAATAGAGATGCTTCCTTTCAAGATCTAAGTGATAACCCATGT AAGGTTGACTTGCATACACAATCTATTGACGGCGCCTGGGGATCAGGAGT GGGCTTCACATTAGTATGTACGGAGGCGCTCATAGCACATC

\section{LJD2737PB}

ATGAGTGGGAGACAGCAAAAGAACTCTGGACACATAAGAAAAGTT GTCCGGAAGGTCAATGTCCATGCTGCATGACAATATCTGAATCAACTGAG AGCGCGCTGCAAGCTCATTTTTCAATCTGTAAGCTAACAAACAGGTTCCAG GAAAATCTAAAAAAATCACTAAAACGCCCAGAAGTACGGAAAGGTTGCTA CAGGACATTAGGAGTTTTCCGGTACAAAAGTAGGTGTTATGTTGGCTTAGT ATGGGGAATTCTCTTGACAACGGAGCTGATTATATGGGCCGCCAGTGCCG AACA-

TTGTGAGTATCAAGGGAAGACAGTGTCTGGATTCCAGCGCATGATGGCAA CTCGAGATTCTTTTCAATCATTCAATGTTACAGAACCACACATTACCAGCA ATCGACTTGAGTGGATTGATCCAGATAGTAGCATTAAAGACCATATCAAC 
ATGGTTTTAAATAGAGATGTTTCCTTTCAAGATCTAAGTGATAACCCATGT AAGGTTGACTTGCATACACAATCTATTGACGGCGCCTGGGGATCAGGAGT GGGCTTCACATTAGTATGTACGGAGGCGCTCATAGCACATC

\section{LJD2836FP}

ATGAGTGGGAGACAGCAAAAGAACTCGAGACACATAAGAAAAGTT GCCCGGAAGGTCAATGTCCATACTGCATGACAATAACTGAATCAACTGAG AGCGCGTTGCAAGCTCATTTTTCAATCTGTAAGCTAACAAACAGGTTCCAG GAAAATCTAAAAAAATCATTAAAACGCCCAGAAGTACGGAAAGGTTGCTA CAGGACATTAGGAGTTTTCCGGTACAAAAGTAGGTGTTATGTTGGCTTAGT ATGGGGAATTCTCTTGACAACGGAGCTGATTATATGGGCCGCCAGTGCCG AA

TTGTGAGTATCAAGGGAAGACAGTGTCTGGATTCCAGCGCATGATGGCAA CTCGAGATTCTTTTCAATCATTCAATGTTACAGAACCACACATTACCAGCA ATCGACTTGAGTGGATTGATCCAGATAGTAGCATTAAAGACCATATCAAC ATGGTTTTAAATAGAGATGTTTCCTTTCAAGATCTAAGTGATAACCCATGT AAGGTTGACTTGCATACACAATCTATTGACGGCGCCTGGGGATCAGGAGT GGGCTTCACATTAGTATGTACGGAGGCGCTCATAGCACATC

\section{LJD2852FP}

ATGAGTGGGAGACAGCAAAAGAACTCGAGACACATAAGAAAAGTT GTCCGGAAGGTCAATGTCCATACTGCATGACAATAACTGAATCAACCGAG AGCGCGTTGCAAGCTCATTTTTCAATCTGTAAGCTAACAAACAGGTTCCAG GAAAATCTAAAAAAATCATTAAAACGCCCAGAAGTACGGAAAGGTTGCTA CAGGACATTAGGAGTTTTCCGGTACAAAAGTAGGTGTTATGTTGGCTTAGT ATGGGGAATTCTCTTGACAACGGAGCTGATTATATGGGCCGCCAGTGCCG AA-

TTGTGAGTATCAAGGGAAGACAGTGTCTGGATTCCAGCGCATGATGGCAA CTCGAGACTCTTTTCAATCATTCAATGTTACAGAACCACACATTACCAGCA ATCGACTTGAGTGGATTGATCCAGATAGTAGCATTAAAGACCATATCAAC ATGGTTTTAAATAGAGATGTTTCCTTTCAAGATCAAAGTGATAACCCATGT AAGGTTGACTTGCATACACAATCTATTGACGGCGCCTGGGGATCAGGAGT GGGCTTCACATTAGTATGTACGGAGGCGCTCATAGCACATC

\section{LJD2860FP}

ATGAGTGGGAGACAGCAAAAGAACTCGAGACACATAAGAAAAGTT GTCCGGAAGGTCAATGTCCATACTGCATGACAATAACTGAATCAACTGAG AGCGCATTGCAAGCTCATTTTTCAATCTGTAAGCTAACAAACAGGTTCCAG GAAAATCTAAAAAAATCATTAAAACGCCCAGAAGTACGGAAAGGTTGCTA CAGGACATTAGGAGTTTTCCGGTACAAAAGTAGGTGTTATGTTGGCTTAGT ATGGGGAATTCTCTTGACAACGGAGCTGATTATATGGGCCGCCAGTGCCG AA-

TTGTGAGTATCAAGGGAAGACAGTGTCTGGATTCCAGCGCATGATGGCAA CTCGAGATTCTTTTCAATCATTCAATGTTACAGAACCACACATTACCAGCA ATCGACTTGAGTGGATTGATCCAGATAGTAGCATTAAAGACCATATCAAC 
ATGGTTTTAAATAGAGATGTTTCCTTTCAAGATCTAAGTGATAACCCATGT AAGGTTGACTTGCATACACAATCTATTGACGGCGCCTGGGGATCAGGAGT GGGCTTCACATTAGTATGTACGGTGGCGCTCATAGCACATC

\section{LJD2865FP}

ATGAGTGGGAGACAGCAAAAGAACTCGAGACACATAAGAAAAGTT GTCCGGAAGGTCAATGTCCATATGGCATGACAATAACTGAATCAACTGAG AGCGCATTGCAAGCTCATTTTTCAATCTGTAAGCTAACAAACAGGTTCCAG GAAAATCTAAAAAAATCATTAAAACGCCCAGAAGTACGGAAAGGTTGCTA CAGGACATTAGGAGTTTTCCGGTACAAAAGTAGGTGTTATGTTGGCTTAGT ATGGGGAATTCTCTTGACAACGGAGCTGATTATATGGGCCGCCAGTGCCG AATTGTGAGTATCAAGGGAAGACAGTGTCTGGATTCCAGCGCATGATGGCAA CTCGAGATTCTTTTCAATCATTCAATGTTACAGAACCACACATTACCAGCA ATCGACTTGAGTGGATTGATCCAGATAGTAGCATTAAAGACCATATCAAC ATGGTTTTAAATAGAGATGTTTCCTTTCAAGATCTAAGTGATAACCCATGT AAGGTTGACTTGCATACACAATCTATTGACGGCGCCTGGGGATCAGGAGT GGGCTTCACATTAGTATGTACGGTGGCGCTCATAGCACATC

\section{LJD2890FP}

ATGAGTGGGAGACAGCAAAAGAACTCGAGACACATAAGAAAAGTT GTCCGGAAGGTCAATGTCCATACTGCATGACAATAACTGAATCAACTGAG AGCGCATTGCAAGCTCATTTTTCAATCTGTAAGCTAACAAACAGGTTCCAG GAAAATCTAAAAAAATCATTAAAACGCCCAGAAGTACGGAAAGGTTGCTA CAGGACATTAGGAGTTTTCCGGTACAAAAGTAGGTGTTATGTTGGCTTAGT ATGGGGAATTCTCTTGACAACGGAGCTGATTATATGGGCCGCCAGTGCCG AA-----------------

TTGTGAGTATCAAGGGAAGACAGTGTCTGGATTCCAGCGCATGATGGCAA CTCGAGATTCTTTTCAATCATTCAATGTTACAGAACCACACATTACCAGCA ATCGACTTGAGTGGATTGATCCAGATAGTAGCATTAAAGACCATATCAAC ATGGTTTTAAATAGAGATGTTTCCTTTCAAGATCTAAGTGATAACCCATGT AAGGTTGACTTGCATACACAATCTATTGACGGCGCCTGGGGATCAGGAGT GGGCTTCACATTAGTATGTACGGTGGCGCTCATAGCACATC

\section{Convict Creek}

ATGAATGTGAGACGGCAAAAGAGCTCGAAACACATAAGAAAAGTT GCCCAGAAGGTCAATGCCCATACTGCATGACAATAACTGAGTCCACTGAG AGTGCATTACAAGCTCATTTTTCAATCTGTAAGCTAACGAACAGGTTCCAG GAAATCTTAAAAAAGTCATTAAAACGTCCAGAAGTAAGGAAAGGTTGTTA CAGGACATTAGGAGTATTCCGCTACAAGAGCAGGTGCTATGTTGGCTTAGT ATGGGGGATCCTCTTGACGACAGAGCTGATTATATGGGCTGCTAGTGCAG • AT-

ATGTGAATATCAAGGTAATACAGTGTCTGGATTCCAACGCATGATGGCAA CTCGAGATTCTTTTCAATCATTCAATGTGACAGAACCACATATTACCAGCA ATCGACTGGAATGGATTGATCCAGATAGTAGTATTAAAGACCATATCAAC 
ATGGTTTTGAATAGAGATGTTTCCTTCCAAGATCTAAGTGATAATCCATGT AAGGTTGATTTGCATACACAATCTATTGATGGGGCTTGGGGATCAGGAGT GGGCTTTACATTAGTATGTACTGTAGGTCTTACAGAGTGTG

SNV (GenBank L25783)

ACGAATGCGAAACAGCAAAAGAACTTGAAACACATAAGAAAAGCT GTCCAGAAGGTCAATGCCCGTATTGTATGACAATAACTGAATCCACTGAG AGTGCTCTCCAAGCCCATTTTGCAATCTGTAAGCTAACAAACAGGTTTCAG GAAAACTTAAAAAAGTCATTGAAACGCCCAGAAGTACGGAAAGGTTGTTA CAGGACACTGGGAGTTTTTAGATACAAGAGCAGATGTTATGTTGGTTTAGT ATGGGGAATTCTTTTAACAACTGAACTGATCATATGGGCAGCCAGTGCAG $\mathrm{AC}-$

ATGTGAGTATCAAGGCAACACAGTGTCTGGATTCCAACGCATGATGGCAA CTCGAGATTCTTTCCAGTCATTCAATGTGACAGAACCACATATCACTAGCA ACCGACTTGAGTGGATTGATCCAGATAGCAGTATCAAAGATCACATTAAT ATGGTTTTAAATCGAGATGTTTCCTTTCAGGATCTAAGTGATAACCCATGC AAGGTTGACCTGCATACACAATCAATTGACGGGGCCTGGGGTTCAGGAGT AGGTTTTACGTTGGTATGTACTGTGGGGCTTACAGAGTGTG

\section{SNV (GenBank L37903)}

ACGAATGCGAAACAGCAAAAGAACTTGAAACACATAAGAAAAGCT GTCCAGAAGGTCAATGCCCGTATTGTATGACAATAACTGAATCCACTGAG AGTGCTCTCCAAGCCCATTTTGCAATCTGTAAGCTAACAAACAGGTTTCAG GAAAACTTAAAAAAGTCATTGAAACGCCCAGAAGTACGGAAAGGTTGTTA CAGGACACTGGGAGTTTTTAGATACAAGAGCAGATGTTATGTTGGTTTAGT ATGGGGAATTCTTTTAACAACTGAACTGATCATATGGGCAGCCAGTGCAG AC-

ATGTGAGTATCAAGGCAACACAGTGTCTGGATTCCAACGCATGATGGCAA CTCGAGATTCTTTCCAGTCATTCAATGTGACAGAACCACATATCACTAGCA ACCGACTTGAGTGGATTGATCCAGATAGCAGTATCAAAGATCACATTAAT ATGGTTTTAAATCGAGATGTTTCCTTTCAGGATCTAAGTGATAACCCATGC AAGGTTGACCTGCATACACAATCAATTGACGGGGCCTGGGGTTCAGGAGT AGGTTTTACGTTGGTATGTACTGTGGGGCTTACAGAGTGTG

New York Virus (GenBank U36803)

ATGAGTGTGAAACAGCAAAAGAGCTAGAAACTCATAAGAAAAGCT GTCCTGAAGGTCAGTGTCCATACTGCATGACAATGACAGAATCCACAGAG AGTGCTCTTCAGGCTCATTTTTCAATTTGTAAGCTTACAAATAGGTTTCAG GAAAATCTAAAAAAATCACTTAAACGCCCTGAAGTTAAACAAGGATGCTA TAGAACATTGGGTGTTTTTAGATATAAGAGTAGGTGTTATGTTGGTCTTGT GTGGGGGGTCCTTCTTACAACTGAACTCATAGTTTGGGCAGCTAGTGCTGA C-

CTGTGAGTATCAAGGTAATACTATCTCGGGATTTCAGAGAATGATGGCTAC AAGAGACTCTTTTCAGTCATTCAATGTAACAGAACCGCACATTACCAGTAA TCGTCTTGAATGGATTGATCCTGATAGCAGTATAAAAGATCATATTAATAT 
GGTCTTAAACCGAGATGTTTCATTTCAGGATTTGAGTGATAATCCCTGTAA AGTGGATCTACATACGCAGTCAATTGATGGAGCTTGGGGCTCTGGGGTAG GTTTCACTCTGGTATGTACAGTGGGACTGACTGAATGTG

Blue River (GenBank AF030552)

ATGATTGTGAGACAGCTAAAGAGCTTGATACACACAAAAAGAGTTG TCCAGAGGGTCAATGCCCTTACTGTATGACCATGACTGAATCAACAGAAA GTGCTCTCCAGGCCCATTTTTCAGTCTGCAAACTTACAAATAGATTTCAAG AAAATTTAAAAAAATCCTTAAAGCACCCAGAAGTAAAACAGGGCTGTTAT AGGACATTAGGAGTATTTAGGTATAAAAGTAGATGTTATGTAGGCCTTGTG TGGGGTTTGCTTCTGACAACTGAACTTATCATCTGGGCAGCTAGTGCAGAA

TTGCGAATATCAGGGCAATACAATTTCAGGATTTAAAAGAATGATGGCCA CAAGAGATTCTTTCCAGTCATTCAATGTTACAGAACCTCATATAACTAGTA ACCGACTAGAATGGATTGACCCTGACAGTAGTATTAAAGACCATATAAAT GTAGTGCTAAATCGTGATGTGTCTTTTCAAGATTTAAGTGATAATCCCTAC AAAGTAGATTTGCACACACAATCTATAGACGGTGCATGGGGTTCTGGGGT AGGTTTTACTCTAGTATGTATTGTGGGTCTTACAGAGTGTG

Bayou virus (GenBank L36930)

ATGAGTGTGAAACAGCAAAAGAACTTGAGGGACATAAAAAAAGCT GTGCTGAAGGGCAGTGCCCTTACTGCATGACTCTTACAGAAGCAACAGAG AGTGCCCTCCAGGCCCATTATGCAGTCTGTAAATTAACTGGGCGGTTTCAA GAGGCATTAAAAAAATCACTTAAGAAGCCAGAAGTCAAGAGAGGCTGTTA TAGAACACTTGGTGTTTTTCGTTACAAGAGTAGGTGTTATGTCGGTCTAGT CTGGTCATTTTTACTGACACTTGAATTAATAGTCTGGGCTGCCAGTGCTGA $\mathrm{C}-$

CTGTGAGTATCAAGGGAATACTGTCTCTGGATTTAAGCGGCTAATGGCAAC TAAAGACTCATTTCAATCATTTAATGTCTCTGAGGCACACATAACAACAAA CAGTCTAGAGTGGGTAGATCCAGACAATAACATAAAAGATCATATCAATC TGGTTCTTAACCGGGATATTTCATTTCAAGATCTGAGTGACAATCCATGTA AGGTGGACCTGTCAACACAATCGATTGATGGTGCATGGGGCTCGGGTGTT GGTTTCACATTGACATGTGTAGTTGGCCTGACAGAATGCC

Black Creek Canal (GenBank L39950)

ATGAATGTGAAACAGCAAAAGAACTTGAAAGCCATAAAAAAAGTT GTGCTGATGGGCAGTGCCCATACTGTATGACTATTACCGAGGCAACTGAG AGTGCTTTACAGGCCCATTATGCTGTATGTAAATTAACAGGGCGCTTTCAT GAGGCTTTAAAAAAATCATTAAAAAAACCAGAGGTTCAGAGGGGTTGTTA TAGAACACTTGGTGTTTTCCGTTATAAGAGTCGTTGCTATGTGGGCTTAGT ATGGATGTGTTTGTTGACTCTTGAGTTGATCGTTTGGGCTGCTAGTGCAGA T

TTGTGAGTATCAAGGGAATACAGTCTCTGGTTTTAAAAGATTGATGGCTAC AAAGGACTCGTTTCAGTCATTTAATGTATCTGAAGTACACATTACAACAAC CAAGCTAGAATGGAGTGATCCTGATAGTAACATCAAAGATCATATAAATT 
TGATTTTAAACCGAGATGTATCATTTCAAGACTTAAGTGACAATCCGTGCA AAGTGGACCTTTCAACACAGGCAATTGATGGTGCATGGGGCTCTGGTGTA GGTTTTACATTGACATGTATAGTGGGATTAACAGAATGCT

Andes Virus (GenBank NC003467)

ATGAGTGTGAAACAGCAAAAGAACTTGAATCACATAGACAGAGTT GTATCAATGGACAATGTCCTTATTGCATGACAATAACTGAAGCAACTGAA AGTGCCTTGCAAGCCCATTATTCCATTTGTAAATTGACAGGAAGATTTCAG GAGGCACTGAAAAAGTCACTTAAAAAGCCAGAGGTAAAAAAAGGTTGTTA CAGAACACTCGGGGTATTTAGATATAAAAGTAGATGTTATGTGGGTTTGGT ATGGTGCCTATTGTTGACATGTGAAATTGTTATTTGGGCCGCAAGTGCAGA G-

TTGTGAATATCAAGGAAATACCATTTCTGGATATAAAAGAATGATGGCAA CAAAAGATTCATTCCAATCATTTAACTTAACAGAACCTCACATCACAACAA ACAAGCTTGAATGGATCGACCCAGATGGGAATACAAGAGACCACGTAAAC CTTGTCTTAAATAGAGATGTCTCATTTCAGGATTTAAGTGATAACCCCTGT AAAGTAGACCTACACACACAAGCAATAGAAGGGGCATGGGGTTCTGGTGT AGGGTTTACACTCACATGTACTGTCGGATTAACAGAGTGCC

Hu39694virus (GenBank AF028023)

ATGAGTGTGAAACAGCAAAAGAATTAGAATCCCATAAACAAAGCT GCATCAACGGGCAATGCCCTTACTGTATGACCATGACAGAGGCAACAGAA AGTGCCTTACAGGCTCATTACTCTATTTGTAAATTAACAGGCAGATTCCAG GAAGCTTTAAAGAAATCACTGAAGAGACCAGAAGTCAAAAAGGGTTGTTA TCGTACATTAGGGGTATTTAGGTACAAGAGTAGATGCTATGTTGGGCTGGT GTGGTGTGTGCTCTTAACATGTGAACTTATCGTATGGGCTGCTAGTGCAGA G-

TTGTGAGTACCAAGGGAACACAATCTCAGGATATAAAAGAATGATGGCCA CAAAGGATTCATTCCAATCTTTCAATTTGACTGATCCACATATTACAACAA ATAAACTTGAATGGATTGACCCTGATGGTAACACACGCGATCATGTAAATT TAGTATTGAATAGAGATGTTTCATTTCAGGATCTAAGTGATAACCCATGTA AGGTTGATCTACACACACAAGCTATAGAAGGAGCATGGGGTTCAGGTGTT GGATTTACATTAACTTGTACTGTCGGATTGACTGAGTGCC

Oran Virus (GenBank AF028024)

ATGAATGTGAGACAGCAAAAGTGTTAGAATCACATAAACAAAGCT GCATTAATGGACAGTGCCCGTACTGCATGACTATGACTGAGGCAACTGAA AGTGCTCTACAAGCTCATTATTCTATCTGCAAATTGACAGGGAGGTTTCAG GAGGCTTTAAAAAAATCATTAAAAAAACCAGAGGTAAAAAGGGGTTGTTA CCGTACCTTAGGTGTATTTAGATACAAGAGTAGATGTTATGTTGGATTAGT TTGGTGTGTGCTTTTAACATGTGAGCTTATTGTCTGGGCAGCTAGTGCGGA A--

ATGTGAGTATCAAGGGAATACCATCTCAGGCTATAAACGAATGATGGCAA CAAAGGATTCATTTCAATCATTTAACCTCACTGACCCACACATCACAACAA ACAAACTTGAATGGATTGACCCTGATGGCAATACAAGGGATCATGTAAAT 
CTCGTTTTAAATAGAGACGTCTCATTTCAGGATTTAAGTGACAACCCGTGT AAGGTTGATTTACATACACAGGCAATAGAAGGGGCATGGGGTTCTGGTGT TGGATTTACACTTACATGTACAGTTGGTTTGACTGAGTGTC

Lechiguanas virus (GenBank AF028022)

ATGAGTGTGAGACAGCCAAAGAATTGGAATCTCATAAGCAAAGTTG CATAAATGGTCAATGTCCATATTGTATGACTATGACTGAGGCAACAGAAA GTGCGCTACAAGCTCATTTTGCTATTTGTAAGCTTACAGGGAGATTCCAGG AAGCCTTAAAAAAATCATTGAAGAAACCAGAAGTCAGAAAGGGCTGTTAC CGTACTCTAGGAGTTTTTAGATACAAGAGCAGGTGCTATGTTGGCTTAGTT TGGTGTGTGTTATTGACATGTGAGCTCATTGTATGGGCTGCTAGTGCAGAG

ATGTGAGTACCAAGGTAACACTATATCTGGATACAAGAGAATGATGGCTA CAAAGGATTCTTTTCAATCATTTAATCTTACTGACCCTCACATAACTACAA ATAAGCTTGAATGGATCGATCCAGATGGCAATACACGAGATCATGTCAAT CTTGTATTGAATAGAGATGTGTCATTTCAAGATTTAAGTGACAATCCTTGT AAGGTTGATTTGCATACACAAGCTATTGAAGGGGCTTGGGGTTCTGGTGTT GGGTTTACATTGACTTGCACTGTTGGTTTAACTGAATGCC

Juquitiba virus (GenBank 168711)

TCTTTAAAGAAACCAGAGATTAAAAGGGGTTGCTATCGCACCATCGGTGTT TTTAGATATAAAAGTAGATGTTATGTGGGGTTAGTATGGTGTTTATTGTTA ACTTTCGAATTGATAGTTTGGGCAGCAAGTGCAGAG-

ATGTGAGTACCAAGGCAATACTGTGTCAGGGTATAAGAGGATGATGGCAA CCAAGGATTCTTTTCAGTCTTTTAATCTAACAGATCCACATATAACAACCA ACAAATTGGAGTGGATAGACCCTGATGGGAATACAAGGGATCATGTCAAC CTTGTATTGAATCGTGATGTGTCTTTCCAGGACCTAAGTGATAACCCTTGT AAGGTAGACCTTCATACACAGGCTATTGAGGGGGCATGGGGCTCAGGTGT TGGTTTCACATTAACATGTGGGGTTGGGCTAACAGAATGCC

Maciel virus (GenBank AF028027)

ACGAATGTGAAACAGCTAAAGAGCTAGAATCACATAGACAGAGTT GTCTTAATGGGCAATGCCCATATTGTATGACAATGACAGAGGCAACAGAA AGTGCTTTGCAAGCTCACTATTCCATTTGCAAGTTAACTAATAGGTTTCAG GAAGCACTAAAGAAGTCATTGAAGAAACCAGAGGTAAGAAGAGGTTGTT ACAAAACATTAGGTGTGTTTAGATACAAAAGTAGATGCTATGTGGGCTTA GTCTGGTGTATATTGCTAACCTTTGAATTGATCATTTGGGCTGTGAGTGCA GAA-

ATGCGAGTATCAAGGGAATACTGTCTCTGGCTATAAAAGATTGATGGGCA CAAAAGGTTCTTTCCAATCATTTAACCTTACAGAACCACATATCACCACAA ATAAACTTGAATGGATTGACCCAGATGGTAATACCCGTGATCATGTAAATC TCATCTTAAATAGAGATGTGTCATTCCAGGATCTGAGTGACAACCCTTGTA AAGTAGATTTGCATACACAAGCAATTGAAGGGGCTTGGGGATCGGGGGTT GGTTTCACACTCACATGTGTTGTCGGTCTCACAGAATGCC 
El Moro Canyon (GenBank U26828)

ATGAATGTGAAACAGCAAAAGAACTCGATTGTCATAAAAAGAGCT GTGCTGAAGGACAATGCCCTTACTGCATGACATTGACAGAATCTACAGAA AGTGCACTTCAAGCACATTTTGCAATCTGCAAACTAACTTCAAGATTTCAG GAGAATTTGAAGAAATCTTTGAAAAGGCAGGATGTTAAACCTGGCTGTTA CAGAACTCTAGGTGTATTTAGATACAAGAGCCGTTGTTATGTCGGGCTTGT ATGGGGCTTTTTACTGACAATTGAGCTTGTTATTTGGGCAGCAAGTGCAGA $\mathrm{T}-$

TTGTGAGTATCAAGGGAATACAGTTTCAGGATATCAAAGGATGATGGCTA CAAAAGATTCTTTTCAGTCATTTAATGTCACGCAACCGCACTTAACAGCCA ATCTACTGGAATGGGTGGACCCAGATAGTACCATCAAAGACCACATTAAC CTAATTCTCAATAGAGATTTATCATTTCAAGACTTGGCAGAGAACCCATGT AAAGTGGATTTACATACCCAAAACATTGATGGAGCATGGGGATCTGGGGT GGGCTTTACATTAACGTGTATTGTAAGCCTGACAGAATGTA

Prospect Hill (GenBank X55129)

AGGAATGTGAAATGGCAAAAGAATTGGAGTCTCACAAGAAAAGTT GTCCTAATGGTATGTGCCCTTACTGCATGAACCCTACAGAGTCTACTGAGT CTGCCCTGCAAGCTCATTTCAAAGTGTGTAAACTTACAACAAGGTTCCAGG AGAACCTTCGCAAGTCACTAAATCCATATGAGCCTAAACGAGGTTGCTAT AGGACACTCTCTGTGTTTAGATATCGGAGTAGGTGCTTTGTGGGCCTTGTT TGGTGCATCCTACTTGTATTAGAACTTGTCATATGGGCTGCAAGTGCGGAT-

ATGTGAGTATGATGGAAACACTCTTTCTGGTTACCAGCGGATGCTTGCAAC AAGGGATTCTTTCCAGTCTTTCAACATTACAGAACCACATATTACCAGCAA TTCACTTGAATGGGTAGATCCAGATAGTTCATTAAAGGATCATATTAATTT GGTAGTTAATCGCGATGTATCATTTCAGGACTTATCTGAAAATCCCTGTCA AGTTGGTGTGGCCGTGTCTTCCATTGATGGTGCATGGGGCTCAGGTGTGGG TTTTAATCTGGTATGTAGTGTGAGTTTGACAGAATGTG

Tula virus (GenBank NC005228)

AAGGATGTGAGACTGCAAAGGAGCTAGAATCTCACAAAAAAAGCT GTCCACATGGACAGTGTCCATACTGTCTCAACCCAACTGAAGCTACTGAGT CTGCCCTACAGGCTCATTTTAAAGTCTGTAAGTTGACAACTAGGTTTCAAG AGAATTTAAAGAAATCATTAAGTACATATGAGCCTAAACGTGGCCTCTATC GAACACTCTCCATGTTTAGATACAAAAGCAAATGCTATGTGGGTTTGGTTT GGTGTATTTTGTTAACTATGGAGTTGATAGTGTGGGCAGCTAGTGCTGAG--

ATGTCAGTATGATGGAAACACTGTATCTGGATATCAGAGAATGATTGCCA CAAAAGATTCATTCCAGTCATTCAATATTACAGAACCCCATATTACAACAA ATTCACTAGAATGGGTTGACCCAGATAGTTCTCTAAAGGACCATGTCAATC TGATAGTAAACCGGGATCTATCTTTCCAAGATTTAGCTGAAAATCCATGTC AGGTTGATTTAAGTGTATCATCAATTGATGGCGCATGGGGCTCAGGTGTGG GGTTTAATTTAGTCTGCTCTGTCAGCCTTACAGAATGTG 
Puumala virus (GenBank NC005223)

ATGAGTGTGAAACTGCTAAGGAGTTAGAATCACATAGAAAGAGCT GTTCAATTGGTTCATGCCCTTATTGCCTTAATCCATCTGAGGCTACACCGTC TGCTCTTCAAGCTCATTTTAAAGTTTGTAAACTAACATCACGGTTTCAGGA AAATTTGAAGAAGTCACTAACAATGTATGAGCCAATGCAGGGTTGTTATC GAACATTATCTCTATTTAGGTACCGCAGTAGATTTTTTGTAGGTTTAGTGTG GTGTATGTTGCTTGTTTTAGAGCTAATTGTATGGGCTGCTAGTGCTGAG-----

ATGTCAGTTTGATGGGAATACAATTTCAGGTTATAAACGGATGGTTGCAAC AAAAGATTCATTTCAATCATTTAATGTAACAGAACCACACATCTCTACAAG TGCATTGGAGTGGATTGACCTAGATAGTTCACTTCGGGATCACATCAATGT GATTGTAAGCCGTGATTTGTCCTTTCAGGATTTAAGTGAAACACCTTGTCA AGTGGATTTGACAACATCAGCTACAGATGGGGCATGGGGTTCAGGAGTTG GTTTTAACCTGGTATGTACTGTTAGTTTAACAGAATGCT

Hantaan virus (GenBank DQ371905)

ATGAGTGTGAGACTTATAAAGAACTAAAGGCACATGGGGTCTCATG CCCACAATCCCAATGCCCTTATTGTTTCACCCACTGTGAGCCCACAGAAGC TGCATTTCAGGCCCATTATAAAGTGTGTCAAGTTACTCATAGATTTAGGGA TGATTTAAAGAAGACAGTTACTCCCCAGAACTTCACACCTGGTTGCTATCG AACATTGAATTTATTCAGATATAAGAGTAGGTGTTATATTTTTACAATGTG GGTTTTTCTTTTAGTCTTAGAATCAATCTTATGGGCAGCCAGTGCATCA-----

TTGTGAATATGATGGGAATATGGTTTCAGGTTATAAAAAAGTAATGGCCA CAATTGATTCTTTTCAATCCTTCAATACAAGCACTATGCATTTCACTGATGA GAGGATAGAATGGAAAGACCCTGATGGGATGTTAAGGGACCATATTAACA TTCTGGTTACAAAGGATATTGACTTTGATAATCTTGGTGAAAATCCCTGCA AGATTGGATTACAAACATCCTCCATTGAAGGGGCATGGGGGTCTGGTGTA GGCTTTACACTTACATGTTTGATATCACTGACAGAATGCT

Dobrava virus (GenBank NC005234)

ATGAGTGTGAAACAGGGAAGGAGCTTAAAGCCCATAATTTATCTTG CCCTCAGTCACAGTGTCCTTATTGCTTTACACACTGTGAGCCTACAGAATC TGCCTTTCAAGCACATTATAAAGTGTGCCAAGCAACACACAGATTTAGGG ATGATTTAAAGAAAACAATAACACCTCAATCAACAAGCCCAGGTTGTTAC CGGACATTAAATCTTTTTAGGTATAAAAGTAGATGTTACATCTTTACAGTG TGGGTGACCTTACTAATCATTGAATCAATCATGTGGGCAGCTAGTGCATCA

GTGCGAGTATGATGGTAATATGGTCTCTGGGTATAAGAAAGTAATGGCAA CTATTGATTCCTTTCAATCATTTAACACTAGTTCAATTCATTATACAGATGA AAGGATTGAGTGGAAAGACCCGGATGGAATGCTTAAGGACCACCTTAATA TACTTGTCACAAAGGATATTGACTTTGAAAACCTTGGAGAGAACCCGTGTA AAGTAGGACTTCAAACATCATCAATAGAAGGTGCATGGGGTTCTGGGGTT GGTTTCACTCTCACTTGTCAAATCTCACTGACAGAGTGTT 
Seoul Virus (GenBank NC005237)

ACGAGTGTGAAACATTAAAGGAATTGAAGGCACATAATCTATCATG TGTTCAAGGGGAATGCCCATATTGCTTTACCCACTGTGAACCGACAGAAAC TGCAATTCAGGCACATTACAAAGTTTGTCAAGCCACCCACCGATTCAGAG AAGATTTAAAAAAGACTGTGACTCCTCAAAATATTGGGCCTGGTTGTTACC GAACATTAAATCTTTTTAGGTATAAAAGTAGGTGTTATATTCTGACAATGT GGACTCTTCTTCTCATTATTGAATCCATTCTCTGGGCAGCAAGTGCAGCA---

TTGTGAATATGATGGGAATATTATCTCAGGCTATAAGAAAGTTCTTGCAAC AATTGATTCTTTCCAATCATTTAACACAAGCAATATACACTTCACTGATGA GAGAATTGAATGGAGAGACCCTGATGGTATGCTTCGGGATCATATTAATA TCGTTATTTCTAAAGATATTGATTTTGAAAATTTGGCTGAGAATCCTTGTA AAGTAGGGCTCCAGGCAGCAAACATAGAAGGTGCCTGGGGTTCAGGTGTC GGGTTTACACTCACATGCCAGGTGTCTCTCACAGAATGCC

Sangassou virus (GenBank DQ268651)

GTGTGAGTACTCAGGTAATATGGTATCTGGTTATAAAAAAGTCATGGCCAC AATTGACTCATTTCAATCATTTAATACAAGTTTAATTCACTTTACAGAAGA GAGGATTGAATGGAAGGATCCTGACGGGATGCTTAGGGACCACCTGAACA TACTAGTAACAAAGGACATCGACTTTGAAAATCTTGGGGAAAACCCTTGT AAAGTAGGGCTTCAAACATCATCT 
Appendix G: Rodent Multiple Sequence Alignment. Sequences of mitochondrial ND3, ND4, ND4L, and arginine tRNA genes aligned with one another by codon reading frame.

\begin{tabular}{|c|c|}
\hline wes. & \\
\hline we11 & \\
\hline wci2 & \\
\hline wC13 & \\
\hline $1 \mathrm{jd} 256$ & \\
\hline 1jd9 304 & \\
\hline jof 9306 & \\
\hline 1jd 3308 & \\
\hline 1jda3.17. & \\
\hline 109324 & \\
\hline 1jब3927 & \\
\hline $1 \mathrm{jd} 9330$ & \\
\hline jod3333 & \\
\hline 1) 93944 & \\
\hline $1 \mathrm{j} 09348$ & \\
\hline $40620^{\circ}$ & \\
\hline LJOT7A & \\
\hline 40886 & \\
\hline 40208 & \\
\hline 401960 & \\
\hline No1669 & \\
\hline UD1524 & \\
\hline 401523 & \\
\hline HD1122 & \\
\hline 402522 & \\
\hline UD1637 & \\
\hline UD1638 & \\
\hline LiD2161 & \\
\hline LD2651 & \\
\hline LD2737 & \\
\hline 402834 & \\
\hline LJO2850 & \\
\hline LD22998 & \\
\hline ED2914 & \\
\hline 402936 & \\
\hline LUO30000 & \\
\hline 402126 & \\
\hline 402138 & \\
\hline $40215 A$ & \\
\hline UJD2158 & \\
\hline LID2836 & \\
\hline UD2852 & \\
\hline LلjQ2860 & \\
\hline 402665 & \\
\hline 402890 & \\
\hline UD?2943 & \\
\hline $\begin{array}{l}\text { Jefferson } \\
\text { ADAMS6 }\end{array}$ & \\
\hline
\end{tabular}

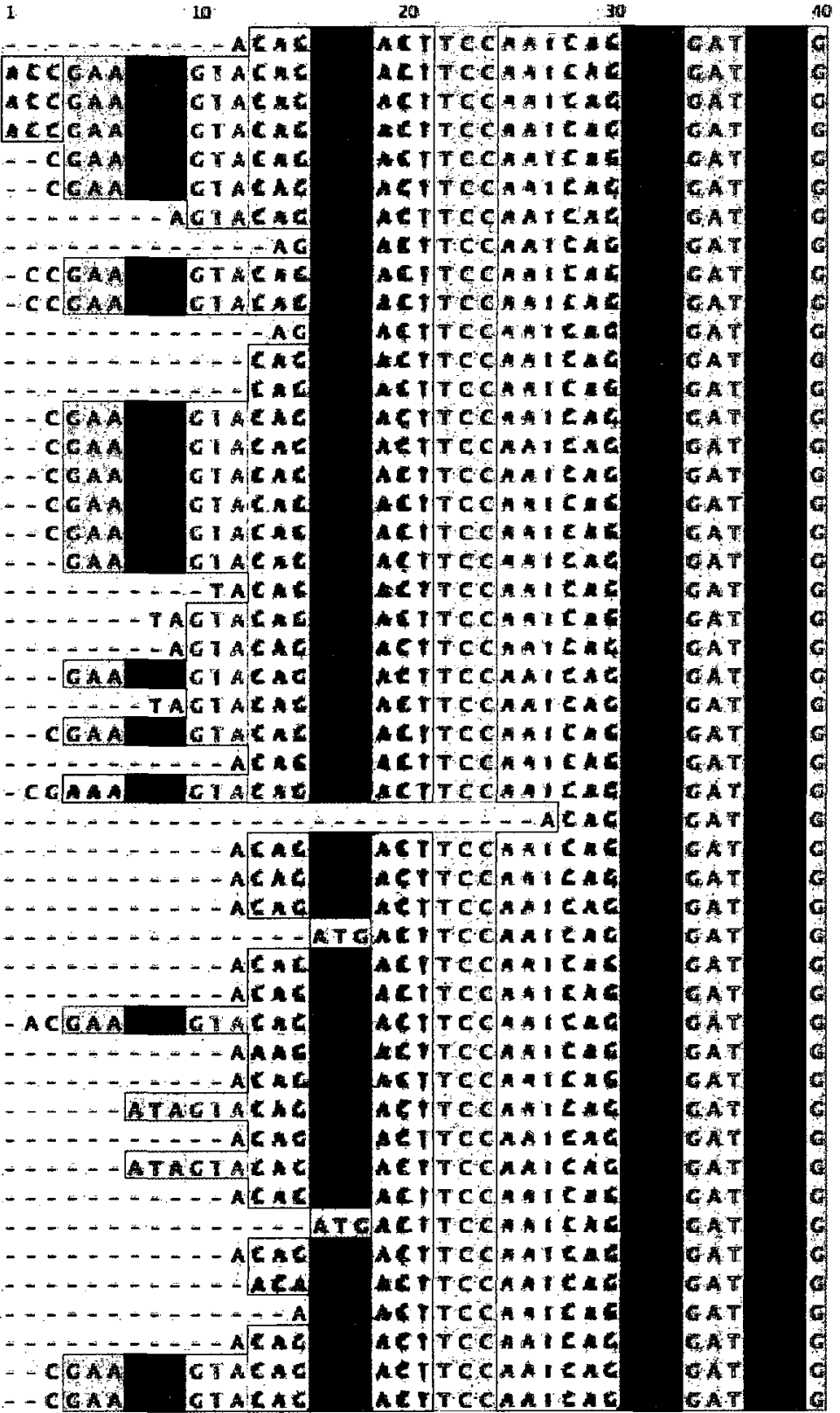


clatsop1

- - - - - - ... - . . - -

clatsop2

clatsop3

clatsop4

LostForest 2

LostForest 3

LostForest4

sj1Pmausterus

sj2P̄mausterus

s]2aPmausterus

sj3Pmausterus

sj5Pmausterus

sj6Pmausterus

sj7Pmausterus

OSM723Pmman

OSM726Pmman

OSM727Pmman

OSM737Pmiman

U40255PsejugSD

U40253PsejugsC

JEB 1309APtruei

AF374578PKEEN

U40252Pleuc

JAM160Pleuc

JAM161Pleuc

JAM162Pleuc

JAM163Pleuc

JAM164Pleuc

JAM168Pmbairdi

JAM 167Pmbairdii

JAM159Pmbairdi

JAM166Pmbairdli

-

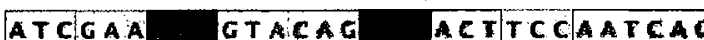

ATCUAA GT GICA ACTTCCAATCÁ

ATCGAA

- ACGAA

G T A $\mathbf{C} A \mathrm{C}$

- - . - . - - CAC

- . - . - ...- $\mathrm{CAC}$

$-\ldots--\ldots+\ldots+-\mathbf{A} \mathbf{C}$

- - - . . . ... - AC

-.........- CA

- . . . - . - -

$---\ldots-\ldots-\ldots--$ CA

- c GAA GTACA

- CCAA

- CGAA

- CGAA

- - - A A

- - - A A

$---\mathbf{A} A$

-. - A A

- - A A

G TACAC

ACTTCCAATCAC

AETTCCAATCAC

AETTCEATEAC

ACT TCCAATEAG

ACTTCEAATEAC

AC TTCCAATEAC

ACTTCCAATCAE

AETJCCAATEA

ACTJCCAATCAO

ACTTCCAATCAC

ACtTCCAATEAG

G TACAGgTGAETTCCA TCA

CT ACAG

GTACA

CTACAC

G TACAG

GTACA A

ACAC

-.... T AGTACAC

............ - $\mathrm{C}$

- - - - - - . - . - -

$\ldots \ldots+. .-\ldots$

-.. A GTACAC

....... GTACA

$\ldots \ldots . . . . .-$ CA

.......... CAC

aETTCCAATEAG

ACTTCCAATEAC

ACTTCCAATEAG

ACTTCCAATCAE

ACYTCGAATCAC

AETTCCAATCAC

AETTCCAATEAC

ACTICCAATCAG

AETTCCAATCAG

AETTCCAATCAG

aCTTCEAATCAG

act TCCAateac

ACTTCCAATCAG

ACTTCCAATCAG

ACIICCLATEAG

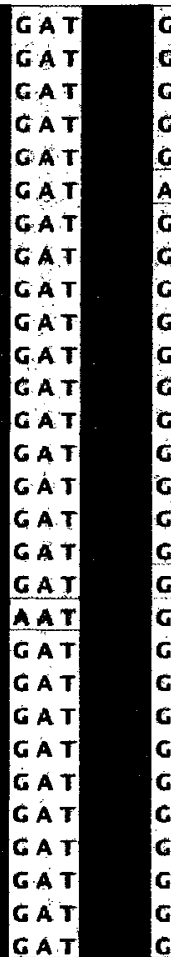

Microtuspennsylvanic

Oryzomyspalustris

Sigmodonhispidus

Reithrodontomysmeg

Rattusnorvegicus

Pinrufinus

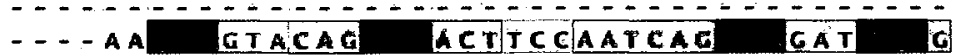


WC7

we11

WC12

wC13

Vd 3256

Id3304

Ijd3306

ljd3308

Jd3317

IJd3324

IJd3327

ljd3330

ljd3333

Jd 3344

IJd3348

LلJD20

LJD774

Lل10886

LJD2088

LJD1960

LJD1669

LJD1524

LJD1523

แJD 1122

LJD2522

LJD1637

LJD1638

LJD2 161

LJO2651

LJD2737

LJD2834

LJD2850

LJD2898

UJD2914

LJD2936

LJD3000

บ02126

LJD2138

LJD2154

LJD2 158

LJD2836

UJD2852

UDD2860

LJD2865

LJD2890

LJD2943

Jefferson 3

ADAM56

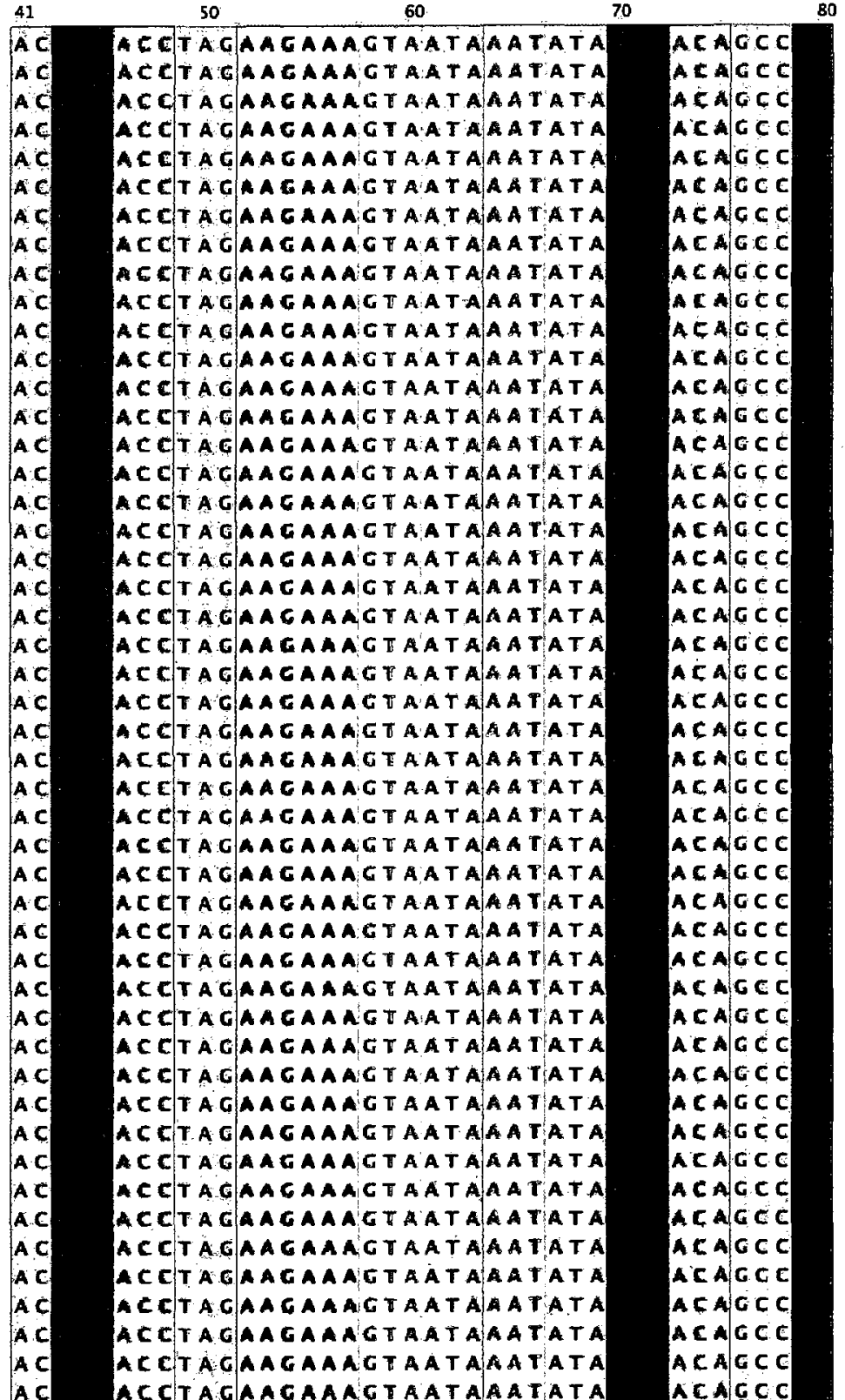




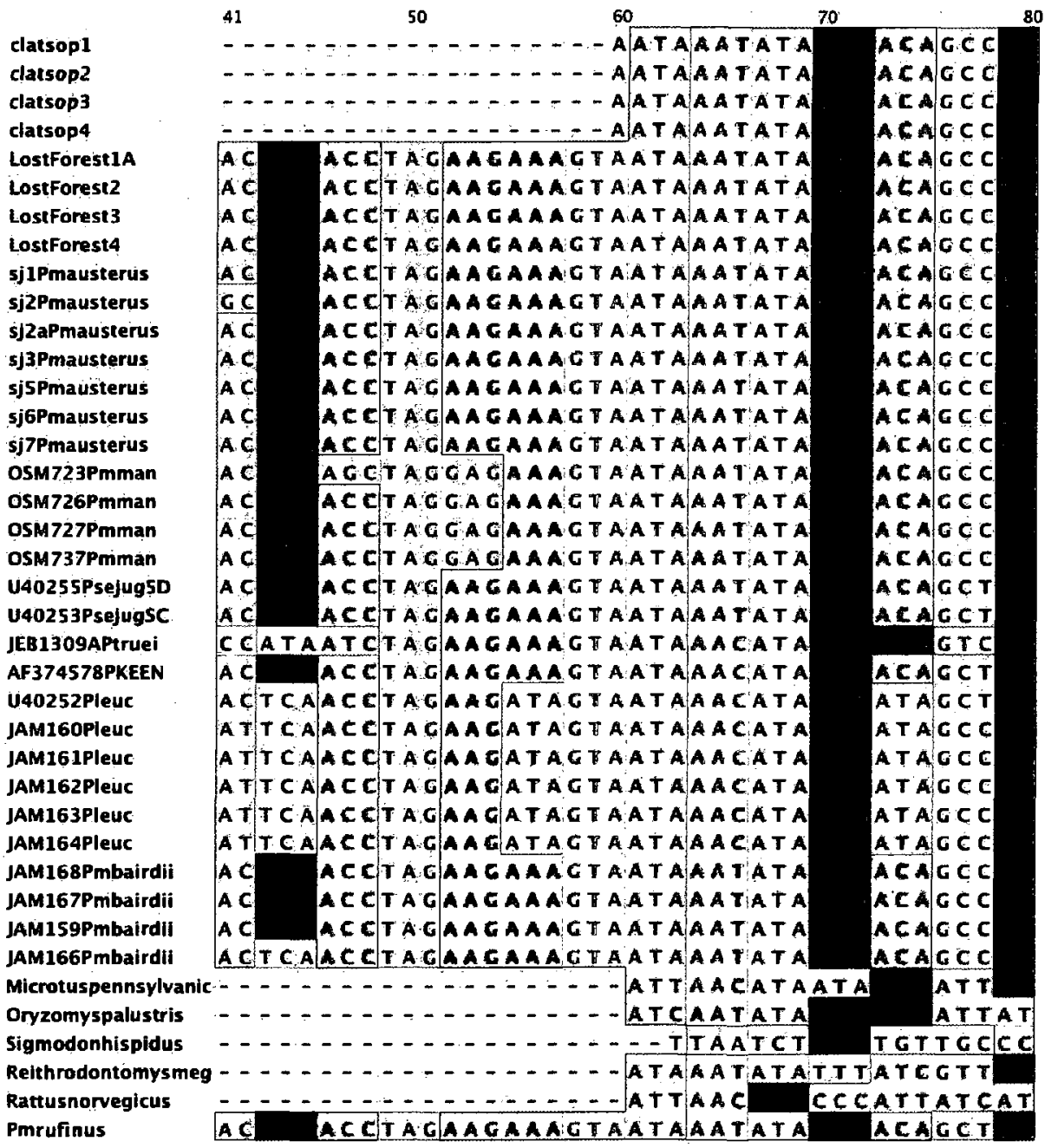


wC7

WC11

wC12

WC13

Jjd3256

Jjd3304

IJd3306

ljd3308

IJd3317

IJd3324

IJd3327

ljd3330

jjd3333

ljd3344

Jd3348

니 620

LJD774

LJD886

LJD2088

LJD1960

니1669

LJD1524

LJD1523

LJD1 122

LJD2522

LDD1637

LJD1638

LJD2 161

LJD2651

LJD2737

LJ02834

LJD2850

LJD2898

LJD2914

LJD2936

LJD3000

LJD2126

LJD2138

LJD2154

LJD2158

LJD2836

LJD2852

LJD2860

LJD2865.

LJD2890

LJD2943

Jefferson 3

ADAMS6

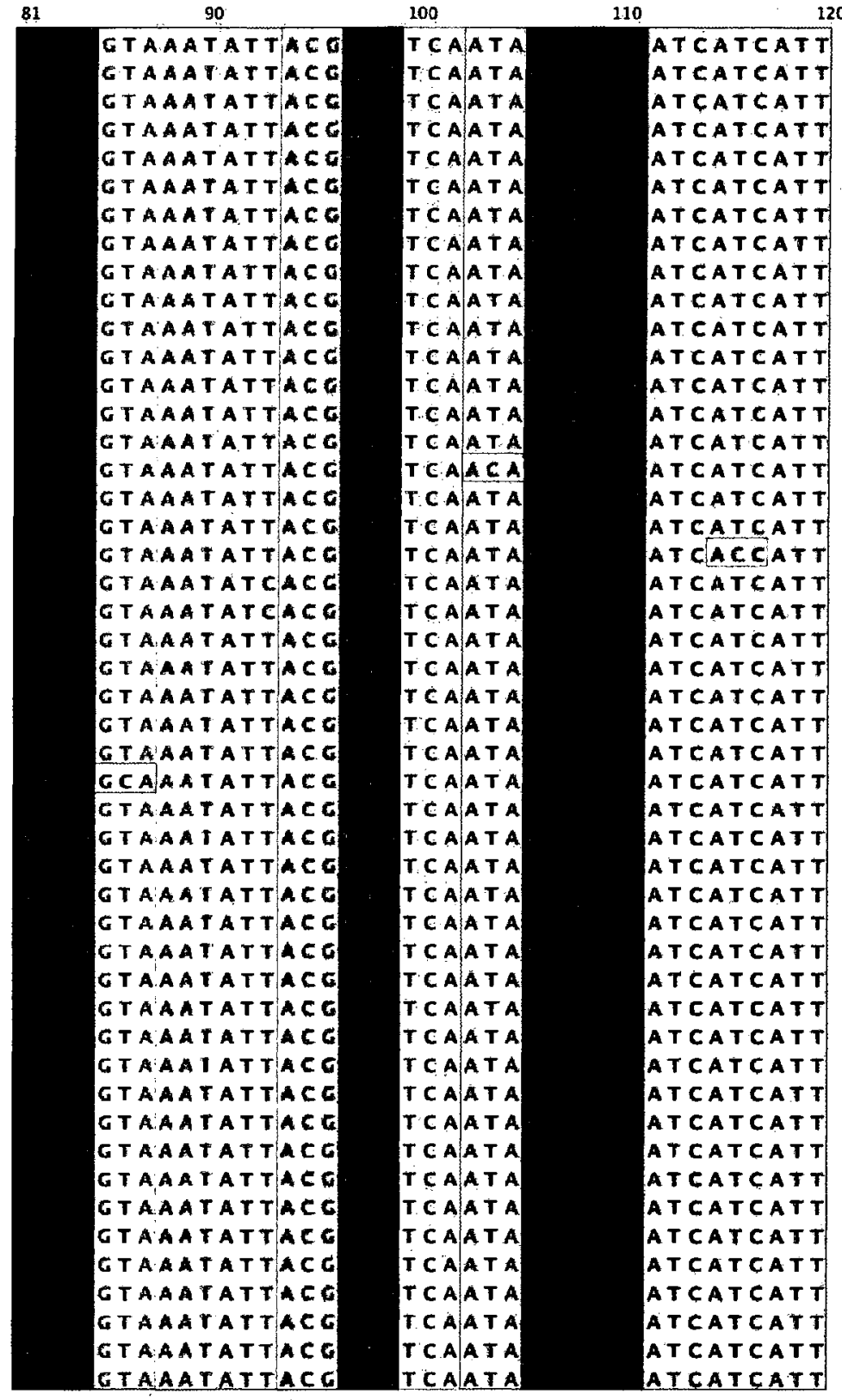




\begin{tabular}{|c|c|c|c|c|c|}
\hline & 90 & & 100 & 110 & \\
\hline clatsop1 & GTAAATATT & $A C \mathrm{C}$ & $T \subset A$ & AT A & ATCATCATT \\
\hline clatsop2 & GTAAATATT & $A \subset G$ & TCA & AT A & ATCATCATT \\
\hline clatsop3 & GTAAATATT & $a \subset d$ & TCA & ATA & ATCATCATT \\
\hline clatsop4 & GTAAATATT & $A<G$ & $T C A$ & A T A & ATCATCATT \\
\hline LostFarest IA & GT AAATATT & 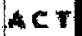 & $T \subset A$ & ATA & AT CA C CAT T \\
\hline LostForest2 & G TAAATATT & act & TCA & $A C A$ & ATCATCATT \\
\hline LostForest 3 & G IAAATATT & $A<c$ & T CA & A T A & АТС СТСАT T \\
\hline LostForest4 & CTAAATATT & $A \in C$ & TEA & $A C A$ & ATCATCATT \\
\hline sj1Pmausterus & GTAAATATT & $A \subset G$ & T.CA & AT A & ATCATCATT \\
\hline sj2Pmausterus & GTAAATATT & $A<$ o & $\mathbf{T} C A$ & A T A & ATCATCATT \\
\hline sj2aPmausterus & G TAAATATT & $A<G$ & TCA & AT A & ATCATCAT T \\
\hline 5j3Pmausterus & GTAAATATT & $A<\theta$ & $T C \dot{A}$ & A T A & ATCATCATT \\
\hline sj5Pmausterus & GTAAATATT & $A C \mathrm{C}$ & $T: C A$ & ATA & ATCATCATT \\
\hline sj6Pmausterus & GT AAATATT & $A C G$ & TCA & & ATСАTCATT \\
\hline sj7Pmausterus & GT AA A TATT & $A \subset G$ & $\ddot{\mathbf{T}} \mathbf{C A}$ & & ATCATCATT \\
\hline OSM723Pmman & CTAAATATT & $A \in A$ & $\mathbf{T} \mathbf{C A}$ & $A T$ & ATCATCATT \\
\hline OSM726Pmman & G TAA I ATT & $A \in A$ & $\mathbf{T} \in \mathbf{A}$ & & ATCATCATT \\
\hline OSM727Pmman & G T AAATATT & $A \subset A$ & $\mathbf{T} \in \mathbf{A}$ & AT A & AT CATCATT \\
\hline O5M737Pmman & G T AA A T AT T & $A \subset A$ & $T<A$ & & A T С T С АT T: \\
\hline U40255PsejugsD & GTAAATATT & $A<G$ & T C A & A.T & ATCATCGTT \\
\hline U40253Psejugse. & GTAAATATT & $A<G$ & $\mathbf{T} \cdot \mathbf{C}$ & & ATCATCG T T: \\
\hline JEBI 309APtruei & TCAGTAAACATT & $A C T$ & $\mathbf{T} \in \mathrm{C}$ & & AT T $\quad$ AT T \\
\hline AF374578PKEEN & GTAAATATT & $A \subset A$ & $\mathbf{T} \subset \mathrm{A}$ & & ATCATCGTT \\
\hline U40252Pleuc & TAGTAAACATC & $A<A$ & $\mathbf{T} \mathbf{C A}$ & ATC & ATCACACTT \\
\hline JAM160Pleuc & A TAGTAAACATC & $A C, A$ & $\mathbf{T} \subset \mathbf{A}$ & AT C & ATCACAGTT \\
\hline JAM161Pleuc & ATAGTAAACATC & $A \subset A$ & $T<A$ & ATC & ATCACAGTT \\
\hline JAM162Pleuc & ATAGTAAACATC & $A \subset A$ & $\mathbf{T C A}$ & A T C & ATCACAGTT \\
\hline JAM163Pleuc & ATAGT AAACATC & & $\mathbf{T C A}$ & & ATCAEA G T T \\
\hline JAM164Pleuc & ATAGTA:A ACATC & $A \in A$ & $\mathbf{T} \in \mathbf{A}$ & ATC & ATCACAGTT: \\
\hline JAMi68PmbairdiI & GTAAATACT & $A \in T$ & $\mathbf{T} \mathbf{C A}$ & AT T & ATCACCATT \\
\hline JAM167Pmbairdii & GTAAATATT & $A \subset T$ & $\mathbf{T C A}$ & A.T T A & ATCACEATT \\
\hline JAM 159Pmbairdii & GTAAATATT & $A C T$ & $\mathbf{T} \subset \mathbf{A}$ & & ATCACEATT \\
\hline JAM166Pmbairdii & GTAAATATT & $A \in T$ & $\mid \mathbf{T} \cdot \mathbf{C} \cdot \mathbf{A}$ & $A T T$ & ATCACSATT \\
\hline Microtuspennsylvanic & ATAAACATC & $G \subset C$ & CCT & GCCATC & ATT T C T \\
\hline Oryzomyspalustris & ACTAACATT & C T A & ICA & & $A T T T \subset C G T A$ \\
\hline Sigmodonhispidus & CATCAACGC & & ATC & CATCC & AAT ACT \\
\hline Reithrodontomysmeg & GTAAATATT & TC.C & GCA & T C CTGT & AT T \\
\hline Rattusnorvegicus & ATTAACATC & $A C C$ & $\mathbf{T C C}$ & TTTATC & AT TTCA|AT T \\
\hline Pmrufinus & GTAAATATT & $A C G$ & ICA & ATA & ATCATCATT \\
\hline
\end{tabular}


we

WC11

WC12

WC13

IJd3256

IJd3304

Jjd3306

ljd3308

Ud 3317

Jjd 3324

Ijd 3327

ljd3330

jjd3333

Jjd 3344

Ijd3348

LلJ620

LلJ 774

ㄴD886

LJD2088

LJD1960

LJD1669

LJD1524

LJD1523

LJD1 122

LJD2 522

니1637

LJD1638

LJD2161

LJD2651

니D2737

LJD2834

LJD2850

LJD2898

LJD2914

LJD2936

LJD3000

ㄴD2 126

LJO2 138

LJD2 154

LJD2 158

LJD2836

LJD2852

LJD2860

LLD2865

LJD2890

LJD2943

Jefferson 3

ADAMS6

\begin{tabular}{|c|c|c|c|c|c|c|}
\hline 121 & & & 15 & 50 & & \\
\hline GCCTTCTGA & CCEGAA & A.T & TAC & $A C T$ & GAA & $|A A A G|$ \\
\hline GCCTTCTCA & cectA & A A T & TAT & $A<T$ & GA A & $A A A C$ \\
\hline GCCTTCTGA & $\operatorname{Ccc} \operatorname{ca}$ & AAT & TAC & $A \subset T$ & GAA & $A \wedge A C$ \\
\hline GCCTTCTGA & $\mid c \operatorname{cc} A$ & $A \cap T$ & TAC & $A C T$ & GA A & $A \wedge A G$ \\
\hline CCCTTCTGA & $\operatorname{cec} \cos$ & A A T & TAT & $\mu \subset T$ & G A A & $A \mathbf{A}$ \\
\hline GCCTTCTCA & $c \in c<A 4$ & A A T & TA T & 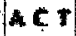 & GAA & AAA \\
\hline$C \subset C T \mathrm{TCT} A \mathrm{~A}$ & $\operatorname{ccc} A A$ & AAT & TAT & $A C T$ & $G A A$ & $A \wedge \sim c$ \\
\hline GCCTTCTGA & $\operatorname{cec} \boldsymbol{C A}$ & A $А T$ & T A T & $\mid A C T$ & GAA & $A \wedge A C$ \\
\hline GCCTTCTGA & $\operatorname{ccc} \in A$ & A A T & T A T & $A C T$ & GA A & $|A \wedge A|$ \\
\hline G C CTTCT G A & ceces & A A T & TAT & $A \in T$ & GA A & 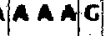 \\
\hline GCCTTCTGA & $\operatorname{cec} A$ & A A T & TAT & $A \in T$ & GA & $|A \wedge A|$ \\
\hline CCCTTCTGA & C C C CAA & A AT & $T A T$ & $A<T$ & GAA & 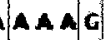 \\
\hline GCCTTCTGA & $\operatorname{cec} \in A$ & A $\mathrm{AT}$ & T AT & $A \in T$ & GAA & $\mid A \wedge A G$ \\
\hline C C CTTCTGA & CCCEA & AAT & T A T & $\mid A<T$ & GA A & $A A$ \\
\hline GCCTICTGA & cecte & AAT & $T A T$ & $a \in T$ & GA A & $A A \wedge C$ \\
\hline$C \subset C T$ T T T A & $\operatorname{ccc} A A$ & $\triangle A T$ & TAT & $A C T$ & GA A & $A A \wedge G$ \\
\hline GCCTTCTGA & $\operatorname{ccc} A$ & A AT & T.AT & $A \subset T$ & GA A & $A \wedge A \mid$ \\
\hline GCCTTCT G A & $\operatorname{ccc} \cos$ & A AT & T A T & $A<T$ & GAA & $|A A A G|$ \\
\hline CCCTICT GA & $\operatorname{cec} A$ & A A T & TAT & $A C T$ & GA A & $A \wedge A G$ \\
\hline C C CTTCTCA & $\operatorname{ccc} A \mathrm{~A}$ & AAT & T A T & $A C T$ & GA A & $A \wedge A C$ \\
\hline$G C C T T C^{\prime} T$ A & $\operatorname{cct} \cos ^{\prime} A$ & A AT & TAT & ACT & $C A A$ & $\mid A \wedge A G^{\prime}$ \\
\hline C C CTTCT GA & $\operatorname{ccc} A A$ & AAT & TAT & A C & G A A & $A \wedge \wedge G_{0}$ \\
\hline GCCT TCT CA & $\operatorname{ccc} c A$ & AAT & TAC & $A C T$ & $G A$ & $\mid A \wedge A G$ \\
\hline GCCTTCTCA & $\operatorname{cec} \in A$ & A A T & TAT & 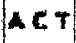 & GA A & $A \wedge A G$ \\
\hline CCCTTCTCA & $\operatorname{ccc} \in A$ & AAT & T A T & $A C T$ & GA A & $A \wedge \wedge d$ \\
\hline GCCTTCTGA & $\operatorname{ccc} \operatorname{ca}$ & AAT & TAT & $A C T$ & GA A & $|A \wedge A G|$ \\
\hline G С СТ T СТG A & $c \in c_{1} A A$ & A AT & T A T & Act & GAA & $A \wedge A G$ \\
\hline C C CTTCTCA & $\operatorname{CECCAA}$ & AAT & $\mathbf{T A T}$ & $A \subset T$ & CA A & $A \wedge A C$ \\
\hline GCCTTCTCA & $\operatorname{ccc} \theta A$ & A A T & T A T & $A \in \mathbf{r}$ & GA A & AA \\
\hline GC.TTCTGA & $\operatorname{ccc} A$ & AAT & T A T & $A C T$ & GA A & $A \wedge A G$ \\
\hline GCCTTCT CA & $\operatorname{cct} A A$ & A A T & TA & $A \in T$ & GA A & $A \wedge A G$ \\
\hline CCCT:TCTCA & $\operatorname{cec} A$ & AAT & T A.T & $A \subset T$ & CAA & $A \wedge A G \mid$ \\
\hline CCCTTCTCA & Ctcen & A A T & TAT & $A \in F$ & GA A & $A \wedge A C$ \\
\hline G C CTTCTG A & $\operatorname{ccctc} A$ & AAT & T A T & ACr & GA A & $A \wedge A G$ \\
\hline GC CTTCTGA & $\operatorname{C\subset C,CAA}$ & AAT & TAT & $A C T$ & GAA & $\wedge \wedge \wedge$ \\
\hline CCCTTCTGA & $\operatorname{cecta}$ & AAT & TA T & $A \in T$ & CAA & $A \wedge A C$ \\
\hline CCCTTCTCA & $\operatorname{ccc} \cos ^{-}$ & AAT & TA T & $A \in Y$ & GAA & $A \wedge A C$ \\
\hline GCCTTCTGA & ccteAs & A.T & TAT & $A \subset T$ & GA A & $A \wedge A C$ \\
\hline GCCTTCT GA & $\operatorname{ccc} \cos$ & AAT & TAT & $A C T$ & G A A & $A \wedge A G \mid$ \\
\hline GCCTTCTGA & $\operatorname{ccc} A A$ & A A T & $\mathbf{T A T}$ & $A C T$ & GA A & $|A \wedge A| G \mid$ \\
\hline GCCTTCTGA & $\operatorname{cccc} A$ & AAT & TAT & $A C T$ & G A A & $|A \wedge A|$ \\
\hline GCCTTCTGA & $\operatorname{ccc} A$ & AAT & TAT & $A \in t$ & GA A & $\mathbf{A} \wedge \mathbf{A} \mathbf{G}^{\prime}$ \\
\hline$G C C T$ T T GA & $\operatorname{cec} \cos$ & AAT & TAT & $A \in T$ & GAA & $A \wedge A G$ \\
\hline GCCTTCTGA & $\operatorname{cct} A A$ & A A T & TAT & $A \subset T$ & GA A & $A \wedge \wedge G$ \\
\hline$C \subset C T$ TCTGA & $\operatorname{ctc} \operatorname{cA} A$ & AAT & T A T & $A<T$ & GA A & $A \wedge A C$ \\
\hline GCCTTCTGA & $\operatorname{ccces} \theta$ & A A T & T A T & $A C T$ & GA A & $A \wedge A C$ \\
\hline CCCTTCTCA & $\operatorname{ccc} \wedge A$ & AAT & T A T & $A<T$ & GA A & $\mid A \wedge A G$ \\
\hline CCCTICTCA & $c \operatorname{ccc} A$ & AAT & TAT & $A C T$ & GAA & $\| A A G$ \\
\hline
\end{tabular}




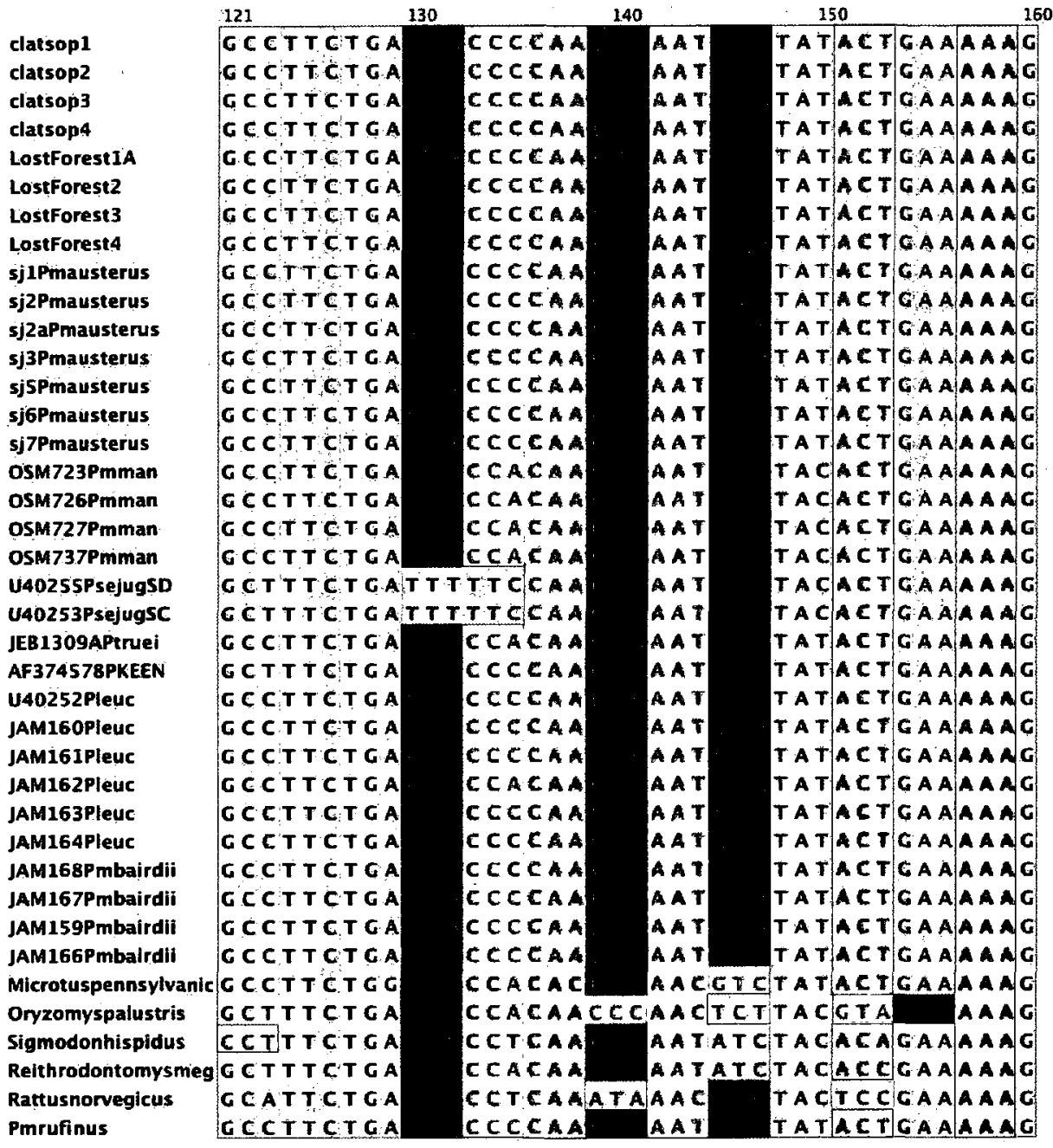


WC7

wel1

WC12

wei3

Ijd3256

Jjd 3304

Jjd3306

tjd3308

Ijd3317

Jjd3324

Jd 3327

jjd 3330

ljd3333

Jjd3344

IJd 3348

UD6 20

UDD774

니 886

LJD2088

LJD1960

LJD1669

LJD1524

LJD1 523

LJD1 122

LJD2522

LJD1637

LJD1638

LJD2161

LJD2651

LJD2737

LJD2834

LJD2850

LJD2898

LJD2914

LJD2936

4) 03000

LJD2126

LJD2138

LJD2154

LJD21 58

LJD2836

LJ02852

t)02860

LJ02865.

LJD2890

LJD2943

Jefferson 3

ADAM56

$161 \quad 170 \quad 180 \quad 190 \quad 200$

CAAACCEGTAT GAGT GCGCATTTGACCCCATAGGCTCTGC CAAACCCETATGACTCCGGATTTGACCCCATACGCTCTGC CAAACCEGTATGACT G CGCATT TCACCCCATAGGCTCTCC CAAACCLCTATGAGTGEGGATTTGACCCCATACGCTCTGC CAAACCCETAT GAGTGCGGATTTGACCCCATAGGCTCTGC CAAACCEATAT GACTGCGGATTTLACCCCATACECTCTGC CAAACCCGTATGAGTGCGGATTTCACCCCATAGGCTCTGC CAAACCCGTATGACTGCGGATTTGACCCCATAGGCTETGC CAAACCCGTATGAGTGCCGATTTGACCCCATAGGCTCTGC CAAACC CATATGAGTGCGGATTTGACCCCATAGCCTCTGC CAAACCCCTATGACTGCGGATTTGACCCEATAGGCTCTGC CAAACCCGTATGAGTGCGGATTTGACCCCATAOGCTCTGC CAAACCCGTATGAGTG CGGATTTGACCCCATAGGCTCTGC CAAACCCETATGAGTGCGGATTTGACCCCATAGGCTCTGC CAAACCCGTATGAGTGCGGATTTGACECCATAAGCTCTGC CAAACCEGTATCAGTGCGGATTTCACCCCATACGCTCTCC CAAACCCGTATGAGTGCGGATTTGACCCCATAGGCTCTGC CAAACCCATATGAGTGCGGATTTGACECCATAGGCTCTGC CAAACCCGTATGAGTGCGGATTTGACECCATAGGCTCTGC CAAACCCCTATGACTGCLEATTTGACCCEATAGCCTCTGC CAAACCCGTATGAGTGCGGATTTGACCCCATAGGCTCTGC CAAACCCGTATGAGTGCGGATTTCACCCCATAGGETCTGC CAAACCCGTATGAGTGCGGATTTGACCCCATAGGCTCTGC CAAACCCGTATGAGTGCGGATTTGACCECATAGGCTCTGC CAAACCCATATGAGTCCGGATTTGACCCCATAGGCTCTGC CAAACC CGTATGAGTGCGGATTTGACLCCATAGGCTCTGC CAAACCEATATGAGTGCGGATTTGACCCCATAGGCTCTGC CAAACCCGTATGACTGCGGATTTCACLCCATAGGCTCTGC CAAACCCGTATGAGTGCGGATTTGACECCATACGCTCTGC CAAACCCGTATGAGTGCGGATTTGACCCCATAG CCTCTGC CAAACCCGTATGAGTGCGGATTTGACCCCATAGGCTCTGC CAAACCCGTATGAGTGCGEATTTGACECCATAGCCTCTGC CAAACCCGTATCAGTGCGGATTTGACCCCATAGGCTCTGC CAAACCCGTATGAGTGCGGATTTGACCCCATAGECTCTGC CAAACCCATATGAGTGCGGATTTGACECCATAGGCTCTGC CAAACCCETATGAGTGCGGATTTGACCCCATAGGCTCTGC CAAACCCGTATGAGTGCCGATTTGACCCCATAAGCTCTCC CAAACCCGTATGAGTGCGGATTTGACCCCATAGGCTCTGC CAAACCCGTATGAGTGCGGATTTGACCCCATAGGCTCTGC CAAACCCGTATGAGTGCGGATTTGACECCATAAGCTCTGC CAAACCCGTATGAGTGCGGATTTGACCCCATAAGCTCTGC CAAACCCGTATGAGTGCGGATTTGACCCCATAGGCTCTGC CAAACCCGTATGAGTGCGGATTTGACCCCATAGGCTCTGC CAAACCCATA T GAGT GCGGATTTGACCCCATAGGCTCTGC CAAACCCGTATGACTGCGEATTTGACECCATAGGCTCTGC CAAACCCCTATGAGTGCGGATTTGACCCCATAGCCTCTGC CAAACCCATATGAGTGCGGATTTGACCCCATAGGCTCTGC CAAACCECTATGAGTGCGCATTTGACCCCATACGCTCTGC 


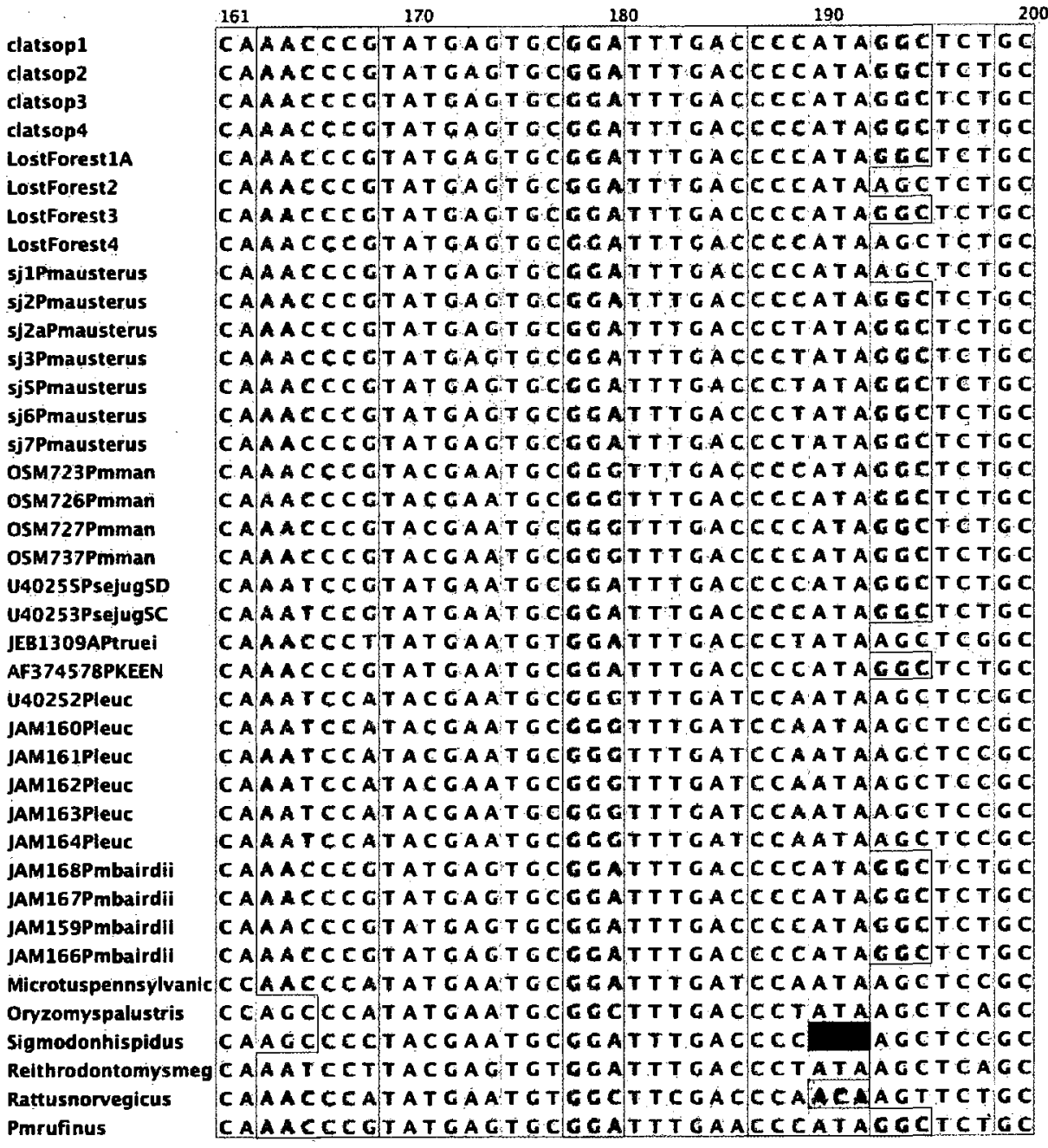


WC7

wC11

wC12

WC13

ijd3256

IJd3304

Ijd3306

ljd3308

Jjd3317

Ijd 3324

1jd3327

ljd3330

ljd3333

IJd3344

Ijd3348

U50620

LJD774

UDD886

UJD2088

LJD1960

UJD1669

UJD1524

LJD1523

4JD1122

LJD2522

UJD1637

LJD1638

WJD2161

LJD2651

LJD27.37

WD2834

LD22850

HJD2898

LJD2914

UJD2936

LJD3000

LJD2126

HJD2138

LJD2154

LJD2158

LJD2836

LJD2852

LJD2860

LJD2865

LJO2890

LJD2943

Jefferson 3

ADAM56
TCG

TC C

$T C G C$

T $\mathbf{C ~ C}$

TC G C

TC G C

TCG

T G C

TCG C

TC G C

TCG

TCG

TCG

TC C C

TC C C

TC G C

T C G C

r C G C

TCC C

r C C

$T C G C$

TC C C

TC G C

TC C C

TC G

TC G

TC C C

TCG

TC C

TC C C

T C G C

TC G C

TC G C

TC C C

TCG

TCG

TC G C

TC G C

T C G C

TC C C

TCC

T C G C

T C C C

$T C G C$

TCG

T C G C

$T C G C$

I C G C
210

220

230

G T AG CAATC

CTAGCAATE

CT AGC AAT C

G T A C AATC

GT AGCAATC

ct alc C Aat C

G TAG CAATC

G T AGCAATC

GT AG CAATC

G TAGCAATC

g Tác Cata

G TAGCAATC

GT AGC AATC

G TAC C A T C

CT AG CAATC

G T AC C A T

GTAGCAATC

G TAG CAATC

G TAG C AATC

G T AIC C AATC

C T AG CAATC

GT AG C A T C

G T ACC AATC

G TAGCAATC

G T AC C AATC

GTAGCAATC

GTAGCAATC

GTACCAATC

GTAGCAATC

GT AUCAATC

GTAGCAATE

CT ACCAATC

G T AGC AATC

G T AUCAATC

G T AG C AATC

GT AUC Aa TC

G T AGCAATC

GTAGCAATC

GT AGCAATC

GT ACC AATC

GTAGCAATC

GT A G C A T C

GTAGCAATC

GT TGCAATC

G T A.G A A TC

GT AIC CA T C

GT AIGCAATC

CCATTCTCAATAAAATTTTTC

CCATTCICAATAAATTTTTC 


\begin{tabular}{|c|c|c|c|c|c|}
\hline & 201 & 210 & 220 & 230 & \\
\hline clatsop 1 & TCG C & CCATTCTCA & А TAAA AT T T TTC & G TACCA & ATC \\
\hline clatsop2 & $T<G C$ & C C A T T CT T A & AT A|A A ATTTTTC & GTAGCA & \\
\hline clatsop3 & 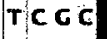 & C CATT CTCA & A T A A A A T T T T T C & $G \Upsilon A|G C A|$ & \\
\hline clatsop4 & $\mathbf{T} \mathbf{C C}$ & C C A T T CT CA & A T A|A A AT T T T TC & $G$ T A C C A & ATC \\
\hline Lostforest IA & TCG C & C C AT TCT C A & AT TA A A AT T T T TC & $G$ T A $G C A$ & A T \\
\hline LostForest2 & 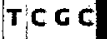 & $\operatorname{CCATTCTCA}$ & А T AA A ATT T T TC & G T A C C A & \\
\hline LostForest 3 & $\mathbf{T} \mathbf{c c c}$ & C CAT TCTCA & A T A A A AT T T T TC & G T A $\mid$ G C A & \\
\hline LostForest4 & $T C G C$ & C C A T T & А T A A A ATT T T TC & C T ACCA & A T C \\
\hline sj1Pmausterus & $\mathrm{T} C \mathrm{CC}$ & C CATTCTCA & A T A A A AT T T T T & G T A C C A & \\
\hline sj2Pmausterus & $\mathrm{T} C \mathrm{CC}$ & C $\subset A T T C T C A$ & AT AA A AT T T T T & C T A C C A & A T \\
\hline sj2aPmausterus & $\mathbf{T} \mathbf{C ~ C ~ C ~}$ & CСAT TCTCA & A T A A A AT T T T TC & G T A G C A & \\
\hline sj3Pmạustẹus & $\mathbf{T} \in \mathbf{C}$ & C C A T T CTCA & AT A A A ATTTTTTC: & C T A C CA: & ATC \\
\hline sj5Pmausterus & TCOC & C CATTCTCA & A T A A A ATT:T T TC & C T A G C A & \\
\hline sj6Pmausterus & $\mathbf{T} \mathbf{C} \mathbf{C}$ & C C A T T C T C A & A T AAA AT T T T T & CTAGCA & \\
\hline sj7Pmausterus & $\mathbf{T} \mathbf{C} \mathbf{c} \mathbf{C}$ & C CATTCTCA & AT A A A ATTTTTC & GT ACCA & \\
\hline OSM723Pmman & TCCC & C C ATT TCTCA & AT AAAGTTTTT & C T A G C A & \\
\hline OSA726Pmman & $T C C C$ & C C A T T C TCA & A T AA A CT T T T TC & C T AG C A & A T \\
\hline OSA727Pmman & $\mathbf{T} \mathbf{C ~ G ~ C ~}$ & C CATTCTCA & ATAAAGTTTT & CT ACCA: & \\
\hline OSM737Pmman & $T C G C$ & C C A T T C TCA & ATAAAGTTTTTC & GTAGCA & \\
\hline U40255Psejug5D & $\operatorname{cct} \mathrm{ct}$ & C & A T A A A AT TCT T & G T A C C A & \\
\hline U40253PsejugSC & clc G T & C C A T T CT $\subset$ A & AT A A A ATTCTTT & C T A C C A & AT T \\
\hline JEB1309APtruei & $\mathrm{T} \mathbf{c c c}$ & CᄃCT TCTCC & A T A|A A ATTCTTC & G T A G C T & AT T \\
\hline AF 374578 PKEEN & $\mathbf{T c c}$ & C C A T T CT C A & A T A A A T T T & G T A C C A & A T C \\
\hline U40252Pleuc & $\operatorname{coc}$ & C C A $: T$ T T T $\mathbf{T}$ & A T A A A AT T T T T T & G T A G C A & \\
\hline JAM160Pleuc & $\mathbf{c} \mathbf{c ~ c ~ c ~}$ & C C A T T T:TCA & A T A A A AT T T T T T & G T A $\mid \mathbf{C}$ C A & \\
\hline JAM161Pleuc & $c c \in c$ & C C A T T T:TCA & AT TA A ATT TT T T & G T A G C A & \\
\hline JAM162Pleuc & $\mathbf{c} \in \mathbf{c}$ & C C A T T T:TCA & АT TA A ATT T T T & G T A G C A & \\
\hline JAMi63Pleuc & $c \in c c$ & C C A T T T:TCA] & AT AAA AT T T T T & G T AC C A & \\
\hline JAM164Pleuc & c c c c & C C A T T T T C A & $\mid \mathbf{A} T \mathbf{T} A$ A A A T T T T T T & $G \backslash A C \subset A$ & \\
\hline JAM168Pmbairdii & $\mathrm{T} C \mathrm{cc}$ & C C A T T C T CA & AT TAA ATTCT TC & G T A C C A & A TC \\
\hline JAM167Pmbairdii & $T<G C$ & C CATT T TCA & А T A $A$ A AT TCT TC & G T A C C A & A T C \\
\hline JAM159Pmbairdii & $\mathbf{T} \mathbf{C ~ C ~ C ~}$ & СС AT TTTCA & $|A T A| A \wedge A T T C T T C$ & G T A G C A & \\
\hline JAM166Pmbairdil & $\mathbf{T} \mathbf{C C}$ & CEATTC:TCA & A T A A A A TTCTTC & C T A C C A & \\
\hline Microtuspennsylyanic & C C GA & C CATTCTCA & A T A|A A AT TT TTC & G TACCA & \\
\hline Oryzomyspalustris & $\mathbf{T} \mathbf{T} \mathbf{C}$ & $\operatorname{ccc} \mathbf{T} \boldsymbol{T} \mathrm{TCA}$ & A T A A A AT T T T T C & G TAGGC & A T \\
\hline Sigmodonhispidus & $T C G A$ & C CTT T CTCC & AT A A A AT TT T TC & GTACCT: & A T T T \\
\hline Relthrodontomysmeg & $\mathbf{T} \mathbf{C C} \mathbf{C}$ & C C A T T C T C T & A T A $\mid$ A ATTTTTC & G TAGCA & ATC \\
\hline Rattusnorvegicus & $A C G C$ & C CTTTTTCA & A T A $A$ A A T T T T T C & C T A C C C & AT T T \\
\hline Pmrufinus & $\mathrm{TCC}$ & CCAT T CITCA & A T A $A$ A ATTTIIT & GIACCA & $A T C$ \\
\hline
\end{tabular}


WC7

wC11

WC12

wC13

IJd 3256

yd3304

|]d3306

tjd3308

Jd3317

IJd3324

Ijd3327

ljd 3330

ljd 3333

Jjd3344

Jjd3348

WD620

UD774

WD886

LJD2088

LJD1960

LلJD1669

LJD1524

LJD1523

LID1122

LJD2522

LلJ1637

LJD1638

니2161

LJD2651

LJD2737

LJD2834

LJD2850

LJD2898

LJD2914

LJD2936

LLD3000

니D2126

LJD2 138

LJD2154

LJD2 158

LJD2836

LJD2852

LJD2860

니D2865

LJD2890

LJD2943

Jefferson 3

ADAMS 6

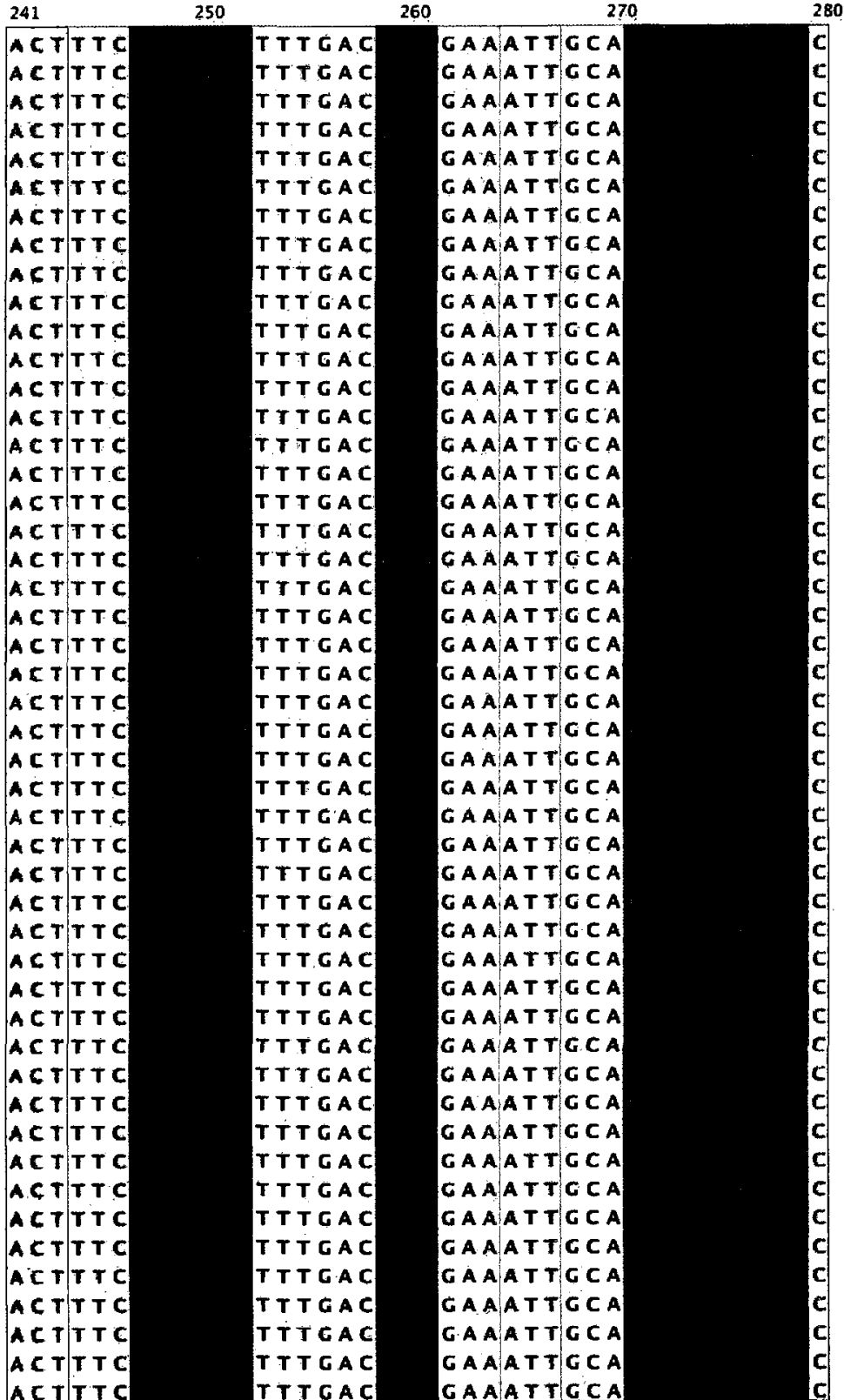




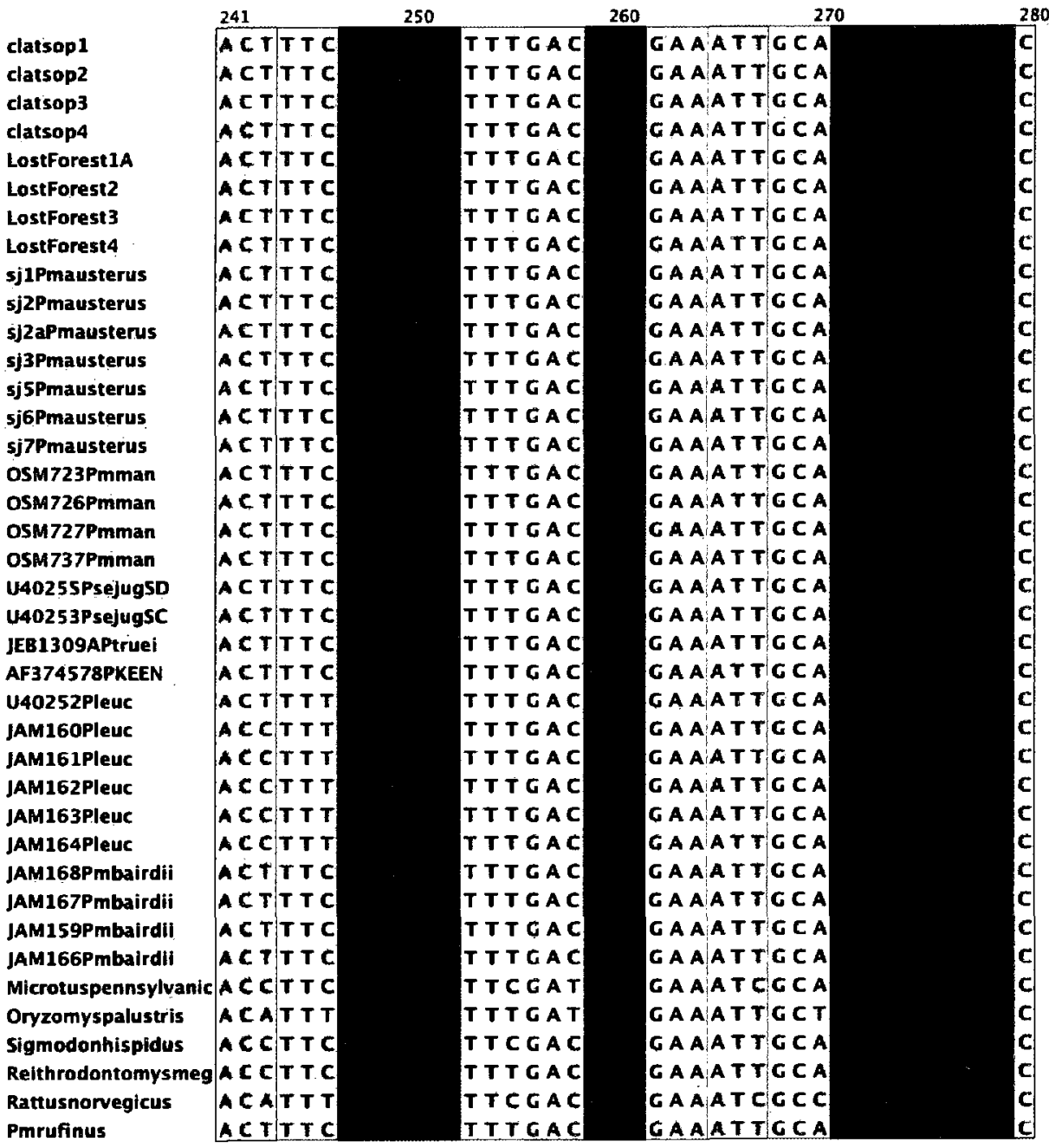


WC7

WC11

WC12

WC13

Jjd3256

IJd3304

ljd3306

ljd3308

IJd3317

IJd3324

ljd3327

ljd3330

jjd3333

IJd 3344

IJd3348

LJD620

LDD774

LDD886

LJD2088

LJD1960

LJD1669

LJD1524

UDD1523

UDi 122

LJD2522

LDD1637

LDD1638

UD2161

LJD2651

LJD2737

LJD2834

LJD2850

LJD2898

LJD2914

LJD2936

LJD3000

LJD2126

LJD2138

LJD2 154

LJD2158

LJD2836

LJD2852

LJD2860

LJD2865

LJD2890

LJD2943

Jefferson 3

ADAMS6
$281 \quad 390 \quad 310 \quad 320$

CA CCATGAGCTATCCAAATATATAATATTAACATCAT

CA CCATGAGCTATCCAAATATATAATATTAACATCAT

CA

CA

CA

CA

CA

CA

C A

CA

CA

C A

CA

CA

$C A$

$C A$

c. A

$C A$

CA

C A

$C A$

CA

$C A$

$C A$

CA

$C A$

CA

CA

C A

CA

CA

C A

C A

C A

C A

$C A$

C A

$C A$

C A

C A

CA

$C A$

$C A$

$C A$

$C A$

$C A$

CA C CAT GAGCTATCCAAATATATAATATTAACATCAT C CATGAGCTATCCAAATATATAATATTAACATCAT C CAT GAGCTATCCAAATATATAATATTAACATCAT CCATGAGCTATCCANATATATAATATTAACATCAT C CATGAGCTAT CICAAATATATAATATTAACATCAT C CATGAGCTATCCAAATATATAATATTAACATCAT C CATGAGCTATCCMAATATATAATAT TAACATCAT CEATGAGCTATCCAAIATATATAATAT TAACATCAT CCATGAGCTATCCAAATATATAATATTAACATCAT C CATGACCTATCCAAATATATAATATTAACATCAT C CATGAGCTAT CCA AIATATATAATATTAACATCAT C CATGAGCTAT CEAAATATATAATATTAACATCAT CCATGAGETATCCAAATATATAATAT TIAACATCAT C CAT GAG CTATCLAAATATATAATATTAACATCAT C CATGAGCTATCLAAATATATAATATTAACATCAT CCATGAGCTATCCAAATATATAATATTAACATCAT C CATGACCTATCCAAATATATAATATTAACATCAT CCATGAGCTATCCAAATATATAATATTAACATCAT C CAT GA G CTATCLAAATATATAATATTAACATCAT C CATGAGCTATCICAAATATATAATATTAACATCAT C CATGAGCTATCCAAATATATAATATTAACATCAT C CATGAGCTATCCAAIATATATAATAT TAACATCAT C CATGAGCTATCCAAATATATAATATTAACATCAT CCATGAGCTATCCAAIATATATAATATTAACATCAT C CATGAGCTATCCAAATATATAATATTAACATCAT C CATGAGCTATCCAAATATATAATATTAACATCAT C CATGAGCTATCCAAATATATAATATTAACATCAT C CAT GAGCTATCCA AIATATATAATATTAACATCAT CCATGAGCTATCEAAATATATAATATTAACATCAT C CATGAGCTATCCAAATATATAATATTAACATCAT C CATGAGCTATCLAAATATATAATATTAACATCAT C CATGAGCTATCCAAATATATAATATTAACATCAT C CATGAGCTATCCAAATATATAATATTAACATCAT CCATGACCTATCCAAATATATAATATTAACATCAT C CATGAGCTATCCAAATATATAATATTAACATCAT C CATGAGCTATCCAAATATATAATATTAACATCAT CEATGAGCTATCCAAATATATAATATTAACATCAT CCATGAGCTATCCAAATATATAATATTAACATCAT CCATGAGCTATCLAAATATATAATATTAACATCAT CCATGAGCTAT CСAAATATATAATATTAACATCAT C CATCAGCTATCCAAATATATAATATTAACATCAT CEATGACCTATCCAAATATATAATATTAACATCAT C CATGAGCTATCLAAATATATAATATTAACATCAT CCATGAGCTATCLAAATATATAATATTAACATCAT CCATGAGCTATCCAAATATATAATATTAACATCAT 


\begin{tabular}{|c|c|c|c|c|}
\hline & 281 & 290 & 300 & 310 \\
\hline clatsopl & & CCATGAGCT & TATCCAATATAT & AATATTAACATCAT \\
\hline clatsop2 & & C CATGAGCT & TATCCAAATATAT & AАTATTAACATCAT \\
\hline clatsop 3 & & CCATGAGCT & ATCL A AAT TAT AT & AATATTAACATCAT \\
\hline clatsop4 & & CCATGAGCT & TATCICAIATATATI & AATATTAACATCAT \\
\hline LostforestIA & & C CATGAGCT & IATCCAAATATAC & AATATTAACATCAT \\
\hline LostForest2 & & C C ATGAGCT: & TATCEAATATAT & A ATATTAACATCAT \\
\hline LostForest 3 & & C CATGAGCT & ATCCA AIATATAT & ААТАТ TAАСАТСАТ \\
\hline LostForest4 & & C CAT GAGCT & I T CCA AIT TAT AT & A ATATTAACATCAT \\
\hline 5j1Pmausterus & & CCATGAGET & IATCICAATA TAT & ААТ АТ ТААСАТСАТ \\
\hline sj2Pmausterus & & C CATGAGET & ГАТ CСААТ T АT АT & AАTATTAACATCAT \\
\hline s]2aPmausterus & & C CATGAGCT & TAT CLA AAT AT AT & АATAT TAACATCAT \\
\hline sj3Pmausterus & & CEATGAGCT & TATCC A AT ATATT & A T TATTAACATCAT \\
\hline sj5Pmausterus & & C CATCAGCT & TATCCAASTATAT| & A ATATTAACATCAT \\
\hline sj6Pmausterus & & C CATGAGCT & TAT CEAAAT AT AT & A А TAT TAACATCAT \\
\hline sj7Pmausterus & & C CAT GAGCT & TА T CС A A T T AT AT & A А А T TA А СATCAT \\
\hline OSM723Pmman & & C CATGAGCT & TATCCAAATAT AT & ААТ АTT:AACAСCAT \\
\hline OSM726Pmman & & C CATGAGCT & TATCC AATATAT & A AT ATTAACACCAT \\
\hline OSM727Pmman & & C CATGAGCT & TAT CIC A AIAT AT AT & AATAT TAACACCAT \\
\hline OSM737Pmman & & C CATCAGCT & IAT CICAAIAT AT AT & A ATATTAACACCAT \\
\hline U40255PsejugSD & & C CATGAGCT & ГАT CС A AAT AT AT & AATACTAACATCAT \\
\hline U40253PsejugSC & & C CATGAGCT & ATCCAAIATATAT & AATACTAACATCAT \\
\hline JEB1309APtruei & & CCCTGAGCT & HTTCANATATAC & AACATTAACACAAC \\
\hline AF374578PKEEN & & C CATGAGCT & - T CCA AIAT AT G T: & AATATIAACATCAT \\
\hline U40252Pleuc & & CCATGAGCC & ATCCAAA T ATAT & AaCACCAACACCAT \\
\hline JAM160Pleuc & & C CATCAGCC & : & AACACEAACACTAT \\
\hline JAM161Pleuc & & C CATGAGCC & $\mid$ ATCCA A|A T AT AT| & AACACCAACACTAT \\
\hline JAM162Pleuc & & CCATGAGCC & A T CCA A T T TAT & AACACCAACACTAT \\
\hline JAM163Pleuc & & C CATGAGCC & ATCCA AIATATAT & AaCACCAACACTAT \\
\hline JAM164Pleuc & & CEATCACCC & $=\mid$ T ClCA A A T ATAT) & A ACACCAACACTAT \\
\hline jAM168Pmbairdii & & CCATGAGCT & ATCEAAITATAT & AATATTAACATCAT \\
\hline JAM167Pmbairdii & & C CATGAGCT & TA TCCA ALT T TAT & AАT AT TAACATCAT \\
\hline JAM159Pmbairdil & & CCATGAGCT & IATCCA AIATAT ATT & A AT AT TAACATCAT \\
\hline JAM166Pribairdii & & CCATGAGCT & F TCCA AATATAT) & AATATTAACATCAT \\
\hline Microtuspennsylvanic & & CCCTGAGCA & ATACA ATTTCCT & AACATAAACAIA \\
\hline Oryzomyspalustris & & C C CT GACCT & AT ACA AT A TACA & GACACACAC AC \\
\hline Sigmodonhispidus & & CCATGAGCC & ATTCAAATCACE & A $A$ TCCGCGAT \\
\hline Reithrodontomysmeg & & CCATGAGCC & ATTCA AATATCA. & A A CATTAAAGCAAC \\
\hline Rattusnorvegicus & & CСATGAGCG & AATTCAAACAACE & AATACEACTACAAT \\
\hline Pmrufinus & & CCAIGACCI & IATCSAAATATAT & AATATTAACATCAT \\
\hline
\end{tabular}


WC

WC11

WC12

WC13

ljd3256

ljd 3304

ljd3306

ljd 3308

ljd 3317

Ijd 3324

ljd3327

ljd 3330

ljd3333

ljd 3344

Ijd3348

LD620

LJD774

LJD886

LD2088

LD1960

LDD1669

UJD1524

LJD15?3

LJD1122

LJD2522

LJD1637

LJD1638

LJD2 161

LJD2651

LJD2737

UJD2834

LD28 50

LD2898

LD22914

LD2936

UD3000

LJD2126

LLD2138

LJD2 154

LJD2 158

LD2836

LJD2852

LJD2860

LJD2865.

LJD2890

LD2943

Jefferson 3

ADAMS6

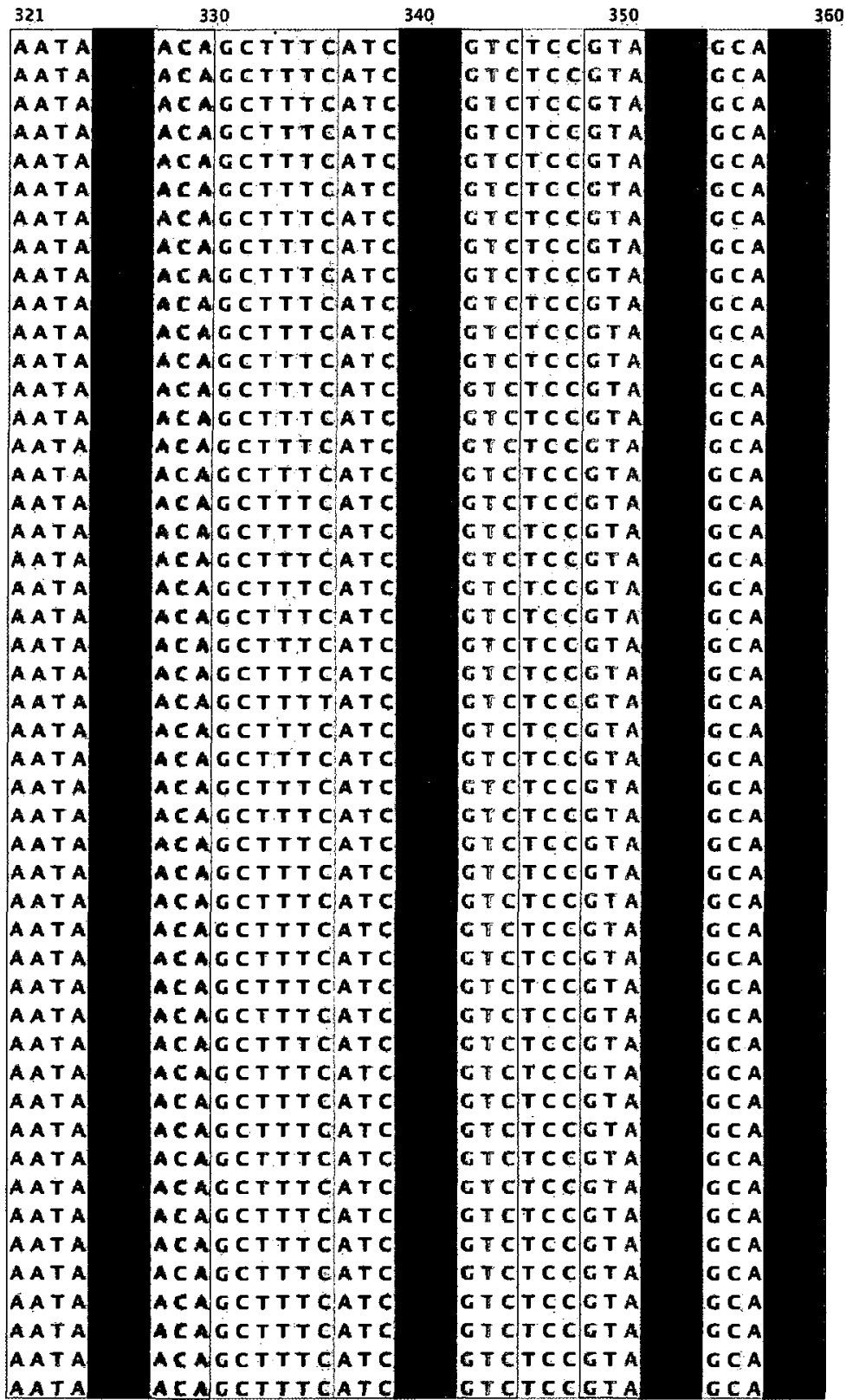




\begin{tabular}{|c|c|c|c|c|c|c|}
\hline & 321 & 330 & 340 & & 350 & \\
\hline clatsop1 & A A T A & ACAGCTTTCATC & GTC & $T C C$ & G T A & G C A \\
\hline clatsop2 & A A T A & $A C A G C T T T C A T C$ & GTC & T C C & G T A & $\mathbf{G C A}$ \\
\hline clatsop3 & A A T A & ACA GCTTTCATC & C TC & T C C & C T A & $\mathbf{G} \mathbf{C}$ \\
\hline clatsopd & A A T A & ACACCTTTCATC & GTC & $\mathbf{T C C}$ & G T A & C C A \\
\hline LostForest1A & A A T A & $A \subset \wedge G C T T$ T CATC & GT $\mathbf{C}$ & $\mathbf{T C C}$ & G T A & GCA \\
\hline LostForest2 & A A T A & ACACCTTTCATC & $\mathbf{G} \mathbf{T} \mathbf{C}$ & $\mathbf{T C C}$ & G T A & GC \\
\hline LostFarest 3 & A A T A & $A \subset A G C T T T C A T C$ & G TC & $T C C$ & G T A & G C A \\
\hline Lostforest 4 & A A T A & ACA GCTTTCATC & Gre & $\mathbf{T C C}$ & G T A & C C A \\
\hline sj1Pmausterus & A A T A & ACACC TT TCA TC & $G \times c$ & $T<C$ & G T A & G C A \\
\hline sj2̇Pmausterus & A A T A & $A \subset A G C T T$ T $A T C$ & G TC & $\mathbf{T C C}$ & G T A & $\mathbf{C C A}$ \\
\hline s\}2aPmausterus & A A T A & $A C A G C T$ T T CATC & C T C & $\mathbf{T C C}$ & G T A & G C A \\
\hline s]3Pmạusterus & A A T A & ACACCTTTCATC & G I & $T \in C$ & G I'A & $C \subset \mathrm{A}$ \\
\hline sj5Pmausterus & A A T A & AC AGCT T TCATC & C T C & $T C C$ & $\mathbf{C} \mathbf{T}$ & $\mathbf{C C A}$ \\
\hline sj6Pmausterus & A A T A & AC $A$ C C T T TCA T C & G T C & TCC & G T A & G C \\
\hline sj7Pmausterus & A A T A & ACAGCTTTCATC & G T C & $\mathbf{T C C}$ & G T A & G C A \\
\hline OSM723Pmman & A АT A & ACAGCTTTCATC & ATC & T C C & G T A & C C A \\
\hline OSM726Pmman & A A T A & ACA GETTTCATC & ATC & T C C & G T A & C C A \\
\hline OSM727Pmman & A A T A & ACACCTTTCATC & A T C & $\mathbf{T C C}$ & $\mathbf{G} \mathbf{T} \mathbf{A}$ & G C \\
\hline OSA737Pmman & A A T A & ACAGCTTTCATC & ATC & T C C & G T A & C C A \\
\hline U40255PsejugSD & A A T A & ACA GCTTTCATC & ATC & $\mathbf{T} \mathbf{C C}$ & C T A & G C A \\
\hline U40253PsejugsC & A A T A & ACACCTTTCATC & ATC & tcc & G T A & G C A \\
\hline JEB1309APtruei & T] A T A & CTATCTTTCATC & ATC & $\mathbf{T C T}$ & A T C & GCT \\
\hline AF374578PKEEN & A A T A & ACAGCTT TCATC & G E & $\mathbf{T C C}$ & G T A & $G C A$ \\
\hline U40252Pleuc & A A T A & ACAGCCT T TATT & G T C & $\mathbf{T C T}$ & $\mathbf{G T}_{\mathrm{T}} \mathrm{G}$ & G C C \\
\hline JAM160Pleuc & A A T A & A CAGCTTTTATT & c Tc & $\mathbf{T}<\mathbf{C}$ & C T C & G c c \\
\hline JAM161Pleur & A A T A & ACA $A$ C T T T TA T T & G $\mathbf{~ C ~}$ & $\mathbf{T C C}$ & $\mathbf{G} \mathbf{T} \mathbf{G}$ & c c c \\
\hline JAM162Pleuc & A A T A & ACAGCTTT TATT & $G T C$ & $\mathbf{T}$ C C & $G T G$ & c C C \\
\hline JAM163Pleuc & A A T A & ACAGCTTTTATT & G T C & $\mathbf{T} \in \mathbf{C}$ & G T G & G C C \\
\hline JAM164Pleuc & A A T A & ACAGCTTTTATT & G & $\mathbf{T C C}$ & G T C & $\mathrm{ccc}$ \\
\hline JAM168Pmbairdii & A A T A & ACACCT T TCATC & G & TCC & C T A & C C A \\
\hline JAM167Pmbairdii & A A T A & ACAGCTTTCAT & GTC & $\mathbf{T C C}$ & G T A & GCA \\
\hline JAM159Pmbairdii & A A T A & AEACCTT TCATC & G TC & $\mathbf{T C C}$ & G T A & $G \subset A$ \\
\hline JAM166Pmbairdii & A ATA & $A \in A C C T T T C A T C$ & c r c & $r \in c$ & G T A & $G \subset A$ \\
\hline Microtuspennsylvanic & A T A $A \subset C$ & ATAGCATTTATC & ATC & $A C A$ & ATT & $G \subset C$ \\
\hline Oryzomyspalustris & C A T AGCT & G C C T C T:T T TA T T & $G \mp C$ & $\mathbf{T C T}$ & ATC & $A C T$ \\
\hline Sigmodonhispidus & AATAATC & ACATCCTTTATC & ATT & $\mathbf{T C A}$ & A T C & G C C \\
\hline Reithrodontomysmeg & A A T A & ACATCATT TA T T & CT $A$ & TCA & G TA & $\mathbf{C C C}$ \\
\hline Rattusnorvegicus & A ATACCA & ACTACCTTT:ATT & $\mathbf{C} \mathbf{c}$ & $A C T$ & A T T & $T C T$ \\
\hline Pmrufinus & AAIA & ACAGCTTTCATC & Grc & $\mathbf{T C C}$ & G T A & G C A \\
\hline
\end{tabular}


we7

wC11

WC12

WC13

IJd3256

IJd 3304

Jjd3306

ljd 3308

IJd3317

Ijd3324

ljd3327

ljd3330

jjd3333

jjd3344

IJd3348

LDD620

LJD774

LD886

LJD2088

LJD1960

LJD1669

LJD1524

LJD1523

LJD1122

LJD2522

LJD1637

LJD1638

LJD2161

LJ02651

LJD2737

LJD2834

LJD2850

UD2898

LJD2914

LJD2936

LJD3000

LJD2126

LJD2138

LJD2154

LJD2158

LJD2836

LJD2852

LJD2860

LJD2865

LJD2890

LJD2943

Jefferson 3

ADAMS6

\begin{tabular}{|c|c|c|c|}
\hline 361 & 370 & 380 & 400 \\
\hline C EC & GCCTACGAGTGA & CAAAAAGGA & GAAT GAA \\
\hline $\operatorname{cec}$ & GCCITACGAGT GA & CAAAAAGA & GAATCAA \\
\hline $\operatorname{ccc}$ & GCCTACGACTGA & CAAAAACGA] & C A A T G A A \\
\hline $\operatorname{cec}$ & GCCTA CGAGTGA & CAAAAAGGA & GAATGAA \\
\hline G G: & G C CI A CGAGTGA & CAAAAAGGA & GAAT GAA \\
\hline Ge & GCCITACGAGTGA & EAAAAACE & GAATGAA \\
\hline & GCCTACGACT GA & CAAAAACG & GAATGA \\
\hline G & GCCT ACG AGT GA & CAAAAGG & GAAT GAA \\
\hline & G C CITACGAGT G A & CAAAAAGG & GAAT GAA \\
\hline G & GCCTACGAGTGA & EAAAAAGG & CAAT G A A \\
\hline & GCCTACGAGTCA & CAAAAACEA & GAATCAA \\
\hline G G & GCCTACGAGTGA & CAAAAAGGA & GAAT GAA \\
\hline & GCCT ACG AGT GA & CAAAAAGGA & GAATGAA \\
\hline & GCCTACG AGT GA & EAAAAACOA & GAATGAA \\
\hline & GC CT ACGACT GA & CAAAAAGEA & GAAT GAA \\
\hline & GCCTACGACTGA & CAAAAACGA & CAAT CAA \\
\hline & GCCT ACGAGTGA & CAAAAAGGA & GAAT GA \\
\hline & GCCTACGACTGA & CAAAAAGCA & GAATT GAA \\
\hline & GCCTACGACTGA & CAAAAAGEA & GAATGAA \\
\hline & GCCTACGACTGA & CAAAAAGGA & CAAT GAA \\
\hline & GCCTACGAGTGA & CAAAAAGGA & GAAT GAA \\
\hline & GCCTACGAGTGA & CAAAAAGGA & G:AAT GAA|A \\
\hline & G C CT ACGACT GA & CAAAAAGGA & GAATGA|A \\
\hline & GCCTACGACTCA & CAAAAAGCA & CAATGAA \\
\hline & GCCT ACGAGT GA & CAAAAAEGA & GAAT GA \\
\hline & G C C T A C C A G T G A & CAAAAAGGA & GAAT GA|A| \\
\hline & GCCTACGAGTGA & CAAAAAGGA & GAAT GA A \\
\hline & C C CTACGAGTGA & CAAAAACGA & CAAT GAA \\
\hline & GCCTACGAGTGA & CAAAAAGCA & GAATGAA \\
\hline & GCCTACGAGTGA & C.AAAAAGGA & GAAT GAA \\
\hline & GCCITACG AGTGA & CAAAAAGGA & GAAT G AA \\
\hline & GCCITACGAGTGA & CAAAAAGEA & GAATGAA \\
\hline & C CCTACGAGTGA & CAAAAAGGA & GAAT GA A \\
\hline & GCCTACGAGTGA & CAAAAAGGA & GAATGAA| \\
\hline & GCCTACGAGTGA & CAAAAAGES & GAATGAA \\
\hline & CCCTACCACTGA & CAAAAACGA & GAATCAA| \\
\hline & GC CI ACCACTGA & CAAAAAGGA & GAATCAA| \\
\hline & GCCITACG AGTGA & CAAAAAGGA & GAATCAA \\
\hline Go & GCCT ACG AG TGA & CAAAAAGGS & GAAT GAA \\
\hline $\mathbf{G ~ C}$ & GCCTACGAGTGA & CAAAAAGGA & GA ATGAA \\
\hline C c & CCCITACCACT G A & CAAAAACGD & CAATCAA \\
\hline 66 & G C CT ACGAAT G A & CAAAAAGG & GAATGAA \\
\hline 660 & GCCTACG AGT GA & CAAAAAGCA & GAATCAA \\
\hline G G C & GCCIACGAGTGA & CAAAAAGGA & GAATGAA \\
\hline $\boldsymbol{C O C}$ & GCCTACGAGTGA & CAAAAAGGA & CA ATGAA \\
\hline cec & GCCTACGAGTGA & CAAAAAGGA & GAATGAA \\
\hline G G C & GCCTACGAGTGA & EAAAAACGA & GAATGAA \\
\hline $\mathrm{GGC}$ & CCCTACGAGTOA & CAAAAAGGA & GAATGAA \\
\hline
\end{tabular}




\begin{tabular}{|c|c|c|c|c|}
\hline & 361 & 370 & 380 & \\
\hline clatsop1 & G G C & GCGTACGAGTGA & TAAAAAGEA & GAAT GAA \\
\hline clatsop2 & $\mathbf{G} \mathbf{6 C}$ & GCCTACGAGTGA & CAAAAACGA & G A A T G A \\
\hline clatsop3 & $6 \mathrm{GC}$ & GCCTACGAGTGA & CAAAAAGGA & GAAT GA \\
\hline clatsop4 & G 4 & GCCITACGACTGA & CAAAAAGGA & GAA TAA \\
\hline LostFarest1A & $\operatorname{coc}$ & GCCT ACG AGTGA & CAAAAACOA & G A A T GA \\
\hline LostForest2 & G C C & GCCT ACG A GTG A & CAAAAACGA & GA AT GAA \\
\hline LostForest 3 & $\operatorname{coc}$ & GCC CACIG AGTG A & CAAAAAGGA & GA ATGA \\
\hline LostForest4 & c c c & GCCITACGACT GA & CAAAAAGGA & CAATCA \\
\hline sj1Pmausterus & 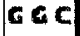 & GC CITACG AGT G A & CAAAAAGA & GA AT GA A \\
\hline sj2Pmausterus & 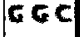 & GCCTACGAGTGA & CAAAAAGGa & GAAT GAA \\
\hline sj2aPmausterus & $G<C$ & GCC TT ACG AGTGA & CAAAAAGGA & GAATUAA \\
\hline sj3Pmausterus & ece & GCCITACG ACT GA & CAAAAAGGA & CA A T GAA \\
\hline sjSPmausterus & $6 \mathrm{c}$ & GCC TACG AGTGA & EAAAAAGGA & GA A T CAA \\
\hline sj6Pmausterus & 66 & GCCST ACG A GTGA & CAAAAAGGA & GAAT GAA \\
\hline sj7Pmausterus & Gc & GCCTACGAGTGA & CAAAAAGGA & GA A T GA \\
\hline OSM723Pmman & $A C C$ & GCCTACGAATGA & CAAAAAGGa & CAAT G A A \\
\hline OSM726Pmman & $A G C$ & GCCITACGAAT GA & CAAAAAGCA & GAATCAA \\
\hline OSM727Pmman & $A C C$ & GCCT ACG A ATG A & $\operatorname{CAAAAAGGA}$ & CAAT CAA \\
\hline OSM737Pmman & A.C.C & GCCTACGAATGA & CAAAAAGGA & GA A T GA \\
\hline U40255PsejugSD & G GE & GCCTACGAATGA & CAAAAAGGA & GAAT GA \\
\hline U40253PsejugsC & ec & GCCTACGAATGA & EAAAAAGCA & GAATEA \\
\hline JEB1309APtruei & $66 \mathrm{G}$ & GCCTACGAATGA & A $\triangle A A A \wedge A \subset \in A$ & G A A T CA A \\
\hline AF374578PKEEN & $\operatorname{cec}$ & GCCTACGAATGA & CAAAAAGGA & GAAT GAA \\
\hline U40252Pleuc & $\mathbf{G} \in \mathbf{C}$ & GCCTACGAATGA & $A C A A A A G G G G$. & GAAT GAA \\
\hline JAM160Pleuc & $c \in c$ & GCCT ACG AATG A & ACAAAAGCGG & G A A T GA \\
\hline JAM161Pleuc & ec & GCCTACGAATGA & ACAAAAGGGG & GAAT GA \\
\hline JAM162Pleuc & GGC & G C.CT A C G A A T G A & ACAAAAGGGG & GAAT С AA \\
\hline JAM163Pleuc & $\operatorname{coc}$ & GCCTACG A ATG A & ACAAAACGGG & GAAT GAA \\
\hline JAM 164Pleuc & coc & GCCTACGAATGA & ACAAAAACGE & CAATCAA \\
\hline JAM168Pmbairdii & $\operatorname{coc}$ & GCCTACG AGTGA & CAAAAAGGA & GAAT GAA \\
\hline JAM167Pmbairdii & c ec & GCCT ACG ACT G A & CAAAAAGGA & GAAT GAA \\
\hline JAM159Pmbalrdii & G G C & GCCTACGAGTGA & CAAAAAGGA & GAAT GAA \\
\hline JAM166Pmbairdii & ect & GCCTACGAGTGA & CAAAAAGGA & GA A T EA A \\
\hline Microtuspennsylvanic & C C & GCCTACGAAT GA & ACAAAAACGC & G A A T G A A \\
\hline Oryzomyspalustris & $\Leftrightarrow a c$ & GCTTATGAGTGA & AATAAAGGO & GAAT GA A \\
\hline Sigmodonhispidus & $6 \in 5$ & ATCT ACG AAT GG & TAAATAAACGC & G A G T G A \\
\hline Relthrodontomysmeg & G C A & G CCTACG A AT GA & CAAAAGGGT & GA A TGA A \\
\hline Rattusnorvegicus & C E C & GCCTACGAATGA & CACAAAAAGGA & GAAT GAA \\
\hline Pmrufinus & $G \leq C$ & GCCCACGACTGA & CAAAAAGGA & GAAT GA \\
\hline
\end{tabular}


wC7

WC11

WC12

wC13

Jjd3256

Ijd3304

Ijd3306

ljd3308

ljd3317

Jd3324

Jjd3327

ljd 3330

Jjd3333

Ijd3344

Jd 3348

LJD620

LJD774

LJD886

LJD2088

LJD1960

LJD1669

LJD1524

LJD1523

LJD1122

LJD2522

LJD1637

LJD1638

LJD2 161

LJD2651

LJD2737

LJD2834

LJD2850

LJD2898

LJD2914

LJD2936

LJD3000

LJD2126

LJD2138

LJD2154

LJD2 158

LJD2836

LJD2852

LJD2860

LJD2865

LJD2890

LJD2943

Jefferson 3

ADAMS6
GAA-TAACTGGTAATTAGTTTAATTAAAACAAATGAT GAA-TAACTGGTAATTAGTTTAATTAAAACAAATGATT GAA-TAACT GGTAATTACTTTAATTAAAACAAATGATT CAA-TAACTGGTAATTAGTTTAATTAAAACAAATGATT GAA-TAACTGGTAATTAGTTTAATTAAAACAAATGATT GAA-TAACTGGTAATTAGTTTAATTAAAACAAATCATT GAA-TAACTGGTAATTAGTTTAATTAAAACAAATCATT GAA-TAACT GGTAATTAGTTTAATTAAAACAAATGATT GAA-TAACTGGTAATTAGTTTAATTAAAACAAATGATT CAA-TAACTGGTAATTAGTTTAATTAAAACAAATGATT GAA- TAACTGGTAATTAGTTTAATTAAAACAAATGATT GAG- TAACTGGTAATTAGTTTAATTAAAACAAATGATT GAA-TAACT G T TATTAGTTTAATTAAAACAAATGATT GAA-TAACTGGTAATTAGTTTAATTAAAACAAATGATT GAG - TAACTGGTAATTAGTTTAATTAAAACAAATGATT CAA-TAACTGGTAATTAGTTTAATTAAAACAAATGATT GAG-TAATTGGTAATTAGTTTAATTAAAACAAATGATT GAA-TAACT GGTAATTAGTTTAATTAAAACAAATGATT GAG-TAACT GCTAATTAGTTTAATTAAAACAAATGATT GAA-TAACTGGTAATTAGTT TAATTAAAACAAATGATT GAC- TAACTGGTAATTACTTTAATTAAAACAAATGATT GAA-TAACTGGTAATTAGTTTAATTAAAACAAATGATT GAA-TAACT GGTAATTAGTTTAATTAAAACAAATGATT GAA-TAACTGGTAATTACTTTAATTAAAACAAATGATT GAA-TAACTGGTAATTAGTTTAATTAAAACAAATGATT GAG-TAACTGGTAATTAGTT TAATTAAAACAAATGATT GAA-TAACTGGTAATTAGTTTAATTAAAACAAATGATT GAA-TAACTGGTAATTAGTTTAATTAAAACAAATGATT GAC- TAATTGGTAATTAGTTTAATTAAAACAAATGATT GAC-TAACT G G TAATTACTTTAATTAAAACAAATGATT GAA-TAACTGGTAATTAGTTTAATTAAAACAAATGATT GAA-TAACTGGTAATTACTTTAATTAAAACAAATGATT GAC - TAACTGGTAATTACTTTAATTAAAACAAATGATT GAA-TAACT GGTAATTAGTTTAATTAAAACAAATGATT GAA-TAACTGGTAATTAGTTTAATTAAAACAAATGATT GAA-TAACTGGTAATTAGTTTAATTAAAACAAATGATT GAG - TAACT GG TAATTACTTTAATTAAAACAAATGATT GAA-TAACTGGTAATTACTTTAAT TAAAACAAATGATT GAA-TAACTGGTAATTAGTTTAATTAAAACAAATGATT GAG-TAACT GGTAATTACTTTAATTAAAACAAAT GATT GAC-TAACTGGTAATTAGTTTAATTAAAACAAATGATT GAC - TAACTGGTAAT TACT T TAAT TAAAACAAATGATT GAA-TAACTGGTAATTACTTTAATTAAAACAAATGATT GAA-TAACTGGTAATTAGTTTAATTAAAACAAATGATT CAC-TAACT GGTAATTACTT TAAT TAAAACAAATGATT GAA-TAACT G GTAATTAGTTTAATTAAAACAAATGATT GAA-TAACTGGTAATTAGTTTAATTAAAACAAATGATT GAC-TAACT GGTAATTAGTTTAATTAAAACAAATGATT 


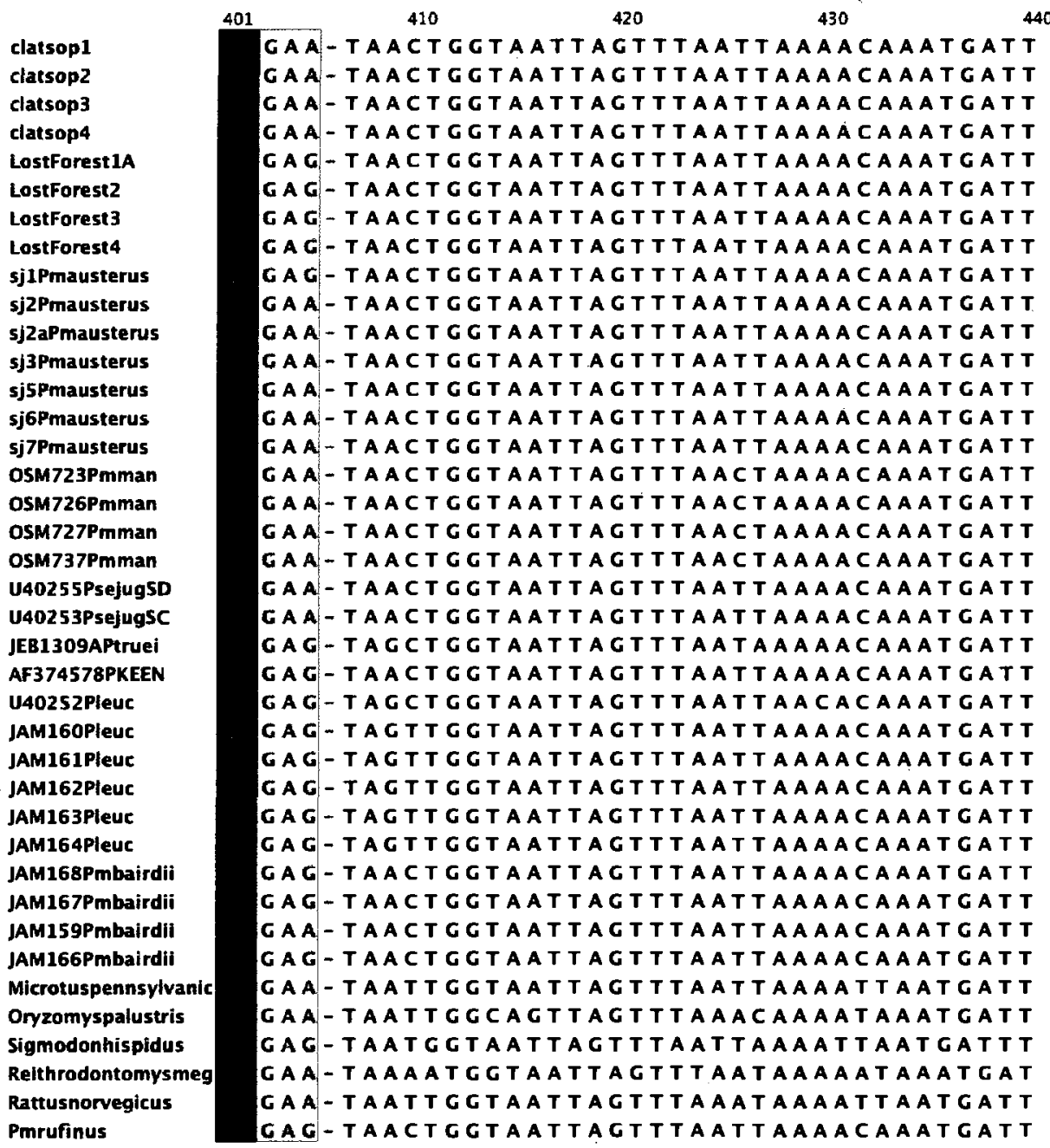


441

450

460

470

480

WC7

we11

wC12

WC13

ljd 3256

ijd 3304

jjd3306

jjd3308

ljd 3317

vid3324

Jjd 3327

Jjd 3330

ljd 3333

Ijd 3344

Ijd3348

LD620

LJD774

LJD886

LJD20B8

LJD1960

LلJ1669

LDD1524

LUD1523

LJD1122

UJD2522

ㄴD1637

LJD1638

UJD2 161

ㄴD2651

LD2737

LD2834

LJD2850

UJD2 898

UJD2914

UJD2936

LJD3000

UJD2126

LJD2138

LDD2154

UJD2 158

UJD2836

UD2852

UDD2 860

LJD2865

WJD2890

UJD2943

Jefferson 3

ADAM56

TCGACT CATTAGATTATGATATACATTATAATTACCAAAA TCGACTCATTAGATTATGATATACATTATAATTACCAAAA TCGACTCATTAGATTATGATATACATTATAATTACCAAAA TCGACT CATTAGATTATGATATACATTATAATTACCAAAA TCGACT CATTAGATTATGATATACATTATAATTACCAAAA TCGACTCATTACATTATGATATACATTATAATTACCAAAA TCGACTCATTAGATTATGATATACATTATAATTACCAAAA TCGACTCATTAGATTATGATATACATTATAATTACCAAAA TCGACTCATTAGATTATCATATACATTATAAT TACCAAAA TCGACTCATTAGATTATGATATACATTATAATTACCAAAA TCGACTCATTAGATTATCATATACATTATAATTACCAAAA TCGACTCATTAGATTATGATATACATTATAATTACCAAAA TCGACTCATTAGATTATGATATACATTATAATTACCAAAA TCGACTCATTAGATTATGATATACATTATAATTACCAAAA TCGACTCATTAGATTATGATATACATTATAATTACCAAAA TCGACTCATTAGATTATCATATACATTATAATTAC CAAAA TCGACTCATTAGATTATGATATACATTATAATTACCAAAA TCGACTCATTAGATTATCATATACATTATAATTAC CAAAA TCGACTCATTACATTATGATATACATTATAATTACCAAAA TCGACTCATTACATTATGATATACATTATAATTACCAAAA TCGACTCATTACATTATCATATACATTATAATTACCAAAA TCGACTCATTAGATTATGATATACATTATAATTACCAAAA TCGACTCATTAGATTATGATATACATTATAATTACCAAAA TCGACTCATTAGATTATGATATACATTATAATTACCAAAA TCGACTCATTAGATTATGATATACATTATAATTACCAAAA TCGACTCATTAGATTATGATATACATTATAATTACCAAAA TCGACTCATTAGATTATGATATACATTATAATTACCAAAA TCGACTCATTAGATTATGATATACATTATAATTACCAAAA TCGACTCATTAGATTATGATATACATTATAATTACCAAAA TCGACTCATTAGATTATCATATACATTATAATTAC CAAAA TCGACTCATTAGATTATCATATACATTATAATTACCAAAA TCGACTCATTAGATTATGATATACATTATAATTACCAAAA TCGACTCATTAGATTATGATATACATTATAATTACCAAAA TCGACTCATTAGATTATGATATACATTATAATTACCAAAA TCGACTCATTAGATTATGATATACATTATAATTACCAAAA TCGACTCATTAGATTATCATATACATTATAATTACCAAAA TCGACTCATTAGATTATCATATACATTATAATTACCAAAA TCGACTCATTAGATTATCATATACATTATAATTACCAAAA TCGACTCATTAGATTATCATATACATTATAATTACCAAAA TCGACTCATTAGATTATCATATACATTATAATTACCAAAA TCGACTCATTAGATTATCATATACATTATAATTACCAAAA TCGACTCATTAGATTATGATATACATTATAATTACCAAAA TCGACTCATTACATTATCATATACATTATAATTACCAAAA TCGACTCATTAGATTATCATATACATTATAATTACCAAAA TCGACTCATTAGATTATGATATACATTATAATTACCAAAA TCGACTCATTAGATTATCATATACATTATAATTACCAAAA TCGACTCATTAGATTATGATATACATTATAATTACCAAAA TCGACTCATTAGATTATGATATACATTATAATTACCAAAA 


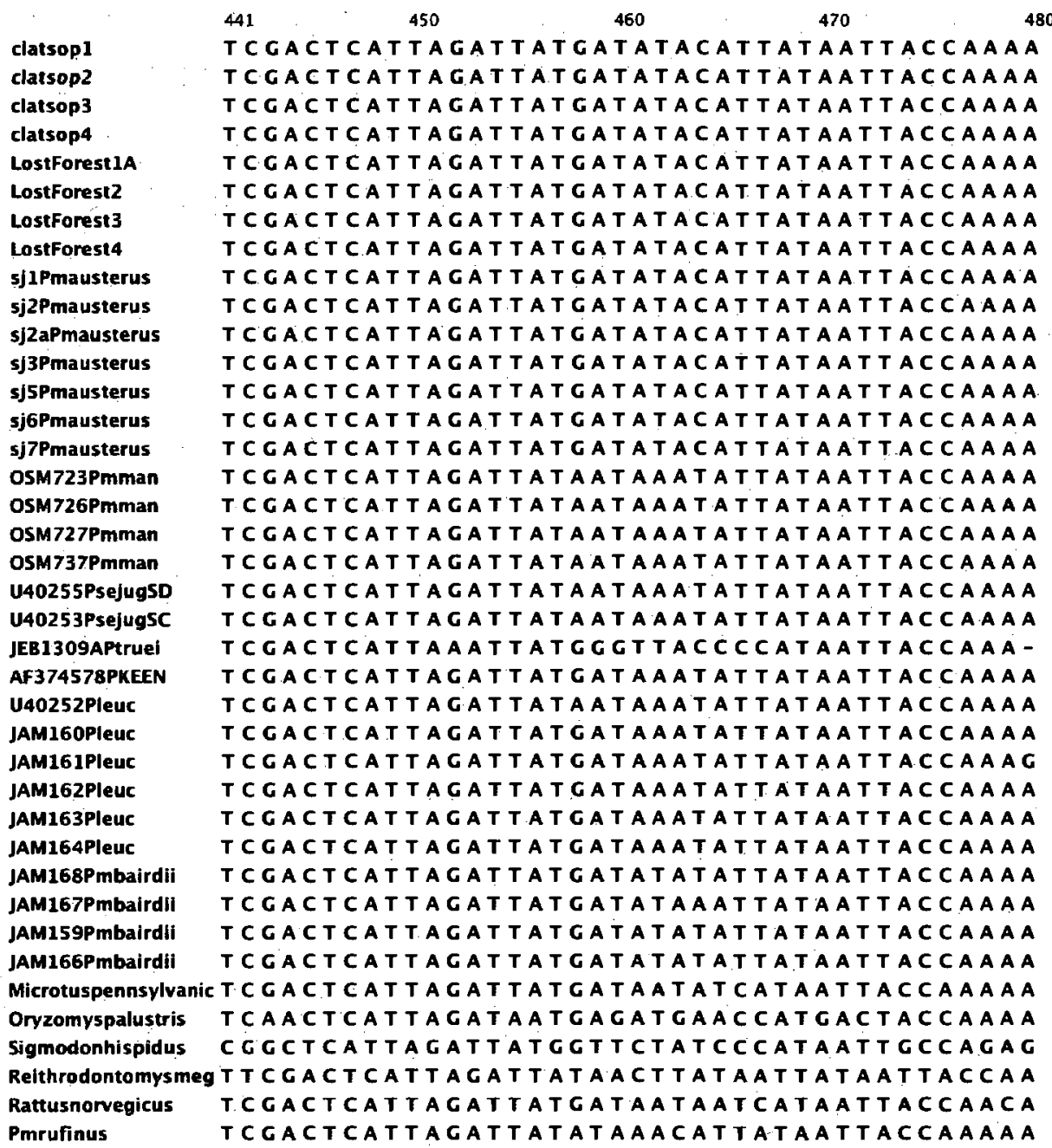


WC7

WC11

WC12

WC13

I]d3256

IJd3304

IJd3306

ljd3308

lid3317

Ijd3324

ljd3327

ljd 3330

ljd3333

ljd3344

IJd3348

LJD620

LJD774

UJD886

LjD2088

LJD1960

LJD1669

LJD1524

LJD1523

LJDI 122

LUD2 522

LJD1637

LJD1638

LJD2161

LJD2651

LJD2737

LJD2834.

LJD2850

LJD2898

LJD2914

LJD2936

LJD3000

LJD2126

LJD2138

LJD2 154

LJD2158

LJD2836

LJD2852

LJD2860

LJD2865

UJD2890

LJD2943

Jefferson 3

ADAMS 6
$481 \quad 490$

- - ATG TCATCTGTAACCTCCAATATTATA

- - ATGTCATCTCTAACETCCAATATtATA

- - ATGTCATCTCTAACCT C CAATATTATA

- - - AT GTCATCTCTAACCTCCAATATTATA

-.. AT GTCATETCTAACCTCCAATATTATA

-.. AT GTCATCTCTAACCTCCAATATTATA

- - A T GTCATCTCTAACCTCCIAATATTATA

-.. - AT GTTCAT CTG TAAC CT C CAATATTATA

- - AT GTCAT CTGTAIACCTCCAATATTATA

- - - AT GT CAT CTGTAACCTCCAATATTATA

- - ATGTCATCTCTAACCTCCAATATTATA

-.. ATGTCAT ETGTAACETCCAATATTATA

-.. - AT GT CATCTGTAACETCCAATATTATA

- - AT TT CATCTCTAACCTCCAATATTATA

-.. ATGTCATCTCTAACETCCAATATTATA

- - AT GTCATCTCTAACCTCCAATATTATA

- - - A T GTCATCTG TAACCTCCAATATTATA

- - A TGTCATCTGTAACCITCCAATATTATA

- - at GTCatctetalacetccaAtattata

-.. - AT CTCATCTGTAACCTCCAATATTATA

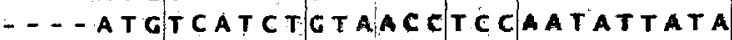

- - - AT GTCATCTGTAIACCTTCAATATTATA

-.. A T GT CATCTGTAACCTCCAATATTATA

-.. A T CTCATCTCTAACCTCCAATATTATA

- - - ATGTCATCT G TAACCTCCAATATTATA

-.. A T GT CATCTG TAACCTTCCAATATTATA

- - A T GTCATCTCTALCCT C CAATATTATA

- - A t CTCAT CTETAaCC|TCCAATATTATA

- - ATGTCATCTCTAACCTCCAatATTATA

- - - ATCTCATCTGTAACCTCCAATATTATA

- - AT GTCATCTG TAACCTCCAATATTATA

-.. A TCTCATCTGYARCCTCCAATATTATA

-.. A TCTCATCTCTAACCTCCAATATTATA

- - - AT GTCATCTGTAACCTCCAATATTATA

-.. - AT GTCATCTGTAACCTCCAATATTATA

- - - AT GTCATCTGTAAC CT CCAATATTATA

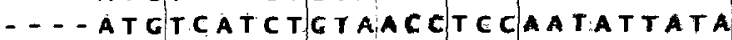

-.- - ATGTCATCTGTAACCTCCAATATTATA

- - - AT GTCAT CTCTAACCTCCAATATTATA

-.. - AT GTCATCTCTAACCTCCAATATTATA

-.. - ATCTCATCTCTAACCTCCAATATTATA

-.. AT GTCAT CTGTAACCTCCAATATTATA

- - - ATGTCATCTGTAACETCCAATATTATA

-.. - AT GT CAT CTG TAACCTCCAATATTATA

- - - AT GTCAT CTCTAACCTCCAATATTATA

- - - ATCTCATCTGTAACCTCCAATATTATA

- - AT GTCATCTGTAACCTCCAatatTata

... A t GTCATCTGTAACCTCCAatatTata

GCATTC

GCATTC

GCATTC

GCATTC

GCATTC

GCATTC

GCATTC

GCATTC

CCATTC

GCATTC

CCATTC

GCATTC

CCATTC

GCATTC

GCATTC

C CATTC

GCATTC

GCATTC

GCATTC

CCATTC

GCATTC

G.CATTC

GCATTC

GCATTC

GCATTC

G C ATTC

GCATTC

CCATTC

GCATTC

GCATTC

GCATTC

CCATTC

CCATTC

GCATTC

GCATTC

GCATTC

CCATTC

GCATTC

GCATTC

GCATTC

CCATTE

GCATTE

GCATTC

GCATTE

CCATTC

GCATTC

GCATTE

GCATTC 


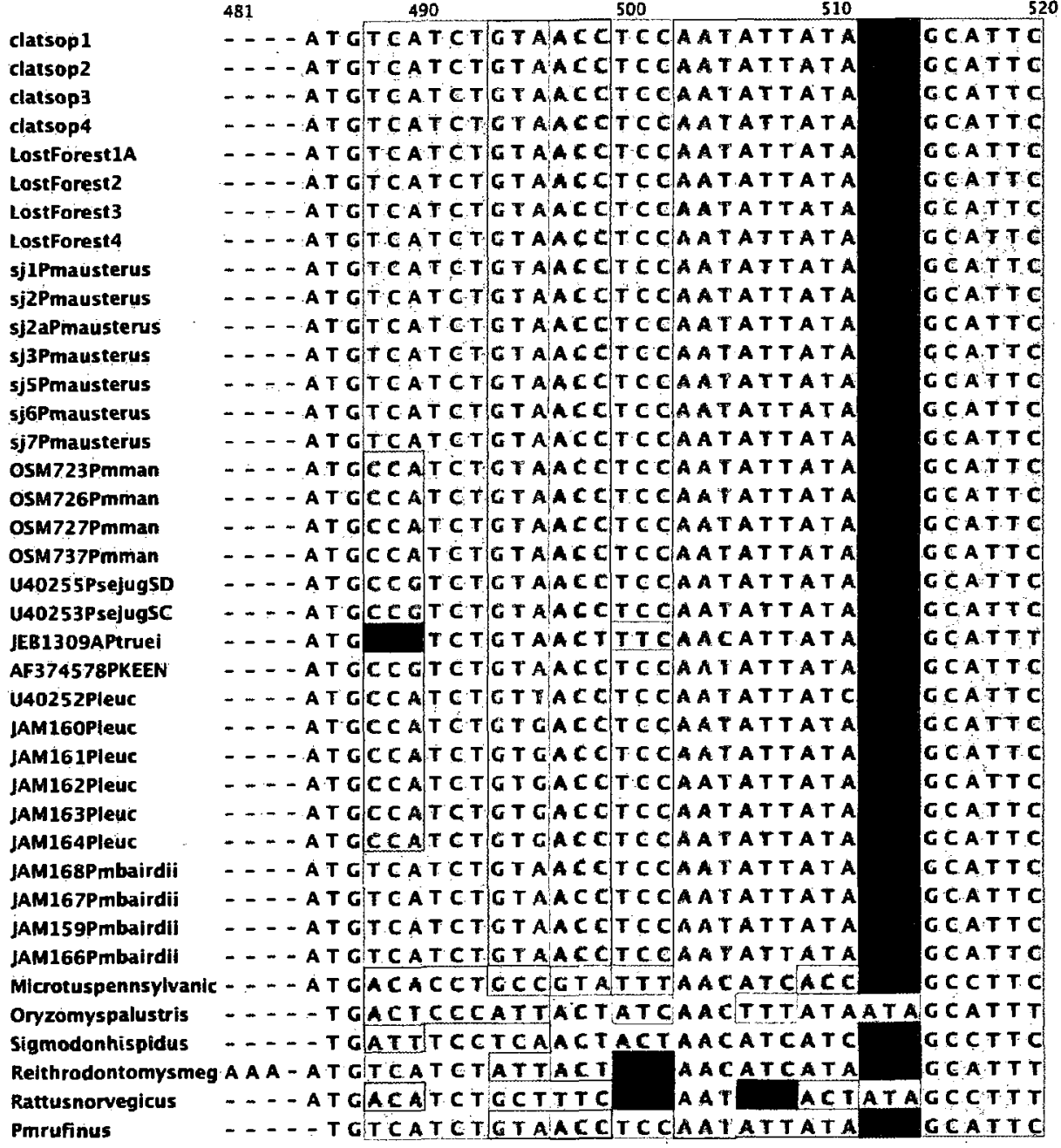


wc7

WCII

WC12

WC 13

Ijd3256

Jjd 3304

Ijd 3306

jjd3308

Jjd3317

Ijd3324

Jjd3327

jjd3330

jjd3333

Ijd3344

Ijd 3348

LJD620

LJD774

LJD886

LJD2088

LJD1960

LJD1669

LJD1524

LJD1523

LJD1122

LJD2522

LJD1637

LJD1638

LJD2161

IJD2651

LJD2737

LJD2834

LJD2850

LJD2898

LJD2914

LJD2936

LJD3000

LJD2126

LJD21.38

LJD2 154

LJD2158

LJD2836

LJD2852

LJD2860

LJD2865

LJD2 890

LJD2943

Jefferson 3

ADAMS6

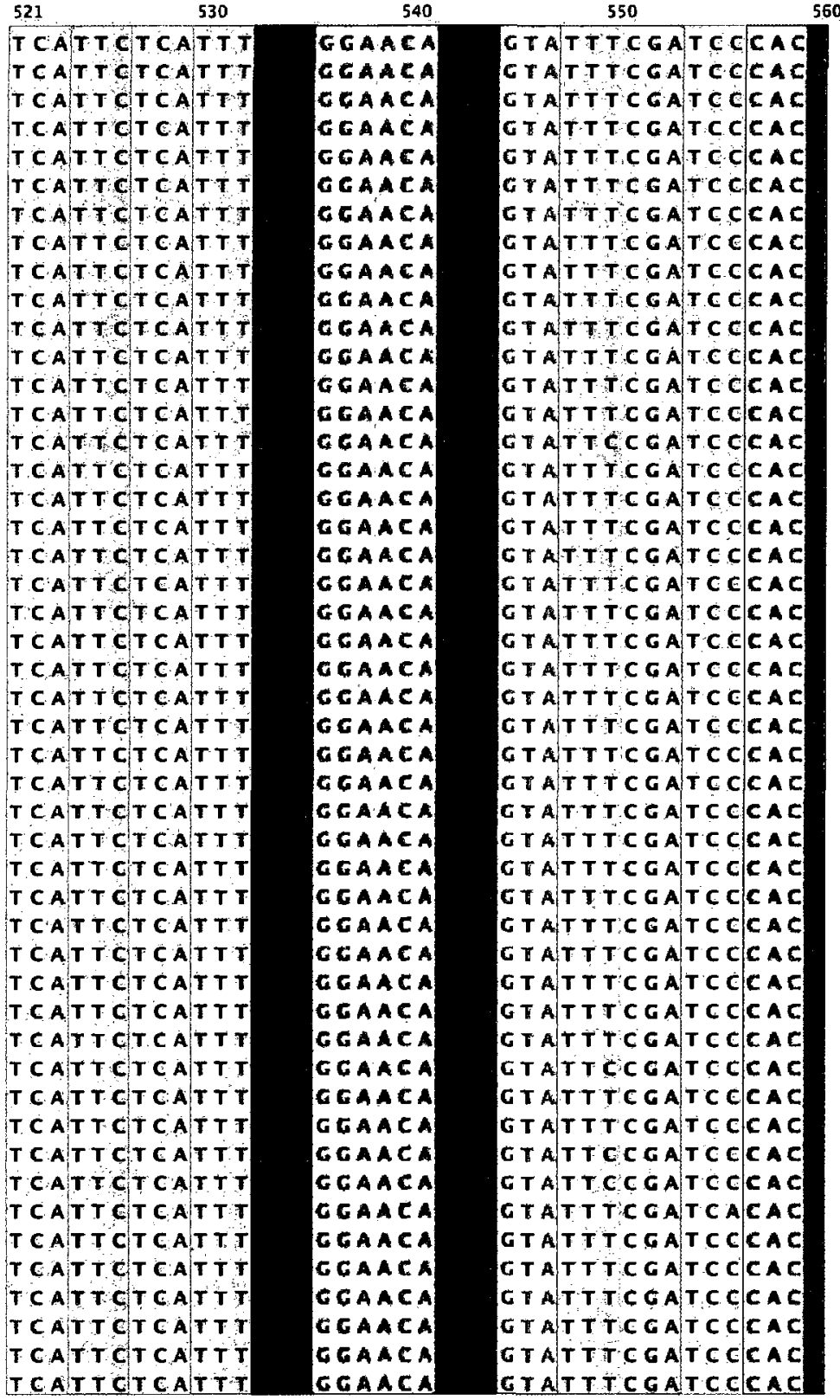




\begin{tabular}{|c|c|c|c|c|}
\hline & 521 & 540 & 550 & \\
\hline clatsop 1 & T CATT CT CATTT & GGAACA & GTATTTCGATCC & $C A C$ \\
\hline clatsop 2 & T CATTCTCATT T & $G \in A A C A$ & G TATTTCGATCC & CAC \\
\hline clatsop 3 & T CATTCTCATTT & $\operatorname{GAACA}$ & C T ATT TCGATCE & CA \\
\hline clatsop4 & TCAT TCT CATT & CGAACA & G TATTTCGATCC & Ca \\
\hline LostForest1A & T C ATT T T T CATT & GGAACA & G T ATTTEGATCC & Ca \\
\hline LostForest2 & T C ATT T CT CATT & $\operatorname{c\in A} A \subset A$ & G T ATT TCGATCC & $C A$ \\
\hline LostForest 3 & T C ATT T CT CATT & CGAACA & G T A T T TCGATCG & CA \\
\hline LostForest4 & TCATTCTCATT & CGAACA & GT ATTTCGATCC & $\mathbf{E A}$ \\
\hline sj1Pmausterus & $\mid \mathrm{T}$ C A T T C T C A & GGAACA & CTATTCCGATCC & CA \\
\hline sj2Pmausterus & T CATT T CTCATT & G GAACA & CTATT TCGATCC & $\mathbf{C A}$ \\
\hline sj2äPmausterus & T CATT T T CA & GGAACA & G TATTTCGATCC & $\mathbf{C A}$ \\
\hline sJ3Pmausterus & TCATT TCT CA & GGAACA & C TATTTCGATCC & Ca \\
\hline sjsPmausterus & T C ATT T CTCAT & G $A A A C A$ & G T ATT TC GATTC C & $\mathbf{C A}$ \\
\hline sj6Pmausterus & T CATT TCT CAT & GGAACA & $G T A T T T C G A T C C$ & $C A$ \\
\hline sj7Pmausterus & T CA:T TCTTCA:T T & GGAACA & GTATTTCGATCC & \\
\hline OSM723Pmman & T C GTTCT CATT & $\operatorname{CGAACA}$ & CIATTTCGATCC & $\mathbf{C A}$ \\
\hline OSM7.26Pmman & 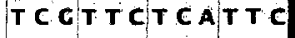 & G $A A A C A$ & CTATTTCGATCC & CA \\
\hline OSM727Pmman & T C GTT CT C ATT T C & GEAACA & CTATTTCGATCC & \\
\hline O5M737Pmman & $\mid \mathbf{T} \in \mathbf{G} T \mathbf{T} \boldsymbol{T} \mathbf{T} \in \mathbf{A}$ & GGAACA & CTATTTCGATCC & \\
\hline U402SSPsejugSD & TCATT TT T CATT T & GGAACA & CTATTTCGAGCC & $C a$ \\
\hline U40253PsejugSC & TCATT TTTCATTT & CEAAEA & GTATTTEGAGCC & CA \\
\hline JEB1309APtruei & ATAT T TT CAT & G GAACA & GTATTCCGATCC & ca \\
\hline AF374578PKEEN & T C ATT T TT CA & GGAACA & GTATTTCGATCC & \\
\hline U40252Pleuc & TCATT TT CATT & $G \in A A C T$ & GTETTTCGCTCT & \\
\hline JAM160Pleuc & T C ATT T T:T C AT & $\operatorname{cecact}$ & CTCTT TCGCTCT & CA \\
\hline JAM16.1Pleuc & $\mathbf{T} \boldsymbol{C A} \mathbf{A} \mathbf{T} \mathbf{T} \mathbf{T} \mathbf{A}$ & GGGACt & G T GT T TCGCTCT & $\mathbf{C A}$ \\
\hline JAM162Pleuc & T C A T T T T T CA:T & GGGACT & G T GTT T C G CTCT & \\
\hline JAM163Pleuc & T C ATT T TT CATT & $\operatorname{G\in CACT}$ & GTGTTTCG CTCT & Ca \\
\hline JAM164Pleuc & ITCATTTTCAT & Geant & C T CTT TCGCTCT & Ca \\
\hline JAM168Pmbairdii & T C ATT T C:T CAT & GecacA & CIATT TCGATCC & $C A$ \\
\hline JAM167Pmbairdii & T C AT T CTCAT & GGGACA & GTATTTCGATCC & $C A$ \\
\hline JAM159Pmbairdil & T C A:T T C:T C ATT T T & $\operatorname{GGACA}$ & GTATTTCGATCC & \\
\hline JAM166Pmbairdil & T C ATT T CT C AT T T & $\operatorname{c\in c} A \subset A$ & GTATTTCGATCC & CAC \\
\hline Microtuspennsylvanic & T CTT T CTCT & CGGACT & ATATTCEGATCA & Ca \\
\hline Oryzomyspalustris & I CCT T CTCC & G A A A T & ATATTCCGCTCC & $\mathbf{C A C}$ \\
\hline Sigmodonhispidus & AT AT TTTCC & GEAACC & ATAT TC C G ATC C & CAC \\
\hline Reithrodontomysmeg & A TTTTTTCCTTT & $\cos \operatorname{ca}$ & А T ATTCC GC|TCT & $C \wedge T$ \\
\hline Rattusnorvegicus & $A C A \quad T C T$ & GETACT & ATATTTCGCTCC & CAC \\
\hline Pmrufinus & ICATICISATT T & GGAACG & GTATTTCGATCE & 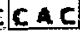 \\
\hline
\end{tabular}


WC7

WC11

WC12

WC13

Ijd3256

ljd 3304

Ijd3306

ljd3308

Ijd 3317

IJd3324

ljd3327

ljd3330

ljd3333

ljd3344

ljd3348

UD620

UD774

UD886

LjD2088

LJD1960

LJD1669

LJD1524

LJD1523

LJD1122

LJD2522

LJD1637

LJD1638

LJD2161

LjD2651

LJD2737

LJD2834

LJD2850

LJD2898

LJD2914

LJD2936

LJD3000

LJD2126

LJD2138

LJD2 154

LPD2158

LJD2836

LJD2852

LJD2860

LJD2865

LJD2890

LJD2943

Jefferson 3

ADAMS6

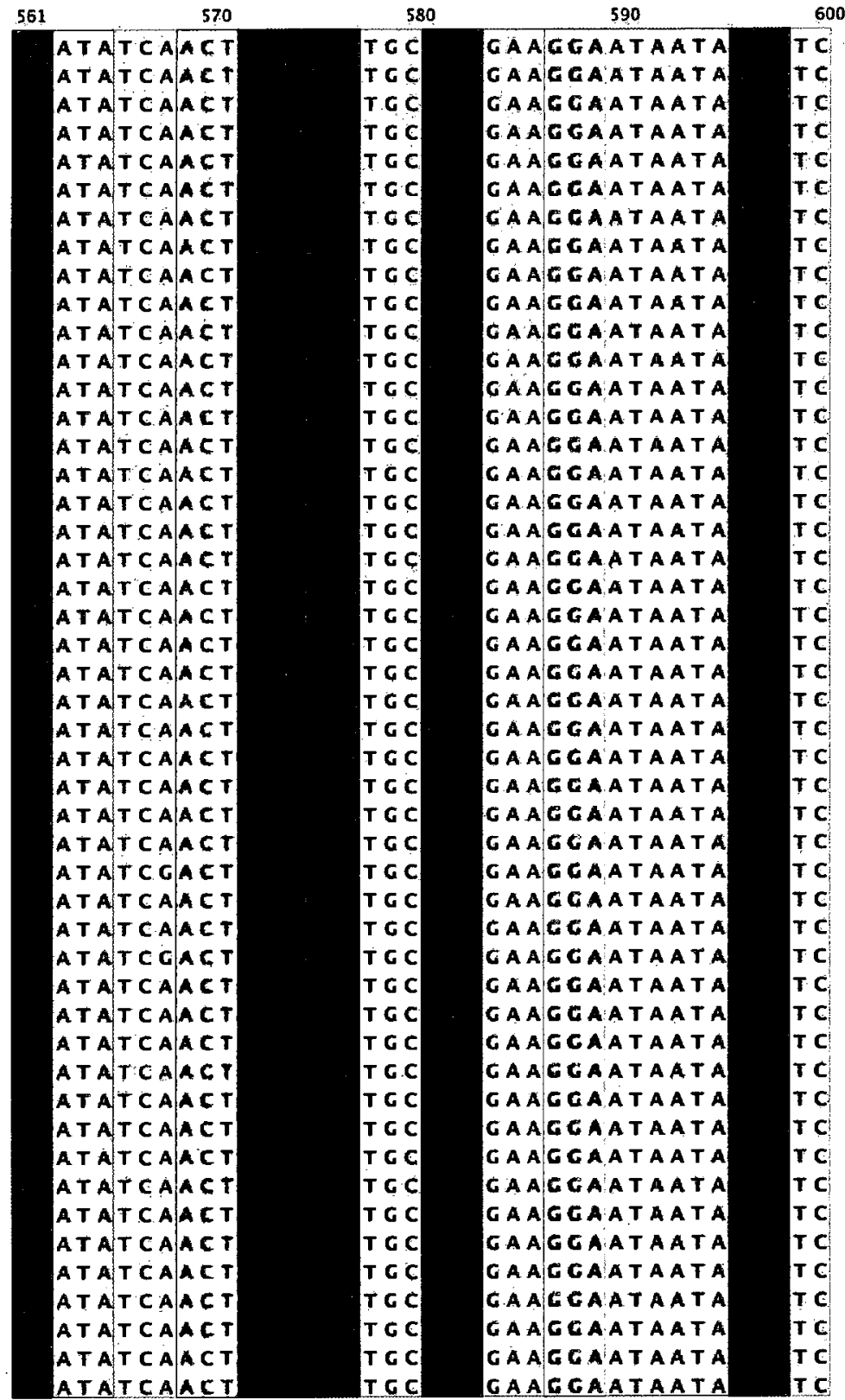




\begin{tabular}{|c|c|c|c|}
\hline & 570 & 580 & 590 \\
\hline clatsop1 & AT ATCAACT & TC C & GAAGGAATAATA \\
\hline clatsop2 & AT ATCAACT & TCC & GAAGGAATAATA \\
\hline clatsop3 & A T A T C A A C T & $\mathbf{T} \mathbf{G} \mathbf{C}$ & GAAOGAATAATA \\
\hline clatsop4 & $A T A T C A \mid A C T$ & $\mathbf{T} \cdot \mathbf{C}$ & САACGAATAATA \\
\hline Lostforest1A & AT ATCALET & $\mathbf{T} \mathbf{G} \mathbf{C}$ & GAAGGAATAATA \\
\hline LostForest2 & AT ATC ALET & $\mathbf{T} \mathbf{G ~ C}$ & GAAGGAATAATA \\
\hline LostFọrest3 & A T ATT C AAC T & T G C & GAAGGAATAATA \\
\hline LostForest4 & A T A T $\in A \mid A C T$ & $\mathbf{T} \mathbf{C} \mathrm{C}$ & GAACGAATAATA \\
\hline sj1Pmausterus & A T ATC C A ACT & $T G C$ & GAAGGAATAATA \\
\hline sj2Pmausterus & AT ATC A|ACT & T G C & GAAGGAATAATA \\
\hline sj2aPmausterus & A T A T C A ACT & T G C & GAA GAATAATA \\
\hline sj3Pmausterus & AT ATCAACT & TCC & GAAGGAATAATA \\
\hline sj5Pmausterus & AT ATC AACT & $\mathbf{T G C}$ & GAAGGAATAATA \\
\hline sj6Pmausterus & A T ATCAACT & T G C & CAAGGAATAATA \\
\hline sj7Pmausterus & A T ATC A ACT & $T: C . C$ & G AAG GAATAATA \\
\hline osM723Pmman & ATATCATAT & T.GC & GAAGGAATAATA \\
\hline OSM726Pmman & AT ATC AlaCT & T.C. C & CAACGAATAATA \\
\hline OSM727Pmman & A T ATC AACT & $\mathbf{T G C}$ & GAAGGAATAATA \\
\hline OSM737Pmman & AT ATCA|ACT & T G C & GAAGGAATAATA \\
\hline U40255PsejugSD & A T AT C G|ACT & T G C & G A GGAATAATA \\
\hline U40253PsejugSC & A T AT C CAC T & $T G C$ & G AAGGAATAATA \\
\hline JEB 1309APtruei & A T GTC T A C A & $T \in C$ & G A CGGAATAATA \\
\hline AF374578PKEEN & A T AT C GACT & T C C & GAAGGAATAATA \\
\hline U40252Pleuc & A T ATC C A C C & $\mathbf{T G C}$ & G A AGGGATAATG \\
\hline JAM160Pleuc & A T ATCCACC & $\mathbf{T} \mathbf{C C}$ & C A AGG GAT A AT G \\
\hline JAM161Pleut & A T A T C C:ACC & $T G C$ & GAAGAGATAATG \\
\hline JAM162Pleuc & A T ATC EACC & T G C & GAAGGGAT AAT G \\
\hline JAM163Pleuc & AT ATCCACC & $\mathbf{T} \in \mathbf{C}$ & GAACGGATAATG \\
\hline JAM164Pleuc & A T ATCCCACC & $\mathbf{T C C}$ & CAACGEATAATG \\
\hline JAM168Pmbairdii & AT T & T G C & GAACGAATAATA \\
\hline JAM167Pmbairdii & A T ATCAACT & $\mathbf{T} \in \mathbf{C}$ & GAACGAATAATA \\
\hline JAM159Pmbairdil & ATATCAACC & $T \in C$ & GAACGAATAATA \\
\hline JAM166Pmbairdli & ATATCAACT & $\mathbf{T C C}$ & GAACCAATAATA \\
\hline Microtuspennsylvanic & AT AT C C ACC & T G C & G A AGCATAATA \\
\hline Oryzomyspalustris & A T AT C TAC C & T C C & GAAGGTATGATG \\
\hline Sigmodonhispidus & $A T G T C T A C C$ & TGC & GAAGGAATAATG \\
\hline Reithrodontomysmeg & AT ATCAACA & $\mathbf{T G C}$ & G A GG GTAT A ATA \\
\hline Rattusnorvegicus & ATATCCACT & TC C & CAAGGAATAATA \\
\hline Pmrufinus & A I ATCAACT & TGC & GAAGGAATAATA \\
\hline
\end{tabular}


WC7

WC11

WC12

WC13

Jjd3256

Jjd3304

Jjd3306

ljd3308

1jd3317

Ijd3324

Ijd 3327

Jjd 3330

ljd3333

IJd3344

IJd3348

LJD620

LJD774

LJD886

LJD2088

LJD1960

LDD1669

LJD1524

LjD1523

LJD1 122

LJD2522

LJD1637

LJD1638

LDD2 161

LJD265 1

LJD2737

LJD2834

LJD2850

LJD2898

LJD2914

LJD2936

LJD3000

LJD2 126

LJD2 138

LJD2154

LJD2158

LJD2836

LJD2852

LJD2860

LJD2865

LJD2890

LJD2943

Jefferson 3

ADAMS6

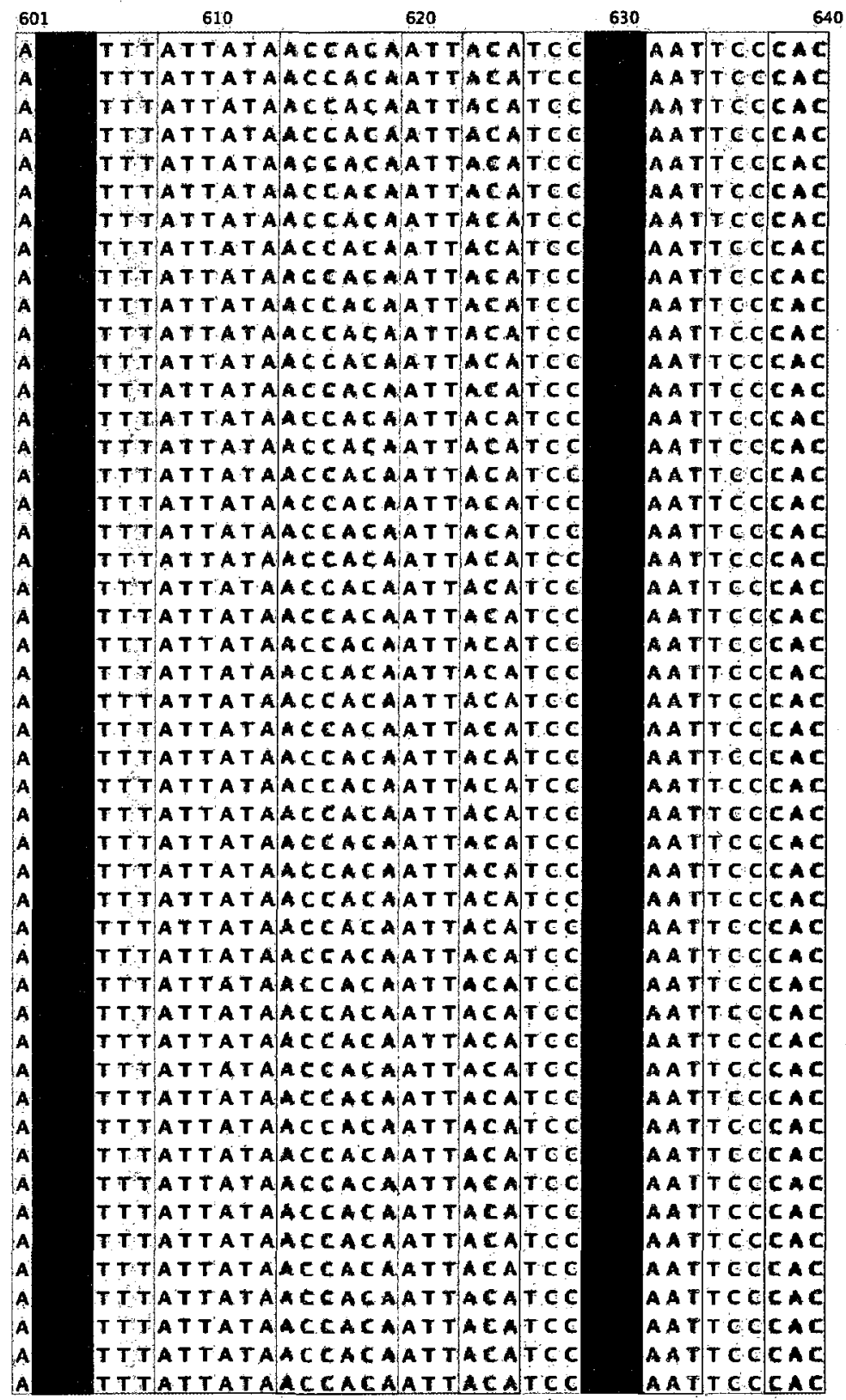


clatsop 1

clatsop2

clatsop3

clatsop 4

LostForest1A

LostForest2

LostForest 3

LostForest 4

sj1Pmausterus

sj2Pmausterus

sj2aPmausterus

sj3Pmaustẹus

sjSPmausterus

sj6Pmausterus

sj7Pmausterus

OSM723Pmman

oSM726Pmmian

OSM727Pmman

OSM737Pmiman

U40255PsejugSD

U40253PsejugSC

JEB 1309APtruei

AF374578PKEEN

U40252Pleuc

JAM160Pleuc

JAM161Pleuc

JAM162Pleuc

JAM163Pleuc

JAM164Pleuc

JAM168Pmbairdii

JAM167Pmbairdi

jAM 159Pmbairdil

JAM 166P mbairdil

601

$\frac{610}{T T T T}$

$610 \quad 620$

T TACATCC TT TATTATAACCACAATTACATCC TTTATTATAACCACALTTACATCC TTTATTATAACEAGAATTACATCE T T TAT TATAACEACAATTAEATCC TITAT TATAACCACAATTACATCC TT TAT TATAACCAEAATTAEATEC TTTATTATAACEACAATTACATCC TATATTATA]ACCACAATTACATCC TTTATTATAACCACAATTACATCC TT TAT TAT AACCACAATTACATCC TTTAT TATAACEACAATTAEATCC TTTATTATAACCACAATTACATCC TTTATTATAACCAEAATTAEATCC TTTATTATAACCACAATTACATCE T TTAT TATAACGACAATTAEATCC TTTATTATAACEACAATTACATCE TTTATTATAACEACAAT TACATCC TTTATTATAACCACAATTACATCC TTTATTATAACEAC ATTACALCC TT TAT TATAACEACAATTACCGTC TTTAT TATAACEACAATTACATCC T TTAT TAT AACCACAATTACATC C T TCATTATAACAACAATTACTICC T TCATTATAACAACAATTACTTCC T T CAT TATAACAACAAT TACTTCC T TCAT TAT AACAACAATCACTTCC TTCATTATANCAACAATTACTTCC T TCATTATAACAACAATTACTTCC TTTATTATAACEACAATTACATCC T T TAT TATAACCACAATTACATCC TT TAT TATAACEACAATTACATCC TTTATTATARCCACAATTACATCC Microtuspennsylvanic TCCETTTATC GCTACTATCACACCC Oryzomyspalustris Sigmodonhispidus Reithrodontomysmeg $A$ Rattusnorvegicus Pmrufinus

630 A TTCCCAC A A TT ClCAC A A TTCCCAC AatT Celcac A A T T C A C AATTCCCAC A A TT C ClCAC A A r TCCCAC AATTCCCAC A A TTCCCAC A ATTCCCAC A A r C C CleAC A A Trecleac A ATCCTCE A A TT C ClCAC A ATTCCCAC A ATTCCCAC A A T TCCAC AatTGCCAC AATICCCAC CACCCECAC Aactecac AC $\mathrm{CTCTAC}$ AactCerac A ACTCCEAC Aactectac A ACTCCTAC Aact celcac A A E TCCEAC Aartcechc A A TTC ClCAC AatTCClenc A A TTCCCAC aAcacacac AACACACAC AGCTCTGA A ATTCTCAT AACTCCAAC AATTCECAC 
wey

wC11

wC12

WC13

Jjd3256

ljd3304

ljd3306

Jjd3308

ljd 3317

Ijd 3324

Ijd 3327

ljd 3330

ljd 3333

Ijd 3344

ljd 3348

L) 0620

LJD774

LJD886

LJD2088

LJD1960

LJD1669

LUD1524

LJD1523

LJD1122

LJD2522

LJD1637

LJD1638

LJD2 161

LJD2651

LJD27.37

LJD2834

LJD2850

LJD2898

LJD2914

LᄂJD2936

LJD3000

LJD2 126

LJD2 138

LJD2 154

LJD2 158

LJD2836

LJD2852

LJD2860

LJD2 865.

LJD2890

LلJ2943

Jefferson 3

ADAMS6

\begin{tabular}{|c|c|c|c|c|}
\hline 641 & 650 & & 660 & \\
\hline$T \subset A$ & A T A A T T A T AT AC & $C \in T$ & CCCATTGTTATT & G T A T \\
\hline$T C A$ & А T А А T T А T AT AC & $C \in \mathrm{T}$ & CCCATTGTTATT & C T A T \\
\hline$T C A$ & A T A A T T AT AIT AC & C C T & CEEATTGTTATT & G TAT \\
\hline$T \dot{C A}$ & A T A A T T A T AT AC & $C<T$ & CCCATTGTTAT & G T A T \\
\hline$T C A$ & ATAATTAT ATAC & $\mid c \in t$ & CCCATTGTTATT & G T A T \\
\hline$T C A$ & AT A A T TAT AT AC & $c \in T$ & CCCATTGTTATT & $\boldsymbol{C} \mathbf{T} \mathbf{A}$ \\
\hline$T C A$ & AT A ATT AT AT AC & Cet & $C C \in A T T G T T A T$ & GT ATT \\
\hline TCA & AT AATTAT AT AC & $C E T$ & CCCATTGTTAT & G T A T \\
\hline$T C A$ & AT AAT T AT AT AC & $C \mathbf{C T}$ & CCCATTGTTAT & G T A T \\
\hline$T C A$ & ATAATT AT AT AC & $C \subset \mathrm{T}$ & CCEATTGTTATT & CT $\mathbf{T} \mathrm{T}$ \\
\hline$T C A$ & AT TATTAT AT AC & $C<\mathrm{T}$ & C C CATTGTTATT & \\
\hline$r \in A$ & AT A AT T AT AT AC & $c<c$ & CCCATTGTTATT & G T A T \\
\hline$T \subset A$ & AT A AT T AT ATAC & $\subset \subset T$ & CCCATTGTTATT & GTAT \\
\hline$T C A$ & AT AAT TAT ATAC & CCT & CCCATTGTTATT & $G \mathrm{G} \cdot \mathrm{T}$ \\
\hline TCA & ATAATTATATAC & C C T & CECATTGTTATT & C T A T \\
\hline$T C A$ & AT A AT T AT AT AC & $c \mathrm{cr}$ & CCCATTGTTATT & $G \mathbf{T} A \mid \mathbf{T}$ \\
\hline$T C A$ & AT A ATTATATAC & C CT & CCCATTG TTATT & G T ATT \\
\hline $\mathbf{T} \mathbf{A}$ & A T A A T T A T AT AC & $C E T$ & C C CATTGTTATT & GT AT T \\
\hline T C:A & AT AATT AT AT AC & $C \in T$ & CCCATTGTTATT & GTAT \\
\hline T CA & AT A AT T AT AT AC & $C \mathrm{CT}$ & CCCATTCTTATT & C T A T \\
\hline$T C A$ & AT A AT TAT ATAC & $C \mathrm{CT}$ & CCCATTGTTATT & G TAT \\
\hline T C A & AT A AT T AT AT AC & $\mathbf{C} \mathbf{C T}$ & C C С T T T T T T T & G T A.T \\
\hline TCA & ATAATTAT AT AC & C C T & CCCATTGTTATT & GT AT \\
\hline T C.A & AT A AT T AT AT AC & C C T & CCCATT TT TATT & G T AT \\
\hline $\mathbf{T} \subset A$ & AT A A T T AT AT AC & $C C T$ & CCCATTGTTATT & $G T A T$ \\
\hline T CA & AT A AT T AT ATAC & $c \in c$ & CCCATTGTTATT & $G T A T$ \\
\hline$T C A$ & AT A AT TAT ATAC & $C \mathbf{C T}$ & ССС ATTGTTATT & G T A $\mathbf{T}$ \\
\hline$T C A$ & A T A A T T AT AT AC & $c \in T$ & CCCATTGTTATT & G T A T \\
\hline T CA & AT A A T TATATAC & C C T & СС С T T CT TATT & G T A T \\
\hline$T C A$ & AT A AT TATAT AC & $C \subset T$ & CCCATTGTTATT & G T A T T \\
\hline TCA & AT A AT TAT AT AC & C C T & C C A TTGTTATT & GTAT \\
\hline T C A & AT A A T T A T A T AC & C C T & CCEATTCTTATT & G T A T \\
\hline TCA & A T A A T T A T AIT AC & $\operatorname{cet}$ & CCCATTGTTATT & $G T A T$ \\
\hline$T \subset A$ & A T A A T T AT A'T AC & $C \mathrm{CT}$ & CCCATTGTTATT & GTAT \\
\hline$T C A$ & AT A AT TATAT AC & $C \subset T$ & CCCATTGTTATT & G T A T \\
\hline$T \subset A$ & AT AAT TAT AT AC & $C \subset T$ & CCCATTGTTATT & $\mathbf{C} \mathbf{T} \mathbf{A}$ \\
\hline $\mathbf{T} C A$ & A T A A T T A T AT AC & C C T & CCCATTGTIATT & G T A T \\
\hline T C A & A T A A T T A T A T AC & CCT & CC CATTGTTATT & G T A T \\
\hline $\mathbf{T} C A$ & A T A A T T AT AT AC & & C C С AT T T: TATT & G T A T \\
\hline T CA & A T A A T T A T AT AC & $C \in T$ & CCCATTGTTATT & G T A T \\
\hline$T C A$ & AT TA T T AT ATT AC & Cet & CCCATTGTTATT & G T $\mathbf{A} T$ \\
\hline T C A & A T A A T T A T AT AC & $C \mathbf{C T}$ & CССATTGTTATT & $\mathbf{G} \mathbf{T} \mathbf{A} \mathbf{T}$ \\
\hline $\mathbf{T C A}$ & A T A A T T A T AT AC & $C \mathbf{C T}$ & CCCATTGTTATT & $\mathbf{G} \mathbf{T} \mathbf{A} \mathbf{T}$ \\
\hline$T C A$ & AT A A T T AT AT AC & $c \in t$ & CECATTGTTATT & G T A T \\
\hline$T C A$ & AT AAT T AT AT AC & C C C & CCCATTCTTATT & C T A T \\
\hline$T C A$ & AT A AT T A T AT AC & $C \in T$ & CCLATTGTTATT & C T A T \\
\hline$T \in A$ & AT AAT TAT AT AC & $C \subset \mathrm{T}$ & CCCATTGTTATT & G T A T T \\
\hline T C A & A TAATTATATAC & CET & CCCATIGTTATT & G T A T \\
\hline
\end{tabular}




\begin{tabular}{|c|c|c|c|c|c|}
\hline & 641 & 650 & 660 & 670 & \\
\hline clatsop 1 & TCA & A T A A T TA T AT AC & $C \subset T$ & CCEATTGTTATT & GT AT \\
\hline clatsop2 & $T C A$ & AT A AT TAT AT AC & C CT & CССATTGTTATT & G T AT \\
\hline clatsop3 & $\mathbf{T} \in \mathbf{A}$ & AT AATTATATAC & $C \in T$ & CCCATTGTTATT & G T AT \\
\hline clatsop4 & $T<A$ & AT AATTATATAC & $C \in T$ & CCCATTGTTATT & G T A T \\
\hline LostForest 1A & TCA & AT A A T T AT AT AC & $\mathbf{C C T}$ & CCCATTGTTATT & C T ATt \\
\hline LostForest2 & $T C A$ & A T A AT T AT AT AC & $c \subset T$ & CCCATTGTTATT & G TAT \\
\hline LostForest 3 & T C A & А T АAT T АТ АТ АC & $\mathbf{C C T}$ & СССАT TСT TAT & G T AT \\
\hline LostForest4 & $\mathbf{T C A}$ & A T A A T T AT AT AC & C C T & CCCATTGTTATT & G T A T \\
\hline sj1Pmausterus & $\mathbf{T} \subset \mathbf{A}$ & AT AAT T AT ATT AC & $\mathbf{C} \mathbf{c t}$ & CCCATTGTTATT & GT AT \\
\hline sj2Pmausterus & $\mathbf{T C A}$ & AT A ATT AT ATAC & $\mathbf{C C T}$ & CССATTGTTATT. & G T A T \\
\hline sj2aPmausterus & T. C A & AT AATTAT AT AC & $C \in T$ & CСCATTGTTATT & G T A:T \\
\hline sj3Pmausterus & TCA & AT AAT TATATAC & $c<T$ & CCEATTGTTATT & C T A $\mathbf{T}$ \\
\hline sjsPmausterus & $T C A$ & AT A ATT AT AT AC & $C \in T$ & C C CATTGTTATT & G Y A T T \\
\hline sj6Pmausterus & $\mathbf{T C A}$ & AT A AT T AT AT AC & $c \in T$ & CCCATTGTTATT & G T AT \\
\hline sj7Pmausterus & $\mathbf{T} \boldsymbol{C A}$ & AT A A T T A T AT AC & $c \in \mathrm{r}$ & CССATTGTTATT & G T ATT \\
\hline OSM723Pmman & $\mathbf{T} \mathbf{C A}$ & AT A.AT T AT AT AC & 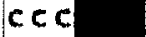 & CCCATTGTTATT & GT AT \\
\hline OSM726Pmman & $\mathbf{T C A}$ & ATAATTATATAC & ecc & CC СATTGTTATT & С T ATt \\
\hline OSM727Pmman & $\mathbf{T} \mathbf{C A}$ & AT AATTATATAC & $c<c$ & CCCATTGTTATT & G T A T \\
\hline OSM737Pmman & $\mathbf{T} \mathbf{C A}$ & AT A AT T А TAT AC & 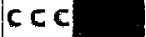 & CCCATTGTTATT & G T A T \\
\hline U40255PsejugSD & $\mathbf{T} \subset \mathbf{A}$ & AT AATTAT AT AC & cec & CECATTGTTATT & $G \backslash A T$ \\
\hline U40253PsejugsC & $\mathbf{T} \subset \mathbf{A}$ & A T AAT TAT AT GE & $\mathrm{ccc}$ & CСTATTCTTATT & G T A T \\
\hline JEB1309APtruei & $\mathbf{T} \subset \mathrm{A}$ & A T AA AT A T AT A C & $C \subset$ & CCCTATTGTTA & G T GT \\
\hline AF374578PKEEN & $\mathbf{T} \mathbf{C} \cdot \mathbf{A}$ & AT AATTAT ATGC & $\operatorname{ccc}$ & CCСATTGTTATT & G T AT \\
\hline U40252Pleuc & $\mathbf{T} \subset \mathbf{A}$ & AT A ATCATATGC & C C TAT G & CECATTGTTATC & G T AT \\
\hline JAM160Pleuc & T C:A & AT A ATCAT ATT AC & CETATC & CCCATTCTTA & C T A \\
\hline JAM161Pleuc & $\mathbf{T C A}$ & АТ А А T АT АT АC & CETATC & CCCATTGTTATC & G А A \\
\hline JAM162Pleuc & $\mathbf{T C A}$ & A T A A T С A AT A C & CCTATC & CCCATTGTTATC & G T A T \\
\hline JAM 163Pleuc & $\mathbf{T C A}$ & AT AAT CATATAC & C C TATC & ECCATTGTTATC & GTAT \\
\hline JAM164Pleuc & $\mathbf{T} C \mathbf{A}$ & AT AATCAT ATTAC & & CCCATTETTATC & C T A T \\
\hline JAM168Pmbairdii & $T \subset A$ & АT A ATTAT AT AC & CCT & CCCATTCTTATT & G TAT \\
\hline JAM167Pmbairdii & TCA & АT A ATTATATAC & $C \subset \mathrm{T}$ & CCCATTGTTATT & $G \mathrm{IA} \mathrm{T}$ \\
\hline JAM159Pmbairdil & T CA & АТ A AT T AT AT AC & CᄃT & CCCATTGTTATT & G T AT \\
\hline JAM166Pmbairdli & $\mathbf{T C A}$ & АТ A AT T AT ATT AC & $\mathbf{C C T}$ & CCCATTGTTATT & G T A T \\
\hline Microtuspennsylvanic & $T \subset A$ & AT A AT T AT AT TT & $\operatorname{cec}$ & CCTATCGTTATC & G T AT T \\
\hline Oryzomyspalustris & $T \in A$ & А T A A TCA СА Т A T & GTAA & CCAATTATTATA & G T ATT \\
\hline Sigmodonhispidus & $\mathbf{T} \subset \mathbf{C}$ & AT A A T С AT ATT AT & ICT & CCTATTGTTATT & G T A.T \\
\hline Reithrodontomysmeg & $\mathbf{T} \mathbf{C} \mathbf{A}$ & AT A AT TATATAT & ACAATT & CСАAT TATСATT & G T A T \\
\hline Rattusnorvegicus & $\mathbf{T} \mathbf{C C}$ & AT A A T CTCCATA & $A C E$ & CCLAATTACCATT & G T TT \\
\hline Pmrufinus & TCA & ATAATTATATGA & $\mathrm{CCT}$ & CCCATTGTTATC & GIAIT \\
\hline
\end{tabular}


WC7

WC11

WC12

WC13

jjd3256

ljd3304

ljd3306

ljd3308

ljd3317

yd3324

ijd3327

ljd 3330

ljd3333

Ijd3344

Ijd3348

LJ0620

LUD774

L50886

LJ02088

LJD1960

LJD1669

LDD1524

LJD1523

LJD1122

LJD2522

LJD1637

LJ01638

LJD2161

LJD2651

LJD2737

LJD2834

LJD2850

LJD2898

LD2914

LJD2936

LJD3000

LD2 126

LJD2138

LJD2154

LJD2158

LJD2836

LJD2852

LJD2860

LJD2865

LJD2890

LJD2943

Jefferson 3

ADAMS6

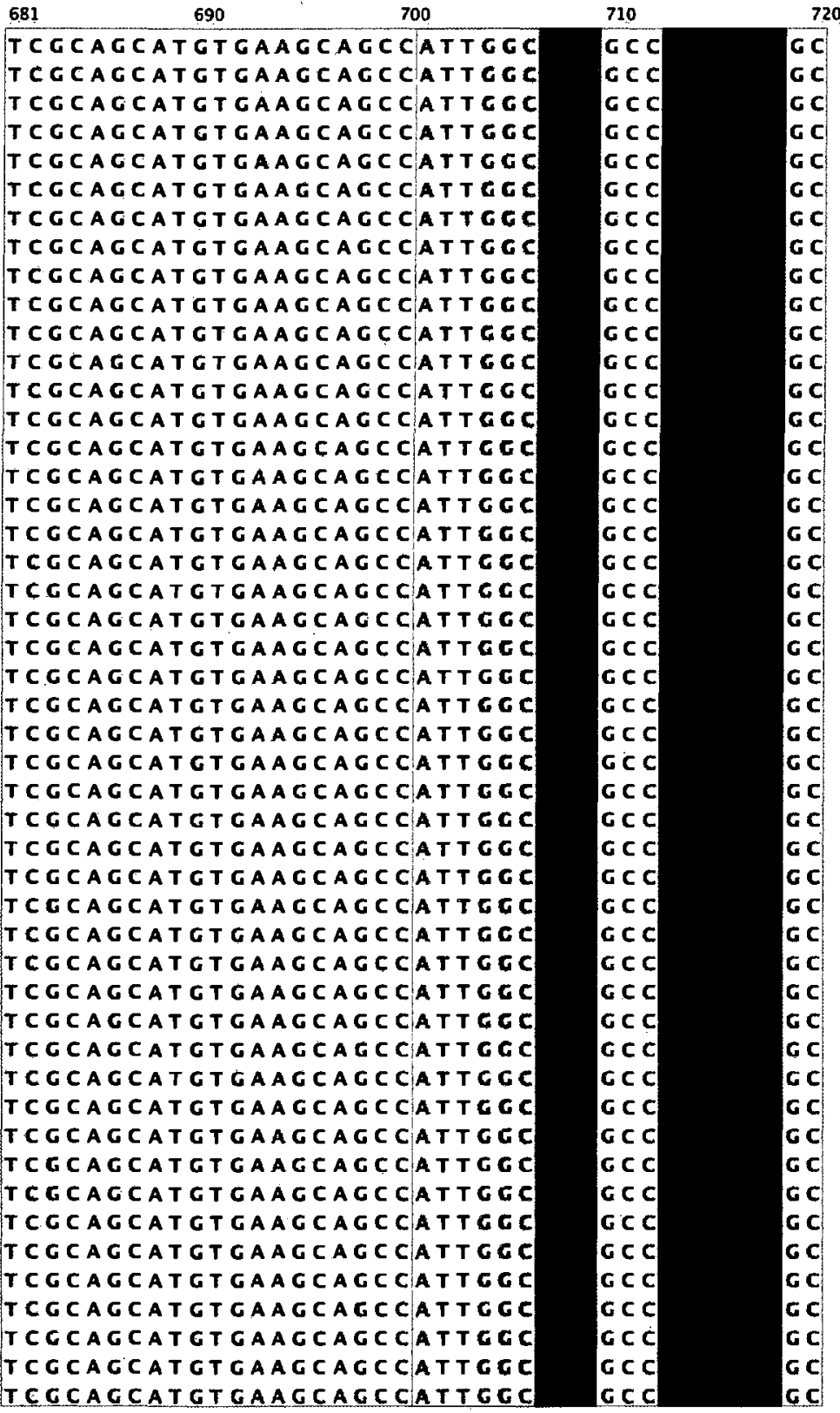




\begin{tabular}{|c|c|c|c|}
\hline & 690 & 710 & 72 \\
\hline clatsop 1 & TCGCAGCATGTGAAGCAGCCATTGGC & G C C & $\mathbf{6 c}$ \\
\hline clatsop2 & T CGCAG CATGT G A AG CAGCCAT TGGC & G C C & T T T G C \\
\hline clatsop3 & TCGCAGCATGTGAAGCAGCCATTGGC & G c c & T T T G C \\
\hline clatsop4 & TCGCAGCATGTGAAGCAGCCATTGGC & G C C & $\mathbf{T G C}$ \\
\hline LostForest 1A & TCGCAGCATGTGAAGCAGCCATTGGC & G C C & - \\
\hline LostForest2 & TCGCAGCATGTGAAGCAGCCATTGGC & G C C & G C \\
\hline LostForest 3 & TCGCAGCATGTGAAGCAGCCATTGCC & G C C & G c \\
\hline LostForest 4 & TCGCAGCATGTGAAGCAGCCATTGGC & G C C & c c \\
\hline sj1Pmausterus & TCGCAGCATGTGAAGCAGCCATTGGC & G C C & G C \\
\hline sj2Pmausterus & TCGCAGCATGTGAAGCAGCCATTGGC & $\mathbf{G C C}$ & GC \\
\hline sj2aPmausterus & TCGCAGCATGTGAAGCAGCCATTGGC & $\mathbf{G C C}$ & $\mathbf{G C}$ \\
\hline sj3Pmausterus & TCGCAGCATGTGAAGCAGCCATTGGC & c C C & $\mathbf{G C}$ \\
\hline sj5Pmausterus & TCGCAGCATGTGAAGCAGCCAT TGGC & $\mathbf{G C C}$ & $\mathbf{G C}$ \\
\hline sj6Pmausterus & TCGCAGCATGTGAAGCAGCCATTGGC & G C C & G C \\
\hline sj7Pmausterus & TCGCAGCATGTGAAGCAGCCAT TGGC & G C C & $\mathbf{G C}$ \\
\hline OSM723Pmman & TCGCAGCATGTGAAGCAGCTATTGGC & G C C & G C \\
\hline OSM726Pmman & TCGCAGCATGTGAAGCAGCTATTGGC & c c c & $\mathbf{c c}$ \\
\hline OSM727Pmman & TCGCAGCATGTGAAGCAGCTATTGGC & GC C & G C \\
\hline OSM737Pmman & TCGCAGCATGTGAAGCAGCTATTGGC & $\mathbf{G C C}$ & $\mathbf{G C}$ \\
\hline U40255PsejugSD & TCGCAGCATGTGAAGCAGCTATTGGC & GC C & $\mathbf{6} \mathbf{c}$ \\
\hline U40253PsejugsC & TCGCAGCATGTGAAGCAGCTATTGGC & c c c & C c \\
\hline JEB1309APtruei & TTGCAGCATGCGAAGCGGCCATTGGC & $\mathbf{G C T}$ & G C \\
\hline AF374578PKEEN & TCGCACCATG TGAAGCAGCCATTGGC & $\mathbf{G C C}$ & G c \\
\hline U40252Pleuc & TCGCACCATGTGAAGCAGCTATTGGC & $\operatorname{ccc}$ & $\mathbf{G C}$ \\
\hline JAM160Pleuc & TCGCACCATGTGAAGCAGCCATTGGC & G C C & $\mathbf{e c}$ \\
\hline JAM161Pleuc & TCGCAGCATG TGAAGCAGCCAT TGGC & G C C & GC \\
\hline JAM162Pleuc & TCGCAGCATGTGAAGCAGCCATTGGC & GCC & $6 \mathrm{C}$ \\
\hline JAM163Pleuc & TCGCACCATGTGAAGCAGCCATTGGC & G C C & G C \\
\hline JAM164Pleuc & TCGCAGCATGTGAAGCAGCCATTGGC & C C C & G C \\
\hline JAM 168Pmbairdii & TCGCAGCATGTGAAGCAGCCATTGGC & G C C & $\mathbf{G} \mathbf{C}$ \\
\hline JAM 167Pmbairdii & TCGCAGCATGTGAAGCAGCCATTGGC & C C C & $\mathbf{G} \mathbf{c}$ \\
\hline JAM 159Pmbairdli & TCGCAGCATGTGAAGCAGCCATTGGC & G C C & $\mathbf{G ~ C}$ \\
\hline JAM166Pmbairdil & TCGCAGCATGTGAAGCAGCCATTGGC & c C c & G C \\
\hline Microtuspennsylvanic & TCGCTGCCTGTGAAGCAGCCGTTGGA & GCC & G C \\
\hline Oryzomyspalustris & TTGCAGCATGTGAAGCAGCCATTGCC & $\mathbf{C ~ C ~ T}$ & G T \\
\hline Sigmodonhispidus & TTCCAGCATGTGAAGCAGCTATTGGT & C C T & G C \\
\hline Reithrodontomysmeg & TTGCAGCATGTGAAGCAGCTATTGGT & G C T & $\mathbf{G} \mathbf{C}$ \\
\hline Rattusnorvegicus & TTCCACCCTGCGAAGCACCACTACCT & G C C & $\mathbf{C} \mathbf{T}$ \\
\hline Pmrufinus & TCGCAGCATGTGAAGCAGCCATTGGC & $\mathbf{G C C}$ & $\mathbf{G C}$ \\
\hline
\end{tabular}


WC7

WC11

WC12

WC13

IJd3256

IJd3304

Ijd3306

jjd3308

ljd3317

IJd3324

Ijd3327

ljd3330

jjd3333

ljd3344

Ijd 3348

LjD620

L50774

LJD8B6

LJD2088

Lل1960

LلD1669

LJD1524

LJD1523

LJDi122

LJD2522

LJD1637

LDD1638

LJD2161

L02651

LJD2737

LJ02834

LJD2850

LJD2898

LD2914

LJD2936

LJD3000

LJD2126

LJD2138

LJD2154

.JD2158

LJD2836

LDD2852

LJD2860

LJD2865

LJD2890

LJ02943

Jefferson 3

ADAMS6
A21 $\frac{730}{740}$ TCAAACTCCTACGGAACATATTATGTGCAAAAT AAAA T.EAAAETCETACOGAACAGATTATCTCEAAAAT A A A A A A A A.A A A)A A.A A A AIA A A A A A A A.A A A A.A A A A A A A A A A A A A A A A A A A A A A A A A A A A A A A A A A A A.A.A A A A A A A A A A A A A A A A A A A A A A A A A A A $A$ A A A A A.AA A A A A $A$ A A A A A A A A A A A A A A A A A A A A A A A A A A A A A A A A A A A A A A A A A A A A A A A A.AA A A A A A A A A TICAAATCCTACGGAACAGATTATGTGCAAAA TCAAACTC CTACGGAACAGATTATGTGCAAAAT TCAAACTC GTACGGAACAGATTATGTGCAAAAC TEAAACTCCTACGGAACACATTATGTGCAAAAT TEAAACTCCTACGGAACAGATTATGTGCAAAAT TCAAACTCCTACGGAACAGATTATGTGCAAAAT TCAAACTCTACGGAACAGATTATGTGCAAAAT TCAAACTC CTACGGAACAGATTATGTGCAAAAT TCAAACTCCTACGGAACAGATTATGTGCAAAAT TCAAACTECTACGGAACAGATTATGTGCAMAAT TCAAACTCCTACGGAACAGATTATGTGCAAAAT TCAAACTCCTACGGAAEAGATTATGTGCAAAAT TCAAACTCCTACOCAACACATTATCTACAAAAT TCAAACTCCTACGGAACACATTATGTGCAAAAT TCAACTCCTACGGAACACATTATGTGCAAAAT TCAAACTCCTACGGAACAGATTATGTGCAAAAT TCAAACTCCTACGCAACAGACTATGTACAAAAC TCAAACTCETACIGAACAGATTATGTCICAAAAT TCAAACTCCTACGGAACAGATTATETGCAAAAT TCAAACTCCTACGGAACAGATTATGTGCAAAAT TEAAACTCCTACGGAACAGATTATGTGCAAAAT TCAAACTCCTACGGAACACATTATCTGCAAAAT TCAAACTCCTACGGAACAGATTATGTGCAAAAC TCAAACACETTACGGAACAGATTATGTGCAAAAT TCAAACTCCTACGGAACAGATTATGTGCAAAAT TCAAACTCCTACGGAACAGATTATGTGCAAAAT TCAAACTC CITACGGAACAGATTATGTGCAAAAT TCAAACTCCTACGGAACAGATTATGTGCAAAAT TCAAACTCCTACGGAACAGATTATGTGCAAAAT TCAAACTCCTACGGAACACATTATGTGCAAAAT TCAAACT CCTACGGAACACATTATGTCCAAAAT TEAAACTCCTACGGAACAGATTATGTGCAAAAT TCAAACTCCTACCGAACAGATTATGTCCAAAAT TCAAACTCCTACGGAACACATTATGTGCAAAAT TCAAACTCCTACCGAACACATTATGTCCAAAAT TCAAACTCCTACGGAACAGATTATGTGCAAAAT TCAAACTCCTACGGAACAGATTATGTGCAAAAT TCAAACTCCTACGGAACAGATTATGTGCAAAAT TCAAACTCCTACIGGAACAGATTATGTGCAAAAT TEAAACTCCTACGGAACAGATTATGTGCAAAAT TCAAACTCCTTCGGAACACATTATGTGCAAAAT TCAAACTCCTACIGGACAGATTATGTGCAAAAT TCAAACTCCTACICGAACACATTATG TGCAAAAT TEAAACTCCTACGGAACAGATTATGTGCAAAAT TEAAACTCCTACGGAACAGATTATGTGCAAAAT TEAAACTCCITACGGAACAGATTATGTGCAAAAT 
clatsop 1

clatsop2

clatsop 3

clatsop4

Lostforest1A

LostForest2

LostForest3

LostForest4

sj1Pmausterus

sj2Pmausterus

sj2aPmausterus

sj3Pmausterus

sj5Pmausterus

sj6Pmausterus

sj7Pmausterius

OSM723Pmman

OSM726Pmman

OSM727Pmman

OSM737Pmman

U40255PsejugsD

U40253PsejugSC

jEB1309APtruei

AF374578PKEEN

U40252Pleur

JAM160Pleuc

JAM161Pleue

JAM162Pleuc

JAM163Pleuc

JAM164Pleuc

JAM 168Pmbairdii

JAM167Pmbairdit

jAM159PmbairdI JAM166Pmbatridi

Microtuspennsylvanic Oryzomyspalustris Sigmodonhispidus Reithrodontomysmeg Rattusnorvegicus

Pmrufinus

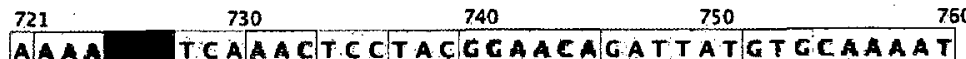

AAAA TCAAACTCCTACGGAACAGATTATGTGCAAAAT

AAAA TCAAACTCCTAGGGAACAGATTATGTGCAAAAT

$\ldots \ldots \ldots$

AAAA TCAAACTCCTACGGAACAGATTATGTGGAAAAT

AAAA TCAAACTCETACEGAACACATTATGTGCAAAAT

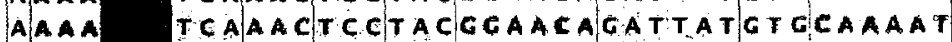

AAAA TEAAACTECTACOGAAEAGATTATGTGCAAAAT

AAAA TCAAAETCCTACGGAACAGATTATGTACAAAAT

AAAA TCAAACTCGTACGGAACAGATTATGTGCAAAAT

AAAA TCGAACTCETACGGAALACTTATGTCCAAAAT

AaAA TCGAACTCETACGCAACAGATTATGTGCAAAAT

ALAA TCGAACTCGTACGGAACAGATTATGTGEAAAAT

AAA TEGAACTCETACGGAACAGATTATGTGCAAAAT

AAAA TEAAACTCETACGGAACAGATTATGTGCAAAAT

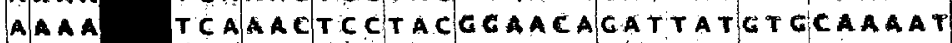

AAAA TCAAACTCCTACGGAACAGATTATGTCEAAAAT

AaA TCAAACTCCTACGgAACAGATTATGTGCAAAAT A A AGTATEAAACTCCTACGGAACACATTATGTGCAAAAC AAAAGTATCAACTCCTACGGAACACATTATGTGCAAAAC A.A A A

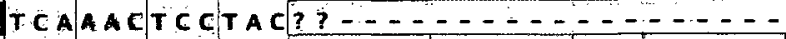

A.AAGTATCAAACTCCTACGGAACAGATTATGTACAAAAT A AAGTATCAAATTCATATGGAACACACTATGTACAAAAC AAAA TCAAACTCCTACGGAACAGATTATGTGCAAAAC

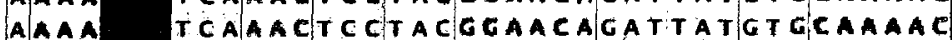
AAAA TCAAACTCCTACGGAACAGATTATGTGCAAAAC A A A

A A A A

A A A A

A A A

A A A

A A A A

TCAMACTC CTA CGGAACAGATTATGT GCAAAAC TCAAACTCETACGGAACAGATTATGTGCAAAAC TCAAACTCCTACICCAACAGATTATGTGCAAAAC TCAAACTCCTACGGAACAGATTATGTGCAAAAC TCAAACTCCTACGGAACACATTATGTGCAAAAC TCAAACTCCTACGCAACACATTATCTGCAAAAC AAAAATCT GAAACACTTATGCGTCTGATTTCGTCCATAAT TATCATTTCTAATACTTACTGCACAGAGTATGTCLAAAAT TATAATTTCCAGCACATACGGAACTGACTACGTTCAAAAC gAAAGTATCAAATTCATACGGCACACACTATGTACAAAAC AAAAATTTCAAATACTTACGGAACAGACTACGTACAAAAC AAAAGTATEAAACICCTACGGAAEAGATTATGTACAAAAT 
WC7

WC11

WC12

WC13

IJd3256

Ijd3304

1jd3306

ljd3308

ljd3317

Ijd 3324

ljd3327

ljd 3330

ljd3333

IJd3344

ljd3348

LD 20

LJD774

LJD886

LJD2088

LJD1960

LJD1669

LJD1524

LJD1523

UD1122

LDD2522

LJD1637

LJD1638

UDD2161

LJD265 1

LJD2737

LD22834

LJD2850

UD2898

LJD2914

LJD2936

LJD3000

LJD2126.

LJD2138

LJD2 154

LJD215B

UD2836

LJD2852

LD2860

LJD2865

LjD2890

UD2943

Jefferson 3

ADAMS6

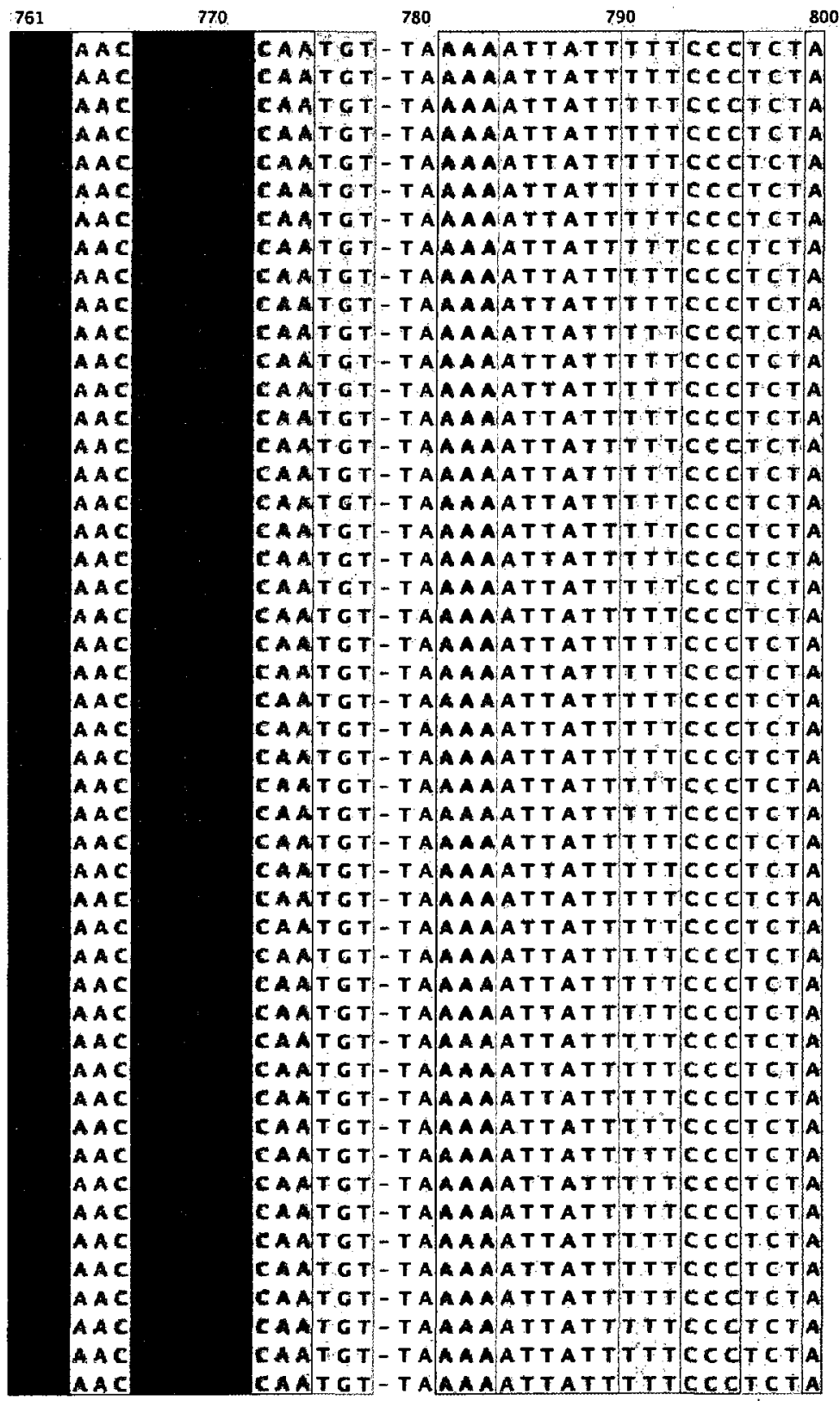




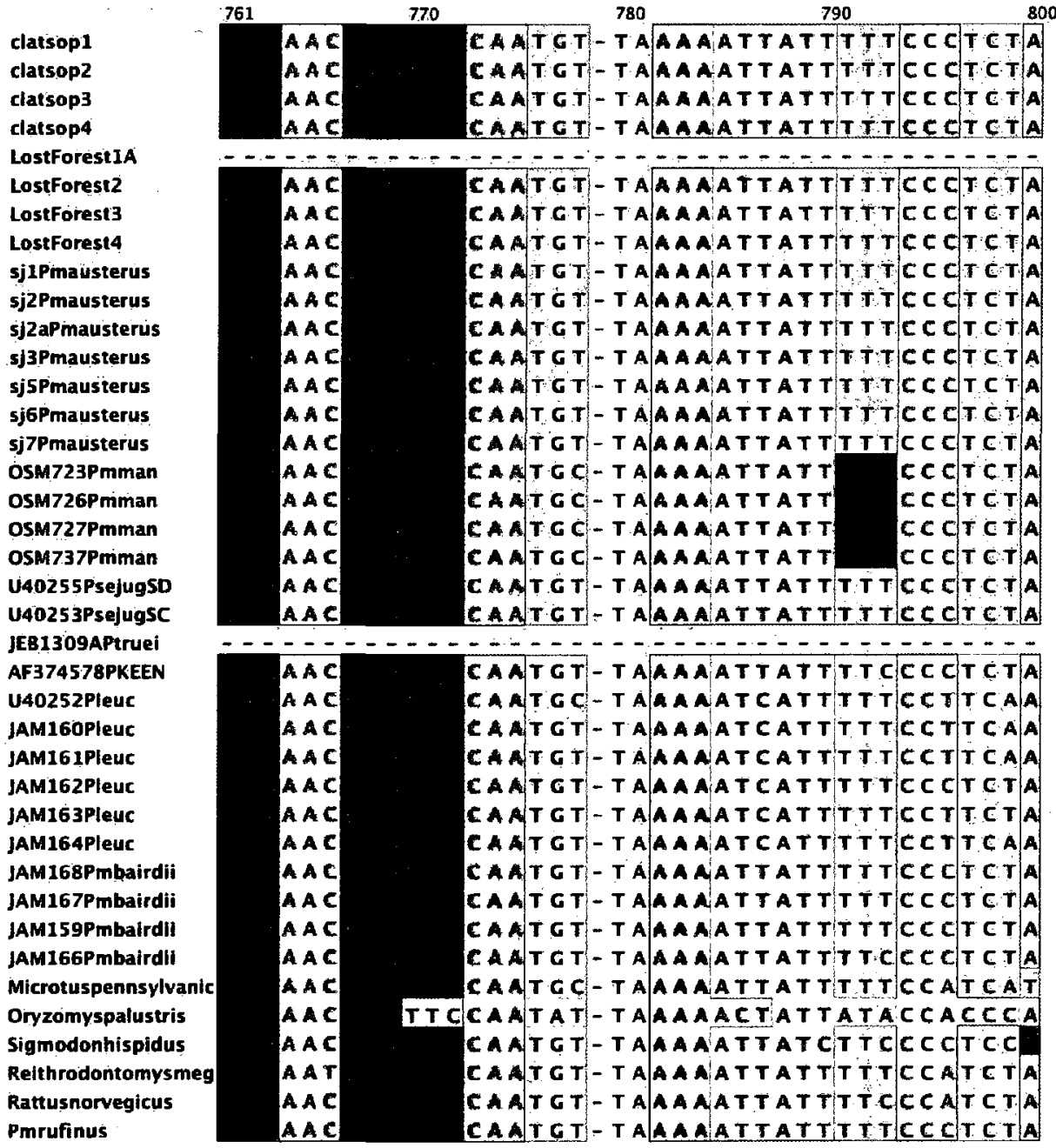


WC7

WC11

WC12

WC13

Ijd3256

Ijd3304

Ijd3306

ljd3308

Ijd3317

IJd3324

Ijd3327

ljd3330

ljd 3333

ljd3344

Ijd 3348

LJD620

LUD774

LJD886

LJD208B

LJD1960

LJD1669

LJD1524

LJD1523

LJD1122

LJD2522

LJD1637

LJD1638

LJD2161

LJD2651

LJD2737

LJD2834

LJD2850

LJD2898

LJD2914

LJD2936

UD3000

LJD2126

LJD2138

LJD2154

LJD2158

LJD2836

LJD2852

LJD2860

LDD2865

LJD2890

UJD2943

Jefferson 3

ADAMSE

\begin{tabular}{ll} 
& \\
\hline &
\end{tabular}




\begin{tabular}{|c|c|c|c|c|}
\hline & 801 & 810 & 820 & 830 \\
\hline clatsopi & TC:A A & CCA & $A C C T G A$ & ICAAACAAAAAAAA \\
\hline clatsop2 & T C A T A & $C \subset A$ & $A C E T G A$ & TCAAACAAAAAAAA \\
\hline clatsop 3 & T.CA T A & C C A & $A \subset \in T G A$ & TCAAACAAAAAAAA \\
\hline clatsop4 & TCATA & CeA & $A<\mathrm{CT} \mathbf{T A}$ & I CAAACAAAAAAAA \\
\hline LostForest1A & $=-$. &.--- &.---- & $-=--1-+-1-\cdots-1-$ \\
\hline LostFonest2 & TCAT & CCA & $A C E T G A$ & TCA AACAAAAAAAA \\
\hline LostForest3 & TCAT & $E \subset A$ & $\operatorname{ACCTGA}$ & TCAAACAAAAAAAA \\
\hline LostForest 4 & TCAT & CCA & $A \in E T A$ & TCAAACAAAAAAAA \\
\hline sj1Pmausterus & TCA & $C \subset A$ & $A C C T G A$ & TCAAACAAAAAAAA \\
\hline sj2Pmausterus & TCA & C CA & $A C E T G A$ & TCAAACAAAAAAAA \\
\hline sj2aPmausterus & TCA T & C C A & $\operatorname{AccTGA}$ & TCAAACAAA A A A A A \\
\hline sj3Pmausterus & $T \subset A T$ & $C \subset A$ & $A \subset \mathbb{Q} \mathrm{T}$ & TCA AACAAAAAAAA \\
\hline sj5Pmausterus & $T \subset A T$ & $C \subset A$ & $A \subset C \backslash G A$ & TCAAACAAAAAAAA \\
\hline sj6Pmausterus & TCAT & $C \subset A$ & $A C E T G A$ & TCA|AACAAAAAAAA \\
\hline sj7Pmausterus & TCAT & $C \subset A$ & $A \in C \rightarrow G A$ & TCAAACAAAAAAAA \\
\hline OSM723Pmman & T CA & & $A \in C T G$ & TCAAACAAAAAAAA \\
\hline OSM726Pmman & T $\subset A .7$ & $c \in A$ & $\operatorname{AcCT} \mathrm{TA}$ & TCA $A$ ACAAA AAAAA \\
\hline OSM727Pmman & T CA T & Cc & $A \subset C T G A$ & TCAAACAAAAAAAA \\
\hline OSM737Pmmàn & $T \in A T$ & C C A & $A \subset C T C A$ & TCAAACAAAAAAAA \\
\hline U40255PsejugSD & $\mathbf{T C A}$ & & $A \in C T G A$ & TCAAACAAAAAAAA \\
\hline U40253PsejugSC. & ICATA & ECA & $A \in C T G A$ & TCA|AACAAAAAAAA \\
\hline JEB1 309APtruei & $\ldots-\cdots$ & $\therefore-$ & $--\cdot-$ & $-\cdots--\cdots---_{-1}$ \\
\hline AF374578PKEEN & $T \subset A$ & $C \subset A$ & $A \subset C \backslash G A$ & TCAAACAAAMAAAA \\
\hline U4025 2Pleuc & T T A T & ect & $\operatorname{acc} \in \mathrm{G}$ & TCA A C A ACAAAAG \\
\hline JAM160PleuC & TCATA & $\operatorname{coc}$ & $A \subset \in T G A$ & TCAAACAACAAAAC \\
\hline JAM161PleuC & $T \subset A T$ & C C G & $A C C T G A$ & TCAAAEAACAAAAG \\
\hline JAM 162Pleiuc & TСATA & $c \in G$ & $A \subset C T G A$ & TCA AACAACAAAAG \\
\hline JAM163PleuC & T C A T A & $c<\mathrm{C}$ & $A \subset \subset G A$ & TCA A A C A A C'AA AAC \\
\hline JAM 164Pleuc & TCATA & $\operatorname{ccs}$ & $A \in C T G A$ & TEAAACAACAAAAC \\
\hline JAM168Pmbairdii & TCAT & $C \subset A$ & $A \in C \backslash G \mathrm{~A}$ & TCAAACAAAAAAAA \\
\hline JAM 167Pmbairdii & TCAT & C CA & $A \subset C T C A$ & TCA|AACAAA AAAAA \\
\hline JAM 159Pmbairdil & TCATA & $C \subset A$ & $A \in C T G A$ & TCAAACIAAAAAAAA] \\
\hline JAM 166Pmbairdil & $\mathbf{T} \subset \mathbf{A T}$ & & $A C C T G A$ & T CAAACAAAAA A AA \\
\hline Microtuspennsylvanic & I TAT & & ACCT GA & TCAACCAATIAAAAA \\
\hline Oryzomyspalustris & $T r A$ & & $A C T \mid T G A$ & TCCAACAACAAAAA \\
\hline Sigmodonhispidus & T T & $\operatorname{ccc}$ & $A \subset G T G A$ & TCTAATAACAAAAA \\
\hline Relthrodontomysmeg & T TA T A & & $A \in C T G A$ & TEAAACAATAAAAA \\
\hline Rattusnorvegicus & TCATA & & ACA $T$ G A & TCAICCC AACAAAAA \\
\hline Pmrufinus & ICATA & CCA & $A \subset C) G A$ & $I C A \triangle A C \wedge A \wedge \wedge \wedge \wedge A A$ \\
\hline
\end{tabular}


WC7

WC11

WC12

WC 13

jjd3256

Ijd3304

jjd3306

jjd3308

Ijd3317

ljd 3324

ijd3327

ljd3330

jjd3333

Ijd3344

ljd3348

LD620

UD774

LD886

LJD2088

LJD1960

LJD1669

LJD1524

LJD1523

LJD1122

LJD2522

LJD1637

LJD1638

LJD2 161

LD265 1

L.JD2737

LJD2834

LJD2850

LJD2898

LJD2914

LJD2936

LJD3000

LJD2126

LJD2138

LJD2154

LJD2158

LJD2836

LJD2852

LJD2860

LJD2865

LJD2890

LJD2943

Jefferson 3

ADAMS6

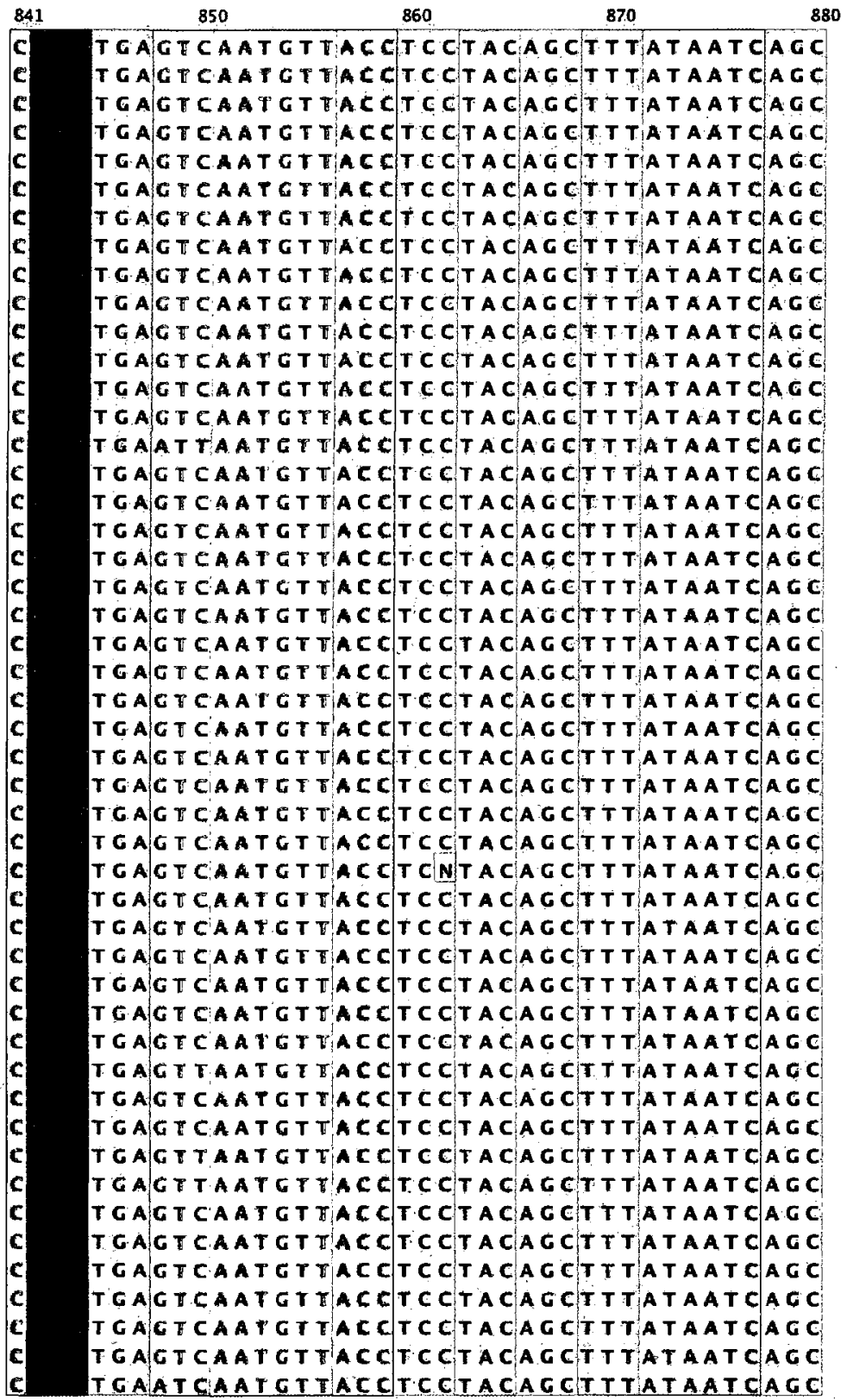




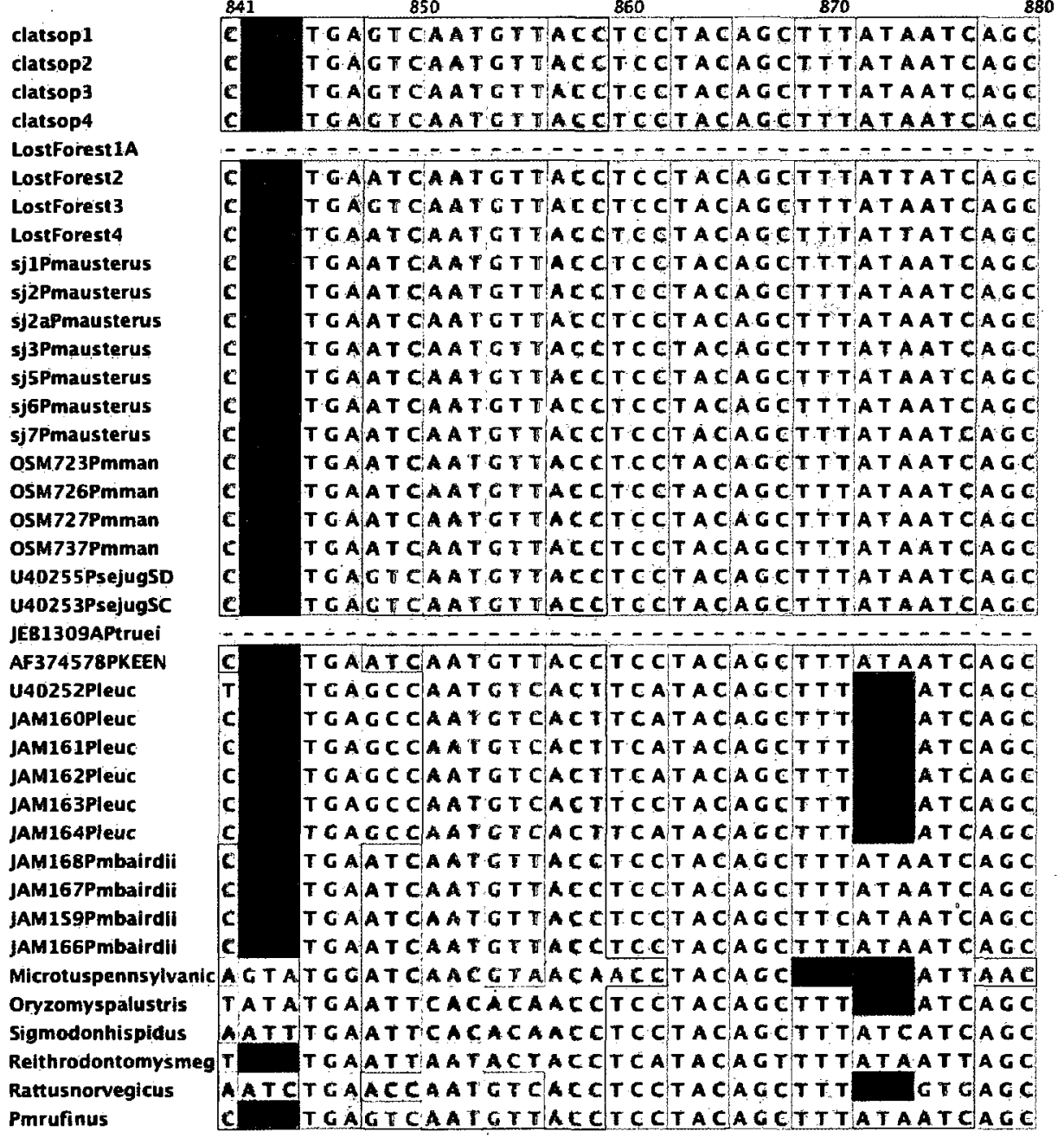


WC7

WC11

WC12

WC13

Ijd3256

ijd 3304

IJd3306

ljd3308

(jd3317

IJd3324

Ijd3327

ljd3330

ljd3333

IJd3344

IJd3348

LلD620

LJD774

LJD886

WD2088

LJD1960

LD1669

LDD 1524

LJD1523

LJD1122

LلJD2522

LJD1637

LDD1638

LJD2 161

LJD2651

LJD2737

LJD2834

LJD2850

LJD2898

LDD2914

LJD2936

LJD3000

LJD2126

LJD2138

LDD2154

LJD2158

LJD2836

LJD2852

LJD2860

LJD2865

LJD2890

LJD2943

Jefferson 3

ADAMS6

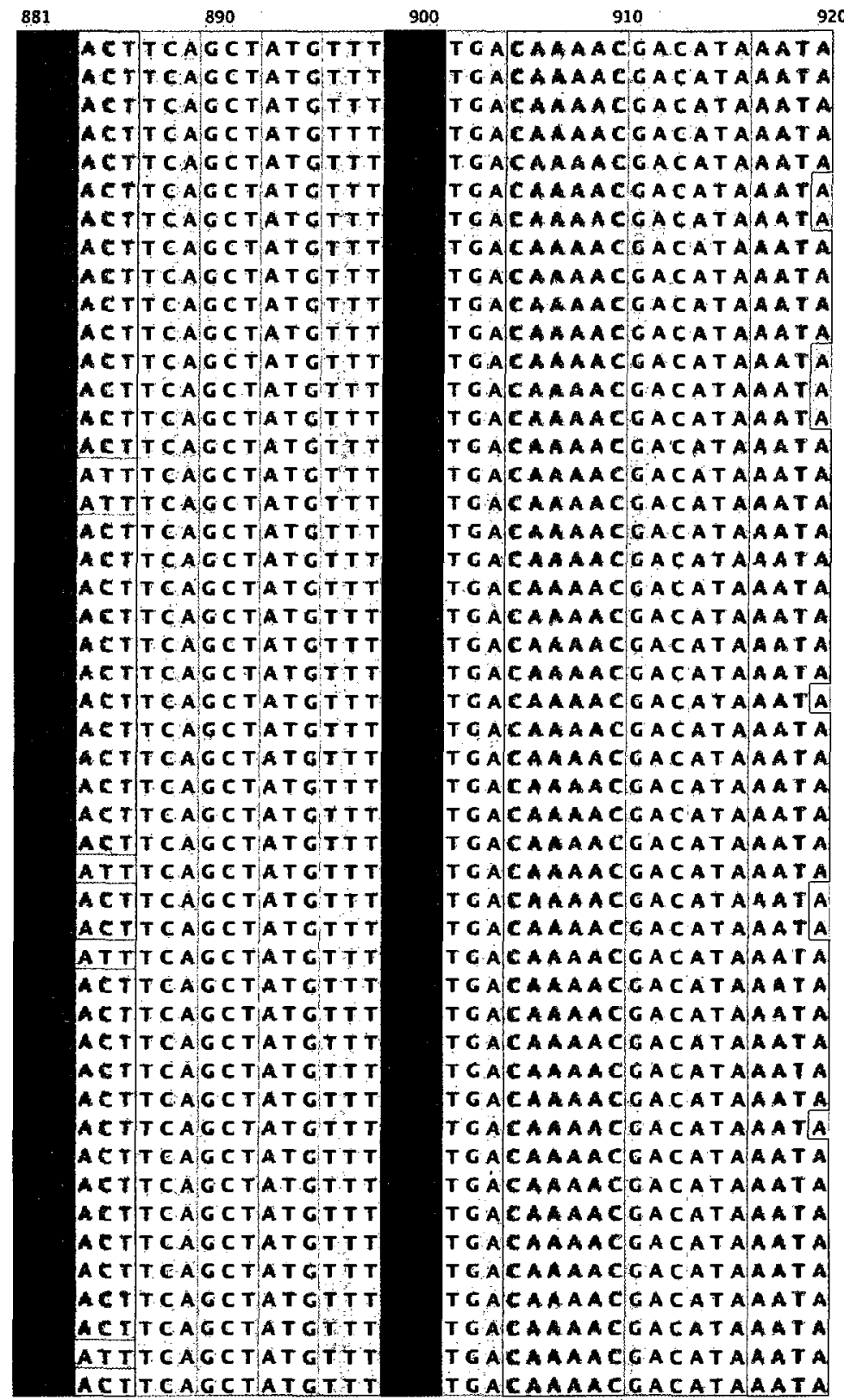




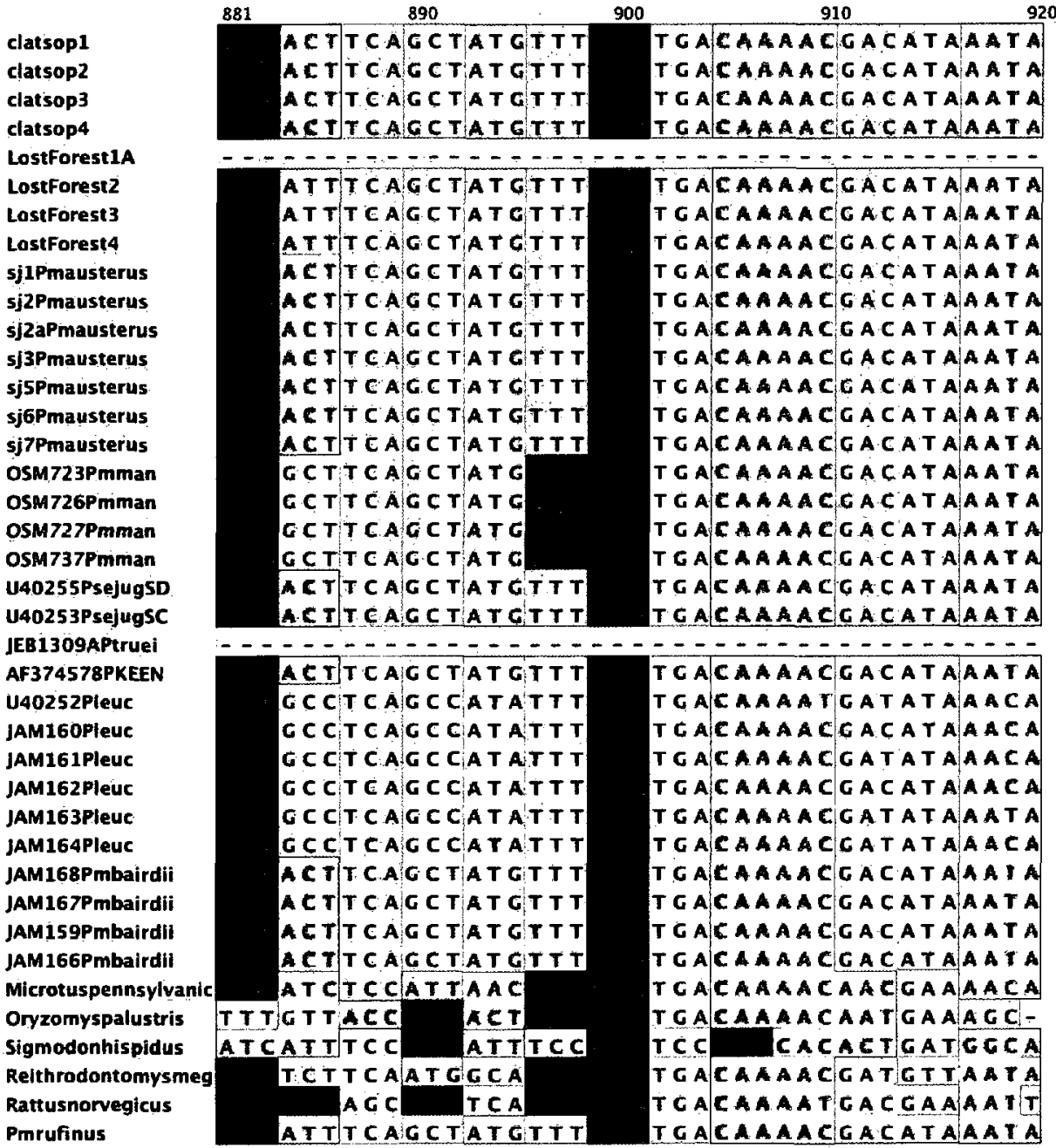


we7

WC11

WC12

WC13

Jjd3256

Ifd3304

Jjd3306

lid3308

ljd 3317

Ijd3324

ljd 3327

ljd 3330

ljd 3333

ljd 3344

Ijd3348

LJD620

LJD774

LJD886

LJD2088

LJD1960

LUD1669

LJD1524

UJD1523

LJD1 122

LJD2522

LJD1637

LJD1638

LJD2161

LjD265 1

UJD2737

니D2834

LJD2850

LJD2898

LJD2914

LJD2936

LJD3000

LJD2126

LJD2 138

LJD2 154

LJD2158

UD2836

LJD2852

UJD2860

LJD2865

LJD2890

LJD2943

Jefferson 3

ADAMS6

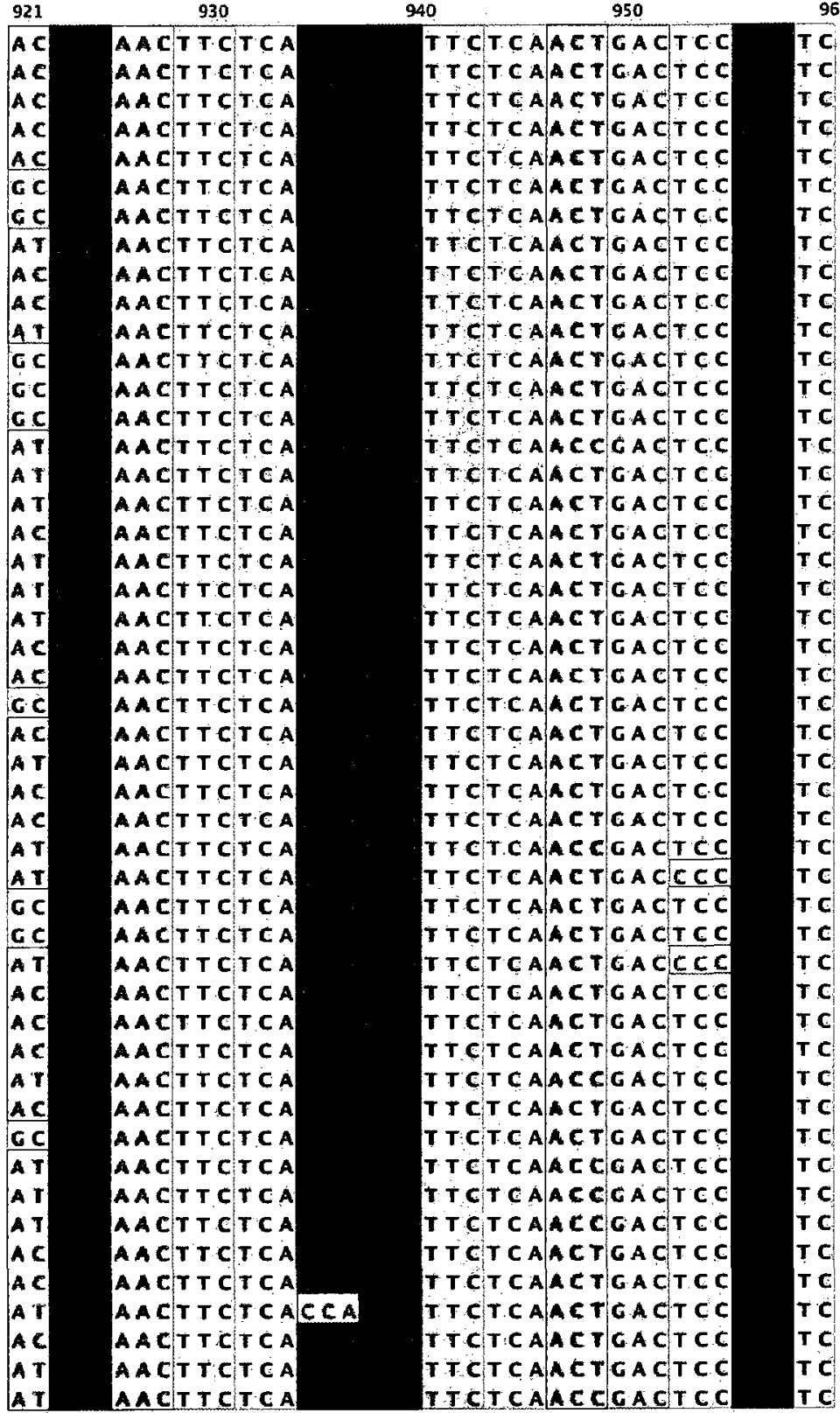




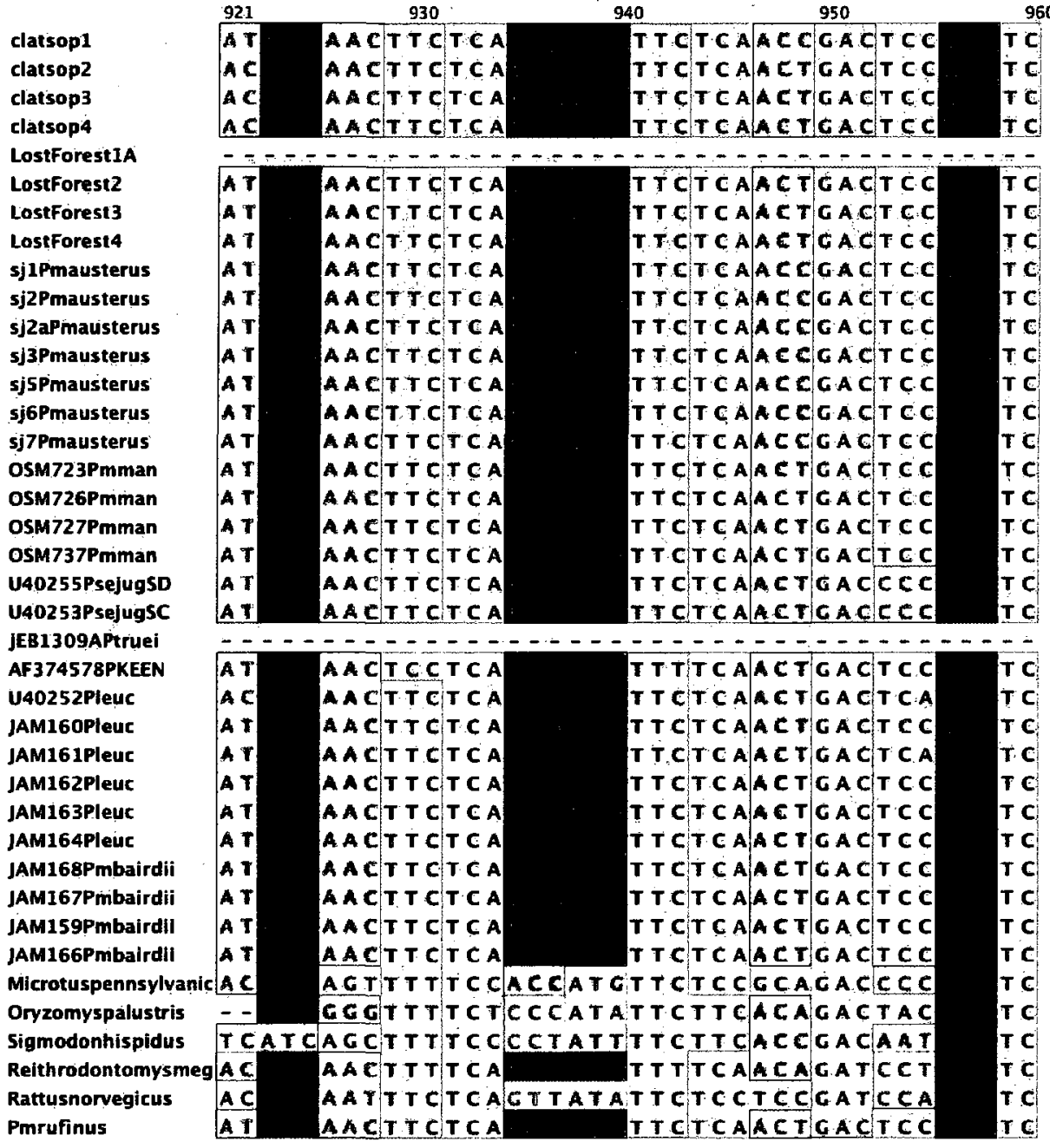


WC7

weI1

WC12

WC13

Jjd 3256

IJd3304

Jjd3306

ljd3308

Ijd3317

Ijd3324

Jd 3327

ljd 3330

ljd3333

Jjd 3344

Jjd3348

WD620

UDD774

UD886

LJD2088

LJDi960

LJD1669

LJD1524

LJD1 523

LJD1 122

LJD2522

LJD1637

LJD1638

LJD2 161

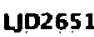

LلJ2737

LJD2834

LJD2850

LJD2898

UD2914

402936

403000

LJD2126

LJD2138

LJD2154

LJD2 1.58

LJD2836

LJD2852

LJD2860

LJD2865

LJD2890

LJD2943

Jefferson 3

ADAMS6

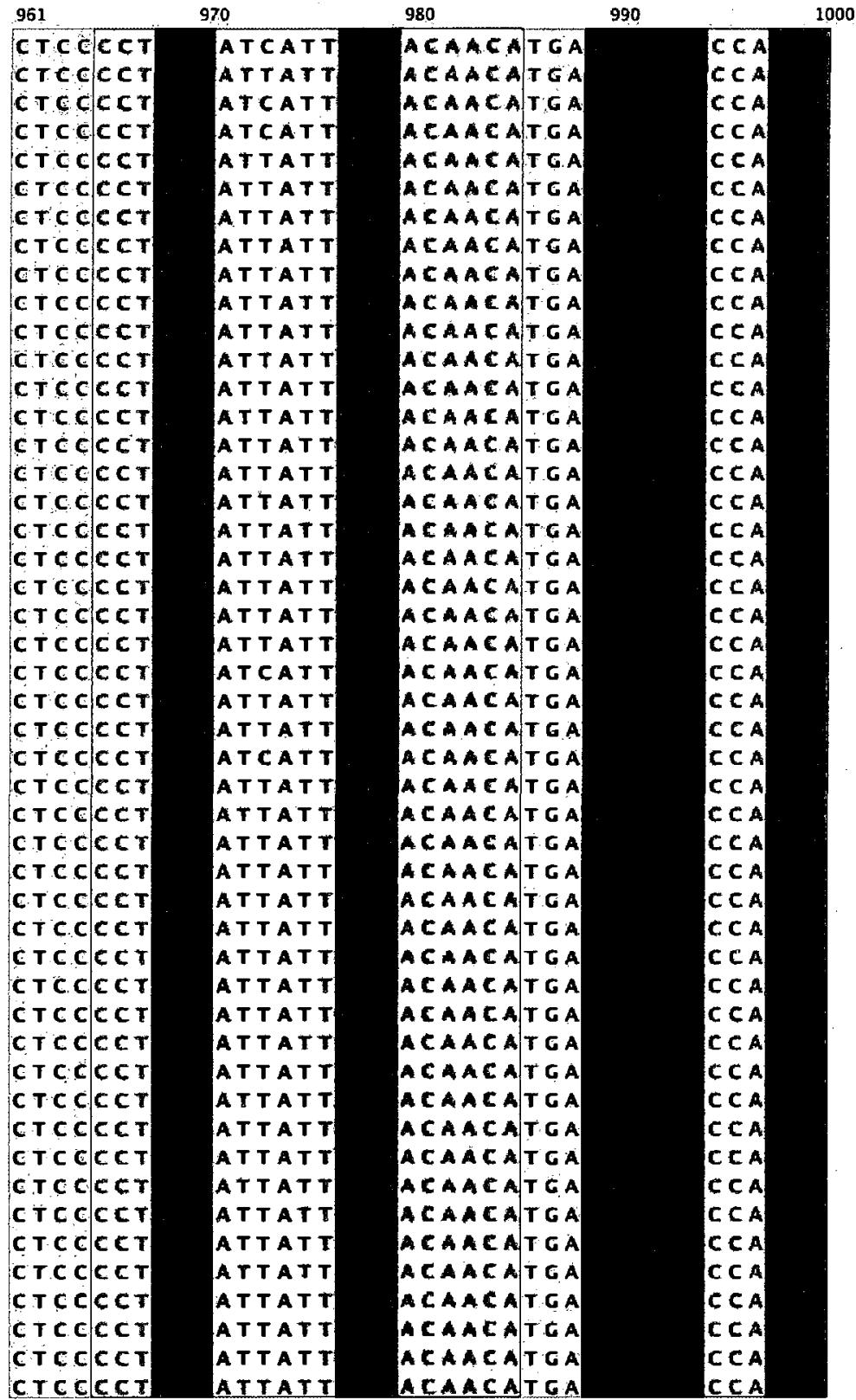




\begin{tabular}{|c|c|c|c|c|c|}
\hline & 961 & & 70 & 980 & \\
\hline clatsop 1 & CrCc & $\operatorname{CCT}$ & ATT AT T & $A C A A C A T G A$ & $C \subset A$ \\
\hline clatsop2 & $C T C C$ & C C T & ATTATT & ACA & $C \subset A$ \\
\hline clatsop3 & $c \mathbf{C C}$ & CCT & ATT ATT & ACAACATGA & C C A \\
\hline clatsop4 & CTCE & C C T & ATTATT & $A \in A \wedge \subset A T G A$ & $\mathrm{CCA}$ \\
\hline LostForest 1A & ---- & $\therefore--$ & ------ & $-1-------=-$ & -- \\
\hline LoștForest2 & C T C C & $C \mathbf{C T}$ & ATTATT & AEAACATGA & $C \subset A$ \\
\hline LostForest3 & C TCE & CET & ATTATT & $\operatorname{ACA} A \subset A T G A$ & $C \in A$ \\
\hline LostForest4 & CTC C & $C \mathrm{CT}$ & AT T ATT & ACAACATGA & c C A \\
\hline sj1Pmausterus & C T C C & $C \subset T$ & ATT ATT & $A E A A C A T G A$ & $C \subset A$ \\
\hline sj2Pmausterus & CTCC & $C \mathrm{CT}$ & ATT AT T & ACA ACATGA & $C \subset A$ \\
\hline s\}2aPmausterus & C TCC & $c<T$ & ATT ATT & ACAACATGA & $C \subset A$ \\
\hline sj3Pmausterus & T T C & 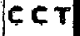 & ATT ATT & $A \subset A A C A T G A$ & $c \in A$ \\
\hline sjSPmausterus & C $\mathbf{T C C}$ & $C<T$ & ATTATT & $A \subset A A C A T G A$ & $\mid c \in A$ \\
\hline sj6Pmausterus & $C \mathrm{TCC}$ & $C<T$ & ATT ATT & $A C A A C A T G A$ & C C A \\
\hline sj7Pmausterus & CT C E & $c \in T$ & ATT ATT & $A \subset A A C A T C A$ & $C \subset A$ \\
\hline OSM723Pmman & C T $\mathbf{C} \mathbf{C}$ & $C \mathrm{CT}$ & АТT АT T & $A \subset A A \subset A T G A$ & $C \subset A$ \\
\hline OSM726Pmman & C T C & CCt & АTTATT & $A \subset A A C A T C A$ & c c $A$ \\
\hline OSM7.27Pmman & $\mathrm{C} \mathrm{T} \mathbf{C}$ & $\mid c t$ & ATT ATT & $A \in A A C A T G A$ & $C \subset A$ \\
\hline OSM737.Pmiman & C T C C & $c<\pi$ & ATT TTT & ACA ACATT GA & $C \subset A$ \\
\hline U40255PsejugSD & $c \mathbf{C} \mathbf{C}$ & C C T & ATT ATT & ACA ACA T GA & $C \subset A$ \\
\hline U40253PsejugSC & CTCC & $C<T$ & ATTATT & $A \subset A A C A T G A$ & $C \subset A$ \\
\hline JEB1309APtruei & $-\cdots$ & $-\cdots$ & $-\cdots \cdots$ & $-\cdots-\cdots-\cdots$ & 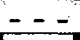 \\
\hline AF374578PKEEN & $\mid C T C C$ & C C $T$ & ATTATT & ACAACATGA & CCA \\
\hline U40252Pleuc & C т с C & $c \in c$ & ATT ATC & ACAACAITCA & $C \subset A$ \\
\hline JAM160Pleuc & C т с C & cec & ATTATT & $A \subset A A C A T C A$ & $C \subset A$ \\
\hline JAM161Pleuc & $C \mathbf{C} \mathbf{C}$ & $C<c$ & A T T A T T & $A \subset A A C A T G A$ & $c \in A$ \\
\hline JAM162Pléuc & C TCC & ecc & AT TATT & ACAACATGA & C C A \\
\hline JAM163Pleuc & C T C C & 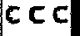 & ATT TATT & $A \in A A G A \mid T G A$ & $C \subset A$ \\
\hline JAM164Pleuc & E T CC & $c \in c$ & ATT A T T) & $A \subset A A \subset A T G A$ & $c \in A$ \\
\hline JAM168Pmbairdii & $|c+c c|$ & CCT & ATTATT & $A \in A A C A T G A$ & C C A \\
\hline JAM167Pmbairdii & $|c \mathbf{c} c|$ & C CT & ATT T T T & $A \subset A A C A T G A$ & C C A \\
\hline JAMi59PmbairdlI & $\mid \mathbf{c}$ T $\mathbf{C ~ C} \mid$ & C C & ATT T TT & $A \subset A A C A T G A$ & $C \subset A$ \\
\hline JAM166Pmbairdli & $C \mathbf{T C}$ & $C \in \mathbf{T}$ & ATTATT & $A \subset A A C A T C A$ & $\operatorname{ceA}$ \\
\hline Microtuspennsylvanic & $A C C T$ & $C C A$ & 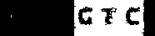 & $A \subset A A C C T G A$ & cec \\
\hline Oryzomyspalustris & $\mathrm{C} \mathbf{T C}$ & $c<c$ & ATT & ACAACCTGA & $c \in c$ \\
\hline Sigmodonhispidus & 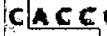 & $c \in c$ & AT T T & $A C A A G C T G A$ & $\operatorname{ccc}$ \\
\hline Relthrodontomysmeg & $\mathrm{CTCC}$ & ecc & ATCATT & ACAAECTGA & $C \subset A$ \\
\hline Rattusnorvegicus & $c \operatorname{ccc}$ & $C C A$ & ATCATT & ACA ACTTGA & $c \in A$ \\
\hline Pmrufinus & CTCCl & CCT & ATTATT & $\triangle \subset A \wedge C A T G A$ & $C C A$ \\
\hline
\end{tabular}


WC7

wC11

WC12

WC13

lJd 3256

IJd3304

Jdd3306

ljd3308

IJd3317

IJd3324

IJd3327

ljd 3330

ljd3333

IJd 3344

IJd3348

LD620

LDD774

LJD886

LD2088

LDD1960

LDD1669

LDD1524

LDD1523

LD1122

LD2522

LJD1637

LD1638

LJD2161

LjD2651

LJD2737

LJD2834

LJD2850

LJD2898

LJD2914

LJD2936

LJD3000

LJD2126

LD2138

LJD2154

LJD2 158

LJD2836

LD2852

LJD2860

LDD2865

LDD2890

LJD2943

Jefferson 3

ADAMS6
$1001 \quad$ GCCACCEAAAACCACATAAAAAAGGAACAC

AT A

A T A

A T A

A.T A

A T A

A T A

AT $\mathrm{A}$

A T A

A T A

A $T A$

A $\mathbf{T}$

A T A

A T A

A T A

A T $A$

A T A

A T A

A T A

A T A

A T A

A $T$ A

A T A

A T A

A TA

A T A

A T A

AT A

A T A

A T A

A T $A$

AT $A$

A T A

A T A

A T A

A T A

A $A$

A T $A$

A T A

A T A

$A T A$

AT $A$

A T A

A T A

A T $A$

A T $A$

A T A

A T A

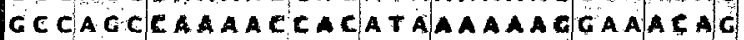
GCCACCEAAACCACATAAAAAAGGAAACAC

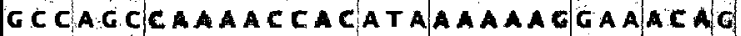

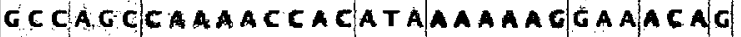
GCCAGCEAAAACCACATAAAAAAGGAAAEAG

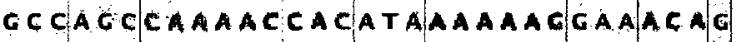
GC CAGCCAAAACCACATAAAAAAGGAAMCAG

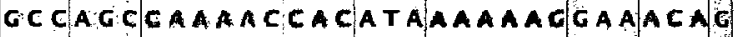
GCCAGCEAAAACEACATAAAAAAGGAAACAG GCCACCEAa aceacataAaAaAgGaAacag GCCAGCEAAAACCACATAAAAAAGGAAACAG GC CAGCCAAAACEACATAAAaAagGaAaCag GC CAGCEAAAACCACATAAAAAAGGAAACAG

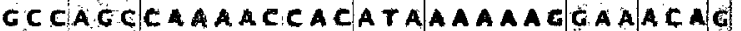
GCCAGCCAAAACCACATAAAAAAGGAAACAG GCCACCCAAAACCACATAAAAAAGGAAACAG G C CA GCEAAaACCACATAaAaAagGaAacag

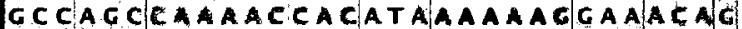

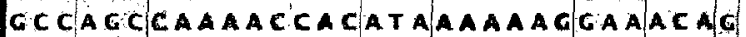
GCCAGCCAAAACEACAT AAAAAAGGAAACAG

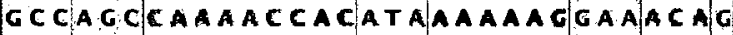
GCCAGCEAAAACCACATAAAAAAGGAAACAG GC CA GCCAAAACCACATAAAAAAGGAaACAG GCCAGCGaAaAceacatalaAaAgGaAacag GCCAGCEAAAACLACATAIAAAAGGAAACAG GCCACCCA AACCACATAAAAAACGAAACAG

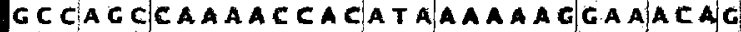

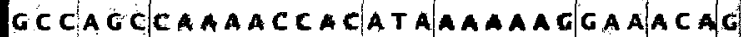
GCCAGCCAAAACGACATAAAAAACGAAICAG GCCAGCEAAAACCACATAAAAAAGGAAACAC G CCAGCEAAAACCACATAAAAAAGGAAACAC GCCAGCEAAAACCACATALAAAAGGAALCAG G C CAGCEAAAACCACATAAAAAAGGAAACAG GCCAGCEAAAACCAEATAAAAAAGGAAICAC G C CAGCCAAAACCACATAAAaAa GaAACAC GCCAGCEAAAACEAEATAAAAAAGGAAACNC G C C GCEAAAACCACATAaAaAacGaAaCag GCCAGCEA AACCACATAAAAAAGGAAACAG

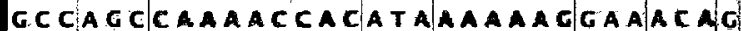
GC CA GCEAAAACEACATAAAAAAGGAAACNG GCCAGCGAAAACCACATAAAAAAGGAAACAG GCCAGCeAaAaCcacataAaAaAdgaAacag GCCAGCCAAAACEACATAMAAAAGGAARCAG

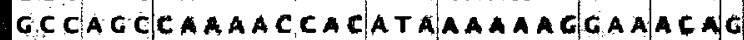
GCCAGCCAAAACCACATAaAaAaGGaAaCaC

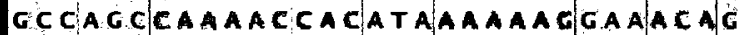
GĆACCEAAAACCACATAAAAAACGAAACAG 


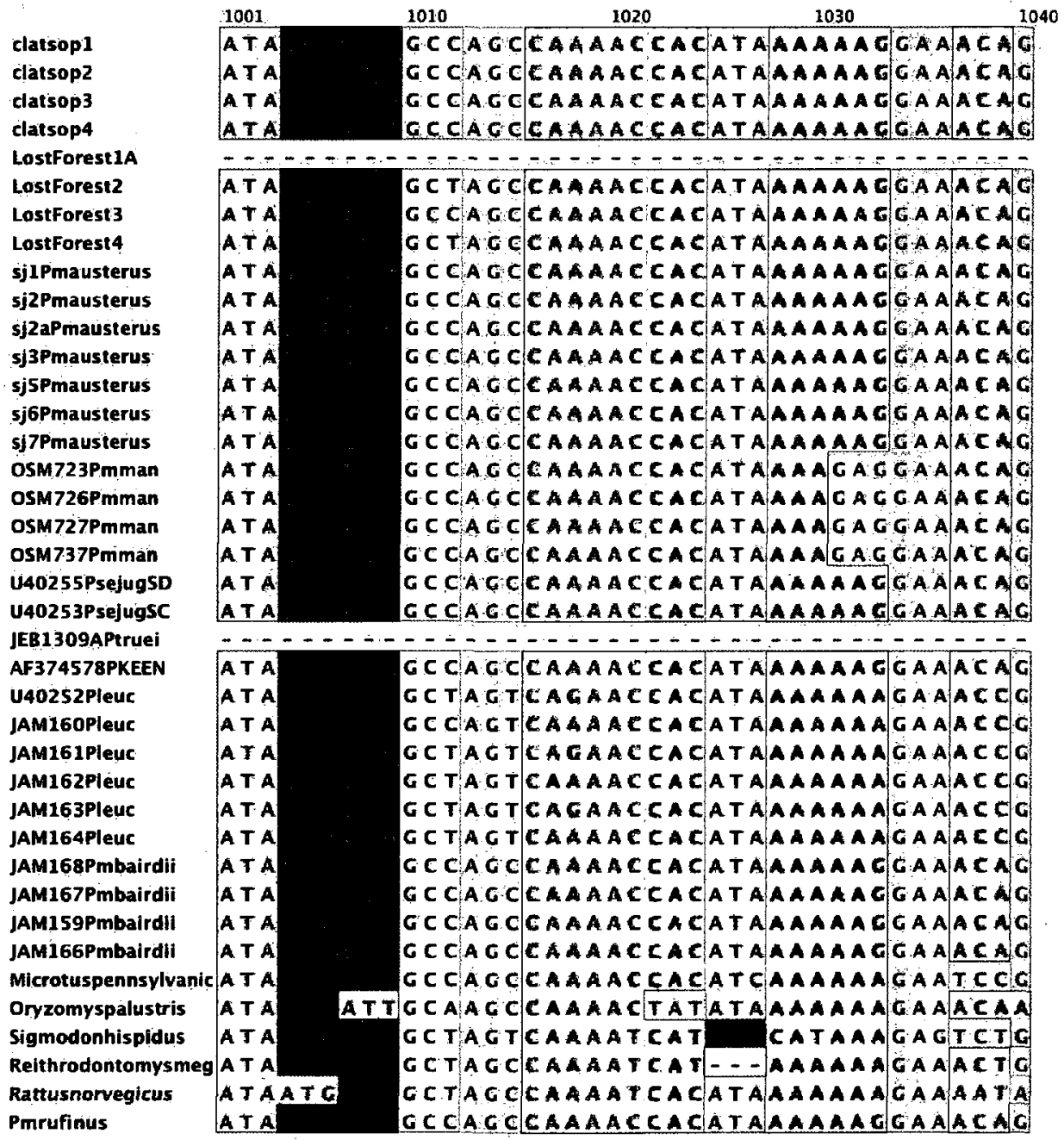


WC7

WC11

WC12

WC13

IJd3256

ljd3304

ljd3306

ljd3308

|jd3317

Ijd3324

ljd3327

ljd3330

ljd3333

Jjd3344

Jjd3348

니D620

UD774

UD886

UJD2088

UDD1960

LJD1669

LD1524

LJD1523

LDD1122

LJD2522

4JD1637

UDD1638

LJD2161

UD265 1

LJD2737

LD2834

LJD2850

LJD2898

LD2914

LJD2936

LDD3000

LJD2126

LJD2138

LJD2 154

LJD2 158

LJD2836

LJD2852

UJD2860

LJD2865.

LJD2890

LJD2943

Jefferson 3

ADAMS6
$1041 \frac{1050}{1060}$ A GT CAAA CAAAAAAACATACATTTCAATA ACT CAAACAAAAAAACATACATTTCAATA A GTCAAACAAAAAAACATACATTTCAATA A GTCAAACAAAAAAACATATATTTCAATA A CTCAAACAAAAAAACATACATTTCAATA A GTCAAACAAAAAAACATACLTTTCAATA A GT CAaACIaAaAaAaCATACATTTCAATA AGTCATACAAMAAAACATACATTTCAATA A GTCAAACAAAAAAACATATATTTCAATA A GTCAAACAAAAAARCATACATTTCAATA A GT CAAACAAAAAAACATACATYTCAATA A GT CAIAACAAAAAAACATACATTTCAATA A GTCAAACAAAAAAACATACATTTCAATA A G AAEAAAAAAACATACATTTCAATA AG T CAACAAAAAAACATACATTTEAATA ACTCALACAAAAAAACATATATTTCAATA A GTCAAACAAAAAAACATATATTTCAATA ACT CAAACAAAAAAACATACATTTCAATA ACT CAAACAAAAAAACATATATTTCAATA A.GTCAAACAAAAAAACATATATTTCAATA A GTCAAACAAAAAAACATACATTTCAATA A GTCAAACAAAAAAACATACATTTCAATA AGTCAAACAAAAAAACATACATTTCAATA A GTCAAACAAAAAAACATATATTTCAATA A G T CAAACA AaAAAACATACATTTTCAATA A GTCAAACAAAAAAACATACATTTCAATA A CTCAAACAAAAAAACATACATTTCAATA A GTCAAACAAAAAAACATACATTTCAATA A GTCAAACAAAAAAACATACATTTCAATA A GTCAAACAAAAAAACATACATTTCAATA ACT CAIAACAAAAAAACATACLATTTCAIATA A GT CALACAAAAAAACATACATTTCAATA A CT CAAAACAAAAAAACATACATTTCAATA A GT CAAACAAAAAAACATATATTT CAATA A GTCALACAAaAaAaCATACATTTCAATA AC AACAAAAAAACATACATTTCAATA $A G$ AACAAAAAAACATACATTTCAATA A GT CAAACAAAAAAACATACATTTCAATA AG AACAAAAAAACATACATTTCAATA AG AaCIAAAAAAAEATACATTTCAATA AGTCAAACAAAAAAACATACATTTCAATA A GTCAAACAAAAAAACATACATTTCAATA A GT CAAACAAAAAAACATATATTTCAATA ACTCAAACAAMAAAACATACIATTTCAATA A GTCAAACAAAAAAACATACATTTCAATA ACTCAAAEIAAAAAAACATATATTTCAATA A GTCAIAACAAAAAAACATACAT TTCAATA
1070

G T C
c $\mathrm{T}$ e TC G T $\mathrm{C}$ G T c r c Te G TC G I c $\mathrm{TC}$ G T G T $C$ G T C G T C c T G T G T C GTC A T C C T C G T C c TC G TC cre c TC G TC c TC C T C CTC G TC c TC 6 T C G $T C$ G T C G TC G TC c T C G TC cr c TC CTC GTC c $T C$ G T G TC GT GTC 


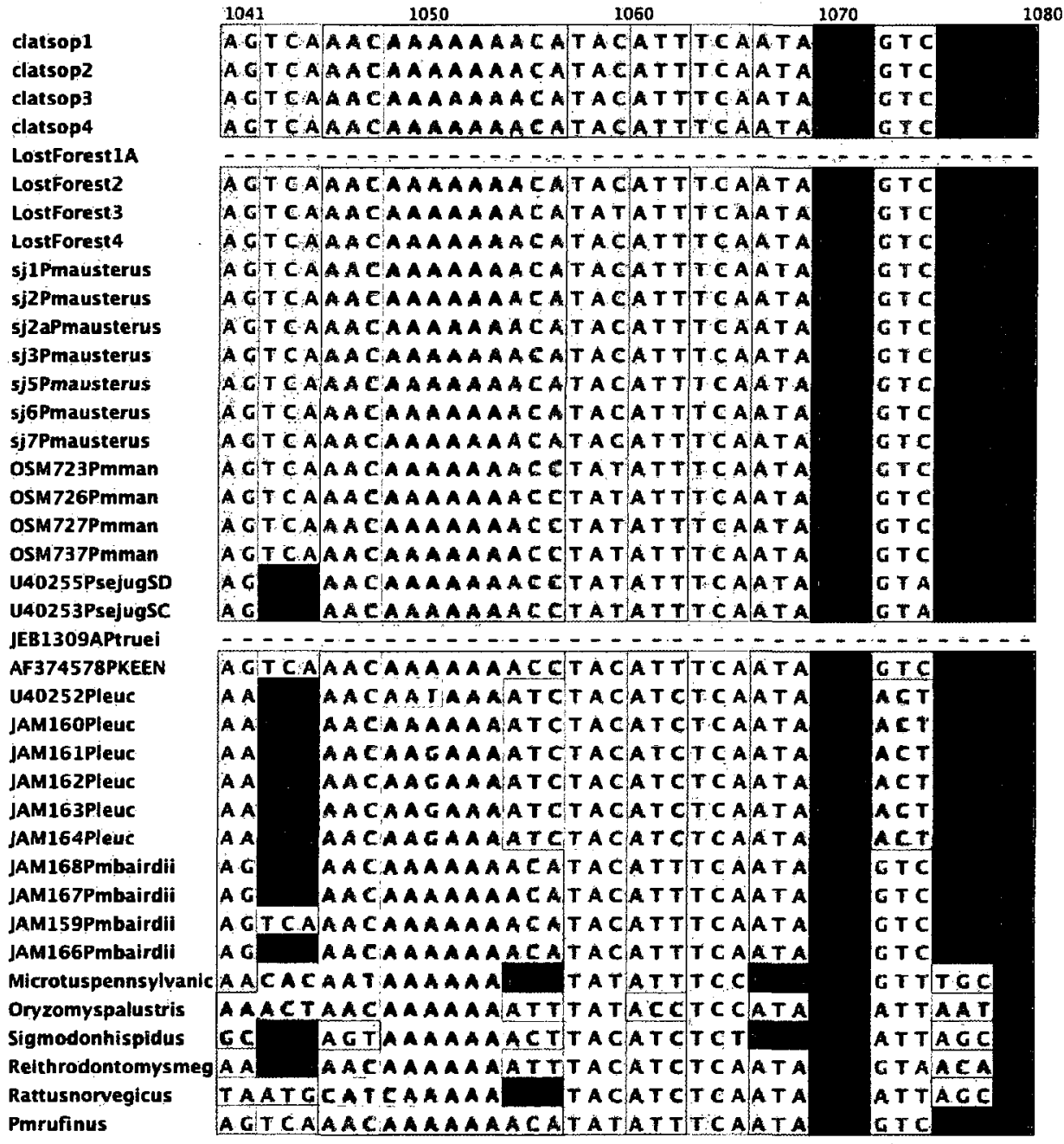


wC7

wC11

wC12

wCis

Ud3256

IJd3304

Jjd3306

Jjd3308

ljd 3317

Jjd3324

ljd 3327

jjd3330

ljd 3333

Jjd3344

IJd3348

LJD620

UJD774

LJD886

LJD2088

LJD1960

LJD1669

LJD1524

LJD1523

LUD1 122

LJD2522

LJD1637

LJD1638

LJD2161

LJD2651

LJD2737

LJD2834

LJD2850

LJD2898

LJD2914

LJD2936

LJ03000

LJD2 126

LUD2138

LJD2 154

니2 158

LJD2 836

LJD2852

LJD2860

LJD2865.

LJD2890

LJD2943

Jefferson 3

ADAMS6

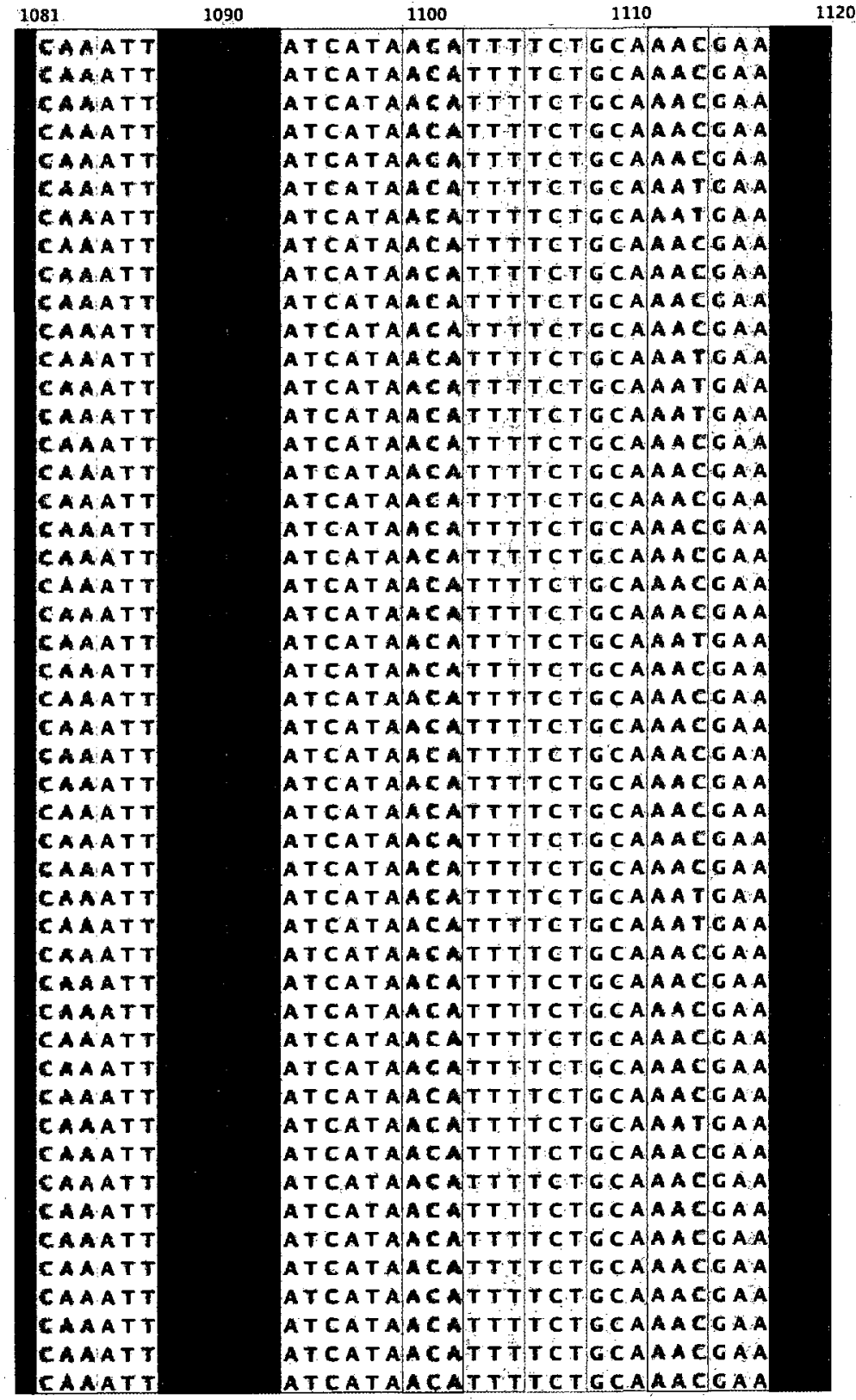




\begin{tabular}{|c|c|c|c|c|c|c|}
\hline & 1081 & 1090 & & 1100 & 1110 & \\
\hline clatsop 1 & EAAATT & & ATCATA & ARATTTCT & GCA & $A A C G A A$ \\
\hline clatsop2 & CAART T & & ATCATA & MEAT TtTCT & GCA & A A T GA \\
\hline clatsop3 & CA AIATT & & ATCATA & ACATTTTCT & $G C A$ & A ATGAA \\
\hline clatsop 4 & CAATI & & ATCATA & LAEATITCT & $\mathbf{C C A}$ & $\triangle A \cap G A A$ \\
\hline Lostfọrest1A & $\ldots-\cdots$ & & ------ & $-1-----$ & $=-$ & ---- \\
\hline LostForest2 & CAAATT & & A T C A T A & ACATITTCT & $G C A$ & $A A C C A A$ \\
\hline LostForest3 & CAAAT T & $\therefore$ & ATCATA & $A A C A T T T C T$ & G C A & $A \triangle C G A A$ \\
\hline LostForest 4 & CAATT & & AT CATA & AC TTTTCT & $G C A$ & $A \triangle C G A A$ \\
\hline sj1Pmausterus & CAAIAT T & & ATCATA & ACATTTTET & $G C A$ & $A A C O A A$ \\
\hline sj2Pmausterus & CAAATT & & ATCATA & CEATTTCT & $\mathbf{C C A}$ & $A \wedge C A A$ \\
\hline sj2aPmausterus & CAAAT T & & ATCATA & $A C A T T T T C T$ & $C \mathrm{CA}$ & A ACGAA \\
\hline sj3Pmausterus & CAAATT & : & ATCATA & AC ATTTICT & $C \subset A$ & $A A C O A A$ \\
\hline sj5Pmausterus & CAAATT & : & ATCATA & $A C A T T T T C T$ & $\mathbf{G} \mathbf{C A}$ & $A \triangle C O A A$ \\
\hline sj6Pmausterus & CAAATT & . & ATCATA & $A \subset A T T T T C T$ & $\mid G \in A$ & A A C CAA \\
\hline sj7Pmausterus & CAAATT & ; & ATCATA & ACATTTTCT & $\mathbf{G C A}$ & A ACAA \\
\hline OSM723Pmman & EAAATT & a & A T CATA & AC $A T \mathrm{TTCT}$ & $\mathbf{G} \mathbf{C} \mathbf{A}$ & AACGAA \\
\hline OSM726Pinman & CAATT & . & AT $T$ A T A & ACATSTTCT & $G \in A$ & $A A C G A A$ \\
\hline OSM727Pmman & CAATT & & $A T \subset A T A$ & $A \in A T T T T C T$ & $G \mathbf{A}$ & $A A C A A$ \\
\hline OSM737Pmiman & CAAATT & . & ATCATA & ACATTTTCT & $\mathbf{G C A}$ & AACGAA \\
\hline U40255PsejugsD & CAATT & $\therefore$ & A T CATA & 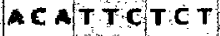 & $\mathbf{C C A}$ & AATCAA \\
\hline U40253PsejugSC & CAAATT & $:$ & $A T C A T A$ & AEATTCTCT & GCA & A A TEAA \\
\hline JEB1309APtruei & ------ & - & $\ldots-\cdots$ & $----\cdots-1-\cdots$ & $-\ldots$ & $-\cdots-\cdots$ \\
\hline AF374578PKEEN & CAAATT & 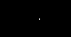 & ATCATA & ACATTCTCT & $\mathbf{G C A}$ & $A A T G A G$ \\
\hline U40252Pleuc & EAAATC & & ATCATA & ACTLTTTCT & G CA & AACIAA \\
\hline JAM160Pleuc & CAAATC & & A T CATA & $A \subset T T \mathbf{T} T \mathbf{T} C \mathrm{~T}$ & $\mathbf{G C A}$ & $A A C A G$ \\
\hline JAM161Pleuc & CAAATC & 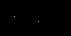 & A T C A T A & $A \in T T T T T \in T$ & $G \mathrm{CA}$ & AAEGAG \\
\hline JAM162Pleuc & CAAATC & 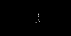 & ATCATA & ACTTTTTCT & $\mid \mathbf{G} \mathbf{C A}$ & AACGAG \\
\hline JAM163Pleuc & CAAATE & : & ATCATA & $A C T T T T T C T$ & $\mathbf{G C A}$ & $A \cap E G A G$ \\
\hline JAM164Pleuc & CAAATC & : & АТ ТАТ A & $\boldsymbol{A C T} \mathrm{T} T \mathrm{TTTCT}$ & $\mathbf{C C A}$ & $A A C O A G$ \\
\hline JAM168Pmbairdij & CAAATT & . & ATCATA & $A C \wedge T T T T C T$ & $G C A$ & A CGAA \\
\hline JAM167Pmbairdii & CAATT & & A TCAT A & $A C A T T T T C T$ & $\mathbf{G C A}$ & AACSGA \\
\hline JAM159PmbairdiI & CAAATT & & ATCATA & ACATTTTCT & $\mathbf{G C A}$ & A ACGAA \\
\hline JAM166Pmbairdii & EAAATT & . & ATEATA & ACATTTTCT & $\mathbf{G C A}$ & AACGAA \\
\hline Microtuspennsylvanic & $C A A A C C$ & & ATCATC & $A \in C T T C T \in T$ & $\mathbf{C C A}$ & AACICA \\
\hline Oryzomyspalustris & EAAATT & $c$ & $A<\operatorname{cit} T$ & ACATTCTCT & G T G & A ATGAA \\
\hline Sigmodonhispidus & CAAATT & & $A \in T A T C$ & ACA T T C T C T & $\mathbf{G C T}$ & $A A \in G A G$ \\
\hline Reithrodontomysmeg & CA AATT & & АТ Т А T & $A C A T T T T C T$ & GCA & $A A C G A A$ \\
\hline Rattusnorvegicus & CAAATT & & ATCATA & ACATTCTCC & $C \mathbf{A}$ & $A \in A G A$ \\
\hline Pmrufinus & CAAATT & & A TCATA & ACAITITCI & $\mathbf{C C A}$ & $A A C G A$ \\
\hline
\end{tabular}


WC7

WC11

WC12

WC13

ijd 3256

yd 3304

yd3306

ljd3308

Ijd3317

Ijd3324

IJd3327

ljd3330

ljd3333

IJd3344

lid 3348

HD620

UDD774

LD886

LJD2088

LJD1960

LJD1669

LJD1524

LJD1523

LJD1122

LJD2522

LJD1637

LJD1638

LJD2 161

LJD2651

LJD2737

LJD2834

LJD2850

LJD2898

LJD2914

LJD2936

LJD3000

LJD2126

LDD2138

LJD2154

LJD2158

LJD2836

LJD2852

LJD2860

LJD2865

LJD2890

LJD2943

Jefferson 3

ADAMS6
$1121 \quad 1130 \quad 1140 \quad 1150$

ATTATATTTTACAT ATTATATTTTACATC ATTATATTTTACATC ATTATATTTTACIATC ATTATATTTTACATC ATTATATTTTACATC ATTATATTTTACATC ATTATATTTTACATC AT TATATTTTACATC ATTATATTTTACATC GTTATATTTTACATC ATTATAITTTTACATC ATTATATTTTACATC ATTATATTTTACATC ATTATATTTTACATC ATTATATTTTACATC ATTATATTTTACATC ATTATATTTTACATC ATTATATTTTACATC ATTATATTTTACATC ATTATATTT TACATC ATTATATTTTACATC ATTATATTTTACATC ATTATATTTTACATC ATTATATTTTACATC ATTATATTTTACATC ATTATATTTTACATC ATT ATATTTTACATC ATTATATTTTACATC ATTATA'T TT TACATC ATTATATTTTACATC ATTATATTTTACATC ATTATATTTTACATC ATTATATT T TACATC ATTATATTTTACATC ATTATATTTTACATC ATTATATTTTACATC ATTATATTTTACATC ATTATATTTTACLATC ATTATATTTTACATC ATTATATTTTACATC ATTATATTTTACATC ATTATATTTTACATC AT TA T ATT T TACATC ATTATATTTTACATC ATTATATTTTACATC ATTATATTTTACATC ATTATATTTTACATC TGAAGCCACE TTTGAACCCACC TTTGAAGCCACC TTTGAAGCCACC TTTGAACCCACE TT T CAAGCCACC TT TGAAGCCACC TTTCAAGCTACC TTTGAAGCCACC TTTGAAGCEACC TTTGAACCTACC TTTCAACCCACC TTTGAACCCACC TTTGAACCCACC TTTCAAGCCACC TTTGAACCCACC TTTGAACCCACC TT TGAAGCCACC TTTGAACCCACC TT TCAACCCACC TTTGAACCCACC TTTGAACECACC TTTGAACCCACC TTTGAACCCACE TTTGAAGCCACC TTTGAACCCACC TTTGAAGCCACC TTTCAACCEACC TTTGAACCCACC TTTGAACCCACC TTTGAAGCCACC TTTGAACCCACC TTTGAACCCACC TTTGAACCCACC TT TGAACCCACE TTTGAACCCACC TT TGAAGCCACC TTTGAAGCCACE TTTGAAGCCACC TT TGAACCCACC TTTCAACCCACC TT TGAAGCACC TTT TAAG C CACC TTTGAaCCCACC TT TGAACCCACC TTTGAACCCACC TTTGAAICCACC tTtGaAGCele
A TCECCA ATCECCA A TECCCA ATCCCCA A TCCCCA A TCECCA AT CECEA ATCCCCA A T CCCCA ATCCCEA ATCECCA A TCCCCA A TCCCEA A TCCCCA ATCCCCA ATCCCCIA A T CCCCA ATCCCCA A T CCCEA A TCCCCA ATCCCCA A TCCCCA ATCECEA ATCCCCA ATCECCA ATCCCCA ATCCCCA ATCCCCA ATCCCC'A ATCCCCA A TCCC.CA ATCECCA ATCCCCA A T CCCCA ATCCCCA ATCCCCA ATCCCCA ATCCCCA ATCCCCA ATCCCCA AT CCCCA ATCCCCA ATCCECA AT TCCCA ATCecé ATCCCCA ATCCCCA ATCECEA 


\begin{tabular}{|c|c|c|c|c|}
\hline & 1121 & 1140 & 11 & \\
\hline clatsop1 & АT TAT ATT TT ACAT & TTTGAAGCC & $A C C$ & ATCECCA \\
\hline clatsop2 & АТ T AT AT T T TACAT & TTTGAACCC & ACC & ATCCCCA \\
\hline clatsop3 & АT T A T AT T T T ACAT & T TTGAACCC) & $A<C$ & ATCCCCA \\
\hline clatsop4 & ATIATATTITACATC & ITIGAACC & $A \subset C$ & ATCCCEA \\
\hline LostForest1A & 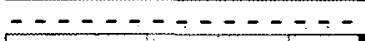 & $--\cdots \cdots$ & $-\ldots$ & $-\ldots-\cdots$ \\
\hline Lostforest2 & ATT AT AT TT ACAT & TTTGAACCC & $\operatorname{Acc}$ & ATCCCCA \\
\hline LostForest 3 & AT T A T ATT T T T AC) T & T T T GAAGCC & A C C & A TCCEC \\
\hline LostForest 4 & АТ TAT АТ TТ TАСАТ & T.T TAAGCC & $\operatorname{ACC}$ & ATeCCCA \\
\hline sj1Pmausterus & AT T A T AT T T T ACLAT & TTTGAAGCC & $A \in C$ & ATCCCCA \\
\hline sj2Pmausterus & АT TAT АT TT TACAT & T T T GAAGCC & $A<C$ & A T C C C C \\
\hline sj2aPmausterus & 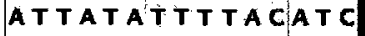 & T T T GAACCC & $A \subset C$ & ATCCCC \\
\hline sj3Pmausterus & АTT АТ АT TTT ACATC & TTTGAAGCC & $A \subset C$ & A TCCCC \\
\hline sj5Pmausterus & AT T AT ATT T TACAT & TTTGAAGCC & $A \in C$ & ATCCCCA \\
\hline sj6Pmausterus & AT TAT AT TT TACAT & TTTGAAGCC & $A<C$ & ATCCCCA \\
\hline sj7Pmausterius & AT T A T AT T T T A CA TC & T T T G A A G C C & $A \subset C$ & A TCCCCA \\
\hline OSM723Pmman & АТ T АT АT T T АCATC & T T T GA ACC & $A \subset C$ & ATCCCCA \\
\hline OSA726Pmman & 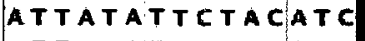 & T TTGAAGCC & $A \subset C$ & A TCCCCA \\
\hline OSM727Pmman & АТ Т АТ АТ T Т Т А А T & T T TGAACCC & $A \subset C$ & $|A T C C C C A|$ \\
\hline OSM737Pmman & АТ T А T АТ T С T АСА TC & T T T G A A C C C & $A \subset C$ & ATCCCC:A \\
\hline U40255PsejugSD & AT T AT ATTT T AT & T T T G A A G C T & $A \subset C$ & ATCCCCA \\
\hline U40253PsejugsC & ATTATATTTTATATC & TTTCAACCT & $A \subset C$ & ATCCCCA \\
\hline JEB 13 09APtruei & $--\cdots-\cdots-\cdots-\cdots$ & $-\cdots---\cdots$ & -- & $\ldots-\cdots$ \\
\hline AF374578PKEEN & А T T AT AT T T T ATATC & T T T GA AGCC & $A \subset C$ & ATTCCTA] \\
\hline U40252Pleuc & AT T AT AT T T T ATATC & T TCGAAG CT & $A \subset \mathbf{A}$ & ATTCCTA \\
\hline JAM160Pleuc & AT TATATTT TATA TC & TT T CAAGCC & $A \subset A$ & A T CECTIA \\
\hline JAM16 IPleuc & АТТАТАТТТТ АТАТС & TTTGAAGCT & $A \subset A$ & A TCCCCA \\
\hline JAM162Pleuc & AT T AT AT T T TATA TC & T T T G A A C C C & $\mathbf{A} \boldsymbol{C A}$ & ATCCCT,A \\
\hline JAM163Pleuc & ATTATATTT TATATC & T T T G A A G C T & ACC & ATCECTA \\
\hline JAM164Pleuc & A T TATATT T TATATC & T T TCA ACC T & $A \subset A$ & A T C C C T:A \\
\hline JAM168Pmbairdii & АТ TAT АT T T T ACATC & TTTGAAGCC & $A C C$ & ATCCCCA \\
\hline JAM167Pmbairdii & АТ TAT АT T T TACATC & TTTGAACCC & $A \subset C$ & $A T C \in C C A$ \\
\hline JAM159Pmbalrdii & АТTATATTTTACATC & TTTGAACCC & $A C C$ & ATCCCCA \\
\hline JAM166Pmbairdii & АT TATATTTTACATC & TTTGAACCC & $A C C$ & A TCCCCA \\
\hline Microtuspennsylvanic & АТ С АT AT TT T ACATC & TTTCAACCC & $A C T$ & $A T C C C A A$ \\
\hline Oryzomyspaluseris & AT T & T T T GAACC C & $A \in T$ & ATTCETA \\
\hline Sigmodonhispidus & АТ Т АТ АТ С TАTATC & T T TGAAGCC & $A \subset A$ & $A T C \subset \subset A_{1} A$ \\
\hline Reithrodontomysmeg & AT TATATT T TACATC & TTTGAAGCT & $A \subset$ & A TCCCcA \\
\hline Rattusnorvegicus & AT T & $T \boldsymbol{T} \in \boldsymbol{G} \boldsymbol{G}^{\prime} \boldsymbol{G C C}$ & $A \subset T$ & ATCCCA:A \\
\hline Pmrufinus & ATTATATTTTACATC & TITGAAGCC & $A C C$ & $A T C C C C A$ \\
\hline
\end{tabular}


WC7

WC11

WC12

WC13

ljd3256

ljd3304

ljd3306

jjd3308

1jd3317

ljd3324

Ijd3327

ljd3330

ljd3333

ljd3344

ljd 3348

LJD620

LJD774

LDD886

LJD2088

LJD1960

LJD1669

LDD1524

LJD1523

LJD1I22

LJD2522

LJD1637

LJD1638

LJD2161

니D2651

LJD2737

LD2834

LJD2850

LJD2898

LJD2914

LJD2936

LJD3000

LJD2126

LJD2138

LJD2154

LJD2158

LJD2836

LJD2852

LJD2860

LJD2865

LJD2890

LJD2943

Jefferson 3

ADAMS6

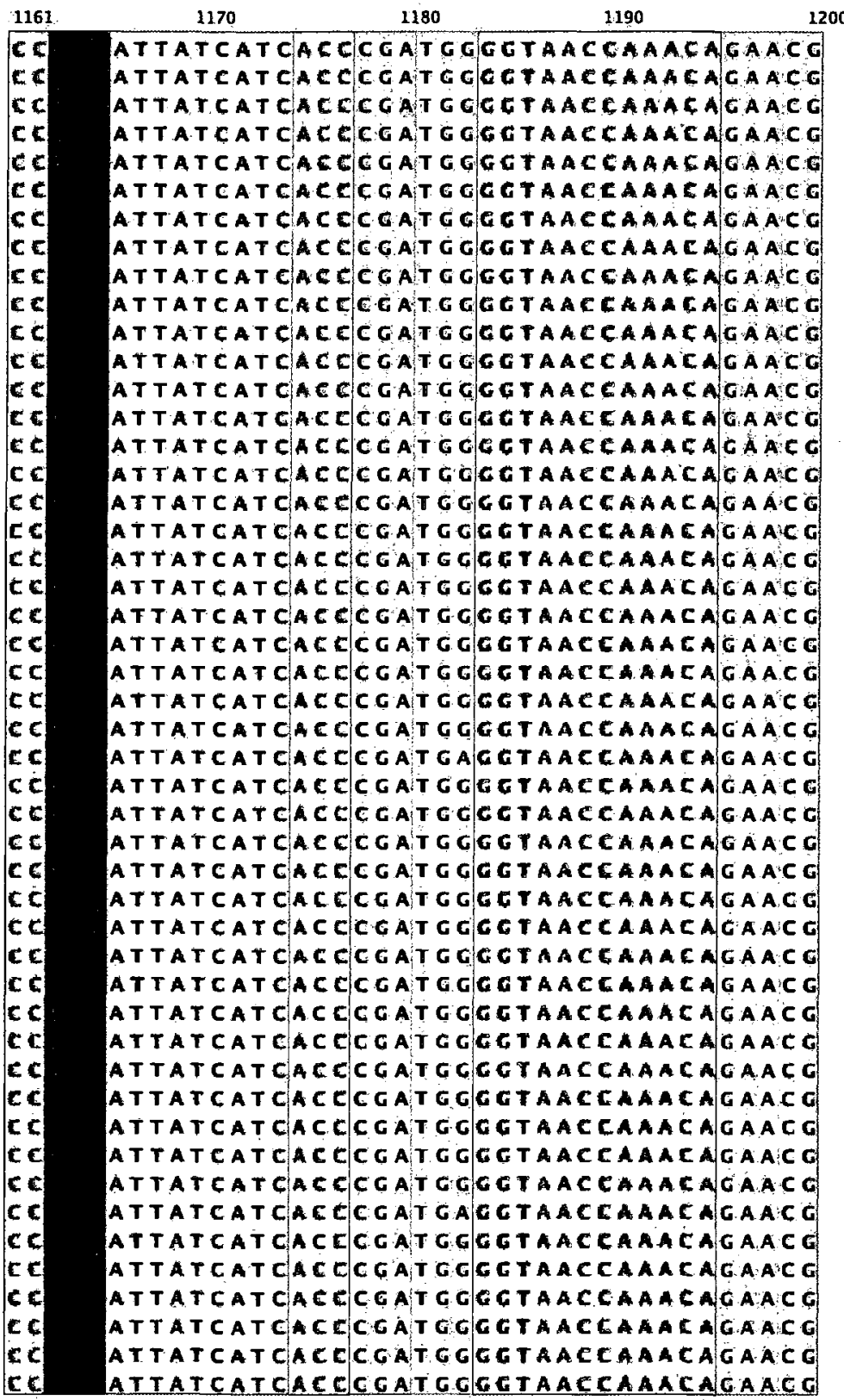




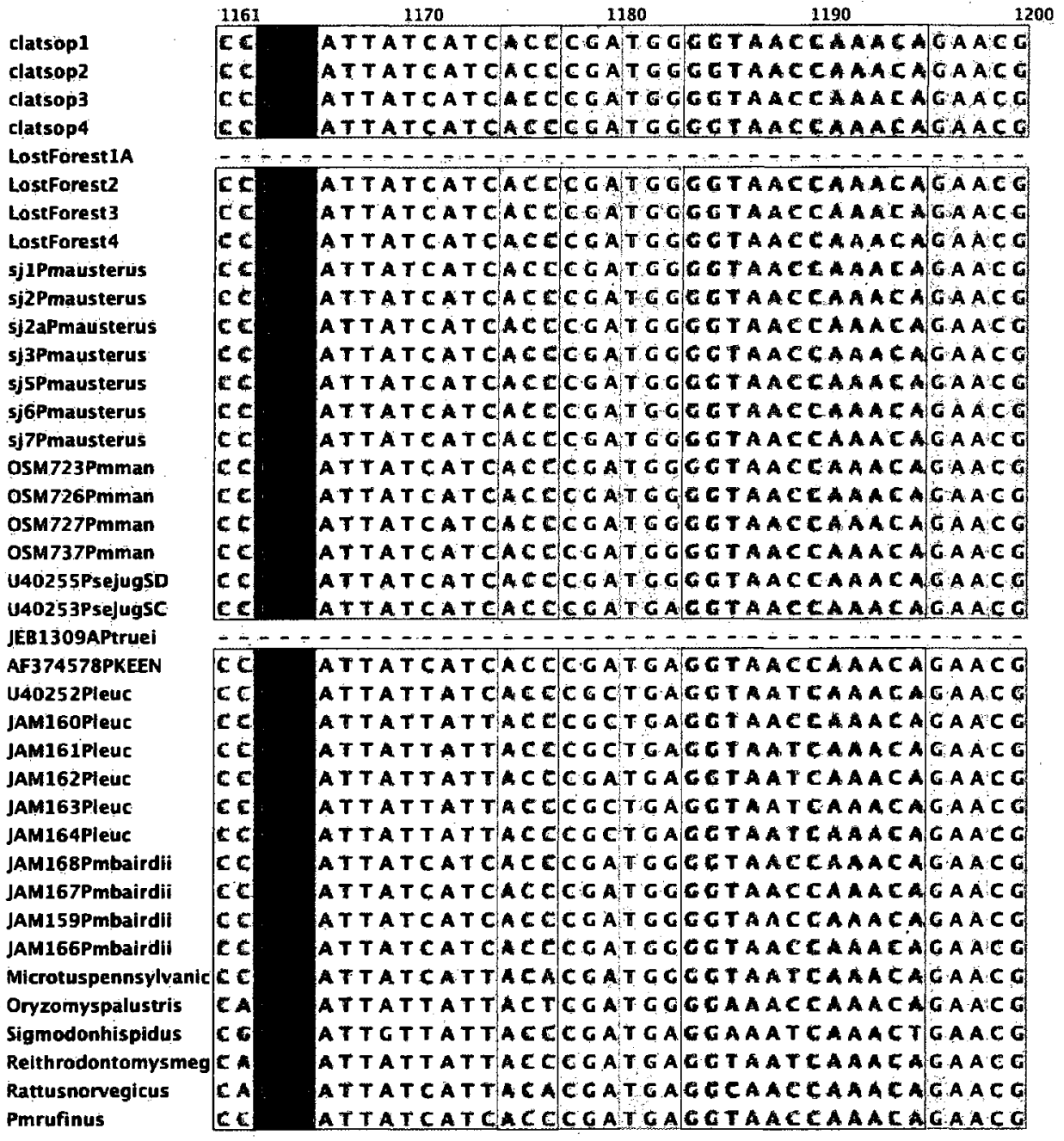


WC7

wC11

WC12

wC13

Ijd3256

IJd3304

IJd3306

ljd3308

|jd3317

Ijd 3324

IJd3327

ljd 3330

ljd 3333

Jdd 3344

JJd3348

니 620

LJD774

LJDB86

LJD2.088

LJD1960

LJD1669

LJD1524

LJD1523

LJD1122

LJD2522

LJD1637

LJD1638

LلJD2161

LJD2651

LJD2737

LJD2834

LJD2850

LلJD2898

LJD2914

LJD2936

LJD3000

LJD2 126

LJD2138

LJD2154

LJD2 158

UD2836

LJD2852

LJD2860

LJD2865

LD2890

LDD2943

Jefferson 3

ADAMS6

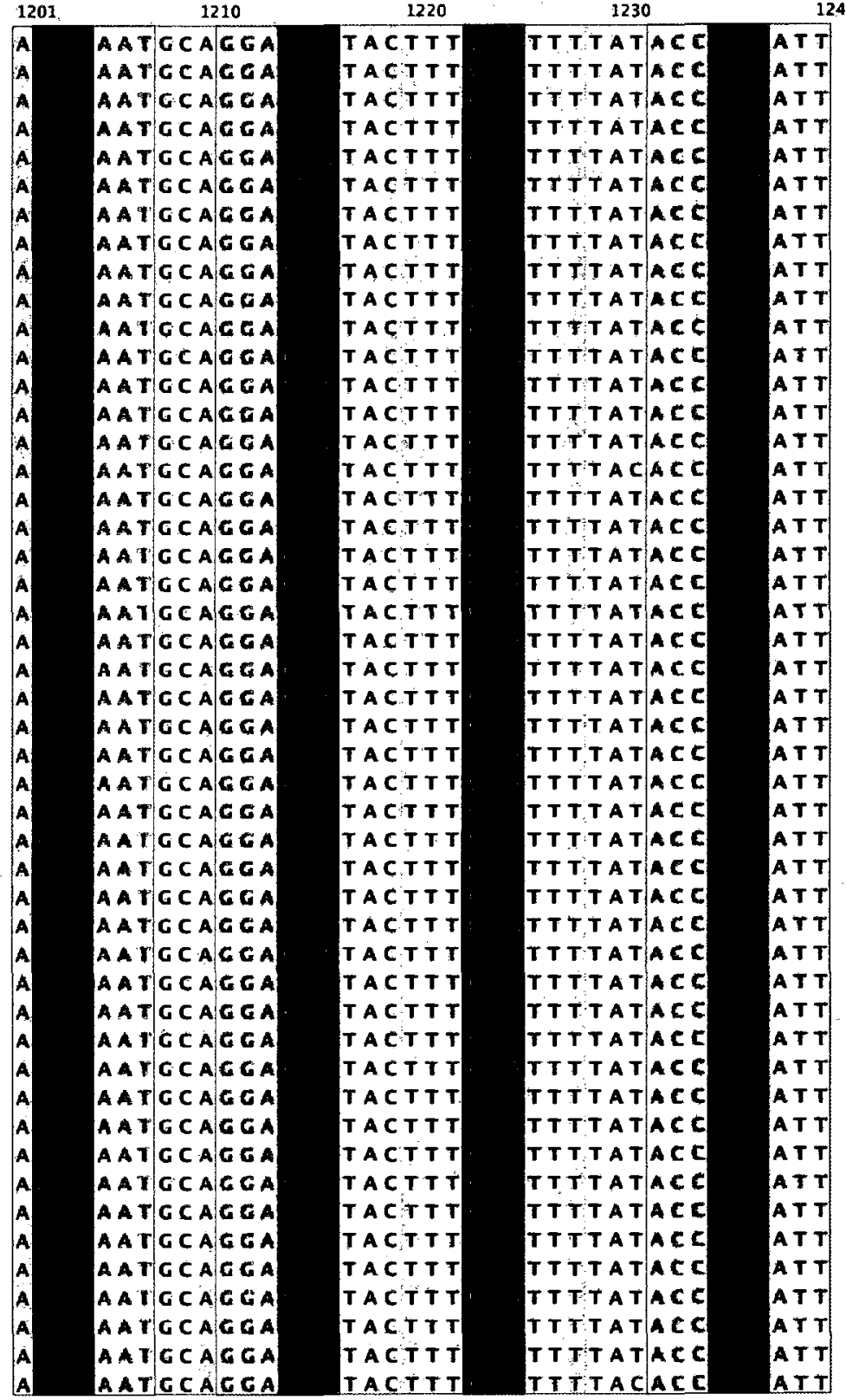


clatsop1

clatsop2

clatsop3

clatsop4

LostForest1A

LostForest2

LostForest 3

LostForest4

sj1Pmausterus

sjzPmausterus

sj2aPmausterus

sj3Pmausterus

sj5Pmausterus

sj6Pmausterus

sj7Pmausterus

OSM723Pmman

OSM726Pmman

OSM727Pmman

OSM737Pmiman

U40255PsejugSD

U40253PsejugSC

JEB1309APtruei

AF374578PKEEN

U40252Pleue

JAM160Pleuc

JAM16ipleuc

JAM162Pleuc

JAM163PleUC

JAM164Pleue

JAM168Pmbairdii

JAM167Pmbairdii

JAM159Pmbairdil

JAM166Pmbairdiı

Microtuspennsylvanic A

Oryzomyspalustris

Sigmodonhispidus

Relthrodontomysmeg

Rattusnorvegicus

Pmrufinus

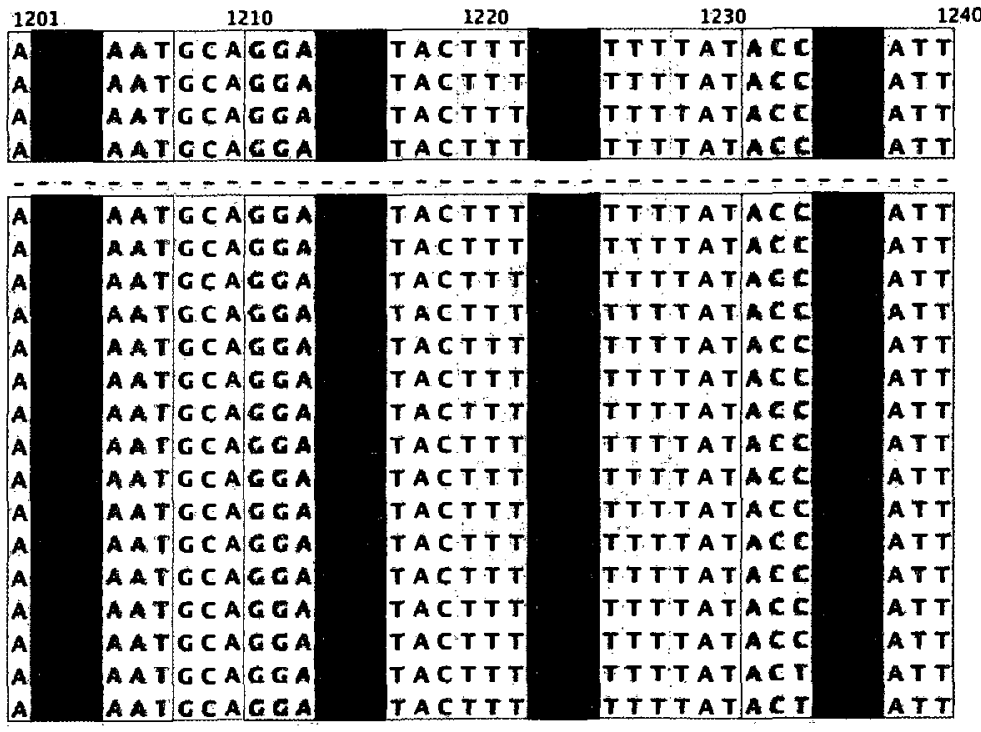

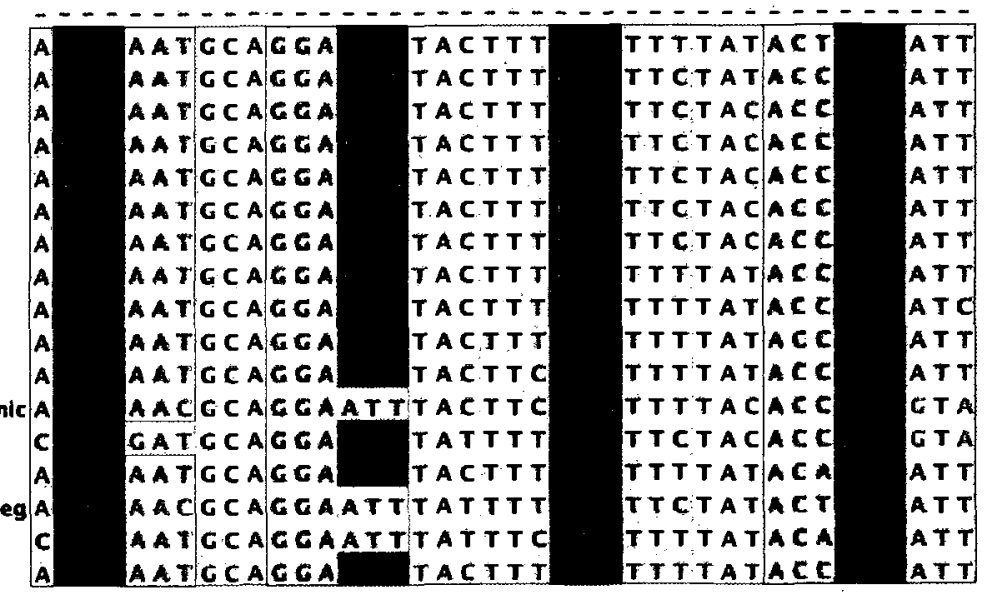


WC7

WC11

WC12

WC13

Ijd3256

ijd3304

ijd 3306

ljd3308

ljd3317

ljd3324

ljd3327

ljd3330

ljd3333

ljd3344

ljd3348

LJD620

LD774

LDD886

LD2088

LJD1960

LJD1669

LJD1524

LJD1523

LJD1122

LJD2522

LJD1637

LJD1638

LJD2 161

LJD2651

LJD2737

LJD2834

LJD2850

LJD2898

LJD2914

LJD2936

LJD3000

LJD2 126

LJD2 138

LJD2154

LJD2 158

LJD2836

LJD2852

LJD2860

LJD2865

LJD2890

LJ02943

Jefferson 3

ADAM56

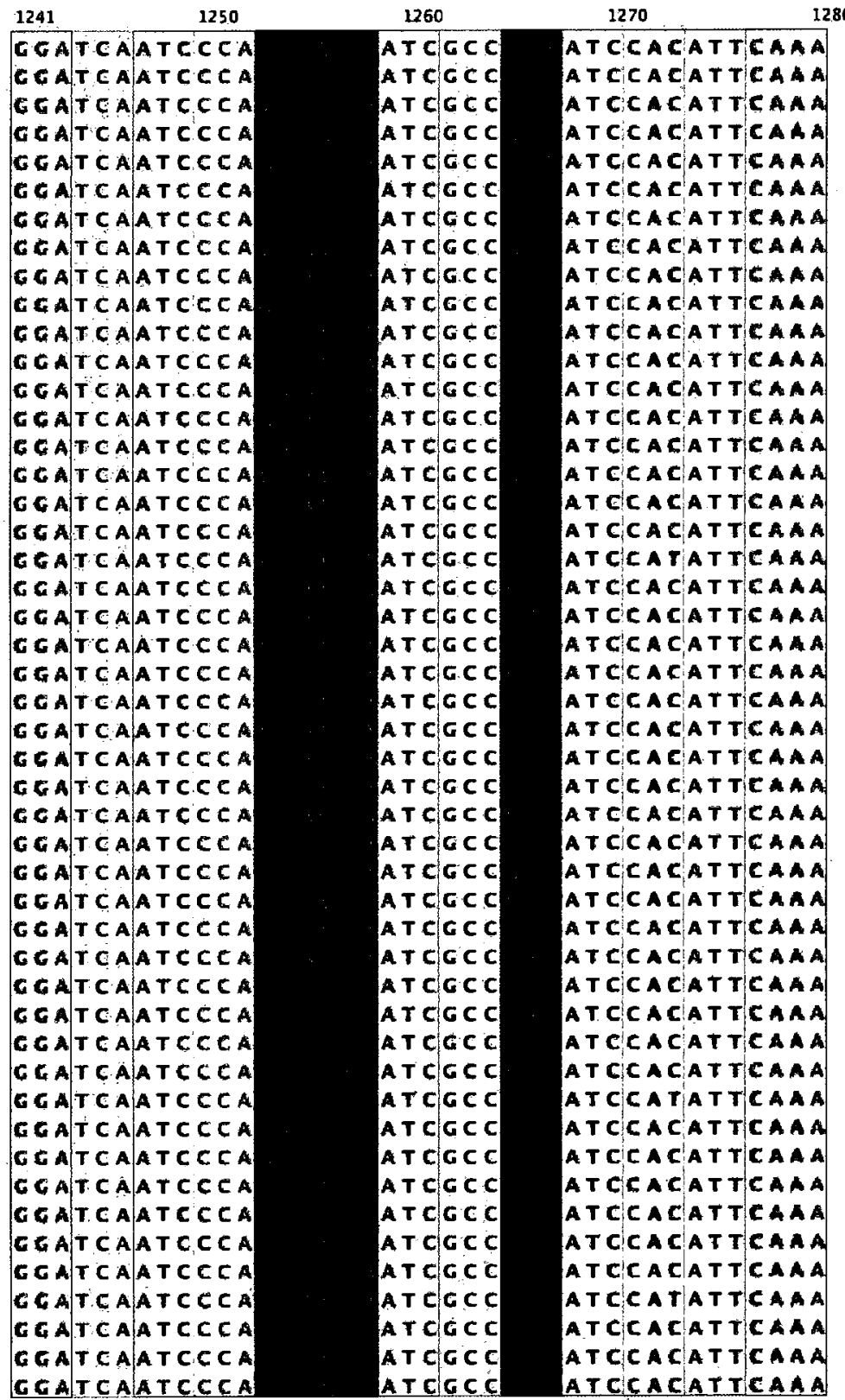




\begin{tabular}{|c|c|c|c|}
\hline & 1241. & 1260 & 1270 \\
\hline clatsop 1 & CGATCA ATCCCA & ATCGC & ATCCACATTCAAA \\
\hline clacsopz & GGATTCAATCCCA & A T COCC & АТCСАCАTTCAAA \\
\hline clatsop3 & GGA|TCA A TCCCA & A T C GCC & ATCCACATTCAAA \\
\hline clatsop4 & GGATCALTCECA & ATCGCC & ATCCACATTCAAA \\
\hline LostForestia & $\ldots-\cdots-\ldots-\cdots$ & $\ldots \ldots$ & $-\cdots-\cdots,-\cdots$ \\
\hline LostForesțt2 & GGATCAATCCCA & ATCGCC & ATTCACATTCAAA \\
\hline LostForest 3 & G G ATCAATCCA & ATCGC & ATCCACATTCAAA \\
\hline LostForest 4 & G GATCA ATCCCA & ATCGEC & ATTCACATTCAAA \\
\hline șj1Pmausterus & G GATCAATCEC & ATCGCC & AT CCACATTCAAA \\
\hline sj2Pmausterus & G GATAATCCCA & ATCGCC & ATCCACATTCAAA \\
\hline sj2aPmausterus & G CATCAATCCCA & ATCGCC & ATCСACATTCAAA \\
\hline sj3Pmausterus & C CA T C A A TCCCA & ATCGC & A T CICACATTCANA \\
\hline sj5Pmausterus & $C \subset A C A A T C C C A$ & ATCGCC & A T CLACATTCAAA \\
\hline sj6Pmausterus & G G ATCAATCCCA & ATCGCC & ATCCACATTCAAA \\
\hline sj7Pmausterus & CEATCA ATCCCA & ATCGCC & ATCCACATTCAAA \\
\hline OSM723Pmman & C CATCAATCCCT & ATTGC.C & ATCTTACATTCAMA \\
\hline OSM726Pmman & C A A T A A TCCE & ATrGC & ATCTACATTCAAA \\
\hline OSM727Pmman & GGATCAATCECT & ATTCCC & ATCTACATTEAAA \\
\hline OSM737Pmman & GGATCAATCCCT & ATTGCC & ATCT ACATTCAAA \\
\hline U40255PsejugSD & CGATCAATCCCA & A T T G C C & А T TIT ACATTCAAA \\
\hline U40253PsejugSC & GGATCAATCCCA & ATTCCC & ATTTACATTCAAA \\
\hline JEB1309APtruei & $---\cdots---\cdots$ & $--\cdots-\cdots$ & $\cdots \cdots-\cdots$ \\
\hline AF374578PKEEN & GGATCAATCCCA & ATtGCC & ATTTATATTCAAA \\
\hline U40252Pleuc & G GA|T CAATCCEC & ATTCCT & A T CT T CATCCAAA \\
\hline JAM160Pleuc & CEATCAATCCCA & ATtGCT & А T CT TC:ATCCAAA \\
\hline JAM161Pleuc & C GATCAATCCCA & ATTGCT & ATCTTCATCCAAA \\
\hline JAM162Pleuc & G G ATCA|ATCCCA & ATTGCT & A T CTT T CAT CCCAAA \\
\hline JAM163Pleuc & C CATCAATCCCA & ATT TCT & ATCTTCAATCCAAA \\
\hline JAM164Pleuc & C CATCAATCCCA & ATTCCT & ATCTTCATCCAAA \\
\hline JAM168Pmbairdii & EGATCAATCCCA & ATCGCS & ATCCACATTCAAA \\
\hline JAM167Pmbairdii & G GATCAATCC CA & A TCGCC & ATCCACATT|CAAA \\
\hline JAM159Pmbairdil & GGA|TCAATCCCA & AтCGC & А T СС АСА T TCAA A \\
\hline JAM166Pmbairdil & C CA T CA A TCC CA & A T C G C C & A TCCACATTCAAA \\
\hline Microtuspennsylvanic & $|C G A| T C A \mid A T C C C A$ & ATTGCE & ATTTATATTCAAA \\
\hline Oryzomyspalustris & CGA|TCA|ATTCCC & ATTECC & ATTTGA \\
\hline Sigmodonhispidus & GGATCCATCCCC & ATT GCC & ATTUCT \\
\hline Reithrodontomysmeg & |CGATCAATTCCA & ATTGCT & A T TT \\
\hline Rattusnoryegicus & CGCTCCATCCCA & ATTCCC & A TTICAAT CCAAA \\
\hline Pmrufinus & GGAISAATCCCA & $A T C \in C C$ & A TCCACAT TICAAA \\
\hline
\end{tabular}


WC7

WC11

WC12

WC13

ijd 3256

IJd3304

IJd3306

ljd3308

IJd3317

ijd3324

IJd3327

ljd 3330

ljd3333

IJd3344

IJd3348

LD620

4JD774

WD886

LJD2088

LJD1960

LJD1669

LJD1524

LJD1523

LJD1122

LJD2522

LJD1637

LJD1638

LJD2161

LJD2651

LJD2737

LJD2834

LJD2850

LJD2898

LJD2914

LJD2936

LJD3000

LJD2126

LJD2138

LJD2154

LJD215B

LJD2836

LJD2852

LJD2860

LJD2865

LJD2890

LJD2943

Jefferson 3

ADAMSE

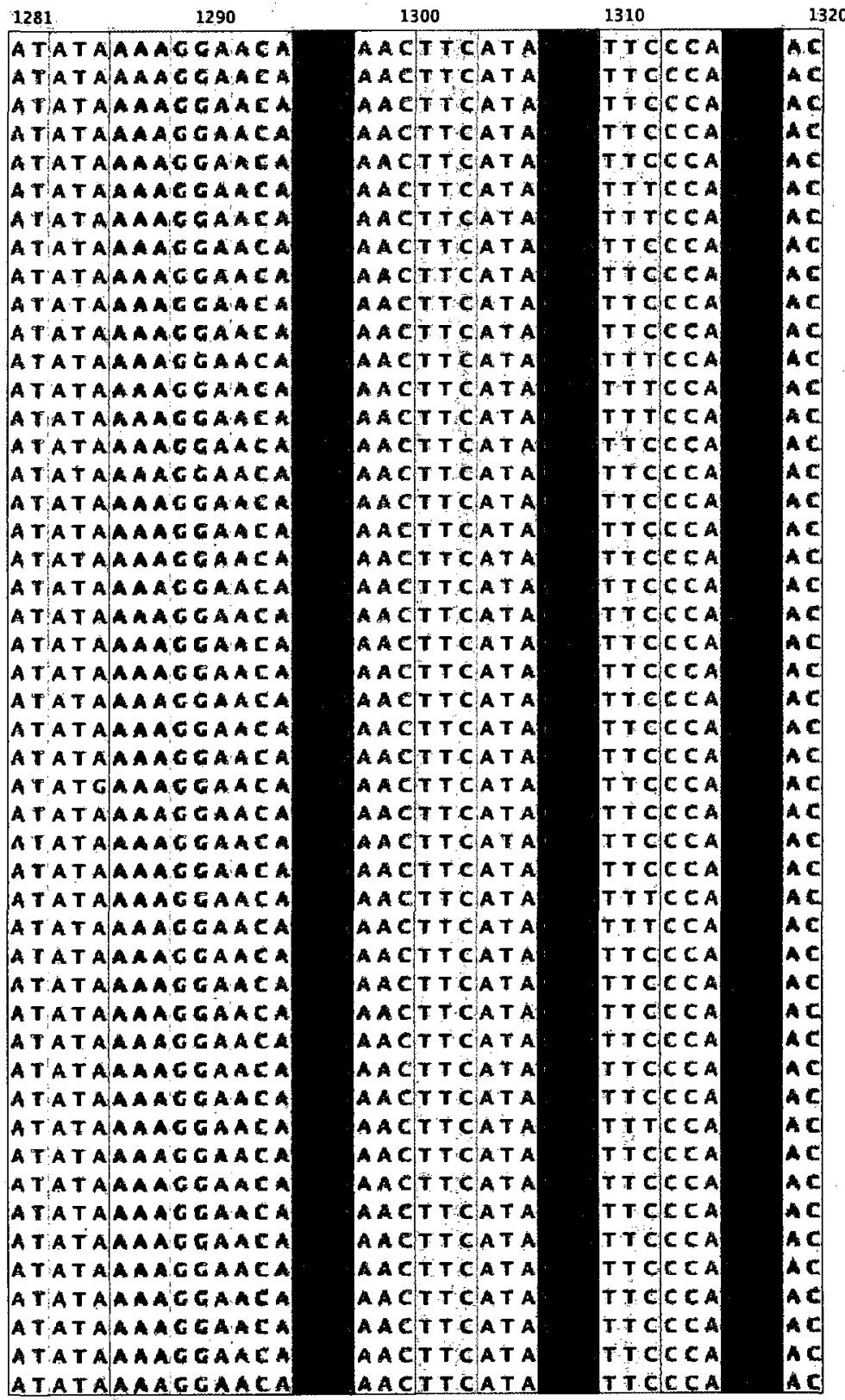


clatsop1

clatsop2

clatsop3

clatsop4

LostForest1A

LostForest2

LostForest 3

LostForest 4

sj1Pmausterus

sj2Pmausterus

sj2apmausterus

sJ3Pmausterus

sj5Pmausterus

sj6Pmausterus

sj7Pmausterus

OSM723Pmman

osM726Pmiman

OSA727Pmiman

OSM737Pmman

U40255PsejugsD

U40253PsejugsC

JEB 1309APtruei

AF374578PKEEN

U40252Pleuc

JAM160Pleuc

JAM161Pleuc

JAM162Pleuc

JAM163Pleuc

JAM164Pleuc

JAM168Pmbairdii

JAM167Pmbairdi

JAM159Pmbairdil

JAM166Pmbairdi

\begin{tabular}{|c|c|c|c|}
\hline 1281 & 1290 & 1300 & 1310 \\
\hline ATATA & WAAGGAACA & AACTTCATA & TTCCCA \\
\hline ATATA & AAAGGAACA & A ACTTC:AT A & $T T C C C A$ \\
\hline ATATA & a a ágane & A ACTTCA & T TCC $\subset A$ \\
\hline ATATA & AAACGAACA & AACTTCATA & $\operatorname{ITCCA}$ \\
\hline
\end{tabular}

A TATA A

AACTTCATA

Tecta

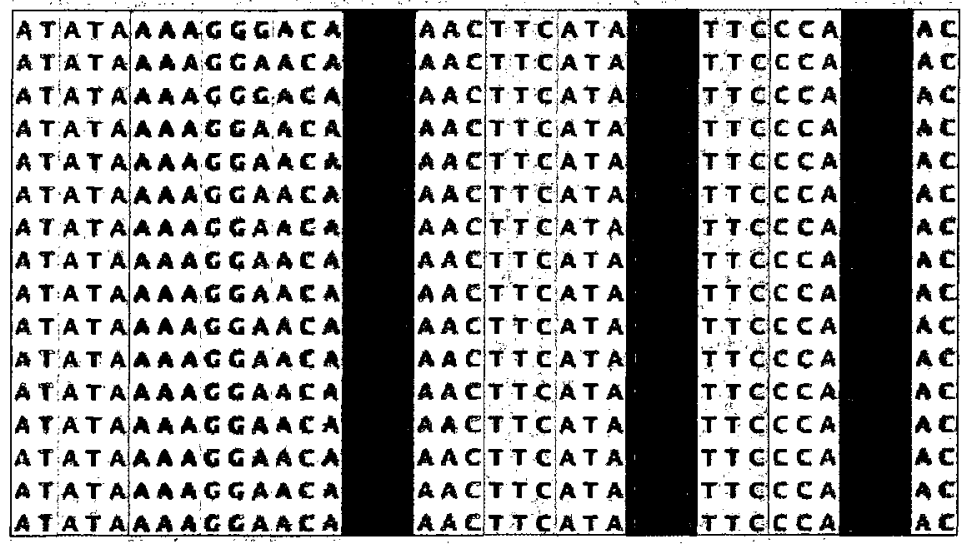

ATATAAAAGGAaCA ACTCAAAAGGAACa a CT CaAaAcGaAca actraAaAgGaÁa A CiCAAAAGGAACA ACTCAAAAGGAAGa A CTCAAAACGAACA

a tia taAaACgaACA ATATAAAAGGALCA ATATAAAAGGAACA ATATAAAACCAACA Microtuspennsylvanic A TTCAICAC CA ACA Oryzomyspalustris ACTAC GGAAAC Sigmodonhispidus AAAAT GECACC Reithrodontomysmeg $A$ T coanta Rattusnorvegicus Pmrufinus A CTCAATACGAACA ATATAIAACOAACA

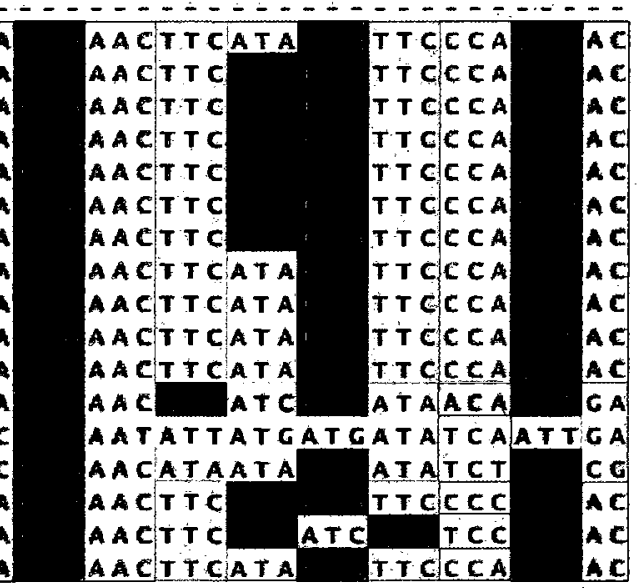


we7

we11

WC12

WC13

Jjd 3256

Ijd 3304

ljd3306

ljd 3308

Jjd3317

Jjd 3324

Ijd 3327

ljd 3330

ljd 3333

Jjd 3344

ljd3348

UD620

LJD774

UDD886

LJD2088

LJD1960

LJD1669

LJD1524

LJD1523

LJD1 122

บJD2522

UDD1637

UDD1638

LJD2 161

LJD2651

LJD2737

LJD2834

LJD2850

LJD2898

UDD2914

LJD2936

UJD3000

4JD2 126

LD2 138

LJD2154

LJD2158

LJD2836

LJD2852

WD2860

LJD2865

LJD2890

LJD2943

Jefferson 3

ADAMS6

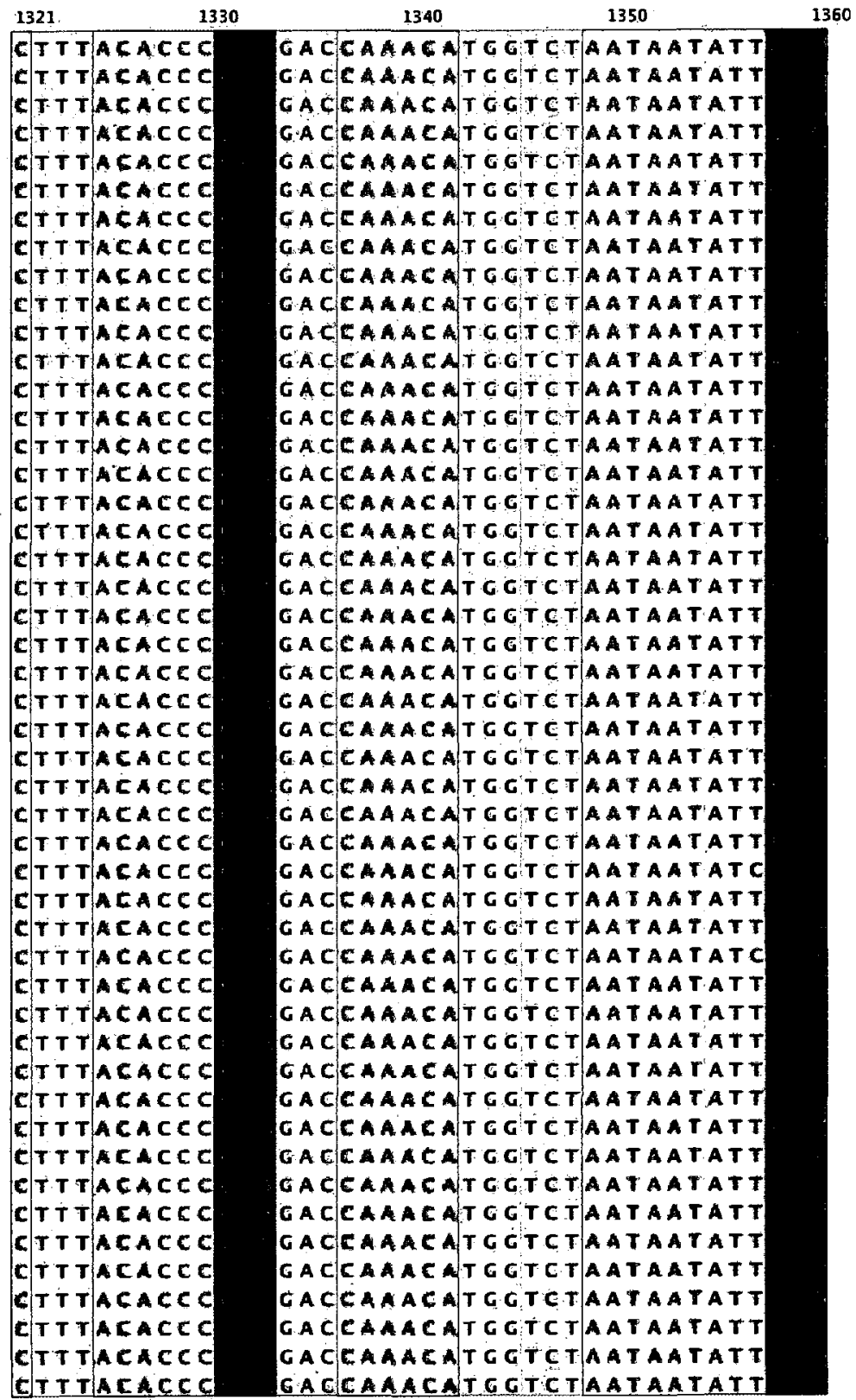




\begin{tabular}{|c|c|c|c|c|}
\hline & 1321 & 1330 & 1340 & 1350 \\
\hline clatsop1 & $\mathbf{C} \mathbf{T} \mathbf{T}$ & $A \in A C C C$ & GACCAAAEATGGTCT & AATAATATT \\
\hline clatsop2. & $\mathbf{C} \mathbf{T} \mathbf{T} \mathbf{T}$ & $A C A C C C$ & GACLAAACATGGTCT & TAATAATATT \\
\hline clatsop3 & $\mathbf{C} \mathbf{T} \mathbf{T}$ & $A C A C C C$ & GACEAAACATGGTCT & IA T A ATAT \\
\hline clatsop 4 & $\mathbf{C T} \mathbf{T}$ & ACACEC & CACEAAACATCETET & IAATAATAT \\
\hline LostförestiA & --- & $\div-----1=$ & $-1--1-1-1-1-1---1$ & ------- \\
\hline LostForest2 & C T T T & $A<A<C c$ & GACEAAACATGGTCT & ГАТАATATT \\
\hline LostForest 3 & $\mathbf{C} \mathbf{T} \mathbf{T} \mathbf{T}$ & $A \subset A C \subset C$ & GACEAAACATGGTCT & ГАТ А А T:ATT \\
\hline LostForest 4 & $\mathbf{E} \mathbf{T} T \mathbf{T}$ & $A C A C C C$ & GACEAAACATGGTCT & A ATAATATT \\
\hline sj1Pmausterus & $\mathrm{CTT}$ & $\operatorname{ACACCC}$ & GACCAAAEATGGTCT & HAVAATATT \\
\hline sj2Pmausterus & $\mathbf{E} \mathbf{T} \mathbf{C}$ & $A \subset A C C C$ & GACXAAACATGGTCT & FATAATAT \\
\hline sj2aPmausterus & $\mathbf{C} \mathbf{T} \mathbf{T} \mathbf{T}$ & $A<A C C C$ & GAC|CAAACATGGTCT & AАT A TAT \\
\hline sj3Pmạusterus & $\mathbf{C} \mathbf{T} \mathbf{C}$ & $\operatorname{ACACCC}$ & CACEAAACATGGTCT & A A Y A TATT \\
\hline sj5Pmausterus & $\mathbf{E}: \mathbf{T} \mathbf{T} \mathbf{T}$ & $A C A C C C$ & GACEAAACATGGTCT & TAATAATATT \\
\hline sj6Pmausterus & C T T T & ACACCC & CACACAACATGGTCT & A \\
\hline sj7Pmausterus & $\boldsymbol{E} \mathbf{T} \mathbf{T}$ & $\operatorname{AcAccc}$ & GACCAAACATGGTCT & НАT A ATAT \\
\hline OSM723Pmman & ETT & $A C A C C$ & CACCAAACATGGTCT & AATAATATT \\
\hline OSM726Pmman & $\mathbf{C} \mathbf{T} \mathbf{T} \mathbf{T}$ & $A \in A C C C$ & CACLAAAEATCGTCT & AAYAATATT \\
\hline O5M7.27Pmman & $\mathbf{C} \mathbf{T} \mathbf{T}$ & ACACCC & GACEAACATGCTET & A $A T A A T T$ \\
\hline O5M737Pmman & $\mathbf{C} \mathbf{T} \mathbf{T} \mathbf{T}$ & $A \subset A \subset C C$ & GACCAAACATGCTCT & A ATA T TAT \\
\hline U40255PsejugSD & C T T T & $A C A C C E$ & GACCAAACATGGTCT & AATAATATT \\
\hline U40253PsejugsC & $\mathbf{C} \mathbf{T} \mathbf{T}$ & $A<A C<C$ & GAClCAAACATGCTCT & AA PAAYATT \\
\hline JEB1309APtruei & $--\infty--$ & $\ldots \ldots$ & $--\cdots-\cdots-\cdots-\cdots$ & $\ldots-\ldots-\cdots$ \\
\hline AF374578PKEEN & $\mathbf{C T \mathbf { T }}$ & $A C A C C C$ & GACJEAAACATGGTCT & A ATTATAT T \\
\hline U40252Pieuc & $\mathbf{T} \mathbf{T} \mathbf{T} \mathbf{C}$ & $A \subset A C C C$ & A ATCAAACATGATCT & A ACAACATT \\
\hline JAM160Pleuc & T T T C & $\operatorname{Actcc}$ & A A TAAACATG ATCTT & $A A C A A C A T T$ \\
\hline JAM16 IPleuc & $\mathbf{r} \mathbf{T} \mathbf{T}$ & $\operatorname{seccc}$ & 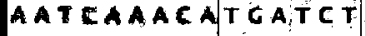 & AACAACAT \\
\hline JAM162Pleuc & $\mathbf{T} \mathbf{T} \mathbf{T} \mathrm{C}$ & $A \subset A C C C$ & ААТСAАACATGATCT & AACAACAT \\
\hline JAM163Pleuc & $\mathbf{T} \mathbf{T} \mathbf{C}$ & $A \subset A c C C$ & A ATCA A AC AT G ATCT & $\operatorname{ACAACATT}$ \\
\hline JAM164Pleuc & $\mathbf{T} \mathbf{T} \mathbf{T} \mathbf{C}$ & $A \subset A C E C$ & AATCAAACATTATCT & AACAACATT \\
\hline JAM168Pmbairdii & $\mathbf{C} \mathbf{T} \mathbf{T}$ & $A \subset A<C c$ & CACXAAACA T G GTCC & $A A T A A T A T T$ \\
\hline JAM167Pmbairdii & CT T C & $\operatorname{Acacc}$ & G ACICAAACATGGTCC & AATAATATT \\
\hline JAM159Pmbairdil & C.T T & $A \subset A C C$ & GACCAAACATGGTCC & AATAATATT \\
\hline JAM166Pmbairdii & C. T. T & $\operatorname{ACACCC}$ & GACEAAACATCGTCC & AATAATATT \\
\hline Microtuspennsylvanic & $C T C$ & TCACCAAT & $A A C C A A A C A T C A T C T$ & A ACA А A TC \\
\hline Oryzomyspalustris & CTC C & CAAACAATA & $|A C T| \quad T C T T G A T C A$ & A ATGATAT T \\
\hline Sigmodonhispidus & $\mathbf{C} \mathbf{T} \mathbf{C} \mathbf{T}$ & CACCCTAT & GACLCCITCTTGATCA & A AT A T T TT \\
\hline Reithrodontomysmeg & CT T C & $A C T \leq A A$ & AATCAAACATGATCT & - AATAACATT \\
\hline Rattusnorvegicus & $A \wedge C A$ & $\operatorname{caccc}$ & CCCI CA A ATUATEC & AACACCATT \\
\hline Pmrufinus & $\mathrm{CTT}$ & $A C A C C C$ & GACLAACATGGTCT & AATAATATI \\
\hline
\end{tabular}


WC7

WC1]

WC12

WC13

Jd 3256

IId3304

IJd3306

Ijd3308

Ijd3317

IJd3324

ljd3327

Ijd3330

ljd 3333

IJd3344

Jjd3348

U.J0620

WD774

4JD886

LJD2088

LD1960

LJD1669

LJD1524

LJD1523

LJD1 122

LJD2522

LJD1637

LJD1638

LJD2161

LJD2651

LJD2737

LJD2834

LJD2850

LJD2898

LJD2914

LJD2936

LJD3000

LJD2126

LJD2138

LJD2 154

LJD21 158

LJD2836

LJD2852

LJD2860

LJD2865

LJD2890

LJD2943

Jefferson 3

ADAM56

$1361 \quad 1370 \quad 1380 \quad 1390 \quad 1400$

TGA GCATGTATAATGGCATTTATGATTAAAATACCA İGA GCATGTATAATGGCATTTATGATTAAAATACEA TG G GCATGTATAATCGCATT TATGATTAAAATACCA GCATGTATAAT GGCATTTATGATTAAAATACCA G CAT GTATAAT GGCATT TATGATTAAAITACCA GCATGTATAATGGCATTTATGATTAAAATACCA G CAT GTATAAT GG CATTTATGATTAAAATACCA G CAT GTATAAT GG CATTTATGATTAAAATACCA GCAT GTATAATGGCATTTATGATTAAAATACCA G CAT GTATAATGGCATTTATGATTAAAATACCA G CAT GTATAAT GG CATTTATGATTAAAATACCA G CAT GTAT AATGGCATTTATGATTAAAATACCA G C AT GTATAAT GCCATTTATGATTAAAATACCA GCAT G TAT AAT GG CAT TTATGATTAAAATACCA GCAT GTATAATCGCATTTATGATTAAAATACCA GCAT G TATAATGGCATT TATGATTAAAATACCA GCAT G TATAATGGCATTTATGATTAAAATACCA GCATGTATAATGGCATTTATGATTAAAATACCA G CAT G TATAATGGCATTTATGATTAAAATACCA GCATGTATAATCGCATTTATAATTAAAATACCA G CAT G TATAATCG CAT T TATAATTAAAATACCA G CAT GTATAATGGCATTTATGATTAAAATACCA GCATG TATAATGGCATTTATGATTAAAATACCA G CAT G TATAATCG CATT TATGATTAAAATACCA G CATG TATAAT GGCATTTATGATTAAAATACCA G CAT CTATAATGGCATTTATGATTAAAATACCA G CAT GTATAATGGCATTTATGATTAAAATACCA GCATGTATAATGCCATTTATCATTAAAATACCA G CATG TATAATAGCATTTATGATTAAAATACCA G CAT G TATAATG CATT TATGATTAAAATAC CA G C ATG TATAATGGCATTTATGATTAAAATACCA GCATCTATAATCG CATTTATGATTAAAATACCA G C AT G TATAAT GGCATTTAT GATTAAAATACCA GCAT GTATAAT GGCATTTATGATTAAAATACCA GCAT GTATAAT GCCATTTATGATTAAAATACCA CCAT GTATAATGCCATTTATCATTAAAATACCA GCATGTATAATGGCATTTATCATTAAAATACCA G CAT GTATAATGGCATTTATGATTAAAATACCA GCAT GTATAATGGCATT TATGATTAAAATAC CA GCATGTATAATGGCATTTATGATTAAAATACCA GCAT GTATAATGGCATTTATGATTAAAATACCA GCAT G TATAATGGCAT T TATGATTAAAATACCA GCAT G TATAAT GG CATTTAT GATTAAAATACCA G CATG TATAAT GGCATTTATGATTAAAATACCA G CAT G TATAAT GG CATTTATCATTAAAATACCA C CAT GTATAATGGCATTTAT GATTAAAATACCA GCATGTATAAT GGCATTTATGATTAAAATACCA $\mathrm{T} A$ GCATGTATAATGGCATTTATGATTAAAATACCA 
clatsop1

clatsop2

clatsop 3

clatsop4

LostForestiA

LostForest2

LostForest 3

Lostforest 4

sj1Pmausterus

sj2Pmausterus

sj2aPmausterus

sj3Pmausterus

sj5Pmausterus

sj6Pmausterus

sj7Pmausterus

oSM723Pmman

oSM726Pmiman

OSM727Pmman

OSM737Pmman

U40255PsejugSD

U40253PsejugSC

JEB1309APtruei

AF374578PKEEN

U40252Pleuc

JAM160Pleuc

JAM161Pleuc

JAM162Pleuc

JAM163Pleuc

JAM 164Pleuc

JAM168Pmbairdii

JAM167Pmbairdii

JAM 159Pmbairdil

JAM166Pmbairdil

Microtuspennsylva

Oryzomyspalustris

Sigmodonhispidus

Relthrodontomysm

Rattusnorvegicus

Pmrufinus \begin{tabular}{|l|l|l|l|}
1361 & 1370 & 1380 & 1390 \\
\hline TGA & CCATGTATAATGGCATTTATGATTAAAATACCA \\
TGA & GCATGTATAATGGCATTTATGATTAAAATACCA \\
TGA & GCATGTATAATGGCATTTATGATTAAAATACCA \\
TGA & GCATGTATAATGGCATTTATGATTAAAATACCA
\end{tabular} GCATGTATAATGGCATTTATGATTAAAATACCA

TGA GCATGTATAATGGCATTTATGATTAAAATACCA TTGA GCATGTATAATGGCATTTATGATTAAAATACCA $\mathbf{T} \mathbf{A}$

$T \in A$

$\mathbf{T} \cdot \mathbf{G}$

$\mathbf{T} \mathbf{A}$

T G A

$T \in A$

$T \in A$

T G A

$T \in A$

T C A

$T G A$

T G A

T $\mathbf{A}$

T GA

G CATGTATAATGGCATTTATGATTAAAATACCA G CAT GTATAATGGCATTTATGATTAAAATACCA G CAT G TATAATGG CATTTATGATTAAAATACCA GCATG TAT AATGGCATTTATGATTAAAATACCA G CAT G TATAAT GCCATTTATGATTAAAATACCA G CAT G TATAAT GCCATT TATGATTAAAATACCA G CAT GTATAAT GGCATT TATGATTAAAATACCA GCAT G TAT AATGG CATTTATGATTAAAATACCA GCATG CATAATGGCATTTATAATTAAAATACCA C CAT GCATAATCG CATTTATAATTAAAATACCA GCATGCATAATGGCATTTATAATTAAAATACCA GCATG CATAATGGCATTTATAATTAAAATACCA GCATC CATAAT GCCATTTATAATTAAAATGECA

TGA GCATGCATAATGGCATTTATGATTAAAATACCA TGA GCCTGCATAATGGCATTCATAATTAAAATACCA TGA CCGTCCATAATGCCATTCATAATTAAAATACCA TGA GCGTGCATAATGGCATTCATAATTAAAATACCA

$\mathbf{T} \mathbf{A}$

T G A

$\mathbf{T} \mathbf{G}$

T $\mathbf{G}$

$\mathbf{T} \mathbf{A}$

$\mathbf{T} \mathbf{A}$

T G A GCGTCCATAAT GCCATTCATAATTAAAATACCA GCGTG CATAATGGCATTCATAATTAAAATACCA C C GT G CATAATCGCATTCATAATTAAAATA'C CA GCAT G TATAATGGCATTTATAATTAAAATACCA G CAT G TATAAT GGCATTTATAATTAAAATACCA GCATGTATAATGGCATTTATAATTAAAATACCA G CAT G TATAATCG CATTTATAATTAAAATACCA ic $\mathbf{T} \mathbf{A}$ GCCTGTATTAT GGCTTTCATAGTTAAAATACCTGCATGCATA GCCTTT GTGAAAATACCCGCGTGCATAATAGCATTT ATCAAAATGCCCGCCTGCATGATAGCATTC ATTAAAATACCTG CAT GTATAATAG CATTTATAATCAAAATAC CA GCATGCATAATGGCATTTATGATTAAAITACCA 
WC7

WC11

WC12

wC13

IJd3256

IJd3304

Jjd3306

ljd3308

IJd3317

Ijd3324

ijd3327

ljd3330

jjd3333

IJd3344

IJd3348

UDD620

UDD774

HD886

UD2088

LD1960

UDD1669

LDD1524

UD1523

LDD1122

UDD2522

UJD1637

UD1638

LD2161

LJD2651

UJD2737

LJD2834

LJD2850

LJD2898

LJD2914

LJD2936

LJD3000

LJD2126

LJD2138

LD2154

UD2158

UJD2836

UD2852

UD2860

LJD2865

LJD2890

LJD2943

Jefferson 3

ADAMS6

\begin{tabular}{|c|c|c|c|c|}
\hline 1401 & 1410 & 14 & 1430 & 14 \\
\hline TAC & GGATCCAC & T G A & cctaAsGc & $\mathbf{C A C}-\cdots$ \\
\hline T AC & CGACTCCAC & TEA & CCTAAAGCC & EACGAG]AA \\
\hline T A C & 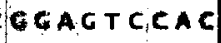 & T G A & CCTAAACEC & $C \ldots$ \\
\hline T AC & GOAGTCCAC & $T G A$ & C CTAAAGCC & CAClg - - - \\
\hline T A C & $\operatorname{cGAGTCCAC}$ & $\mathbf{T} \mathbf{G} \mathbf{A}$ & CETAAAGCC & $\operatorname{CACG}-\ldots$ \\
\hline TAC & CGACTCtec & $\mathbf{T} \mathbf{G}$ & CCTAAAGCC & $|\boldsymbol{C A C}| \mathrm{C}_{-\cdots}$ \\
\hline TA C & GCACTCEAC & T G A & CСTAAAGCC & C ACG $-\cdots$ \\
\hline TAC & CoAGTCAC & $\ddot{T} G A$ & C C TAA AGC C & $\mathbf{C A C} \mathbf{G}-\cdots$ \\
\hline TAC & GGAGTCCAC & T G A & CCTAAAGCC & CACGTAG \\
\hline TAC & teactecac & T G A & $C \subset T A A A G C C$ & CA $-\ldots$ \\
\hline T AC & CEAGTCCAC & T G A & CCTAAACCC & CACGTA- - \\
\hline TAC & G GAGTCCAC & T G A & CС TAAAGCC & C A c $\mathbf{G}$ T - - \\
\hline TAC & CGAGTCEAC & T G A & CСTAAAGCC & $|<A C| \cdots-$ \\
\hline T A C & GGA T TCAC & $T \in A$ & CETAAAGCC & CACG T - - \\
\hline TAC & Genetecac & T G A & $\operatorname{CCTAAACCC}$ & $C A=-\cdots-$ \\
\hline T AC & CGAGTCCAC & $T G A$ & CCTAAACCC & CACG TAGA \\
\hline TAC & GGAGTCCAC & $T G A$ & $C \in T A A A G C C$ & CACGTAGA \\
\hline T A C & CGACTCCAC & $T \in A$ & $\operatorname{CCT} A \wedge A \mid C C$ & $C A C G T A G A$ \\
\hline TAC & CaActeac & T G A & CeтAAAGCC & CACGTAL- \\
\hline T A C & CGACTCCAC & T G A & CCTAAAGCC & CA C:C T A.CA \\
\hline T AC & GAGTCEAC & T G A & CETAAAGCC & CACGTAGA \\
\hline T A C & CGAGTecac & T G A & $C \subset T \wedge A A G C C$ & $\operatorname{CACGTA--}$ \\
\hline TAC & CGAGTCEAC & $T \in A$ & CCTAAAGCC & $C A C \ldots$ \\
\hline TAC & CGACTCAC & 广 $\mathbf{C A}$ & ectaA $\operatorname{sic} c$ & CAclu A - - - \\
\hline $\mathbf{T}$ A C & CEACTCCAC & $\mathbf{T} \mathbf{C} \mathbf{A}$ & CCTAAAGEC & CACGTAGA \\
\hline T A C & CGAGTCEAC & T G A & CСTAAAGCC & $\ldots \ldots \ldots$ \\
\hline T A C & Gactecac & T G A & $C \subset T_{A \wedge} \wedge \subset C$ & $c \wedge \mathrm{C} \mathrm{C}_{--\cdots}$ \\
\hline TAC & CGaGtecac & T G A & C CTAAAG-- & $\because--\cdots$ \\
\hline T A C & CGAGTCCAC & $T \in A$ & CCTAAAGCC & CAC \\
\hline T A C & G6G6TCCAC & $T \in A$ & CСTAAAGCC & $C A C G$ I A G \\
\hline T A C & CGACT CCAC & $T G A$ & CCTAAAGCC & $\mathrm{CAC}-\cdots$ \\
\hline TAC & CGAGTCCAC & T G A & CETAAAGCC & $|C A C| \cdots$ \\
\hline T A C & $\operatorname{cec} T \operatorname{coc}$ & $T \in A$ & C C TA A A C C & CAC $\ldots .$. \\
\hline T A C & $\operatorname{coA} \mathrm{TCCAC}$ & TGA & CETAAAGCC & $|\operatorname{cAc}| \cdots-\cdots$ \\
\hline $\mathbf{T A C}$ & CGACTCCAC & $T \in A$ & $C \subset T^{\prime A A A G C C}$ & CAC] - . - \\
\hline TAC & GCACTCCAC & $T \in A$ & CCTAAACCC & $C A-\cdots$ \\
\hline TAC & Geacrcenc & $\mathbf{T} \in \mathbf{A}$ & $C \subset T_{A} A$ ACC & $\operatorname{CACGTA-}$ \\
\hline $\mathbf{T} \mathbf{A C}$ & CGACTCCAC & $T \in A$ & CCTAAAGCC & $|\operatorname{cac}|-\cdots$ \\
\hline T A C & gGagtceac & $T \in A$ & CCTAA TACC & $\operatorname{CAC}=-\cdots$ \\
\hline TAC & GGA TCCAC & $T \cdot \mathbf{A}$ & $C \subset T \wedge A \wedge G \subset C$ & $\mathbf{c A c}]_{-\cdots}$ \\
\hline T A C & GGACTCEAC & $\mathbf{T} C A$ & CCTAAACCC & $c \ldots-\cdots$ \\
\hline TAC & GGATCCAC & T G A & CGTAAAGEC & CAC $\ldots \cdots$ \\
\hline T A C & GGAGTCCAC & $T G A$ & $c \in T A A \wedge G C C$ & $|c \wedge c| \ldots$ \\
\hline T A C & G GAGTCAC & $T \in A$ & c craAAGCC & {$[\mathbf{C A C} \mid \cdots$} \\
\hline TAC & CGACTCCAC & $T \in A$ & $C \in T_{A} A C_{C-}$ & $\ldots-\cdots$ \\
\hline TA C & CGAGTECAC & T G A & CCTAA $\sqrt{G C C}$ & $\ldots \ldots$ \\
\hline TAC & GGAGTCCAC & T. A & ectaA TGec & CACGTAGA \\
\hline TAC & GGAGICCAC & TGA & CCTAAAGCC & CACIGTAGA \\
\hline
\end{tabular}




\begin{tabular}{|c|c|c|c|c|}
\hline & 1410 & 1420 & 1430 & \\
\hline clatsop1. & TACGGAGTCEAC & $T G A$ & CCTAAACCC & CACGTAAA \\
\hline clatsop2 & TACGGAGTCICAC & T G A & ECTAAAGCC & CACGTAAA \\
\hline clatsop3 & TacGGátceac & $\mathbf{T} \mathbf{A}$ & CCTAAAGCC & $|C A C G T A \wedge A|$ \\
\hline clatsop 4 & TACCEACTCCAC & T. A A & CEAAAGCC & EAEGTAAa \\
\hline LostForest1A & $----------1-7$ & $---=$ & $m-\cdots-\cdots-$ & $=-\ldots-\ldots$ \\
\hline LostForest2 & TACGGAGTCEAC & $\mathbf{T} \in \mathbf{A}$ & CCTAAAGCC & CACLGAA \\
\hline LostForest 3 & TACGGACTCCAC & T G A & CCTAAAGCC & CACG I A.CA \\
\hline LostForest 4 & TACCGAG TCCAC & TGA & CCTAAAGCC & CACGTAGA \\
\hline sj 1Pmausterus & TACGGAGTCEAC & TCA & CCTAAAGCC & CACG -- \\
\hline sj2Pmausterus & TACGGAGTCEAC & $T \in A$ & CCTAAACCC & CAC $\quad G A$ \\
\hline sj2aPmausterus & TACGGAGTCAC & TGA & CCTAAAGCC & EA $\mathbf{c}$ - $-\cdots$ \\
\hline sj3Pmausterus & TACGAAGTCCAC & TGA & CCTAAAGCC & $\mid \mathbf{C A C}-\cdots$ \\
\hline sj5Pmausterus & TACGCAG TC:CAC & T G A & CCTAAAGCC & CA $-\cdots$ \\
\hline sj6Pmausterus & TACGGAGTCEAC & $T G A$ & CСTAAAGCC & CAC $-\cdots$ \\
\hline sj7Pmausterus & TACGGAG TCCAC & TOA & C CTAAAGCC & CA A $\mathbf{G T}=-$ \\
\hline OSM723Pmman & TACCGAGTCCAC & TCA & $C \in T^{\prime} A A A G C C$ & CACGTAGA \\
\hline OSM726Pmiman & TACGGAGTCCAC & T G A & CCTAAACC & CACGTAGA \\
\hline OSM727Pmman & TACGGAGTCCAC & $T G A$ & CCTAAAGCC & $\operatorname{CacGTa}$ \\
\hline OSM737Pmiman & TACGGAGTCCAC & $T G A$ & С С TAAAGCC & CACGTAGA] \\
\hline U40255PsejugSD & T ACGGACTC:CAC & T G A & CСTAAACCC & CACGTAGA] \\
\hline U40253PsejugsC & TACCEACTCEAC & TCA & CetAAACCC & CACGTAGA \\
\hline JEB 1309APtruei & $--\ldots-\cdots-\ldots-\cdots$ & $-\ldots$ & $--\ldots-\ldots-\ldots$ & $-\ldots-\cdots--$ \\
\hline AF374578PKEEN & TACGOAG YCEAC & $T G A$ & C CTAAACCC & CACGTAGA \\
\hline U40252Pleuc & TACGGGGTCEAC & T.GA & $\operatorname{CCCAAAGCC}$ & CACGTAGA \\
\hline JAM160Pleuc & TACCECGTCAC & $\mathbf{T} \in \mathbf{A}$ & CCCAAACCC & CAECTALCA \\
\hline JAM161Pleuc & TACGGGGTECAC & $T \in A$ & C C CAAAGCC & CACGTAGA \\
\hline JAM162Pleuc & TACGGGGTCCAC & T G A & $\operatorname{cccAAACC}$ & CAC[G:- - \\
\hline JAM163Pleuc & TACGGGCTCCAC & $T G A$ & $\operatorname{ccc} A \wedge A G \subset c$ & CACGTAGA \\
\hline JAM164Pleuc & Tacecoctecac & T C A & $\operatorname{cccha} \operatorname{Gcc}$ & $\operatorname{CACGTA} G$ \\
\hline JAM168Pmbairdii & TACLCACTECAC & T GA & CCTAAAGCC & $\operatorname{CAC} G_{--\cdots}$ \\
\hline JAM167Pmbairdii & TACGGAGTCCAC & T G A & $C \subset T A \wedge A G \subset C$ & $\mathbf{C} \wedge \mathrm{C}-{ }_{-}--$ \\
\hline JAM159Pmbairdii & TACGEAGTCCAC & $T G A$ & CCTAAAGCC & CACGIA:- \\
\hline JAM166Pmbairdil & TACGGAGTCCAC & TCA & CETAAAGCC & CAC $\cdots$ \\
\hline Microtuspennsylva & & & $\ldots-\cdots$ & $\cdots \cdots$ \\
\hline Oryzomyspalustris & & & -------- & $-----\cdot$ \\
\hline Sigmodonhispidus & $\cdots-\cdots-\cdots-\cdots$ & & $-\cdots-\cdots$ & $\cdots \cdots$ \\
\hline Relthrodontomysm & 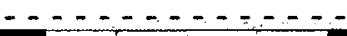 & & $----\ldots-\cdots$ & $----2--$ \\
\hline Rattusnorvegicus & TACGGACTCCAT & $\mathbf{T} \mathbf{G}$ & CEAAAAGCC & CACG TAGA \\
\hline Pmrufinus & TACGGAGTCEAC & $\mathbf{T} \mathbf{A} A$ & CCTAAAGCC & CACGTAGA \\
\hline
\end{tabular}


wc7

wC11

wC12

WC13

ijd 3256

ljd3304

jd 3306

jjd3308

|jd 3317

Ijd3324

Hd 3327

tjd 3330

ijd 3333

ljd 3344

ljd3348

UD6 20

LjD774

LJD886

LD2088

LJD1960

LJD1669

LJD1524

LJD1523

LDD1122

LjD2 522

LJD1637

LJD1638

LJD2161

LD2651

LJD2737

LJD2834

LJD2850

LJD2898

LJD2914

LJD2936

LJD3000

LJD2126

LDD2138

LJD2 154

LDD2158

LJD2836

LJD2852

UJD2860

LJD2865

LJD2890

LJD2943

Jefferson 3

ADAMS6
1441 1449

AGCTICCAAA

$\therefore--\therefore=-1-$

- - - - - - - - -

- - - - - . -

- $-\overline{-}-\mathrm{C} \cdot-$

$-\cdots--\cdots$

GGCTICCCA

- - - - - - ..

- - - - - - - -

-... - - - -

- - - - - - - -

- $-\cdots$

..........

$A C G T C C A-$

AGCTeCA - -

AGETCCA

AACGTCA

AACECOA

- - - - - - -

- - - - - - -

- - - - - -

B - - - - -

- - - - - - -

- - - -.. - - .

- + . . . . .

ACTCCAA -

- - - - - -

- - - - - -

- - - - - - -

- - - - - -

- - . - - . -

........

- . . . . .

-........

- . - . - - -

..........

- $-. . \cdot-\cdot$

- - - - - - -

- - - . - - - -

.. - . . -

- - . - - - -

Fotrect.-

ACETCCA - 


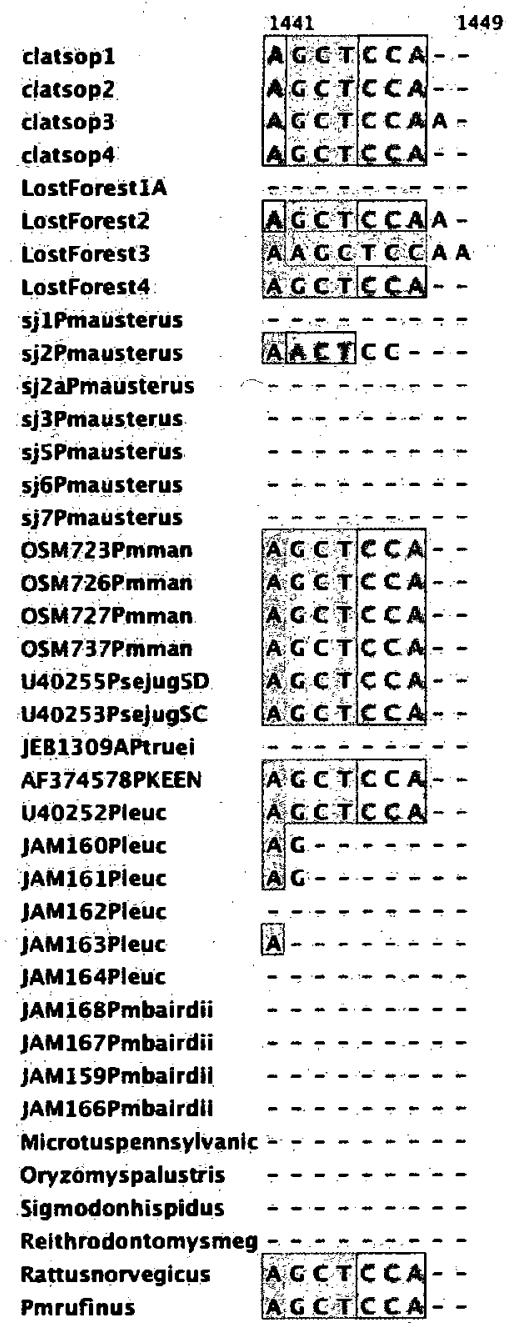


Appendix H: Hantavirus Multiple Sequence Alignment. Sequences of M segment G1/G2 glycoprotein precursor gene aligned with one another and adjusted to codon reading frame. $T^{*}=$ Troutdale, $\mathrm{WC}=$ Washington County, $\mathrm{WCps}=$ Washington County patient sample, $\mathrm{LJD}^{*}=$ Portland area specimens collected for this research.

\begin{tabular}{|c|c|c|c|c|c|c|c|c|}
\hline & 1 & 10 & & & 20 & & 30 & \\
\hline$T 7$ & A T T & G AGTG GGAG & A.CA & $\mathbf{C} \subset \mathbf{A}$ & AAAGAA & T G. & $A \subset A$ & CATAAGA \\
\hline T8 & A T & G AGTGGG AG & $A \subset A$ & $\mathbf{G} \mathbf{C} \mathbf{A}$ & A A A GA & T G G & $A C A$ & CATA A \\
\hline WC4 & A T & GAGTG GGAG & $A \subset A$ & $G C A$ & AAAGAA & $G \in A^{\prime}$ & $A \subset A$ & CATAAGA \\
\hline WCps & A T & G AGT G GG AC & $|A C A|$ & C C A & $A A \wedge C A A$ & G CA & $A C A$ & CATAAG \\
\hline wic7 & A T & $G A \dot{E} T C G C A C$ & $A \subset A$ & $G \in A$ & AAAGAA & $G 6 \mathrm{~A}$ & $A C A$ & CATAAG \\
\hline WC12 & $\mathbf{A} \mathbf{T}$ & GAGTGGGAG & ACA & $C \subset A$ & AAAGAA & G F A & $A C A$ & CATAAGS \\
\hline wC13 & A T & GAGTGGGAG & $|A \subset A|$ & GCA & $A \wedge A G A A$ & GA & $\mathbf{A C A}$ & CATARGA \\
\hline LJD1523FP & A T & G ACTG GG AG & $A \subset A$ & G E A & A A A A A & $\mathbf{T G}$ & $A \subset A$ & CATAAGA \\
\hline ㄴJD2126FP & $\mathbf{A T}$ & GAGTGGGAC & $|A \in A|$ & $|C \subset A|$ & A A ACA A & GA E & ACA & CATAAGA \\
\hline LJD2138FP. & A T & G A G T GG AC & $A \subset A\}$ & G C A A & $\triangle A A G A A$ & GA G & $A<A$ & CATAAGA \\
\hline LJD2 154FP & AT & G AGTG CGAC & $A C A$ & C C A & $A \wedge A G A \cdot A$ & GA & $A \subset A$ & CATARAA \\
\hline LJJD2493FP. & $\mathbf{A} \mathbf{T}$ & GAGTGGCAC & $A \subset A \mid$ & $\mathbf{G C A}$ & A A ACA A & G A G & $A \subset A$ & CATAACA \\
\hline LJJD2494FP & A T & GAGT G GGAC & $A \subset A\}$ & $|C \subset A|$ & $A A A C A A$ & G AC: & $A \subset \mathbf{A}$ & CATAAGA \\
\hline LJD2497FP. & A T & GAGTEGGAG & $A \subset A$ & $G \subset A$ & $A A A C A A$ & GAG & $A \subset \wedge$ & CATAAGA \\
\hline LJD2499FP & $\mathbf{A} \mathbf{T}$ & GA GTGGGAC & $|A C A|$ & $\mathbf{C} \subset \mathbf{A}$ & $A A A G A A$ & G A G & $A \subset A$ & CATAAGA \\
\hline UJD2520FP & A T & GAGTGGGAG & $A C A \mid$ & GCA & $A A \wedge G A A$ & G AG & $A C A$ & $C A T A A G A$ \\
\hline LJD2522FP & A T & G A G T G G: A & $A \in A$ & $\mathbf{C} \subset \mathbf{A}$ & $A A A C A A$ & G A C & $A<A$ & CATAAG \\
\hline LJD2737PB & A T & GACT G GCAC & $A \subset A$ & $\mathbf{C C A}$ & $A \cap A G A A$ & T G C & Aca & CATAACA \\
\hline LjD2836FP & $\dot{\mathbf{A}} \mathbf{T}$ & G AGTCGGAG & & $C \subset A$ & $A \wedge A G A A$ & G A C & $A<A$ & CATAAGA \\
\hline LJD2852FP & A T & G A G TG GGAG & & & A A A $A$ A & C A G & $A \subset A$ & CATAAG \\
\hline LJD2860FP & A T & G A GTG GGAG & $C A$ & GCA & $A A A G A A$ & C A G & $A<A$ & CATAAGA \\
\hline LJD2865FP & A T & G AGT G GG AG & $A \subset A$ & GCA & $A A A G A A$ & CA C & $A \subset A$ & CATAAGA \\
\hline LJD2890FP. & A T & G AG T, G G A C & & $G \subset A$ & $\triangle A A \subset A A$ & G A G & $A \subset A$ & CATAAGA \\
\hline convictcreek & $\mathbf{A} \mathbf{T}$ & G A A T G TGA G & & & $A A A G A G$ & & $A C A$ & CATAAGA \\
\hline SNVL25783 & AC & G A A T C CG A A & & & $A A A C A A$ & GA A & $A<a$ & CATAAGA \\
\hline SNVL37903 & $A C$ & CAATGCUA A & $A \subset A$ & $\mathrm{cc}$ & A A ACA A & G A A & $A \in A$ & CATAA \\
\hline NewYorkVirusU3680 & A T & G A G T.C TG A A & & $\mathbf{G C A}$ & A A A A G & CA A & $A C T$ & $\operatorname{CATAAGA}$ \\
\hline BlueRiverAF030552 & A T & $\mathbf{G} \mathbf{A} \mathbf{T}: \mathbf{G} \mathbf{T} \mathbf{G} \mathbf{A} \mathbf{C}$ & & G C T & $A A \wedge G A G$ & G A T & $A C A$ & $C A C A A \wedge A$ \\
\hline BayouvirusL36930 & $\mathbf{A} \mathbf{T}$ & G AGTGTGA A & & & $A A A C A$ & G A G & GGA & CATAAAA \\
\hline BlackCreekCanalk 39 ! & $\mathbf{A} \mathbf{T}$ & GAATGTGA A & $A C A$ & G C A & A A A $A$ A & GA A & ACC & CATAAAA \\
\hline AndesVirusNCOO346 & $\dot{A} \mathbf{T}$ & G AG T G T $G A$ & $A \subset A$ & $G \in A$ & $A \cap A C A A$ & CA A & $\mathbf{T} \subset \mathbf{A}$ & CATAGAC \\
\hline Hu39694virusAF028 & A T & G A G TG TGAA & $A \subset A$ & GCA & $A A \wedge(G A A$ & GA A & $\mathbf{T} \mathbf{C C}$ & CATAAAC \\
\hline OranVirusAF028024 & A T & GAATGTGAG & $A \subset A$ & GCA & A A $A \mathrm{G}$ & GA A & $\mathbf{T} \subset \mathbf{A}$ & CATAAAC \\
\hline Lech!guanasvirusAFC & & GAGTGTGAC & & & & CA A & $\mathbf{T C T}$ & CAIAACE \\
\hline Juquitibalikevirus 16 . & & -------- & $-\ldots$ & 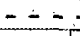 & $----\cdots$ & $-=-$ & --- & $-\infty-\cdots-$ \\
\hline MacielvirusAF02802: & AC C & G A A T G TIGAA & $A \subset A$ & $\mathbf{G} \mathbf{c}$ & AAAGAG & G A A & $T \subset A$ & CATAG A.C \\
\hline ElMoroCanyonU2682 & & GAATG TGA A & $A C A$ & & AA ACAA & G AT: & $\mathbf{T} \mathbf{C T}$ & CATAAAA \\
\hline ProspectHillX55129 & A G & GAATG TGAA & A T & & $A \triangle A C A A$ & GAC & $\mathbf{T C T}$ & CAEAACA \\
\hline Tulavirus NCD05228 & $\mathbf{A} \mathbf{A}$ & GATCTOAC & $A C T$ & $G \subset A$ & AAGCAC & $\mathrm{CAA}$ & $\mathbf{T C T}$ & CACAAAA \\
\hline PuumalavirusNCO05: & $\mathbf{A} \mathbf{T}$ & G AGT G TG A A & $A \subset T$ & $G \subset T$ & $A A G G A G$ & GAA & TCA & CATAG AIA \\
\hline Hantaanvirus DQ371! & A T & G AGTG TGAG & a C T & $\mathbf{T A T}$ & AAACAA & $A \wedge \in$ & GCA & CATGGGG \\
\hline DobravavirusNC005z & $\mathbf{A T}$ & GAGTG TGAA & & & & $A \wedge A$ & Gec & CAIAAT \\
\hline SeoulVirus NCOOS237 & & GAGTGTGAA & $A \subset A$ & & $A A G G A A$ & $A \wedge G$ & GEA & CATAAT: \\
\hline
\end{tabular}




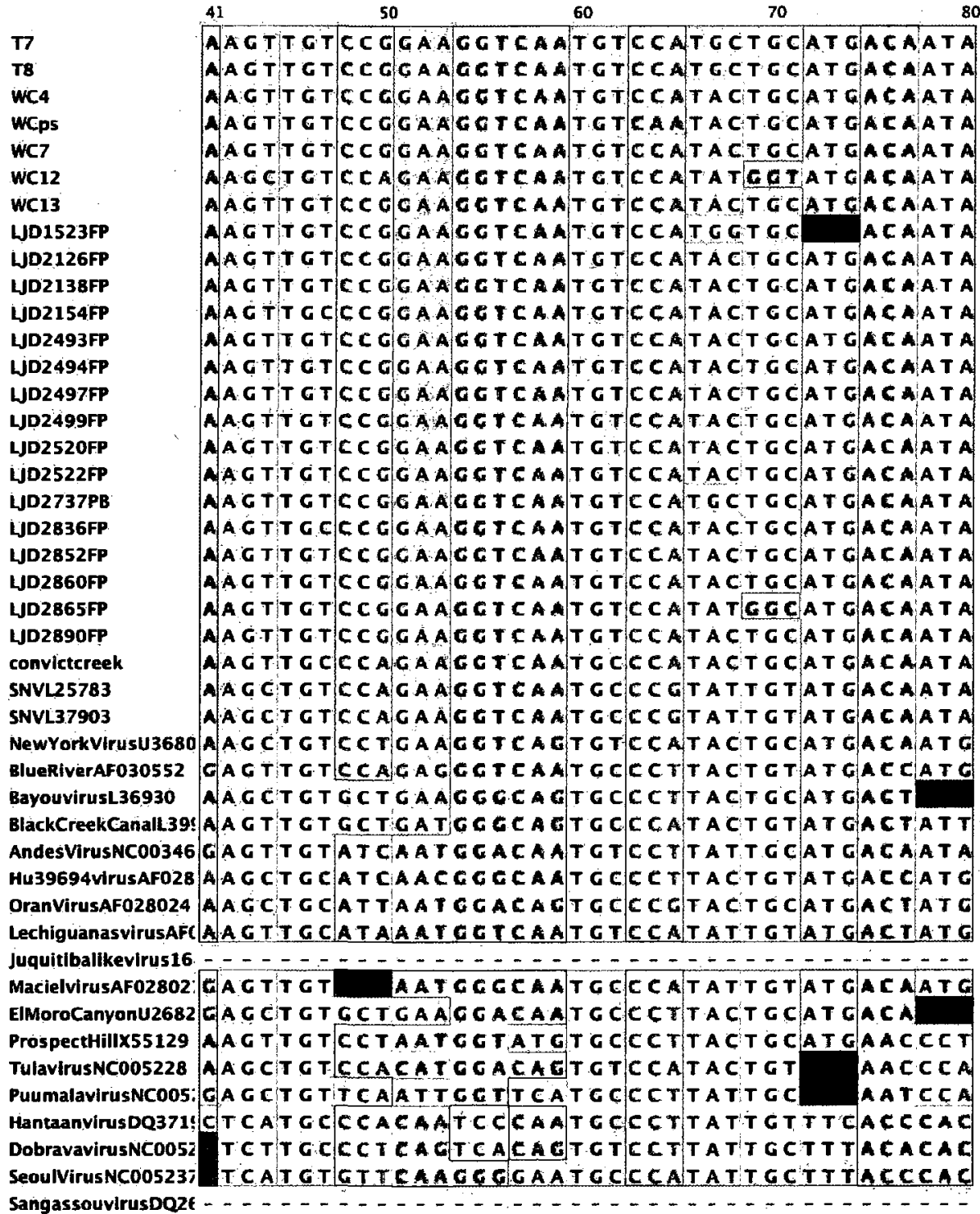




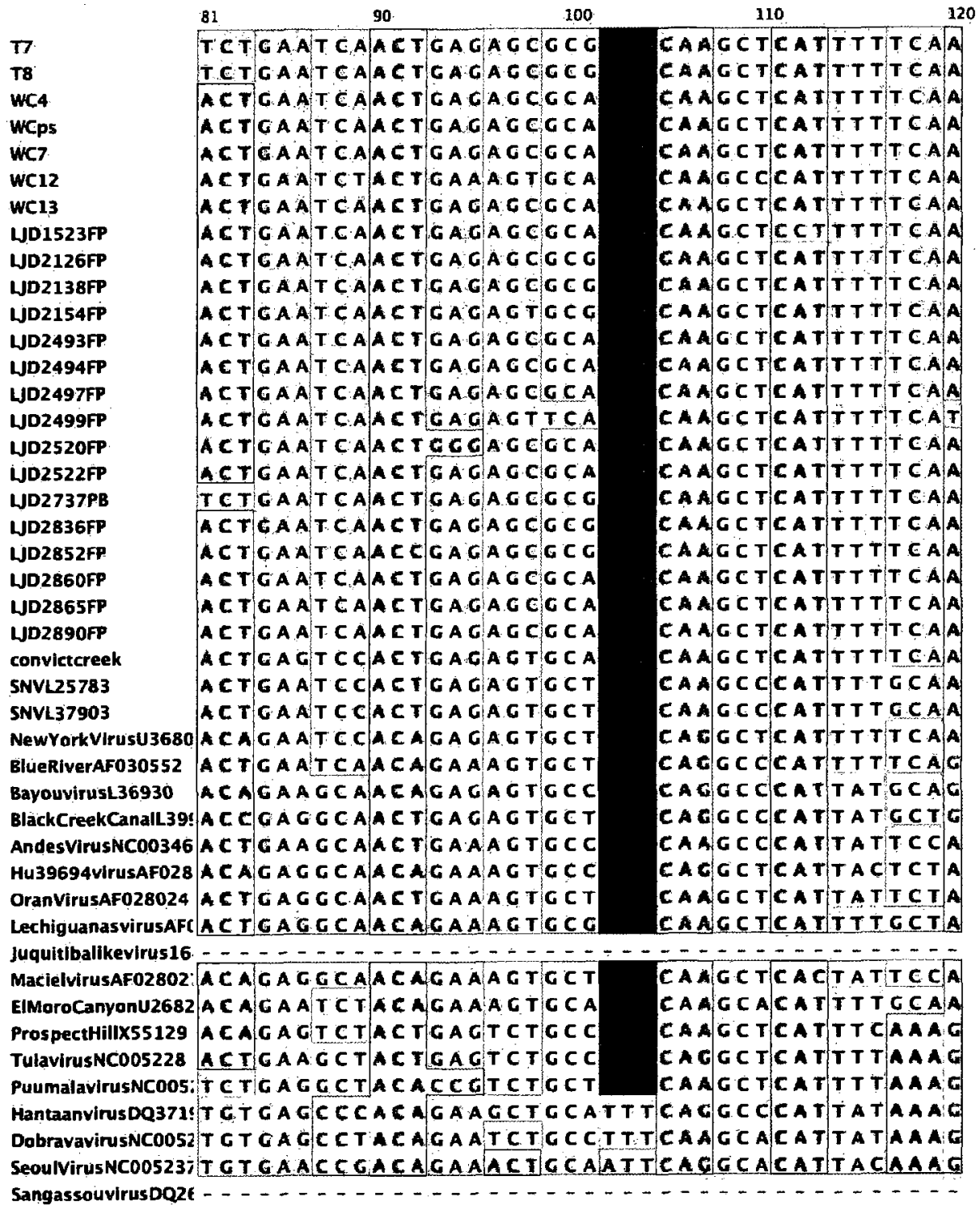




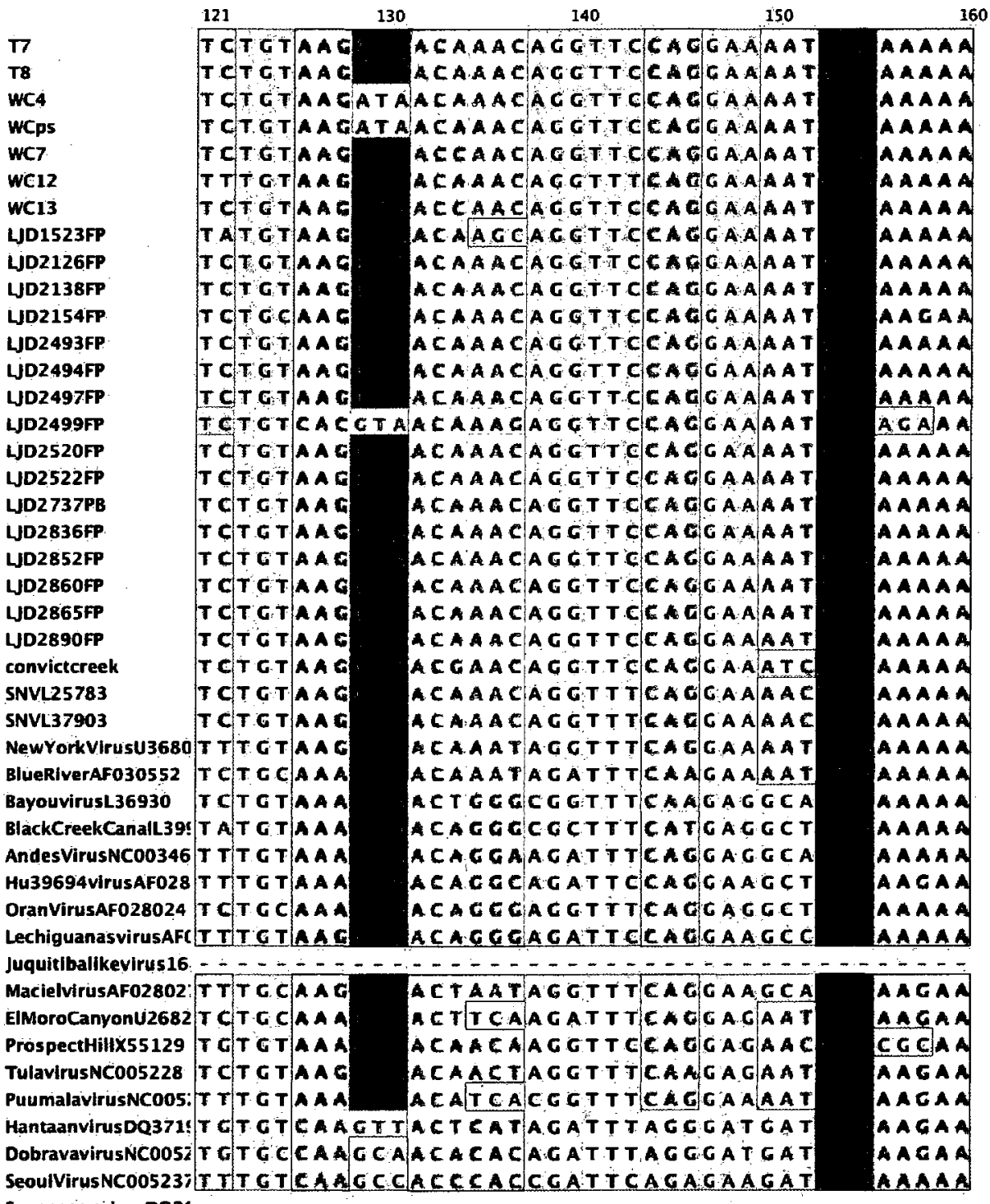

SangassouvlrusDO2t _. $\ldots \ldots \ldots \ldots \ldots \ldots \ldots$ 
17

T8

WC4.

wCps

WC7

wC12

WC13

LJD1523FP

LJO2126FP

LJD2138FP

LJD2154FP

UJD2493FP

LJD2494FP

LJD2497FP

CjD2499FP

LJD2520FP

LJD2522FP

LJD2737PB

LJD2836FP

LJD2852FP

LJD2860FP

LJD2865FP

LJD2890FP

convlctcreek

SNVL25783

SNVL37903

NewYortirusU3680 TC BlueRiverAF030552 A T C C BayouvirusL36930 A T C A BlackCreekCanalL39! A T C A AndesVirus NCO0346 C T C A Hu39694virusAF028 A T C A OranVirusAF028024 A T C A LechiguanasvirusAFt $A$ T C A Juquitibalikevirus16 - T C T MacielvirusAF02802:GT C A ElMoroCanyonU2682 A T C T ProspectHillx55129 C.T C A TulavlrusNCO05228 ATC A PuumalavirusNCO05: C T C A ACATGTATGAGECAATGCAGGGTTGTTATCGA Hantaanvirus DQ371!CACACT TACTECE'CACAACTTCACACCTGCTTGCTATCGA DobravavirusNC005ZAACAATAACACETCAATCAACAAGCCCAGGTTGTTACCGG SeoulvirusNC005237GACTGTGACTCCTEAAAATATTGGGCCTGETTGTTACCGA SangassouvirusdQ2f $\ldots \ldots \ldots \ldots$

AaÁc CCCAGAAGTACGGAAAGGTTGETACAGG AA ACGCCCAGAALTACGGAAAGGTTGCTACAGC

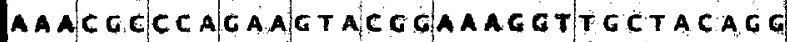
AaACGCCEAGAAGTACGGAAAGGTTGCTACAGG AAAGGCCCAGAAGTACGGAAAGGTTGCTACAGG A A ACGTCCAGAGG TACGGAAAGGTTGCTACAGA AANCGCECAGAAGTACGGAAAGGTTGCTACAGG AAACGCEC AG AAGTAGGGAAAOGTTGETACAGG AAACGCCCAGAAGTACGGAAAGGTTGCTACAGG AAACGCCCACAAGTACGGAAAGETTGCTACAGG AA ACGCCEACAACTACGCAAAGGTTGCTATACG AAACGCCCAGAAGTACGgAAAGGTTGCTACAGG AancGCCEAGAAGTACGGAAACGTTGCTACAGG ATCA AAACGCCCAGAAGTACGGAAACGTTGCTACAGG
A.TCACATCAA CCACAAGTACGCAAAGCTTGCTACAGG ATCA AAACGCCCAGAAGTAGGGAAAGGTTGCTACAGG ATCA AAAEGGCCAGAAGTACGGAAAGGTTGCTACACG ATCA AAACGCCLAGAACTACGCAAAGGTTGCTACAGG

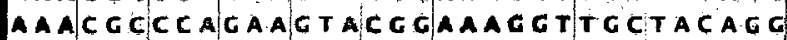
AAACGCGCAGAAGTACGGAAAGGTTGCTACAGG AaACGCECAGAAGTACGGAAAGGTTGCTACAGG AAACGCCCAGAAGTACGGAAAGGTTGCTACAGG

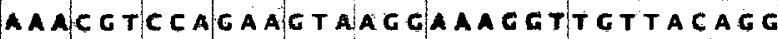
A AACGCCCAGAAGTACG CIAAGGTTGTTACAGG AaACGCCCAGAAGTACGGAAAGGTTGTTACAGG A AACGCECTIGAAGT TIAAACAAGGATGCTATAGA AAGCACCCACAAGT AaAACAGGGCTGTTATAGG AaGAaGCCAGAAGT CAAGAGAGGCTGTTATAGA

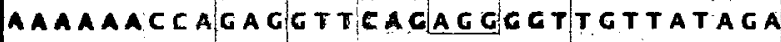
AAAAAGCCAGACGTAAAAAAAGGTTGTTACAGA A A GAGCCACAAGTCAAAAACGGTTGTTATCGT A AAAAACCAGACGTAMAAAGGGGTTGTTACCGT AagaAacta|caAG T CAGAAAGGGCTGTTACCGT A G GAACCAGACATTAAAAGGGGTTGCTATCGC A ACAAACCAGACGT AIAGAAGAGGTTGTTACIAAA AAAAGGCACGATG T AAACCTGGCTGTTACAGA AATCCATATGACGCTAAACGAGGTTGCTATAGC AGTACATATGAGCCTAAACGTGGC TATCGA

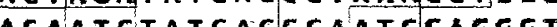




\begin{tabular}{|c|c|c|c|c|c|}
\hline & 201 & 210 & & 230 & \\
\hline $\mathbf{T 7}$ & & GGAGTTTTCEGGTAC & A A A ACT & AGGTGTTAT & CT T G \\
\hline$\Gamma 8$ & & CEACTTTTECGCTACD & A A A A A T T & TACGTGTTAT & crta \\
\hline WC4 & & $\mid G G A C T T T T C C G G T A C D$ & $A A A \mid A G T$ & AG GTGT TAT & C T T C \\
\hline wCps & $A C$ & G GAGTT|TTECGGTACS & A A A A G T & AGGTGTTAT & G T T G \\
\hline wc7 & & GGAGTTTTCCGTACH & A A A A C T & AGGT GT TAT & CTTC \\
\hline wC12 & & GEGGTTTTCCGGTATA & A A A.A.GT & A G G T T TAT & CrTt \\
\hline wC13 & & CGAGTTTTCCGGTACA & A A A A G T & A G G T GT TAT & E T T C \\
\hline LJD1523FP & A A & CGAGTTTTCGGTACA & A A AAGT & A G GT GT TAT & С T T G \\
\hline LJO2126FP & $A C A$ & GGAGTTTTCCGTACA & A AAAGT & A G GT GT TAT & GTT'G \\
\hline LJD2 138FP & $A C A$ & GGAGTTTTCCGGTACD & A A A A GT & A G GT GT T A T & G T $\mathbf{C}$ \\
\hline LJO2 $154 F P$ & AC: & G GAGTTTTCCGGTATA & A ACA CT T & A G GT GT TAT & G T T $C$ \\
\hline LJD2493FP & & GGAGTTTCEGGTACA & A A AAGT & AGCTCTTAT & C IT \\
\hline LJD2494FP & & CGAGTTTTCCGGTACA & AAAACT & A G GT GT T A T & G Tre \\
\hline LJD2497FP & & CGAGTVTTCCGGTACA & AAAACT & A G GT GT T AT & CT TO \\
\hline LJD2499FP & & CGAG TTTTCCGGTACA & AAAACT & A G GT GT TAT & c T r t \\
\hline LJD2520FP & & CGAGTITCCGGTACA & A A A A CT & A G GT G T TAT & C T T G \\
\hline LJD2522FP & & CGAGTTTTCCGGTACA & $A A A A G T$ & A G GT GT TAT & \\
\hline LJD2737PB & & CGAGTTTTCCGETACA & $A \wedge A$ A G T & A G GT G T T A T & \\
\hline LJD2836FP. & & GGAGTTTTCCGGTACA & A A A A GT & A G G T G T A T & \\
\hline LJD2852FP & & GGAGTTTTCCGGTACA & A A A ACT & AC GT GT T A T & GTTC \\
\hline LJD2860FP & & GGAGTTTTCCGGTACA & $\triangle A A A T$ & A G GTGTTAT & C T r c \\
\hline LJO2865FP & & GGAGTTTTCEGGTACA & $\operatorname{AAACT}$ & A G GT G T TAT & G T T \\
\hline LJD2890FP & & GGAGTTTTCCGGTACA & $A \cap A A C T$ & ACGTCTTAT & GT T \\
\hline convictcreek & & GGACTATTCCGCTACA & $A A G A C C$ & ACCTCCTAT & CTTG \\
\hline SNVL25783 & & GGAGTTTTTAGATACS & $\triangle A G A G C$ & AGATCTTAT & G T T \\
\hline SNVL37903 & & CGAGTTTTTAGATACD & A A C A G C & AGATGTTAT & CT T G \\
\hline NewYarkVirusU3680 & & G ETGTTTTTAGATATA & $A \wedge C A C T$ & AC CT GTT T AT & СT T C \\
\hline BlueRiverAF030552 & & CGACTATTTACGTATA & AAAACT & ACATCTTAT & CTAC \\
\hline BayouvirusL36930 & & CGTGTTTTTCGTTACA & AAGAGT & AG CT GTTAT & G rcd \\
\hline BlackCreekCanalL39! & & GGTGTTTTCCGTTATA & AAGAGT & CGTTGCTAT & CTGC \\
\hline AndesVirusNCO0346 & & GGGGTATTTAGATATA & A A A A G T & AGATGTTAT & GTG \\
\hline Hu39694virusAF028 & & GEGGTATTTAGGTACA & $A \wedge C \mid A C T$ & AGATGCTAT & CTT \\
\hline OranVirusAF028024 & & CGTGTATTTAGATACA & $A A C A G T$ & AGATGTTAT & \\
\hline echiguanasvirusAFC & & GGACTTTTTAGATACA & A A C A C C & AGGTCCT & \\
\hline uquitibalikevirus 16. & & CET GTTTTTAGATATA & A A A A C T & AGATGTTAT & \\
\hline lacielvirusAF02802: & & CGTGTGTTTAGATAC|A & A A A A G T & AC ATTCTAT & \\
\hline IMoroCanyonU2682 & & CGTGTATTTAGATAC:A & A ACAACC & C G TT G TTAT & \\
\hline ProspectHillX55 129 & & TCTGTGTTTAGATATC & CGCACT & AGGTGCTT T & G T G \\
\hline Tulavirus NC005228 & & TCCATGTTTAGATACL & $\triangle \wedge \wedge A C C$ & AAATGCTAT & G T G \\
\hline PuumalavirusNCOO5: & & TCT TTTAGGTACO & CGCAGT & A G A T T T T T T & G T A.C \\
\hline HantaanvirusDQ371! & & TTCACATATA & AACACT & A G GT G T TAT & A T T \\
\hline obravavirusNCOO5z & & TT TAGGTATA & A A A A C T & A G AT G T TAC & ATC \\
\hline oulVirusNCOOS23i & & TTTACGTATA & $\triangle A A A C T$ & AGGTCTTA & AT T \\
\hline
\end{tabular}

SangassouvirusDO2E - 


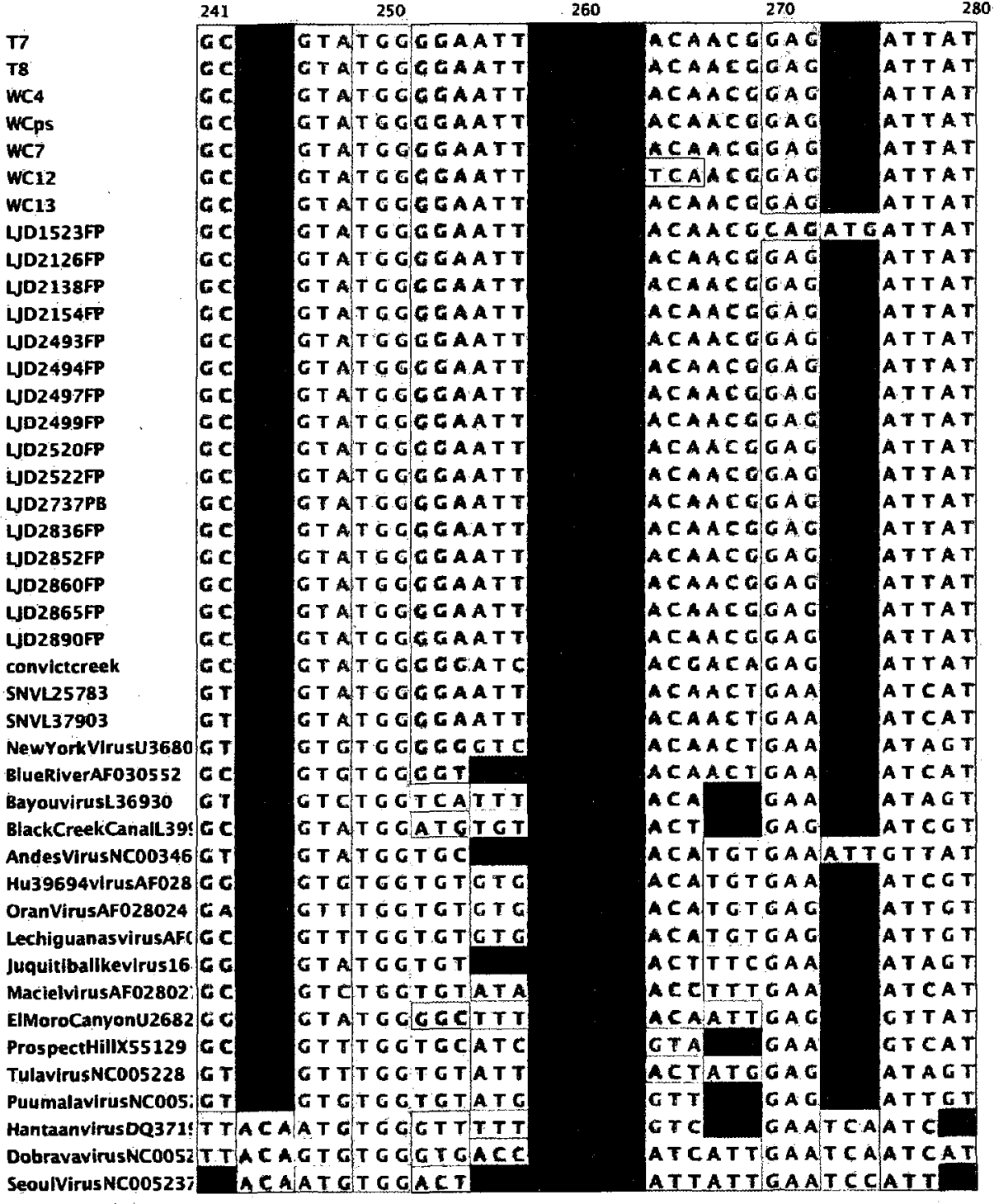

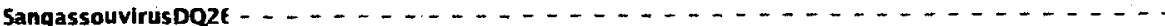




\begin{tabular}{|c|c|c|}
\hline & 290 & 310 \\
\hline T7 & TGGGCCGCCAGTGCCGAA & $-\ldots \ldots \ldots-\cdots, \ldots$ \\
\hline T8 & ATG G G C CCCAGTGCCGAA & $\ldots-\cdots,-\cdots, \cdots,-\cdots$ \\
\hline wC4 & AT G G GCCGCCAGTGCCGAA: & $\ldots-\cdots+\cdots, \cdots, \cdots$ \\
\hline WCps & AT G G C C GCCACTGCC G A A & $\ldots \ldots-\cdots \cdots \cdots$ \\
\hline WC7 & A T G G A A GCCAGTGCCGAA & $\ldots-\cdots-\cdots, \ldots-\cdots$ \\
\hline wC12 & AT G G G C G G C C A G T G C C G A A & $\ldots \ldots \ldots \ldots \ldots \ldots$ \\
\hline wC13 & AST G GACGCCAGTGCCGAA & $-\cdots-\cdots,-\cdots,-\cdots$ \\
\hline LJD1523FP & AT G G C C G C C A G T G C C G A A & $\ldots \ldots \ldots \ldots \ldots$ \\
\hline LJD2126FP & $A T G G G C C G C C A G T G C C G A A$ & $\cdots-\cdots \cdots \cdots-\cdots, \cdots$ \\
\hline LJD2138FP & AT G G GCCGCCAGTGCCGAA & $\rightarrow-\cdots-\cdots-\cdots-\cdots-\cdots$ \\
\hline LJD2 154FP & AT C G C C G C A A T G C & - CAAGATGTGCTATGCCGCCT \\
\hline UJD2493FP & $A T G G G C C G C C A C T G C C G A A$ & $--\infty-\cdots--\infty-\cdots$ \\
\hline UJD2494FP & ATGGCCCGCCAGTGCCGAA & $-\cdots-\cdots,-\cdots$ \\
\hline WD2497FP & AT TGGCCGCCACTCCCGAA & $\cdots \cdots \cdots-\cdots \cdots$ \\
\hline WJD2499FP & AT T G GCCGCCAGTGCC & $\cdots-\cdots \cdots \cdots$ \\
\hline UDD2520FP & A T G G G C C G C C A G T G C C G A A & $\ldots-\cdots-\ldots, \ldots-\cdots$ \\
\hline LلJD2522FP & ATGG GCCGCCAGTGCCGAA & $-\cdots-\cdots-\cdots-\cdots-\cdots$ \\
\hline LJD2737PB & AT G G G C C G C C A G T G C C G & $\ldots-\cdots \cdots \cdots-\cdots$ \\
\hline UJD2836FP & ATG TGCCGCCAGTGCCGA A & $\cdots-\cdots \cdots \cdots-\cdots$ \\
\hline UJD2852FP & AT G G G C C G C C A G TC C C G A A & -- \\
\hline LJD2860FP & ATGGGCCGCCAGTGCCGAA & $-\cdots, \cdots-\cdots, \cdots,-\cdots$ \\
\hline LJD2865FP & A T G G G C C G C C A G T G C C C & $\cdots---\cdots---\cdots-\cdots$ \\
\hline UJD2890FP & AT G G G C G C A A T GCCGAA & $\cdots \cdots-\cdots \cdots-\cdots$ \\
\hline convictcreek & 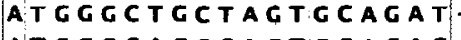 & $-\ldots-\cdots$ \\
\hline SNVLZ5783 & AT G G G C A G C C A G T G C A & $-\cdots-\cdots-\cdots-\cdots-\cdots$ \\
\hline SNVL37903 & AT G G G C A GCCAGTCC & $\ldots--\cdots-\cdots,-\cdots,-\cdots$ \\
\hline NewYorkVIrusu3680 & TTG G GCA GCT A T TCCT GAC) & $\ldots-\cdots-\cdots$ \\
\hline BlueRiverAf030552 & CTGGGCAGCTAGTGCAGAA. & $-\cdots-\cdots,-\cdots,-\cdots$ \\
\hline BayouvirusL36930 & CT G C G T G C C A G TGCT G AC & 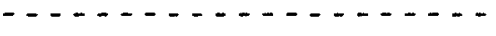 \\
\hline BlackCreekCanalL39! & T: T G G G C T G C T A G T C & $-\cdots--\cdots,--\cdots,-\cdots$ \\
\hline AndesVlrusNC00346 & T T G GGCCGCAAGTGCAGAG & $-\cdots--\cdots-\cdots-\cdots-\cdots$ \\
\hline HuJ9694virusAF028 & AT C G G C T G C T A C T G C A G AG & $---\ldots-\cdots-\cdots-\cdots$ \\
\hline OranVirusAF028024 & CTGGGCAGCTAGTGCGGAA. & $-\cdots-\cdots-\cdots-\cdots-\cdots$ \\
\hline LechiguanasvirusAFC & A T G G G C T G C T A G T C C & 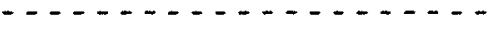 \\
\hline Juquitlbalikevirus16 & T T G G G AGCAAGT G & $\cdots-\ldots \ldots \ldots-\cdots$ \\
\hline MacielvirusAF02802: & TIT G G G C TG T GA G T G C A GAA & $\cdots-\cdots+\cdots+\cdots+\cdots$ \\
\hline ElMoroCanyonU2682 & TTGGGCAGCAAGTGCAGAT & 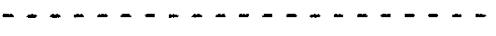 \\
\hline ProspectHillX55129 & ATGG GCTGCAAGTGCGGAT. & $-\cdots---\cdots--\cdots-\cdots$ \\
\hline TulavirusNC005228 & G T G G G C A G C T A G T G C T G A G & --------- \\
\hline uumalavirusNC005: & A T G C G C T G C T A G T G C T G A G & $---\cdots---$ \\
\hline HantaanvirusDQ37 1! & TGGGCAGCCAGTGCATC & $-\cdots-1$ \\
\hline $\begin{array}{l}\text { DobravavirusNC005i } \\
\text { SeoulVirusNCO05237 }\end{array}$ & $\begin{array}{l}\text { GT GGGCAGCTAGTGCATC } \\
\text { TGGGCAGCAAGTGCACC }\end{array}$ & 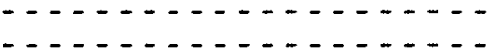 \\
\hline 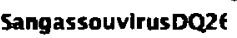 & 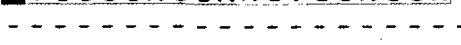 & $\ldots \ldots$ \\
\hline
\end{tabular}


321
17

78

WC4

WCps

WC7

WC12

WC13

LJD1523FP

LJD2126FP

LJD2138FP

LJD2154.FP

LJD2493FP

LJD2494FP

LJD2497FP

LJD2499FP

LJD2520FP

LJD2522FP

LJD2737PB

LJD2836FP

LJD2852FP

LJD2860FP

LJD2865FP

LJD2890FP

convictcireek

SNVL25783

SNVL37903
330

340

350

360

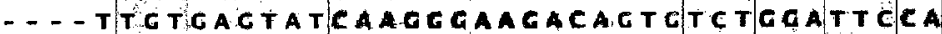

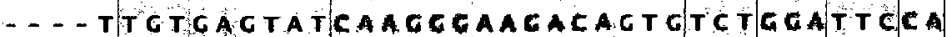

- - TTGTGAGTATCAAGgGaAGACÁtGTCTGGATTECA - - - TTGTGAGTATCAAGGGAAGACAGTGTCTGGATTCCA - - TtG TGAGTATCAACGgaAgacagtGTCTGgatTCCA - - TTG TGAGTATCAAGGgAAGARACTGTCTGGATTCEA -... TTG TGAGTATCAAGGGAAGACAGTGTCTGGATTCEA -.. TTGTGAGTATCAAGGGAAGACAGTGTCTGGATTCCA - TTGTGAGTATCAAGGGAAGACACTCTCTGGATTCCA CCGTTTGTCAGTATCAAGCGAAGACACTATCTGGATTECA -.. TTG TCAGTATCAAGGGAAGACACTGTCTGGATTCCA - - TTGTGAGTATCAACGCAAGACAGTGTCTGGATTECA -.. TTGTGAGTATEAAGGGAAGACAGTGTCTGGATTCEA -. - tTGTGACTATCAAGGGAACACAGTGTCTGGATtCEA - - - TTGTGAGTATCAAGGGAAGACAGTGTCTGGATTCEA - - TTGTGAGTATEAAGGGAAGACAGTGTCTGGATTECA -.. TTGTGAGTATCAAGGGAAGACAGTCTCTGGATTCCA -. - TTGTGAGTATCAAGGGAACACAGTCTCTCOATTCCA -.. TTG TCAGTATCAAGGGAAGACACTGTCTGGATTCCA -.. TTGTGAGTATCAAGGGAAGACACTGTCTGGATTCCA - - - TTGTG TAGTATCAAGgQAaGACAGTGTCTGGATTCCA - - TTGTGAGTATCAAGGGAAGACAGTGTCTGCATTCCA -.. ATGTGAATATCAAGCTAATACACTCITCTGCATTCCA - - ATGTGAGTATCAAGGCAACACAGTGTCTGGATTCCA

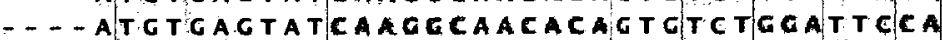
NewYorkVIrusU3680-... CTT GTCAGTATEAAGGTAATACTATCTCGGGATTTCA BlueRIVEAAF030552 -.. - TTG CGAATATCAGCCCAATACAATTTCACGATTTAA BayouvirusL36930 - - - CTG TGAGTATCAAGCCAATACTGTCTCTGGATTTAA BlackCreekCanalL39! - - - TT GTGAGTATCAAGGCAATACAGTCTCTGGTTTTAA AndesVirusNC00346 - - - TTG TCAATATCAAGCAAATACCATTTCTGGATATAA Hu39694virusAF028 - - - TTGTGAGTACCAAGGGAACACAAT CTCACGATATAA OranVirusAF028024 -... A T GTCAGTATCAACGGAATACCATCTCAGGCTATAA LechiguanasvirusAFT - . - ATGTGAGTACCAAGGTAACACTATATCTCGATACAA Juquitibalikevirus16. - - AT G TGAGTACEAAGGCAATACTGTGTCAGGETATAA MacielvirusAF02802: -... ATC CGAGTATCAAGGCAATACTGTCTCTGCCTATAA EIMoroCanyonU2682 - - - TT GTGACTATCAACG GAATACAGTTTCACGATATCA ProspectHillX55129 - - - ATGTGAGTATGATGGAAACACT TCTGGTTACEA TulavirusNC005228 - - - AT GTCAGTATGATGGAAACACTGTATCTGGATATCA PuUmalavirusNCOO5: - - - ATGTCAGTTTGATGGGAATACAATTTCAGGTTATAA HantaanvirusDQ371! - - - TTGTGAATÁTCATGCCAATATCETTTCAGGTTATAA DobravavirusNCO05:-..- GT G GGAGTATGATGGTAATATGCTCTCTGEGTATAA SeoulVirusNC00523i-... TT GTGAATATGATGGGAATATTATCTCAGCETATAA SangassouvirusDQ2f - - GTGTGAGTACT CACGTAATATGCTATCTGGTTATAA 


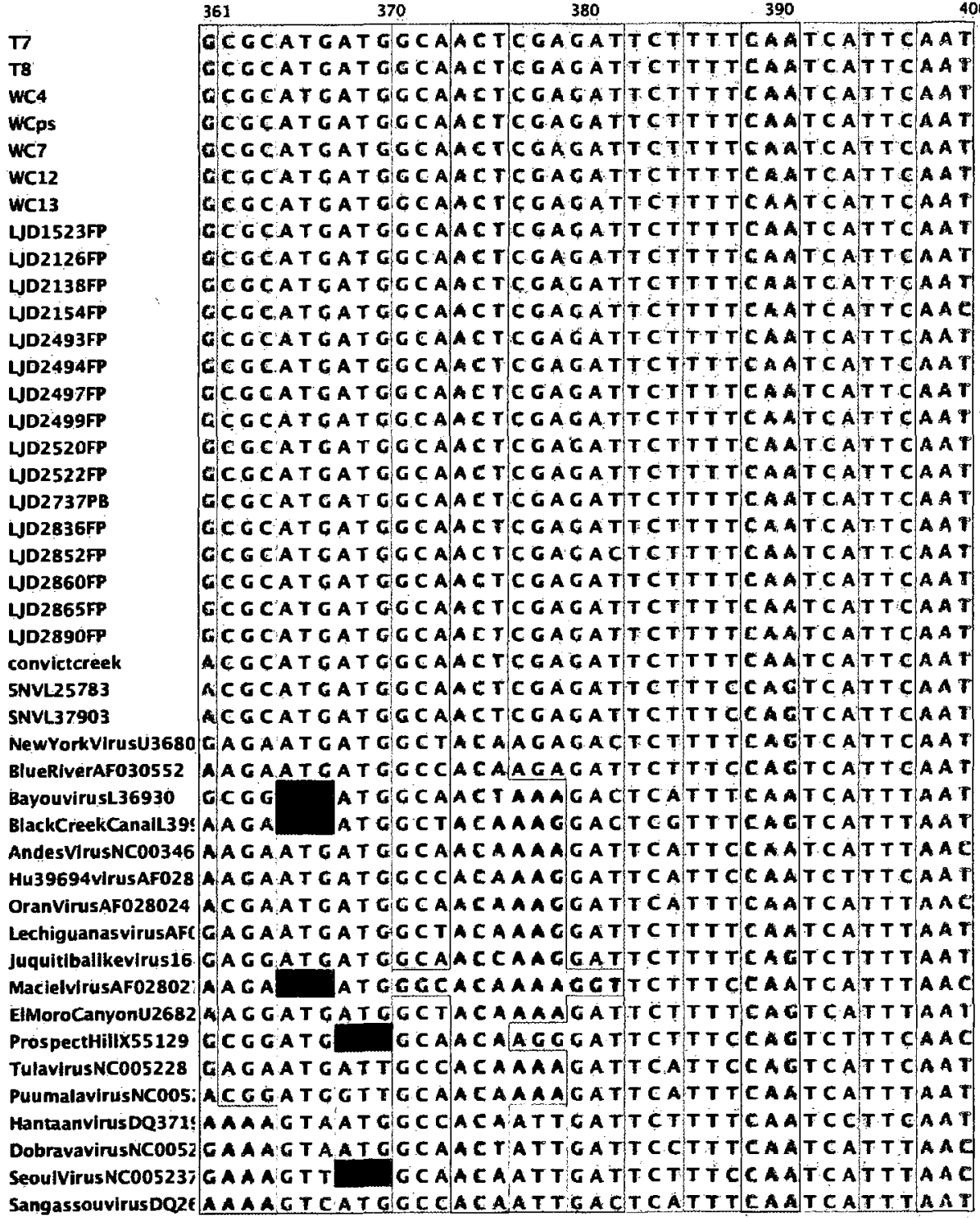




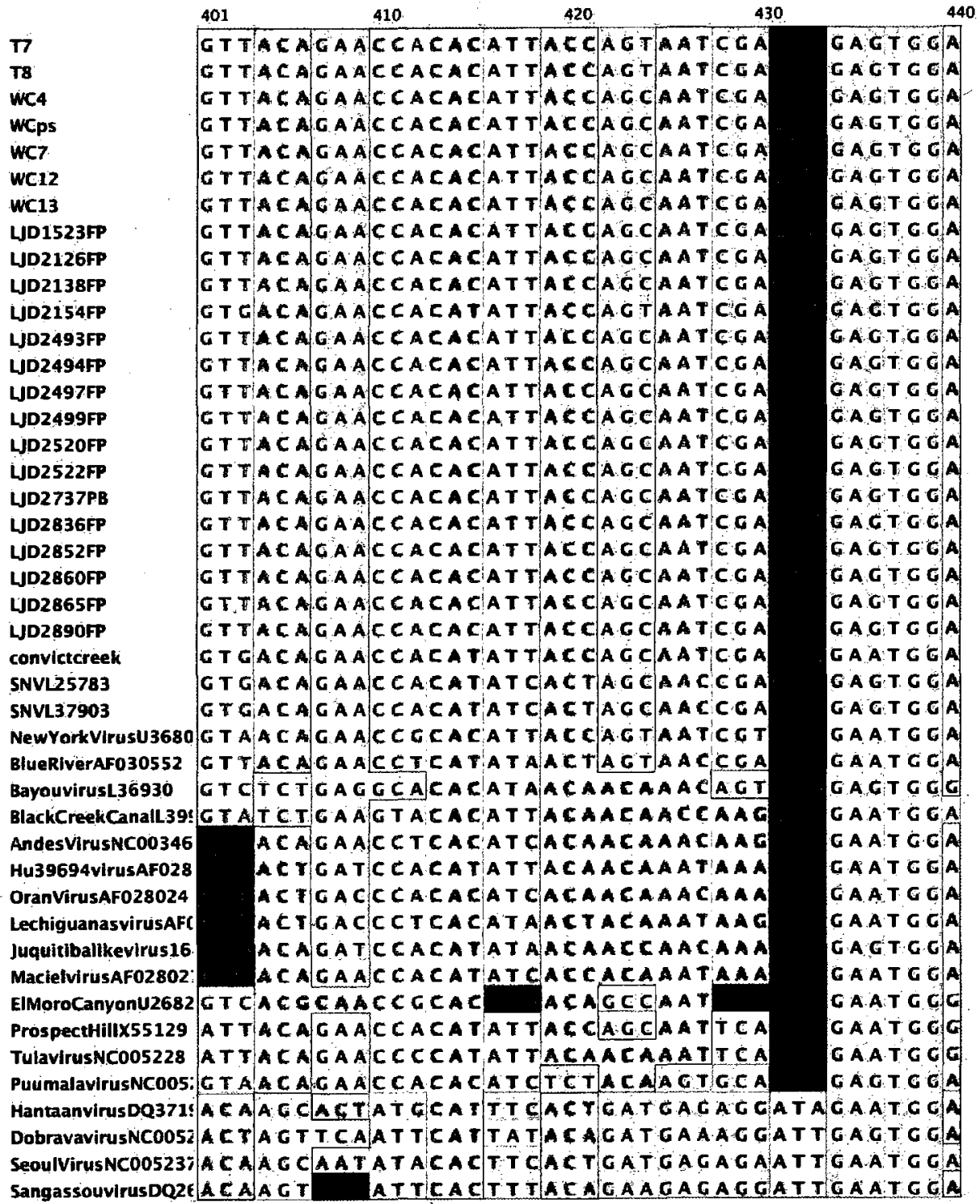




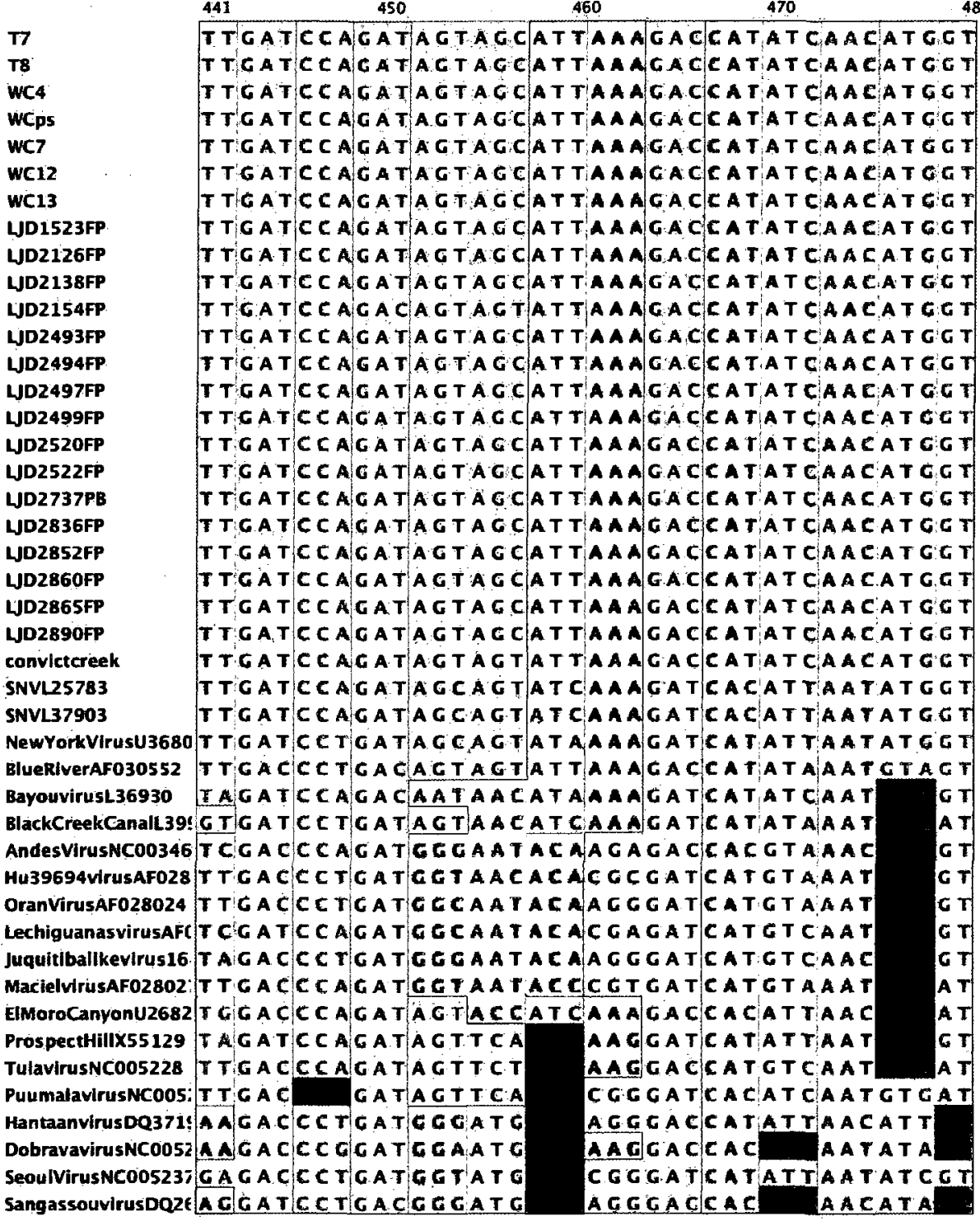




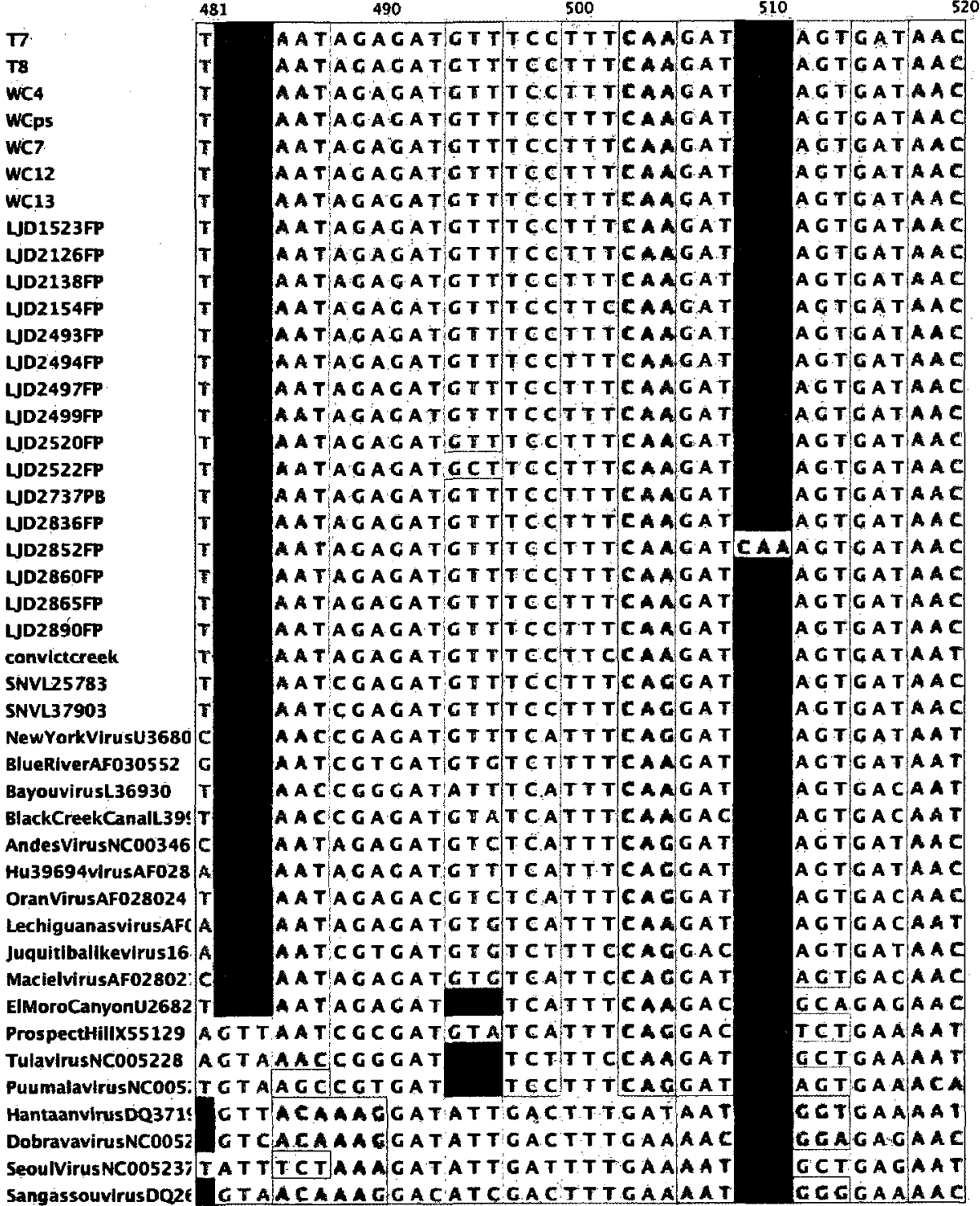




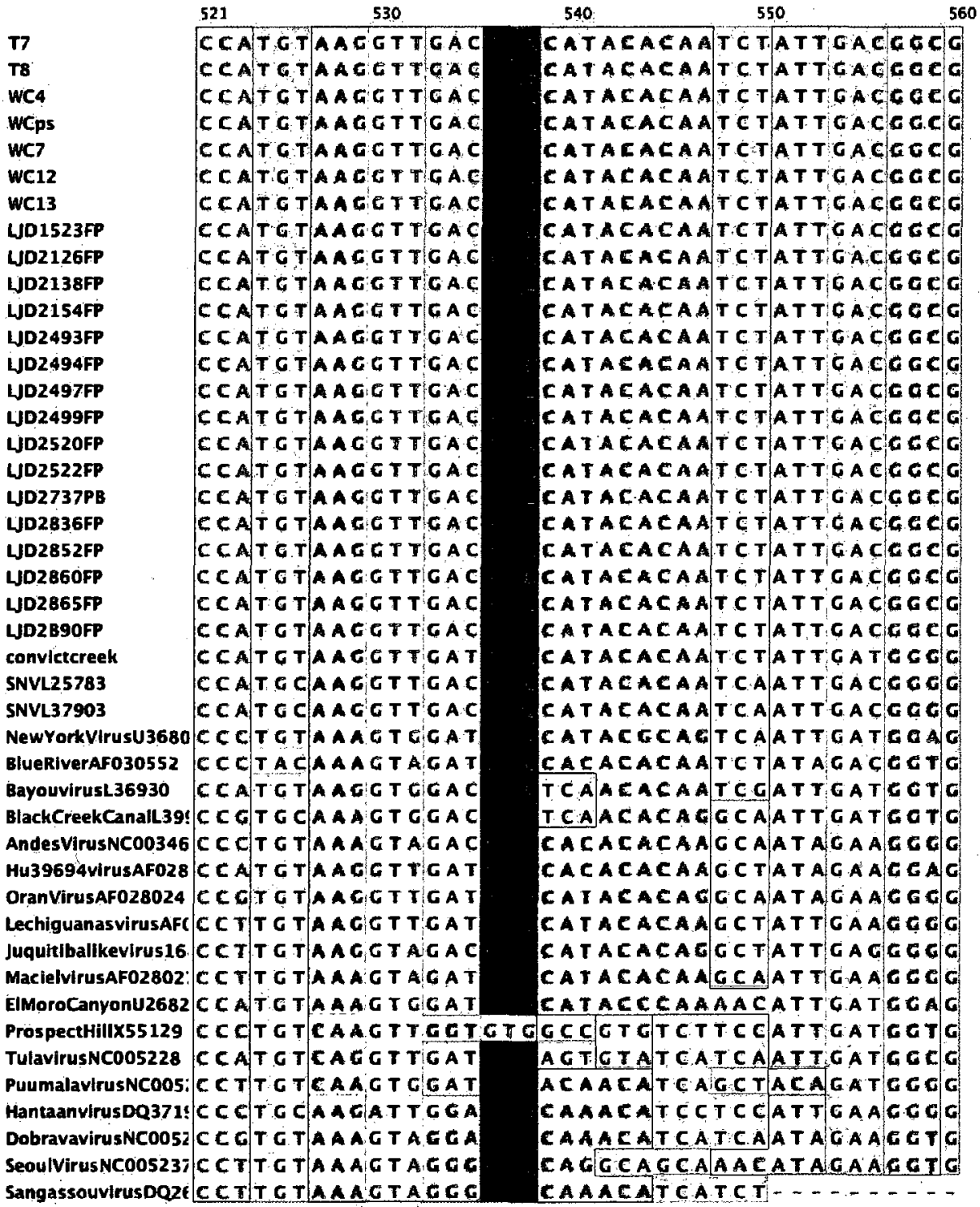




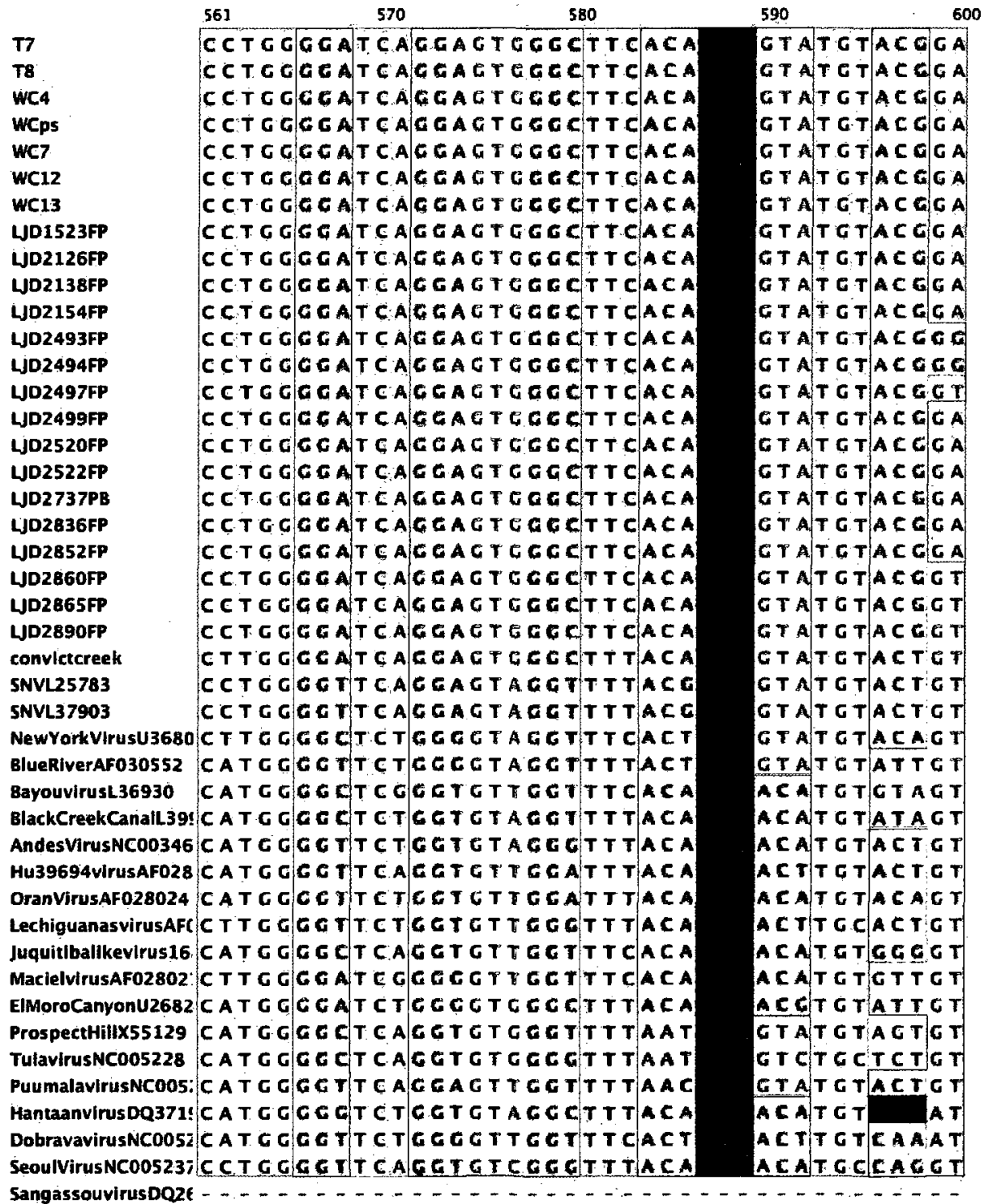




\begin{tabular}{|c|c|c|}
\hline & 601 & 610 \\
\hline$T 7$ & GGCG & ATAGCACAT \\
\hline $\mathrm{TB}$ & G G C C & ATAGCACAT \\
\hline wC4 & $\operatorname{CGCC}$ & ATAGCACA \\
\hline Weps & $\operatorname{coc}$ & ATA C C A|CAT \\
\hline wC7 & G G C G & ATACCACAT \\
\hline wC12 & $\operatorname{coc} G$ & ATAGCA|CAT \\
\hline wC13 & C G C C & ATACCACAT \\
\hline LJD1523FP & GCCT & ATCGCACAT) \\
\hline LJD2126FP. & G G C T & ATCGCACAT) \\
\hline LJD2138FP & C G C G & $\mid$ A T A $\mid$ G C A CA T $\mid$ \\
\hline LJD2154FP & G G G & AT AIGCACAT \\
\hline LJD2493FP & C C C & A T AIC C ACATC \\
\hline LJD2494FP & C G C G & ATACCACATC \\
\hline LD2497FP & EGCT & A T AIG C ACATC \\
\hline LD2499FP & G G C C & (A T A|GCACAT) \\
\hline LJD2520FP & $\operatorname{coc} 0$ & A T A|C C A|CAT \\
\hline LJD2522FP & G G C G & $\mid$ AT A|C C A|CAT \\
\hline LJD2737PB & $G \in \subset G$ & A T A|C CA|CATC \\
\hline LDD2836FP & G G C G & A T A|C C A|CAT \\
\hline LJD2852FP & $\operatorname{coc} 0$ & A T A|C C A C A T \\
\hline LJD2860FP & 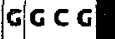 & A T A GCA|C AT \\
\hline LD2865FP & $\mathbf{c} \mathbf{G} \mathbf{c} \mathbf{G}$ & A T ACCACATC \\
\hline LJD2890FP & $c \in c G$ & ATACCACATIC \\
\hline convictcreek & $A G C T$ & ACAGAGTGTO \\
\hline 5NVL25783 & GGG & ACA A AGT GT \\
\hline SNVL.37903 & G G G C & ACAGGACT TO \\
\hline NewYorkVIrusU3680 & GGeA & ACTGAATGTO \\
\hline BlueRiverAF030552 & G G G T & $A \subset A G A C T C T C$ \\
\hline BayouvirusL36930 & Toc C & ACAIGAATGCO \\
\hline BlackCreekCanall 39! & $\operatorname{GGGA}$ & ACAGA AT G \\
\hline AndesVIrusNC00346 & CGCA & ACACAGTCCO \\
\hline Hu39694virusAF028 & $\mid c \in A$ & ACTCAGTGCOC \\
\hline OranVirusaf028024 & $r \in C T$ & ACTCACTCT \\
\hline LechiguanasvirusAFt & $r \in 0 t$ & ACTGAATGCC \\
\hline Juquitibalikevirus16 & $T \in G$ & ACAGAATGCO \\
\hline MacielvirusAF02802 & $\operatorname{cect}$ & ACAGAATCCO \\
\hline ElMoroCanyonU2682 & $A \widehat{A G C}$ & $A \subset A G A A T G T A$ \\
\hline ProspectHillx55129 & CA G T & AEAGA AT GTC \\
\hline TulavirusNCO05228 & CA G C & ACAGA ATGTC \\
\hline PuumalavirusNCOOS: & T/AGT & ACAGAATG CT \\
\hline Hantaanvirus DQ371! & ATTCA & ACACA ATGC \\
\hline Dobravavirus $\mathrm{NCO} 52$ & $C T \subset A$ & ACAGAGTGT \\
\hline SeoulVirusNC00523i & $\mathrm{GTCT}$ & ACAGAATGC \\
\hline
\end{tabular}

